University of Nebraska - Lincoln

DigitalCommons@University of Nebraska - Lincoln

Faculty Publications from the Harold W. Manter Laboratory of Parasitology

$12-1983$

\title{
A Checklist of Parasites of California, Oregon, and Washington Marine and Estuarine Fishes
}

\author{
Milton S. Love \\ University of California - Santa Barbara, love@lifesci.ucsb.edu \\ Mike Moser \\ University of California - Berkeley, mmoser@berkeley.edu
}

Follow this and additional works at: https://digitalcommons.unl.edu/parasitologyfacpubs

Part of the Aquaculture and Fisheries Commons, Biodiversity Commons, Marine Biology Commons, Other Ecology and Evolutionary Biology Commons, and the Parasitology Commons

Love, Milton S. and Moser, Mike, "A Checklist of Parasites of California, Oregon, and Washington Marine and Estuarine Fishes" (1983). Faculty Publications from the Harold W. Manter Laboratory of Parasitology. 750.

https://digitalcommons.unl.edu/parasitologyfacpubs/750

This Article is brought to you for free and open access by the Parasitology, Harold W. Manter Laboratory of at DigitalCommons@University of Nebraska - Lincoln. It has been accepted for inclusion in Faculty Publications from the Harold W. Manter Laboratory of Parasitology by an authorized administrator of DigitalCommons@University of Nebraska - Lincoln. 
The National Marine Fisheries Service (NMFS) does not approve, recommend or endorse any proprietary product or proprietary material mentioned in this publication. No reference shall be made to NMFS, or to this publication furnished by NMFS, in any advertising or sales promotion which would indicate or imply that NMFS approves, recommends or endorses any proprietary product or proprietary material mentioned herein, or which has as its purpose an intent to cause directly or indirectly the advertised product to be used or purchased because of this NMFS publication. 


\section{CONTENTS}

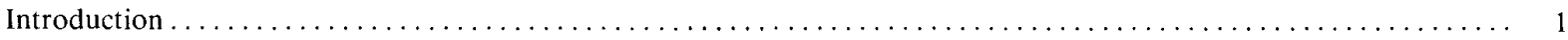

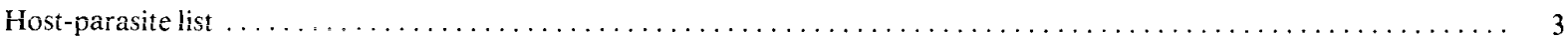

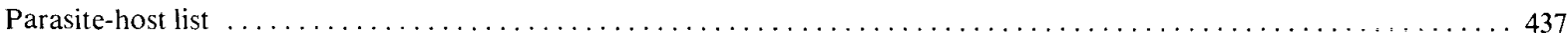

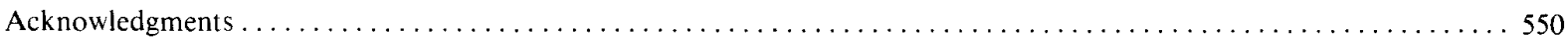

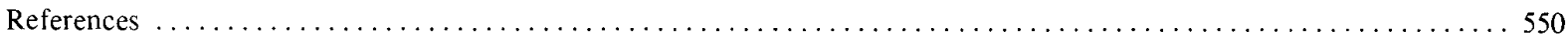

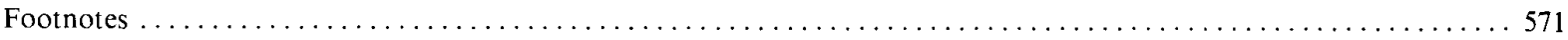

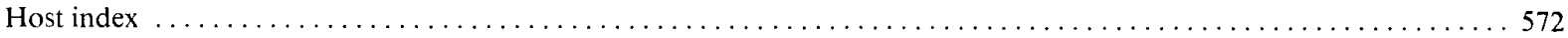




\title{
A Checklist of Parasites of California, Oregon, and Washington Marine and Estuarine Fishes
}

\author{
MILTON S. LOVE' and MIKE MOSER ${ }^{2}$
}

\begin{abstract}
This report is a summary of the published records of parasites found from the marine and estuarine fishes of California, Oregon, and Washington. Coverage has not been limited to the western United States, but rather includes parasite reports from throughout each fish's range. Included is a host-parasite list and parasite-host crossindex.
\end{abstract}

\section{INTRODUCTION}

We have produced this survey because we felt there was a need for a single source which would list all the parasites infecting marine and estuarine fish from California, Oregon, and Washington. Pertinent sources are scattered over a number of journals, covering many years. Workers find it time consuming and laborious to search through the literature. Moreover, it is a task that many workers in the field have to duplicate. We hope this paper will shorten the job considerably.

We use "marine and estuarine" in a broad sense and include anadromous species in this survey. We have also included records from landlocked populations of anadromous fish, such as Salmo gairdneri, the rainbow trout, and $S$. clarki, the cutthroat trout. Though this survey (which encompasses works up to and including mid-1978) covers marine and estuarine fishes of California, Oregon, and Washington, we have included citations of parasites recorded from individual fish outside of this range, if the fish species has been reported from within it. Our reasoning is that many parasites found in hosts outside the California-Washington boundaries have subsequently been found to infect the same host species within the boundaries. We have not included all citations of a particular parasite from a particular host, as this is beyond the scope of our work.

The taxonomic status of various parasites is under debate in numerous cases. In listing these parasites we have, out of necessity, chosen one side of an argument over others. We make no pretense of being experts in the field of systematics and taxonomy. Many of the choices we made, however, were based on discussions with individuals more qualified than ourselves. No matter what choices were made, we realize there will be those who disagree. We are always open to suggestions on changes and corrections, and we hope they will be forthcoming. Both host names and their order in this publication follow Bailey et al. (1970).

We have included some unpublished data, primarily in the form of personal communications. We recognize that some workers believe that the possibility of errors in these circuinstances is higher than in published works. It is our feeling that

'Department of Biology, Occidental College, 1600 Campus Road, Los Angeles, CA 90041.

${ }^{2}$ Long Marine Laboratory, University of California, Santa Cruz, CA 95064. workers using this list are capable of exercising their discretion as to which citation to accept or reject.

An example of the format for each citation is shown below, followed by an explanation of usages.

\section{Squalus acanthias Spiny Dogfish}

CESTODA

\section{LACISTORHYNCHIDAE}

Lacistorhynchus tenuis (Van Beneden 1858) Dollfus 1929.

France. Joyeux and Baer (1936); Massachusetts. Spiral valve. Linton (1924) as Rhynchobothrium bulbifer.

Squalus acanthias--Host.

CESTODA-We have generally used either classes or subclasses for the initial heading. We have tried to use headings commonly used and familiar to most workers.

LACISTORHYNCHIDAE-Generally families are used below classes or subclasses.

Lacistorhynchus tenuis (Van Beneden 1858) Dollfus 1929. Genus and species are given as in the cited paper, along with the original describer(s) and redescriber(s). Within each family, genera and species are not listed alphabetically.

France-If available, the geographic location of the host is given. Joyeux and Baer (1936)-This is the citation and will be found in the References section.

Massachusetts-If the parasite has been found from the same host in more than one geographic location, we often give multiple listings. We have not, as a rule, listed every citation.

Spiral valve-This is the site of infection within the host. In this instance, the initial citation (Joyeux and Baer 1936) did not mention site of infection.

Rhynchobothrium bulbifer-If the source listed used a name later supplanted, we have listed it for ease of reference.

We have marked with an asterisk $\left(^{*}\right)$, some revisions and comments on parasite taxonomy which have lately come to our attention. Deardorff and Overstreet (1981) have resurrected the genus Hysterothylacium to include the genus Thynnascaris and those members of Contracaecum which mature in fishes.

Among the copepods, Z. Kabata (pers. commun.) has the following comments. There are no authenticated records for Bomolochus solae from America. Though there is an old re- 
port of Caligus curtus from Squalus acanthias, this is a very doubtful record. Pseudocaligus apodus is properly placed in Caligopsis. Clavella inversa should not be within this genus.
Koyeria trecai, Neobrachiella oblonga, and Pennella costai are nomen nudum. Perissopus oblongatus is a composite taxon. Haemobaphes theragrae is species inquirenda. 


$$
\begin{gathered}
\text { HOST-PARASITE LIST } \\
\text { MYXINIDAE -- Hagfishes } \\
\text { Eptatretus stouti - Pacific Hagfish }
\end{gathered}
$$

PROTOZOA (MYXOSPORIDA)

\title{
CERATOMYXIDAE
}

Ceratomyxa californica Jameson 1929. Central California. Gallbladder. Jameson (1929).

Leptotheca galeata (Jameson 1929) Kudo 1933. Central

California. Gallbladder. Jameson (1929) as Ceratomyxa g.

\author{
PETROMYZONTIDAE -- Lampreys \\ Lampetra tridentata - Pacific Lamprey
}

CESTODA

PHYLLOBOTHRI IDAE

Phyllobothrium sp. Immature adults. British Columbia.

Digestive tract. Bangham and Adams (1954).

DIGENEA

NANOPHYETIDAE

Nanophyetus salmincola (Chapin 1926) Chapin 1927. Metacercaria. Oregon. Gebhart et a7. (1966).

NEMATODA

ACANTHOCHEILIDAE

Pseudanisakis tricupula Gibson 1973. Oregon. Shaw (1947) as Acanthocheilus.

DIOCTOPHYMATIDAE

Eustrongylides sp. Larva. British Columbia. Bangham and Adams (1954). 


\section{HEXANCHIDAE -- Cow Sharks \\ Hexanchus griseus - Sixgill Shark}

CESTODA

LACISTORHYNCHIDAE

Grillotia acanthoscolex Rees 1944. Atlantic. Intestine. Rees (1944). Grillotia musculara (Hart 1936) Dollfus 1942. Washington.

Hart (1936a) as Tentacularia m.

Grillotia scolecina (Rudolphi 1819). Washington; Mediterranean.

Yamaguti (1959b) [poorly described - Dollfus (1942)].

Grillotia heptanchii (Vaullegeard 1899) Dollfus 1942. Chile.

Carvaja 1 (1971); France. Joyeux and Baer (1936) as

G. instituta; British Columbia. Intestine. Wardle (1933b)

as G. erinaceus.

ONCOBOTHRI IDAE

Acanthobothrium paulum Linton 1890 . France. Williams (1969) [Species incertae sedis - Goldstein (1967)].

Calliobothrium verticillatum (Rudolphi 1819) Van Beneden 1850.

Southwe17 (7925); France. Joyeux and Baer (7936).

PHYLLOBOTHRI IDAE

Phyllobothrium centrurum Southwe17 1925. Atlantic. Williams (1968).

Phyllobothrium dohrnif (Oerley 1885) Zschokke 7888 . Chile. Carvajal (1974); Britain. Spiral valve. Williams (1958); Central California. Riser (1955) as Orygmatobothrium d.

Phyllobothrium rotundum (Klaptocz 1906). Triest. Yamaguti (1959b).

Phyllobothrium triacis Yamaguti 1952. Mediterranean. Williams (1968) [=Crossobothrium? t. - Williams (1968)].

Phyllobothrium lactuca Van Beneden 1850. France. Laubier et a). (1966).

Phyllobothrium sinuosiceps Williams 1959. Chile. Carvajal (1974); Britain. Spiral valve. Williams (1959).

Anthobothrium auriculatum (Rudolphi 1819) Diesing, 1863 . France. Joyeux and Baer (1936).

\section{TENTACULARIIDAE}

Tentacularia megalobothrida Hart 1936. Washington. Spiral valve. Hart (1936a).

Tetrarhynchus sp. Larva. Washington, Alaska. Below pyloric valve. Hart (1936a). [May be Nybelinia surmenicola Dollfus (1942)]. 
Hexanchus griseus (Continued)

COPEPODA

EUDACTYLINIDAE

Bariaka pamelae (Laubier 1966) Cressey. France. Branchial

rays. Laubier et al. (1966) as Protodactylina p.

\section{PANDARIDAE}

Demoleus heptapus (0tto 1821) Heller 1865. Central California. ExternaT. WiTson (1935b) as D. paradoxus; France. Laubier et ai. (1966).

\section{DIGENEA}

\section{AZYGIIDAE}

Otodistomum veliporum (Creplin 1837) Stafford 1904. Washington.

Pratt and McCauley (1961); Stomach. British Columbia. Margolis ${ }^{3}$. Otodistomum plicatum Kay 1947. Washington. Pyloric caeca.

Kay (1947).

Otodistomum scymni (Risso 1826) Guiart 1938. France. Laubier et a). (1966).

\section{MONOGENEA}

\section{HEXABOTHRI IDAE}

Erpocotyle taschenbergi Maillard and 01iver 1966. France.

Gills. Laubier et aT. (1966).

Erpocotyle grisea (Cerfontaine 1899). France. Laubier et al.

(1966); Washington. Bonham (1950) as Squalonchocotyle g.

NEMATODA

PHYSALOPTERIDAE

Proleptus elegans (0erley, 1885). Stomach, intestine. Yamaguti (1961).

Notorynchus maculatus - Sevengi11 Shark

\section{CESTODA}

DASYRHYNCHIDAE

Floriceps saccatus Cuvier 1817. Baja California. Heinz and Dailey (1974). PHYLLOBOTHRIIDAE

Anthobothrium cornucopia Van Beneden 1850. Northern California.

$$
\text { Spiral valve. Pappast. }
$$


Notorhynchus maculatus (Continued)

\title{
TENTACULARI IDAE
}

Nybelinia sp. Northern California. Spiral valve. Pappas (1970). COPEPODA

\section{PANDARIDAE}

* Perissopus oblongatus (Wilson 1908) Cressey 1967. California. Pandarus bicolor Leach (1912a) as Achtheinus dentatus. surface. Russo (1975).

HIRUDINOIDEA

PISCICOLIDAE $\frac{\text { Branchellion lobata Moore 1952. Northern California. }}{\text { Claspers, buccal cavity, fins. Russo (1975). }}$

\author{
HETERODONTIDAE -- But Thead Sharks \\ Heterodontus francisci - Horn Shark
}

CESTODA

ONCOBOTHRI IDAE

Acanthobothrium bajaensis Appy and Dailey 1973. Baja California. SpiralvaTve. Appy and Dailey (1973).

TENTACULARI IDAE

Nybelinia anthicosum Heinz and Dailey 1974. Baja California. ISOPODA

\section{CYMOTHOIDAE}

$\frac{\text { Nerocila californica Schioedte and Meinert 1887. California. }}{\text { Dorsal fin. Richardson (1905). }}$ NEMATODA

GNATHOSTOMATIDAE

Echinocephalus pseudouncinatus Millemann 1951. Southern (1963).
(19ulf Gulf of California. Spiral valve. Millemann 
Heterodontus francisci (Continued)

PHYSALOPTERIDAE

Proleptus acutus Dujardin 1845. Southern California. Stomach. Specian et aT. (1975).

\author{
RHINCODONTIDAE -- Whale Sharks \\ Rhincodon typus - Whale Shark
}

COPEPODA

PANDARIDAE

Prosaetes rhinodontis (Wright 1874) Wilson 1907. Seychelles

Islands, Indian Ocean. GiTls. Wilson (1907b).

\author{
ODONTASPIDIDAE -- Sand Tigers \\ Odontaspis ferox - Ragged-tooth Shark
}

\title{
CESTODA
}

LITOBOTHRIDAE

Litobothrium gracile Dailey 1971. Southern California. Spiral valve. Dailey (1971).

COPEPODA

EUDACTYLINIDAE

Nemes is lamna Risso 1826. Mediterranean. Yamaguti (1963a) from odontaspis sp. Likely this is $\underline{0}$. ferox.

\author{
ALOPIIDAE -- Thresher Sharks \\ Alopias superciliosus - Bigeye Thresher
}

CESTODA

LITOBOTHRIDAE

Litobothrium alopias Dailey 1969. Southern California. Spiral valve. Dailey (T969a). 
Litobothrium coniformis Dailey 1969. Southern California. Spiral vàive. Dailey (1969b).

Litobothrium daileyi Kurochkin and Slankis 1973. Spiral

valve. Kurochkin and Slankis (1973).

Renyxa amplifica Kurochkin and Slankis 1973. Spiral valve. Kurochkin and Slankis (1973).

\section{SPHYRIOCEPHALIDAE}

Sphyriocephalus viridis Wagener 1854. Southern California. Heinz and Dailey (1974).

Sphyriocephalus pelorosoma Heinz and Dailey 1974. Southern California. Stomach. Heinz and Dailey (1974).

COPEPODA

\section{EUDACTYLINIDAE}

$\frac{\text { Bariaka alopiae Cressey 1966. Indian Ocean. Gill filaments. }}{\text { Cressey (1967a). }}$

\section{PANDARIDAE}

Dinemoura discrepans Cressey 1967. Madagascar, Pacific Ocean. Body surface. Cressey (1967c).

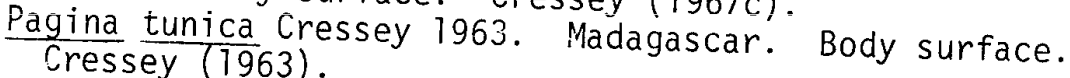

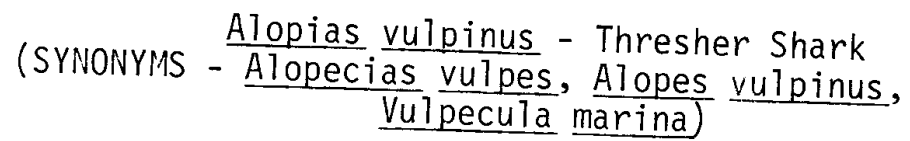

\section{CESTODA}

GYMNORHYNCHIDAE

$\frac{\text { Molicola uncinatum (Linton 1924). Southern California. }}{\text { Heniz and Dailey (1974). }}$ HEPATOXYLIDAE

Hepatoxylon squali Bosc 1811. Southern California. Heinz and Dailey (1974). [= . E trichiuri - Dollfus (1942)].

\section{LACISTORHYNCHIDAE}

Lacistorhynchus tenuis (Van Beneden 1858) Dollfus 1929. Larva. Massachusetts. Spiral intestine. Linton (1924) as
Rhynchobothrium bulbifer. 


\section{ONCOBOTHRI IDAE}

Phoreiobothrium lasium Linton 1889. Massachusetts. Yamaguti (1959b).

Acanthobothrium coronatum (Rudolphi 1819) Van Beneden 1849. South Atlantic. Digestive tract. Parukhin (1968).

\section{PHYLLOBOTHRI IDAE}

Anthobothrium exiquum Yamaguti 1935. Japan. Spiral valve. Yamaguti (1934b) as A. parvum.

Marsupiobothrium alopias Yamaguti 1952. Pacific coast of Japan. Interior. Yamaguti (1952).

Crossobothrium angustum (Linton 1889) Linton 1901. Chile. Carvajal (1974); France. Euzet (1956); Pacific coast of Japan. Yamaguti (1952) as Phyllobothrium filiferum.

Pithophorus vulpeculae Yamaguti 1952. Japan. Yamaguti (1952).

SPHYRIOCEPHALIDAE

Sphyriocephalus tergestinus Pinter 1913. France. Joyeux and Baer (1936).

\section{COPEPODA}

\section{CAL IGIDAE}

Caligus elongatus Nordmann 1832. England. Leigh-Sharpe (1936) as C. rabidus.

\section{EUDACTYLINIDAE}

Nemes is robusta (Van Beneden 1851) Brian 1906. England. Leigh-Sharpe (1936); Massachusetts. Gills. Wilson (1932); New Zealand. Hewitt (1969c).

Nemesis aggregata Cressey 1967. Indian Ocean. Gill filaments. Cressey (1967a).

Nemesis atlantica Wilson 1922. Massachusetts. Gills. Wilson (1924).

Nemes is lamna Risso 1826. Yamaguti (1963a) as $\mathrm{N}$. mediterranea.

\section{PANDARIDAE}

Dinemoura producta (Müller 1785) Latreille 1829. Faroe Is lands. Exterior. Yamaguti (1963a); Africa. Barnard (1955).

Dinemoura latifolia Steenstrup and Lütken 1861. Italy. Hewitt (1967).

Dinemoura discrepans Cressey 1967. Indian Ocean. Body surface. Cressey (1967c).

Echthrogaleus denticulatus Smith 1874. Indian 0cean. Body surface. Cressey (1967c). 
Pandarus cranchii Leach 1819. Indian Ocean. Body surface. Cressey (1967a).

Pandarus smithii Rathbun 1886. Indian Ocean. Body surface. Cressey (7967a).

*Perissopus oblongatus (Wilson 1908) Cressey 1967. Southern

California. Exterior. Wilson (1921) as Achthenius dentatus. Gangliopus pyriformis Gerstaecker 1854. Indian Ocean. Gill filaments. Cressey (1967a).

\section{LAMNIDAE -- Mackerel Sharks \\ Carcharodon carcharias - White Shark}

\section{CESTODA}

HEPATOXYLIDAE

Hepatoxylon trichiuri (Holten 1802) Dollfus 1942. New Zealand. Spiral valve. Robinson (1959a); Postlarva. Atlantic. Dollfus (1942).

PHYLLOBOTHRI IDAE

Phyllobothrium tumidum Linton 1922. Southern California. Spiral valve. Riser (1955).

Phyllobothrium lactuca Van Beneden 1850. New Zealand. Spiral valve. Robinson (1959a).

Dinobothrium septaria Van Beneden 1889. Massachusetts. Yamaguti $(1959 \mathrm{~b})$.

Dinobothrium plicitum Linton 1922. Baylis (1950).

TENTACULARI IDAE

Tentacularia coryphaenae Bosc 1802. Brazil. Stomach. Dollfus (1942).

\section{COPEPODA}

\section{DICHELESTHIIDAE}

Anthosoma crassum (Abi1dgaard 1794) Gould 1841. Massachusetts. Gills. WiTson (1924); Hawaii. Lewis (1966); New Zealand.

Buccal cavity. Hewitt (1968c).

\section{EUDACTYLINIDAE}

Nemes is robusta (van Beneden 1851) Brian 1906. Massachusetts. GiTls. WiTson (1932).

Nemesis lamna Risso 1826. Florida. Cressey (1970); Mediterranean.

Wilson (1932); Indian Ocean. Cressey (1967a); New Zealand. Hewitt
(1969C). 


\section{PANDARIDAE}

Pandarus cranchij Leach 1819. West coast of Florida. Pectora1, caudal fins. Cressey (1970); Massachusetts. Cressey (1967C); Indian Ocean. Cressey (1967a).

Pandarus smithii Rathbun 1886. West coast of Florida. Body surface. Cressey (1970).

Pandarus floridanus Cressey 1967. West coast of Florida. Body surface. Cressey (1970); Massachusetts. Cressey (1967c).

Pandarus sinuatus Say 1817. Massachusetts, North Carolina. Fins. Wilson (1907b).

Nesippus orientalis Heller 1865. West coast of Florida. Mouth, gill arches. Cressey (1970); Indian Ocean. Cressey (1967a); South Africa. Barnard (1955) as N. alatus.

Nesippus crypturus Heller 1865. West coast of Florida. Mouth. Cressey (1970).

Echthrogaleus denticulatus Smith 1874. Massachusetts. Wilson (1932).

Dinemoura latifolia Steenstrup and Lütken 1861. Massachusetts. Body surface. Wilson (1932); New Zealand. Buccal cavity, gills, body surface. Hewitt (1967); Hawaii. Lewis (1966). Dinemoura producta (Mül ler 1785) Latrei11e 1829. Maine. Cressey (T967c); New Zealand. Body surface. Hewitt (1967).

\section{Cetorninus maximus -- Basking Shark \\ SYNONYM - Selache maximus}

CESTODA

PHYLLOBOTHRI IDAE

Dinobothrium septaria Van Beneden 1889. France. Joyeux and Baer (7936).

Gastrolecithus planus (Linton 1922) Yamaguti 1952. Central

California. Spiral valve. Riser (1955); Great Britain. Sproston (1948), both as Dinobothrium planum.

Reesium paciferum (Sproston 1948) Euzet 1956. Central

California. Spiral valve. Riser (1955) as Dinobothrium spinosum; France. Euzet (1956).

Anthobothrium variabile (Linton 1839) Southwe11 1925. Southwe17 (1925). 
COPEPODA

DICHELESTHI IDAE

Anthosoma crassum (Abildgaard 1794) Gould 1841. Africa.

Between teeth. Barnard (1955).

EUDACTYLINIDAE

Nemes is lamna Risso 1826. Florida. Cressey (1970); Africa. Barnard (1955); New Zealand. Hewitt (1969c); Britain. Leigh-Sharpe (1933) as N. vermi.

PANDARIDAE

Dinemoura producta (Müiler 1785) Latreille 1829. Amsterdam. Cressey (1967c); Africa. Barnard (1955); New Zealand. Hewitt (1967).

CRUSTACEA

LEPADIDAE

Conchoderma auritum. Gills. Delamare-Deboutteville (1948). DIGENEA

SPIRORCHIDAE

Genera and species. Great Britain. Blood vessels. Sproston (1948).

NEMATODA

ANISAKIDAE

Contracaecum plagiostomorum (Linstow 1905). Ceylon. Gills. Bay is (1936).

Isurus oxyrinchus -- Shortfin Mako

SYNONYMS - Isurops is glauca, Isurus tigris, Isurus glaucus, Isurus dekayi, Isurus nasus

CESTODA

GYMNORHYNCHIDAE

Gymnorhynchus isuri Robinson 1959. New Zealand. Spiral valve. Robinson (1959a). 
Gymnorhynchus gigas (Cuvier 1817). Southern California. Heinz and Dailey (1974).

Molicola horridus (Goodsir 1841). Southern California. Heinz and Dailey (1974).

HEPATOXYLIDAE

Hepatoxylon trichiuri (Holten 1802) Dollfus 1942. Postlarva. New Zealand. Body cavity. Robinson (1959a).

ONCOBOTHRI IDAE

Ceratobothrium xanthocephalum Montice11i 1892. Japan.

Yamaguti (1959); France. Euzet (1956); New Zealand.

Spiral valve. Robinson (1959a).

PHYLLOBOTHRI IDAE

Phyllobothrium tumidum Linton 1922. France. Euzet (1956). PROSOBOTHRIIDAE

Platybothrium parvum Linton 1901. Massachusetts. Yamaguti (1959b).

SPHYRIOCEPHALIDAE

Sphyriocephalus tergestinus Pintner 1913. Japan. Dollfus (1942).

TENTACULARI IDAE

Nybelinia pintneri Yamaguti 1934. Southern California. Heinz and Dailey (1974).

Nybelinia lingual is (Cuvier 1817) Dollfus 1929. Mediterranean. Intestine. Do11fus (1942).

Nybelinia robusta (Linton 1890) Dollfus 1930. Massachusetts. Spiral valve. Dollfus (1942).

COPEPODA

CAL IGIDAE

Caligus coryphaenae Steenstrup and Lütken 1861. Japan. Body surface. Shitino (1959e).

\section{DICHELESTHI IDAE}

Anthosoma crassum (Abildgaard 1794) Gould 1841. Southern California, Central Pacific, New Jersey, Indian Ocean, North Atlantic, Chile, Peru. Between teeth. Cressey (1968); New Zealand. Buccal cavity. Hewitt (1968c); South Africa. Barnard (1955). 


\section{EUDACTYLINIDAE}

Nemesis lamna Risso 1826. Southern California, Indian Ocean, Chile, Peru, Mediterranean. Gill filaments. Cressey

(1968); New Zealand. Hewitt (1969c).

\section{EURYPHORIDAE}

Euryphorus brachypturus (Gerstaecker 1853) Heegaard 1972. Japan. Inner surfaces of gill operculums. Shiino (1959e) as E. hemiptera.

\section{PANDARIDAE}

Pandarus bicolor Leach 1816. Pacific. Hewitt (1967).

Pandarus satyrus Dana 1852. South America. Mouth, gill arches. Cressey (1968); Japan. Shi ino (1954e); New Zealand. Buccal cavity, body surface. Hewitt (1967) as P. cranchii.

Pandarus smithii Rathbun 1886. North Atlantic, Indian Ocean. Mouth, body surface. Cressey (1968).

Pandarus katoi Cressey 1967. South America. Mouth, gill arches. Cressey (1968).

Echthrogaleus coleoptratus (Guerin 1817) Wilson 1907. Japan. Buccal cavity, body surface. Shiino (1954e); Massachusetts. Wilson (1932).

Echthrogaleus denticulatus Smith 1874. Southern California, Central Pacific, Chile, Peru, Indian Ocean. Body surface. Cressey (1968); Japan. Buccal cavity, body surface. Shi ino (1954e).

Nesippus borealis (Steenstrup and Lütken 1861) Wilson 1907. Body surface. Hewitt (1967).

Gangliopus pyriformis Gerstaecker 1854. South America. Cressey (1968).

Phyllothyreus cornutus (Edwards 1840) Bassett - Smith 1899. South America. Gill chambers. Cressey (1968); New Zealand. Hewitt (1967).

Dinemoura latifolia Steenstrup and Lütken 1861. Western North America, Caribbean Sea, Indian Ocean, west coast South America, central Pacific. Body surface. Cressey (1968); New Zealand. Buccal cavity, gills. Hewitt (1967).

Dinemoura producta (Mül7er 1785) Latreille 1829. North Atlantic, Southern California, Chile, Peru. Fins. Cressey (1968).

\section{NEMATODA}

\section{ANISAKIDAE}

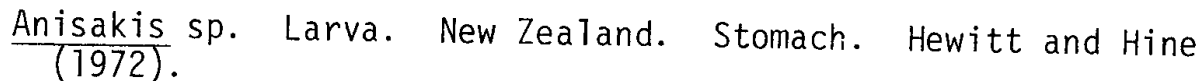




\section{Lamna ditropis -- Salmon Shark \\ SYNONYMS - Lamna nasus, L.}

CESTODA

PHYLLOBOTHRI IDAE

Dinobothrium septaria Van Beneden 1889. Central California. Spiral valve. Riser (1955).

Dinobothrium simile Van Beneden 1889. Central California. Spiral valve. Riser (1955).

Phyllobothrium tumidum Linton 1922. Central California. Spiral valve. Riser (1955).

\section{TENTACULARI IDAE}

Nybelinia sp. Central California. Spiral valve. Riser (1955).

Nybelinia palliata (Linton 1924) Dollfus 1929. Southern California. Young (1954).

Nybelinia surmenicola Okada 1929. Alaska. Stomach.

$$
\text { Shimazu (1975). }
$$

COPEPODA

\section{DICHELESTHIIDAE}

Anthosoma crassum (Abildgaard 1794) Gould 1841. Central California. Outer surface. Wilson (1935b); Japan. Buccal cavity. Shiino (1955c); New Zealand. Hewitt (1968c).

\section{EUDACTYLINIDAE}

Nemesis lamna Risso 1826. Southern California. Gills. Wi 1 son (1935b).

\section{PANDARIDAE}

Echthrogaleus coleoptratus (Guerin 1817) Wilson 1907. Japan. Body surface, fins. Shiino (1957); British Columbia. Bere $^{5}$; New Zealand. Hewitt (1967).

Dinemoura latifolia (Steenstrup and Lutken 1861). Central California. Outer surface. Wilson (1935b); Japan. Shiino (1957).

Dinemoura producta (Muller 1785) Latreille 1829. Japan. Body surface, fins. Shiino (1957) as Dinematura a. 


\section{SCYLIORHINIDAE -- Cat Sharks \\ Apristurus brunneus - Brown Cat Shark}

NEMATODA

ACANTHOCHEILIDAE

Acanthocheilus quadridentatus Molin 1858. Northern California. Free in coelomic cavity, encysted in stomach wal1. Pappas ${ }^{4}$. PROTOZOA (MYXOSPORIDA)

Genera and species. Southern California. Gallbladder. Noble (1966a).

$$
\text { Cephaloscyllium ventriosum -- Swell Shark }
$$

ASPIDOCOTYLEA

ASPIDOGASTERIDAE

Multicalyx cristata (Faust and Tang 1936) Stunkard 1962. Southern

California. Bile ducts. Hendrix and Overstreet (1977).

$$
\begin{aligned}
& \text { CARCHARHINIDAE -- Requiem Sharks } \\
& \text { Carcharhinus leucas - Bull Shark }
\end{aligned}
$$

CESTODA

CATHETOCEPHALIDAE

Cathetocephalus thatcheri Dailey and Overstreet 1973. Florida.

Spiral valve. Dailey and Overstreet (1973).

DASYRHYNCHIDAE

Dasyrhynchus giganteus (Diesing 1850) Pintner 1929. Florida. Intestine. Dollfus (1969b).

DISCUL ICIPITIDAE

Disculiceps pileatus (Linton 1890) Joyeux and Baer 1935. Gulf of Mexico. Chand7er (1954).

ONCOBOTHRIIDAE

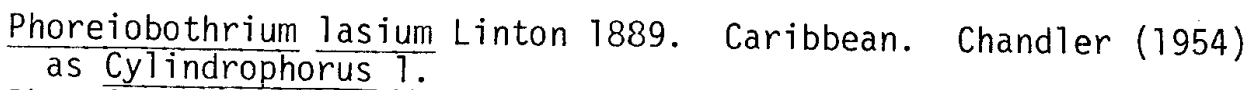
Phoreiobothrium exceptum Linton 1924. Texas. Chandier (1924) as Cylindrophorus e. 
OTOBOTHRI IDAE

Otobothrium penetrans Linton 1907. Caribbean. Chandler (1954).

Otobothrium crenacolle Linton 1890. Caribbean. Chandler (1954).

PHYLLOBOTHRI IDAE

Phyllobothrium musteli (Van Beneden 1850). Caribbean. Chandler (1954).

Anthobothrium laciniatum Linton 1890. Caribbean. Chandler (1954).

COPEPODA

\section{EUDACTYLINIDAE}

Nemes is robusta (Van Beneden 1851) Brian 1906. Madagascar. Gi11 filaments. Cressey (1967a).

Nemes is atlantica Wilson 1922. West Florida. Gills. Cressey (1970).

EURYPHOR IDAE

Alebion carchariae Krøyer 1863. West Florida. Body surface. Cressey (1970).

Alebion gracilis Wilson 1905. Indian Ocean. Body surface. Cressey (1967a).

Paralebion elongatus Wilson 1911. West Florida. Body surface. Cressey (1970); Indian Ocean. Cressey (1967a); West Africa. Caudal fin. Vaissiere (1959).

KROYERI IDAE

Kroyeria gracilis Wilson 1932. Madagascar, Indian Ocean. Gill filaments. Cressey (1967a).

PANDARIDAE

Nesippus orientalis Heller 1865. West Florida. Body surface. Cressey (1970); Indian Ocean. Cressey (1967a).

Nesippus crypturus Heller 1865. West Florida. Mouth. Cressey (1970); Indian 0cean. Cressey (1967a).

Perissopus dentatus Steenstrup and Lütken 1861. West Florida. Exterior. Cressey (1970); Indian Ocean. Cressey (1967a).

Pandarus smithii Rathbun 1886. West Florida. Exterior. Cressey (1970).

Pandarus cranchii Leach 1819. West Florida. Tail, pectoral fins. Cressey (1970).

Pandarus sinuatus Say 1817. West Florida. Exterior. Cressey (1970).

Pandarus carcharini Ho 1960. Madagascar. Cressey (1967c). 
HIRUDINOIDEA

PISCICOLIDAE

Stibarobdel1a macrothela (Schmarda 1861) Llewellyn 1966.

Florida. External surface, mouth. Sawyer et al. (1975).

MONOGENEA

HEXABOTHRIIDAE

Heteroncocotyle leucas Hargis 1955. Louisiana. Hargis (1955b).

Carcharhinus longimanus - Oceanic Whitetip Shark

CESTODA

DASYRHYNCHIDAE

Dasyrhynchus talismani Dollfus 1935. Pacific Ocean Northern Mexico. Heinz and Dailey (1974).

TENTACULARI IDAE

Tentacularia coryphaenae Bosc 1802. Pacific Ocean Northern Mexico. Heinz and Dailey (1974).

COPEPODA

EURYPHORIDAE

Alebion gracilis Wilson 1905. Indian Ocean. Body surface. Cressey (1967a).

KROYERI IDAE

Kroyeria gracilis Wilson 1932. Indian Ocean. Gill filaments. Cressey (1967a).

\section{PANDARIDAE}

Pandarus cranchii Leach 1819. Indian Ocean. Body surface, fin. Cressey (1967a).

Nesippus crypturus Heller 1865. Indian Ocean. Mouth. Cressey (1967a). 
HIRUDINOIDEA

PISCICOLIDAE

Stibarobde 11a macrothela (Schmarda 1861) Llewellyn 1966.

Atlantic. External surface, mouth. Sawyer et a1. (1975).

\author{
Carcharhinus obscurus - Dusky Shark \\ SYNONYM - Carcharias obscurus
}

CESTODA

DASYRHYNCHIDAE

Floriceps saccatus Cuvier 1817. Atlantic. Dollfus (1942). Ca7litetrarhynchus gracilis (Rudolphi 1819) Pintner 1931. Yamaguti (1959b) as Callotetrarhynchus $\mathrm{g}$.

DISCULICIPIDAE

Disculiceps pileatus (Linton 1897) Joyeux and Baer 1936. Massachusetts. Intestine wa11. Linton (1891) as Discocephalum $\mathrm{p}$.

LACISTORHYNCHIDAE

Grillotia erinacea (Van Beneden 1858) Guiart 1927. Massachusetts. Linton (1924) as Rhynchobothrium imparispine.

ONCOBOTHRIIDAE

Phoreiobothrium lasium Linton 1889. Massachusetts. Spiral valve. Linton (1889).

Phoreiobothrium triloculatum Linton 1901. Massachusetts. Linton (1924).

OTOBOTHRI IDAE

Otobothrium crenacolle Linton 1891. North Carolina, Massachussetts, Gulf of Mexico. Spiral valve. Stomachs. Linton (1924). Otobothrium dipsacum Linton 1897 . North Carolina. Linton (1905) as $\underline{\text { insigne. }}$

PHYLLOBOTHRIIDAE

Phyl lobothrium foliatum Linton 1891. Massachusetts. Linton (1891).

Anthobothrium laciniatum Linton 1891. Massachusetts. Linton (1891). 
Crossobothrium angustum (Linton 1889) Linton 1901.

Massachusetts. Linton (1924).

PROSOBOTHRI IDAE

Platybothrium cervinum Linton 1891. Massachusetts. Spiral valve. Linton (1891).

PTEROBOTHRI IDAE

Pterobothrium heteracanthum Diesing 1850. Larva. Massachusetts. Cysts in stomach. Linton (1924) as Synbothrium filicolle.

\section{TENTACULARI IDAE}

Nybelinia bisulcata (Linton 1889) Dollfus 1929. Massachusetts. Intestines. Linton (1889) as Rhynchobothrium bisulcatum. Nybelinia robusta (Linton 1891 ) Dollfus 1930. Massachusetts. Dolifus (1942).

Tentacularia coryphaenae Bosc 1302. Postlarva and adult. Massachusetts. Dollfus (1942).

TRYPANORHYNCHA

Tetrarhynchus bicolor (Bartels 1832) Wagener 1854.

Massachusetts. Linton (1924). [=Tentacularia coryphaenae Dollfus (1942).] [Species incertae sedis - Yamaguti (1961).] Rhynchobothrium tumidulum Linton 1905. Massachusetts. Spiral valve. Linton (1924).

COPEPODA

DICHELESTHI IDAE

Anthosoma crassum (Abi1dgaard 1794) Gould 1841. Wi1son (1932).

\section{EUDACTYLINIDAE}

Nemesis robusta (van Beneden 1851) Brian 1906. Massachusetts. Wilson (1932); Gulf of Mexico. Gills. Bere (1936) as N. pallida.

Nemesis spinulosus Cressey 1970. Wesi Florida. Gills. Cressey (1970).

ivemesis atlantica Wilson 1922. Massachusetts. Gi1ls. Wilson (1924).

Eudactylina spinifera Wilson 1932. Gulf of Mexico. Gills. Bere $(1936)$.

\section{EURYPHORIDAE}

Paralebion elongatus Wilson 1911. Gulf of Mexico. Mouth. Bere (1936). 
Alebion carchariae Krøyer 1863. West Florida. Body surface. Cressey (1970); Massachusetts. Wilson (1932); West Africa. Pectoral fin. Vaissiere (1959).

Alebion gracilis Wilson 1905. Indian Ocean. Body surface, fin. Cressey (1967a); Laysan Island. Wilson (1932).

\section{KROYERI IDAE}

Kroyeria gracilis Wilson 1932. West Florida. Wilson (1932).

\section{PANDARIDAE}

Nesippus oriental is Heller 1865. Angola. Surface. NunesRuivo (1956); GuTf of Mexico. Mouth and body surface. Bere (1936); Massachusetts. Wilson (1932) as $N$. alatus.

Nesippus gracilis Wilson 1935. Gulf of Mexico. Mouth and body surface. Bere (1936).

Pandarus smithii Rathbun 1886. West Florida. Body surface. Cressey (1970). Massachusetts. Wilson (1932).

Pandarus cranchii Leach 1819. West Florida. Tail and pectoral fin. Cressey (1970).

Pandarus satyrus Dana 1852. Gulf of Mexico. Body surface. Bere (1936).

Pandarus sinuatus Say 1817. Gulf of Mexico. Body surface. Bere (1936); Massachusetts. Wi1son (1907b);

North Africa. Exterior on fin. Capart (1953) as P. affinis.

Perissopus communis Rathbun 1887. Massachusetts. Rathbun (1887); Gulf of Mexico. Body surface. Bere (1936).

Perissopus dentatus Steenstrup and Lütken 1861. Indian Ocean. Body surface. Cressey (1967a).

\section{SPHYRI IDAE}

Paeon elongatus Wilson 1932. Massachusetts. Gi1i cavity. WiTs on (1932).

Opimia exilis Wilson 1908. Gulf of Mexico. Gills. Bere $(1936)$.

\section{DIGENEA}

BUCEPHAL IDAE

Prosorhynchoides arcuata (Linton 1900) n. comb. Massachusetts. Do11 fus (1937).

HIRUDINOIDEA

PISCICOLIDAE

Stibarobdella macrothela (Schmarda 1861) Llewellyn 1966.

Atlantic. External surface, mouth. Sawyer et al. (1975). 
MONOGENEA

MONOCOTYLIDAE

Cathariotrema selachii (MacCal7um 1916) Johnston and Tiegs 1922. Massachusetts. Olfactory organs. MacCallum (1916).

NEMATODA

\section{ANISAKIDAE}

Terranova brevicapitata (Linton 1901) Musgovoy 1951. North America. Yamaguti (1961).

Galeocerdo cuvieri - Tiger Shark

SYNONYMS - Galeocerdo arcticus, Galeocerdo tigrinus, Galeocerdo tygrinus

CESTODA

BALANOBOTHRI IDAE

Balanobothrium parvum Southwel1 1925. Ceylon. Southwell (1930).

\section{ONCOBOTHRI IDAE}

Pedibothrium longispine Linton 1909. Ceylon. Southwell (1930).

Pedibothrium hutsoni (Southwe11 1911) Southwe11 1924. Ceylon. Southwel1 (1930)

Phorejobothrium lasium Linton 1839. Massachusetts. Yamaguti (1959b).

Thysanocephalum rugosum Chandler 1942. Florida. Chandler (1954).

Thysanocephalum crispum (Linton 1889) Linton 1891. Massachusetts. Spiral valve. Linton (1889); India. Subhapradha (1955).

OTOBOTHR I IDAE

Otobothrium curtum (Linton 1909) Dollfus 1942. Caribbean. Chandier (1954).

PHYLLOBOTHRI IDAE

Monorygma galeocerdonis MacCa11um 1921. Massachusetts. Williams (1968) [=Phyllobothrium? g. - Williams (1968)]. 
Phyllobothrium dagnallum Southwel1 1927. Ceylon. Southwell (1930). Phyllobothrium lactuca van Beneden 1850. Ceylon. Southwell (1930). Phy I lobothrium musteli (van Beneden 1850). Yamaguti (1959b).

PTEROBOTHRIIDAE

Pterobothrium heteracanthum Diesing 1850. Massachusetts. Stomach. Dollfus (1942).

\section{TENTACULARI IDAE}

Tentacularia coryphaenae Bosc 1802. Postlarva, adults. Massachusetts. Stomach, pyloric caeca. Dollfus (1942).

Tentacularia macropora (Shipley and Hornell 1906) Southwel1 1930. Ceylon. Southweil (1930).

Nybelinia bisulcata (Linton 1889) Dollfus 1929. Massachusetts. Stomach. DolTfus (1942).

TRYPANORHYNCHA

Rhynchobothrium exile Linton 1909. Caribbean. Chandler (1954). COPEPODA

EUDACTYLINIDAE

Eudactylina pusilla Cressey 1967. West Florida. Gills. Cressey (1970); Indian Ocean. Cressey (1967a).

Nemesis robusta (van Beneden 1851) Brian 1906. Massachusetts. Gills. Wilson (1932) as N. pallida.

EURYPHORIDAE

Alebion carchariae Kroyer 1863. West Florida. Body surface. Cressey (1970); Australia. Pectoral fins. Heegaard (1962). Alebion gracilis Wilson 1905. West Florida. Cressey (1970).

KROYERIIDAE

Kroyeria papillipes Wilson 1932. Massachusetts. Gills. Wilson (1932). Kroyeria dispar WiTson 1935. West Florida. Gills. Cressey (1970); Madagascar. Cressey (1967a). Kroyerina elongata Wilson 1932. Massachusetts. Gi17s. Wilson (1944).

\section{PANDARIDAE}

Nesippus orientalis Heller 1865. West Florida. Body surface. Cressey (1970); Madagascar. Cressey (1967c).

Nesippus crypturus Heller 1865. West Florida. Mouth. Cressey (1970); Madagascar. Cressey (1967c).

Nesippus tigris Cressey 1965. West Florida. Nasal cavity. Cressey (1967a); Madagascar. Cressey (1967c).

Pandarus cranchii Leach 1819. Eniwetok Ato11. Body surface. Lewis (1968); West Africa. Cressey (1967c). 
Galeocerdo cuvieri (Continued)

NEMATODA

ACANTHOCHEILIDAE

Acanthocheilus nudiflex Linton 1900. Massachusetts. Yamaguti (1961). ["Should not be in this genus" - Yamaguti (1961)].

\section{ANISAKIDAE}

Terranova brevicapitata (Linton 1901) Musgovoy 1951. North America. Yamaguti (1961).

Terranova galeocerdonis (Thwaite 1927) Johnston and Mawson 1945. Ceylon. Baylis (1936) as Porrocaecum g.

\section{PHYSALOPTERIDAE}

Proleptus australis Baylis 1933. Queensland. Baylis (1933). PROTOZOA (MYXOSPORIDA)

CERATOMYXIDAE

Ceratomyxa lunata Davis 1917. North Carolina. Galibladder. Kudo (1920).

\section{Galeorhinus zyopterus Soupfin Shark}

\section{CESTODA}

\section{PHYLLOBOTHRI IDAE}

Scyphophyllideum giganteum (Van Beneden 1858) Woodland 1927. Central California. Spiral valve. Riser (1955).

Orygmatobothrium musteli (Van Beneden 1850) Diesing 1854. California. Riser (7955).

COPEPODA

EUDACTYLINIDAE

Eudactylinodes uncinata (Wilson 1908) Wilson 1932. Gills. Wilson (1908a) as EudactyTina $u$.

\section{PANDARIDAE}

$\frac{\text { Pandarus }}{(1908 a)}$ cranchii (Leach 1819) Wilson 1907. California. Wilson

Pandarus bicolor Leach 1816. Northern California. Russo (1975). SPHYRI IDAE

Opimia exilis Wilson 1908. Southern California. Mouth, gill cavity. Wilson (1908a). 
Galeorhinus zyopterus (Continued)

HIRUDINOIDEA

PISCICOLIDAE

Branchellion lobata Moore 1952. Northern California. Claspers, buccal cavity, fins. Russo (1975).

MONOGENEA

HEXABOTHRI IDAE

Erpocotyle squali (MacCallum 1931) Price 1942. Northern California. Gills. Pappas:

PROTOZOA (MYXOSPORIDA)

SPHAEROSPORIDAE

Chloromyxum ovatum Jameson 1929. Central California. Gallbladder. Jameson (1929).

Mustelus californicus Gray Smoothhound

(Synonym - Galeus californicus)

CESTODA

EUTETRARHYNCHIDAE

Eutetrarhynchus macrotrachelus Heinz and Dailey 1974. Southern

California. Spiral valve. Heinz and Dailey (1974).

Eutetrarhynchus litocephalus Heinz and Dailey 1974 . Southern

California. Spiral valve. Heinz and Dailey (1974).

LACISTORHYNCHIDAE

Lacistorhynchus tenuis (Van Beneden 1858) Dollfus 1929. Southern California. Heinz and Dailey (1974).

ONCOBOTHRIIDAE

Calliobothrium pellucidum Riser 1955. Southern California. Riser (1955).

COPEPODA

LERNAEOPODIDAE

Pseudocharopinus bicaudatus (Krøyer 1837) Kabata 1964. Central

California. Spiracles. Wilson (1935b) as Charopinus b. 
Mustelus californicus (Continued)

\section{PANDARIDAE}

*Perissopus oblongatus (Wiison 1908) Cressey 1967. Southern

California. Flesh. Ho (1975) as Achtheinus o.

\section{DIGENEA}

HETEROPHYIDAE

Stictodora sawakines is Looss 1899. Metacercaria. USSR.

Ginetsinskaya (1955).

NEMATODA

PHYSALOPTERIDAE

Proleptus acutus Dujardin 1845. Southern California. Stomach . Specian et al. (1975).

PROTOZOA (MYXOSPORIDA)

SPHAEROSPORIDAE

Chloromyxum ovatum Jameson 1929. Southern California. Gallbladder. Jameson (1937).

Mustelus henlei Brown Smoothhound

\section{CESTODA}

\section{LACISTORHYNCHIDAE}

Lacistorhynchus tenuis (Van Beneden 1858) Dollfus 1929. Southern California. Heinz and Dailey (1974).

\section{PHYLLOBOTHRIIDAE}

Pithophorus p. Northern California. Spiral valve. Pappas 4 .

Anthobothrium parvum Stossich 1895. Northern California. Spiral valve. Pappas4.

Phyllobothrium radioductum Kay 1942. Northern California. Spiral valve. Pappas ${ }^{4}$.

Gastrolecithus planus (Linton 1922) Yamaguti 1952. Northern

California. Spiral valve. Pappas 4.

Orygmatobothrium musteli (Van Beneden 1850) Diesing 1854.

California. Riser (1955). 
Mustelus henlei (Continued)

COPEPODA

PANDARIDAE

Pandarus bicolor Leach 1816. Northern California. Body surface. Russo (1975).

*Perissopus oblongatus (Wilson 1908) Cressey 1967. Northern

California. Body surface. Russo (1975).

HIRUDINOIDEA

PISCICOLIDAE

Brancheilion lobata Moore 1952. California. Moore (1952).

MONOGENEA

HEXABOTHRI IDAE

Pseudohexabothrium rajae Brinkman 1952. Northern California. Gil1s. Pappas 4 .

Erpocotyle squali (MacCallum 1931) Price 1942. Northern California. Gi11s. Pappas.

NEMATODA

ACANTHOCHEILIDAE

Acanthocheilus quadridentatus Mol in 1858. Northern California. Encysted in stomach wal1. Pappas ${ }^{4}$.

PHYSALOPTERIDAE

Proleptus obtusus Dujardin 1845. Northern California. Attached to internal stomach 7 ining. Pappas 4.

Proleptus acutus Dujardin 1845. Southern California. Stomach . Specian et al. (1975).

PROTOZOA (MYXOSPORIDA)

SPHAEROSPORIDAE

Chloromyxum ovatum Jameson 1929. Southern California. Gallbladder. Jameson (1937).

Mustelus lunulatus Sicklefin Smoothound

COPEPODA

PANDARIDAE 
Mustelus lunulatus (Continued)

*Perissopus oblongatus (Wilson 1908) Cressey 1967. Southern

California. Exterior. Wilson (1921) as Achtheinus dentatus.

\section{DIGENEA}

GORGODERIDAE

Probolitrema richiardii (Lopez 1888) Looss 1902. Gulf of California. Coelomic cavity. Marke11 (1956) as P. mexicana.

\section{Prionace glauca Blue Shark}

\section{CESTODA}

\section{DASYRHYNCHIDAE}

Floriceps saccatus Cuvier 1817. Japan. Spiral intestine. Do71fus (1942).

Callitetrarhynchus gracilis (Rudolphi 1819) Pintner 1931. Southern California. Heinz and Dailey (1974).

HEPATOXYLIDAE

Hepatoxylon trichiuri (Holten 1802) Dollfus 1942. Postlarva. Mediterranean, Japan. Dollfus (1942); New Zealand. Body cavity. Hewitt and Hine (1972).

Hepatoxylon squali Bosc 1811. Postlarva. Off Northern California. Liver. Pappas (1970). [= H. trichiuri - Dollfus (1942)].

\section{ONCOBOTHRI IDAE}

Cylindrophorus posteroporus Riser 1955. Central California. Riser (7955).

Acanthobothrium coronatum (Rudolphi 1819) Van Beneden 1849. South Atlantic. Digestive tract. Parukhin (1968).

Calliobothrium verticillatum (Rudolphi 1819) Van Beneden 1850. Chile. Carvajal (1974).

\section{PHYLLOBOTHRIIDAE}

Anthobothrium laciniatum Linton 1890. Central California. Riser (1955); New Zealand. Spiral valve. Alexander (1963). Anthobothrium minutum Guiart 1935. Cape Verde Island. Yamaguti (1959b).

Anthobothrium cornucopia Van Beneden 1850. France. Joyeux and Baer (1936).

Anthobothrium auriculatum (Rudolphi 1819) Diesing 1863. France. Joyeux and Baer (1936).

Phyllobothrium dagnallium Southwel1 1927. Newfoundland.

Threlfal1 (1969). 
Phy110bothrium prionacis Yamaguti 1934. Japan. Yamaguti (1934b). [ = Crossobothrium p. = Wil1iams (1968)].

Dinobothrium septaria Van Beneden 1889. Bayl is (1950).

Dinobothrium keilini Sproston 1948. English Channel. Intestine. Sproston (1948).

Crossobothrium angustum (Linton 1889). Central California.

Riser (1955) as Scyphophyllidium a.; Massachusetts. Spiral valve. Linton (1924); Chile. Carvajal (1974).

Pelichnibothrium speciosum Monticelli 1889. Northern California.

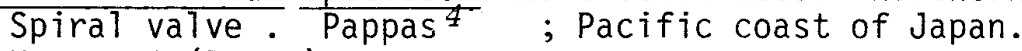

Yamaguti (1959b).

\section{PROSOBOTHRIIDAE}

Platybothrium auriculatum Yamaguti 1952. Chile. Carvajal (1974); Pacific Coast, Japan. Yamaguti (1952).

Platybothrium cervinum Linton 1891 . Massachusetts. Spiral valve. Linton (1924).

Platybothrium parvum Linton 1901. Newfoundland. Threlfall (1969).

PTatybothrium baeri Euzet 1952. Naples. Yamaguti (1959b).

Prosobothrium japonicum Yamaguti 1934. Japan. Yamaguti (1934b); California. Riser (1955).

Prosobothrium armigerum Cohn 1902. Central California. Spiral valve. Riser (1955); France. Joyeux and Baer (1936).

Prosobothrium adherans (Linton 1924) Riser 1955. Massachusetts.

Spiral valve. Linton (1924) as Ichthyotaenia a.

\section{TENTACULARIIDAE}

Tentacularia coryphaenae Bosc 1802. Pacific Japan. Yamaguti (1959b). Nybelinia pintneri Yamaguti 1934. Pacific coast of Japan. Yamaguti (1934b).

Nybelinia lingualis (Cuvier 1817) Dollfus 1929. Mediterranean. Spiral valve. Do 71 fus (1942).

\section{COPEPODA}

\section{DICHELESTHIIDAE}

Anthosoma crassum (Abildgaard 1794) Gould 1841. Japan. Buccal cavity. Shitino (1955c).

\section{EUDACTYLINIDAE}

Nemesis lamna Risso 1826. Great Britain. Gills. Sproston (1948). Nemesis robusta (Van Beneden 1851) Brian 1906. Mediterranean. Delamare-Deboutteville and Nunes-Ruivo (1953). Nemesis Carchariaeglauci (Hesse 1883) Wilson 1922. Northern California. GiTls. Pappas 4 ; Mediterranean. Delamare-Deboutvi1 le and Nunes-Ruivo (1953) both as Kroyeriá c. Species Inquirae, may be $\underline{N}$. robusta. 


\section{KROYERI IDAE}

Kroyeria aculeata (Gerstaecker 1854) Brian 1906. Mediterranean. Delamare-Deboutteville and Nunes-Ruivo (1953) as Lonchidium aculeatum.

Kroyeria gracilis Wilson 1932. Massachusetts. Nostrils. WiTson (1932).

Kroyerina elongata Wilson 1932. Massachusetts. Gills. Wilson (1932).

\section{PANDARIDAE}

Pandarus bicolor Leach 1816. North Atlantic, Mediterranean. Hewitt (1967).

Pandarus satyrus Dana 1852. Japan. Body surface, fins. Kabata and Gusev (1966); Indian Ocean. Cressey (1967a); West Pacific, Western North Atlantic. Cressey (1967c).

Gangliopus pyriformis Gerstaecker 1854. Indian Ocean, North Atlantic. Gills. Cressey (1967c).

Phy110thyreus cornutus (Edwards 1840) Bassett-Smith 1899. Indian Ocean, Western North Atlantic. Gill chambers. Cressey (1967c); Hawai i. Lewis (1966).

Dinemoura producta (Müller 1785) Latreille 1829. Maine. Cressey $(1967 \mathrm{c})$.

Dinemoura latifolia Steenstrup and Lütken 1861. Lewis (1966).

Echthrogaleus coleoptratus (Guerin 1837) Wilson 1907. Northern California. Body surface, fins. Pappas 4 ; Western North Atlantic. Cressey (1967c); Indian Ocean. Cressey (1967a); Australia. Heegaard (1962).

\section{DIGENEA}

\section{PTYCHOGONIMIDAE}

Ptychogonimus megastomus (Rudolphi 1819) Lühe 1900. Stomach . Nicol1 (1915).

\section{NEMATODA}

\section{ANISAKIDAE}

Anisakis sp. Post larva. New Zealand. Stomach. Hewitt and Hine (1972).

\section{Triakis semifasciata Leopard Shark}

\section{CESTODA}


Eutetrarhynchus 1itocephalus Heinz and Dailey 1974. Southern California. Spiral valve. Heinz and Dailey (1974).

\section{LACISTORHYNCHIDAE}

Lacistorhynchus tenuis (Van Beneden 1858) Dollfus 1929. Southern California. Young (1954).

\section{PHYLLOBOTHRI IDAE}

Phyllobothrium radioductum Kay 1942. Southern California. Young (1954).

Phyllobothrium lactuca Van Beneden 1850. Central California. Spiral valve. Riser (1955).

Anthobothrium parvum Stossich 1895. Northern California. Spiral valve. Pappas4.

Dinobothrium planum Linton 1922. Northern California. Spiral valve. Pappas4.

Orygmatobothrium musteli (Van Beneden 1850) Diesing 1854. California. Riser (1955).

\section{TENTACULARI IDAE}

Nybelinia anthicosum Heinz and Dailey 1974. Southern California. Stomach, spiral valve. Heinz and Dailey (1974).

COPEPODA

\section{EUDACTYLINIDAE}

Nemes is carchariaglauci (Hesse 1883) Wilson 1922. Northern California. GilTs. Pappas 4 , as Kroyeria C. Species Inquirae, may be $\mathrm{N}$. robusta.

\section{LERNAEOPODIDAE}

Lernaeopoda galei Krøyer 1837. Northern California. Head. Russo (1975) as ㄴ. scylicola.

\section{PANDARIDAE}

Pandarus cranchii Leach 1819. Northern California. External behind last gi17 slits. Pappas ${ }^{4}$.

Pandarus bicolor Leach 1816. Northern California. Body surface. Russo (1975).

*Perissopus oblongatus (Wilson 1908) Cressey 1967. Southern

California. Wilson (1908a) as Achtheinus oblongus.

Echthrogaleus coleoptratus (Guerin 1837) Wilson 1907. Northern California. Body, fins. Russo (1975).

\section{SPHYRIIDAE}

Paeon elongatus Wilson 1932. Northern California. Gill chambers. Pappas ${ }^{4}$. 
Triakis semifasciata (Continued)

HIRUDINOIDEA

PISCICOLIDAE

Branchellion lobata Moore 1952. Northern California. Claspers, buccal cavity, fins. Russo (1975).

ISOPODA

CYMOTHOIDAE

Nerocila californica Schioedte and Meinert 1881. California.

Fins. Richardson (1905).

MONOGENEA

HEXABOTHRI IDAE

Erpocotyle squali (MacCallum 1931) Price 1942. Northern California. Gills. Pappas4.

Genera and species. Eastern Pacific. Kunnenkeri and Martin (1963).

NEMATODA

PHYSALOPTERIDAE

Proleptus obtusus Dujardin 1845. Northern California. Internal stomach lining. Pappas4 .

PROTOZOA (MYXOSPORIDA)

CERATOMYXIDAE

Ceratomyxa jamesoni (Jameson 1931) Kudo 1933. Southern California. Gal7bladder. Jameson (1937) as $\underline{C}$. habena.

$$
\text { SPHYRNIDAE - Hammerhead Sharks }
$$

Sphyrna lewini Scalloped Hammerhead

COPEPODA

EURYPHORIDAE

Alebion crassus Wilson 1932. Hawaii. External surface.

Lewis (1966) as $\underline{E}$. echinatus.

KROYERI IDAE

Kroyeria praelongacicula Lewis 1966. Hawaii. Gill cavity. Lewis $(\overline{1966)}$. 
Sphyrna lewini (Continued)

PANDARIDAE

Pandarus cranchij Leach 1819. Hawaii. External surface. Lewis (7966).

\title{
SPHYRI IDAE
}

Paeon vaissierei ? Delamare-Deboutteville and Nunes-Ruivo 1953. Hawaii. Gi11 cavity. Lewis (1966).

MONOGENEA

HEXABOTHRIIDAE

Erpocotyle sphyrnae (MacCallum 1931) Price 1942. Hawaii. Gills. Yamaguti (1968b).

NEMATODA

ANISAKIDAE

Terranova galeocerdonis (Thwaite 1927) Johnston and Mawson 1945. West Africa. Campana-Rouget (1955).

\author{
Sphyrna tiburo - Bonnethead \\ SYNONYMS - Reniceps tiburo, Sphyrna tibulo, Cestracion tiburo
}

CESTODA

TRYPANORHYNCHA

Dibothriorhynchus maccallumi MacCallum 1921. Australia. Yamaguti (1959b) [Species Incertae Sedis - Yamaguti (1959b)].

Rhynchobothrium plicatum Linton T905. Yamaguti (1959b) [Species Incertae Sedis - Yamaguti (1959b)].

COPEPODA

BOMOLOCHIDAE

Taeniacanthus albidus Wilson 1911. North Carolina. Wilson (1911). CALIGIDAE

Caligus praetextus Bere 1936. Gulf of Mexico. Bere (1936). EUDACTYLINIDAE

Nemesis robusta (Van Beneden 1851) Brian 1906. Florida. Bere (1936) as $\underline{E}$. pallida. 
Sphyrna tiburo (Continued)

Eudactylina longispina Bere 1936. West Florida. Gills. Bere (1936).

Eudactylinodes nigra (Wilson 1908) Wilson 1932. North Carolina. Gi11 filaments. Wilson (1908b) as Eudactylina $n$.

\section{PANDARIDAE}

Pandarus sinuatus Say 1817. Texas. Body surface. Causey (1955). Perissopus dentatus Steenstrup and Lütken 1861. West Florida. Body surface. Cressey (1970). Perissopus communis Rathbun 1887. Gulf of Mexico. On body surface. Nesippus orientalis Heller 1865. Texas. Pearse (1952); North Carolina. Throat. Wilson (1932) both as N. alatus.

\section{SPHYRI IDAE}

Paeon ferox Wilson 1919. Texas. Gills. Pearse (1952).

\section{MONOGENEA}

HEXABOTHRI IDAE

Erpocotyle tiburonis (Brooks 1934) Yamaguti 1963. Florida. Price (1942) as Neoerpocotyle $t$.

\section{NEMATODA}

\section{CYSTIDICOLIDAE}

Parascarophis galeata (Linton 1905) Campana-Rouget 1955. North Carolina. Airbladder, esophagus. Yamaguti (1961).

\section{PROTOZOA (MYXOSPORIDA)}

\section{CERATOMYXIDAE}

Ceratomyxa mesospora Davis 1917. North Carolina. Galtbladder. Kudo (1920).

\section{SPHAEROSPORIDAE}

Chloromyxum sphyrnae Cunha and Fonseca 1918. Brazil. Gallbladder. Kudo and Meglitsch 6 .

Chloromyxum leydigi Mingazzini 1890. North Carolina. Gallbladder. Kudo (1920).

Sphyrna zygaena - Smooth Hammerhead

SYNONYM - Cestracion zygaena 
CESTODA

ONCOBOTHRI IDAE

Phoreiobothrium exceptum Linton 1924. Massachusetts. Linton (1924); France. Euzet (1956).

Phoreiobothrium pectinatum Linton 1924. Massachusetts. Spiral valve. Linton (1924); France. Euzet (1956).

Phoreiobothrium lasium Linton 1889. Massachusetts. Linton (1924). Thysanocephalum crispum (Linton 1889) Linton 1891. Massachusetts. Spiral valve. Linton (1924); France. Euzet (1956).

\section{OTOBOTHRIIDAE}

Otobothrium crenacolle Linton 1891. Massachusetts. Spiral valve. Linton $(18 \overline{9} 1)$.

Otobothrium penetrans Linton 1907. Massachusetts. Spiral valve. Linton (1924).

Otobothrium propecysticum Dollfus 1969. Mediterranean. Intestine. Do11fus (1969a).

Otobothrium pephrikos Dollfus 1969. Mediterranean. Intestine. Dollfus (1969a).

\section{PHYLLOBOTHRIIDAE}

Anthobothrium laciniatum Linton 1891. France. Euzet (1956); Massachusetts. Linton (1924).

Marsupiobothrium forte (Linton 1924) Yamaguti 1959. Massachusetts. Spiral valve. Linton (1924) as Orygmatobothrium f.

\section{PROSOBOTHRIIDAE}

Platybothrium parvum Linton 1901. Massachusetts. Spiral valve. Linton (1924); France. Euzet (1956).

Prosobothrium adhaerens (Linton 1924) Riser 1955. Massachusetts. Linton (1924) as Ichthyotaenia a.

\section{TENTACULARI I DAE}

Nybelinia bisulcata (Linton 1889) Dollfus 1929. Yamaguti (1959b). Nybelinia palliata (Linton 1924) Dollfus 1929. Florida. Chandler (1954); Massachusetts. Spiral valve. Linton (1924) as Tetrarhynchus palliatus.

Nybelinia sphyrnae Yamaguti 1952. Japan. Yamaguti (1952). Nybelinia syngenes Pintner 1928. Japan, Florida. Yamaguti (1959b). Tentacularia coryphaenae Bosc 1802. Bonin Islands, Pacific. Stomach, intestine. Dollfus (1942).

COPEPODA

BOMOLOCHIDAE

Taeniacanthus flagellans Wilson 1913. Jamaica. Gill cavity. Wilson (1913). 
Sphyrna zygaena (Continued)

CALIGIDAE

Lepeophtheirus longispinosus Wilson 1908. North America. Inside gil1 cavity. Wilson (1908b).

ERGASILIDAE

Ergasilus myctarothes Wilson 1913. Jamaica. Nasal tubes. Wilson (1913).

\section{EUDACTYLINIDAE}

Nemesis versicolor Wilson 1913. Jamaica. Wilson (1913).

Nemes is robusta (van Beneden 1851) Brian 1906. Angola.

Nunes - Ruivo (1956); Gulf of Mexico. Gi11s. Bere (1936)

as $\underline{\text { N. pallida. }}$

EURYPHORIDAE

Alebion echinatus Capart 1953. Japan. Shi ino (1955a).

Alebion crassus Wilson 1932. Japan. Cressey (1972); Massachusetts. Wilson (1932).

KROYERI IDAE

Kroyeria gracilis Wilson 1932. Gulf of Mexico. Gills. Bere (1936).

Kroyeria lineata Van Beneden 1853. Massachusetts. Gills. Wilson (1924); Mediterranean. Delamare-Deboutteville and Nunes-Ruivo (1958).

Kroyeria papillipes Wilson 1932. Massachusetts. Gills. Wilson (1932).

Kroyeria echinata Rangnekar 1956. India. Body surface. Rangnekar (1956).

Kroyeria sphyrnae Rangnekar 1957. India. Pillai (1967).

* Kroyeria trecai Delamare - Deboutteville and Nunes - Ruivo 1958. Senega 7 . Yamaguti (1963a).

PANDARIDAE

Perissopus dentatus Steenstrup and Lütken 1861. Hewitt and Hine (1972).

* Perissopus oblongatus (Wilson 1908) cressey 1967. Gulf of California. Body surface. Wilson (1944) as Achtheinus d.

Pandarus satyrus Dana 1852. Japan. Body surface. Shi ino (1954e).

Pandarus smithii Rathbun 1886. Brazi1. Cressey (1967c); Japan. Yamaguti (1963a).

Pandarus cranchi $i$ Leach 1819. Formosa. Body surface, fins. Cressey (1967c).

Nesippus crypturus Heller 1865. Java; Puerto Rico. Lewis (1966). Nesippus orientalis Heller 1865. South Africa. Mouths, gi11 arches. Cressey (1967c); Angola. Nunes-Ruivo (1956). 
Nesippus orientalis Heller 1865. North Carolina, Massachusetts. Throat. Wilson (1932) as N. alatus.

Echthrogaleus denticulatus Smith 1874. Japan. Body surface. Shiino (1959e).

Phyllosthyreus cornutus Bassett - Smith 1899. Massachusetts. Gills. Wilson (1924) as Parapandarus nodulosus.

Pannosus japonicus. Japan. Body surface. Shiino (1960b) às Gangliopus j.

\section{MONOGENEA}

\section{CAPSALIDAE}

Tristoma coccineum Cuvier 1817. Massachusetts. Price (1939). HEXABOTHRI IDAE

Erpocotyle sphyrnae (MacCa11um 1931) Price 1942. Massachusetts. Gi77s. Price (1942).

Erpocotyle microstoma (Brooks 1934) Yamaguti 1963. North Carolina. Price (7942) as Neoerpocotyle m.; North Carolina. Gi11s. Brooks (1934) as Squalonchocotyle microstoma.

Erpocotyle grisea (Cerfontaine 1899). Atlantic. Price (1942) as Neoerpocotyle.

Neoerpocotyle platens is Mañe-Garzón 1968. Uruguay. Gills. Mane-Garzon and Hoffman-Spector (1968).

\section{LOIMOIDAE}

Loimosina wilsoni Manter 1944. Jamaica. Gills. Yamaguti (1963b). MONOCOTYLIDAE

Cathariotrema selachii (MacCallum 1916) Johnston and Tiegs 1922. Massachusetts. Nasal glands. MacCallum (1916) as Monocotyle selachii.

\section{NEMATODA}

\section{ACANTHOCHEILIDAE}

Acanthocheilus nudiflex Linton 1900. Massachusetts. Yamaguti (1961) ["Should not be referred to this genus" - Yamaguti (1961)].

\section{ANISAKIDAE}

Terranova galeocerdonis (Thwaite 1927) Johnston and Mawson 1945. West Africa. Campana-Rouget (1955).

\section{PROTOZOA (MYXOSPORIDA)}

\section{CERATOMYXIDAE}


Sphyrna zygaena (Continued)

Ceratomyxa recurvata Davis 1917. North Carolina. Gallbladder. Kudo $(1920)$.

Ceratomyxa mesospora Davis 1917. North Caroina. Gailibiadder. Kudo $(1920)$.

Leptotheca fusiformis Davis 1917. North Carolina. Gallbladder. Kudo $(1920)$.

SPHAEROSPORIDAE

Chloromyxum sp. India. Gallbladders. Tripathi (1953).

Chloromyxum leydigi Mingazzini 1890. North Carolina. Gallbladder. Kudo $(1920)$.

\author{
SQUALIDAE -- Dogfish Sharks \\ Euprotomicrus bispinatus - Pygmy Shark
}

CESTODA

SPHYRIOCEPHALIDAE

Sphyriocephalus tergestinus Pintner 1913. Pacific and Indian Oceans. Stomach. Hubbs et a]. (1967).

Sphyriocephalus sp. North central Pacific; Indian Ocean, South Pacific. Stomach. Dollfus (1967).

\author{
Somniosus pacificus - Pacific Sleeper Shark \\ SYNONYMS - Somniosus microcephalus, Somniosus brevipinna
}

CESTODA

PHYLLOBOTHRI IDAE

Phyl lobothrium magnum Hart 1936. Washington. Hart (1936b)

[= Monogyra m. - Williams (1968)].

Monorygma perfectum (Van Beneden 1853) Diesing 1863. Alaska.

Stomach. Linton (1924).

MONOGENEA

HEXABOTHRI IDAE

Erpocotyle somniosi (Causey 1926) Price 1942. Alaska. Gills.

Guberlet (1936a) as Squalonchocotyle s. 
SYNONYMS - Acanthias vulgaris, Squalus lebruni, Squalus kirki, Squalus suckleyi

\section{ACANTHOCEPHALA}

ECHINORHYNCHIDAE

Echinorhynchus gadi Zoega in Müller 1776. Golvan (1969).

CESTODA

EUTETRARHYNCHIDAE

Eutetrarhynchus ruficollis (Eysenhardt 1829) Pintner 1913. France. Joyeux and Baer (1936).

\section{GILQUINIDAE}

Gilquinia squali (Fabricius 1794) Dollfus 1930. Southern California. Heinz and Dailey (1974); British Columbia. Margol is ${ }^{3}$; Newfoundland. Threlfal1 (1969); Sea of Japan. Zhukov (1960a).

HEPATOXYLIDAE

Hepatoxylon trichiuri (Holten 1802) Dollfus 1942. Postlarva. New Zealand. Body cavity. Robinson (1959a); Mediterranean. Dollfus (1942).

\section{LACISTORHYNCHIDAE}

Grillotia erinacea (Van Beneden 1858) Guiart 1927. Massachusetts. Spiral valve. Linton (1924) as Rhynchobothrium imparispine. Lacistorhynchus tenuis (Van Beneden 1858) Dollfus 1929. France. Joyeux and Baer (1936); Massachusetts. Spiral valve. Linton (1924) as Rhynchobothrium bulbifer.

\section{ONCOBOTHRI IDAE}

Acanthobothrium coronatum (Rudolphi 1819) Van Beneden 1849. France. Joyeux and Baer (1936).

Calliobothrium verticillatum (Rudolphi 1819) Van Beneden 1850. Massachusetts. Spiral valve. Linton (1924); France. Joyeux and Baer (1936).

\section{OTOBOTHRI IDAE}

Otobothrium crenacolle Linton 1890. Larva. Massachusetts. Stomach. Linton (1924). 
PHOREIOBOTHRIIDAE

Trilocularia acanthiaevulgaris (01sson 1867) 01sson 1869. France. Euzet (1956); immature. Iceland. Stomach, spiral valve. Manger (1972); New Zealand. Alexander (1963); Newfoundland. Threlfall (1969) as I. gracilis.

\section{PHYLLOBOTHRIIDAE}

Phyllobothrium gracile Wed1 1855. Sea of Japan. Zhukov (1960a). Phyllobothrium thridax Van Beneden 1849. Sea of Japan. Zhukov (1960a).

Phyllobothrium squali Yamaguti 1952. Japan. Yamaguti (1952); France. Euzet (1956) as Crossobothrium s. [=Crossobothrium s. Williams (1968)].

Phyllobothrium lactuca Van Beneden 1850. France. Joyeux and Baer (1936).

Phyllobothrium sp. Newfoundland. Threlfall (1969).

Scyphophyllideum giganteum (Van Beneden 1858) Woodland 1927. Newfoundland. ThrelfalT (1969).

Rhodobothrium pulvinatum Linton 1889. Massachusetts. Yamaguti $(1959 b)$.

Monorygma sp. Massachusetts. Linton (1924).

Anthobothrium pulvinatum Linton 1891. Massachusetts. Linton (1924).

PROSOBOTHRIIDAE

Prosobothrium armigerum Cohn 1902. Attantic. Cohn (1902); France. Joyeux and Baer (1936).

\section{PTYCHOBOTHRIIDAE}

Clestobothrium crassiceps (Rudolphi 1808) Lühe 1899. Massachusetts. "Introduced with Food". Linton (1924).

\section{TENTACULARIIDAE}

Nybelinia bisulcata (Linton 1889) Dollfus 1929. Massachusetts. Stomach. Dollfus (1942).

TRYPANORHYNCHA

Tetrarhynchus tetrabothrium Van Beneden 1849. Central California. Pintner (1930).

COPEPODA

CALIGIDAE

*Caligus curtus Mü1ler 1785. Scandanavia. Parker et a1. (1968). Caligus elongatus Nordmann 1832. New England. External surface. Wilson (1905b); Greenland, North Sea. Wilson (1932) both as C. rapax. 
Caligus coryphaenae Steenstrup and Lütken 1861. Indian Ocean. Fins. Wilson (1923); South Africa. Barnard (1955). Lepeophtheirus cuneifer Kabata 1974. Alaska. Kabata (1974). Questionable host.

\section{CECROPIDAE}

Philorthagoriscus serratus (Krøyer 1863) Horst 1897. Newfoundland. Yamaguti (1963a).

\section{EUDACTYLINIDAE}

Eudactylina acuta Van Beneden 1853. Britain. Gills. Leigh-Sharpe (1933).

Eudactylina acanthii Scott 1901. Angola. Nunes-Ruivo (1956); British Columbia. Bere (1930); New Brunswick. Gills. Bere (1930).

Eudactylina valei Nunes-Ruivo 1956. Angola. Nunes-Ruivo (1956).

EURYPHORIDAE

Alebion gracilis Wilson 1905. Layman Island, Pacific. Outside surface. Wilson (1932).

Alebion glaber Wilson 1907. Massachusetts. Exterior. Wilson $(1907 a)$.

\section{LERNAEOPODIDAE}

Lernaeopoda galei Krøyer 1837. England. Exterior. BassettSmith $(1896)$.

Pseudocharopinus bicaudatus (Krøyer 1837) Kabata 1964.

Massachusetts. WiTson (1932); Britain. Delamare-Deboutteville and Nunes (1952) both as Charopinus bicaudatus.

\section{PANDARIDAE}

Demoleus latus Shiino 1954. New Zealand. Hewitt (1967).

Demoleus heptapus (0tto 1821) Heller 1865. Britain. Body surface . Leigh-Sharpe (1933) as D. paradoxus.

Pandarus spinaciiacantias Hesse 1883. Wilson (1907b) as Nogagus $s-a$ (Species Inquirae).

Pandarus bicolor Leach 1816 . North Atlantic, Netherlands. Body surface. Cressey (1967c); Central California. Wilson (1935b); New Zealand. Hewitt (1967).

* Perissopus oblongatus (Wilson 1908) Cressey 1967. Angola. Nunes-Ruivo (1956) as Achtheinus dentatus.

Nogagus ambiguus Scott 1907 . Britain. Body surface . Leigh-Sharpe (1933) [A male of some species of Perissopus Yamaguti (1963a)].

Echthrogaleus coleoptratus (Guerin 1837) Wilson 1907. Newfoundiand. Yamaguti (7963a). 
Squalus acanthias (Continued)

TREB I IDAE

Trebius caudatus Krøyer 1838. Lewis (1966).

\section{DIGENEA}

\section{AZYGIIDAE}

Otodistomum veliporum (Creplin 1837) Columbia. Stomach. Margol is3;

Stafford 1904. British Newfoundland. Threlfall (1969).

\section{BUCEPHALIDAE}

Prosorhynchus squamatus Odhner 1905. Gulf of St. Lawrence. Myers (1959).

\section{GORGODERIDAE} Body cavity. Manter (1954a) as P. phillipi.

\section{HEMIURIDAE}

Genarchops is muelleri (Levinsen 1881) Yamaguti 1954. Sea of Japan. Stomach. Zhukov (1960a) as Genarches m.

Derogenes varicus (Mül ler 1784) Looss 1901. Newfound and.

Threlfalt (1969).

Hemiurus levinseni Odhner 1905. Gulf of St. Lawrence.

$$
\text { Myers (1959). }
$$

\section{ZOOGONIDAE}

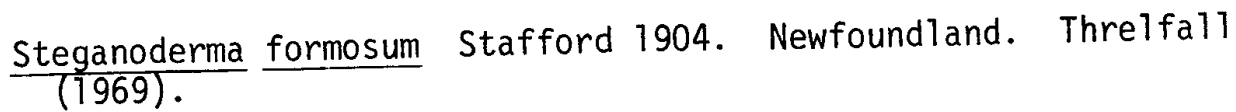

HIRUDINOIDEA

\section{PISCICOLIDAE}

Branchellion lobata Moore 1952. Central Ealifornia. Body surface.

Calliobdella vivida (Verrill 1872) Sawyer, Lawyer and Overstreet 1975. Newfoundland. Threlfal1 (1969) as $\underline{C}$. nodulifera.

MONOGENEA

\section{DACTYLOGYRIDAE} $\frac{\text { Amphibdelloides maccallumi (Johnston and Tiegs 1922) Price } 1937 .}{\text { Massachusetts. }}$ 
Squalus acanthias (Continued)

\section{HEXABOTHRI IDAE}

Hexabothrium appendiculata (Kuhn 1829) Nordmann 1840. Britain. Gills. Nic011 (1915); Canadian Atlantic. Stafford (1904) both as Onchocotyle a.

Erpocotyle abbreviata 01sson 1876. Washington. Guberlet (1932); Newfoundland. Threlfall (1969); Norway. Brinkman (1952) as Squaloncocotyle a.

Erpocotyle squali (MacCallum 1931) Price 1942. Massachusetts. Gills. Price (1942); New Zealand.

Gills. Dillon and Hargis (1968).

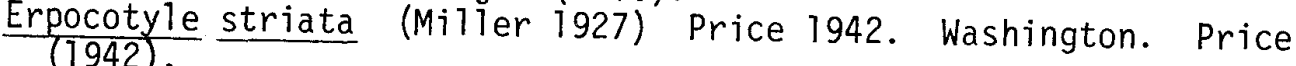

\section{MICROBOTHRI IDAE} $\frac{\text { Microbothrium apiculatum }}{\text { Dawes (1947). }}$.

\section{MONOCOTYLIDAE}

Heterocotyle minima (MacCallum 1916) Price 1938. Massachusetts.

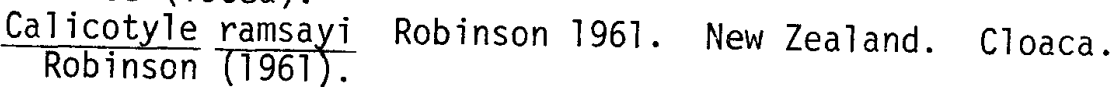

\section{NEMATODA}

\section{ACANTHOCHEILIDAE}

Pseudanisakis tricupola Gibson 1973. Newfoundland. Threlfall (1969) as Eustoma rotundatum.

Acanthocheilus quadridentatus Mol in 1858. Northern California. Stomach waTT. Pappas (1970).

Acanthocheilus bicuspis (Wed7 1855) York and Maplestone 1926.

Mediterranean. Digestive tracts. Nikolaeva and Naidenova (1964).

\section{ANISAKIDAE}

Anisakis sp. Larva. British Columbia. Stomach. Margolis³;

New Zealand. Hewitt and Hine (1972).

* Thynnascaris clavata (Rudolphi 1809) Hartwich 1957. Newfoundland. Threlfal1 (1969) as Contracaecum C.

$\frac{\text { Phocanema sp. Larva. Newfoundland. Threlfall (1969) as Porrocaecum }}{\text { sp. }}$

\section{PHILOMETRIDAE}

Phlyctainophora squali Mudry and Dailey 1961. Southern California. Subcutaneous tissues. Mudry and Dailey (1969). 
Squalus acanthias (Continued)

Proleptus obtusus Dujardin 1845. Atlantic Coast. Yamaguti (1961). Proleptus acutus Dujardin 1845. Mediterranean. Stomach.

Nikolaeva and Naidenova (1964) as $\underline{P}$. robustus.

PROTOZOA (MYXOSPORIDA)

SPHAEROSPORIDAE

Chloromyxum leydigi Mingazzini 1890. England. Dunkerly (1920). Chloromyxum ovatum Jameson 1929. Central California. Gallbladder.

PROTOZOA (SPOROZOA)

EIMER I IDAE

Eimeria lucida (Labbe 1893) Reichenow 1921. Spiral valve. Pellerdy (7965).

Eimeria squali Fitzgerald 1975. Washington. Fitzgerald (1975).

\title{
HAEMOGREGARINIDAE
}

Haemogregarina delagei Laveran and Mesnil 1902. New Brunswick. Blood. Laird and Bullock (T969).

\author{
SQUATINIDAE -- Angel Sharks \\ Squatina californica - Pacific Angel Shark
}

CESTODA

LACISTORHYNCHIDAE

Grillotia smarisgora (Wagener 1854) Dollfus 1947. Southern

California. Heinz and Dailey (1974).

DIGENEA

AZYGI IDAE

Otodistomum veliporum (Crepl in 1837) Stafford 1904. Southern California. Stomach. Caballero and Caballero (1969b).

HIRUDINOIDEA

PISCICOLIDAE

Branchellion lobata Moore 1952. Central California. Body surface. Moore $(1952)$. 
Squatina californica (Continued)

PROTOZOA (MYXOSPORIDA)

SPHAEROSPORIDAE

Chloromyxum levigatum Jameson 1931. Southern California. Ga1Tbladder. Jameson (1931).

\author{
RHINOBATIDAE -- Guitarfishes \\ Platyrhinoidis triseriata - Thornback
}

CESTODA

EUTETRARHYNCHIDAE

Prochristianella minima Heinz and Dailey 1974. Southern California. Spiral valve. Heinz and Dailey (1974).

ONCOBOTHRI IDAE

Acanthobothrium goldsteini Appy and Dailey 1973. Southern California. Spiral valve. Appy and Dailey (1973).

DIGENEA

GORGODERIDAE

Probolitrema richiardij (Lopez 1888) Looss 1902. California.

Stunkard $(1935)$ as P. californiense.

NEMATODA

PHYSALOPTERIDAE

Proleptus acutus Dujardin 1845. Southern California. Stomach. Specian et a1. (1975).

Rhinobates productus - Shovelnose Guitarfish

CESTODA

EUTETRARHYNCHIDAE

Eutetrarhynchus schmidti Heinz and Dailey 1974. Southern California. Spiral valve. Heinz and Dailey (1974).

Prochristianella fragilis Heinz and Dailey 1974. Southern

California. Spiral valve. Heinz and Dailey (1974).

Parachristianella monomegacantha Kruss 1959. Southern California.

Heinz and Dailey (7974). 
Rhinobatos productus (Continued)

LACISTORHYNCHIDAE

Lacistorhynchus tenuis (Van Beneden 1858) Dollfus 1929. Southern California. Heinz and Dailey (1974).

ONCOBOTHRIIDAE

Acanthobothrium dujardinii Van Beneden 1849. Southern California. Young (1954).

Acanthobothrium rhinobati Alexander 1953. Southern California. Spiral valve. Alexander (1953).

Acanthobothrium robustum Alexander 1953. Southern California. Spiral valve. Alexander (1953).

Acanthobothrium olseni Dailey and Mudry 1968. Southern California. Spiral vaTve. Dailey and Mudry (1968).

\section{TETRAPHYLLIDEA}

Genera and species. Red pigmented plerocercoid. Southern California. Stomachs. Kunnenkeri and Martin (1962).

COPEPODA

\section{PANDARIDAE}

*Perissopus oblongatus (Wilson 1908) Cressey 1967. Southern California. Flesh. Ho (1975) as Ächtheinus obiongus.

\section{DIGENEA}

\section{GORGODERIDAE}

Probolitrema richiardij (Lopez 1888) Looss 1902. California. Body cavity. Stunkard (1935) as P. californiense.

\section{MONOGENEA}

\section{HEXABOTHRI IDAE}

Rhinobatonchocotyle cyclovaginatus Doran 1953. Southern California. Gil7s. Doran (1953).

\section{MONOCOTYLIDAE}

Spinuris lophosoma Doran 1953. Southern California. Gills. Doran (1953). Anoplocotyloides papillata (Doran 1953) Young 1967. Southern California. Gills. Doran (1953) as Heterocotyle p. Genera and species. Eastern Pacific. 01factory cavities. Kunnenkeri and Martin (1963). 
TORPEDINIDAE -- Electric Rays

Torpedo californica - Pacific Electric Ray

SYNONYM - Tetronarce californica

CESTODA

ONCOBOTHRI IDAE

Acanthobothrium hispidum Riser 1955. Central California. Spiral valve. Riser (1955).

\section{MONOGENEA}

DACTYLOGYRIDAE

Amphibdelloides maccallumi (Johnston and Tiegs 1922) Price 1937. Southern California. Gills. Alexander (1954).

PROTOZOA (MYXOSPORIDA)

SPHAEROSPORIDAE

Chloromyxum leydigi Mingazzini 1890. Central California. Galibladder. Jameson (1929).

Chloromyxum ovatum Jameson 1929. Central California. Gallbladder. Jameson (1929).

$$
\begin{gathered}
\text { RAJIDAE -- Skates } \\
\text { Raja binoculata - Big Skate }
\end{gathered}
$$

CESTODA

ONCOBOTHRIIDAE

Acanthobothrium dujardinii Van Beneden 1849. Washington. Hart 1936b). $\frac{\text { Acanthobothrium brachyacanthum Riser 1955. Central California. }}{\text { Riser (1955). }}$

Pinguicollum pinguicollum (Sleggs 1927) Riser 1955. Central California. Riser (7955).

PHYLLOBOTHRI IDAE

Echeneibothrium myzorhynchum Hart 1936. Central California. Riser (1955). ["Provisionally accepted as a valid species." Williams 1966)].

Echeneibothrium octorchis Riser 1955. Central California. Riser (1955). 
Raja binoculata (Continued)

Phyllobothrium radioductum Kay 1942. Washington. Kay (1942a); Central California. Spiral valve. Riser (1955).

COPEPODA

CALIGIDAE

Lepeophtheirus parviventris Wilson 1905. British Columbia. Fraser (1920); Kabata (1973).

Lepeophtheirus cuneifer Kabata 1974. Alaska. Kabata (1974).

Lepeophtheirus pravipes Wilson 1912. British Columbia. Kabata (1973).

\section{LERNAEOPODIDAE}

Schistobrachia tertia Kabata 1970. British Columbia. Gills and buccal cavity. Kabata (1970c).

Pseudocharopinus dentatus (Wilson 1912) Kabata 1964. Oregon. Surface. Wilson (1935b); British Columbia. Fraser (1920) both as Charopinus d.

Lernaeopoda gale Krøyer 1837. NorthernCalifornia. Head. Russo (1975) as L. Scylicola.

\section{PANDARIDAE}

*Perissopus oblongatus (Wilson 1908) Cressey 1967. California. Wilson (1911) as Achtheinus dentatus.

\section{DIGENEA}

\section{AZYGIIDAE}

Otodistomum veliporum (Creplin 1837) Stafford 1904. Washington. Stomach. Lloyd (1938); Oregon. Pratt and McCauley (1961).

HIRUDINOIDEA

\section{PISCICOLIDAE}

Branchellion lobata Moore 1952. Central California. Body surface. Moore (1952).

Orientobdella sp. Oregon. Exterior surface. Burreson?

\section{MONOGENEA}

\section{ACANTHOCOTYLIDAE}

A1 lacanthocotyla pugetensis (Guberlet 1934) Yamaguti 1963. Washington. GiTls, nasaT filaments. Robinson (1961) as Acanthocotyle p; Northern California. Crane (1972).

Pseudacanthocotyla pacifica (Guberlet 1937) Yamaguti 1963. Washington. On body, gi17s, nasal filaments. Robinson (1961) as Acanthocotyle p. 
Raja binoculata (Continued)

HEXABOTHRI IDAE

Rajonchocotyle sp. Washington. Guberlet (1936a).

Rajonchocotyle batis Cerfontaine 1899. Washington. Gills. Robinson (1961).

MONOCOTYLIDAE

Merizotyle pugetensis Kay 1942. Washington. Nostrils. Kay (1942a). PROTOZOA (SARCOMASTIGOPHORA)

TRYPANOSOMATIDAE

Trypanosoma gargantua Laird 1951. Oregon. Blood. Burreson?.

$$
\begin{aligned}
& \text { Raja inornata - California skate } \\
& \text { SYNONYM - Raja montereyensis }
\end{aligned}
$$

\section{CESTODA}

ONCOBOTHRI IDAE

Acanthobothrium brachyacanthum Riser 1955. Central California. Riser (1955).

Pinguicollum pinguicollum (Sleggs 1927) Riser 1955. Central California. Riser (1955).

PHYLLOBOTHRI IDAE

Discobothrium (=Echeneibothrium) fallax Van Beneden 1870. Central California. Spirla valve. Riser (1955). "Generic position and systematic identity unclear" - Williams (1966)

Echeneibothrium octorchis Riser 1955. Central California. Riser (1955).

Echeneibothrium macrascum Riser 1955. Central California. Riser (1955).

Phyllobothrium radioductum Kay 1942. Central California. Spiral valve. Riser (1955).

\section{COPEPODA}

\section{LERNAEOPODIDAE}

Bendrata cameroni longiclavata Kabata and Gussev 1966. British CoTumbia. Skin. Kabata (1970c). 


\section{Raja rhina - Longnose Skate}

CESTODA

LACISTORHYNCHIDAE

Grillotia musculara (Hart 1936) Dollfus $1942 . \quad$ Washington.

Spiral valve. Hart (1936a) as Tentacularia m.

ONCOBOTHRI IDAE

Pinguicollum pinguicollum (Sleggs 1927) Riser 1955. Central California. Riser (1955).

PHYLLOBOTHRIIDAE

Discobothrium [=Echeneibothrium] fallax Van Beneden 1870. Central California. Spiral valve. Riser (1955) ["Generic position and systematic identity unclear" - Williams (1966)].

Echeneibothrium octorchis Riser 1955. Central California. Riser (7955).

Echeneibothrium dolichoophorum Riser 1955. Central California. Riser (1955).

Echeneibothrium dubium abyssorum Campbe11 1977. Western North Atlantic. Spiral valve. Campbe11 (1977).

Phyllobothrium radioductum Kay 1942. Central California. Spiral valve. Riser (1955).

COPEPODA

CAL IGIDAE

Lepeophtheirus parviventris Wilson 1905. British Columbia. Kabata (1973).

\section{LERNAEOPODIDAE}

Schistobrachia tertia Kabata 1970. British Columbia.

Gi71s and buccal cavity. Kabata (1970c).

Lernaeopodina pacifica Kabata and Gussev 1966. British Columbia. GiT7s. Kabata (1970 c).

Pseudocharopinus dentatus (Wilson 1912) Kabata 1964. British

Columbia. Buccal cavity, gills, skin, claspers. Kabata (1970c).

\section{DIGENEA}

\section{AZYGI IDAE}

Otodistomum veliporum (Creplin 1837) Stafford 1904.

British Columbia. Stomach. Margolis ${ }^{3}$.

\section{MONOGENEA}


Raja rhina (Continued)

\section{ACANTHOCOTYLIDAE}

Pseudacanthocotyla pacifica (Guberlet 1937) Yamaguti 1963. Washington. Bonham and Guberlet (1938).

NEMATODA

ANISAKIDAE

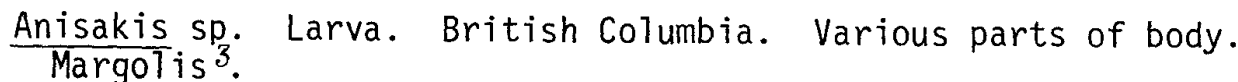

$$
\text { Raja stellulata - Starry Skate }
$$

MONOGENEA

ACANTHOCOTYLIDAE

Pseudacanthocotyla pacifica (Guberlet 1937) Yamaguti 1963. Washington. Bonham and Guberlet (1938) as Acanthocotyla p.

HEXABOTHRI IDAE

Rajonchocotyie batis Cerfontaine 1899. Washington. Price (1942).

$$
\begin{gathered}
\text { DASYATIDAE -- Stingrays } \\
\text { Dasyatis violacea - Pelagic Stingray }
\end{gathered}
$$

CESTODA

ONCOBOTHRI IDAE

Acanthobothrium magnum (Euzet 1956) Goldstein 1967. France. Euzet (1956) as A. crassicalle.

Acanthobothrium benedeni Lönnberg 1889. Mediterranean. Williams (1969).

PHYLLOBOTHRI IDAE

Rhinebothrium palombi Baer 1948. France. Euzet (1956).

TETRAGONOCEPHALIDAE

Tetragonocephalum trygonis Shipley and Hornel1 1905. France. Euzet (1956). 
Dasyatis violacea (Continued)

CAPSAL IDAE

Entobdella diadema (Monticelli 1902) Johnston 1929. Mediterranean. Yamaguti $(1963 b)$.

Gymnura marmorata - California Butterfly Ray

SYNONYM - Pteroplatea marmorata

CESTODA

ONCOBOTHRI IDAE

Acanthobothrium parviuncinatum Young 1954. Southern California. Young (1954).

COPEPODA

TREBI IDAE

Trebius latifurcatus Wilson 1921. Southern California. Exterior. WiTson (1927).

$$
\begin{aligned}
& \text { Urolophus halleri - Round Stingray } \\
& \text { SYNONYM - Urobatis halleri }
\end{aligned}
$$

CESTODA

EUTETRARHYNCHIDAE

Christianella trygonbrucco (Wagener 1854) Joyeux and Baer 1936. California. Young (1954).

Parachristianella trygonis Dollfus 1946. Southern California. Young (1954).

Eutetrarhynchus schmidti Heinz and Dailey 1974. Southern California. Spiral valve. Heinz and Dailey (1974).

Mecistobothrium myliobati Heinz and Dailey 1974. Southern

California. Spiral valve. Heinz and Dailey (1974).

Prochristianella minima Heinz and Dailey 1974. Southern California.

Spiral valve. Heinz and Dailey (1974).

ONCOBOTHRI IDAE

Acanthobothrium olseni Dailey and Mudry 1968. Southern California. Appy and Dailey (1973).

Acanthobothrium dujardini Van Beneden 1849. Southern California. Young (1954).

Acanthobothrium crassicolle Wed1 1855. Southern California. Young (1954). 
PHYLLOBOTHRI IDAE

Anthobothrium laciniatum Linton $189 \%$. Southern Cal ifornia. Young (1954), California. Linton (1891).

Anthobothrium oligorchidum Young 1954. Southern California. Young (1954).

Rhinebothrium flexile Linton 1891. Southern California. Young (1954) as Echeneibothrium $f$.

Rhinebothrium tumidulum Rudolphi 1819. Southern California. Young (1954) as Echeneibothrium $t$.

Rhinebothrium ditesticulum Appy and Dailey 1977. Southern

California. Spiral valve. Appy and Dailey (1977).

Rhinebothrium minimum Euzet 1956. Southern California. Young (1954) as Echeneibothrium m.

Rhinebothrium bilobatum (Young 1955) Appy and Dailey 1977.

California. Yamaguti (1959b) as Echeneibothrium b.

Rhinebothrium urobatidium (Young 1955) Appy and Dáiley 1977.

Southern California. Yamaguti (1959b) as Echeneibothrium $u$.

Caulobothrium multorchidum (Young 1954) Appy and Dailey 1977. Southern California. Young (1954) ["Not in this genus" Williams (1966)] as Echeneibothrium $\mathrm{m}$.

\section{TETRAPHYLLIDEA}

Genera and species. Red pigmented plerocercoid. Southern California. Spiral valve. Kunnenkeri and Martin (1962).

\section{COPEPODA}

CALIGIDAE

Lepeophtheirus parviventris Wilson 1905. California. Wilson (1924).

\section{TREBIIDAE}

Trebius tenuifurcatus Rathbun 1887. Southern California. Exterior. WiTson (1908a).

Trebius latifurcatus Wilson 1921. Southern California. Exterior. WiTson (1927).

\section{MONOGENEA}

CAPSALIDAE

Entobdella guberleti Caballero and Bravo-Holl is 1962. West Coast of Mexico. Gills. Caballero and Bravo-Holl is (1962).

PROTOZOA (MYXOSPORIDA)

SPHAEROSPORIDAE

Chloromyxum ovatum Jameson 1929. California. Gallbladder. Jameson (1931). 


\section{MYLIOBATIDAE -- Eagle Rays}

Myliobatis sp.

ISOPODA

CYMOTHOIDAE

Nerocila californica Schioedte and Meinert 1881. California.

Fins. Richardson (1905). Probably from M. californica.

Myliobatis californica - Bat ray

SYNONYMS - Aetobatus californicus, Holorhinus californicus

CESTODA

ECHINOBOTHRI IDAE

Caulobothrium tetrascaphium Riser 1955. Central California. Riser (1955). Caulobothrium opisthorchis Riser 1955. Central California. Riser (1955).

EUTETRARHYNCHIDAE

Mecistobothrium myliobati Heinz and Dailey 1974. Southern

California. Spiral valve. Heinz and Dailey (1974).

\section{LECANICEPHALIDAE}

Discobothrium myliobatidis Dailey and Mudry 1968. Southern California. Spiral valve. Dailey and Mudry (1968).

ONCOBOTHRIIDAE

Acanthobothrium holorhini Alexander 1953. Southern California. Spiral valve. Alexander (7953).

Acanthobothrium microcephalum Alexander 1953. Southern California. Spiral valve. Alexander (1953).

Acanthobothrium unilateraīis Āi exander 1953. Southern California. Spiral valve. Alexander (1953).

Acanthobothrium maculatum Riser 1955. Central California. Riser (1955).

\section{PHYLLOBOTHRI IDAE}

Echeneibothrium myzorhynchum? Hart 1936. Northern California. Sparks and Chew (1966).

Rhinebothrium flexile Linton 1890. Southern California. Young (1954) as Echeneibothrium $f$.

Inermiphyllidium brachyascum Riser 1955. Central California. Riser (1955). 
Myliobatis californica (Continued)

COPEPODA

TREBI IDAE

Trebius caudatus Krøyer 1838. California. Body surface. Guber 7 et (1936a).

Trebius latifurcatus Wilson 1921. Southern California. Body surface. Wilson (1921).

\title{
DIGENEA
}

GORGODERIDAE

Probolitrema californiense Stunkard 1935. Central California. Coelom. Stunkard (1935).

MONOGENEA

CAPSALIDAE

Pseudoentobdella pacifica (Guberlet 1936) Yamaguti 1963.

California. Price (1939) as Benedenia p.; Guberlet (1936b)

as Epibdella p.

UDONELLIDAE

Udonella caligorum Johnston 1835. On parasitic copepods Trebius caudatus, I. latifurcatus. Central California. Price (1938a).

NEMATODA

GNATHOSTOMATIDAE

Echinocephalus pseudouncinatus Millemann 1951. Gulf of California. Spiral valve. Millemann (1963).

\author{
MOBUL IDAE -- Mantas \\ Mobula lucasana - Smoothtail Mobula
}

COPEPODA

CALIGIDAE

Pupulina brevicauda Wilson 1952. Southern California. Wilson (1952).

Pupulina minor Wilson 1952. Southern California. Mouth, anterior end of body. Wilson (1952). 
CHIMAERIDAE -- Chimaeras

Hydrolagus colliei - Ratfish

ASPIDOCOTYLEĀ

ASPIDOGASTRIDAE

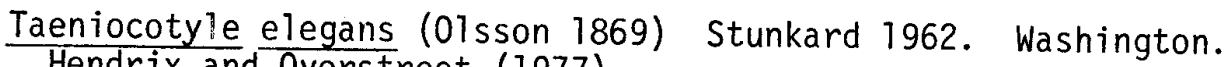
Hendrix and Overstreet (1977).

RUGOGASTRIDAE

Rugogaster hydrolagi Schell 1973. Washington. Rectal gland. Sche11 (1973a).

\section{CESTODARIA}

GYROCOTYLIDAE

Gyrocotyle fimbriata Watson 1911. Washington. Intestine. Lynch (1945); British Columbia. Wardle (1932) as G. urna; Southern California. 01 sori $^{8}$.

Gyrocotyle parvispinosa (Lynch 1945) Land and Dienske 1968. Washington. Intestine. Lynch (1945) as $\underline{G}$. urna parvispinosa.

COPEPODA

\section{CÁLIGIDAE}

Caligus sp. Southern California. Wilson (1908a) as C. gurnardi.

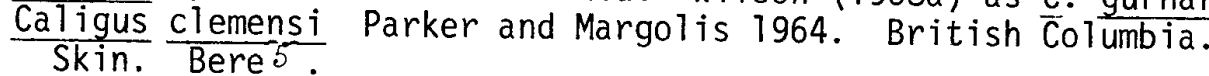

\section{CHONDRACANTHIDAE}

Acanthochondria epachthes (Wilson 1908) 0akley 1930. Central and Southern California. Wilson (1935b); British Columbia. Claspers. Kabata (1968) as A. holocephalarum.

Acanthochondria rectangularis (Fraser 1920) Markevich 1957.

British Columbia. Bere 5 as Chondracanthus r. from "ratfish".

\section{DIGENEA}

\section{AZYGI IDAE}

Otodistomum hydrolagi Schell 1972. Washington. Coelom.

CEPHALOPORIDAE

$\frac{\text { Plectognathotrema hydrolagi }}{01 \text { son et al. (1970). }}$. Hanson and Pratt 1970. Oregon. 
Hydrolagus colliei (Continued)

HIRUDINOIDEA

PISCICOLIDAE

Branchellion lobata Moore 1952. Southern California. 01son ${ }^{8}$. ISOPODA

AEGI IDAE

Rocinela belliceps (Stimpson 1864) Richardson 1899. Richardson (1905).

MONOGENEA

CHIMAERICOLIDAE

Chimaericola leptogaster (Leuckart 1830) Brinkman 1942.

Washington. Bonham (1950) as Octobothrium 1.

NEMATODA

ANISAKIDAE

Anisakis sp. Larva. British Columbia. Smedley (1934) as Contracaecum sp.

PROTOZOA (MYXOSPORIDA)

CERATOMYXIDAE

Leptotheca fisheri (Jameson 1929) Kudo 1933. Central California. GaTlbladder. Jameson (1929) as Ceratomyxa f.

$$
\begin{gathered}
\text { ACIPENSERIDAE -- Sturgeons } \\
\text { Acipenser sp. }
\end{gathered}
$$

CESTODA

AMPHILINIDAE

Amphilina bipunctata Riser 1948. Oregon. Coelom. Riser (1948). Acipenser medirostris - Green Sturgeon

CESTODA

AMPHILINIDAE 
Acipenser medirostris (Continued)

Amphilina japonica Goto and Ishii 1936. Amur Basin, USSR. Body cavity. Dubinina (1971).

MONOGENEA

CAPSALIDAE

Nitzchia quadritestes Pratt and Herrmann 1962. Columbia River. Tip of gill filaments, anterior parts of mouth. Pratt and Herrmann (1962).

DICLYBOTHRIIDAE

Paradiclybothrium pacificum Bykhovsky and Gussev 1950. Tartar Strait, USSR. Gills. Bykhovsky and Gussev (1950).

$$
\text { Acipenser transmontanus - White Sturgeon }
$$

CESTODA

AMPHILINIDAE

Amphilina bipunctata Riser $1948 . \quad$ British Columbia. Body cavity. Margol is 3 .

COPEPODA

CALIGIDAE

Lepeophtheirus saimonis (Krøyer 1838). British Columbia. Kabata (1973).

DIGENEA

DEROPROSTIDAE

Cestrahelmins rivularis Becker 1971. Columbia River. Intestine. Becker (1971).

HEMIURIDAE

Tubulovesicula lindbergi (Layman 1930) Yamaguti 1934. Washington. Stomach. Becker (1970b).

MONOGENEA

CAPSALIDAE

Nitzschia quadritestes Pratt and Hermann 1962. Columbia River. Tips of gill filaments, anterior parts of mouth. Pratt and Herrmann (1962). 
Acipenser transmontanus (Continued)

NEMATODA

ANISAKIDAE

Anisakis sp. Larva. British Columbia. Many places in body. Margot is ${ }^{3}$.

CYSTOOPSIDAE

Cystoopsis acipenseri Wagner 1867. Oregon. Cutaneous vesicles. Yamaguti (7967).

$$
\begin{aligned}
& \text { ALBULIDAE -- Bonefishes } \\
& \text { Albula vulpes - Bonefish }
\end{aligned}
$$

ACANTHOCEPHALA

RHADINORHYNCHIDAE

Illiosentis africanus Golvan 1956. West Africa. Golvan (1956) as I. furcatus africanus.

ASPIDOCOTYLEA

ASPIDOGASTERIDAE

Lobatostoma albulae Yamaguti 1968. Hawaij. Intestine. Yamaguti (1968b).

\section{DIGENEA}

\section{ALBULATREMATIDAE}

Albulatrema ovale Yamaguti 1965. Hawaii. Swim bladder.

$$
\text { Yamaguti }(\overline{\overline{1965 a}}) \text {. }
$$

BOTULISACCIDAE

Botulisaccus pisceus Caballero, Bravo-Hollis, and Grocott 1955. Panama (Pacific Coast). Intestine. Caballero et al. (1955); Florida. Overstreet (1969).

\section{CRYPTOGONIMIDAE}

Claribulla longula Overstreet 1969. Florida. Intestine. Overstreet (1969). 
Albula vulpes (Continued)

HEMIURIDAE

Gonocercella trachinoti (MacCallum 1913) Yamaguti 1954. Florida. Stomach, upper intestine. Overstreet (1969). Lecithochirium albulae Yamaguti 1970. Hawaii. Stomach. Yamaguti (1970).

\title{
MONORCHI IDAE
}

Lasiotocus albulae Overstreet 1969. Florida. Intestine. Overstreet (1969).

Lasiotocus oculatus (Manter and Pritchard 1961) Yamaguti 1970. Hawaii. Intestine. Manter and Pritchard (1961) as Genolopa oculata.

ZOOGONIDAE

Diphtherostomum albulae Overstreet 1969. Florida. Overstreet (1969).

\section{MONOGENEA}

\section{PTERINOTREMATIDAE}

Pseudopterinotrema albulae Yamaguti 1966. Hawaii. Gills. Yamaguti (1966).

Pterinotrema macrostomon Caballero, Bravo-Hollis, and Grocott 1954. Panama. Gills. Caballero et al. (1954).

Pterinotrema mirabil is Bykhovsky and Nagibina 1959. South China Sea. Gills. Bykhovsky and Nagabina (1959).

NEMATODA

CYSTIDICOLIDAE

Spinitectus beaveri Overstreet 1970. Florida. Mucosa of stomach. Overstreet (1970).

\author{
MURAENIDAE -- Morays \\ Gymnothorax mordax - California Moray \\ SYNONYM - Lycodontis mordax
}

COPEPODA

HATSCHEKI IDAE

Hatschekia pinguis Wilson 1908. Southern California. Gills. Wilson (1908a). 
Gymnothorax mordax (Continued)

DIGENEA

BUCEPHALIDAE

Dollfustrema californiae Montgomery 1957. Southern California. Intestine. Montgomery (1957); Baja California. Stomach.

Reid et a1. (1965).

HEMIURIDAE

Magniscyphus taboganus (Sogandres-Bernal 1959) Reid, Coil and Kuntz (1965). Southern California. Intestine. Reid et al. (1965).

Lecithophyl lum intermedium (Manter 1934) Margol is 1958. Baja California. Intestine. Arai (1962).

Sterrhurus taboganus Sogandares-Bernal 1959. Baja

California. Stomach. Arai (1962).

HIRUDINOIDEA

PISCICOLIDAE

Aestabdella abditovesiculata (Moore 1952) Burreson 1976.

California. Burreson (1976a).

\author{
NEMICHTHYIDAE -- Snipe Eels \\ Serrivomer sector - Sawtooth Snipe Ee1
}

\title{
NEMATODA
}

\section{ANISAKIDAE}

Anisakis sp. Larva. Southern California; Baja California. Mesentery, intestine. Collard 9 .

Phocanema sp. Larva. Baja California. Mesentery.

Col1ard 9 as Terranova sp.

CYSTIDICOLIDAE

Ascarophis sp. Larva. Southern California. Mesentery. Collard ${ }^{9}$. PROTOZOA (MYXOSPORIDA)

Genera and species. Southern California. Gallbladder. Noble (1966a). 


\section{CLUPEIDAE -- Herrings \\ Alosa sapidissima - American Shad}

BRANCHIURA

ARGULIDAE

Argulus canadensis Wilson 1916. Connecticut. Opercula.

Davis $(7956)$.

CESTODA

PHYLLOBOTHRI IDAE

Phyllobothrium caudatum (Zschokke and Heitz 1914). Northern

California. Intestine. Hensley and Nahhas (1975) as

Pelichnibothrium speciosum.

TETRAPHYLLIDEA

Genera and species, Red pigmented plerocercoids. Southern California.

Intestine, pyloric caeca. Kunnenkeri (1962).

COPEPODA

\section{CALIGIDAE}

Caligus elongatus Nordmann 1832. New England. External surface. Wilson (1905b) as C. rapax.

\section{DIGENEA}

\section{CLINOSTOMAT IDAE}

C1 inostomum marginatum (RudoTphi 1819) Braun 1899. Metacercaria. Maryland. Throughout body. Holl is and Coker (1948).

\section{NEMATODA}

\section{ANISAKIDAE}

Raphidascaris sp. Northern California. Coelom, mesentery. Hensley and Nahhas (1975).

Contracaecum brachyurum (Ward and Magath 1917). Northern Cal ifornia. Intestine, caeca. Hensley and Nahhas (1975).

\section{Clupea harengus harengus - Atlantic Herring}

$$
\text { and C. h. pallasij, Pacific Herring }
$$

Hubbs (pers. comm.) states that the Atlantic (C. $\underline{h}$. harengus) and Pacific forms (c.h. pallasij) are treated various $\overline{T y}$ as conspecific or distinct species by different authorities. We have lumped them under a single species with two subspecies. Fish taken from the Bering Sea or Pacific Ocean are C. h. pallasii and those from the Atlantic Ocean, North Sea, Baltic Sea or White Sea are $\underline{C}$. $\underline{h}$. harengus. 
Clupea harengus (Continued)

\section{ACANTHOCEPHALA}

\section{ECHINORHYNCHIDAE}

Echinorhynchus gadi Zoega in Müller 1776. Baltic Sea. Intestine. Dollfus (1956); Kamchatka, USSR. Strelkov (1960).

Metechinorhynchus salmonis (Mü11er 1784) Petrotchenko 1956.

Finland. Intestine. Dollfus (1956) as Echinorhynchus s.

Pseudoechinorhynchus clavula (Dujardin 1845) Petrotchenko 1956.

Finland. Intestine. Dol7fus (1956) as Echinorhynchus c.

Acanthocephalus lucii? (Müller 1776) Lühe 1911. Golvan Ti969).

POLYMORPHIDAE

Corynosoma strumosum (Rudolphi 1802) Lühe 1904. Larva. Kamchatka, USSR. Skryabina (1963); Baltic Sea. Peritoneum. Dollfus (1956). Corynosoma semerme (Forsse11 1904) Lühe 1911. Larva. Baltic Sea. Body cavity. Dollfus (1956).

\section{POMPHORHYNCHIDAE}

Pomphorhynchus kostylewi Petrochenko 1956. Baltic Sea. Intestine. Rokicki (7973).

Pomphorhynchus laevis (Zoega in Müller 1776) Van Cleave 1924. Baltic Sea. Intestine. Dollfus (1956).

\section{RHADINORHYNCHIDAE}

Serrasentis sagittifer (Linton 1889) Linton 1932. Postlarva. U.S. Atlantic. Abdomen. Dollfus (1956) as Serrasentis socialis.

BRANCHIURA

ARGULIDAE

Argulus alosae Gould 1912. Western North Atlantic. Body surface. Sindermann (1957).

CESTODA

\section{AMPHI COTYLIDAE}

Eubothrium crassum (BToch 1779) Nybelin 1922. Plerocercoid. Finland. Stomach, intestine. Dollfus (1956).

\section{BOTHRIOCEPHALIDAE}

Bothriocephalus scorpij (Müller 1776) Rudolphi 1808. Massachusetts. Intestine. Do 11fus (1956).

\section{LACISTORHYNCHIDAE}

Lacistorhynchus tenuis (Van Beneden 1858) Do11fus 1929. Larva.

Massachusetts. Muscle, viscera. Dollfus (1956). Grillotia erinacea (Van Beneden 1858) Guiart 1927. Larva. Massachusetts. Stomach. Dollfus (1956). 
Clupea harengus (Continued)

PHYLLOBOTHRIIDAE

Phyllobothrium sp. Larva. British Columbia. Intestine. Ârai (1969b).

PSEUDOPHYLLIDEA

Genera and species. Kamchatka, USSR. Skryabina (1963).

TETRAPHYLLIDEA

Scolex pleuronectis Müiler 1788. Sweden. Digestive tube. Dollfus (1956); Sea of Japan. Intestine, gallbladder. Zhukov (1960a);

California. Kunnenkeri (1962).

COPEPODA

BOMOLOCHIDAE

Bomolochus cuneatus Fraser 1920. British Columbia. Fraser (1920).

CALIGIDAE

Caligus clemensi Parker and Margolis 1964. British Columbia. External. Parker and Margolis (1964); Fraser (1920) as C. gurnardi.

Caligus elongatus Nordman 1832. Scotland. Dol1fus (1956) as C. rapax. Lepeophtheirus pollachius Bassett-Smith 1896. Scotland. Buccál cavity. Dollfus $(1956)$.

PENNELLIDAE

Lernaeenicus sprattae (Sowerby 1806) 01sson 1869. Britain. Dollfus $(1956)$.

\section{DIGENEA}

BUCEPHALIDAE

Bucephalus polymorphus Baer 1827. Yamaguti (1971).

Prosorhynchoides gracilescens (Rudolphi 1819) n. Comb. Sea of Japan. Mesentery. Zhukov (1960a) as Bucephalopsis g.

FELLODISTOMATIDAE

Pseudopentagramma petrowi (Layman 1930) Yamaguti 1971. British Columbia. Pyloric caeca, intestine. Margol is and Ching (1965) as Pentagramma p. HEMIURIDAE

Lecithaster confusus Odhner 1905. Massachusetts. Linton (1940); North Sea. Yamaguti (1971).

Lecithaster gibbosus (Rudolphi 1802) Lühe 1901. British Columbia. Intestine. Arai (1969b); Baltic, Scotland, Canada. Dollfus (1956). 
Parahemiurus merus (Linton 1910) Woolcock 1935. British Columbia. Stomach, intestine. Arai (1969b); Central California. Nahhas 10. Hemiurus levinseni Odhner 1905. Kamchatka, USSR. Stomach. Pyloric caeca. Skryabina (1963); Canadian Atlantic. Dollfus (1956).

Hemiurus luehei Odhner 1905. Sweden. Looss (1907a). Derogenes varicus (Müiler 1784) Looss 1901. Britain, New Brunswick. Viscera, muscle. Dollfus (1956); Kamchatka, USSR. Stomach, intestine. Skryabina (1963).

Brachyphallus crenatus (Rudolphi 1802) Odhner 1905. Kamchatka, USSR. Stomach. Skryabina (1963); Massachusetts. Linton (1940). Brachyphallus amuriens is Babaskin 1928. USSR. Yamaguti (1971). Pronopyee ocreata (RudoTphi 1802) Looss 1899. Europe. Stomach. Yamaguti (1971).

\section{HETEROPHYIDAE}

Cryptocotyle lingua (Crepl in 1825) Lühe 1899. Metacercaria. Maine. Skin, muscle. Sindermann and Rosenfield (1954).

\section{LEPOCREADI IDAE}

Opechona bacillaris (Molin 1859) Looss 1907. Britain. Stomach. Do11fus (1956).

\section{OPECOEL IDAE}

Podocotyle reflexa (Creplin 1825) Odhner 1905. Kamchatka, USSR. Stomach, intestine, pyloric caeca. Skryabina (1963).

\section{RENICOLIDAE}

Renicola sp. Metacercaria. North Sea. Pyloric caeca. MacKenzie (1975).

\section{MONOGENEA}

\section{GYRODACTYLIDAE}

Gyrodactylus harengi Malmberg 1956. Bering Sea, USSR. Gi1ls. Zhukov (1960b); Sweden. Yamaguti (1971).

Gyrodactylus dogieli Zhukov 1960. Bering Sea. Gills. Zhukov (1960b). Gyrodactyloides baueri Kulachkova 1970. White Sea. Nasal cavity. KuTachkova (1970).

Gyrodactyloides petruschewski i Bykhovsky 1947. Bering Sea. Gi11s. Zhukov (1960b).

\section{GYRODACTYLOIDEA}

Genera and species. British Columbia. Gills, body surface. Arai (1969b). 


\section{MAZOCRAEIDAE}

Mazocraeoides georgei Price 1936. Massachusetts. Gills. Price (1961b).

Mazocraes harengi (Van Beneden and Hesse 1863) Nicoll 1915.

France. Gills. Dollfus (1956).

\section{NEMATODA}

\section{ANISAKIDAE}

Anisakis sp. Larva. North Sea. Body cavity. Khalil (1969); British Columbia. Margolis ${ }^{3}$; Kamchatka, USSR. Intestine, abdominal wal1, body cavity. Skryabina (1963).

* Thynnascaris adunca (Rudolphi 1802) Hartwich 1957. Adults. Larva. Belgium. Digestive tube. Dollfus (1956) as Contracaecum a.

Phocanema decipiens (Krabbe 1878) Myers 1959. Larva. U.S. At lantic. Intestine. Dollfus (1956) as Porrocaecum d.

Phocanema sp. Larva. Kamchatka, USSR. Strelkov (1960) as Porrocaecum sp.

Contracaecum hypomesi Fujita 1932. Larva. Japan. Yamaguti (1961). Contracaecum sp. Larva. British Columbia. Body cavity. Arai $(1969 \mathrm{~b})$; Japan. Kagei et a). (1970).

Contracaecum sp.? California. Kunnenkeri (1962).

\section{CYSTIDICOLIDAE}

Ascarophis pacificus Zhukov 1960. Kamchatka, USSR. Strelkov (1960). PROTOZOA (CILIOPHORA)

\section{URCEOLARI IDAE}

Trichodina ploveri Zhukov 1964. Bering Sea, USSR. Gills. Zhukov (1964).

PROTOZOA (MYXOSPORIDA)

\section{CERATOMYXIDAE}

Ceratomyxa acadiensis Mayor 1915. Canadian Atlantic. Gallbladder. E1Tis (1930) Tentative identification.

Ceratomyxa oriental is (Dogiel 1948) Shulman 1953. USSR, Sea of Japan. Gallbladder. Kudo and Meglitsch6.

Ceratomyxa auerbachi Kabata 1962. North Sea. Gallbladder. Kabata (1962).

\section{SPHAEROSPORIDAE}

Sphaerospora oriental is Shulman and Shulman - Albova 1953. White Sea. Urinary bladder. Shulman (1955). 
Clupea harengus (Continued)

TETRACAPSULIDAE

Kudoa clupeidae (Hahn 1917) Meglitsch 1947. Massachusetts. Muscle. Kudo (1920) as Chloromyxum s.

PROTOZOA (SARCOMASTIGOPHORA)

HEXAMITIDAE

Hexamita salmonis (Moore 1922). Sanzin (1965).

PROTOZOA (SPOROZOA)

\section{EIMERI IDAE}

Eimeria nishin Fujita 1934. Japan. Testes. Pellerdy (1965) "Probabiy C. h. pallasi."

Eimeria sardinae (Thélohan 1890) Reichenow 1921. North Atlantic. Gonads. Dollfus (1956).

Eimeria clupearum (Thélohan 1894) Doflein 1909. North Atlantic. Liver. Dollfus (1956).

\section{Dorosoma petenense - Threadfin Shad}

\section{ACANTHOCEPHALA}

\section{NEOECHINORHYNCHIDAE}

Tanaorhamphus longirostris (Van Cleave 1913) Ward 1918. Oklahoma. Hopkins $(1966)$.

Gracilisentis gracilisentis (Van Cleave 1913) Van Cleave 1919. Oklahoma. Hopkins (1966).

\section{COPEPODA}

\section{ERGASILIDAE}

Ergasilus clupeidarum Johnson and Rogers 1972. Alabama. Gill filaments. Johnson and Rogers (1972).

\section{DIGENEA}

\section{CLINOSTOMATIDAE}

Clinostomum marginatum (Rudolphi 1819) Braun 1899. Southern California. Skin, muscle, coelom, mesentery, orbit of eyes. Miller et a1. (1973).

NEMATODA

\section{ANISAKIDAE}

Contracaecum sp. Juveniles. Oklahoma. Hopkins (1966). 
Dorosoma petenense (Continued)

CAMALLANIDAE

Camallanus sp. Juvenile. Okilahoma. Hopkins (1966).

Camallanus oxycephalus Ward and Magath 1917. Louisiana. Arnold et al. (1966).

PROTOZOA (CILIOPHORA)

URCEOLARI IDAE

Trichodina microdenticulata Wellborn 1967. Louisiana. Body surface. WelTborn (1967).

PROTOZOA (SARCOMASTIGOPHORA)

CRYPTOBI IDAE

Cryptobia sp. California. Hoffman (1967).

$$
\text { Sardinops sagax - Pacific Sardine }
$$

CESTODA

TETRAPHYLLIDEA

Genera and species. Red pigmented plerocercoid. Southern California. Intestine, pyloric caeca. Kunnenkeri (1962).

\section{DIGENEA}

HEMIURIDAE

Parahemiurus merus (Linton 1910) Woolcock 1935. Central California. Intestine. Nahhas 10 .

Parahemiurus noblei King 1962. Baja California. Intestine. King (1962).

Myosaccium ecaude Montgomery 1957. California. Kunnenkeri (1962). NEMATODA

ANISAKIDAE

Anisakis sp. Not clear if larva or adult. California. Kunnenkeri (1962).

Contracaecum sp. Not clear if larva or adult. California. Kunnenkeri (1962).

PROTOZOA (MYXOSPORIDA)

CERATOMYXIDAE

Ceratomyxa orientalis (Dogiel 1948) Shulman 1953. USSR. Gallbladder. Kudo and Meglitsch6. 
Ceratomyxa filiformis Dogiel 1948. USSR. Gallbladder. Kudo and MegTitsch6.

\author{
ENGRAULIDAE -- Anchovies \\ Cetengraul is mysticetus - Anchoveta
}

ISOPODA

CYMOTHOIDAE

Nerocila californica Schioedte and Meinert 1881. Gulf of California. Gi7ls. Brusca (1977).

\title{
Engraul is mordax - Northern Anchovy
}

CESTODA

TENTACULARI IDAE

Nybelinia sp. Larva. Southern California. Coelom. Mizrahi11.

TETRAPHYLLIDEA

Genera and species. Red pigmented plerocercoid. Southern California. Intestine, pyloric caeca. Kunnenkeri (1962).

COPEPODA

\section{CALIGIDAE}

Caligus klawei Shiino 1959. Southern California. External. Shiino (1959b).

Caligus bonito Wilson 1905. Southern California. External. Mizrahi11 as . . kuroshio.

\section{DIGENEA}

\section{DIDYMOZOIDAE}

Genera and species. Larva. Southern California. Coeloms-viscera. Mizrahi ${ }^{-11}$ as Torticaecum fenestratum.

\section{HEMIURIDAE}

Parahemiurus merus (Linton 1910) Woolcock 1935. Southern California. Intestine. Mizrahi 11 . 
Engraulis mordax (Continued)

ISOPODA

CYMOTHOIDAEE

Lironeca californica Schioedte and Meinert 1884. Southern California. Gi17 chambers. Mizrahi11.

Nerocila californica Schioedte and Meinert 1881. Southern California. Gi11 chambers. Mizrahi11.

MONOGENEA

DICLIDOPHORIDAE

Cyclobothrium sp. Southern California. Gill chambers. Mizrahi11.

NEMATODA

ANISAKIDAE

Anisakis sp. Larva. Southern California. Coelom. Mizrahi11.

Contracaecum sp. Larva. Southern California. Coelom. Mizrahi11

Raphidascaris sp. Larva. Southern California. Coelom. Mizrahi11.

PROTOZOA (MYXOSPORIDA)

MYXIDIIDAE

Sphaeromyxa reinhardti Jameson 1929. Central California.

Gallbladder. Jameson (1929).

PROTOZOA (SPOROZOA)

EIMERI IDAE

Eimeria clupearum (Thélohan 1894) Doflein 1908. Southern California.

Liver and other visceral organs. Mizrahi11.

\author{
SALMONIDAE -- Trouts \\ Oncorhynchus gorbuscha - Pink Salmon
}

ACANTHOCEPHALA

ECHINORHYNCHIDAE

Echinorhynchus gadi Zoega in Mü7ler 1776. Datta, USSR. Intestine. Bogdanova (1963); British Columbia. Ekbaum (1938). 
Oncorhynchus gorbuscha (Continued)

POLYMORPHIDAE

Corynosoma strumosum (Rudoiphi 1802) Lühe 1904. Juvenile. North Pacific. Margolis (1958b).

Corynosoma villosum Van Cleave 1953. Juvenile. Alaska. Margolis (7958b).

Bolbosoma caenoforme (Heitz 1920) Meyer 1933. Larva. Kamchatka, USSR. Body cavity. Trofimenko (1962).

RHADINORHYNCHIDAE

Rhadinorhynchus trachuri Harada 1935. Kamchatka, USSR. Mamaev et a1. (1959).

BRANCHIURA

ARGUL IDAE

Argulus coregoni Thorel1 1864. Amgun River, USSR. Gills. Bogdanova (1963).

CESTODA

AMPHICOTYLIDAE

Eubothrium crassum (B1och 1779) Nybel in 1922. Kamchatka, USSR. Digestive tract. Trofimenko (1962).

CYATHOCEPHAL IDAE

Bothrimonus sturionis Duvernoy $1842 . \quad$ Alaska. Intestine. Burt and Sandemann (1969).

DIPHYLLOBOTHRIIDAE

Diphyllobothrium sp. Larva. Amgun River, USSR. Muscles. Bogdanova (1963).

Diphyllobothrium latum (Linneus 1758) Matz 1892. Larvã. Japan. Muscles. Eguchi (1932).

PHYLLOBOTHRI IDAE

Phyllobothrium sp. Larva. British Columbia. Intestine. Boyce (1969).

Phyllobothrium caudatum (Zschokke and Heitz 1914). Larva. Amgun River, USSR. Intestine. Bogdanova (1963): British

Columbia. Stomach, pyloric caeca. Margol is 3 as $\underline{\text { P. ketae. }}$ 
Oncorhynchus gorbuscha (Continued)

PROTEOCEPHAL IDAE

Gangesia parasiluri Yamaguti 1934. USSR. Yamaguti (1959b).

\section{TENTACULARI IDAE}

Nybelinia sp. Larva. British Columbia. Stomach. Wardle (1932). Nybelinia surmenicola Okada 1929. Larva. Amgun River, USSR. Body cavity. Bogdanova (1963).

TETRAPHYLLIDEA

Scolex pleuronectis Müller 1788. Amur Basin, USSR. Gallbladder. Dubinina (1971).

Scolex polymorphus Rudolphi 1819. Kamchatka, USSR. Akmerov (1954a). COPEPODA

BOMOLOCHIDAE

Bomolochus cuneatus Fraser 1920. Gulf of Alaska. Gills. Vervoort (1964) as Parabomolochus c.

\section{CALIGIDAE}

Caligus clemensi Parker and Margolis 1964. British Columbia. Body surface. Parker and Margolis (1964).

Lepeophtheirus salmonis (Krøyer 1838). Alaska. Gills and gill cavity. Wilson (1905b); Japan. Yamaguti (1939b).

ERGASILIDAE

Ergasilus wilsoni Markevich 1933. Amgun River, USSR. Body surface. Bogdanova (1963).

Ergasilus briani Markevich 1934. Amgun River, USSR. Gills. Bogdanova (1963).

\section{LERINAEOPODIDAE}

Genera and species. Nasal cavity. Smirnova et al. (1964).

\section{DIGENEA}

\section{ALLOCREADI IDAE}

Crepidostomum ussuriensis Layman 1930. Primore Region, USSR. Intestine. Bogdanova (1963). 
BUCEPHAL IDAE

Prosorhynchoides basargini (Layman 1930) Margolis and Arthur 1979. Kamchatka, USSR. Intestine. Akhmerov (1955) as Bucephalops is b. Prosorhynchoides gracilescens (Rudolphi 1819) n. comb. Kamchatka, USSR. Digestive tract. Trofimenko (1962).

\section{FELLODISTOMATIDAE}

Pseudopentagramma petrowi (Layman 1930) Yamaguti 1971. British Columbia. Intestine. Boyce (1969) as Pentagramma p.

HEMIURIDAE

Parahemiurus merus (Linton 1910) Woolcock 1935. British Columbia. Stomach. Boyce (1969); Primore Region, USSR. Intestine. Bogdanova (1963).

Derogenes varicus (Mül ler 1784) Looss 1901. USSR. Intestine. Bogdanova (1963).

Genolinea anura (Layman 1930) Yamaguti 1954. USSR. Stomach. Bykhovskaya-Pavlovskaya et al. (1962).

Genolinea oncorhynchi Margol is and Adams 1956. British Columbia. Margolis and Adams (1956).

Brachyphallus amuriensis Babaskin 1928. Kamchatka, USSR. Akhmerov (1954b).

Brachyphallus crenatus (Rudolphi 1802) Odhner 1905. Kamchatka, USSR. Digestive tract. Trofimenko (1962).

Genarchopsis muelleri (Levinsen 1881) Yamaguti 1954. Kamchatka, USSR. Digestive tract. Trofimenko (1962) as Genarches m.

Lecithaster gibbosus (Rudolphi 1802) Lühe 1901. British Côlumbia. Intestine, pyloric caeca. Margol is and Boyce (1969); Kamchatka, USSR. Trofimenko (1962).

Lecithaster stellatus Looss 1907. USSR. Intestine. BykhovskayaPavlovskaya et al. (1962).

Tubulovesicula lindbergi (Layman 1930) Yamaguti 1934. British Columbia. Intestine. Margol is and Boyce (1969); Primore Region, USSR. Intestine. Bogdanova (1963).

Hemiurus levinseni Odhner 1905. British Columbia. Margol is (1956a); Primore Region, USSR. Intestine. Bogdanova (1963).

Lecithophyllum botryophorum (01sson 1868) Odhner 1905. British

Columbia. Stomach. Margolis (1958a) as $\underline{L}$. anteroporum.

HETEROPHY IDAE

Apophallus sp. Larva. USSR. Gills. Bogdanova (1963). Galactosomum phalacrocoracis Yamaguti 1939. Metacercaria. British Columbia. Encysted in viscera? Pearson et al. (1978).

\section{LAMPRITREMATIDAE}

Lampritrema miescheri (Zschokke 1890) Margolis 1962. Pacific 0cean, USSR. Margolis (1962). 
Oncorhynchus gorbuscha (Continued)

OPECOELIDAE

Podocotyle enophrysi Park 1937. Washington. Intestine.

Aldrich 12 .

Podocotyle gibbonsiae Johnson 1949. Washington. Intestine.

Aldrich 12 .

Podocotyle atomon (Rudolphi 1802) Odhner 1905. Sea of Japan.

Stomach. Zhukov (1960a).

\section{SYNCOELIDAE}

Syncoelium filiferum (Leuckart, in Sars 1885) Odhner 1911. Washington. Branchial cavity. Lloyd and Guberlet (1936).

HIRUDINOIDEA

GLOSSOPHONIDAE

Hemiclepsis marginata (Müiler 1774) Vezhdovskii 1844. Kamchatka, USSR. Lukin (1962).

\section{PISCICOLIDAE}

Piscicola salmositica Meyer 1946. Washington. Pectoral and pelvic fins, gi ils. Becker and Katz (1965a).

MOLLUSCA

UNIONIDAE

Genera and species. Amgun River, USSR. Gill cavity. Bogdanova (1963).

MONOGENEA

GYRODACTYLIDAE

Gyrodactyloides strelkowi Bykhovsky and Polyanski 1953. Kurile Islands, USSR. Bykhovsky and Polyanski (1953).

NEMATODA

ANISAKIDAE

Anisakis sp. Larva. British Columbia. Body cavity. Arai (1969b); Kamchatka, USSR. Trofimenko (1962). Contracaecum hypomesi Fujita 1932. Larva. Japan. Body cavity. Fujita (1939).

Contracaecum robustum Chandler 1935. Larva. Japan. Intestine. Fujita (1939).

Contracaecum sp. Larva. British Columbia. Intestine. Boyce (1969). 
*Thynnascaris adunca (Rudo1phi 1802) Hartwich 1957. Larva. Amgun River, USSR. Intestine. Bogdanova (1963); adult. Kamchatka, USSR. Trofimenko (1962) both as Contracaecum $\underline{\text { a. }}$

\section{CUCULLANIDAE}

Truttaedacnitis trutta (Fabricius 1774) Petter 1974. Kamchatka, USSR. Intestine. Strelkov (1960) as Cucullanus t.; USSR. Bykhovskaya-Pavlovskaya et al. (1962) as Cucullanus laevis.

CYSTIDICOLIDAE

Cystidicola farionis Fisher 1798. Soviet far east; Alaska. Swimbladder. Margol is (1967a) "probable host".

Ascarophis skrjabini (Layman 1933) Poljansky 1952. USSR. Intestine. Ko and Anderson (1969).

\section{PHILOMETRIDAE}

Philonema oncorhynchi Kuitunen-Ekbaum 1933. Kamchatka, USSR. Mamaev et a]. (1959).

\section{PROTOZOA (CILIOPHORA)}

OPHRYOGLENIDAE

Ichthyopthirius multifillis Fouquet 1876. Amgun River, USSR.

Gills. Bogdanova (1963).

\section{URCEOLARI IDAE}

Trichodina truttae. Sakhalin, USSR. Fins, gills, body surface. Bogdanova and Stein (1963).

Trichodina gracilis Polyanski 1955. Sakhalin, USSR. Bogdanova and Stein (1963).

Tripartiella californica (Davis 1947). Rivers of South Sakhalin, USSR. GiT1s. Bogdanova and Stein (1963).

PROTOZOA (HAPLOSPORIDA)

Genera and species. British Columbia. Kidneys. Hoskins et al $?^{3}$ PROTOZOA (MICROSPORIDA)

Genera and species. Rivers leading into White Sea, USSR. Kidneys. Grozdilova (1968).

NOSEMATIIDAE

Glugea takedai Awakura 1974. Japan. Muscles. Awakurà (1974). 
PROTOZOA (MYXOSPORIDA)

\section{CERATOMYXIDAE}

Leptotheca krogiusi Konovalov and Shulman 1965. Kamchatka, USSR. Urinary bladder. Shulman (1966).

\section{MYXIDI IDAE}

Myxidium oviforme Parisi 1912. USSR. Gallbladder. BykhovskayaPavlovskaya et al. (1962).

Myxidium obscurum Konovalov 1966. Kamchatka, USSR. Intestine. Urinary bladder. Shulman (1966)

Zschokkella orientalis Konovalov and Shulman 1966. USSR. Gallbladder. SchuTman (1966).

MYXOBOL IDAE

Henneguya salminicola Ward 1919. Alaska. Cysts in muscles. Fish (1939). MYXOSOMATIDAE

Myxosoma cerebralis (Hofer and Plehn 1903) Kudo 1933. Sakhalin Island, USSR. Cartilage of head, gill arches and bones. Bogdanova (1960a).

\section{SPHAEROSPORIDAE}

Chloromyxum giganteum Fujita 1923. Japan. Gallbladder. Fujita (1923).

Chloromyxum quadiforme Fujita 1923. Gal1bladder. Fujita (1923).

Chloromyxum orientalis Shulman 1962. Kamchatka, USSR. Vinnichenko et a1. (1971).

PROTOZOA (SARCOMASTIGOPHORA)

CRYPTOBIIDAE

Cryptobia salmositica Katz 1951. Washington. Blood. Becker and Katz (1965b).

Cryptobia makeevi (Akhmerov 1959) Bykhovskaya-Pavlovskaya 1962.

Amur River, USSR. Blood. Bykhovskaya-Pavlovskaya et al. (1962).

\section{TETRAMITIDAE}

Ichthyobodo necatrix (Henneguy 1884). Kola Peninsula, USSR. Bogdanova (7967) as Costia $n$. 
Oncorhynchus keta - Chum Salmon

SYNONYM - Salmo keta

ACANTHOCEPHALA

ECHINORHYNCHIDAE

Acanthocephalus echigoensis Fujita 1920. Japan. Fujita (1920); Kamchatka, USSR. Mamaev et al. (1959) as A. aculeatus.

Echinorhynchus gadi Zoega in Müt ler 1776. Amur Basin, USSR. Intestine. Sokolovskaya (1971); British Columbia. Ekbaum (1938).

NEOECHINORHYNCHIDAE

Neoechinorhynchus rutili (Mü7ler 1780) Hamann 1892. British Columbia. Intestine. Arai (1969b).

POLYMORPHIDAE

Corynosoma villosum Van Cleave 1953. Larva. USSR. Coelom. Bykhovskaya-Paviovskaya et al. (1962).

Bolbosoma caenoforme (Heitz 1920) Meyer 1933. Amur Basin, USSR. Body cavity. Sokolovskaya (1971).

RHADINORHYNCHIDAE

Rhadinorhynchus trachuri Harada 1935. Okhotsk Sea, USSR. Intestine. Boğuanova (7963).

BRANCHIURA

ARGULIDAE

Argulus coregoni Thorell 1864. Amgun River, USSR. Gills. Bogdanova (1963).

CESTODA

AMPHICOTYLIDAE

Eubothrium crassum (Bloch 1779) Nybel in 1922. Kamchatka, USSR. Intestine. Bogdanova (1963); British Columbia. Margolis 3 as E. oncorhynchi.

DIPHYLLOBOTHRI IDAE

Diphyllobothrium sp. Larva. Amgun River, USSR. Muscles. Bogdanova (1963).

Diphyl lobothrium latum (Linneaus 1758) Matz 1892. Plerocercoid. Japan. Muscles. Eguchi (1932). 
Oncorhynchus keta (Continued)

\section{HEPATOXYLIDAE}

Hepatoxylon trichiuri (Holten 1802) Dollfus 1942. Larva. Amur Basin, USSR. Muscles. Dubinina (1971).

\section{PHYLLOBOTHR I IDAE} $\frac{\text { Phyllobothrium caudatum (Zschokke and Heitz 1914). Larva. Okhotsk }}{\text { Sea, Amgun River, USSR. Intestine, anus. Bogdanova (1963); Alaska. }}$ Canavan (1928); British Columbia. Stomach, pyloric caeca. Margolis 3 latter two as $\underline{\text { P. ketae. }}$.

Phyllobothrium sp. Larva. British Columbia. Intestine. Araj (1969b).

\section{PROTEOCEPHALIDAE}

Gangesia parasiluri Yamaguti 1934. USSR. Yamaguti (1959b).

Proteocephalus exiguus La Rue 1911. Kamchatka, USSR. Mamaev et al. (1959).

\section{TENTACULARI IDAE}

Nybelinia sp. Larva. British Columbia. Stomach. Wardle (1932).

Nybelinia surmenicola Okada 1929. Larva. Amgun River, USSR. Body cavity. Bogdanova (1963).

\section{TETRAPHYLLIDEA}

Scolex pleuronectis Müller 1788. Amur Basin, USSR. Gallbladder. Dubinina (1971).

TRIAENOPHORIDAE

Triaenophorus crassus Forel 1868. Larva. Hoffman (1967). COPEPODA

\section{CALIGIDAE}

Caligus clemensi Parker and Margolis $1964 . \quad$ British Columbia. Fraser (1920) as C. gurnardi; British Columbia. Body surface.

Parker and Margolis (1964).

Lepeophtheirus salmonis (Krфyer 1838). Japan. Body surface. Shiino (1959e); USSR. Bykhovskaya-Pavlovskaya et al. (1962). 
Oncorhynchus keta (Continued)

\section{LERNAEOPODIDAE}

Salmincola saimoneus (Linneus 1761) Wilson 1915. USSR. Gills. Bykhovskaya-Pavlovskaya et al. (1962) as S. salmonea.

Salmincola californiensis (Dana 1853) Wilson 1915 . USSR. Gills. Kabata (1969c).

\section{DIGENEA}

\section{ALLOCREADI IDAE}

Crepidostomum salmonis Fujita 1921. Japan. Fujita (1921b).

\section{BUCEPHALIDAE}

Prosorhynchoides gracilescens (Rudolphi 1819) n. comb. Kamchatka, USSR. Mamaev et al. (1959).

\section{ISOPARORCHI IDAE}

Isoparorchis hypselobagri (Billet 1898) Ejsmont 1932. Juvenile. Amur Basin, USSR. Akhmerov (1961).

\section{HEMIURIDAE}

Hemiurus levinseni Odhner 1905. Kamchatka, USSR. Mamaev et a1. (1959).

Parahemiurus merus (Linton 1910) Woolcock 1935. Kamchatka, USSR. Mamaev et at. (1959).

Brachyphallus amuriensis Babaskin 1928. Kamchatka, USSR. Akhmerov (1954b).

Brachypha17us crenatus (Rudolphi 1802) Odhner 1905. British Columbia. Stomach. Margoli is ${ }^{3}$; Kamchatka, USSR. Trofimenko (1962); Japan. Machida et ai. (1972).

Tubulovesicula lindbergi (Layman 1930) Yamaguti 1934. British Columbia. Intestine. Margol is and Boyce (1969); USSR. Bykhovskaya-Pavlovskaya et al. (1962).

Lecithaster gibbosus (Rudolphi 1802) Lühe 1901. British Columbia. Intestine. Margol is and Boyce (7969); Kamchatka, USSR.

Trofimenko (1962); Japan. Intestine. Yamaguti (1934a) as L. Salmonis.

Lecithaster stellatus Looss 1907. USSR. Intestine. BykhovskayaPavlovskaya et a1. (1962).

Genarchopsis muelleri (Levinson 1881) Yamaguti 1954. Kamchatka, USSR. Trofimenko (1962) as Genarches $\mathrm{m}$.

\section{HETEROPHYIDAE}

Apophallus sp. Larva. Amgun River, USSR. Gills. Bogdanova (1963). Galactosomum sp. Larva. British Columbia. Gills (encysted). Arai (1969b). 
Oncorhynchus keta (Continued)

NANOPHYETIDAE

Nanophyetus saiminicồa (Chappin 1926) Chappin 1927. Metacercaria. Oregon. Simms et a1. (1931); USSR. Filimonova (1964) as N. schikhobalowi.

HIRUDINOIDEA

PISCICOLIDAE

Piscicola salmositica Meyer 1946. Washington. Pectoral and pelvic fins, gi11 lamel1ae. Becker and Katz (1965a). Trachelobdella okae Moore 1924. Amur Basin, USSR. Epshtein (1964).

MOLLUSCA

UNIONIDAE

Unio sp. Larva. Amgun River, USSR. Gi11s. Bogdanova (1963). MONOGENEA

GYRODACTYLIDAE

Gyrodactylus lenoki Gussev 1953. Sakhalin Island, USSR. Gills. Bogdanova (7960b).

Gyrodactyloides strelkowi Bykhovsky and Polyanski 1953.

Kamchatka, USSR. Mamaev et a1. (1959).

NEMATODA

ANISAKIDAE

Contracaecum benimasu Fujita 1932. Larva. Japan. Fukui (1958). Contracaecum Tongispiculum Fujita 1940. Larva. Japan. Body cavity. Fujita (1940).

Contracaecum oshoroense Fujita 1940. Japan. Body cavity. Fujita $(1940)$.

Contracaecum tridentatum Fujita 1939. Larva. Japan. "In body." Fujita (1939).

Contracaecum unidentatum Fujita 1939. Larva. Japan. Body cavity. Fujita (1939). Contracaecum hypomesi Fujita 1932. Larva. Japan. Body cavity. Fujita (1939).

Contracaecum sp. Larva. British Columbia. Body cavity. Arai (1969b).

*Thynnascaris adunca (Rudolphi 1802) Hartwich 1957. Larva. Okhotsk Sea, Amgun River, USSR. Livers, body cavity. Bogdanova (1963) as Contracaecum a.

Anisakis sp. Larva. British Columbia. Body cavity. Arai (1969b); Kamchatka, USSR. Trofimenko (1962).

Goezia oncorhynchi Fujita 1940. Larva. Japan. Intestine. Fujita $(1940)$. 
CYSTIDICOLIDAE

Ascarophis skrjabini (Layman 1933) Polyanski 1952. Kamchatka, USSR. Mamaev et a 7 . (1959).

Salvelinema ishii (Fujita 1941) Margolis 1968. Japan. Intestine.

Fujita (1941) as Metabronema i.

Salvelinema salmonicola (Ishii T916) Margolis 1966. Japan to USSR.

Swimbladder. Margolis (1967a).

PHILOMETRIDAE

Philonema oncorhynchi Kuitunen-Ekbaum 1933. Kamchatka, USSR.

Akhmerov (1954b).

Philonema kondai Fujita 1939. Body cavity. Fujita (1939).

THELAZI IDAE

Rhabdochona oncorhynchi (Fujita 1921) Fujita 1927. Japan. Intestine.

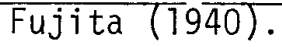

PROTOZOA (CILIOPHORA)

DENDROSOMATIDAE

Trichophyra intermedia Prost 1952. Kola Peninsula, USSR. Bogdanova (1967). OPHRYOGLENIDAE

Ichthyophthirius multifillis Fouquet 1876. Amgun River, USSR. Gills. Bogdanova (7963).

SCYPHIDIIDAE

Scyphidia arctica Zhukov 1960. Kola Peninsula, USSR. Bogdanova (1967). Glossatella conica Timofeev 1962. Kola Peninsula, USSR. Bogdanova (1967). URCEOLARI IDAE

Trichodina gracilis Polyanski 1955. Sakhalin Island, USSR. Bogdanova and Stein (1963).

Irichodina truttae. Sakhalin Island, USSR. Gills, fins, body surface. Bogdanova and Stein (1963).

Trichodina domerguei $f$. latispina Dogiel 1940. Sakhalin, USSR. Bogdanova and Stein (1963).

Tripartiella californica (Davis 1947). Sakhalin Island, USSR. Gills. Bogdanova and Stein (1963).

PROTOZOA (MICROSPORIDA)

NOSEMATIDAE 
Oncorhynchus keta (Continued)

Plistophora sp. Japan. Muscles of head. Awakura (1965).

PROTOZOA (MYXOSPORIDA)

\section{CERATOMYXIDAE}

Ceratomyxa shasta Noble 1950. British Columbia. Lesions in kidneys, pyloric caeca, intestine, muscle. Margolis and Evelyn (1975). Leptotheca krogiusi Konovalov and Shulman 1965. Kamchatka, USSR. Urinary bTadder. Shulman (1966).

\section{MYXIDIIDAE}

Myxidium oviforme Parisi 1912. USSR. Gallbladder. BykhovskayaPavlovskaya et al. (1962).

Myxidium obscurum Konovalov 1966. Kamchatka, USSR. Anterior part of intestine, urinary bladder. Kudo and Meglitsch 6 .

Myxidium minteri Yasutake and Wood 1957. Oregon. Gailbladder. Sanders and Fryer (1970).

MYXOBOLIDAE

Henneguya salminicola Ward 1919. Alaska. Cysts in muscles. Fish (1939).

\section{MYXOSOMATIDAE}

Myxosoma cerebral is (Hofer and Plehn 1903) Kudo 1933. Sakhal in Island, USSR. Cartilage of head, gill arches, bones. Bogdanova (1960a). Myxosoma salmonis Hoshina 1949. Japan. Cysts in skin. Hoshina (1949).

Myxosoma soldatovi (Akhmerov 1960) Kudo and Meglitsch 6 . Amur River, USSR. Cysts under scales. Akhmerov (1960) as Myxobolus s.

Myxosoma dermatobia (Ishii 1916) Hoshina 1952. Tissue. Kudo and Meglitsch.6.

Myxosoma squamal is Iverson 1954. Washington. Body surface. Iverson $(1954)$.

\section{SPHAEROSPORIDAE}

Chloromyxum orientalis Schulman 1962. Kamchatka, USSR. Vinnichenko et al. (1971).

Chloromyxum chitosense Fujita 1923. Japan. Gallbladder. Fujita $\frac{\text { Chloromyxum quadriforme Fujita 1923. Japan. Gallbladder. Fujita }}{(1923) \text {. }}$

Chloromyxum tanakai Fujita 1936. Japan. Gallbladder. Fujita (1936). 


\section{PROTOZOA (SARCOMASTIGOPHORA)}

\section{CRYPTOBI IDAE}

Cryptobia makeevi (Akhmerov 1959) Bykhovskaya-Pavlovskaya et al. 1962. Amur River Basin, USSR. Blood. Bykhovskaya-Pavlovskaya et a1. (1962).

Cryptobia salmositica Katz 1951. Washington. Becker and Katz (1965b).

\section{TETRAMITIDAE}

Ichthyobodo necatrix (Henneguy 1884). Kamchatka, USSR. Bogdanova (1967) as Costia n.

\section{Oncorhynchus kisutch - Silver Salmon}

\section{ACANTHOCEPHALA}

\section{ECHINORHYNCHIDAE}

Metechinorhynchus salmonis (Müller 1784) Petrotchenko 1956. Lake Michigan. Intestine. Amin and Burrows (1977).

Metechinorhynchus lateral is (Leidy 1851) Golvan 1969. Humboldt Bay, California. Intestine. Haderlie (1953) as Echinorhynchus 1.

Echinorhynchus gadi Zoega in Müiler 1776. British Columbia. Intestine. Arai (1969b); Kamchatka, USSR. Strelkov (1960).

\section{NEOECHINORHYNCHIDAE}

Neoechinorhynchus rutili (Müller 1780) Hamann 1892. British Columbia. Intestine. Arai (1969b); USSR. Bykhovskaya-Pavlovskaya et al. (1962).

Neoechinorhynchus cristatus Lynch 1936. Kamchatka, USSR. Intestine. Akhmerov (1955).

\section{POLYMORPHIDAE}

Bolbosoma caenoforme (Heitz 1920) Meyer 1933. Larva. Kamchatka, USSR. Intestine. Strelkov (1960).

Corynosoma sp. Juvenile. British Columbia. Intestine. Arai (1969b).

\section{POMPHORYNCHIDAE}

Pomphorhynchus bulbocol1i Linkins in Van Cleave 1919. British CoTumbia. Bangham and Adams (1954).

\section{RHADINORHYNCHIDAE}

Rhadinorhynchus trachuri Harada 1935. Kamchatka, USSR. Mamaev et aT. (1959); Oregon. 01son (1978a) as Nipporhynchus $t$. 
Oncorhynchus kisutch (Continued)

BRANCHIURA

ARGULIDAE

Argulus pugettensis Dana 1852. Pacific Coast. Wilson (1908a).

\section{CESTODA}

AMPHICOTYLIDAE

Eubothrium crassum (Bloch 1779) Nybel in 1922. Kamchatka, USSR. Strelkov (1960); British Columbia. Pyloric caeca. Wardle (1932) as $E$. oncorhynchi.

Eubothrium salvelini (Schrank 1790) Nybel in 1922. Alberta, Leong and Holmes (1974); Kamchatka, USSR. Intestine. Konovalov (1971).

BOTHRIOCEPHALIDAE

Bothriocephalus sp. Larva. Oregon. Intestine. 01son (1978a).

CYATHOCEPHALIDAE

Cyathocephallus truncatus (Pallas 1781) Kessler 1868. USSR. Intestine. Bykhovskaya-Pavlovskaya et al. (1962).

DIPHYLLOBOTHRIIDAE

Diphy 7 lobothrium cordiceps (Leidy 1871) Matz 1892. Larva. Montana. Body cavity. Simms and Shaw (1931) as Dibothrium c.

Diphyllobothrium sp. Larva. British Columbia. Body cavity. Arai (1969b).

PHYLLOBOTHRI IDAE

Phyllobothrium sp. Larva. British Columbia. Intestine. Arai (1969b). Phyl10bothrium caudatum (Zschokke and Heitz 1914). Kamchatka, USSR. Mamaev et a . (1959).

PROTEOCEPHAL IDAE

Proteocephalus sp. Larva. British Columbia. Intestine. Arai (1969b). Proteocephalus salmonidicola Alexander 1951. Washington. Intestine. Becker (1967a).

Proteocephalus ambloplitis (Leidy 1887) Benedict 1900. Plerocercoid. Washington. Becker (1967a).

Proteocephalus exiquus La Rue 1911. Kamchatka, USSR. Konovalov (1971).

TENTACULARI IDAE

Nybelinia surmenicola Okada 1929. Kamchatka, USSR. Mamaev et al. (1959).

Nybelinia sp. Larva. British Columbia. Stomach. Wardle (1932). 
Oncorhynchus kisutch (Continued)

TETRAPHYLLIDEA

Scolex pleuronectis Mül 1er 1788. Kamchatka, USSR. Intestine. Strelkov (1960).

Scolex polymorphus Rudolphi 1819. Kamchatka, USSR. Akhmerov (1954a).

TRIAENOPHORIDAE

Triaenophorus nodulosus (Pallas 1760) Rudolphi 1819. Plerocercoid. Canada. Leong and Holmes (1974).

COPEPODA

CALIGIDAE

Lepeophtheirus salmonis (Krøyer 1838). British Columbia. Skin surface. Margolis ${ }^{3}$; Oregon. 01 son (1978a).

Caligus clemensi Parker and Margol is 1964. British Columbia. Body surface. Parker and Margol is (1964).

ERGASILIDAE

Ergasilus auritus Markevich 1940. Alberta. Gi11s. Leong and Holmes (1974).

Ergasilus nerkae Roberts 1963. Alberta. Gills. Leong and Holmes (1974).

\section{LERNAEOPODIDAE}

Salmincola californiensis (Dana 1853) Wilson 1915. British Columbia. Pectoral fins. Arai (1969b).

\section{DIGENEA}

\section{ALLOCREADI IDAE}

Crepidostomum farionis (Müller 1780) Lühe 1909. British Columbia. Bangham and Adams (1954).

\section{DIDYMOZOIDAE}

Genera and species. Larva. Kamchatka, USSR. Mamaev et al. (1959) as Monilicaecum ventricosum.

\section{DIPLOSTOMATIDAE}

Diplostomum sp. British Columbia. Bangham and Adams (1954); Kamchatka, USSR. Mamaev et a1. (1959).

Spathaceum indistinctum (Guberlet 1923) Hughes 1929. Metacercaria. Washington. Eyes. Becker (1967a) as Diplostomum flexicaudum.

HERMIURIDAE

Hemiurus levinseni Odhner 1905. Kamchatka, USSR. Intestine. Strelkov (1960). 
Brachyphallus crenatus (Rudolphi 1802) Odhner 1905. Kamchatka, USSR. Strelkov (1960); Oregon. Stomach. 01son (1978a). Deropegus aspina (Ingles 1936) McCauley and Pratt 1961. Oregon. Stomach. McCauley and Pratt (1961).

Parahemiurus merus (Linton 1910) Woolcock 1935. British Columbia. Intestine, pyloric caeca. Arai (1969b).

Derogenes sp. Pratt and McCauley (1961).

Lecithaster gibbosus (Rudolphi 1802) Lühe 1901. British Columbia. Intestine, pyloric caeca. Arai (1969b); Kamchatka, USSR. Strelkov (1960); Washington. Ching (1960a) as L. salmonis.

Tubulovesicula lindbergi (Layman 1930) Yamaguti 1934. Washington. Ching (1960a).

\section{HETEROPHYIDAE}

Apophallus donicus (Skrjabin and Lindtrop 1919) Price 1931. Metacercaria. Oregon. Skins. Niemi and Macy (1974).

\section{NANOPHYET IDAE}

Nanophyetus salmincola (Chapin 1926) Chapin 1927. Metacercaria. Oregon. Simms et al. (1931).

OPECOELIDAE

Podocotyle sp. Oregon. Intestine. 01 son (1978a).

Plagioporus shawi (McIntosh 1939) Margolis 1970. Oregon. Intestine. McIntosh (1939) as Podocotyle s.

\section{STRIGEIDAE}

Cotylurus erraticus (Rudolphi 1809) Szidat 1928. Metacercaria. Montana. Pericardial cavity. 01 son (1970).

\section{SYNCOELIIDAE}

Syncoelium filiferum (Leuckart in Sars 1885) Odhner 1911. Kamchatka, USSR. Mamaev et a1. (1959); Oregon. Gills. 01son (1978a) as S. katuwo.

\section{HIRUDINOIDEA}

\section{PISCICOLIDAE}

Piscicola salmositica Meyer 1946. British Columbia, Washington, Oregon. Pectoral, pelvic fins, gills. Becker and Katz (1965a). MONOGENEA 
CAPSALIDAE

Encotyllabe masu Ishi i and Sawada 1938. Japan. Gills. Sproston $(7946)$. Tehaonchus

\section{GYRODACTYLIDAE}

Gyrodactyloides strelkowi Bykhovsky and Polyanski 1953. Kamchatka, USSR. Mamaev et a]. (1959).

GYRODACTYLOIDEA

Genera and species. British Columbia. Gills. Arai (1969b).

NEMATODA

ANISAKIDAE

Anisakis sp. Larva. British Columbia. Body cavity. Arai (1969b); Kamchatka, USSR. Strelkov (1960); Oregon. 01son (1978a). Contracaecum crassicaudatum Fujita 1939. Larva. Japan. Body cavity. Fujita (1939).

Contracaecum hippoglossi Fujita 1932. Larva. Japan. Fujita (1932). Contracaecum sp. Larva. British Columbia. Body cavity. Arai (1969b).

* Thynnascaris sp. Oregon. Intestine. 01son (1978a).

* Thynnascaris adunca (Rudolphi 1802) Hartwich 1957. British Columbia. Stomach, intestine. Margolis 3 ; USSR. Bykhovskaya-Pavlovskaya et al. (1962). Both as Contracaecum a.

\section{CAPILLARI IDAE}

Capillaria sp. British Columbia. Stomach. Arai (1969b).

Skrjabinocapillaria bakeri (Mueller and Van Cleave 1932) Skrjabin and Schikhobalova 1954. Bangham and Adams (1954) as Hepaticola bakeri.

CUCULLANIDAE

Cucullanus sp. British Columbia. Intestine. Arai (1969b).

Truttaedacnitis trutta (Fabricius 1794) Petter 1974. Kamchatka, USSR. Strelkov (1960) as Dacnitis t.

\section{CYST IDICOLIDAE}

Ascarophis sebastodis 01 sen 1952. British Columbia. Intestine. Arai (1969b).

Cystidicoloides tenuissima (Zeder 1800) Rasheed 1966. British Columbia. Margolis (1965); California. Intestine.

Haderlie (1953). Both as Metabronema salvelini. 
Oncorhynchus kisutch (Continued)

Cystidicola farionis Fischer 1798. Soviet Far East. Swimbladder. Margolis (1967a). "Probable host".

Salvelinema walkeri (Ekbaum 1935) Margolis 1967. California. Swimbladder. Margol is (1967a); British Columbia. Ekbaum (1935) as Cystidicola w; California. Haderlie (1953) as Cystidicola stigmatura.

DIOCTOPHYMATIDAE

Eustrongylides sp. Larva. British Columbia. Viscera. Godfrey14.

\section{PHILOMETRIDAE}

Philonema oncorhynchi Kuitunen-Ekbaum 1933. Kamchatka, USSR. Mamaev et a T. (1959); Larva. British Columbia. Bangham and Adams (1954). Philonema agubernaculum Simon and Simon 1936. Canada. Leong and Holmes (1974).

Philonema sp. British Columbia. Swimbladder wall. Arai (1969b).

THELAZI IDAE

Rhabdochona kisutchi Margolis, Moravec and McDonald 1975. British Columbia. Intestine, pyloric caeca. Margolis et al. (1975).

PROTOZOA (CILIOPHORA)

DENDROSOMATIDAE

Trichophyra sp. Washington. Gills. Becker (1967a). URCEOLARI IDAE

Trichodina sp. Washington. Gills. Becker (1967a). PROTOZOA (HAPLOSPORIDA)

Genera and species. British Columbia. Kidneys. Hoskins et a1. ${ }^{13}$ PROTOZOA (MICROSPORIDA)

NOSEMATIDAE

Plistophora salmonae Putz, Hoffman and Dunbar 1965. California.

Gills. Leitritz and Lewis (1976).

PROTOZOA (MYXOSPORIDA)

CERATOMYXIDAE

Leptotheca krogiusi Konovalov and Shulman 1965. Kamchatka, USSR. Urinary bladder. Shulman (1966).

Ceratomyxa shasta Noble 1950. Columbia River. Sanders et a1. (1970). 
MYXIDI IDAE

Myxidium minteri Yasutake and Wood 1957. Washington, Oregon. Renal tubuTes. Yasutake and Wood (1957); gallbladder. Sanders and Fryer (1970).

Zschokkella oriental is Konovalov and ShuTman 1966. USSR. Gallbladder. Shulman (1966).

MYXOBOLIDAE

Myxobolus squamae Keysselitz 1908. Oregon. Flesh. Shaw et al. (1934).

Myxobolus kisutchi Yasutake and Wood 1957. Washington. Spinal cord. Yasutake and Wood (1957).

Myxobolus neurobius Schuberg and Schroder 1905. Kamchatka, USSR. Cysts in nervous tissues. Kudo and Meglitsch6.

Myxobolus insidiosus Wyatt and Pratt 1963. Washington. Muscles. Wyatt (1978).

Henneguya salminicola Ward 1919. Alaska, Washington. Cysts in muscles. Fish (1939); Kamchatka, USSR. Zschokke and Heitz (1914) as H. zschokkei.

\section{MYXOSOMATIDAE}

Myxosoma squamalis Iverson 1954. Washington. Body surface. Iverson (1954); British Columbia. Margolis ${ }^{3}$.

Myxosoma dermatobia (Ishi i 1916) Hoshina 1952. USSR. Skin. Bykhovskaya-Pavlovskaya et a1. (1962).

\section{PROTOZOA (SARCOMASTIGOPHORA)}

\section{CRYPTOBIIDAE}

Cryptobia borreli (Laveran and Mesnil 1901). Northern California. BTood. WaTes and Wolf (1955).

Cryptobia salmositica Katz 1951. Oregon. Blood. Davison and Breese (1954); British Columbia, Washington. Becker and Katz (1965b); USSR. Bykhovskaya-Pavlovskaya et a1. (1962).

\section{THECAMOEB IDAE}

Thecamoeba hoffmani Sawyer, Hnath, and Conrad 1974. Oregon, Washington. Gil1s. Sawyer et a1. (1974).

\section{Oncorhynchus nerka - Sockeye Salmon}




\section{ECHINORHYNCHIDAE}

Metechinorhynchus sajimonis (Müi ler 1784) Petrotschenko 1956. Lake Huron. Intestine. Co7Tins and Dechtiar (1974); Kamchatka, USSR. Konovalov et al. (1970).

Echinorhynchus gadi Zoega in Müller 1776. Kamchatka, USSR. Trofimenko (1962).

Acanthocephalus echigoensis Fujita 1920. Japan. Van Cleave (7937) as A aculeatus.

AcanthocephaTus jacksoni Bullock 1962. Lake Huron. Intestine. Collins and Dechtiar (1974).

\section{LEPTORHYNCHOIDIDAE}

Leptorhynchoides thecatus (Linton 1891) Kostylew 1924. Lake Huron. Intestine. ColTins and Dechtiar (1974).

\section{NEOECHINORHYNCHIDAE}

Neoechinorhynchus rutili (Müller 1780) Hamann 1892. British Columbia. Intestine. Arai (1969) ; Oregon. Intestine. Merritt and Pratt (1964); Kamchatka, USSR. Trofimenko (1962); Lake Huron. Collins and Dechtiar (1974).

Neoechinorhynchus tumidus Van Cleave and Bangham 1949. Lake Huron. Intestine. Collins and Dechtiar (1974).

Neoechinorhynchus pungitius Dechtiar 1971. Lake Huron. Intestine. Collins and Dechtiar (1974).

POLYMORPHIDAE

Bolbosoma caenoforme (Heitz 1920) Meyer 1933. Kamchatka, USSR. Akhmerov (7954b); Washington to Alaska. Margol is (1963). Corynosoma strumosum (Rudolphi 1802) Lühe 1904. Juvenile. North Pacific. Margolis (1958b); Kamchatka, USSR. Trofimenko (1962). Corynosoma semerme (Forssel1 1904) Lühe 1911. Juvenile. Alaska. Margolis (7958b).

Corynosoma wegeneri Heinze 1934. Juvenile. Alaska. Margolis (1958b).

\section{POMPHORHYNCHIDAE}

Pomphorhynchus bulbocolli Linkins in Van Cleave 1919. British Columbia. Bangham and Adams (1954); Lake Huron. Intestine. Collins and Dechtiar (1974).

\section{RHADINORHYNCHIDAE}

Rhadinorhynchus trachuri Harada 1935. Washington. Margolis (1963) as Nipporhynchus t. 


\section{HYDRACHNIDAE}

Hydrachna sp. Lake Huron. Gills. Collins and Dechtiar (1974).

\section{CESTODA}

\section{AMPHICOTYLIDAE}

Eubothrium crassum (BToch 1779) Nybel in 1922. Kamchatka, USSR. Mamaev et a T. (1959). Eubothrium salvelini (Schrank 1790) Nybel in 1922. Washington to Alaska. Intestine. Smith (1973); Kamchatka, USSR. Konovalov et a1. (1970). Eubothrium sp. British Columbia, Alaska. Margolis (1963).

\section{CYATHOCEPHALIDAE}

Cyathocephalus truncatus (Pallas 1781) Kessler 1868. USSR. Intestine. Bykhovskaya-Pavlovskaya et a7. (1962). Bothrimonus sturionis Durvernoy 1842. Alaska. Margolis (1963) as Diplocotyle olkirki.

\section{DIPHYLLOBOTHRI IDAE}

Diphyllobothrium ursi Rausch 1954. Pleroceroid. Alaska. Intestine. Rausch (1954).

Diphyllobothrium sp. Larva. British Columbia. Body cavity. Arai (1969b); Kamchatka, USSR. Body cavity. Trofimenko (1962);

California. Haderlie (1953).

Diphyllobothrium latum (Linnaeus 1758) Matz 1892. Larva. Alaska. Merre11 (1964).

Diphy1 lobothrium cordiceps (Leidy 1871) Matz 1892. Larva. Canada. Body cavity. Mudry and Anderson 15.

\section{LIGULIDAE}

Schistocephalus sp. Larva. British Columbia. Bangham and Adams 1954).

\section{PHYLLOBOTHRI IDAE}

Pelichnibothrium sp. Larva. Japan. Seki (1975).

Phyllobothrium sp. Larva. British Columbia. Intestine. Arai (1969b). Phyl1obothrium caudatum (Zschokke and Heitz 1914). Larva. Kamchatka, USSR. Digestive tract. Trofimenko (1962) as Pelichnibothrium speciosum; Washington. Margolis (1965).

\section{PROTEOCEPHALIDAE}

Proteocephalus arcticus Cooper 1921. British Columbia. Wardle (1933b). [Species Inquirae Freze (1965)].

ProteocephaTus exiquus La Rue 1911. British Columbia. Bangham and Adams (1954); Kamchatka, USSR. Akhmerov (1954b). 
ProteocephaTus 1aruei Faust 1920. Hoffman (1967).

Proteocephalus sp. Larva. Washington to Alaska. Margolis (1963).

\section{TENTACULARIIDAE}

Nybelinia sp. Larva. British Columbia. Stomach. Wardle (1932).

\section{TETRAPHYLLIDEA}

Scolex pleuronectis Müller 1788. Kamchatka, USSR. Intestine. Strelkov (1960).

Scolex polymorphus Rudolphi 1819. Kamchatka, USSR. Intestine. Akhmerov (1955).

\section{TRIAENOPHORIDAE}

Triaenophorus crassus Forel 1868. Alaska. Margolis (1967b).

Triaenophorus nodulosus (Pallas 1760) Rudolphi 1819. Larva. Lake Huron. Liver. Collins and Dechtiar (1974).

COPEPODA

\section{CALIGIDAE}

Lepeophtheirus salmonis (Krøyer 1838). British Columbia. Margolis' Pacific Coast. Wilson (1908a) as L.. pacificus.

\section{ERGASILIDAE}

Ergasilus auritus Markevich 1940. Alaska, British Columbia. Roberts (1963); Kamchatka, USSR. Konovalov et al. (1970).

Ergasilus nerkae Roberts 1963. British Columbia. Gills. Roberts (1963).

Ergasilus turgidus Fraser 1920. Alaska. Gills, fins. Cope (1959). Ergasilus caeruleus Wilson 1911. Lake Huron. Gills. Collins and Dechtiar (1974).

\section{LERNAEOPODIDAE}

Salmincola edwardsii (01sson 1869) Wilson 1915. Kamchatka, USSR. Akhmerov (1954b).

Salmincola californiensis (Dana 1853) Wilson 1915. Oregon, Washington, Idaho, British Columbia. Gills. Kabata (1969c); Japan. Mouth. Yamaguti (1939c); USSR. Bykhovskaya-Pavlovskaya et al. (1962) as Salminicola falculata.

Salmincola carpionis (Krøyer 1837) Wilson 1915. British Columbia. Foerster (1929).

\section{DIGENEA}

\section{ALLOCREADI IDAE}


Crepidostomum farionis (Müller 1780) Lühe 1909. British Columbia. Bangham and Adams (1954); Kamchatka, USSR. Trofimenko (1962);

California. Intestine, pyloric caeca. Haderlie (1953); Lake Huron. Collins and Dechtiar (1974).

\section{BUCEPHALIDAE}

Prosorhynchoides gracilescens (Rudolphi 1819) n. comb. Kamchatka, USSR. Mamaev et a1. (1959); Alaska. Margolis (1963).

\section{DIPLOSTOMATIDAE}

Diplostomum sp. Larva. Kamchatka, USSR. Mamaev et al. (1959). Diplostomum spathaceum (Rudolphi 1819) 01sson 1876. Metacercaria. Lake Huron. Eyes. Collins and Dechtiar (1974).

Tetracotyle sp. Larva. North Pacific. Margolis (1963).

\section{FELLODISTOMATIDAE}

Pseudopentagramma petrowi (Layman 1930) Yamaguti 1971. Alaska. caeca, intestine. Margolis and Ching (1965) as Pentagramma p.

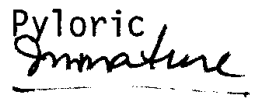

GORGODERIDAE

Phy1lodistomum conostomum (01sson 1876) Odhner 1902. Kamchatka, USSR. Konovalov et al. (1970).

HEMIURIDAE

Genolinea laticauda Manter 1925. British Columbia. Stomach. Arai (1969b).

Parahemiuris merus (Linton 1910) Woolcock 1935. British Columbia. Intestine. Arai (1969b).

Sterrhurus sp. Kamchatka, USSR. Akhmerov (1954b).

Hemiurus levinseni Odhner 1905. Kamchatka, USSR. Intestine. Strelkov (1960); Alaska, British Columbia. Margol is (1963).

Derogenes varicus (Müi ler 1784) Looss 1901. Kamchatka, USSR. Mamaev et a1. (1959); British Columbia, Alaska. Margolis (1963).

Brachyphallus crenatus (Rudolphi 1802) Odhner 1905. Kamchatka, USSR. Digestive tract. Trofimenko (1962); Washington to Alaska. Margol is (1963).

Genarchopsis muelieri (Levinsen 1881) Yamaguti 1954. Kamchatka, USSR. Digestive tract. Trofimenko (1962) as Genarches m.

Lecithophyllum botryophorum (01sson 1868) Odhner 1905. British Columbia, ATaska. Stomach. Margol is (1963) as L. anteroporum.

Tubulovesicula lindbergi (Layman 1930) Yamaguti 1934. Washington. Margolis (1965). 
Oncorhynchus nerka (Continued)

Lecithaster gibbosus (Rudolphi 1802) Lühe 1901. British Columbia. Intestine. Arai (1969b); Kamchatka, USSR. Trofimenko (1962).

\section{LAMPRITREMATIDAE}

Lampritrema miescheri (Zschokka 1890) Margol is 1962. Okhotsk Sea, USSR. Stomach, gills. Margolis (1962).

OPECOELIDAE

Plagioporus shawi (McIntosh 1939) Margolis 1970. Washington. Intestine. Margolis (1970b).

STRIGEIDAE

Cotylurus erraticus (Rudo7phi 1809) Szidat 1928. Metacercaria. Montana. Pericardial cavity. 01 son (1970).

\section{SYNCOELIIDAE}

Syncoelium fil iferum (Leuckart, in Sars 1885) Odhner 1911. Washington. Branchial cavity. Lloyd and Guberlet (1936); British Columbia. Margol is (1963) as $\underline{\text { S. katuwo. }}$

HIRUDINOIDEA

PISCICOLIDAE

Piscicola salmositica Meyer 1946. British Columbia, Washington.

Pectoral, pelvic fins, gills. Becker and Katz (1965a).

MOLLUSCA

UNIONIDAE

Anodonta beringiana Middendorf. Glochidium. Alaska. Gills, body surface. Cope (1959).

MONOGENEA

GYRODACTYLIDAE

Gyrodactyloides strelkowi Bykhovsky and Polyanski 1953. Kamchatka,

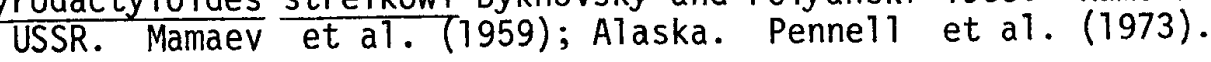

\section{TETRAONCHIDAE}

Tetraonchus alaskensis Price 1937. Alaska. Margolis (1963).

NEMATODA

ANISAKIDAE 
Contracaecum hypomesi Fujita 1932. Larva. Japan. Body cavity. Fujita (1939).

Contracaecum benimasu Fujita 1932. Larva. Japan. Yamaguti (1961). Contracaecum elongatum Fujita 1939. Larva. Japan. Body cavity.

Fujita (1939).

Contracaecum hippoglossi Fujita 1932. Larva. Japan. Fujita (1932). Contracaecum ochotense Fujita 1932. Larva. Japan. Fujita (1932). Contracaecum sp. Larva. British Columbia. Body cavity. Arai (1969b); Japan. Seki (1975).

Contracaecum spiculigerum (Rudolphi 1809). Railliet and Henry 1912. Larva. British Columbia. Bangham and Adams (1954).

* Thynnascaris adunca (Rudolphi 1802) Hartwich 1957. British Columbia. Stomach, intestine. Margolis3; Kamchatka, USSR. Trofimenko (1962) both as Contracaecum a.

Ânisakis sp. Larva. British Columbia. Body cavity. Arai (1969b);

Washington. Margolis (1965); Kamchatka, USSR. Trofimenko (1962).

Raphidascaris sp. Japan. Fukui (1958).

\section{CAPILLARI IDAE}

Capillaria sp. Alaska. Margolis (1963).

Thominx catenata (Van Cleave and Mueller 1932) Skrjabin and Schikhobalova 1954. Lake Huron. Intestine. Collins and Dectiar (1974) as Capillaria c.

\section{CUCULLANIDAE}

Truttaedacnitis trutta (Fabricius 1794) Petter 1974. Kamchatka, USSR. Akhmerov (1954b) as Dacnitis truttae.

\section{CYSTIDICOL IDAE}

Ascarophis sebastodis 01 sen 1952. British Columbia. Intestine. Arai $(1969 \mathrm{~b})$.

Cystidicola farionis Fisher 1798. Japan Sea, USSR to Bering Sea, Alaska. Swimbladder. Margol is (1967a).

Cystidicola stigmatura (Leidy 1886) Ward and Magath 1916. Lake Huron. SwimbTadder. Collins and Dechtiar (1974).

Salvelinema salmonicola (Ishi i 1916) Margol is (1966). Japan, USSR, Alaska. Swimbladder. Margolis (1967a); Kamchatka, USSR. Trofimenko (1962) as $\underline{S}$. cristata.

Cystidicoloides tenuissima (Zeder 1800) Rasheed 1969. Probably Russian waters. Intestine. Ko and Anderson (1969); British Columbia. Bangham and Adams (1954); USSR. Stomach, intestine. Byhovskaya-Paviovskaya et al. (1962) latter two as Metabronema salvelini.

Metabronema laticauda Fujita 1939. Kamchatka, USSR. Intestine. Fujita (1939).

Spinitectus gracilis Ward and Magath 1917. Lake Huron. Intestine. Collins and Dechtiar (1974). 


\section{PHILOMETRIDAE}

Philonema oncorhynchi Kuitunen-Ekbaum 1933. British Columbia. Peritoneum, pyloric caeca, gonads, mesentery. Dombroski (1955); Kamchatka, USSR. Trofimenko (1962).

\section{THELAZI IDAE}

Rhabdochona fujii (Fujita 1921) Fujita 1927. Japan. Intestine. Fujita (1927a) as Cystidicola $f$.

Rhabdochona cascadilla Wigdor 1978. British Columbia. Bangham and Adams (1954); Lake Huron. Intestine. Collins and Dechtiar (1974).

\section{PROTOZOA (CILIOPHORA)}

\section{DENDROSOMATIDAE}

Trichophrya piscium Bütsch1i 1889. Kamchatka, USSR. Konovalov et al. (1970).

\section{URCEOLARI IDAE}

Trichodina nigra kamchatka. Kamchatka, USSR. Konovalov et al. (1970). PROTOZOA (MICROSPORIDA)

\section{NOSEMATIDAE}

Plistophora sp. Northern California. Gills. Wales and Wolf (1955); Muscles of head. Awakura (1965). Glugea takedai Awakura 1974. Japan. Muscles. Awakura (1974).

\section{PROTOZOA (MYXOSPORIDA)}

\section{CERATOMYXIDAE}

Leptotheca krogiusi Konovalov and Shulman 1965. Kamchatka, USSR. Urinary bladder. Shulman (1966).

\section{MYXIDI IDAE}

Myxidium oviforme Parisi 1912. USSR. Gallbladder. BykhovskayaPavlovskaya et al. (1962).

Myxidium obscurum Konovalov 1966. Kamchatka, USSR. Anterior intestine, urinary bladder. Shulman (1966). 
MYXOBOLIDAE

Henneguya salminicola Ward 1919. Kamchatka, USSR. Muscles. Akhmerov

(1954a); British Columbia. Muscles. Margolis (1963).

Myxobolus neurobius Schuberg and Schroder 7905. Kamchatka, USSR.

Cysts in nervous tissues. Bykhovskaya-Pavlovskaya et al. (1962). Myxobolus $\frac{\text { krokhini Konovalov and Shulman 1966. Kamchatka, USSR. }}{\text { Konovalov et aT. (1970). }}$

MYXOSOMATIDAE

$\frac{\text { Myxosoma }}{\text { Halliday }} \frac{\text { cerebral is }}{(1976) \text {. }}$ (Hofer and Plehn 1903) Kudo 1933. Asia. Brain.

\section{SPHAEROSPORIDAE}

Chloromyxum wardi Kudo 1920. Alaska, USSR. Gallbladder. Kudo (1920). Chloromyxum corregoni Bauer 1948. USSR. Gallbladder. Kudo and

\section{PROTOZOA (SARCOMASTIGOPHORA)}

CRYPTOBIIDAE

Cryptobia salmositica Katz 1951. Becker (1970a).

Cryptobia branchialis. Japan. Gills. Sano (1970).

\section{HEXAMITIDAE}

Hexamita salmonis (Moore 1922). British Columbia. Duff (1934).

\section{TETRAMITIDAE}

Ichthyobodo necatrix (Henneguy 1884). British Columbia. Gills. Hoskins et a 1.13 as Costia necatrix.

$$
\text { Oncorhynchus tshawytscha - Chinook Salmon }
$$

\section{ACANTHOCEPHALA}

\section{ECHINORHYNCHIDAE}

Echinorhynchus gadi Zoega in Müiler 1776. British Columbia. Intestine. Arai (1969b); Kamchatka, USSR. Trofimenko (1962).

Metechinorhynchus salmonis (Mülier 1784) Petrotchenko 1956. Lake Michigan. Intestine. Amin and Burrows (1977).

\section{NEOECHINORHYNCHIDAE}

Neoechinorhynchus cristatus Lynch 1936. Kamchatka, USSR. Akhmerov (1954b). POLYMORPHIDAE

Corynosoma strumosum (Rudolphi 1802) Lühe 1904. Larva. Kamchatka, USSR. Trofimenko (1962).

Bolbosoma caenoforme (Heitz 1920) Meyer 1933. USSR. Trofimenko (1962). 
Oncorhynchus tshawytscha (Continued)

BRANCHIURA

ARGULIDAE

Argulus sp. Oregon. Shaw (1947).

CESTODA

AMPHICOTYLIDAE

Eubothrium sp. Oregon. Shaw (1947).

Eubothrium crassum (Bloch 1779) Nybel in 1922. Kamchatka, USSR.

Trofimenko (1962); British Columbia. Pyloric caeca.

Wardle (1932) as E. oncorhynchi.

BOTHRIOCEPHALIDAE

Bothriocephalus sp. Larva. Oregon. Intestine. 01son (1978a).

DIPHYLLOBOTHRI IDAE

Diphy7lobothrium sp. Larva. Kamchatka, USSR. Mamaev et a1. (1959). HEPATOXYLIDAE

Hepatoxylon trichuri (Holten 1802) Do11fus 1942. Post 1arva.

Kamchatka, USSR. Zschokke and Heitz (1914) as Coenomorphus grossus.

PHYLLOBOTHRIIDAE

Phyllobothrium caudatum (Zschokke and Heitz 1914). Larva. Kamchatka, USSR. Mamaev et aT. (1959).

Phyllobothrium sp. Larva. British Columbia. Intestine. Arai (1969b); Oregon. Olson (1978a).

PROTEOCEPHALIDAE

Proteocephalus exiguus La Rue 1911. Kamchatka, USSR. Intestine. Akhmerov (1955).

TENTACULARI IDAE

Nybelinia sp. Larva. British Columbia. Stomach. Wardle (1932).

Nybel inia surmenicola Okada 1929. Larva. Kamchatka, USSR. Strelkov (1960).

Tentacularia coryphaenae Bosc 1802. Larva. Kamchatka, USSR. Zschokke and Heitz (1914) as Tetrahynchus quadirostris.

\section{TETRAPHYLLIDEA}

Scolex pleuronectis Müller 1788. Kamchatka, USSR. Intestine. Streikov (1960). 
Oncorhynchus tshawytscha (Continued)

Scolex polymorphus Rudolphi 1819. Kamchatka, USSR. Akhmerov (1954a).

\section{CALIGIDAE}

\section{CALIGIDAE}

Caligus sp. Central California. Body surface. Wilson (1908a) as C. gurnardi.

Lepeophtheirus salmonis (Krøyer 1838). British Columbia. Margolis ${ }^{3}$; Central California. Gills. Wilson (1908a).

\section{LERNAEIDAE}

Lernaea cyprinacea Linnaeus 1758. Oregon. Eyes. Uzmann and Rayner (1958).

\section{LERNAEOPODIDAE}

Salmincola californiensis (Dana 1853) Wilson 1915. California, Oregon. Gills, Kabata (7969c); Bykhovskaya-Pavlovskaya et al. (1962) as Salminicola falculata.

\section{THERODAMASIDAE}

Paeonodes nemaformis Hewitt 1969. New Zealand. Hewitt (1969a).

\section{DIGENEA}

AZYGIIDAE

Azygia robusta Odhner 1911. Kamchatka, USSR. Trofimenko (1962). BUCEPHALIDAE

Prosorhynchoides gracilescens (Rudo1phi 1819) n. comb, Kamchatka, USSR. Trofimenko (1962).

HEMIURIDAE

Deropegus aspina (Ingles 1936) McCauley and Pratt 1961. Oregon. Stomach. McCauley and Pratt (1967). Genolinea Tintoni Skrjabin and Guschanskaja 1955. California. Stomach. Linton (1940) as Genarches sp.

Brachyphallus crenatus (Rudolphi 1802) Odhner 1905. Washington. Stomach. Lloyd (1938); Oregon. Shaw (1947); Kamchatka, USSR. Trofimenko (1962).

Derogenes sp. Pratt and McCauley (1961). Derogenes infirmus (Linton 1940) Yamaguti 1971. Northern California. Stomach, esophagus. Linton (1940) as Genarches i. Lecithaster gibbosus (Rudolphi 1802) Lühe 1901. Washington. Intestine. Lloyd (7938); British Columbia. Intestine. Arai (1969b). TubuTovesicula Tindbergi (Layman 1930) Yamaguti 1934. Oregon. Stomach. Mccauley (1960); British Columbia. Stomach. Margolis 3 as I. nanaimoensis. 
NANOPHYETIDAE

Nanophyetus salmincola (Chapin 1926) Chapin 1927. Metacercaria. Oregon. Simms et a1. (1931); Oregon. Kidneys. Millemann et al. (1964).

HIRUDINOIDEA

PISCICOLIDAE

Piscicola salmositica Meyer 1946. Washington, Oregon. Pectoral, pelvic fins, gills. Becker and Katz (1965a); California. Haderlie (1953).

MOLLUSCA

\section{UNIONIDAE}

Anodonta beringiana Middendorf. Glochidium. Alaska. Skin, fins, mouth. Cope (1959).

Margaritifera margaritifera (Linnaeus). Glochidium. Washington. Gills. Davis (1953).

NEMATODA

\section{ANISAKIDAE}

* Thynnascaris sp. Oregon. Intestine. 01son (1978a).

* Thynnascaris adunca (Rudolphi 1802) Hartwich 1957. Kamchatka, USSR. Intestine. Strelkov (1960) as Contracaecum a. Contracaecum sp. Larva. British Columbia. Body cavity. Arai (1969b); Kamchatka, USSR. Strelkov (1960). Contracaecum spiculigerum (Rudolphi 1809) Railliet and Henry 1912. Larva. Oregon. Intestine. Shaw et a1. (1934).

Rhaphidascaris sp. Oregon. Shaw (1947). Anisakis sp. Larva. British Columbia. Many places in body. Margolis ${ }^{3}$; Kamchatka, USSR. Trofimenko (1962); Oregon. 01 son (1978a).

\section{CAPILLARI IDAE}

Capillaria sp. British Columbia. Stomach. Arai (1969b).

CUCULLANIDAE

Truttaedacnitis trutta (Fabricius 1794) Petter 1974. Kamchatka, USSR. Mamaev et al. (1959) as Dacnitis $t$.

\section{CYSTIDICOLIDAE}

Salvelinema walkeri (Ekbaum 1935) Margolis 1967. British Columbia. Swimbladder. Godfrey 14. 
Oncorhynchus tshawytscha (Continued)

PHILOMETRIDAE

Philonema oncorhynchi Kuitunen-Ekbaum 1933. British Columbia. Body cavity. Margol is ${ }^{3}$.

Philonema sp. Larva. British Columbia. Swimbladder wal1. Arai $(1969 b)$.

PROTOZOA (CILIOPHORA)

CHLAMYDODONTIDAE

Chilodonella cyprini (Moroff 1902). California. Skin. Leitritz and Lewis (1976).

URCEOLARI IDAE

Tripartiella californica (Davis 1947). USSR. Body surface, fins, gills. Bykhovskaya-Pavlovskaya et a1. (1962); Sacramento River, California. Davis (1947) as Trichodina c.

PROTOZOA (HAPLOSPORIDA)

Dermocystidium salmonis Davis 1947. Northern California. Gills. Davis (1947).

PROTOZOA (MICROSPORIDA)

NOSEMATI IDAE

Plistophora salmonae Putz, Hoffman and Dunbar 1965. California. Gills. Leitritz and Lewis (1976).

Glugea takedai Awakura 1974. Japan. Muscles. Awakura (1974).

PROTOZOA (MYXOSPORIDA)

CERATOMYXIDAE

Ceratomyxa shasta Noble 1950. Oregon. Sanders et a1. (1970).

Leptotheca krogiusi Konovalov and Shulman 1965. Kamchatka, USSR. Urinary bladder. Shulman (1966).

\section{MYXIDI IDAE}

Myxidium minteri Yasutake and Wood 1957. Washington. Renal tubules. Yasutake and Wood (1957); Oregon. Gallbladder. Sanders and Fryer (1970).

Myxidium oviforme Parisi 1912. USSR. Gallbladder. BykhovskayaPavlovskaya et al. (1962).

Zschokkella orientalis Konovalov and Shuiman 1966. USSR. Gallbladder. ShuIman (1966). 
MYXOBOLIDAE

Henneguya saiminicola Ward 1919. Alaska. Muscles (cysts). Fish (1939). MyxoboTus insidiosus Wyatt and Pratt 1963. Oregon. Muscles. Wyatt and Pratt (1963).

Myxobolus kisutchi Yasutake and Wood 1957. Washington. Brain. Wyatt $(1978)$.

\section{SPHAEROSPORIDAE}

Chloromyxum majori Yasutake and Wood 1957. Washington. Glomeruli. Yasutake and Wood (1957).

PROTOZOA (SARCOMASTIGOPHORA)

\section{CRYPTOBI IDAE}

Cryptobia salmositica Katz 1951. Northern California. Blood. Davison and Breese (1954); Washington. Becker and Katz (1965b). Cryptobia borreli (Laveran and Mesnil 1901). Northern California. Blood. Wasles and Wolf (1955).

\section{HEXAMITIDAE}

Hexamita salmonis (Moore 1922). Washington. Intestine. Uzmann et al. $(1965)$.

\section{THECAMOEBIDAE}

Thecamoeba hoffmani Sawyer, Hnath and Conrad 1974. Washington, Oregon. Gi17s. Sawyer et a1. (1974).

$$
\text { Salmo clarki - Cutthroat Trout }
$$

\section{ACANTHOCEPHALA}

\section{ECHINORHYNCHIDAE}

Metechinorhynchus lateralis (Leidy 1851) Golvan 1969. Northern California. Intestine. Haderlie (1953) as Echinorhynchus 1.

\section{NEOECHINORHYNCHIDAE}

Neoechinorhynchus rutili (Müller 1780) Hamann 1892. British Columbia. Bangham and Adams (1954); Wyoming. Intestine. Heckmann (1971). Neoechinorhynchus crassus Van Cleave 1919. USSR. Intestine. Bykhovskaya - Pavlovskaya et al. (1964).

\section{POMPHORHYNCHIDAE}

Pomphorhynchus bulbocolli Linkins in Van Cleave 1919. British Columbia. Bangham and Adams (1954). 
Salmo clarki (Continued)

AMPHICOTYLIDAE

Eubothrium salvelini (Schrank 1790) Nybelin 1922. British Columbia. Bangham and Adams (1954).

CYATHOCEPHAL IDAE

Cyathocephalus sp. Washington. Neiland (1952).

DIPHYLLOBOTHRI IDAE

Diphy110bothrium cordiceps (Leidy 1871) Matz 1892. Larva. Montana. Body cavity. Simms and Shaw (1931) as Dibothrium c.

Diphyll lobothrium sp. Larva. Washington. Becker and Brunson (1967).

PROTEOCEPHALIDAE

Proteocephalus primaverus Neiland 1952. Washington. Neiland (1952).

ProteocephaTus ambToplitis (Leidy 1887) Benedict 1900. Plerocercoid. Washington. Becker (1967a).

Proteocephalus arcticus Cooper 1921. British Columbia. Wardle (1933b) [Species Inquirae - Freze (1965)].

Proteocephalus laruei Faust 1920. Immature. Wyoming. Digestive tract. Bangham (1951).

Proteocephalus salmonidicola Alexander 1951. British Columbia. Bangham and Adams (1954); Washington. Intestine. Becker (1967a).

COPEPODA

CALIGIDAE

Lepeophtheirus salmonis (Krøyer 1838). British Columbia. Skin. Margolis $(1958 \mathrm{c})$.

\section{LERNAEOPODIDAE}

Salmincola edwardsii (01sson 1869) Wilson 1915. British Columbia. Beres as $\underline{\text { s. bicauliculata. }}$

DIGENEA

\section{ALLOCREADI IDAE}

Crepidostomum farionis (Mü11er 1780) Lühe 1909. Northern

California. Gallbladder. Haderlie (1953); Hyoming. Heckmann (1971).

Crepidostomum cooperi Hopkins 1931. Wyoming. Probably rectum. Hunninen and Hunter (1933) as C. fausti.

Crepidostomum transmarinum (NicoT1 $\overline{1909)}$. North America. Hunninen and Hunter (1933).

Allocreadium lobatum Wallin 1909. Wyoming. Bangham (1951). 
Salmo clarki (Continued)

\section{CLINOSTOMATIDAE}

Clinostomum marginatum (Rudolphi 1819) Braun 1899. Metacercaria. Washington. Throughout body. Uzmann and Douglass (1966).

\section{DIPLOSTOMATIDAE}

Diplostomum sp. British Columbia. Bangham and Adams (1954). Neascus sp. British Columbia. Bangham and Adams (1954). Posthodiplostomum minimum (MacCallum 1921) Dubois 1936. Larva. Wyoming. Bangham (1957).

\section{HEMIURIDAE}

Derogenes sp. Pratt and McCauley (1961).

Deropegus aspina (Ingles 1936) McCauley and Pratt 1961. Oregon. Stomach. McCauley and Pratt (1961).

\section{HETEROPHYIDAE}

Apophallus sp. Larva. Wyoming. Bangham (1951).

\section{NANOPHYETIDAE}

Nanophyetus salmincola (Chapin 1926) Chapin 1927. Metacercaria. Oregon. Simms et a1. (1931).

\section{OPECOELIDAE}

Plagioporus siliculus Sinitsin 1931. Oregon. Sinitsin (1931). Plagioporus shawi (McIntosh 1939) Margolis 1970. Yamaguti (1971) as Peracreadium s.

\section{SANGUINICOLIDAE}

Cardicola davisi (Wales 1958) Meade and Pratt 1965. Oregon. Davis et a1. (1961) as Sanguinicola d.

Cardicola alseae Meade and Pratt 1965. Oregon. Blood. Meade and Pratt (1965).

Cardicola klamathensis (Wales 1958) Meade and Pratt 1965. Oregon. Wales (1958) as Sanguinicola $k$.

HIRUDINOIDEA

\section{PISC ICOLIDAE}

Piscicola salmositica Meyer 1946. Wyoming. Fins. Heckmann (1971). Myzobdel1a sp. Wyoming. Bangham (1951) as Illinobdella sp. 
Salmo clarki (Continued)

MOLLUSCA

UNIONIDAE

Margaritifera margaritifera (Linnaeus). Glochidia. Wyoming. Bangham (1957).

MONOGENEA

GYRODACTYLIDAE

Gyrodactylus elegans Nordmann 1832. Washington. Wood and Mizelle (1957).

TETRAONCHIDAE

Tetraonchus alaskensis Price 1937. Alaska. Gi1ls. Price (1937a). NEMATODA

ANISAKIDAE

Contracaecum sp. Larva. British Columbia. Bangham and Adams (1954).

\section{CAPILLARI IDAE}

Skrjabinocapillaria bakeri (Mueller and Van Cleave 1932) Skrjabin and Schikhobalova 1954. Wyoming. Bangham (1951); British Columbia. Bangham and Adams (1954) both as Hepaticola b.

Thominx catenata (Van Cleave and Mueller 1932) Skrjabin and Schikhobalova 1954. Idaho. Fritts (1959).

\section{CYSTIDICOLIDAE}

Salvel inema walkeri (Ekbaum 1935) Margol is 1967. Northern California. Intestine. Haderlie (1953) as Cystidicola stigmatura.

Cystidicoloides tenuissima (Zeder 1800) Rasheed 1966. Wyoming. Bangham (1951) as Metabronema salvelini; Oregon. Shaw (1947) as Ascarophis hardwoodi.

Cystidicoloides sp. Idaho. Fritts (1959).

Genera and species. Larva. Wyoming. Bangham (1951) as Agamospirua sp.

\section{CUCULLANIDAE}

Truttaedacnitis trutta (Fabricius 1794) Petter 1974. Oregon. Shaw (1947) as Cucu17anus t; Wyoming. Intestine, liver, mesentery. Bangham (1951) as Bulbodacnitis scotti; British Columbia. Bangham and Adams (1954) as B. globosa. 
Salmo clarki (Continued)

DIOCTOPHYMATIDAE

Eustrongylides sp. Larva. Oregon. Muscies. Shaw et a1. (1934); British Columbia. Bangham and Adams (1954).

PHILOMETRIDAE

Philonema onchorhynchi Kuitunen-Ekbaum 1933. British Columbia. Bangham and Adams (1954).

Philometra sp. Oregon. Shaw (1947).

THELAZI IDAE

Rhabdochona sp. Idaho. Fritts (1959). $\frac{\text { Rhabdochona }}{\text { and Adams }} \frac{\text { cascadilla Wigdor 1918. British Columbia. Bangham }}{(1954) \text {. }}$

PROTOZOA (CILIOPHORA)

DENDROSOMATIDAE

Trichophyra sp. Washington. Gi11s. Becker (1967a).

URCEOLARIIDAE

Trichodina truttae. Oregon. Body surface. Davis (1947).

Trichodina sp. Wyoming. Gills. Heckmann (1971).

PROTOZOA (MYXOSPORIDA)

CERATOMYXIDAE

Ceratomyxa shasta Noble 1950. Oregon. Sanders et al. (1970). MYXIDI IDAE

Myxidium minteri Yasutake and Wood 1957. Columbia River.

Gallbladder. Sanders and Fryer (1970).

MYXOSOMATIDAE

Myxosoma cerebralis (Hofer and Plehn 1904) Kudo 1934. USA. Brain. Halliday (1976).

PROTOZOA (SARCOMASTIGOPHORA)

HEXAMITIDAE

Hexamita salmonis (Moore 1922). British Columbia. Duff (1934). 
Salmo clarki (Continued)

\section{TETRAMITIDAE}

Ichthyobodo necatrix (Henneguy 1884). Hoffman (1967) as Costia $n$. Costia pyriformis Davis 1943. Wyoming. Gi1ls. Heckmann (1971).

PROTOZOA (SPOROZOA)

HAEMOGREGARINIDAE

Haemogregarina sp. Wyoming. Blood. Heckmann (1971).

Salmo gairdneri -- Rainbow Trout

SYNONYMS - Salmo irideus, Salmo shasta

\section{ACANTHOCEPHALA}

\section{ECHINORHYNCHIDAE}

Metechinorhynchus lateral is (Leidy 1851) Golvan 1969. Newfoundland. Intestine. Sandemann and Pippy (1967) as Echinorhynchus 1. Metechinorhynchus salmonis (Mü1 ler 1784) Petrotschenko 1956. Yugoslavia. Zitnan and Cankovic (1970); USSR. Intestine. Bykhovskaya-Pavlovskaya et a1. (1962); Lake Michigan. Amin and Burrows (1977).

Metechinorhynchus truttae (Schrank 1788) Petrotschenko 1956. Yugoslavia. Zitnan and Cankovic (1970); Ireland. Intestine. Kane (1966); USSR. Bykhovskaya-Pavlovskaya et al. (1962). Metechinorhynchus leidyi (Van Cleave 1924) Golvan 1969. Lake Huron, Canada-USA. Bangham (1944).

Acanthocephalus echigoens is Fujita 1920. Japan. Van Cleave (1931) as $A$. acerbus.

Acanthocephalus anguillae (Müller 1780) Lühe 1911. USSR. Intestine. Bykhovskaya-Pavlovskaya et al. (1962).

Acanthocephalus jacksoni Bullock 1962. New England. Bullock (1962).

Acanthocephalus minor Yamaguti 1935. Japan. Awakura (1972).

Acanthocepha7us opsariichthys Yamaguti 1935. Japan. Intestine. Nakaj ima and Egusa (1975a).

Acanthocephalus parksidei Amin 1975. Wisconsin. Amin (1975). Pseudorhadinorhynchus samegaiens is Nakajima and Egusa 1975. Japan. Intestine. Nakajima and Egusa (1975b).

Pseudoechinorhynchus clavula (Dujardin 1845) Petrotschenko 1956. USSR. Petrushevski ánd Shuiman (1955) as Echinorhynchus c.

\section{LEPTORHYNCHOIDIDAE}

Leptorhychoides thecatus (Linton 1891) Kostylev 1924. Golvan (1969). 
Salmo gairdneri (Continued)

\section{NEOECHINORHYNCHIDAE}

Neoechinorhynchus rutili (Mülier 1780) Hamann 1892. Oregon. Intestine. Merritt and Pratt (1964).

\section{RHADINORHYNCHIDAE}

Rhadinorhynchus sp. Northern California. Intestine. Edwards and Nahhas (7968).

\section{POMPHORHYNCHIDAE}

Pomphorhynchus laevis (Zoega in Müller 1776) Van Cleave 1924. Ireland. Rectum. Kane (1966); Yugoslavia. Zitnan and Cankovic (1970).

Pomphorhynchus bulbocolli Linkins in Van Cleave 1919. British Columbia. Bangham and Adams (1954).

\section{BRANCHIURA}

ARGUL IDAE

Argulus pugettensis Dana 1852. British Columbia. External. Wi Tson (1912b).

Argulus foliaceus (Linnaeus 1758). Great Britain. Fins, body surface. Wootten (1973b).

\section{CESTODA}

\section{AMPHICOTYLIDAE}

Eubothrium sp. Washington. Intestine. Griffith (1953).

Eubothrium crassum (Bloch 1779) Nybelin 1922. Ireland. Intestine. Kane $(1966)$.

Eubothrium salvelini (Schrank 1790) Nybelin 1922. British

Columbia. Bangham and Adams (1954); Newfoundland. Intestine.

Sandemann and Pippy (1967).

CYATHOCEPHALIDAE

Cyathocephalus truncatus (Pa11as 1781) Kessler 1868. USSR.

Petrushevski (1955); France. Joyeux and Baer (1936).

\section{DIPHYLLOBOTHRI IDAE}

Diphyllobothrium sp. Plerocercoid. Alaska. Outer surface of stomach, intestine. Needham and Behnke (1965); California. Haderlie (1953); Ireland. Chappell and Owen (1969). 
Diphyllobothrium cordiceps (Leidy 1871) Matz 1892. Plerocercoid. Montana. Simms and Shaw (1931); Oregon. Shaw (1947).

Diphyllobothrium latum (Linnaeus 1758 ) Matz 1892. USSR.

Petrushevski and Shulman (1955).

Diphyllobothrium dendriticum (Nitzsch 1824) Matz 1892. Plerocercoid. Europe. Chappe 71 and Owen (1969).

Diphyl lobothrium ditremum (Creplin 1825) Matz 1892. Plerocercoid. Canada. Viscera. Mudry and Anderson 15.

\section{LIGULIDAE}

Schistocephalus sp. Larva. British Columbia. Bangham and Adams (1954).

Schistocephalus solidus (Müller 1776) Steenstrup 1857. Plerocercoid. Ireland. Chappel1 and Owen (1969).

Ligula intestinalis (Linneaus 1758). Larva. Hoffman (1967).

\section{PHYLLOBOTHRI IDAE}

Pelichnibothrium sp. Northern California. Edwards and Nahhas (1968).

\section{PROTEOCEPHALIDAE}

Proteocephalus ambloplitis (Leidy 1887) Benedict 1900. Plerocercoid. Washington. Becker (1967a).

Proteocephalus pinguis La Rue 1911. Immature. Wisconsin.

Digestive tract. Bangham (1944).

Proteocephalus longicollis (Zeder 1800) Nufer 1905. USSR.

Petrushevski ảnd Shilman (1955).

Proteocephalus salmonidicola Alexander 1951. Oregon. Alexander

(1951); California. Intestine. Haderlie (1953); British

Columbia. Bangham and Adams (1954).

Proteocephalus sp. Idaho. Fritts (1959).

Proteocephalus percae (Müller 1780) Kailliet 1889. Larva. Great Britain. Intestine. Wootten (197.3b).

\section{TRIAENOPHORIDAE}

Triaenophorus lucii (Müller 1776). Plerocercoid. USSR. Petrushevski and Shulman (1955).

COPEPODA

\section{CALIGIDAE}

Caligus elongatus Nordmann 1832. Atlantic. Skin. Sutterlin et aT. (1976).

Caligus clemensi Parker and Margol is 1964. British Columbia. Fraser (1920) as C gurnardi.

Lepeophtheirus salmonis (Krdyer 1838). British Columbia. Fraser $(1920)$. 
Salmo gairdneri (Continued)

ERGASILIDAE

Ergasilus nerkae Roberts 1963. British Columbia. Bangham and Adams (17954) as E. caeruleus.

Ergasilus sieboldi Nordmann 1832. Poland. Hoffman (1967).

\section{LERNAE IDAE}

Lernaea cyprinacea Linnaeus 1758. Oregon. Eyes. Uzmann and Rayner (1958); Great Britain. Skin. Kennedy (1975).

\section{LERNAEOPODIDAE}

$\frac{\text { Salmincola }}{\text { Oregon. }} \frac{\text { Gilliforniensis }}{\text { Gills. Kabata }}(1969 \mathrm{C}$ ). Salmincola edwardsii (01sson 1869) Wilson 1915. Northern

California. Kabata (1969c); British Columbia. Bangham and
Adams (1954).

\section{DIGENEA}

\section{ALLOCREADI IDAE}

$\frac{\text { Bunodera }}{\text { Anderson }} \frac{\text { luciopercae }}{(1977) \text {. }}$. Crepidostomum metoecus (Braun 1900) Braun 1900. Yugoslavia. Rukavina and DeTic (1965).

$\frac{\text { Crepidostomum canadense Hopkins 1931. Oregon. Shaw (1947) as }}{\text { C. laureatum. }}$ Crepidostomum cooperi Hopkins 1931. Oregon. Shaw (1933).

Crepidostomum farionis (Müller 1780) Lühe 1909. Oregon. Shaw (1947); British Columbia. Bangham and Adams (1954); USSR. Petrushevski and Shulman (1955); California. Intestine and pyloric caeca; Haderlie (1953); Newfoundland. Sandemann and Pippy (1967).

$\frac{\text { Allocreadium lobatum Wall in 1909. British Columbia. Bangham }}{\text { and Adams }}$

\section{AZYGI IDAE}

$\frac{\text { Azygia lucij (Müller 1776) Lühe 1909. Yugoslavia. Zitnan and }}{\text { Cankovic (1970). }}$

\section{CLINOSTOMATIDAE}

Clinostomum marginatum (Rudolphi 1819) Braun 1899. Metacercaria. Washington. Throughout body. Uzmann and Douglas (1966);
California. Haderlie (1953).

\section{DIPLOSTOMAT IDAE}

Diplostomum spathaceum (Rudolphi 1819) 01sson 1876. Metacercaria. Europe. ChappelT and Owen (1969); Finland. Eyes. Tiainen (1966). 
Spathaceum indistinctum (Guberlet 1923) Hughes 1929. Metacercaria. Washington. Eyes. Becker (1967a) as Diplostomum flexicaudum.

Tylodelphys clavata (Nordmann 1832) Diesing 1850. Larva. Great Britain. Eyes. Wootten (1973b).

Tylodelphys podicipina Kozicka and Niewiadomska 1960. Larva. Great Britain. Eyes. Wootten (1973b).

\section{ECHINOSTOMATIDAE}

Echinochasmus milvi Yamaguti 1939. Metacercaria. Washington. Gi17 tissues. Uzmann and Hayduk (1964).

\section{GORGODERIDAE}

Phyllodistomum lachancei Choquette 1947. Hoffman (1967).

\section{HEMIURIDAE}

Aponurus sp. Oregon. Shaw (1947).

Deropegus aspina (Ingles 1936) McCauley and Pratt 1961. Oregon. Stomach. McCauley and Pratt (1961).

Derogenes sp. Shaw (1947).

\section{HETEROPHY IDAE}

Apophallus donicus (Skrjabin and Lindtrop 1919) Price 1931. Metacercaria. Oregon. Skin. Niemi and Macy (1974).

\section{NANOPHYETIDAE}

Nanophyetus salminicola (Chapin 1926) Chapin 1927. Metacercaria. Oregon. Simms et a7. (1931).

\section{OPECOELIDAE}

Plagioporus shawi (McIntosh 1939) Margol is 1970. Oregon. Intestine. McIntosh (1939); Washington. Griffith (1953) both as Podocotyle s.

Plagioporus augusticolle (Hausmann 1896) Dobrovolny 1939. Washington. Intestine. Becker (1967a); Northern California. Intestine. Haderlie (7953).

Crowcrocaecum testiobliqum (Wisniewski 1932) Skrjabin and Koval 1956. Yugos Tavia. Zitnan and Cankovic (1970) $[=$ Nicolla I. Yamaguti (1971)].

Crowcrocaecum wisniewskii Slusarki 1958. Europe. Slusarki (1958).

\section{SANGUINICOLIDAE}

Cardicola davisi (Wales 1958) Meade and Pratt 1965. California. Blood vessels of gills. Wales (1958); Oregon. Davis et al. (1961) both as Sanguinicola d.

Cardicola alseae Meade and Pratt 1965. Oregon. Vascular system. Meade and Pratt (1965). 
Salmo gairdneri (Continued)

Sanguinicola idahoens is Schell 1974. Idaho. Blood vessels, eyes, brain surface. Sche11 (1974).

STEGANODERMATIDAE

Pseudochetosoma salmonicola Dollfus 1951. Bulgaria. Gallbladder. Kakatcheva-Avramova (7966).

\section{STRIGEIDAE}

Cotylurus erraticus (Rudolphi 1809) Szidat 1928. Metacercaria. Europe and North American. Pericardial cavity. Wootten (1973a).

HIRUDINOIDEA

PISCICOLIDAE

Piscicola salmositica Meyer 1946. Washington. Pectoral and pelvic fins, gill Tamellae. Becker and Katz (1965a); California. Meyer (1946).

Piscicola geometra (Linneaus 1761). USSR. Petrushevski and Shulman (7955). Myzobdel7a sp. British Columbia. Bangham and Adams (1954) as Illinobdella sp.

MOLLUSCA

UNIONIDAE

Margaritifera margaritifera (Linnaeus). GTochidia. California. GiTIs. Davis (T953).

Anodonta sp. Larva. Great Britain. Gills. Wootten (1973b).

\section{MONOGENEA}

\section{DISCOCOTYLIDAE}

Discocotyle salmonis Schaffer 1916. Long Island. Gills. Shaffer $(1976)$.

Discocotyle sagittata (Leuckart 1842) Diesing 1850 . Europe.

Gills. Chappel1 and Owen (1969).

\section{GYRODACTYLIDAE}

Gyrodactylus elegans Nordmann 1832. California. Gills, skin. Haderlie (1953); Washington. Wood and Mizelle (1957).

Gyrodactylus colemanensis Mizelle and Kritsky 1967. Northern

California. Skin. Mizelle and Kritsky (1967c).

Gyrodactylus salaris Malmberg 1956. Yugoslavia. Zitnan and Cankovic $(1970)$.

Gyrodactylus brevis Crane and Mizelle 1967. Northern California.

Skin. Crane and Mizeīle (1967). 


\section{NEMATODA}

\section{ANISAKIDAE}

Goezia ascaroides (Goeze 1782). France. Dolifus (1935a); USSR. Bykhovskaya-Pavlovskaya et a1. (1962).

Raphidascaris acus (Bloch 1779) Railliet and Henry 1915. Italy. Hoffman (1967).

Contracaecum spiculigerum (Rudolphi 1809) Rail1iet and Henry 1915. Larva. Northern California. Mesentery, peritoneum, stomach wal1. Haderlie (1953); Oregon. Stomach, intestine. Shaw (1947).

Contracaecum sp. Larva. New Zealand. Stomach. Hewitt and Hine (1972).

\section{CAMALLANIDAE}

Cama11anus lacustris (Zoega 1776) Rai11iet and Henry 1915. USSR. Petrushevski and ShuIman (1955); Ireland. Kane (1966).

\section{CAPILLARI IDAE}

Capillaria salvelini Polyanski 1952. Yugoslavia. Zitnan and Cankovic (1970).

Capillaria sp. Japan. Seki (1975).

Skrjabinocapi ilaria bakeri (Mueller and Van Cleave 1932) Skrjabin

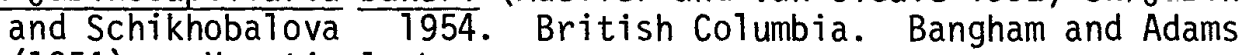
(1954) as Hepaticola b.

\section{CUCULLANIDAE}

Bulbodacnitis ampullastoma Maggenti 1971. Northern California. PyToric caeca, duodenum. Maggenti (1971).

Truttaedacnitis trutta (Fabricius 1794) Petter 1974. Oregon. Shaw (1947) as Cucu17anus t. and C. globosus; British Columbia. Bangham and Adams (1954) as Bulbodacnitis globosa; California. Haderlie (1953) as Bulbodacnitis occidentalis.

\section{CYSTIDICOLIDAE}

Cystidicoloides tenuissima (Zeder 1800) Rasheed 1966. British Columbia. Bangham and Adams (1954) as Metabronema salvelini; Northern California. Haderlie (1953) as Metabronema salvelini; Yugoslavia. Rukavina and Delic (1965) as Sterliadochona t.; USSR. Stomach. Zitnan (1968) as Metabronema truttae.

Cystidicola farionis Fischer 1798. USSR. Zitnan (1968); Ireland. Chappe17 and Owen (1969).

Salvelinema walkeri (Ekbaum 1935) Margol is 1967. British Columbia. Bangham and Adams (1954) as Cystidicola stigmatura.

Ascarophis skrjabini (Layman 1933) Poly anski 1952. USSR. Intestine. Bykhovskaya-Pavlovskaya et a1. (1962). 
Spinitectus gordani Cordero-del-Campillo and Alvarez-Pellitero 1976. Spain. Esophagus and stomach. Cordero-del-Campillo and AlvarezPelitero (1976).

Spinitectus gracilis Ward and Magath 1917. Hoffman (1967).

\section{DIOCTOPHYMATIDAE}

Eustrongylides sp. Larva. New Zealand. Stomachs, mesenteries, intestine. Hewitt and Hine (1972).

\section{PHILOMETRIDAE}

Philometra sp. Oregon. Shaw (1947).

Philonema oncorhynchi Kuitunen-Ekbaum 1933. Body cavity. Meyer (1960); Northern Cal ifornia. Haderlie (1953); Larva. British Columbia. Bangham and Adams (1954).

Philonema agubernaculum Simon and Simon 1936. Wyoming. Body cavity. Simon and Simon (1936) [=Philonema oncorhynchi - Baylis (1936)].

Philonema sp. Alaska. Needham and Behnke (T965); Larva.

Washington. Abdominal cavity. Becker (1967a).

THELAZI IDAE

Rhabdochona cascadilla Wigdor 1918. British Columbia. Bangham and Adams (1954).

Rhabdochona denudata (Dujardin 1845) Railliet 1916. USSR. Bykhovskaya-Pavlovskaya et al. (1962).

Sterliadochona pedispicula Maggenti and Paxman 1971. Northern

California. Stomach and esophagus. Maggenti and Paxman (1971).

PROTOZOA (CILIOPHORA)

CHLAMYDONTIDAE

Chilodonella cyprini (Moroff 1902). California. Skin. Leitritz and Lewis (1976); USSR. Petrushevski and Shulman (1955).

DENDROSOMATIDAE

Trichophyra piscium Butsch1i 1889. Newfoundland. Gil1s. Sandemann and Pippy (7967).

Trichophyra sp. Washington. Gills. Becker (1967a).

\section{EPISTYLIDAE}

Epistylis sp. California. Skin. Leitritz and Lewis (7976).

OPHRYOGLENIDAE

Ichthyophthirius multifillis Fouquet 1876. USSR. Petrushevski and Shulman (1955). Yugoslavia. Zitnan and Cankovic (1970); California. Skin.

Leitritz and Lewis (1976). 
Salmo gairdneri (Continued)

SCYPHIDIIDAE

Scyphidia sp. Washington. Gills. Becker (1967a).

Glosssatella USSR. Bajljozov et a1. (1964).

URCEOLARI IDAE

Trichodina salmincola Wellborn 1967. North Carolina. Fins, body surface. Wellborn (1967).

Trichodina fultani Davis 1947. West Virginia. Body surface, fins, gil1s. Davis (1947).

Trichodina domerguei. USSR. Petrushevski and ShuIman (1955).

PROTOZOÁ (HAPLOSPORIDÁ)

Dermocystidium salmonis Davis 1947. California. Gills, skin. Leitritz and Lewis (1976).

PROTOZOA (MICROSPORIDA)

NOSEMATIDAE

Glugea takedai Awakura 1974. Japan. Muscles. Awakura (1974). Plistophora sp. Northern California, British Columbia. Gills. Wales and Wolf (1955); Japan. Muscles of head. Awakura (1965). Plistophora salmonae Putz, Hoffman and Dunbar 1965. California. Gills. Putz et aT. (1965).

PROTOZOA (MYXOSPORIDA)

CERATOMYXIDAE

Ceratomyxa shasta Noble 1950. California. Viscera. Noble (1950). MYXIDIIDAE

Myxidium minteri Yasutake and Wood 1957. Oregon. Renal tubules. Yasutake and Wood (1957); Gallbladder. Sanders and Fryer (1970). Myxidium oviforme Parisi 1912. California. Gallbladder. Jameson (1931). Zschokkella nova Klokacawa 1914. USSR. Petrushevski and Shulman (1955). MYXOBOLIDAE

Myxobolus kisutchi Yasutake and Wood 1957. Oregon. Epidermal scales. Yasutake and Woods (1957).

MYXOSOMATIDAE

Myxosoma squamalis Iverson 1954. Washington. Body surface. Iverson $(1954)$.

Myxosoma cerebralis (Hofer and Plehn 1903). New Zealand. Cartilage of head. Hewitt and Hine (1972); USSR. Petrushevski and Shulman (1955). 
Salmo gairdneri (Continued)

SPHAEROSPORIDAE

Chioromyxum majori Yasutake and Wood 1957. Washington. Glomerulus. Yasutake and Wood (1957).

Chloromyxum truttae Leger 1961. USSR. Petrushevski and Shuiman (1955).

PROTOZOA (SARCOMASTIGOPHORA)

CRYPTOBIIDAE

$\frac{\text { Cryptobia salmositica Katz 1951. Washington. Blood. Becker and }}{\text { Katz }(7965 \mathrm{~b}) .}$

Cryptobia borreli (Laveran and Mesnil 1901). Northern California.

Blood. Wales and Wolf (1955).

HEXAMIT IDAE

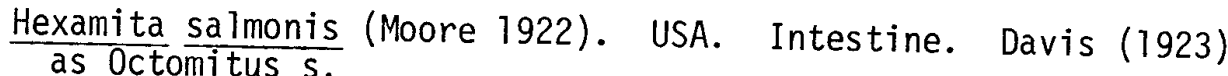

Octomitus truttae Schmidt 1920. USSR. Intestine. Bykhovskaya-

Pavlovskaya et al. (1962); Yugoslavia. Zitnan and Cankovic (1970).

TETRAMITIDAE

Costia pyriformis Davis 1943. West Virginia. Gills. Davis (1943).

Ichthyobodo necatrix (Henneguy 1884). USSR. Petrushevski and Shuiman (1955); Yugoslavia. Zitnan and Cankovic (1970) both as Costia $\underline{n}$.

\section{THECAMOEBIDAE}

Thecamoeba hoffmani Sawyer, Hnath and Conrad 1974. Michigan. Gills. Sawyer et al. (1974).

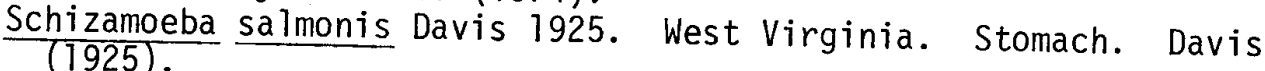

\section{Salvelinus malma - Dolly Varden}

ACANTHOCEPHALA

ECHINORHYNCHIDAE

Acanthocephalus minor Yamaguti 1935. Japan. Awakura (1972).

Echinorhynchus gadi Zoega in Muller 1776. British Columbia. Intestine. Arai (1969b); Kamchatka, USSR. Zhukov (1963).

NEOECHINORHYNCHIDAE

Neoechinorhynchus rutili (Muller 1780) Hamann 1892. British Columbia. Intestine. Bangham and Adams (1954). 
POLYMORPHIDAE

Bolbosoma caenoforme (Heitz 1920) Meyer 1933. Kamchatka, USSR. Zhukov (1963).

Corynosoma strumosum (Rudolphi 1802) Lühe 1904. Larva. Kamchatka, USSR. Intestine. Zhukov (1963).

\section{CESTODA}

AMPHICOTYLIDAE

Eubothrium crassum (B1och 1779) Nybelin 1922. Kamchatka, USSR. Zhukov (7963).

Eubothrium salvelini (Schrank 1790) Nybelin 1922. British Columbia. Zhukov (1963).

\section{CYATHOCEPHALIDAE}

Bothrimonus sturionis Duvernoy 1842. Canada. Intestine. Cooper $(1921)$.

\section{DIPHYLLOBOTHRI IDAE}

Diphy110bothrium cordiceps (Leidy 1871) Matz 1892. Larva. Canada. Body cavity. Mudry and Anderson 15 .

Diphyllobothrium dalli i Rausch 1956. Larva. Kamchatka, USSR. Zhukov (1963).

Diphyllobothrium latum. (Linnaeus 1758) Matz 1892. Larva. Canada. Mudry and Anderson 15 .

PHYLLOBOTHRI IDAE

Phyllobothrium sp. Larva. British Columbia. Intestine. Araj $(1969 \mathrm{~b})$.

\section{PROTEOCEPHALIDAE}

Proteocephalus salmonidicola Alexander 1951. British Columbia. Bangham and Adams (1954).

TETRAPHYLLIDAE

Scolex pleuronectis Müller 1788. Kamchatka, USSR. Zhukov (1963). COPEPODA

\section{LERNAEOPODIDAE}

Salmincola californiensis (Dana 1852) Wilson 1915. Canada. Gills. Wilson (1920).

Salmincola carpionis (Krøyer 1837) Wilson 1915. Alaska. Kabata (1969c). 
Salvelinus ma1ma (Continued)

Salmonicola edwardsii (01sson 1869) Wilson 1915. British Columbia. Bangham and Adams (1954).

\section{DIGENEA}

\section{ALLOCREADIIDAE}

Crepidostomum metoecus (Braun 1900) Braun 1900. Japan. Seki (1975). Crepidostomum farionis (Müller 1780) Lühe 1909. British Columbia. Intestine. Arai (1969b); Kamchatka, USSR. Zhukov (1963). Neophas is oculata (Levinsen 1881) Miller 1941. Kamchatka, USSR. Zhukov (1963).

Bunodera luciopercae (Müller 1780) Lühe 1909. Oregon. Mudry and Anderson (1977).

\section{AZYGI IDAE}

Azygia lucij (Müler 177ó) Lühe 1909. Japan. Seki (1975).

\section{BUCEPHALIDAE}

Prosorhynchoides gracilescens (Rudölphi 1819) n. comb. Kamchatka, USSR. Intestine. Zhukov (1963).

Prosorhynchoides ozakii (Ozaki 1928) Margolis and Arthur 1979. British Columbia. Bangham and Adams (1954) as Bucephalops is o.

Prosorhynchus crucibuium (Rudoīphi 1819) Õdhner 1905. Kamchatka, USSR. Zhukov (1963).

\section{FELLODISTOMATIDAE}

Pseudopentagramma petrowi (Layman 1930) Yamaguti 1971. Kamchatka, USSR. Zhukov (1963) as Bacciger p.

\section{HEMIURIDAE}

Brachyphallus crenatus (Rudolphi 1802) Odhner 1905. Kamchatka, USSR. Strelkov (1960); British Columbia. Bangham and Adams (1954).

Genarchopsis mue1leri (Levinsen 1881) Yamaguti 1954. Kamchatka, USSR. Zhukov (7963).

Genolinea anura (Layman 1930) Yamaguti 1954. Kamchatka, USSR. Zhukov (1963).

Derogenes varicus (Mü11er 1784) Looss 1901. Kamchatka, USSR. Zhukov (1963).

Hemiurus levinseni Odhner 1905. Kamchatka, USSR. Zhukov (1963). Lecithaster gibbosus (Rudolphi 1802) Lühe 1901. British Columbia. Intestine, pyloric caeca. Arai (1969b); Kamchatka, USSR. Zhukov (1963).

Tubulovesicula lindbergi (Layman 1930) Yamaguti 1934. British Columbia. Stomach. Arai (1969b). 
Salvelinus malma (Continued)

OPECOELIDAE

Podocotyle atomon (Rudolphi 1802) Odhner 1905. Kamchatka, USSR. Zhukov (1963).

Podocotyle reflexa (Creplin 1825) Odhner 1905. Kamchatka, USSR. Zhukov (1963).

MONOGENEA

DISCOCOTYLIDAE

Discocotyle sagittata (Leuckart 1842) Diesing 1850. British Columbia. Bangham and Adams (1954).

NEMATODA

Tetraonchus aladensis Prici 1937 Alaska Psie (1237)

\section{ANISAKIDAE}

Anisakis sp. Larva. Kamchatka, USSR. Strelkov (1960).

Contracaecum sp. Larva. British Columbia. Body cavity. Arai (7969b).

* Thynnascaris adunca (Rudolphi 1802) Hartwich 1957. Kamchatka, USSR. Strelkov (1960) as Contracaecum a.

CAP ILLARI IDAE

Capillaria sp. Japan. Seki (1975).

CUCULLANIDAE

Truttaedacnitis trutta (Fabricius 1794) Petter 1974. Kamchatka, USSR. Zhukov (1963); British Columbia. Bangham and Adams (1954) both as Cucullanus t.

Cucullanus sp. Japan. Seki (1975).

CYSTIDICOLIDAE

Ascarophis pacificus Zhukov 1960. Kamchatka, USSR. Zhukov (1963). Cystidicola sp. Canada. Ko and Anderson (1969).

Cystidico1oides tenuissima (Zeder 1800) Rasheed 1966. British Columbia. Bangham and Adams (1954) as

Metabronema salvelini.

DIOCTOPHYMATIDAE

Eustrongyloides sp. Larva. British Columbia. Bangham and Adams (1954).

PHILOMETRIDAE

Philonema oncorhynchi Kuitunen-Ekbaum 1933. Kamchatka, USSR. Zhukov (1963); British Columbia. Bangham and Adams (1954). 
Salvelinus malma (Continued)

\title{
THELAZIIDAE
}

Rhabdochona milleri (Choquette 1951) Moravec and Arai 1971. Canada. Mudry and Anderson (1977).

PROTOZOA (MICROSPORIDA)

NOSEMATIIDAE

Glugea takedai Awakura 1974. Japan. Muscles. Awakura (1974). PROTOZOA (MYXOSPORIDA)

MYXIDIIDAE

Myxidium oviforme Parisi 1912. Kamchatka, USSR. Zhukov (1964). MYXOSOMATIDAE

Myxosoma cerebralis (Hofer and Plehn 1903) Kudo 1933. USA. Brain. Halliday (1976).

\author{
OSMERIDAE -- SMeìts \\ Hypomesus pretiosus - Surf Smelt
}

DIGENEA

FELLODISTOMATIDAE

Pseudopentagramma petrowi (Layman 1930) Yamaguti 1971. British Columbia. Pyloric caeca, intestine. Margolis and Ching (1965) as Pentagramma $\mathrm{p}$.

HEMIURIDAE

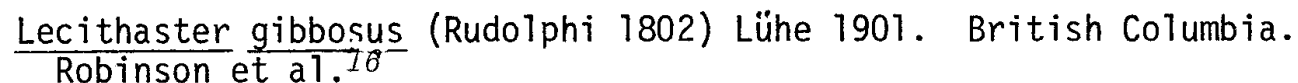
NEMATODA

ANISAKIDAE

Anisakis sp. Larva. Washington. Loose in body cavity and encysted in liver. Ki Tambi and DeLacy (1967).

Contracaecum sp. Larva. British Columbia. Mesentery, stomach, liver. Margolis 3 .

* Thynnascaris adunca (Rudolphi 1802) Hartwich 1957. British

Columbia. Stomach, intestine. Margolis 3 as Contracaecum a. 


\section{Mallotus villosus - Capel in}

CESTODA

TETRAPHYLLIDEA

Scolex pleuronectis Müiler 1788. Kamchatka, USSR. Zhukov (1963). DIGENEA

FELLODISTOMATIDAE

Pseudopentagramma petrowi (Layman 1930) Yamaguti 1971. British Columbia. Margolis and Ching (1965) as Pentagramma p.

HEMIURIDAE

Lecithaster gibbosus (Rudolphi 1802) Lühe 1901. Kamchatka, USSR. Zhukov (1963).

NEMATODA

ANISAKIDAE

Anisakis sp. Larva. British Columbia. Margolis3.

* Thynnascaris adunca (Rudolphi 1802) Hartwich 1957. Kamchatka, USSR. Zhukov (1963).

Spirinchus thaleichthys - Longfin Smelt

SYNONYM - Spirinchus dilatus

DIGENEA

FELLODISTOMATIDAE

Pseudopentagramma petrowi (Layman 1930) Yamaguti 1971. British Columbia. Pyloric caeca, intestine. Margolis and Ching (1965) as Pentagramma $\mathrm{p}$.

Thaleichthys pacificus - Eulachon

CESTODA

PHYLLOBOTHRI IDAE

Phyllobothrium sp. Larva. British Columbia. Intestine. Arai $(7969 b)$. 
Thaleichthys pacificus (Continued)

COPEPODA

\section{PENNELLIDAE}

Haemobaphes disphaerocephalus Grabda 1976. Washington, Oregon. Gill cavity. Grabda (1976).

Haemobaphes diceraus Wilson 1917. British Columbia. Gills. Hoskins et a 7.13

DIGENEA

HEMIURIDAE

Lecithaster gibbosus (Rudolphi 1802) Lühe 1901. British Columbia. Intestine. Arai (1969b).

NEMATODA

ANISAKIDAE

Contracaecum sp. Larva. British Columbia. Body cavity. Arai (1969b).

$$
\text { ALEPOCEPHALIDAE -- STickheads }
$$

Bajacal ifornia burragei

\section{PROTOZOA (MYXOSPORIDA)}

MYXIDIIDAE

Myxidium bajacalifornium Noble 1966. Southern California.

GallbTadder. Noble (1966a).

$$
\text { BATTHYLAGIDAE -- Deepsea Smelts }
$$

Bathylagus ochotensis - Popeye Blacksmelt

\section{PROTOZOA (SARCOMASTIGOPHORA)}

\section{CRYPTOBIIDAE}

Cryptobia sp. Noble and Collard (1970). 


\section{PROTOZOA (SARCOMASTIGOPHORA)}

CRYPTOBI IDAE

Cryptobia sp. Noble and Collard (1970).

$$
\text { Keuroglossus stilbius - California Smoothtongue }
$$

COPEPODA

\section{PENNELLIDAE}

Cardiodectes medusaeus (Wilson 1908) Yamagutj 1963. Central California. Flesh (embedded). Anderson. ${ }^{17}$

\section{DIGENEA}

\section{HEMIURIDAE}

Aponurus californicus Noble and Orias 1970. Southern California; Mexico. Stomach. Noble and Orias (1970).

PROTOZOA (MYXOSPORIDA)

MYXOBOLIDAE

Myxobolus sp. Southern California. Gallbladder. Noble (1966a). PROTOZOA (SARCOMASTIGOPHORA)

CRYPTOBI IDAE

Cryptobia stilbia Noble 1968. Southern California. Stomach. Noble (1968).

$$
\text { GONOSTOMATIDAE -- Lightfishes }
$$

Cyclothone acclinidens - Benttooth Bristlemouth

NEMATODA

ANISAKIDAE

Paranisakis sp. Larva. Southern California. Cysts in mesentery. Collard 9 . 


\section{STERNOPTYCHIDAE -- Hatchetfishes \\ Argyropelecus pacificus - Pacific Hatchetfish}

\section{ACANTHOCEPHALA}

Genera and species. Larva. Southern California. Intestine. Collard ${ }^{9}$.

DIGENEA

DIDYMOZOIDAE

Genera and species. Larvae. Southern California. Gills. Collard?

Sternoptyx diaphana- Longspine Hatchetfish

\section{CESTODA}

\section{TETRAPHYLLIDEA}

Genera and species. Plerocercoid. Southern California. Pyloric caeca, intestine. Collard ${ }^{9}$.

$$
\begin{aligned}
& \text { STOMIATIDAE -- Scaly Dragonfishes } \\
& \text { Stomias atriventer - Blackbelly Dragonfish }
\end{aligned}
$$

CESTODA

TETRAPHYLLIDEA

Genera and species. Plerocercoid. Southern California; Baja California. Intestine. Collard ${ }^{9}$.

$$
\text { SYNODONTIDAE -- Lizardfishes }
$$

Synodus lucioceps - California Lizardfish

\section{BRANCHIURA}

ARGUL IDAE

Argulus borealis Wilson 1912. Southern California. Jensen ${ }^{18}$. CESTODA

BOTHRIOCEPHALIDAE 
Synodus lucioceps (Continued)

Anatrum histocephalum Jensen and Heckmann 1977.

California. Intestine. Jensen and Heckmann (1977a).

DASYRHYNCHIDAE

Callitetrarhynchus gracilis (Rudolphi 1819) Pintner 1931. Larva. Southern California. Viscera. Jensen ${ }^{18}$.

\section{LACISTORHYNCHIDAE}

Lacistorhynchus tenuis (Van Beneden 1858) Dollfus 1929. Larva. Southern California. Viscera. Jensen 18.

Grillotia smarisgora (Wagener 1854) Dollfus 1947. Larva.

Southern California. Mesentery.' Jensen ${ }^{18}$.

ONCOBOTHRI IDAE

Acanthobothrium sp. Larva. Southern California. Intestine. Jensen ${ }^{18}$. COPEPODA

CALIGIDAE

Caligus hobsoni Cressey 1969. Southern California. Jensen ${ }^{18}$.

DIGENEA

OPECOELIDAE

Helicometrina nimia Linton 1910. Intestine. Arai (1962).

ISOPODA

CYMOTHOIDAE

Lironeca vulgaris Stimpson 1857. California. Turner et a1. (1969). NEMATODA

ANISAKIDAE

Anisakis sp. Larva. Southern California. Dailey et al. (in press).

Contracaecum sp. Larva. Southern California. Dailey et al. (in press).

Phocanema sp. Larva. Southern California. Dailey et al. (in press).

*Thynnascaris adunca (Rudolphi 1802) Hartwich 1957. Southern California. Jensen 18 .

PROTOZOA (MYXOSPORIDA)

CERATOMYXIDAE

Ceratomyxa sp. Southern California. Gallbladder. Jensen ${ }^{18}$. 
SEARSIIDAE -- Tubeshoulders

Sagamichthys abei - Shining Tubeshoulders

PROTOZOA (MYXOSPORIDA)

MYXIDIIDAE

Myxidium melanostigmum Noble 1966. Southern California. Gallbladder. Noble (1966a). 


\section{ALEPISAURIDAE -- Lancetfishes \\ Alepisaurus ferox - Longnose Lancetfish}

CESTODA

PHYLLOBOTHRI IDAE

Phyllobothrium caudatum (Zschokke and Heitz 1914). Larva. Central California. Riser (1956); Medeira. Yamaguti (1959b).

TENTACULARI IDAE

Tentacularia coryphaenae Bosc 1802. Larva. Central California. Musculature. Riser (7956).

DIGENEA

BOTULIDAE

Botulus alepidosauri Guiart 1938. Mediterranean. Stomach. Guiart $(7938 a)$.

\section{PARALEPIDIDAE -- Barracudinas}

Macroparalepis sp.

CESTODA

TETRAPHYLLIDEA

Genera and species. Larva. Southern California. Caeca. Collard ${ }^{9}$. NEMATODA

\section{ANISAKIDAE}

Anisakis sp. Larva. Southern California. Mesentery. Collard 9 .

Phocanema sp. Larva. Southern California. Mesentery. Collard $\dot{9}$. Contracaecum sp. Larva. Southern California. Intestine. Collard.

$$
\text { MYCTOPHIDAE -- Lanternfishes }
$$

Ceratoscopelus townsendi-Dogtooth Lampfish

\section{CESTODA}

\section{PSEUDOPHYLLIDEA}

Genera and species. Larva. Baja California. Pyloric caeca. Collard 9 . 
Ceratoscopelus townsendi (Continued)

TETRAPHYLLIDEA

Genera and species. Larva. Collard ${ }^{9}$.

COPEPODA

Genera and species. Intermediate stage. Baja California. Dorsal fin. Collard ${ }^{9}$.

DIGENEA

FELLODISTOMATIDAE

Genera and species. Equatorial Pacific. Stomach. Collard ${ }^{9}$.

HEMIURIDAE

Genera and species. Equatorial Pacific; Baja California. Stomach, intestine. Collard ${ }^{9}$.

NEMATODA

ANISAKIDAE

Anisakis sp. Larva. Equatorial Pacific. Intestine. Collard ${ }^{9}$.

Phocanema sp. Larva. Equatorial Pacific. Liver, mesentery. Collard 9 as Terranova

Contracaecum sp. Larva. Collard 9 .

Diaphus theta - California Headlight Fish

CESTODA

PSEUDOPHYLLIDEA

Genera and species. Larva. Southern California. Intestine, colon/rectum, pyloric caeca. Collard9.

TETRAPHYLLIDEA

Genera and species. Larva. Southern California. Intestine. Collard 9 . COPEPODA

PENNELLIDAE

Cardiodectes medusaeus (Wilson 1908) Yamaguti 1963. Southern California. Heart. Collard ${ }^{9}$. 
NEMATODA

ANISAKIDAE

Anisakis sp. Larva. Southern California. Pyloric caeca. Collard 9 . Contracaecum sp. Larva. Southern California. Pyloric caeca, coelom, mesentery. Collard ${ }^{9}$.

\section{Lampanyctus regal is - Pinpoint Lampfish}

NEMATODA

ANISAKIDAE

Anisakis sp. Larva. Southern California. Coelom. Collard.

Lampanyctus ritteri - Broadfin Lampfish

CESTODA

PSEUDOPHYLLIDEA

Genera and species. Larva. Southern California. Intestine. Collard 9 .

TETRAPHYLLIDEA

Genera and species. Larva. Southern California. Heart, pyloric caeca, intestine. Collard 9 .

COPEPODA

PENNELLIDAE

Cardiodectes medusaeus (Wilson 1908) Yamaguti 1963. Southern California. Heart. Moserto.

MONOGENEA

MACROVALVITREMATIDAE

Genera and species. Southern California. Gills. Collard?. NEMATODA

\section{ANISAKIDAE}

Anisakis sp. Larva. Southern California, Oregon. Liver, heart, mesentery, kidneys, coelom, peritoneum. Collard 9 .

Paranisakis sp. Larva. Southern California. Gonads. Collard?.

Contracaecum sp. Larva. Southern California. Mesentery. Collard?. 
Parvilux ingens - Giant Lampfish

NEMATODA

ANISAKIDAE

Anisakis sp. Larva. Southern California. Coelom. Collard ${ }^{9}$.

Stenobrachius leucopsarus - Northern Lampfish

SYNONYM - Nannobrachium leucopsarium

\section{ACANTHOCEPHALA}

Genera and species. Larva. Southern California. Intestine. Collard 9 .

CESTODA

PSEUDOPHYLLIDEA

Genera and species. Plerocercoid. Southern California. Colon, intestine. Collard ${ }^{9}$.

TETRAPHYLLIDEA

Genera and species. Plerocercoid. Southern California, Oregon. Colon, mesentery. Collard 9 .

COPEPODA

\section{PENNELLIDAE}

Cardiodectes medusaeus (WiTson 1908) Yamaguti 1963. Southern California, Oregon. Heart. Collard 9 ; Central California. Flesh (embedded). Wilson (1908a) as Lernaeenicus $\mathrm{m}$.

\section{DIGENEA}

\section{DIDYMOZOIDAE}

Genera and species. Larva. Southern California. Collard 9 as Monil icaecum.

\section{NEMATODA}

\section{ANISAKIDAE}

Anisakis sp. Larva. Southern California. Coelom, liver, colon, mesentery. Collard9.

Contracaecum sp. Larva. Southern California, Oregon, Alaska. Coelom, mesentery, liver, pyloric caeca, kidneys. Collards. Phocanema sp. Larva. Southern California. Intestine. Collard 9 as Terranova sp. 


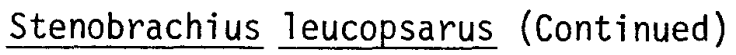

CYSTIDICOLIDAE

Ascarophis sp. Larva. California. Collardg.

PROTOZOA (MYXOSPORIDA)

Genera and species. Southern California. Gallbladder. Noble (1966a).

Symbolophorus cal iforniensis - Cal ifornia Lanternfish

\section{CESTODA}

PSEUDOPHYLLIDEA

Genera and species. Larva. Southern California. Colon, rectum. Collard 9 .

TETRAPHYLLIDEA

Genera and species. Larva. Southern California. Colon, rectum. Collard ${ }^{9}$.

NEMATODA

ANISAKIDAE

Anisakis sp. Larva. Southern California. Coelom. Collard 9 .

Tarletonbeania crenularis - Blue Lanternfish

CESTODA

Genera and species. Larva. Southern California, Oregon. Stomach, liver. Collara ${ }^{9}$.

NEMATODA

ANISAKIDAE

Anisakis sp. Larva. Southern California. Coelom. Collard 9 .

Contracaecum sp. Larva. Southern California. Coelom. Collard ${ }^{9}$.

Triphoturus mexicanus - Mexican Lampfish

CESTODA

PSEUDOPHYLLIDEA

Genera and species. Larva and adults. Southern California. Intestine. Collard 9 . 
Triphoturus mexicanus (Continued)

NEMATODA

ANISAKIDAE

Anisakis sp. Larva. Southern California. Mesentery. Collard ${ }^{9}$. Contracaecum sp. Larva. Southern California. Coelom. Collard 9 . PROTOZOA (MYXOSPORIDA)

Genera and species. Trophic stages. Noble and Collard (1970).

$$
\begin{aligned}
& \text { BATRACHOIDIDAE -- Toadfishes } \\
& \text { Porichthys sp. }
\end{aligned}
$$

\section{DIGENEA}

HEMIURIDAE

Aponurus trachinoti Manter 1940. Southern California. Stomach. Manter and Van CTeave (1951).

Brachadena pyriformis Linton 1910. Southern California. Stomach. Manter and Van Cleave (1951). Probably P. myriaster.

Lecithaster gibbosus (Rudolphi 1802) Lühe T901. Washington. Intestine. Ching (1960b) as $\underline{L}$. salmonis.

$$
\text { Porichthys notatus - Plainfin Midshipman }
$$

\section{DIGENEA}

\section{HEMIURIDAE}

Derogenes varicus (Müller 1784) Looss 1901. Washington. Ching (1960a).

Lecithaster gibbosus (Rudo1phi 1802) Lühe 1901. Washington. Ching (1960a) as L. salmonis.

Tubulovesicula lindbergi (Layman 1930) Yamaguti 1934. British Columbia. Stomach. Arai (1969b).

\section{LEPOCREADI IDAE}

Opechona alaskensis Ward and Fillingham 1934. Alaska. Ching (1960a).

\section{OPECOELIDAE}

Podocotyle atomon (Rudolphi 1802) Odhner 1905. Washington. Intestine. ATdrich ${ }^{1}$ ? 
Porichthys notatus (Continued)

PROTOZOA (MYXOSPORIDA)

CERATOMYXIDAE

Ceratomyxa elegans Jameson 1929. Central California. Gallbladder. Jameson (1929); Washington. Kuris 20 .

SINUOL INEIDAE

Davisia sp. Washington. Urinary bladder. Kuris 20.

Davisia bidens (Jameson 1931) Laird 1953. California. Urinary bladder. Jameson (1931) as Sinuolinea b.; Washington. Kuris 20 . Davisia cella (Jameson 1931) Laird 1953. California. Urinary bladder. Jameson (1931) as Sinuolinea c.

Sinuolinea dimorpha (Davis 1917) Kudo 1920 . California. urinary bladder. Jameson (1931) as Sphaerospora d.

\author{
GOBIESOCIDAE -- Clingfishes \\ Gobiesox maeandricus - Northern Clingfish
}

SYNONYM - CauTarchus maeandricus

DIGENEA

HEMIURIDAE

Sterrhurus exodicus (McFarlane 1936) Yamaguti 1958. Central

CaTifornia. Stomach. Nahhas ${ }^{10}$.

\title{
OPECOELIDAE
}

Helicometrina elongata Noble and Park 1937. Northern California. Small intestine. Noble and Park (1937).

Podocotyle gibbonsiae Johnson 1949. Central California.

Digestive tract. Johnson (1949).

\section{NEMATODA}

\section{PHILOMETRIDAE}

Philometra americana Kuitunen-Ekbaum 1933. British Columbia. Body cavity, lateral wal1s. Kuitunen-Ekbaum (1933a).

PROTOZOA (MYXOSPORIDA)

MYXIDIIDAE

Zschokkeīla sp. Washington. Urinary bladder. Kuris 20 .

Sphaeromyxa balbianii Thélohan 1895. Washington. Gallbladder. Kuris ${ }^{2}$. 
Gobiesox maeandricus (Continued)

SINUOLINE IDAE

Davisia sp. Washington. Urinary bladder, Kuris 20.

Gobiesox rhessodon - California Clingfish

PROTOZOA (MYXOSPORIDA)

MYXIDI IDAE

Sphaeromyxa ovula Noble 1939. Southern California. Gallbladder. Nob7e (1939).

$$
\text { Rimicola eigenmanni - Slender Clingfish }
$$

PROTOZOA (MYXOSPORIDA)

\section{CERATOMYXIDAE}

Leptotheca compressa Noble 1939. Southern California. Urinary bladder. Noble (1939).

$$
\text { GADIDAE -- Codfishes }
$$

Gadus macrocephalus - Pacific Cod

SYNONYM - Gadus morhua macrocephalus

\section{ACANTHOCEPHALA}

\section{ECHINORHYNCHIDAE}

Echinorhynchus gadi Zoega in Müller 1776. British Columbia. Intestine. Margol is 3 ; Kamchatka, USSR. Skryabina (1963).

POLYMORPHIDAE

Corynosoma strumosum (Rudolphi 1802) Lühe 1904. Larva. Kamchatka, USSR. Skryabina (1963); Washington. Van Cleave (1953).

\section{CESTODA}

\section{AMPHICOTYLIDAE}

Abothrium gadi Van Beneden 1871. Kamchatka, USSR. Intestine. Skryabina (7963); British Columbia. Margolis ${ }^{3}$.

BOTHRIOCEPHALIDAE

Bothriocephalus sp. Larva. Kamchatka, USSR. Pyloric caeca, intestine. Skryabina (1963). 
Gadus macrocephalus (Continued)

PHYLLOBOTHRI IDAE

Phyilobothrium sp. Larva. British Columbia. Intestine. Arai (1969b).

\section{TENTACULARI IDAE}

Nybelinia surmenicola Okada 1929. Larva. Sea of Japan. Zhukov (1960a).

\section{TETRAPHYLLIDEA}

Scolex pleuronectis Müller 1788. Kamchatka, USSR. Intestine, gal1bladder. Skryabina (1963).

COPEPODA

\section{CALIGIDAE}

Lepeophtheirus parviventris Wilson 1905. Alaska, British Columbia. Exterior. Townsend (1938) as $L$. septentrional is; USSR. Wilson (1908a).

Lepeophtheirus hospitalis Fraser 1920. British Columbia. Fraser (1920).

Lepeophtheirus dissimulatus WiIson 1905. Eastern Pacific. Lewis (1967).

Lepeophtheirus cuneifer Kabata 1974. Alaska. Kabata (1974) Questionable host.

\section{CHONDRACANTHIDAE}

Chondracanthus palpifera Wilson 1912. British Columbia. Gill cavity. Fraser (1920).

\section{LERNAEOPODIDAE}

Clavella adunca (Strøm 1762) Dollfus 1953. British Columbia. Gills, branchial cavity. Kabata (1970c); Japan. Gills. Yamaguti (1939c) as C. uncinata.

\section{NAOBRANCHI IDAE}

Naobranchia occidental is Wilson 1915. Alaska. Gills. Wilson (1915).

\section{DIGENEA}

\section{ACANTHOCOLPIDAE}

Stephanostomum baccatum (Nicoll 1907) Manter 1934. Sea of Japan. Intestine. Zhukov (1960a). 
Gadus macrocephalus (Continued)

BUCEPHAL IDAE

Prosorhynchus crucibulum (Rudolphi 1819) Odhner 1905. Sea of Japan. Intestine. Zhukov (1960a).

\section{FELLODISTOMATIDAE}

Pseudopentagramma petrowi (Layman 1930) Yamaguti 1971. Pacific Ocean-USSR. Margolis and Ching (1965) as Pentagramma p.;

Kamchatka, USSR. Skryabina (1963) as Bacciger p.

Stenakron vetustum Stafford 1904. Kamchatka, USSR. Strelkov (1960).

\section{HEMIURIDAE}

Tubulovesicula a lindbergi (Layman 1930) Yamaguti 1934. Sea of Japan. Stomach, intestine. Zhukov (1960a).

Brachyphallus crenatus (Rudolphi 1802) Odhner 1905. Sea of Japan. Stomach. Zhukov (1960a).

Lecithaster gibbosus (Rudolphi 1802) Lühe 1901. British Columbia. Intestine, pyloric caeca. Arai (1969b); Sea of Japan. Zhukov (1960a).

Parahemiuris merus (Linton 1910) Woolcock 1935. British Columbia. Intestine, stomach, pyloric caeca. Arai (1969b).

Hemiurus levinseni Odhner 1905. Kamchatka, USSR. Stomach, caeca. Skryabina (1963).

Derogenes varicus (Müiler 1784) Looss 1901. Kamchatka, USSR. Stomach, intestine. Skryabina (1963).

\section{LEPOCREADI IDAE}

Lepidapedon gadi (Yamaguti 1934) Acena 1947. Japan. Intestine. Acena (1947); Kamchatka, USSR. Skryabina (1963).

\section{OPECOELIDAE}

Podocotyle atomon (Rudolphi 1802) Odhner 1905. Kamchatka, USSR. Intestine. Strelkov (1960).

Podocotyle reflexa (Creplin 1825) Odhner 1905. Kamchatka, USSR. Stomach, intestine, caeca. Skryabina (1963).

\section{ZOOGONIDAE}

Steganoderma formosum Stafford 1904. Kamchatka, USSR. Intestine. Strelkov (1960).

HIRUDINOIDEA

PISCICOLIDAE

Beringobde11a rectangulata (Levinsen 1882) Caballero 1974. Alaska. Gi17s, giTT cavity. Moore and Meyer (195i) as genus? $\underline{r}$. 
Gadus macrocephalus (continued)

MONOGENEA

GYRODACTYLIDAE

Gyrodactylus marinus Bykhovsky and Polyanski 1953. Sea of Japan. Gills. Bykhovsky and Poly anski (1953).

NEMATODA

\section{ANISAKIDAE}

Anisakis sp. Larva. British Columbia. Body cavity. Arai (1969b); Japan. Saito et a1. (1970); Kamchatka, USSR. Body cavity, intestine, abdominal wa11. Skryabina (1963).

* Thynnascaris adunca (Rudolphi 1802) Hartwich 1957. British Columbia. Stomach, intestine. Margolis ${ }^{3}$; Sea of Japan. Zhukov (1960a) both as Contracaecum a.

Contracaecum sp. Japan. Kagei et al. (1970); Kamchatka, USSR. Intestine, Tiver, kidney, body cavity. Skryabina (1963).

Phocanema sp. Larva. British Columbia. Cysts in musculature. Margolis ${ }^{3}$; Kamchatka, USSR. Muscle, body cavity. Skryabina (1963). Both as Porrocaecum sp.

Phocanema decipiens (Krabbe 1878) Myers 1959. Larva. British Columbia. Margolis (7956b) as Terranova d.

CYSTIDICOLIDAE

Ascarophis pacificus Zhukov 1960. Sea of Japan. Stomach. Zhukov (1960a). Ascarophis filiformis Polyanski 1952. Sea of Japan. Stomach. Zhukov(1960a). PROTOZOA (CILIOPHORA)

URCEOLARI IDAE

Trichodina murmanica Polyansky 1955. USSR. Gills. Zhukov (1964). [Nomen dubium Lom and Laird (1969)].

PROTOZOA (MYXOSPORIDA)

TETRACAPSULIDAE

Kudoa sp. British Columbia. Muscle. Hoskins et ar. ${ }^{13}$

MYXIDIIDAE

Myxidium ochotense Fujita 1923. Japan. Gallbladder. Fujita (1923).

$$
\text { Merluccius productus - Pacific Hake }
$$

CESTODA

BOTHRIOCEPHALIDAE

Bothriocephalus ospariichthydis Yamaguti 1934. Northeast Pacific. Kovalenko (1970).

Bothriocephalus sp. Northeast Pacific. Kovalenko (1970). 
DIPHYLLOBOTHRIIDAE

Diphyllobothrium sp. Northeast Pacific. Kovalenko (1970).

PTYCHOBOTHRIIDAE

Clestobothrium crassiceps (Rudo7phi 1808) Lühe 1899. British

Columbia. Intestine. Margol is ${ }^{3}$.

TENTACULARI IDAE

Nybel inia surmenicola Okada 1929. Post larva. British Columbia. Stomach wall. Margol is ${ }^{3}$.

TRYPANORHYNCHA

Genera and species. Larva. Northeast Pacific. Kovalenko (1970). COPEPODA

\section{CALIGIDAE}

Lepeophtheirus dissimulatus Wilson 1905. Baja California. Causey (1960).

Caligus sp. British Columbia. Lee (1975).

\section{CHONDRACANTHIDAE}

Chondracanthus palpifera Wilson 1912. British Columbia. Bere Acanthochondria sp. Northeast Pacific. Kovalenkc

\section{LERNAEOPODIDAE}

Neobrachiella robusta (Wi1son 1912) Kabata 1979. British Columbia. Gi17 arches. Kabata (1970b) as Brachiel1a 1. Neobrachiella sp. Northeast Pacific. Kovalenko (1970) as Parabrachiella. Wilson 1915. British Columbia. Lee (1975).

PENNELLIDAE

Haemobaphes sp. Northeast Pacific. Kovalenko (1970).

\section{DIGENEA}

\section{FELLODISTOMATIDAE}

Fellodistomum furcigerum (01sson 1867) Yamaguti 1954 . Northeast Pacific. Kovalenko (1970). 
Merluccius productus (Continued)

HEMIURIDAE

Lecithophyllum botryophorum (01sson 1868) Odhner 1905. British Columbia. Margolis (1958a) as L. anteroporum.

Aponurus argentini Polyanski 1952. Northeast Pacific. Kovalenko (1970).

Derogenes varicus (Müller 1784) Looss 1901. Northeast Pacific. Kovalenko (1970).

Derogenes macrostoma Yamaguti 1938. Northeast Pacific. Kovalenko (1970).

Lecithochirium sp. Northeast Pacific. Kovalenko (1970).

SANGUINICOLIDAE

Aporocotyle margolisi Smith 1967. British Columbia. BuTbus arteriosus. Smith (1967).

\section{MONOGENEA}

\section{DISCOCOTYLIDAE}

Anthocotyle americana (MacCa11um 1916) Price 1943. Northern California. Gills. Crane (1972); Northeast Pacific. Kovalenko (1970).

Anthocotyle merlucci Van Beneden and Hesse 1863. Northeast Pacific. Kovalenko (7970).

NEMATODA

ANISAKIDAE

Anisakis sp. Larva. British Columbia. Many places in body. Margolis 3; Southern California. Dailey et a7. (in press).

*Thynnascaris adunca (Rudolphi 1802) Hartwich 1957. Northeast Pacific. Kovalenko (1970) as Contracaecum a.

* Thynnascaris melanogrammi (Smedley 1934). Northeast Pacific. Kovalenko (1970).

Contracaecum sp. Larva. Northeast Pacific. Kovalenko (1970).

PROTOZOA (MYXOSPORIDA)

SINUOLINEIDAE

Conispora renalis Sankurathri 1977. British Columbia. Kidney tubules. Sankurathri (1977).

Microgadus proximus - Pacific Tomcod

ACANTHOCEPHALA

ECHINORHYNCHIDAE 
Microgadus proximus (Continued)

Echinorhynchus gadi Zoega in Müller 1776. Canada. Van Cleave (1920). Metechinorhynchus Teidyi (Van Cleave 1924) Golvan 1969. Canada. Van Cieave (1920) as E. Salvelini.

COPEPODA

\section{CALIGIDAE}

Lepeophtheirus cuneifer Kabata 1974. Southeast Alaska. Kabata (1974). Questionable host.

\section{LERNAEOPODIDAE}

Clavella adunca (Strøm 1762) Dollfus 1953. British Columbia. Gills, branchiâ cavity. Kabata (1970c).

Clavella canaliculata Wilson 1915. Pectoral fins. Wilson (1915).

\section{DIGENEA}

\section{HEMIURIDAE}

Derogenes varicus (Mü17er 1784) Looss 1901. Oregon. McCauley (1960). Hemiurus levinseni Odhner 1905. Oregon. McCauley (1960).

\section{MONOGENEA}

\section{GYRODACTYLIDAE}

Gyrodactylus californiensis Mizelle and Kritsky 1967. Northern California. Skin. Mizelle and Kritsky (1967a). Gyrodactylus cranei Mizelle and Kritsky 1967. Northern California. Skin. MizeT7e and Kritsky (1967a).

Gyrodactylus pacificus Mizelle and Kritsky 1967. Northern California. Skin. Mize11e and Kritsky (1967a).

Gyrodactylus rogersi Mizelle and Kritshy 1967. Northern California. Skin. Mize $17 e$ and Kritsky (1967a).

Gyrodactylus elongatus Mizelle and Kritsky 1967. Northern California. Skin. Mizelle and Kritsky (1967a).

\section{PROTOZOA (MYXOSPORIDA)}

MYXIDIIDAE

Sphaeromyxa maiyai Morrison and Pratt 1973. Oregon. Gallbladder. Morrison and Pratt (1973).

$$
\text { Theragra chalcogramma - Walleye Pollock }
$$

\section{ACANTHOCEPHALA}

\section{ECHINORHYNCHIDAE}

Echinorhynchus gadi Zoega in Mü17er 1776. British Columbia. Arai (1969b); Kamchatka, USSR. Skryabina (1963). 
POLYMORPHIDAE

Bolbosoma nipponicum Yamaguti 1939. Kamchatka, USSR. Intestine. Mamaev and Baeva (1963).

Corynosoma strumosum (Rudolphi 1802) Lühe 1904. Larva. Kamchatka, USSR. Mesentery, intestine. Mamaev and Baeva (1963); British Columbia. Margolis (1956b).

CESTODA

AMPHICOTYLIDAE

Abothrium gadi Van Beneden 1871. British Columbia. Intestine. Margolis 3; Kamchatka, USSR. Mamaev and Baeva (1963).

BOTHRIOCEPHALIDAE

Bothriocephalus scorpi i (Müller 1776) Rudoiphi 1808. Larva. British Columbia. Intestine. Arai (1969b); Kamchatka, USSR. Intestine, pyloric caeca. Skryabina (1963).

DIPHYLLOBOTHRI IDAE

Pyramicocephalus phocarum (Fabricius 1780). Larva. Kamchatka, USSR. Intestine, body cavity. Mamaev and Baeva (1963).

Diphyllobothrium sp. Larva. Western Kamchatka, USSR. On digestive tract. Tsimbalyuk and Semeshko (1971).

\section{LACISTORHYNCHIDAE}

Grillotia erinacea (Van Beneden 1858) Guiart 1927. Kamchatka, USSR. Body cavity. Mamaev and Baeva (1963).

PHYLLOBOTHRI IDAE

Phyllobothrium sp. Larva. British Columbia. Intestine. Arai (1969b). PSEUDOPHYLLIDEA

Genera and species. Larva. Kamchatka, USSR. Intestine, abdominal wal1. Mamaev and Baeva (1963).

TENTACULARI IDAE

Nybelinia surmenicola Okada 1929. Larva. British Columbia. Intestine, stomach. Arai (1969b); Kamchatka, USSR. Body cavity, abdominal wall. Skryabina (1963).

TETRAPHYLLIDEA

Scolex pleuronectis Müller 1788. Kamchatka, USSR. Gallbladder, intestine. Skryabina (1963). 
Theragra chalcogramma (Continued)

COPEPODA

CÂLIGIDAE

Lepeophtheirus cuneifer Kabata 1974. Alaska. Kabata (1974), questionable host.

Lepeophtheirus parviventris WiTson 1905. Japan, USSR. Kabata (1973).

Caligus clemensi Parker and Margolis 1964. British Columbia. Body surface. Parker and Margol is (1964).

CHONDRACANTHIDAE

Chondracanthus theragrae Yamaguti 1939. Japan. Gil1s. Yamaguti $(1939 \mathrm{c})$.

\section{LERNAEOPODIDAE}

Clavella perfida Wilson 1915. British Columbia. Gills. Kabata (1970c); A7aska. Wilson (1915).

Clavel1a adunca (Strom 1792) Dollfus 1953. North Pacific. Akhmerov (1951) as $\underline{\mathrm{C}}$. irina and $\underline{\mathrm{C}}$. uncinata.

PENNELLIDAE

* Haemobaphes theragrae Yamaguti 1939. Japan. Yamaguti (1939b). Haemobaphes diceraus Wilson 1917. North Pacific. Gills. Grabda (1975).

\section{DIGENEA}

\section{ALLOCREADIIDAE}

Anisorchis opisthorchis Polyanski 1955. Kamchatka, USSR. Intestine. Strelkov (1960).

\section{BUCEPHALIDAE}

Prosorhynchus crucibulum (Rudolphi 1819) Odhner 1905. Sea of Japan. Intestine. Zhukov (1960a).

Prosorhynchoides gracilescens (Rudolphi 1819) n. comb. Kamchatka, USSR. Intestine. StreTkov (1960) as Bucephalopsis $g$.

\section{FELLODISTOMATIDAE}

Fellodistomum furcigerum (01sson 1867) Yamaguti 1954. Kamchatka, USSR. Strelkov (1960) as Steringophorus $f$.

\section{HEMIURIDAE}

Hemiurus communis Odhner 1905. Washington. Stomach. Ching (1960b). Hemiurus odhneri Yamaguti i934. Japan. Stomach. Yamaguti (1934a). Hemiurus Tevinseni Odhner 1905. Washington. Ching (1960a); Kamchatka, USSR. Stomach, pyloric caeca. Skryabina (1963). 
Lecithaster gibbosus (Rudolphi 1802) Lühe 1901. British Columbia. Intestine. Arai (1969b); Kamchatka, USSR. Mamaev and Bąeva (7963). Tubulovesicula Tindbergi (Layman 1930) Yamaguti 1934. Washington. Ching (1960a). Genolinea anura (Layman 1930) Yamaguti 1954. Sea of Japan. Stomach. Zhukov (1960a). Lecithophyllum sphaerolecithum (Manter 1925) Odhner 1927. Kamchatka, USSR. Stomach. Mamaev and Baeva (1963). Derogenes varicus (Müller 1784) Looss 1901. Kamchatka, USSR. Esophagus, stomach. Mamaev and Baeva (7963).

Brachyphal Tus crenatus (Rudolphi 1802) Odhner 1905. Kamchatka, USSR. Stomach. Mamaev and Baeva (1963).

\title{
LEPOCREADI IDAE
}

Opechona alaskensis Ward and Fillingham 1934. Sea of Japan. Intestine. Zhukov (1960a). Lepidapedon microtyleum (Odhner 1905) Yamaguti 1958. Washington. Intestine. Ching (1960a); British Columbia. Arai (1969b). Lepidapedon gadi (Yamaguti 1934) Acena 1947. Kamchatka, USSR. Digestive tract. Mamaev and Baeva (1963); Japan. Machida et a1. (1972).

\section{OPECOELIDAE}

Podocotyle reflexa (Crepl in 1825) Odhner 1905. Kamchatka, USSR. Digestive tract. Mamaev and Baeva (1963).

Podocotyle sinusacca Ching 1960. British Columbia. Intestine. Arai (1969b) as Neopodocotyloides s.

Opecoel ina theragrae Lloyd 1938. Washington. Intestine. Lloyd (1938).

\section{SANGUINICOLIDAE}

Aporocotyle theragrae Ichihara 1970. Japan. Blood vessels. Ichihara (7970).

Aporocotyle simplex Odhner 1900. Kamchatka, USSR. Mamaev and Baeva (1963).

\section{ZOOGONIDAE}

Steganoderma formosum Stafford 1904. Kamchatka, USSR. Digestive tract. Mamaev and Baeva (1963).

\author{
HIRUDINOIDEA
}

\section{PISCICOLIDAE}

Beringobde11a rectangulata (Levinsen 1882) Caballero 1974. North Pacific. Akhmerov (1957). 


\section{GYRODACTYLIDAE} Gyrodactylus marinus Bykhovsky and Poly anski 1953. Sea of Japan.
Gills. Bykhovsky and Poly anski (1953).

Gyrodactylus pterigialis Bykhovsky and Polyanski 1953. USSR. Bykhovsky (1957).

\section{NEMATODA}

\section{ANISAKIDAE}

Anisakis sp. Larva. British Columbia. Arai (1969b).

Contracaecum sp. Larva. British Columbia. Arai (1969b); Japan. Kagei et a 1. (1970); Kamchatka, USSR. Many places in body. Skryabina (1963).

* Thynnascaris adunca (Rudoiphi 1802) Hartwich 1957. British Columbia. Stomach, intestine. Margolis ${ }^{3}$; larva. Kamchatka USSR. Body cavity, muscles, etc. Mamaev and Baeva (1963). Both as Contracaecum a. Both as Contracaecum a.
Phocanema $\frac{\text { Larva. British Columbia. Intestines. Arai (1969b); }}{\text { Sp. Larva }}$ (1963) as
Kamchatka, USSR. Muscles, body cavity. Skryabina (1963) as Porrocaecum sp. Porrocaecum sp.
Phocanema decipiens (Krabbe 1878) Myers 1959. Larva. Bering Sea,
USSR. Muscles. Schiller (1954) as Terranova d.

\section{CYSTIDICOLIDAE} Ascarophis skrjabini (Layman 1933) Polyanski 1952. Kamchatka, USSR.
Stomach, intestine. Mamaev and Baeva (1963).
Stomach. Stomach, intestine. Mamaev and Bhukov 1960 . Sea of Japan. Stomach.
Ascarophis pacificus Zhukov (1960a).

PROTOZOA (MICROSPORIDA)

NOSEMATIDAE $\frac{\text { Glugea punctifera }}{(1955) \text {. Thélohan 1895. Sea of Japan. Muscles. Dogie1 }}$

PROTOZOA (MYXOSPORIDA)

MYXIDI IDAE $\frac{\text { Myxidium theragrae Fujita 1923. Japan. Gallbladder. Fujita }}{(1923) .}$

OPHIDIIDAE -- Cusk-eels and Brotulas

Parabassogigas grandis 
Parabassogigas grandis (Continued)

DIGENEA

HEMIURIDAE

Gonocerca oregonens is McCauley, Pequegnat and Brownel1 1970.

Oregon. Stomach. McCauley et al. (1970).

$$
\begin{gathered}
\text { ZOARCIDAE -- Eelpouts } \\
\text { Aprodon cortezianus - Bigfin Eelpout }
\end{gathered}
$$

ACANTHOCEPHALA

ECHINORHYNCHIDAE

Echinorhynchus gadi Zoega in Müller 1776. British Columbia. Intestine. Arai (1969b).

CESTODA

BOTHRIOCEPHALIDAE

Bothriocephalus scorpii (Müller 1776) Rudolphi 1808. British Columbia. Intestine. Arai (1969b).

PHYLLOBOTHRI IDAE

Phyllobothrium sp. Larva. British Columbia. Intestine. Arai $(1969 b)$.

TENTACULARI IDAE

Nybelinia surmenicola Okada 1929. British Columbia. Intestine. Arai (7969b).

DIGENEA

HEMIURIDAE

Lecithaster gibbosus (Rudolphi 1802) Lühe 1901. British Columbia. Intestine. Arai (1969b).

Parahemiuris merus (Linton 1910) Woolcock 1935. British Columbia. Intestine. Arai (1969b).

OPECOELIDAE

Podocotyle sp. Immature. British Columbia. Intestine. Arai $(7969 \mathrm{~b})$.

NEMATODA 
Aprodon cortezianus (Continued)

ANISAKIDAE

Contracaecum sp. Larva. British Columbia. Body cavity. Arai (1969b).

CAPILLARIIDAE

Capillaria sp. British Columbia. Stomach. Arai (1969b).

Lycodapus fierasfer - Blackmouth Eelpout

COPEPODA

PENNELLIDAE

Haemobaphes enodis Wilson 1917. California. Gills. Wilson (1917).

Lycodapus mandibularis - Pallid Eelpout

COPEPODA

PENNELLIDAE

Cardiodectes medusaeus (Wilson 1908) Yamaguti 1963. Central California. Flesh (embedded). Anderson 17.

$$
\text { Lycodes brevipes - Shortfin Eelpout }
$$

DIGENEA

ZOOGONIDAE

Zoogonus dextrocirrus A1drich 1961. Washington. Intestine. A7drich (1961).

\section{NEMATODA}

\section{ANISAKIDAE}

Anisakis sp. Larva. British Columbia. Margolis ${ }^{3}$.

CAPILLARI IDAE

Capillaria sp. British Columbia. Margolis ${ }^{3}$.

$$
\text { Lycodopsis pacifica - Blackbelly Eeelpout }
$$

DIGENEA 
OPECOELIDAE

Pseudopecoelus vulgaris (Manter 1934) Vañ Wicklen 1946. Washington. Intestine. LToyd (1938) as Cymbepha17us v.

Podocotyle gibbonsiae Johnson 1949. Washington. Intestine. ATdrich ${ }^{2}$.

Podocotyle enophrysi Park 1937. Washington. Intestine. Aldrich 22 as $\underline{P}$. pacifica.

ZOOGONIDAE

Zoogonus dextrocirrus A1drich 1961. Washington. Intestine. Aldrich ${ }^{72}$.

MONOGENEA

MICROCOTYLIDAE

Microcotyle pacifica Crane 1972. Northern and Southern California.

GiT1s. Crane (1972).

PROTOZOA (MICROSPORIDA)

Genera and species. Noble and Collard (1970).

Maynea californica - Persimmon Eelpout

ACANTHOCEPHALA

ECHINORHYNCHIDAE

Echinorhynchus canyonensis Huffman and KTiever 1977. Centra1

California. Intestine. Huffman and Kliever (1977).

COPEPODA

CHONDRACANTHIDAE

Acanthochondria cornuta (Mü17er 1777) Oakley 1927. Central California. Kîjever'il.

Melanostigma pammelas - Midwater Eelpout

DIGENEA

FELLODISTOMATIDAE

Fellodistomum melanostigum Noble and Orias 1975. Southern California. Stomach, intestine. Noble and Orias (1975).

HEMIURIDAE

Aponurus pacificus Noble and Orias 1975. Southern California. Stomach. Nob7e and Orias (1975). 
Melanostigma pammelas (Continued)

Aponurus californicus Noble and Orias 1970. Southern California. Stomach. Noble and Orias (1975).

Aponurus sp. Southern California. Stomach. Noble and Orias (1975).

Dissosaccus sp.? Southern California. Intestine. Noble and

Orias (1975).

NEMATODA

\section{ANISAKIDAE}

Anisakis sp. Larva. Southern California. Coiled in mesentery. Noble and Orias (1975).

Contracaecum sp. Larva. Southern California. Coiled in mesentery, intestine. Noble and Orias (1975).

PROTOZOA (MYXOSPORIDA)

MYXIDI IDAE

Myxidium melanostigmum Noble 1966. Southern California. Gallbladder. Noble $(1966 a)$.

$$
\begin{gathered}
\text { MORIDAE -- Codlings } \\
\text { Antimora microlepis - Finescale Codling }
\end{gathered}
$$

COPEPODA

\section{PHILICHTHYIDAE}

Sarcotaces komaii Shi ino 1953. Japan. Avdeev and Avdeev (1975).

\section{DIGENEA}

\section{LEPOCREADI IDAE}

Lepidapedon antimorae McCauley 1968. Oregon. Intestine. McCauley (1968).

\section{MONOGENEA}


Antimora microlepis (Continued)

\title{
DICLIDOPHORIDAE
}

Choriocoty?e oregonensis McCauley and Simoker 1969. Oregon.

Gills. McCauley and Smoker (1969).

\author{
MACROURIDAE -- Grenadiers \\ Coelorinchus scaphopsis - Shoulderspot Grenadier
}

PROTOZOA (MYXOSPORIDA)

CERATOMYXIDAE

Leptotheca informis Auerbach 1910. Pacific - Mexico. Gallbladder. Yoshino and Moser (1974).

MYXIDIIDAE

Zschokkel1a rovignens is Nemeczek 1922. Mexico. Urinary bladder and kidneys. Moser and Noble (1977d).

MYXOBOLIDAE

Myxobolus mexicanus Yoshino and Nobie 1973. Gulf of California. Kidneys. Yoshino and Noble (1973).

\section{Coryphaenoides sp.}

DIGENEA

LEPOCREADIIDAE

Lepidapedon lutem abyssensis McCauley 1968. Oregon. Intestine. McCauley (1968).

Coryphaenoides acrolepis - Pacific Rattail

SYNONYMS - Hemimacrurus acrolepis, Macrurus acrolepis

COPEPODA

CHONDRACANTHIDAE

Lateracanthus quadripedis Kabata and Gusev 1966. Aleutian deep. Walls of branchial cavity. Kabata and Gusev (1966).

LERNAEOPODIDAE 
Neobrachiella annulata (Markevich 1940) Kabata 1979. East of Japan. Buccal cavity. Kabata and Gusev (1966) as Brachiella a.

\section{DIGENEA}

\section{HEMIURIDAE}

Dinosoma oregonensis McCauley and Pequegnat 1968. Oregon. Stomach. Mccauley and Pequegnat (1968).

Dinosoma pectoral is McCauley and Pequegnat 1968. Oregon. Stomach. McCauTey and Pequegnat (1968).

\section{LEPOCREADIIDAE}

Lepidapedon Tuteum abyssensis McCauley 1968. Oregon. Intestine. McCauTey (1968).

\section{MONOGENEA}

\section{DISCOCOTYLIDAE}

Cyclocotyloides pinguis (Linton 1940) Price 1943. Oregon. Gills, mouths. McCauTey and Smoker (1969).

\section{PROTOZOA (MYXOSPORIDA)}

\section{CERATOMYXIDAE}

Leptotheca armatura Yoshino and Moser 1974. Southern California. Gallbiadder and bile duct. Moser and Noble (1976b).

Leptotheca minima Meglitsch 1960. Northern California. Urinary bladder, kidney tubules. Yoshino and Moser (1974).

Leptotheca informis Auerbach 1910.

California. Gallbladder. Yoshino and Noble (1973).

Ceratomyxa hokarari Meg1itsch 1960.

California. Gallbladder. Yoshino and Noble (1973); Northern Cal ifornia. Yoshino and Moser (1974).

\section{MYXIDI IDAE}

Zschokkella globulosa Davis 1917. Northern California. Kidneys. Moser and Noble (1977d).

\section{SINUOL INEIDAE}

Davisia pectoralis Moser and Noble 1975. Northern California. Urinary bladder and kidneys. Moser and Noble (1977a). Davisia coryphaenoidia Yoshino and Noble 1973. Southern California. Urinary bladder. Yoshino and Noble (1973); Northern California. Yoshino and Moser (1974). 
Sinuolinea magna Yoshino and Noble 1973. Southern California. Urinary bladder. Yoshino and Noble (1973).

Sinuolinea triangulata Schulman 1966. Northern Mexico to Oregon. Urinary b7adder and kidneys. Moser and Noble (1977c).

Myxoproteus californicus Yoshino and Noble 1973. Southern California. Urinary bladder. Yoshino and Noble (1973); Northern California. Yoshino and Moser (1974).

Myxoproteus rosenblatti Moser and Nob7e 1977. Oregon. Galibladder. Moser and Nob7e (7977b).

Myxoproteus abyssus Yoshino and Moser 1974. Southern California, British Columbia. Gallbladder. Yoshino and Moser (1974).

\section{SPHAEROSPORIDAE}

Auerbachia pulchra Lom, Noble and Laird 1975. Northern California. GalTbladder. Moser and Noble (1977a).

Chloromyxum kabatai Moser and Noble 1977. Oregon. Gallbladder. Moser and Nob7e (1977a).

\section{PROTOZOA (SARCOMASTIGOPHORA)}

\section{CRYPTOBIIDAE}

Cryptobia coryphaenoideana Noble 1968. Mexico. Stomach. Noble 7968.

\section{Coryphaenoides armatus}

SYNONYMS - Malacocephalus abyssorum, Coryphaenoides abyssorum

\section{CESTODA}

\section{LACISTORHYNCHIDAE}

Grillotia (Paragrillotia) rowei Campbel1 1977. Plerocercoid.

$$
\text { Western North AtTantic. Liver, mesentery, etc. Campbel1 (1977). }
$$

COPEPODA

\section{CHONDRACANTHIDAE}

Chondracanthodes rickettsi Wilson 1935. California. Gill cavity. WiTson (1935b).

Chondracanthodes deflexus Wilson 1932. Southern California. Gi11 cavity. Ho (1970) from Malacoecephalus abyssorum. Hubbs (pers. comm.) states that M. abyssorum does not exist and he cannot find any reference to it. He suggests that it may be a gross misstating of $\underline{C}$. abyssorum. 


\section{DIGENEA}

\section{HEMIURIDAE}

Glomericirrus ulmeri Campbe11 and Munroe 1977. Western North Atlantic. Stomach and intestine. Campbel1 and Munroe (1977). Gonocerca phycidis Manter 1925. Western North Atlantic. Stomach. Campbe17 and Munroe (1977).

Gonocerca haedrichi Campbel1 and Munroe 1977. Western North Atlantic. Ureter and urinary bladder. Campbel1 and Munroe (1977).

\section{PROTOZOA (MYXOSPORIDA)}

\section{CERATTOMYXIDAE}

Leptotheca minima Meglitsch 1960. Oregon. Central California. Urinary bladder and duct, kidney tubules. Yoshino and Moser (1974).

Leptotheca armatura Yoshino and Moser 1974. Southern California. Gallbladder. Yoshino and Moser (1974).

Leptotheca coelorhynchus Yoshino and Noble 1973. South Australia. GaTlbladder. Moser and Noble (1976b).

\section{MYXIDIIDAE}

Myxidium pearcyi Moser, Noble and Lee 1976. Mexico, California. Gallb7adder. Moser et a1. (1976).

Myxidium coryphaenoidium Noble 1966. Oregon, Central California. Ga71bTadder. Yoshino and Moser (1974).

Zschokkella globulosa Davis 1917. Oregon, Central California. Urinary bladder and duct, kidney tubules. Yoshino and Moser (1974).

Zschokkella russelli Tripathi 1948. Oregon. Urinary bladder. Yoshino and Moser (1974).

Zschokkella meglitschi Moser and Noble 1977. Oregon. Urinary bladder and kidneys. Moser and Noble (1977d).

MYXOBOLID̄ĀE

Myxobolus mexicanus Yoshino and Noble 1973. Northern California. Kidneys. Moser and Noble (1977c).

\section{SINUOL INEIDAE}

Sinuolinea triangulata Schulman 1966. Mexico-Oregon. Urinary bladder and kidneys. Moser and Noble (1977c).

Myxoproteus abyssus Yoshino and Moser 1974. Northern California. Urinary bladder and duct. Yoshino and Moser (1974). Myxoproteus rosenblatti Moser and Noble 1977. Costa Rica. Urinary bladder and kidneys. Moser and Noble (1977b). 
Coryphaenoides armatus (Continued)

SPHAEROSPORIDAE

Chloromyxum subsphaericum Shulman 1966. Costa Rica. Gallbladder. Moser and Noble (1977a).

\section{Coryphaenoides filifer - Threadfin Grenadier}

DIGENEA

HEMIURIDAE

Gonocerca oregonens is McCauley, Pequegnat and Browne11 1970.

Oregon. Stomach. McCauley et al. (1970).

\section{LEPOCREADIIDAE}

Lepidapedon Tuteum abyssensis McCauley 1968. Oregon. Intestine. McCauley (1968).

Lepidapedon yaquina McCauley 1968. Oregon. Intestine. McCauley (1968).

Lepidapedon cascadens is McCauley 1968. Oregon. Intestine. McCauTey (1968).

Lepidapedon oregonensis McCauley 1968. Oregon. Intestine. McCauTey (1968).

Lepidapedon filiformis McCauley 1968. Oregon. Intestine. McCauley (1968).

MONOGENEA

DISCOCOTYLIDAE

Cyclocotyloides pinguis (Linton 1940) Price 1943. Oregon.

ATeutian Islands. Gills, mouth. McCauley and Smoker (1969).

PROTOZOA (MICROSPORIDA)

Genera and species. Noble and Collard (1970).

PROTOZOA (MYXOSPORIDA)

CERATOMYXIDAE

Ceratomyxa sp. Noble and Collard (1970).

Leptotheca informis Auerbach 1910. Oregon. Gallbladder.

Yoshino and Moser (1974). 
MYXIDIIDAE

Myxidium coryphaenoidium Noble 1966. Oregon. Gallbladder. Yoshino and Moser (1974).

Zschokkella russelli Tripathi 1948. Oregon. Urinary bladder.

Yoshino and Moser (1974).

Zschokkella meglitschi Moser and Noble 1977. Oregon. Urinary

bladder. Moser and Noble (1977d).

SINUOLINEIDAE

Myxoproteus abyssus Yoshino and Moser 1974. Oregon. Urinary bladder. Moser and Noble (1977b).

Myxoproteus rosenblatti Moser and Noble 1977. Canada. Gallbladder. Moser and Noble (1977b).

Sinuolinea triangulata Schulman 1966. Oregon. Urinary bladder. Yoshino and Moser (1974).

SPHAEROSPORIDAE

Auerbachia pulchra Lom, Noble and Laird 1975. Oregon-Canada. Gallbladder. Moser and Noble (1977a).

PROTOZOA (SARCOMASTIGOPHORA)

CRYPTOBIIDAE

Cryptobia sp. Noble and Collard (1970).

Coryphaenoides leptolepis - Ghostly Grenadier

DIGENEA

HEMIURIDAE

G1omericirrus U1meri Campbe11 and Munroe 1977. Western North Atlantic. Stomach, intestine. Campbell and Munroe (1977).

\section{LEPOCREADI IDAE}

Lepidapedon luteum abyssensis McCauley 1968. Oregon. Intestine. McCauley (1968).

Lepidapedon yaquina McCauley 1968. Oregon. Intestine. McCauley (1968).

Lepidapedon cascadens is McCauley 1968. Oregon. Intestine. McCauley (1968).

Lepidapedon oregonensis McCauley 1968. Oregon. Intestines. McCauley (1968).

PROTOZOA (MYXOSPORIDA)

CERATOMYXIDAE

Leptotheca armatura Yoshino and Moser 1974. Central California. Urinary bladder and kidney tubules. Yoshino and Moser (1974). 
MYXIDIIDAE

Myxidium pearcyi Moser, Noble and Lee 1976. California to Washington. Gallbladder. Moser et al. (1976).

Myxidium coryphaenoidium Noble 1966. Washington, Oregon, Centra 7 California. Gallbladder. Yoshino and Moser (1974).

Myxidium orientalis Schulman 1962. Central California. GaT1bTadder. Yoshino and Moser (1974).

Zschokkella globulosa Davis 1917. Washington, Oregon, Central California. Urinary bladder and ducts, kidney tubules. Yoshino and Moser (1974).

SINUOL INEIDAE

Sinuolinea triangulata Schulman 1966. Oregon. Urinary bladder. Yoshino and Moser (1974).

\section{Coryphaenoides pectoralis - Giant Grenadier \\ SYNONYM - Albatrossia pectoral is}

\section{COPEPODA}

\section{LERNAEOPODIDAE}

Neobrachiella nitida (Wilson igi5) Kabata 1979. Northern Pacific. Mouth. Wilson (1935b) as Brachiel1a n.

\section{DIGENEA}

\section{HEMIURIDAE}

Dinosoma oregonensis McCauley and Pequegnat 1968. Oregon. Stomach. McCauTey and Pequegnat (1968).

Dinosoma pectoral is McCauley and Pequegnat 1968. Oregon. Stomach. McCauTey and Pequegnat (1968).

Gonocerca oregonensis McCauley, Pequegnat and Brownel1 1970. Oregon. Stomach. McCauley et al. (1970).

Gonocerca oshoro Shimazu 1970. Gulf of Alaska. Ovaries. Shimazu (1970).

\section{MONOGENEA}

DICLIDOPHORIDAE

Cyclocotyloides pinguis (Linton 1940) Price 1943. Northwest Pacific. Mouth. Linton (1940) as Diclidophora p.; Oregon, Alaska. McCauley and Smoker (1969).

PROTOZOA (MYXOSPORIDA) 


\section{CERATOMYXIDAE}

Leptotheca armatura Yoshino and Moser 1974. Washington, Cal ifornia. Urinary bladder, kidney tubules. Yoshino and Moser (1974).

Leptotheca informis Auerbach 1910. Washington, Central and Northern California. Gallbladder. Yoshino and Moser (1974).

\section{MYXIDI IDAE}

Zschokkella meglitschi Moser and Noble 1977. Central California. Urinary bladder. Moser and Noble (1977d).

\section{SINUOLINEIDAE}

Sinuolinea magna Yoshino and Noble 1973. Northern California. Urinary bTadder. Yoshino and Moser (1974).

Sinuolinea triangulata Schulmar 1966. Northern California. Kidneys. Moser and Noble (1977c).

Davisia coryphaenoidia Yoshino and Noble 1973. Northern California. Urinary bladder and kidneys. Moser and Noble (1977a).

Davisia pectoralis Moser and Noble 1975. Northern California. Urinary bladder, kidney tubules. Moser and Noble (1975).

Myxoproteus Californicus Yoshino and Noble 1973. Washington, Northern California. Urinary bladder, kidney tubules. Yoshino and Moser (1974).

\section{SPHAEROSPORIDAE}

Auerbachia pulchra Lom, Noble and Laird 1975. Northern California. Moser and NobTe (1977a).

\section{Nezumia 1iolepis - Smooth Grenadier}

\section{PROTOZOA (MYXOSPORIDA)}

\section{MYXIDIIDAE}

Myxidium coryphaenoidium Noble 1966. Southern California. Ga17b1adder. Moser et a1. (1976). Zschokkella globulosa Davis 1917. Southern California. Urinary bladder and kidney. Moser and Noble (1977d).

$$
\text { Nezumia stelgidolepis - California Rattail }
$$

PROTOZOA (MYXOSPORIDA) 
Nezumia stelgidolepis (Continued)

Ceratomyxa hopkinsi Jameson 1929. Southern California.

Gallbladder. Moser and Noble (1976a).

MYXIDIIDAE

Zschokkella meglitschi Moser and Noble 1977. Southern California. Urinary bladder. Moser and Noble (1977d).

Zschokkella globulosa Davis 1917. Southern California. Urinary bladder. Moser and Noble (1977d).

Myxidium coryphaenoidium Noble 1966. Southern California. Gallbladder. Yoshino and Noble (1973).

\section{SCOMBERESOCIDAE -- Sauries \\ Cololabis saira - Pacific Saury}

ACANTHOCEPHALA

ECHINORHYNCHIDAE

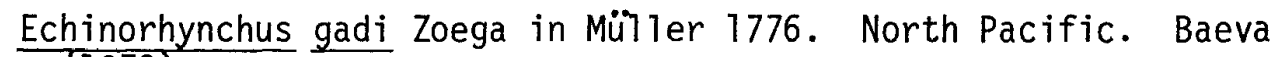
(1970).

POLYMORPHIDAE

Bolbosoma caenoforme (Heitz 1920) Meyer 1933. North Pacific. Baeva (1970).

RHADINORHYNCHIDAE

Rhadinorhynchus selkirki Van Cleave 1921. Japan. Internal. Yamaguti $(1963 \mathrm{c})$. Rhadinorhynchus cololabis Laurs and McCauley 1964. Oregon. Intestine. Laurs and McCauley (1964).

Rhadinorhynchus trachuri Harada 1935. Sea of Japan. Baeva (1965a).

CESTODA

PHYLLOBOTHRI IDAE

Genera and species. Plerocercoid. Southern California. Stomach, intestine. Collard ${ }^{9}$.

TETRABOTHRI IDAE

Priapocephalus sp. Larva. North Pacific. Intestine. Baeva $(1965 a)$.

\section{TETRAPHYLLIDEA}

Scolex pleuronectis Müller 1788. North Pacific. Intestine. Baeva (1965a). 
Cololabis saira (Continued)

COPEPODA

BOMOLOCHIDAE

Bomolochus decapteri Yamaguti 1936. North Pacific. Baeva (1970).

CALIGIDAE

Caligus macarovi Gussev 1951. Oregon, Washington, British Columbia. Hughes (1973); Japan. Body surface. Shiino (1954a) as $\underline{C}$. fulvipurpureus.

\section{PENNELLIDAE}

Pennella sp. Oregon, Washington, British Columbia. Body surface. Hughes (1973).

\section{DIGENEA}

DIDYMOZOIDAE

Genera and species. Larva. North Pacific. Baeva (1970) as Torticaecum metacercaria, Monilicaecum ventricosum metacercaria.

\section{FELLODISTOMATIDAE}

Lintonium novikovi Baeva 1965. North Pacific. Intestine. Baeva (1965a) ["not referable to Lintonium" - Yamaguti (1971)].

\section{HEMIURIDAE}

Genera and species. Southern California. Stomach, intestine. Collard9; North Pacific. Baeva (1970).

Brachyphallus crenatus (Rudolphi 1802) Odhner 1905. North Pacific. Baeva (1970).

Lecithaster gibbosus (Rudolphi 1802) Lühe 1901. Peter the Great GuTf, USSR. Akhmerov (1955).

Lecithaster sayori Yamaguti 1938. North Pacific. Intestine. Baeva (1965a).

\section{NEMATODA}

\section{ANISAKIDAE}

Anisakis sp. Japan. Gut. Otsuru et a1. (1965); North Pacific. MuscTe. Baeva (1965a).

*Contracaecum fabri (Rudolphi 1819). North Pacific. Digestive tract. Baeva (1965a).

*Thynnascaris adunca (Rudoiphi 1802) Hartwich 1957. North Pacific. Baeva (1970).

Spinitectus sp. North Pacific. Intestine. Baeva (1965a). Spinitectus pacificus Sobolev and Belogurov 1968. Pacific. Sobolev and Belogurov (1968). 
Cololabis saira (Continued)

PROTOZOA (MYXOSPORIDA)

MYXIDIIDAE

Sphaeromyxa parva Dogie1 1948. Bay of Peter the Great, USSR. GaT1bladder. Dogie1 (1948).

PROTOZOA (SPOROZOA)

EIMERI IDAE

Eimeria auxidis Dogiel 1948. Peter the Great Gulf, USSR. Kidneys. Pellerdy (1965).

$$
\text { CYPRINODONTIDAE -- Killifishes }
$$

Fundulus parvipinnis - California Killifish

COPEPODA

\section{ERGASILIDAE}

Ergasilus sp. Southern California. Gills. Baker ${ }^{22}$.

\section{DIGENEA}

\section{CYATHOCOTYLIDAE}

Mesostephanus appendiculatus (Ciurea 1916) Lutz 1935. Metacercaria. Southern California. Muscle. Martin (1961).

DIPLOSTOMATIDAE

Posthodiplostomum minimum (MacCa17um 1921) Dubois 1936.

Metacercaria. Southern California. Egg sacs. Yoshino (1972).

GORGODERIDAE

Phyllodistomum sp. Manter and Van Cleave (1951).

\section{HETEROPHYIDAE}

Euhaplorchis californiensis Martin 1950. Metacercaria. Southern California. Brain surface. Yoshino (1972).

Ascocotyle angrense Travassos 1976. Metacercaria. Southern California. Gill filaments. Yoshino (1972).

Ascocotyle sexidigita Martin and Steele 1970. Metacercaria. Southern California. Gut wall lateral musculature. Yoshino (1972); California. Martin and Steele (1970).

Phocitremoides ovale Martin 1950. Metacercaria. Southern California. Scales. Yoshino (1972). 
Fundulus parvipinnis (Continued)

Stictodora hancocki (Martin 1950) Witenberg 1953. Metacercaria. Southern California. Muscle, brain case, etc. Martin (1950) as Parastictodora $h$.

Pygidiopsoides spindalis Martin 1951. Metacercaria. Southern California. Gills. Martin (1951).

\section{RENICOLIDAE}

Renicola cerithidicola Martin 1971. Metacercaria. Southern California. Gills. Martin (1971).

Renicola buchanani (Martin and Gregory 1951). Metacercaria. Southern California. Liver. Martin (1971).

MONOGENEA

DACTYLOGYRIDAE

Urocleidus sp. Southern California. Gill filaments. Yoshino (1972).

GYRODACTYLIDAE

Gyrodactylus sp. Southern California. Gills. Baker 22 . NEMATODA

CAMALLÃNIDAE

Spirocamallanus pereirai (Annereaux 1946) 01 sen 1952. Larva and adult. Southern California. Stomach, intestine. Noble and King (1960).

PROTOZOA (CILIOPHORA)

URCEOLARI IDAE

Trichodina sp. Southern California. Gills. Baker 22.

$$
\text { ATHERINIDAE -- Silversides }
$$

Atherinops affinis - Topsmelt

COPEPODA

BOMOLOCHIDAE

Bomolochus constrictus Cressey in Cressey and Collette 1970.

Southern California. Gil1s. Hobson (1971) as Parabomolochus c.

CALIGIDAE

Caligus serratus Shiino 1965. Southern California. Body surface. Hobson (1971). 
Atherinops affinis (Continued)

ERGAS ILIDAE

Ergasilus sp. Southern California. Body surface. Baker 22 .

PENNELLIDAE

Peniculus fissipes Wilson 1917. Southern California. Embedded in fins. Hobson (1971).

\section{DIGENEA}

HETEROPHYIDAE

Ascotyle angrense Travasssos 1916. Metacercaria. Southern California. Baker ${ }^{2}$.

Cercarioides humbargari (Park 1936) Dubois and Mahon 1959.

Metacercaria. Southern California. 01 son .

ISOPODA

CYMOTHOIDAE

Nerocila californica Schioedte and Meinert 1881. Southern California. Gills. Brusca (1977). Speculative host.

MONOGENEA

GYRODACTYLIDAE

Archigyrodactylus atherinops Mizelle and Kritsky 1967. Northern

California. Skin. Mizelle and Kritsky (1967b).

Gyrodactylus sp. Southern California. Gills. Baker22.

POLYOPISTHOCOTYLEA

Genera and species. Southern California. Gills. Baker22. NEMATODA

ANISAKIDAE

Contracaecum sp. Immature. Southern California. $01 \operatorname{son}^{8}$.

\section{CAMALLANIDAE}

Spirocamallanus pereirai (Annereaux 1946) 01 sen 1952. Southern

California. Intestine. Noble and King (1960).

PROTOZOA (CILIOPHORA)

URCEOLARIIDAE

Trichodina sp. Southern California. Gills. Baker ${ }^{22}$. 
CESTODA

TETRAPHYLLIDEA

Genera and species. Yellow pigmented plerocercoid. Southern

California. Encysted in rectal mesentery. Kunnenkeri (1962).

COPEPODA

CALIGIDAE

Caligus serratus Shiino 1965. Southern California. Shiino (1965b).

\section{DIGENEA}

\section{LEPOCREADIIDAE}

Lepocreadium sp. Central California. Intestine. Chapa ${ }^{23}$.

\section{MONORCHI IDAE}

Asymphylodora atherinopsidis Annereaux 1947. Northern Cal ifornia. Intestine. Annereaux (1947).

ISOPODA

\section{CYMOTHOIDAE}

Nerocila californica Schioedte and Meinert 1881. Southern California. Gills. Brusca (1977). Speculative host.

\section{NEMATODA}

\section{ANISAKIDAE}

Anisakis sp. Larva. Southern California. Dailey et al. (in press). Contracaecum sp. Larva. Southern California. Dailey et al. (in press).

\section{CAMALLANIDAE}

Spirocamallanus pereirai (Annereaux 1946) 01 sen 1952. Northern California. Intestine. Annereaux (1946) as Procamallanus p.

$$
\text { Leuresthes tenuis - California Grunion }
$$

\section{BRANCHIURA}

ARGULIDAE

Argulus melanostictus Wilson 1935. Southern California, Baja California. Fins. A. C. 01son (1972). 
CESTODA

\section{LACISTORHYNCHIDAE}

Lacistorhynchus tenuis (Van Beneden 1858) Dollfus 1929.

Plerocercoid. Southern California. Muscles, abdominal areas. $01 \operatorname{son}^{8}$.

COPEPODA

BOMOLOCHIDAE

Bomolochus cuneatus Fraser 1920. Southern California. Gill chambers. A. C. 01son (1972) as Bomolochus pectinatus.

CALIGIDAE

Caligus olsoni Pearse 1953. Southern California. Skin. Pearse $(1953)$.

\section{LERNAEOPODIDAE}

Clavellopsis sp. Southern California and Baja California. Mouth, pharynx. A. C. 01son (1972).

\section{DIGENEA}

BUCEPHALIDAE

Prosorhynchoides labiatus (Manter and Van Cleve 1951) n. comb. Metacercaria. Southern California. Muscle of heart ventricle. A. C. 01son (1975) as Bucephalops is 1.

DIDYMOZOIDAE

Genera and species. Larva. Southern California. Coelom. 01son 8 as Torticaecum fenestratum.

HETEROPHYIDAE

Cercarioides humbargari (Park 1936) Dubois and Mahon 1959. Metacercaria. Southern California. Coelom-viscera. 01 son $^{8}$ LEPOCREADI IDAE

Lepocreadium manteri 01son 1978. Southern California and Baja California. Intestine. Olson (1978).

MONORCHIIDAE

Asymphylodora atherinosidis Annereaux 1947. Southern California. Intestine. 07 son $^{8}$. 
ISOPODA

\section{CYMOTHOIDAE}

Nerocila californica Schioedte and Meinert 1881. California. Body surface. A. C. OTson (1972).

Lironeca californica Schioedte and Meinert 1884. California. Gill chambers.A. C. OTson (1972).

MONOGENEA

HETERAXINIDAE

Leuresthicola olsoni Price 1962. Southern California. Gills.Price (1962b). NEMATODA

\section{ANISAKIDAE}

Contracaecum sp. Larva. Southern California. Coelom-viscera. 01 son $^{8}$. CAMALLANIDAE

Spirocamallanus pereirai (Annereaux 1946) 01sen 1952. Southern California.

\section{CUCULLANIDAE}

Cucullanus sp. Larva. Southern California. Coelom. 01 son $^{8}$.

$$
\begin{gathered}
\text { ZEIDAE -- Dories } \\
\text { Zenopsis nebulosa - Mirror Dory }
\end{gathered}
$$

CESTODA

LACISTORHYNCHIDAE

Lacistorhynchus tenuis (Van Beneden 1858) Dollfus 1929. Larva. New Zealand. Body cavity. Robinson (1959a).

TRIAENOPHORIDAE

Eubothrioides lamellatum Yamaguti 1952. Japan. Yamaguti (1952). COPEPODA

\section{CALIGIDAE}

Lepeophtheirus goni isti Yamaguti 1936. Japan. Body surface. Shiino (1959e).

Anuretes quadrilaterus Shiino 1954. Japan. Body surface. Shiino (1954d). Caligus glandifer Shiino 1954. Japan. Shiino (1954b). 
Zenopsis nebulosus (Continued)

CHONDRACANTHIDAE

Chondracanthus distortus Wilson 1922. Japan. Mouth. Yamaguti (1939c).

DIGENEA

OPECOELIDAE

Pseudopecoelus japonicus (Yamaguti 1938) Von Wicklen 1946. Japan. Intestine. Yamaguti ${ }^{24}$ as Cymbephallus $j$.

NEMATODA

ANISAKIDAE

* Contracaecum zenops is Yamaguti 1941. Japan. Sma11 intestine. Yamaguti (1947); New Zealand. Hewitt and Hine (1972). Contracaecum sp. Larva. New Zealand. Stomach, intestine, body cavity. Hewitt and Hine (1972).

Anisakis sp. Larva. New Zealand. Viscera, mesentery, under peritoneum. Hewitt and Hine (1972).

$$
\begin{aligned}
& \text { LAMPRIDIDAE -- Opahs } \\
& \text { Lampris regius - Opah }
\end{aligned}
$$

CESTODA

PHYLLOBOTHRIIDAE

Phyllobothrium caudatum (Zschokke and Heitz 1914). Pacific coast of Japan. Intestine. Yamaguti (1963a) as Pelichnibothrium speciosum.

COPEPODA

\section{LERNAEOPODIDAE}

Clavellistes shoyoae Shitno 1963. Pacific. External, around the eyes. Shiino (1963c).

Neobrachiella regius (Lewis 1967) Kabata 1979. Hawaii. Gill arches. Lewis (1967) as Brachiella r.

\section{DIGENEA}

DIDYMOZOIDAE

Lamprididymozoon Tampridis (Yamaguti 1940) Yamaguti 1971. Pacific coast of Japan. Gi11 filaments, arches. Yamaguti (1940) as Nematobothrium 1. Metadidymocystis cymbiformis Yamaguti 1970. Hawaii. Muscles. Yamaguti (1970). HEMIURIDAE

Lampritrema nipponicum Yamaguti 1940. Baja California. Intestine. Arai (1962); Japan. Stomach. Yamaguti (1940).

Lampritrema hawaiiense Yamaguti 1970. Hawaii. Stomach. Yamaguti (1970). 165 
Lampris regius (Continued)

NEMATODA

ANISAKIDAE

Anisakis sp. Larva. New Zealand. Viscera. Hewitt and Hine (1972).

$$
\text { GASTEROSTEIDAE -- Sticklebacks }
$$

Aulorhynchus flavidus - Tube-snout

CESTODA

BOTHRIOCEPHALIDAE

Bothriocephalus sp. Larva. British Columbia. Intestine. Arai (1969b).

PHYLLOBOTHRI IDAEE

Phyllobothrium sp. Larva. British Columbia. Intestine. Arai (1969b).

\section{DIGENEA}

HEMIURIDAE

Lecithaster gibbosus (Rudolphi 1802) Lühe 1901. British Columbia. Intestine. Arai (1969b).

\section{OPECOELIDAE}

Podocotyle atomon (Rudolphi 1802) 0dhner 1905. British Columbia. Intestine. Arai (1969b).

\section{NEMATODA}

\section{ANISAKIDAE}

Anisakis sp. Larva. British Columbia. Body cavity. Arai (1969b). *Thynnascaris adunca (Rudolphi 1802) Hartwich 1957. Larva. British Columbia. Body cavity. Arai (1969b) as Contracaecum a.

\section{CYSTIDICOLIDAE}

Ascarophis sebastodis 01sen 1952. Larva. British Columbia. Intestine. Arai $(196 \overline{9 b})$.

Gasterosteus aculeatus - Threespine Stickleback

ACANTHOCEPHALA

\section{ECHINORHYNCHIDAE}

Metechinorhynchus salmonis (Mü17er 1784) Petrotschenko 1956. USSR. Intestine. Bykhovskaya-Pavlovskaya et a1. (1962).

Metechinorhynchus lateralis (Leidy 185i) Goivan. Newfoundiand, Labrador. Hanek and Threlfall (1970); USSR. BykhovskayaPavlovskaya et al. (1962).

Echinorhynchus gadi Zoega in Müller 1776. Barents Sea, USSR. Intestine. Polyanski (1955b). 
Pseudoechinorhynchus clavula (Dujardin 1845) Petrotschenko 1956. USSR. Bykhovskaya-Pavlovskaya et a 1. (1962); Britain. Intestine. Chubb (19700) as Acanthocephalus c.

Acanthocephalus lucii (Müller 1776) Lühe 1911. USSR. Intestine. Bykhovskaya-Paviovskaya et a 1. (1962).

Acanthocephalus minor Yamaguti 1935. Japan. Awakura (1972).

\section{NEOECHINORHYNCHIDAE}

Neoechinorhynchus cristatus Lynch 1936. Kamchatka, USSR. Intestine. Skryabina (1963).

Neoechinorhynchus rutili (Müller 1780) Hamann 1892. Newfoundland, Labrador. Hanek and Threlfal1 (1970); British Columbia. Bangham and Adams (1954); USSR. Bykhovskaya-Pavlovskaya et a?. (1962).

\section{POLYMORPHIDAE}

Corynosoma strumosum (Rudolphi 1802) Lühe 1904. Larva. USSR. Bykhovskaya-Pavlovskaya et al. (1962).

Corynosoma semerme (Forssel1 1904) Lühe 1911. Larva. USSR. Bykhovskaya-Pav7ovskaya et a7. (1962).

\section{POMPHORHYNCHIDAE}

Pomphorhynchus laevis (Zoega in Müller 1776) Van Cleave 1924. Larva. IreTand. Intestine. Kane (1966); USSR. BykhovskayaPavlovskaya et al. (1962).

\section{RHADINORHYNCHIDAE}

Telosentis exiquus (Von Linstow 1901) Van Cleave 1921. B1ack Sea. Intestine. Yamaguti (1963c).

\section{BRANCHIURA}

\section{ARGUL IDAE}

Argulus foliaceus (Linnaeus 1758). USSR. Body surface. Bykhovskaya-Pavlovskaya et al. (1962).

Argulus canadensis Wilson 1916. Newfoundland. Body surface. Thre? fal1 (1968).

Argulus alosae Gould 1912. Gulf of St. Lawrence. Body surface. Wilson (1917).

Argulus japonicus Thiele 1900. Ukraine, USSR. Sukhenko (1966).

\section{CESTODA}

\section{AMPHICOTYLIDAE}

Eubothrium crassum (Bloch 1779) Nybel in 1922. Larva. Norway. Liver, viscera, body cavity. Vik (1963). Eubothrium sp. England. Chappel1 and Owen (1969). 
BOTHRIOCEPHALLIDAE

Bothriocephalus? claviceps (Goeze 1782) Rudoiphi 1810. England. Chappe11 and Owen (1969).

Bothriocephalus scorpii (Mül1er 1776) Rudo1phi 1808. Larva. British Columbia. Intestine. Arai (1969b); USSR. BykhovskayaPavlovskaya et al. (1962).

Bothriocephalus sp. Labrador, Newfoundland. Hanek and Threlfall (1970).

\section{CYATHOCEPHALIDAE}

Cyathocephalus truncatus (Pallas 1781) Kessler 1868. British Columbia. Intestine. Lester (1974); Norway. Vik (1954).

\section{DIPHYLLOBOTHRIIDAE}

Diphyllobothrium sp. Plerocercoid. British Columbia. Liver. Lester (1974).

Diphyllobothrium dendriticum (Nitzsch 1824) Matz 1892. Plerocercoid. Ireland. Kane (1966); USSR. Bykhovskaya-Pavlovskaya et a1. (1962).

Diphyl lobothrium ditremum (Crep1 in 1825) Matz 1892. Plerocercoid. Britain. Chubb (1970a); USSR. Bykhovskaya-Pavlovskaya et al. (1962) as D. norvegicum.

Diphyllobothrium vogeli Kuhlow 1952. Plerocercoid. West Germany. Liver. Vik et a1. (1969).

\section{LIGUL IDAE}

Schistocephalus solidus (Müller 1776) Steenstrup 1857. Plerocercoid. England. Body cavity. Pennycuick (1971); Labrador, Newfoundland. Hanek and Threlfall (1970); USSR. Bykhovskaya-Pavlovskaya et al. (1962); British Columbia. Lester (1974); California. Haderlie (1953).

Schistocephalus sp. Larva. British Columbia. Bangham and Adams (1954).

\section{PHYLLOBOTHRI IDAE}

Phyllobothrium sp. Larva. British Columbia. Intestine. Arai $(1969 b)$.

\section{PROTEOCEPHALIDAE}

Proteocephalus filicollis (Rudolphi 1802) Weinland 1858. England. Intestine. Chappel1 (1969); Labrador, Newfoundland. Hanek and Threlfall (1970); USSR. Ginetsinskaya (1955).

Proteocephalus pugetensis Hoff and Hoff 1929. British Columbia. Bangham and Adams (1954).

Proteocephalus longicollis (Zeder 1800) Nufer 1905. USSR. Intestine. Freze (1965). Possible host.

Proteocephalus percae (Müiler 1780) Kaill iet 1889. USSR. Intestine. Freze (1965). Possible host. 
TETRAPHYLLIDEA

Scolex polymorphus Rudolphi 1819. Barents Sea, USSR. Polyanski $(1955 a)$.

TRAIAENOPHORIDAE

Triaenophorus crassus Forel 1868. Europe. Intestine. Yamaguti $(1959 b)$.

Triaenophorus Tucii (MU1ler 1776). Plerocercoid. USSR. Petrushevski

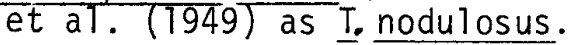

COPEPODA

BOMOLOCHIDAE

Bomolochus cuneatus Fraser 1920. British Columbia. Kabata (1971).

CALIGIDAE

Caligus lacustris Steenstrup and Lütken 1861. USSR. Skin, gi11s. Bykhovskaya-Pavlovskaya et al. (1962).

Caligus clemensi Parker and Margol is 1964. British Columbia. Body surface. Parker and Margolis (1964).

\section{ERGASILIDAE}

Ergasilus globosus Krøyer 1838-40. Yamaguti (1963a) [Species inquirae - Yamaguti (1963a)].

Ergasilus turgidus Fraser 1920. British Columbia. Probably gills. Fraser (1920).

Ergasilus auritus Markevich 1940. British Columbia. Gills, body surface. Arai (1969b); Labrador, Newfoundland. Hanek and Threlfall (1970); USSR. Bykhovskaya-Pavlovskaya et al. (1962). Ergasilus manicatus Wilson 1911. Massachusetts. Roberts (1970). Ergasilus sieboldi Nordmann 1832. Poland. Gil1s. Grabda (1963). Thersitina gasterostei (Pagenstecher 1861) Normon 1905. Newfoundland, Labrador. Hanek and Threlfall (1970); Baltic Sea. Kozikowska (1965); British Columbia. Lester (1974).

\section{LERNAEIDAE}

Lernaea cyprinacea Linnaeus 1758. Britain. Skin. Kennedy (1975); USSR. Skin, gills. BykhovskayaPavlovskaya et al. (1962) as L. esocina.

\section{DIGENEA}

\section{ALLOCREADIIDAE}

Allobunodera mediovitellata (Tsimbalyuk and Roytman 1965) Yamaguti 1971. British Columbia. Lester (1974). 
Bunodera luciopercae (Mül ler 1776) Lühe 1909. Labrador, Newfoundland. Hanek and Threlfal1 (1970).

Bunoderináa eucaliae Milier 1936. British Columbia. Bangham and Adams (1954); Oregon. Pratt and McCauley (1961) both as Bunodera.

Cainocreadium gasterostei (Bovien 1932). Yamaguti (1971).

Crepidostomum cornutum (Osborn 1903) Stafford 1904. Oregon. Pratt and McCauley (1961).

Crepidostomum cooperi Hopkins 1931. Newfoundland. Hanek and Threlfal1 (1969b).

Crepidostomum farionis (Mül ter 1780) Lühe 1909. Newfoundland. Threlfall (1968).

\section{CYATHOCOTYLIDAE}

Holostephanus luhei Szidat 1936. Metacercaria. England. Erasmus (1962).

\section{DIPLOSTOMATIDAE}

Posthodiplostomum brevicaudatum (Nordmann 1832) Wisniewski 1858. Metacercaria. Europe. Yamaguti (1971).

Posthodiplostomum cuticola (Nordmann 1832) Dubois 1936. Larva. USSR. Skin, fins. Bykhovskaya-Pavlovskaya et al. (1962).

Posthodiplostomum minimum (MacCallum 1921) Dubois 1936. British Columbia. Bangham and Adams (1954).

Diplostomum scudderi (01ivier 1841) Dubois 1936. Metacercaria. Biritish Columbia. Eyes. Lester (1974).

Diplostomum pungitius Shigin 1965. Metacercaria. USSR. Eyes. Shigin (1965).

Diplostomum gobiorum Shigin 1965. Metacercaria. USSR. Eyes. Shigin (1965).

Diplostomum sp. Larva. British Columbia. Bangham and Adams $(1954)$.

Diplostomum spathaceum (Rudolphi 1819) 01sson 1876. Newfoundland, Labrador. Hanek and Threlfall (1970); Metacercaria. Scotland. Eyes. Williams (1966); USSR. Bykhovskaya-Pavlovskaya et al. (1962); British Columbia. Lester (1974).

Diplostomum clavatum (Ciurea 1928). Metacercaria. USSR. Getsevichiute (1958).

Diplostomum gasterostei Williams 1966. Metacercaria. England. Retinal layer of eyes. Pennycuick (1971).

Tetracotyle sp. British Columbia. Bangham and Adams (1954).

Neascus sp. Metacercaria. Northern California. Skins. Haderlie (1953).

Neascus brevicaudatus (Nordmann 1832). Larva. USSR. Eyes. Getsevichiute (1958).

Tylodelphys clavata (Nordmann 1832) Diesing 1850. Larva. England. Eyes. Wootten (1973b).

\section{ECHINOSTOMATIDAE}

Patasiger cf. pungens (Linstow 1894). Metacercaria. France. Gills, stomach. Dol1fus (1961) as Echinostomum p. 
FELLODISTOMATIDAE

Faustula gasterostei Schel1 1973. Washington. Intestine. Sche17 (1973b).

\section{GORGODERIDAE}

Phyllodistomum folium (01fers 1816) Braun 1899. England. Urinary bladder, ureter. Dawes (1947).

\section{HEMIURIDAE}

Lecithaster gibbosus (Rudolphi 1802) Lühe 1901. Barents Sea, USSR. Intestine. Polyanski (1955b); British Columbia. Intestine. Arai (1969b); Labrador, Newfoundland. Hanek and Threlfall (1969a); Washington. Ching (1960a).

Tubulovesicula lindbergi (Layman 1930) Yamaguti 1934. Washington. Ching (7960a).

Brachyphal1us crenatus (Rudolphi 1802) Odhner 1905. Labrador, Newfoundland. Hanek and Threlfall (1969a); USSR. BykhovskayaPaviovskaya et al. (1962).

Parahemiurus merus (Linton 1910) Woolcock 1935. Washington. Gut. Ching $(1960 a)$.

Derogenes varicus (Mü1 ler 1784) Looss 1901. Washington. Ching (1960a); Barents Sea, USSR. Intestine. Polyanski (1955b); British Columbia. Intestine. Arai (1969b); Labrador, Newfoundland. Hanek and Threlfall (1969a).

\section{HETEROPHYIDAE}

Apophallus bacalloti (Morozov 1852) Balozet and Callot 1939. Larva. France. Body surface. Timon-David (1963). Apopha1lus brevis Ransom 1920. Metacercaria. Quebec. Gills, skin. Hanek and Molnar (1974).

Cryptocotyle concavum (Creplin 1825) Lühe 1899. Metacercaria. Centrd? California. Wootton (1957).

\section{OPECOEL IDAE}

Podocotyle apodichthysi Park 1937. California. Intestine. Park (7937b).

Podocotyle enophrysi Park 1937. Washington. Intestine. Ching (7960a) as P. pacifica.

Podocotyle atomon (RudoTphi 1802) Odhner 1905. Labrador, Newfound land. Hanek and Threlfal1 (1969a); USSR. BykhovskayaPavlovskaya et a1. (1962).

Podocotyle reflexa (Crepl in 1825) Odhner 1905. Barents Sea, USSR. Intestine. Polyanski (1955b).

Podocotyle sinusacca Ching 1960. British Columbia. Intestine. Arai (1969) as Neopodocotyloides s.

Podocotyle simplex (Rudolphi 1809) Stafford 1904. Canadian Atlantic. Intestine. Stafford (1907). 
STRIGEIDAE

Cotylurus pileatus (Rudolphi 1802) Dubois 1937. Larva. USSR. Internal organs. Bykhovskaya-Pavlovskaya et al. (1962).

Apatemon gracilis (Rudolphi 1819) Szidat 1928. Metacercaria. British Columbia. Intestine. Lester (1974).

HIRUDINOIDEA

PISCICOL IDAE

Piscicola geometra (Linnaeus 1761). USSR. Bykhovskaya-Pavlovskaya et al. (1962).

MONOGENEA

DACTYLOGYRIDAE

Dactylogyrus sp. British Columbia., Gills. Lester (1974).

DISCOCOTYLIDAE

Diplozoon paradoxum Nordmann 1832. Europe. Gi11s. Dawes (1947).

GYRODACTYLIDAE

Gyrodactylus elegans Nordmann 1832. Washington. Guberlet (1936a); England. Gills. Dawes (1947). Gyrodactylus alexanderi Mizelle and Kritsky 1967.

California. Skin. Mizelle and Kritsky (1967d); British Columbia. Lester (1974).

Gyrodactylus arcuatus Bykhovsky 1933. Barents Sea. Gills, fins, skin. PoTyanski (T955b); Britain. Chubb (1970a).

Gyrodactylus bychowskyi Sproston 1946. Barents Sea, Baltic Sea, Sea of Japan. Gills. Polyanski (1955b).

Gyrodactylus avalonia Hanek and Threlfall 1969. Ontario, Canada.

Fins. Hanek and Threlfal1 (1969c).

Gyrodactylus canadens is Hanek and Threlfall 1969. Newfoundland.

Gills. Hanek and Threlfall (1969c).

Gyrodactylus lairdi Hanek and Threlfall 1969. Newfoundland.

Fins. Hanek and Threlfall (1969c).

Gyrodactylus memorial is Hanek and Threlfall 1969. Newfoundland.

Fins. Hanek and Threlfall (1969c).

Gyrodactylus aculeati Malmberg 1956. Sweden. Gi11s. Yamaguti $(1963 \mathrm{~b})$.

Gyrodactylus rarus Wegener 1907. England. Body surface, gills.

Chappel1 (1969); Baltic Sea. Bykhovsky and Poljansky (1953). Gyrodactylus pungitii Malmberg 1964. Britain. Chubb (1970a).

\section{GYRODACTYLOIDEA}

Genera and species. British Columbia. Gills, body surface. Arai (1969b). 
MOLLUSCA

UNIONIDAE

Anodonta beringiana Middendorf. Glochidum. Alaska. Gills, mouth body surface. Cope (1959).

Genera and species. USSR. Bykhovskaya-Pavlovskaya et al. (1962); British Columbia. Lester (1974).

NEMATODA

ACUARI IDAE

Synhimantus brevicaudatus (Dujardin 1845). Larva. Sea of Azov, USSR. Stomach, Iiver, intestine. Mozgovi et aĩ. (1965).

\section{ANISAKIDAE}

Raphidascaris cristata (Linstow 1872). Juvenile. Britain. Chubb (1970a).

Raphidascaris gracillima (Linstow 1890). Baltic, Britain. Yamaguti (1961).

Raphidascaris acus (BToch 1779) Railliet and Henry 1915. USSR. Intestine. Bykhovskaya-Pavlovskaya et al. (1962).

Raphidascaris sp.? Juvenile. England. Chappel1 and Owen (1969).

* Thynnascaris adunca (Rudolphi 1802) Hartwich 1957. Adult, larva. Barents Sea, USSR. Intestine, body cavity, mesentery. Polyanski (1955b) as Contracaecum a.

Contracaecum sp. NewfoundTand, Labrador. Hanek and Threlfall (1970).

Anisakis sp. Larva. Barents Sea, USSR. Body cavity and mesentery. Polyanski (1955b); British Columbia. Arai (1969b).

\section{CAMALLANIDAE}

Cama1lanus lacustris (Zoega 1776) Rai 11 iet and Henry 1915. USSR. Intestine. Bykhovskaya-Pavlovskaya et al. (1962).

Camallanus truncatus (Rudolphi 1814) Railliet and Henry 1915. USSR. Intestine. Bykhovskaya-Pavlovskaya et a1. (1962).

\section{CAPILLARI IDAE}

Hepaticola petruschewskij Schulman 1948. USSR. Liver. Getsevichiute (1958).

\section{CYSTIDICOL IDAE}

Ascarophis morrhuae Van Beneden 1871. Newfoundland, Labrador. Hanek and Threlfall (1970).

Cystidicoloides tenuissima (Zeder 1800) Rasheed 1966. Poland. Wierzbicki (1962) as Sterliadochona $t$.

Cystidicola farionis? Fischer 1798. USSR. Swimbladder. BykhovskayaPavlovskaya et al. (1962). 
DIOCTOPHYMATIDAE

Eustrongvlides sp. Larva. British Columbia. Bangham and Adams (7954).

\section{PHILOMETRIDAE}

Philonema agubernaculum Simon and Simon 1936. Quebec. Viscera. Hanek and Molnar (1974).

PROTOZOA (CILIOPHORA)

\section{AMPHILEPTIDAE}

Litonotus branchiarum (Weinrich 1925). USSR. Gilis, skin. Bykhovskaya-Pavlovskaya et a1. (1962) as Hemiophrys b.

\section{DENDROSOMATIDAE}

Trichophyra intermedia Prost 1952. Neva Gulf, USSR. Gills. Bykhovskaya-Pavlovskaya et a1. (1962).

\section{EPISTYLIDAE}

Epistylis Iwoffi Fauré-Fremiet 1943. British Columbia. Lester (1974); Europe. On Apiosoma [on gills, body surface]. Lom (1966).

\section{OPHRYOGLENIDAE}

Ichthyophthirius multifillis Fouquet 1876. Ireland. Body surface. Kane (1966); British Columbia. Lester (1974). "May be lab infected."

Ichthyophthirius sp. Newfoundland. Threlfal1 (1968).

\section{SCYPHIDIIDAE}

GTossatella amebae (Grenfel1 1887). Neva Gulf, USSR. Body surface, gi17s. Bykhovskaya-Pavlouskaya et al. (1962).

Apiosoma sp. British Columbia. Gills, body surface. Lester (1974).

\section{URCEOLARI IDAE}

Trichodina tenuidens Fauré-Fremiet 1943. Newfoundland. Gills. Lom and Laird (1969); British Columbia. Gill chamber walls. Lester (1974); England. Chubb (1970b).

Trichodina domerguei subsp. domergei Haider 1964. USSR. Poland. Gills, skin. Bykhovskaya-Pavlovskaya et al. (1962); British Columbia. Fins, body surface. Lester (1974).

Trichodina gracilis Polyanski 1955. Barents Sea, USSR. Body surface, gills. Polyanski (1955b).

Trichodina megamicronucleata Dogiel 1950. Ireland. Body surface. Kane $(1966)$.

Trichodina sp. England. Body surface. Chappe11 (1969). 
Gasterosteus aculeatus (Continued)

PROTOZOA (HAPLOSPORIDA)

Dermocystidium gasterostei Elkan 1962. England. Cysts on fins. Elkan $(7962)$.

PROTOZOA (MICROSPORIDA)

NOSEMATIDAE

Glugea anomala (Moniez 1887) Gurley 1893. Europe. Tissues. Putz et a1. (1965); Alaska. Cope (1959); British Columbia. Lester (1974).

PROTOZOA (MYXOSPORIDA)

CERATOMYXIDAE

Ceratomyxa sp. British Columbia. Intestine. Lester (1974). MYXIDIIDAE

Myxidium gasterostei Noble 1943. California. Gallbladder. Noble (1943); USSR. Bykhovskaya-Pavlovskaya et a1. (1962); British Columbia. Lester (1974).

MYXOBILATIDAE

Myxobilatus gasterostei (Parisi 1912) Davis 1944. USSR.

Bykhovskaya-Pav1ovskaya et a1. (1962); British Columbia. Lester (1974). Myxobilatus medius (Thélohan 1892) Gurley 1894. USSR. BykhovskayaPavlovskaya et a?. (1962).

Myxobilatus sp. British Columbia. Kidney tubules Lester (1974).

MYXOBOLIDAE

Myxobolus physophilus Reuss 1906. USSR. Body cavity. Getsevichiute (1958).

Henneguya sp. Great Britain. Kennedy (1974).

SPHAEEROSPORIDAE

Sphaerospora elegans Thëlohan 1892. USSR. Kidneys, Urinary bladder. Bykhovskaya-Pavlovskaya et a1. (1962); British Columbia. Urinary tubules. Lester (1974).

Unicauda brevis (Thélohan 1892). France. Renal tubules, kidneys, ovaries. Kudo and Meglitsch6.

PROTOZOA (SARCOMASTIGOPHORA)

CRYPTOBI IDAE

Cryptobia branchialis (Nie in Chen 1956). USSR. BykhovskayaPavlovskaya et al. (1962). 
Gasterosteus aculeatus (Continued)

Cryptobia salmositica Katz 1951. Washington. Blood. Becker and Katz (1965b).

\section{HEXAMITIDAE}

Hexamita salmonis (Moore 1922). British Columbia. Intestine. Lester (1974).

\section{TETRAMITIDAE}

Ichthyobodo necatrix (Henneguy 1884). British Columbia. Gills. Lester $(1974)$.

\section{TRYPANOSOMATIDAE}

Trypanosoma occidentalis Becker 1967. Washington. Blood. Becker $(1967 \mathrm{~b})$.

PROTOZOA (SPOROZOA)

\section{EIMERI IDAE}

Eimeria gasterostei (Thélohan 1890) Doflein 1909. USSR. Liver. Bykhovskaya-Pavlovskaya et al. (1962); British Columbia. Lester (1974).

$$
\begin{aligned}
& \text { SYNGNATHIDAE -- Pipefishes and Seahorses } \\
& \text { Syngnathus leptorhynchus - Bay Pipefish } \\
& \text { SYNONYM - } \underline{\text { S. griseolineatus }}
\end{aligned}
$$

CESTODA

PHYLLOBOTHRI IDAE

Phyllobothrium sp. Larva. British Columbia. Intestine. Arai $(1969 \mathrm{~b})$.

COPEPODA

PENNELLIDAE

Haemobaphes sp. Juvenile. British Columbia. Gills. Arai (1969b). DIGENEA

HEMIURIDAE

Lecithaster gibbosus (Rudolphi 1802) Lühe 1901. British Columbia. Intestine. Arai (1969b). 
Syngnathus leptorhynchus (Continued)

Parahemiuris merus (Linton 1910) Woolcock 1935. British Columbia. Intestine. Arai (1969b).

Tubulovesicula lindbergi (Layman 1930) Yamaguti 1934. British Columbia. Stomach. Arai (1969b).

\title{
LEPOCREADIIDAE
}

Opechona occidentalis Montgomery 1957. British Columbia. Intestine. Arai (1969b).

OPECOELIDAE

Podocotyle atomon (Rudolphi 1802) Odhner 1905. British Columbia. Intestine. McFarlane (1936).

Podocotyle reflexa (Creplin 1825) Odhner 1905. British Columbia. Intestine. McFarlane (1936).

Podocotyle enophrysi Park 1937. British Columbia. Intestine. Arai $(196 \overline{9 b})$.

Podocotyle sinusacca Ching 1960. British Columbia. Intestine. Arai (1969b) as Neopodocotyloides $\underline{s}$.

MONOGENEA

GYRODACTYLOIDEA

Genera and species. British Columbia. Gills. Arai (1969b). NEMATODA

\section{ANISAKIDAE}

Anisakis sp. Larva. British Columbia. Body cavity. Arai (1969b). Contracaecum sp. Larva. British Columbia. Body cavity. Arai $(1969 b)$.

\author{
PERCICHTHYIDAE -- Temperate Basses \\ Morone saxatilis - Striped Bass \\ SYNONYMS - Labrax lineatus, Roccus lineatus, Roccus saxatil is
}

ACANTHOCEPHALA

ECHINORHYNCHIDAE

Echinorhynchus gadi Zoega in Müiler 1776. Raney (1952).

LEPTORHYNCHOIDIDAE

Leptorhynchoides thecatus (Linton 1891) Kostylew 1924. Hoffman (1967). 
Morone saxatilis (Continued)

POMPHORHYNCHIDAE

Pomphorhynchus rocci Cordonnier and Ward 1967. North Carolina.

Intestine. Cordonnier and Ward (1967).

Pomphorhynchus laevis (Zoega in Muller 1776) Van Cleave 1924. Raney (1952).

BRANCHIURA

ARGULIDAE

Argulus bicolor Bere 1936. Chesapeake Bay. Skin and gills. Paperna and Zwerner (1976).

\section{CESTODA}

DASYRHYNCHIDAE

Callitetrarhynchus gracilis (Rudolphi 1819) Pintner 1931. Cysts in viscera. Raney (1952) as Rhynchobothrium speciosum.

LACISTORHYNCHIDAE

Lacistorhynchus tenuis (Van Beneden 1858) Dollfus 1929. Larva. Massachusetts. Intestine. Linton (1924) as Rhynchobothrium bulbifer.

PROTEOCEPHALIDAE

Genera and species. Larva. Chesapeake Bay. Mesenteries and liver. Paperna and Zwerner (1976).

TETRAPHYLLIDEA

Scolex pleuronectis Rudolphi 1819. Chesapeake Bay. Intestine.

Paperna and Zwerner (1976).

\section{TRYPANORHYNCHA}

Genera and species. Larva. Chesapeake Bay. Mesenteries. Paperna and Zwerner (1976).

COPEPODA

\section{CALIGIDAE}

Caligus sp. Chesapeake Bay. Gills. Paperna and Zwerner (1976). ERGASILIDAE

Ergasilus labracis Krøyer 1863. Virginia. Roberts (1970); Massachusetts. Gills. Wilson (1917).

Ergasilus lizae Kroyyer 1863. Chesapeake Bay. Gills. Paperna and Zwerner (1976).

\section{LERNAEOPODIDAE}

Achtheres lacae Krøyer 1863. Virginia. Wilson (1915). 
Morone saxatilis (Continued)

DIGENEA

ACANTHOCOLPIDAE

Stephanostomum tenue (Linton 1898) Martin 1938. Massachusetts. Linton (1940).

\section{CLINOSTOMATIDAE}

Clinostomum marginatum (Rudolphi 1819). Metacercariae. Chesapeake Bay. Connective tissue and muscle. Paperna and Zwerner (1976).

\section{CRYPTOGONIMIDAE}

Neochasmus sogandaresi Overstreet 1971. Mississippi. Intestine, pyloric caeca. Overstreet (1971a).

\section{DIPLOSTOMATIDAE}

Diplostomum sp. Metacercariae. Chesapeake Bay. Spleen. Paperna and Zwerner (1976).

Neascus sp. Metacercaria. Chesapeake Bay. Skin. Paperna and Zwerner (1976).

\section{LEPOCREADI IDAE}

Lepocreadium cal ifornianumin Edwards and Nahhas 1968. Northern California. Intestine. Edwards and Nahhas (1968).

Lepocreadium setiferoides (Miller and Northup 1926) Martin 1938. Chesapeake Bay. Intestine. Paperna and Zwerner (1976).

Lepocreadium areolatum (Linton 1900) Stunkarrd 1969. Tentative identification. Chesapeake Bay. Intestine. Paperna and Zwerner (1976).

HIRUDINOIDEA

\section{PISCICOLIDAE}

Myzobdella lugubris Leidy 1851. Chesapeake Bay. Paperna and

$$
\text { Zwerner }(1976) \text {. }
$$

ISOPODA

\section{CYMOTHOIDAE}

Aegathoa cf. oculata (Sey 1818). Chesapeake Bay. Skin. Paperna and Zwerner (1976).

Lironeca ovalis (Sey 1818). New York. Inside of operculums. Alperin (1966).

MOLLLUSCA

Genera and Species. Glochidia. Chesapeake Bay. Gills. Paperna and and Zwerner (1976). 
Morone saxatilis (Continued)

MONOGENEA

CAPSALIDAE

Benedenia mellani (MacCallum 1927) Johnston 1929. Raney (1952) as Epibdella $\mathrm{m}$.

DACTYLOGYRIDAE

Aristocleidus hastatus Mueller 1936. Florida. Gills. Yamaguti (1963b).

Cleidodiscus pricei Mueller 1936. Northern California. Gills. Hensley and Nahhas (1975).

DIPLECTANIDAE

Diplectanum collinsi (Mueller 1936) Price 1937. Florida. Yamaguti (1963b).

\section{GASTROCOTYLIDAE}

Lithidocotyle acanthophallus (MacCallum and MacCallum 1913)

Sproston 1946. New York. Hargis (1956c).

GYRODACTYLIDAE

Gyrodactylus sp. Chesapeake Bay. Gilis. Paperna and Zwerner (1976). MICROCOTYLIDAE

Microcotyle eueides MacCallum and MacCallum 1913. Atlantic (USA). Yamaguti (7963b).

Microcotyle macroura MacCallum and MacCallum 1913. Chesapeake Bay. Gills. Paperna and Zwerner (1976).

NEMATODA

ANISAKIDAE

Contracaecum brachyurum (Ward and Magath 1917). Northern California. Intestine, caeca. Hensley and Nahhas (1975).

Goezia sp. Larva. Chesapeake Bay. Intestine. Paperna and Zwerner (1976).

Goezia annulata (Molin 1859). Raney (1952).

CYSTIDICOLIDAE

Benedenia melleni (MacCallum 1927) Johnston 1929. Raney (1952) as Epibdella m.

CUCULLANIIDAE

Cucullanus sp. Chesapeake Bay. Intestine. Paperna and Zwerner (1976). 
Morone saxatilis (Continued)

GNATHOSTOMATIDAE

Spiroxys sp. Larva. Northern California. Intestine, stomach, caeca, mesentery. Hensley and Nahhas (1975).

PHILOMETRIDAE

Philometra sp. Chesapeake Bay. Body cavity. Bier et al. (1974). Philometra rubra (Leidy 1856). Chesapeake Bay. Intestine. Paperna and Zwerner (1976).

PROTOZOA (CILIOPHORA)

DENDROSOMATIDAE

Trichophyra sp. Chesapeake Bay. Gills. Paperna and Zwerner (1976). EPISTYLIDAE

Epistylis sp. Chesapeake Bay. Gills. Paperna and Zwerner (1976). SCYPHIDIIDAE

Glossatella sp. Chesapeake Bay. Gills. Paperna and Zwerner (1976). Scyphidia sp. Chesapeake Bay. Gills. Paperna and Zwerner (1976). URCEOLARI IDAE

Trichodina davisi Wellborn 1967. North Carolina. Fins, body surface, gills. Wellborn (1967).

Trichodinella sp. Chesapeake Bay. Skin and gills. Paperna and Zwerner (1976).

PROTOZOA (MICROSPORIDIA)

NOSEMATI IDAE

Nosema sp. Chesapeake Bay. Gills. Paperna and Zwerner (1976). PROTOZOA (MYXOSPORIDA)

MYXOSOMATIDAE

Myxosoma sp. Chesapeake Bay. Cartilage, bones. Paperna and Zwerner (1976).

TETRACAPSULIDAE

Kudoa cerebral is Paperna and Zwerner 1974. Chesapeake Bay. Connective tissue of nervous tissue. Paperna and Zwerner (1974). 
Morone saxatilis (Continued)

PROTOZOA (SARCOMASTIGOPHORA)

BLÁSTODDINIIDÁE

Oodinium sp. Chesapeake Bay. Gills. Paperna and Zwerner (1976).

BODINIDAE

Colponema sp. Chesapeake Bay. Gills. Paperna and Zwerner (1976).

$$
\text { Stereolepis gigas - Giant Sea Bass }
$$

CESTODA

DASYRHYNCHIDAE

Callitetrarhynchus gracilis (Rudolphi 1819) Pintner 1931. Larva. Southern California. Viscera. Jensen ${ }^{18}$.

COPEPODA

\section{CAL IGIDAE}

Lepeophtheirus longipes Wilson 1905. Southern California. Exterior. WiTson (1921).

ISOPODA

\section{CYMOTHOIDAE}

Nerocila californica Schioedte and Meinert 1881. Baja California. Gills. Brusca (1977). Speculative host.

MONOGENEA

\section{DIPLECTANIDAE}

Diplectanum americanum Price 1937. West coast of Mexico. Gills. Caballero and Bravo-Hollis (1961).

$$
\text { SERRANIDAE -- Sea Basses }
$$

Epinephelus analogus - Spotted Cabrilla 
Epinephelus analogus (Continued)

DIGENEA

BUCEPHALIDAE

Prosorhynchus gonoderus Manter 1940. Pacific Panama. Intestine. Sogandares-Bernal (1959).

Prosorhynchus ozakii Manter 1934. Pacific Panama. Intestine. Sogandares-Bernat (1959).

\section{LEPOCREADIIDAE}

Lepidapedon epinepheli Bravo-Holl is and Manter 1957. West coast of Mexico. PyToric caeca, intestine. Bravo-Hollis and Manter (1957). Lepidapedon hancocki Manter 1940. West coast of Mexico. Intestine. Yamaguti (1971).

\section{OPECOELIDAE}

Helicometrina nimia Linton 1910. Pacific Panama. Pyloric caeca. Sogandares-Bernat (1959).

MONOGENEA

\section{CAPSALIDAE}

Trochopus pseudomarginatus Bravo-Hollis 1957 . West coast of Mexico. Gills. Bravo-Hollis (1957).

$$
\text { Epinephelus niveatus - Snowy Grouper }
$$

DIGENEA

ACANTHOCOLPIDAE

Stephanostomum microstephanum Manter 1934. Florida. Intestine. Manter (1934).

BUCEPHALIDAE

Prosorhynchus ozakii Manter 1934. Tortugas; Florida. Intestine. Manter (1934).

LEPOCREADI IDAE

Lepidapedon nicol1i Manter 1934. Florida. Intestine. Yamaguti (1971). 


\section{Hemanthias peruanus - Splittail Bass}

COPEPODA

CHONDRACANTHIDAE

Blias prionoti Krøyer 1863. Gills. Ho (1970).

Mycteroperca sp.

Manter and Van Cleave (1951) reported examining Mycteroperca pardal is from La Jolla, California. Except for this record, M. pardalis has not been recorded from California, and we feel this record is in question. We therefore designate the fish Mycteroperca $\mathrm{sp}$. for the time being.

\section{DIGENEA}

LEPOCREADIIDAE

Lepidapedon sp. Southern California. Manter and Van Cleave (1951).

$$
\text { Mycteroperca xenarcha - Broomtail Grouper }
$$

COPEPODA

\section{CALIGIDAE}

Lepeophtheirus dissimulatus Wilson 1905. Albermarle Islands. Skin. WiTson $(1937 a)$.

\section{DIGENEA}

BUCEPHAL IDAE

Bucephalus heterotentaculatus Bravo-Hollis and Sogandares-Bernal 1956. Baja California. Intestine. Arai (1962).

Prosorhynchus ozakii Manter 1934. Galapagos Islands. Caeca. Manter (1940a).

Prosorhynchus pacificum Manter 1940. Galapagos Islands. Caeca. Manter (1940a).

\section{LEPOCREADI IDAE}

Lepidapedon hancocki Manter 1940. Galapagos Islands. Intestine. Manter (19 $90 a)$.

\section{OPECOELIDAE}

Hamacreadium mutabile Linton 1910. Galapagos Islands. Intestine. Yamaguti (1971). 
Mycteroperca xenarcha (Continued)

ISOPODA

CYMOTHOIDAE

Nerocila californica Schioedte and Meinert 1881. Baja California. Gi17s. Brusca (1977). Speculative host.

$$
\text { Paralabrax clathratus - Kelp Bass }
$$

COPEPODA

BOMOLOCHIDAE

*Bomolochus solae Claus 1864. Baja California. Causey (1960). Holobomolochus Tongicaudus (Cressey 1969) Kabata 1971. Southern California. Gill cavity. Cressey (1969b).

Taeniastrotos californiensis Cressey 1969. Southern California. Schultze 25 .

\section{CALIGIDAE}

Lepeophtheirus constrictus Wilson 1908. Southern California. Schuttze 25

Lepeophtheirus longipes Wilson 1905. Southern California. Body surface. Wil son (1921).

Caligus productus Dana 1853. Baja California. Causey (1960).

Caligus mutabilis Wilson 1905. Gulf of California. Causey (1960); Southern California. Schultze 25 .

HATSCHEKIIDAE

Hatschekia albirubra Wilson 1913. Baja California. Causey (1960). Hatschekia pacifica Cressey 1970. Southern California. Schultze 25. LERNAEOPODIDAE

Clavella parva Wilson 1912. Southern California. Schultze 25. PENNELLIDAE

Peniculus fissipes Wilson 1917. Southern California. Schultze 25.

\section{DIGENEA}

\section{HEMIURIDAE}

Elytrophallus mexicanus Manter 1940. Southern California. Intestine. Manter and van Cleave (1951).

\section{MONORCHI IDAE}

Monorcheides alexanderi Arai 1962. Guadalupe Island, Mexico. Pyloric caeca. Arai (1962). 
Paralabrax clathratus (Continued)

OPECOELIDAE

Helicometrina nimia Linton 1910. Intestine. Arai (1962).

Plagioporus issaitschikowi (Layman 1930) Yamaguti 1938. Southern

California. Intestine. Manter and Van Cleave (1951).

ISOPODA

CYMOTHOIDAE

Nerocila californica Schioedte and Meinert 1881. California. Brusca (1977). Speculative host.

MONOGENEA

MACROVALVITREMATIDAE

Pseudotagia clathratus Crane 1972. Southern California. Gills. Crane $(1972)$.

NEMATODA

ANISAKIDAE

Anisakis sp. Larva. Southern California. Viscera. Dailey et al. (in press).

Phocanema sp. Larva. Southern California. Viscera. Dailey et al.

(in press).

SPIRURIDAE

Dollfusnema piscicola Caballero 1974. Baja California. Cabal7ero (1974).

Paralabrax maculatofasciatus - Spotted Sand Bass

COPEPODA

BOMOLOCHIDAE

Holobomolochus longicaudus (Cressey 1969) Kabata 1971. Southern California. Schultze 25 .

Taeniastrotos californiensis Cressey 1969. Southern California. Schultze25.

\section{CALIGIDAE}

Sciaenophilus bennetti (Causey 1953) Yamaguti 1963. Baja California. Mouth. Causey (1960) as Caligus b.

Lepeophtheirus constrictus Wilson 1908 . Southern California. Body surface. Wilson (1908a). 
Paralabrax maculatofasciatus (Continued)

Caligus productus Dana 1853. Gulf of California. Causey (1960). Caligus mutabilis Wilson 1905. Gulf of California. Causey (1960); Southern California. Schultze 25 .

\section{HATSCHEKI IDAE}

Hatschekia pacifica Cressey 1970. Southern California. Schultze 25 .

\section{LERNAEOPODIDAE}

Clavella parva Wilson 1912. Southern California. Schultze25. PSEUDOCYCNIDAE

Pseudocycnus appendiculatus Heller 1865. Gulf of California. Causey $(1960)$.

\section{DIGENEA}

\section{HEMIURIDAE}

Dinurus barbatus (Cohn 1902) Looss 1907. Baja California. Intestine. Arai (1962).

Magniscyphus taboganus (Sogandares-Bernal 1959) Reid, Coil and Kuntz 1965. Baja California. Intestine. Arai (1962) as Sterrhurus taboganus.

OPECOELIDAE

Opecoelus mexicanus Manter 1940. Baja California. Intestine. Arai (1962).

Helicometrina nimia Linton 1910. Intestine. Arai (1962).

Helicometrina fasciata (Rudolphi 1819) Odhner 1902. Baja California. Intestine. Arai (1962).

Helicometra pretiosa Bravo-Hollis and Manter 1957. West coast of Mexico. Intestine. Bravo-Holl is and Manter (1957).

Dactylostomum winteri Caballero and Caballero 1971. Baja California. Intestine. Cabal7ero and Caballero (1971a).

Hamacreadium bolivari Caballero and Caballero 1970. Gulf of

California. Intestine. Caballero and Caballero (1970).

MONOGENEA

CAPSALIDAE

Benedenia melleni (MacCallum 1927) Johnston 1929. New York Aquarium. Eyes, skin. Sproston (1946).

DIPLECTANIDAE

Diplectanum amplidiscatum Bravo-Hollis 1954. West coast of Mexico. Gills. Bravo-Hollis (1954a). 
COPEPODA

BOMOLOCHIOAE

Taeniastrotos californiensis Cressey 1969. Southern California. Body surface. Cressey (1969b).

Holobomolochus longicaudus (Cressey 1969) Kabata 1971. Southern

California. Gill cavity. Cressey (1969b).

* Bomolochus solae Claus 1864. Baja California. Causey (1960).

\section{CALIGIDAE}

$\frac{\text { Lepeophtheirus }}{\text { Schultze25 }}$ constrictus Wilson 1908. Southern California. Lepeophtheirus dissimulatus WiIson 1905. Baja California. Causey $(1960)$.

Caligus mutabilis Wilson 1905. Gulf of California. Causey (1960); Southern California. Schultze 25 .

HATSCHEKIIDAE

Hatschekia pacifica Cressey 1970. Southern California. Schultze25. LERNAEOPODIDAE

Clavella parva Wilson 1912. Southern California. Schultze25. PENNELLIDAE

Peniculus fissipes Wilson 1917. Pacific coast of Baja California. Fins. Schultze 25 .

\section{DIGENEA}

\section{OPECOELIDAE}

Opecoelus inimici Yamaguti 1934. Cerros Island, Mexico. Intestine. Manter (1940a).

Helicometrina nimia Linton 1910. Cerros Island, Mexico. Intestine. Manter (1940a).

ISOPODA

CYMOTHOIDAE

$\frac{\text { Lironeca vulgaris Stimpson 1857. Southern California. Turner }}{\text { et al. }}$ NEMATODA

\section{ANISAKIDAE}

Anisakis sp. Southern California. Dailey et al. (in press). Phocanema sp. Southern California. Dailey et al. (in press). 
Paralabrax nebulifer (Continued)

PROTOZOA (CILIOPHORA)

OPHRYOGLENIDAE

Cryptocaryon irritans Brown 1951. New York Aquarium. Skin, gills, and eyes. NigrelTi and Ruggieri (1966).

BRANCHIOSTEGIDAE -- Tilefishes

Caulolatilus princeps - Ocean Whitefish

COPEPODA

LERNAEOPODIDAE

Neobrachiella gracilis (Wilson 1908) Kabata 1978. Baja California. Causey (1960) as Brachiella $\mathrm{g}$.

DIGENEA

HOMALOMETRIDAE

Myzotus vitellosus Manter 1940. Galapagos Islands. Intestine. Manter (1940a) probably from $\underline{C}$. princeps.

MONOGENEA

DICLIDOPHORIDAE

Choricotyle caulolatili (Meserve 1938) Sproston 1946. Galapagos

Islands. Gil1s. Meserve (1938) as Diclidophora c.

PROTOZOA (MYXOSPORIDA)

CERATOMYXIDAE

Ceratomyxa sp. Southern California. Gallbladder. Jensen ${ }^{18}$.

\author{
ECHENEIDAE -- Remoras \\ Echeneis naucrates - Sharksucker
}

ACANTHOCEPHALA

RHADINORHYNCHIDAE

Rhadinorhynchus tenuicornis (Linton 1891) Van Cleave 1918.

Massachusetts. Cable and Linderoth (1963). 
Echeneis naucrates (Continued)

Gorgorhynchus medius (Linton 1908) Chandler 1934. Parukhin (1970) as G. gibberum.

Serrasentis sagittifer (Linton 1889) Linton 1932. South China Sea. Parukhin (1967) as $\underline{\text { s. }}$ socialis.

BRANCHIURA

ARGULIDAE

Argulus varians Bere 1936. Gulf of Mexico. Bere (1936).

\section{CESTODA}

TENTACULAR IIDAE

Nybelinia robusta (Linton 1897) Dollfus 1930. Larva. Massachusetts. Stomach. Linton (1924) as Tetrarhynchus $\underline{r}$.

Tentacularia coryphaenae Bosc 1802 . Postlarva. Massachusetts.

Encapsulated in stomach, mesentery. Linton (1924) as

Tetrarhynchus bicolor.

COPEPODA

\section{CALIGIDAE}

Lepeophtheirus longipes Wilson 1905. Africa. Barnard (1955).

Tuxophorus caligodes Wilson 1908. North Carolina. Wilson (1908b); Gulf of Mexico. Exterior. Bere (1936).

Caligus praetextus Bere 1936. Gulf of Mexico. Mouth, branchial cavity, outer surface. Bere (1936) from "Sucking fish", Tikely

E. naucrates.

DIGENEA

ACANTHOCOLPIDAE

Tormopsolus echenei Parukhin 1966. Gulf of Tonkin. Intestine:

Parukhin (1966).

Stephanostomum imparispine (Linton 1905) Manter 1940. South China Sea. Parukhin (1967).

DIDYMOZOIDAE

Didymozoides sp. Larva. Red Sea. Gill cavity. Nikolaeva (1965). HEMIURIDAE

Tubulovesicula lindbergi (Layman 1930) Yamaguti 1934. South China Sea. Parukhin (1967).

Sterrhurus floridens is Manter 1934. Tortugas: Florida. Stomach. Manter (1934).

Lecithochirium montice11ii (Linton 1898) Crowcroft 1946.

Massachusetts. Stomach. Linton (1940) as Sterrhurus $\mathrm{m}$. 
Echeneis naucrates (Continued)

Parahemiurus merus (Linton 1910) Woolcock 1935. Jamaica. Stomach. Nahhas and Cable (1964).

LEPOCREADI IDAE

Echeneidocoelium indicum Simha and Pershad 1964. India. Intestine. Madhavi (1970).

MONOGENEA

DIONCIDAE

Dioncus remorae (MacCa11um 1916) Price 1938. New York Aquarium. Gills. MacCallum (1916) as Acanthodiscus remorae; West Indies. Johnston and Tiegs (1922) as Dionchotrema $\frac{r}{r}$.; Australia. Young (1970); South Atlantic. Parukhin (1968).

Dioncus agassizi Goto $1899 . \quad$ India. Gills. Rao and Madhavi (1967).

NEMATODA

ANISAKIDAE

Raphidascaris sp. South China Sea. Parukhin (1967).

CAMALLANIDAE

Spirocamallanus olseni Campana-Rouget and Razarihelissoa 1965. Sea of Nossi-Bé. Stomach. Campana-Rouget and Razarihelissoa (7965).

CAPILLARI IDAE

Capillaria echenei Parukhin 1967. South China Sea. Parukhin (1967). CYSTIDICOLIDAE

Ascarophis sp. South China Sea. Parukhin (1967).

Spinitectus echenei Parukhin 1967. South China Sea. Parukhin (1967).

Remora australis - Wha lesucker

COPEPODA

CALIGIDAE

Lepeophtheirus crassus (Wilson and Bere 1936) Shiino 1960.

Pacific. Skin. Shiino (1963a). 


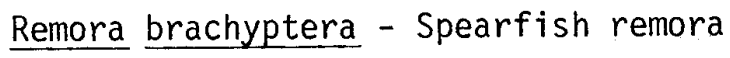

MONOGENEA

DIONCIDĀE

Dioncus agassizi Goto 1899. Rhode Island, Massachusetts. Gills. Yamaguti (1963b).

$$
\begin{aligned}
& \text { Remora remora - Remora } \\
& \text { SYNONYM - Echeneis remora }
\end{aligned}
$$

CESTODA

BOTHRIOCEPHAL IDAE

Bothriocephalus sp. Plerocercoid. Massachusetts. Linton (1941).

DASYRHYNCHIDAE

Callitetrarhynchus gracilis (Rudolphi 1819) Pintner 1931.

Plerocercoid. Massachusetts. Peritoneum. Dollfus (1942).

TENTACULARI IDAE

Nybelinia robusta (Linton 1891 ) Dollfus 1930. Larva. France.

Linton (1891) as Tetrarhynchus robustum.

Nybelinia bisulcata (Linton 1889) Dol1fus 1929. Larva. Massachusetts.

Linton (1924) as Tetrarhynchus b.

TETRAPHYLLIDEA

Scolex polymorphus Rudolphi 1819. Massachusetts. Linton (1924).

COPEPODA

\section{CALIGIDAE}

Caligus elongatus Nordmann 1832. New England. External surface. Wilson (1905b) as C. rapax. Lepeophtheirus crassus (Wilson and Bere 1936) Shiino 1960.

Indian Ocean. External surface. Lewis et àl. (1969).

PANDARIDAE

* Perissopus oblongatus (Wilson 1908) Cressey 1967. Atlantic.

Vilson (1911) as Achtheinus dentatus.

PENNELLIDAE

Pennella remorae Murray 1856. New Zealand. Heegaard (1962); Atlantic, Mediterranean. In flesh. Brian (1906). 
Remora remora (Continued)

DIGENEA

DIDYMOZOIDAE

Genera and species. Larva. Massachusetts. Linton (1940) as Distoma fenestratum.

HEMIURIDAE

Brachyphallus crenatus (Rudolphi 1802) Odhner 1905. Massachusetts. Linton $(1940)$.

Lecithochirium monticellii (Linton 1898) Crowcroft 1946.

Massachusetts. Stomachs. Linton (1940) as Sterrhurus monticelli.

LEPOCREADIIDAE

Echeneidocoelium indicum Simha and Pershad 1964. India. Intestine. Simha and Pershad (1964).

MONOGENEA

DIONCIDAE

Dionchus agassizi Goto 1899. Massachusetts. Gi11s. Linton (1940).

$$
\begin{aligned}
& \text { CARANGIDAE -- Jacks and Pompanos } \\
& \text { Caranx caballus - Green Jack } \\
& \text { SYNONYM - Paratractus caballus }
\end{aligned}
$$

\section{DIGENEA}

ACANTHOCOLPIDAE

Stephanostomum filiforme Linton 1940. Massachusetts. Linton (1940). FELLODISTOMATIDAE

Tergestia laticollis (Rudolphi 1819) Stossich 1899. Costa Rica. Intestine. Manter (1940a).

\section{MONOGENEA}

\section{HETERAXINIDAE}

Cemocotyle borinquenensis Price 1962. Puerto Rico. Yamaguti (1963b). 
ACANTHOCEPHALA

RHADINORHYNCHIDAE

Gorgorhynchoides sp. Florida. Cable and Linderoth (1963).

CESTODA

TENTACULARI IDAE

Nybelinia bisulcata (Linton 1889) Dollfus 1929. Larva. North Carolina. Cysts on sides of stomach. Dollfus (1942).

TRYPANORHYNCHA

Rhynchobothrius carangis MacCallum 1921. USA. Yamaguti (1959b). [Species Incertae sedis - Yamaguti (1959b).]

\section{COPEPODA}

CALIGIDAE

Caligus coryphaenae Steenstrup and Lütken 1861. Texas.. Gills. Causey (1953b) as C. aliuncus.

Caligus constrictus Heller 1865. Panama. Exterior. Wilson

(1937a); Mexico. Causey (1960).

Caligus praetextus Bere 1936. Louisiana. Causey (1953a).

Caligus rectus Pearse 1952. Texas. Exterior. Pearse (1952).

CaTigus spinosurculus Pearse 1951. Bahama Islands. Exterior. Pearse (1951).

Caligus tenax Heller 1865. Texas. Gills. Causey (1953b).

Caligus diaphanus Nordmann 1832. Panama Bay. Exterior. Wilson $(1937 a)$.

Caligus elongatus Nordmann 1832. Texas. Gills. Causey (1953b) as C. rapax.

Caligus confusus Pillai 1961. Panama. Wilson (1937a) as

C. constrictus.

CaTigus robustus Bassett-Smith 1898. Gulf of Mexico. Body surface, branchial cavity. Bere (1936).

Lepeophtheirus edwardsi Wilson 1905. Massachusetts.

External surface. Wilson (1905a).

Anuretes heckelij (Kollar in Kroyer 1863) Heller 1865. North America. Yamaguti (1963a).

Dentigryps longicauda Cressey 1966. Madagascar. Body surface. Cressey $(1966)$.

HATSCHEKIIDAE

Hatschekia oblonga Wilson 1913. Panama. Gills. Wilson (1937a). 
Caranx hippos (Continued)

LERNANTHROPIDAE

Lernanthropus giganteus Krøyer 1863. Texas. Pearse (1952); West Africa. Delamare - Deboutteville and Nunes - Ruivo (1954).

\section{PANDARIDAE}

Pandarus sinuatus Say 1817. Texas. Body surface. Causey (1953b). PENNELLIDAE

Lernaeenicus longiventris Wilson 1917. Western North Atlantic. Flesh. Wilson (1917); Texas. Causey (1953b); Panama Bay. Wilson (1937a).

\section{DIGENEA}

\section{ACANTHOCOLPIDAE}

Stephanostomum ditrematis (Yamaguti 1939) Manter 1947. Florida. Intestine. Nahhas and Short (1965); Linton (1940) as $\underline{S}$. filiforme. Stephanostomum longisomum Manter 1940. Ecuador, Mexico. Intestine. Manter (1940a).

Stephanostomum megacephalum Manter 1940. Panama, Ecuador, Mexico. Gills, intestine. Manter (1940a); Louisiana. Sparks (1958). Stephanostomum hispidum (Yamaguti 1934) Manter 1940. Colima, Mexico. Intestine. Bravo-Holl is (1954b).

Manteria brachyderus (Manter 1940) Caballero 1950. Ecuador. Intestine. Cabal1ero (1950).

\section{BUCEPHALIDAE}

Prosorhynchoides gracilescens (Rudolphi 1819) n. comb. North Carolina. Linton (1905) as Gasterostomum $g$.

Prosorhynchoides arcuata (Linton T900) n. comb. North Carolina. Linton (1905) as Gasterostomum arcuatum.

Bucephalus introverus Manter 1940. Columbia. Stomach. Manter (1940a).

Bucephalus varicus Manter 1940. West coast of Mexico. Intestine. Bravo-Hol is and Sogandares-Bernal (1956); Florida. SogandaresBernal and Hutton (1959).

\section{FELLODISTOMATIDAE}

Tergestia pectinata (Linton 1905) Manter 1940. Jamaica-Curacao. Nahhas and Cable (1964); Florida. Intestine. Nahhas and Short (1965).

Monascus typicus (Odhner 1911) Yamaguti 1954. West coast of Mexico. Intestine. Lamothe-Argumedo (1969a). 
Caranx hippos (Continued)

HEMIURIDAE

Sterrhurus zeloticus (Travassos, Freitas and Bührnheim 1966)

Yamaguti 1971. Brazi1. Stomach. Travassos et a1. (1966) as Separogermiductus z.

Sterrhurus musculus Looss 1907. Florida. Stomach. Overstreet $(1.969)$.

Ectenurus virgulus Linton 1910. Jamaica- Curacao. Intestine. Nahhas and Cable (1964); Florida. Overstreet (1969).

Ectenurus americanus (Manter 1947) Manter and Pritchard 1960. Jamaica-Curacao. Stomach. Nahhas and Cable (1964).

Ectenurus yamagutii Nahhas and Powe11 1971. Florida. Stomach. Nahhas and Powe11 (1971).

Parahemiurus merus (Linton 1910) Woolcock 1935. Jamaica-Curacao. Stomach. Nahhas and Cable (1964); Florida. Overstreet (1969). Lecithochirium parvum Manter 1947. Curacao-Jamaica. Stomach. Nahhas and Cable (1964).

OPECOELIDAE

Pseudopecoeloides carangis (Yamaguti 1938) Yamaguti 1940. Mexican Pacific. Intestine. Yamaguti (1938b) as Cymbephallus carangi; West coast of Mexico. Bravo-Hollis (1954b).

Alloanomalotrema ghanens is (Fischthal and Thomas 1970) Yamaguti 1971. Ghana. Sma11 intestine. Fischthal and Thomas (1970b) as Poracanthium $\mathrm{g}$.

ISOPODA

CYMOTHOIDAE

Lironeca ovalis (Sey 1818). Long Island Sound, USA. Gills. Briggs (1970).

MONOGENEA

ALLOPYRAGRAPHORIDAE

Allopyragraphorus hippos (Hargis 1956) Yamaguti 1963. Gulf of Mexico. Gills. Hargis (1956a) as Pyragraphorus $h$.

DIONCIDAE

Dioncus remorae (MacCallum 1916) Price 1938. New York Aquarium. Gi17s. Hargis (1955c).

\section{DISCOCOTYLIDAE}

Allodiscocotyla mexicana Caballero and Bravo-Hollis 1963 . West coast of Mexico. Gills. Caballero and Bravo-Hollis (1963). [In Allodiscotylidae - Caballero and Bravo-Hollis (1963).] 
Vallisia riojai Caballero and Bravo-Hollis 1963. West coast of Mexico. Gills. Caballero and Bravo-Hollis (1963). [In Anthocotylidae - Caballero and Bravo-Hollis (1963).]

GASTROCOTYLIDAE

Pseudomazocraes monsivaisae Caballero and Bravo-Hollis 1955. West coast of Mexico. Caballero and Bravo-Hollis (1955).

\section{HETERAXINIDAE}

Helixaxine winteri Caballero and Bravo-Hollis 1965. Caribbean Mexico. Gills. Caballero and Bravo-Hollis (1967).

Zeuxapta seriolae (Meserve 1938) Price 1962. Mexico. LamotheÁrgumedo (1970).

Cemocotyle noveboracensis Price 1962. New York. Gills. Price (1962b); Caribbean Mexico. Caballero and Bravo-Hollis (1967).

\section{MICROCOTYLIDAE}

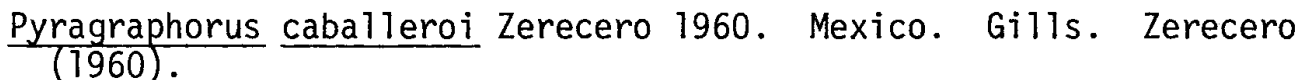

\section{PROTOMICROCOTYLIDAE}

Abortipedia indica Unnithan 1962. India. Gills. Unnithan (1962). Protomicrocotyle manteri Bravo-Holi is 1967. Mexican Gulf of Mexico. Gills. Caballero and Bravo-Hollis (1967).

Protomicrocotyle mirabilis (MacCal1um 1918) Johnston and Tiegs 1922. Florida. Hargis (1957).

NEMATODA

\section{CUCULLANIDAE}

Cucullanus carangis (MacCallum 1921) Tornquist 1931. New York Aquarium. Intestine. Yamaguti (1961).

PROTOZOA (SPOROZOA)

HAEMOGREGARINIDAE

Haemogregarina bigemina Laveran and Mesnil 1901. Puerto Rico. Blood. Saunders (1966).

$$
\text { Chloroscombrus orqueta - Pacific Bumper }
$$

ISOPODA

CYMOTHOIDĀE

Lironeca convexa Richardson 1905. Pacific. Mouth, gill chambers. Menzies et al. (1955). 
ACANTHOCEPHALA

RHADINORHYNCHIDAE

Serrasentis lamelliger (Diesing 1854) Meyer 1932. Yamaguti (1963C). CESTODA

\section{TENTACULARI IDAE}

Nybelinia lingualis (Cuvier 1817) Dollfus 1929. France. Joyeux and Baer (1936).

COPEPODA

CALIGIDAE

Caligus productus Dana 1853. Indian 0cean. Lewis et a1. (1969); Puerto Rico. Exterior, gills. Wilson (1935a) as $\underline{\text { C. lobatus. }}$

DIGENEA

ACANTHOCOLPIDAE

Stephanostomum naucrotis Nagaty 1957. Red Sea. Nagaty (1957). ACCACOEL I IDAE

Genera and species. Metacercaria. Mediterranean. Nikolaeva (1968a). HIRUDINELLIDAE

Hirudinella ventricosa (Pallas 1774) Baird 1853. Canary Islands. Gibson (1976).

MONOGENEA

CAPSAL IDAE

Ancyrocotyle vallei (Parona and Perugia 1895) Parona and Monticelli 1903. Mediterranean. Gi11s. Dawes (1947).

Ancyrocotyle bartschi Price 1934. Puerto Rico Deep. Gills. Price $(1934)$.

Benedenia melleni (MacCallum 1927) Johnston 1929. New York Aquarium. Eyes, skin. Sproston (1946).

Nematistius pectoralis - Roosterfish

COPEPODA

CALIGIDAE

Caligus tenuifurcatus Wilson 1937. Panama Bay. Gill cavity. Wilson (1937a). 
CESTODA

BOTHRIOCEPHALIDAE

Bothriocephàlus scorpii (Müli Ter 1776) Rudo7phi 1808 . Massachusetts. Linton (1947).

COPEPODA

CALIGIDAE

Caligus bonito Wilson 1905. Gulf of Mexico. Body surface. Bere (1936).

\section{DIGENEA}

ACANTHOCOLPIDAE

Stephanostomum sp. Massachusetts. Linton (1940).

Manteria brachydera (Manter 1940) Caballero 1950. Florida. Hutton (1964); Ecuador. Intestine. Manter (1940a) as Dihemistephanus $\underline{b}$.

\section{FELLODISTOMATIDAE}

Tergestia pectinata (Linton 1905) Manter 1940. Jamaica. Intestine. Nahhas and Cable (1964).

HEMIURIDAE

Lecithochirium monticelli (Linton 1898) Crowcroft 1946. Massachusetts. Stomach. Linton (1940) as Sterrhurus m. Lecithochirium microstomum Chandler 1935. Florida. Stomach. Overstreet (1969).

Mecoderus oligoplitus Manter 1940. Ecuador. Stomach. Manter (1940a).

Ectenurus lepidus Looss 1907. Brazil. Intestine. Travassos

$$
\text { et â. (1969). }
$$

MONOGENEA

DISCOTYLIDAE

Vallisia oligoplites Hargis 1957. Florida. Gills. Hargis (1957). HETERAXINIDAE

Heteraxinoides oligoplitis (Meserve 1938) Price 1962. Ecuador. Gi17s. Meserve (1938) as Axine o. 
CESTODA

DASYRHYNCHIDÃE

Callitetrarhynchus gracil is (Rudolphi 1819) Pintner 1931. Larva. Southern California. Viscera. Jensen ${ }^{18}$.

COPEPODA

CALIGIDAE

Caligus productus Dana 1853. Baja California. Causey (1960). Caligus chorinemi Kollar in Krøyer 1863. Columbia; Costa Rica. WiTson (1937a).

Caligus tenuicaudatus Shi ino 1959. Baja California. Exterior. Shiino (1959a).

Caligus lunatus Wilson 1928. Panama. Gills. Wilson (1937a).

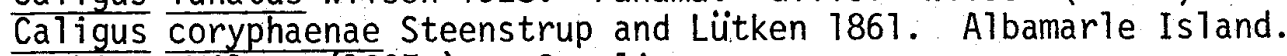

Skin. Wilson (1937a) as $\underline{C}$. aliuncus.

PENNELLIDAE

Lernaeenicus longiventris Wilson 1917. West coast of Mexico. Causey $(1960)$.

\section{DIGENEA}

ACANTHOCOLPIDAE

Stephanostomum hispidum (Yamaguti 1934) Manter 1940. Mexico; Panama. Intestine. Manter (1940a).

BUCEPHALIDAE

Bucephalus introversus Manter 1940. Columbia; Mexico. Stomach. Manter (1940a).

HEMIURIDAE

Lecithochirium magnaporum Manter 1940. Galapagos. Stomach. Manter (1940a).

\section{MONOGENEA}

HETERAXINIDAE

Zeuxapta seriolae (Meserve 1938) Price 1962. Galapagos Island. Gil1s. Meserve (1938) as Axine seriolae. 


\section{Trachinotus rhodopus - Gafftopsail Pompano}

\section{DIGENEA}

ÁCCÁCOELIIIDÁE

Tetrochetus proctocolus Manter 1940. Galapagos Is 1ands. Rectum. Manter (1940a).

HEMIURIDAE

Gonocercella pacifica Manter 1940. Columbia. Stomach. Manter (1940a).

Lecithophyllum trachinoti (Manter 1940) Yamaguti 1971. Mexico. Manter (1940a) as Aponurus $t$.

LEPOCREADIIDAE

Opechona pharyngodactyla Manter 1940. Mexico. Intestine. Manter $(1940 a)$.

OPECOELIDAE

Opegaster parapristipomatis Yamaguti 1938. Galapagos Islands. Digestive tract. Yamaguti (1938).

$$
\text { Trachurus symmetricus - Jack Mackere1 }
$$

\section{ACANTHOCEPHALA}

RHADINORHYNCHIDAE

Rhadinorhynchus sp. California. Intestine. Dailey (1969a).

\section{CESTODA}

\section{DASYRHYNCHIDAE}

Dasyrhynchus sp. Plerocercus. California. Abdominal wall tissue. Dailey (1969a).

\section{TETRAPHYLLIDEA}

Scolex pleuronectis Müller 1788. California. Intestine, caeca, body cavity. Dailey (1969a).

Scolex polymorphus Rudolphi 1819. California. Intestine, caeca, body cavity. Dailey (1969a).

COPEPODA

CALIGIDAE

Caligus pelamydis Krøyer 1863. Southern California. Body surface. Shiino (1965b). 
Trachurus symmetricus (Continued)

DIGENEA

HEMIURIDAE

Lecithochirum magnaporum Manter 1940. California. Body cavity. Dailey (1969a).

OPECOELIDAE

Opegaster sp. California. Intestine. Dailey (1969a).

ISOPODA

CYMOTHOIDAE

Lironeca ovalis Stimpson 1857. California. Turner et al. (1969). NEMATODA

\title{
ANISAKIDAE
}

Contracaecum sp. Larva. California. Mesenteric fat surrounding stomach. Dailey (1969a).

Phocanema sp. Larva. California. Wall of stomach. Dailey (1969a) as Porrocaecum sp.

Anisakis sp. Larva. California. Body cavity, stomach wall. Dailey (1969a).

\author{
CORYPHAENIDAE -- DOIphins \\ Coryphaena hippurus - Dolphinfish
}

\section{ACANTHOCEPHALA}

\section{RHADINORHYNCHIDAE}

Serrasentis sagittifer (Linton 1889) Linton 1932. Atlantic. Yamaguti (1963c) as Serrasentis social is.

Rhadinorhynchus pristis (Rudolphi 1802) Lühe 1911. Curacao. Cable and Linderoth (1963); Japan. Yamaguti (1963c) as R. katsuwonis.

CESTODA

DASYRHYNCHIDAE

Callitetrarhynchus gracil is (Rudolphi 1819) Pintner 1931. Plerocercoid. North Carolina. Stomach. Dollfus (1942). 
Coryphaena hippurus (Continued)

HEPATOXYLIDAE

Hepatoxylon trichiuri (Holten 1802) Do11fus 1942. Postlarva. Brazi1; Azores. Stomach. Dollfus (1942).

OTOBOTHRI IDAE

Otobothrium crenacolle Linton 1891. Larva. North Carolina. Linton (1924).

\section{TENTACULARIIDAE}

Tentacularia coryphaenae Bosc 1802. Plerocercoid. Florida. Body cavity. Ward (1954).

Nybelinia bisulcata (Linton 1889) Dollfus 1929. Plerocercoid. Florida. Viscera. Ward (1954).

\section{TRYPANORHYNCHA}

Tetrarhynchus papillosus Rudo7phi 1809. Yamaguti (1959b) [Species incertae sedis - Yamaguti (1959b)].

\section{COPEPODA}

\section{CALIGIDAE}

Caligus coryphaenae Steenstrup and Lütken 1861. Hawai i; Ecuador. On body. Shiino (1963a); Texas. Pearse (1952); Japan. Shiino (1960b); Indian Ocean. Lewis et a1. (1969).

Caligus constrictus Heller 1865. Panama. Body surface. Wilson $(1937 a)$.

Caligus quadratus Shi ino 1954. Indian Ocean. Lewis et al. (1969); Japan. Shiino (1959e); Hawaii. Buccal cavity, body surface. Lewis (1967).

Caligus productus Dana 1853. Indian Ocean. Gill cavity. Lewis et a1. (1969); Gulf of Mexico. Pearse (1952); Eastern Pacific. Shiino (1959d).

Caligus confusus? Pillai 1961. Panama. Lewis (1968).

Caligus belones Krøyer 1863. Florida. Gills. Burnett-Herkes $(1974)$.

Caligus bonito Wilson 1905. Florida. Gills. Burnett-Herkes $(1974)$.

Caligus patulus Wilson 1937. Florida. Gi11s. Burnett-Herkes (1974).

\section{EURYPHORIDAE}

Euryphorus nordmanni Edwards 1840. Indian Ocean. Gill cavity. Lewis et a1. (1969); Hawaii. Lewis (1967); Ryukyu Island. Kabata and Gusev (1966) as E. nympha; Texas. Causey (1953b) as E. coryphaenae. 


\section{LERNAEOPODIDAE}

Charopinopsis quaternius (Wiison 1935) Yamaguti 1963. South India. Gills. Pillai (1962); Hawaii. Lewis (1967); Texas. Pearse (1952) both as Brachiella c.

\section{PENNELLIDAE}

Lernaeeicus longiventris Wilson 1917. Western North America. Flesh. Wilson (1932); Texas. Pearse (1952).

\section{DIGENEA}

\section{ACANTHOCOLPIDAE}

Stephanostomum coryphaenae Manter 1947. Brazi1. Stomach, intestine. Travassos et a1. (1967); Bimini. Rectum. Sogandares-Berna 1 (1959).

\section{ACCACOELIDAE}

Tetrochetus coryphaenae Yamaguti 1934. Louisiana. Sparks (1958); Japan. Yamaguti (1934a); Bimini; Pacific Panama. Rectum. Sogandares-Bernal (1959).

\section{BATHYCOTYLIDAEE}

Bathycotyle branchial is Darr 1902. Florida. Gi1ls, buccal cavity. Burnett-Herkes (1974).

Bathcotyle coryphaenae Yamaguti 1938. Pacific coast of Japan. Gil1s. Yamaguti (1938); Louisiana. Sparks (1958).

\section{DIDYMOZOIDAE}

Genera and species. Larva. Gulf of Aden. Intestine. Nikolaeva (1965).

\section{HEMIURIDAE}

Dinurus barbatus (Cohn 1902) Looss 1907. Panama. Stómach. Manter (1940a); Jamaica-Curacao. Nahhas and Cable (1964). Dinurus longisinus Looss 1907. Panama. Stomach. Manter (1940a); Hawaji. Yamaguti (1970); Bimini. Sogandares-Bernal (1959); Pacific Japan. Stomach. Yamaguti (1934a) as D. coryphaenae. Dinurus tornatus (Rudolphi 1819) Looss 1907. AtTantic, BraziT, Red Sea. Stomach. Looss (1907b); Louisiana. Sparks (1958); Jamaica-Curacao. Stomach. Nahhas and Cable (1964).

Dinurus breviductus Looss 1907. Louisiana. Sparks (1958); Jamaica-Curacao. Stomach. Nahhas and Cable (1964).

HIRUDINELLIDAE

Hirudinella ventricosa (Pallas 1774) Baird 1853. Gulf of Mexico. Manter (1954b); AtTantic. Stomach. Manter (1940b) as H. clavata. 
Coryphaena hippurus (Continued)

\section{OPECOELIDAE}

Helicometrina nimia Linton 1910. Intestine. Arà (1962).

Opecoeloides sp. Brazil. Intestine. Travassos et a1. (1969).

MONOGENEA

CAPSALIDAE

Capsala Taevis (Verrill 1874) Johnston 1929. Brazil. Price (1938b). Benedenia hendorffi (Linstow 1889) Odhner 1905. Chile. Price (1938b).

GASTROCOTYLIDAE

Neothroacocotyle coryphaenae (Yamaguti 1938) Hargis 1956. Japan.

Gil7s. Yamaguti (1938) as Thoracocotyle c.

NEMATODA

ANISAKIDAE

Contracaecum sp. Larva. Formosa. Myers and Kuntz (1962).

PROTOZOA (MYXOSPORIDA)

TETRACAPSALIDAE

Kudoa sp. Muscle. Lom (1970a).

PROTOZOA (SPOROZOA)

HAEMOGREGAR INIDAE

Haemogregarina bigemina Laveran and Mesnil 1901. Bermuda; Florida Keys. Blood. Saunders (1966).

$$
\begin{aligned}
& \text { MELÂMPHAIDÄE -- Bigscales } \\
& \text { Melamphaes acanthomus - Shoulderspine Bigscales }
\end{aligned}
$$

DIGENEA

HEMIURIDAE

Genera and species. Collard?

NEMATODA

ANISAKIDAE

Contracaecum sp. Larva. Southern California. Coelom. Collard ${ }^{9}$. 
DIGENEA

HEMIURIDAE

Genera and species. Southern California. Mouth, heart, stomach. Collard ${ }^{9}$.

\author{
BRAMIDAE -- Pomfrets \\ Brama japonica - Pacific Pomfret
}

DIGENEA

LAMPRITREMATIDAE

Lampritrema miescheri (Zschokke 1890) Margolis 1962. Gulf of Alaska. Gills. Margolis (1962).

SYNCOELIDAE

Capiatestes thyrsitae Crowcroft 1948. Pacific 0cean. Gills, esophagus. Lebedev (1968). Uncertain host.

Syncoelium sp. Gulf of Alaska. Gills. Margolis (1962).

$$
\begin{gathered}
\text { POMADASYIDAE -- Grunts } \\
\text { Anisotremus davidsoni - Sargo }
\end{gathered}
$$

\title{
DIGENEA
}

HEMIURIDAE

Brachadena pyriformis Linton 1910. Southern California. Stomach. Manter and Van Cleave (1951).

MONORCHIDAE

Lasiotocus longicaecum (Manter 1940) Yamaguti 1954. Southern

California. Intestine. Manter and Van Cleave (1951) as Proctotrema 1.; Panama. Bravo-Holl is (1956).

$$
\text { Xenistius californiensis - Salema }
$$

\section{DIGENEA}

HEMIURIDAE

Lecithophyllum intermedium (Manter 1934) Margol is 1958. Baja California. Intestine. Arai (1962). 
Xenistius californiensis (Continued)

OPECOELIDAE

Opecoelus xenistii Manter 1940. Galapagos Islands. Intestine. Manter (1940a).

Helicometrina nimia Linton 1910. Intestine. Arai (1962).

\author{
SPARIDAE -- Porgies \\ Calamus brachysomus - Pacific Porgy
}

COPEPODA

CALIGIDAE

Caligus productus Dana 1853. West coast of Mexico. Causey (1960).

Caligus mutabilis Hilson 1905. Gulf of California. Causey (1960).

\title{
DIGENEA
}

HEMIURIDAE

Lecithochirium microstomum Chandler 1935. Galapagos Islands. Stomach. Manter (1940b).

OPISTHOLEBETIOAE

Pachycreadium gastrocotylum (Manter 1940) Manter 1954. Galapagos Islands. Intestine. Manter (1940b) as Plagioporus $\mathrm{g}$.

\author{
SCIAENIDAE -- Drums \\ Cheilotrema saturnum - Black Croaker
}

NEMATODA

ANISAKIDAE

Anisakis sp. Larva. Southern California. Dailey et al. (in press).

Cynoscion nobilis - White Seabass

COPEPODA

CALIGIDAE

Lepeophtheirus abdominis Shiino 1960. Southern California. Exterior. Shiino (1960 c). 
Cynoscion nobilis (Continued)

Lepeophtheirus thompsoni Baird 1950. Southern California. Exterior. Wilson (1908a); many places in Mexico. Causey (1960).

\section{LERNAEOPODIDAE}

Neobrachiella gracilis (Wilson 1908) Kabata 1978. Southern California. Mouth. Wilson (1908a) as Brachiella g.

CESTODA

DASYRHYNCHIDAE

Callitetrarhynchus gracilis (Rudolphi 1819) Pintner 1931. Larva. Southern California. Viscera. Jensen 18 .

\section{LACISTORHYNCHIDAE}

Grillotia smarisgora (Wagener 1854) Dollfus 1947. Larva. Southern California. Mesentery. Jensen 18 .

Lacistorhynchus tenuis (Van Beneden 1858) Dollfus 1929. Larva. Southern California. Viscera. Jensen ${ }^{18}$.

\section{DIGENEA}

\section{ACANTHOCOLPIDAE}

Pleorchis magniporus Arai 1962. Baja California. Intestine. Arai (1962). Pleorchis californiensis Manter and Van Cleave 1951. Southern California. Intestine. Manter and Van Cleave (1951).

\section{MONOGENEA}

Genera and species. Eastern Pacific. Kunnenkeri and Martin (1963). NEMATODA

\section{ANISAKIDAE}

Anisakis sp. Larva. Southern California. Dailey et a]. (in press). PROTOZOA (MYXOSPORIDA)

CERATOMYXIDAE

Ceratomyxa venusta Jameson 1931. Southern California. Gallbladder. Jameson (1931).

\section{TETRACAPSULIDAE}

Kudoa clupeidae (Hahn 1917) Meglitsch 1947. Southern California. Muscle. Jensen 18 . 
DIGENEA

ÂCĀNTTHOCOLLPIDÂE

Pleorchis magniporus Arai 1962. Baja California. Intestine. Arai (1962).

DIPLANGIDAE

Diplangus mexicanus Bravo-Hollis and Manter 1957. Baja California. Intestine and pyToric caeca. Arai (1962).

$$
\text { Genyonemus lineatus - White Croaker }
$$

CESTODA

LACISTORHYNCHIDAE

Lacistorhynchus tenuis (Van Beneden 1858) Dollfus 1929. Larva. Southern California. Viscera. Jensen 18 .

COPEPODA

BOMOLOCHIDAE

Holobomolochus attenuatus (Wīison 1913) Vervoort 1969. Baja

California. Causey (1960) as Bomolochus a.

CAL IGIDAE

Lepeophtheirus sp. Southern California. Jensen ${ }^{18}$.

LERNAEOPODIDAE

Neobrachiella gracilis (Wilson 1908) Kabata 1979. Mexico. Causey (1960) as Brachiella $\mathrm{g}$.

\section{DIGENEA}

ACANTHOCOLPIDAE

Stephanostomum cal ifornicum Manter and Van Cleave 1951. California. Intestine, stomach. Manter and Van Cleave (1951).

\section{NEMATODA}

ANISAKIDAE

Anisakis sp. Larva. Southern California. Dailey et al. (in press). 
ACANTHOCEPHALA

RHADINORHYNCHIDAE

Illiosentis cetratus Van Cleave 1945. Southern California. Intestine. Van Cleave (1945).

COPEPODA

BOMOLOCHIDAE

* Bomolochus solae Claus 1864. Baja California. Causey (1960).

\section{CALIGIDAE}

Caligus mutabilis Wilson 1905. Baja California. Causey (1960). Lepeophtheirus sp. Southern California. Jensen ${ }^{18}$.

\section{HATSCHEKI IDAE}

Hatschekia albirubra Wilson 1913. Baja California. Causey (1960).

\section{DIGENEA}

BUCEPHALIDAE

Prosorhynchus crucibulum (Rudo1phi 1819) Odhner 1905. Baja

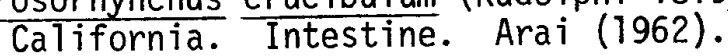

\section{DIPLANGIDAE}

Diplangus triradiatus Manter and Van Cleave 1951. Southern California. Intestine. Manter and Van Cleave (1951).

\section{MONORCHI IDAE}

Postmonorchis donacis Young 1953. Southern California. Intestine. Young (1953).

OPECOELIDAE

Helicometrina nimia Linton 1910. Intestine. Arai (1962).

$$
\text { Roncador stearnsi - Spotfin Croaker }
$$

\section{ACANTHOCEPHALA}

\section{RHADINORHYNCHIDAE}

I11iosentis cetratus Van Cleave 1945. Southern California. Intestine. Van CTeave (1945). 
Roncador stearnsi (Continued)

CESTODA

TETRAPHYLLIDEA

Genera and species. Red pigmented plerocercoid. Southern

California. Intestine; pyloric caeca. Kunnenkeri (1962).

COPEPODA

BOMOLOCHIDAE

Holobomolochus attenuatus (Wilson 1913) Vervoort 1969. Baja

California. Causey (1960) as Bomolochus a.

\section{LERNAEOPODIDAE}

Neobrachiella gulosa (Wilson 1915) Kabata 1979. Baja California. Causey (1960) as Brachiella g.

Neobrachiella sciaenophila (HeTler 1865) Kabata 1979. Baja California. Causey (1960) as Brachiella s. ("Host not well identified:")

\section{DIGENEA}

DIPLANGIDAE

Diplangus triradiatus Manter and Van Cleave 1951. Southern California. Intestine. Manter and Van Cleave (1951).

\section{MONORCHI IDAE}

Postmonorchis donacis Young 1953. Southern California. Intestine. Young (1953).

\section{OPECOELIDAE}

Pseudopecoelus umbrinae Manter and Van Cleave 1951. Southern

California. Intestine. Manter and Van Cleave (1951).

$$
\text { Seriphus politus - Queenfish }
$$

NEMATODA

\section{ANISAKIDAE}

Anisakis sp. Southern California. Dailey et al. (in press). 


\section{ACANTHOCEPHALA}

POL YMORPHIDAE

Arhythmorhynchus macracanthus Ward and Winter 1952. Juvenile. California. Mesentery. Ward and Winter (1952).

Corynosoma obtuscens Lincicome 1943. Juvenile. Southern

California. Mesentery. Ward and Winter (1952).

Corynosoma osmeri Fujita 1921. Juvenile. Southern California. Mesentery. Ward and Winter (1952).

\section{RHADINORHYNCHIDAE}

I1liosentis cetratus Van Cleave 1945. Southern California. Intestine. Van Cleave (1945).

\section{DIGENEA}

ACANTHOCOLPIDAE

Stephanostomum californicum Manter and Van Cleave 1951. Southern

California. Intestine. Manter and Van Cleave (1951).

\section{DIPLANGIDAE}

Diplangus triradiatus Manter and Van Cleave 1951. Southern

California. Intestine. Manter and Van Cleave (1951).

\section{OPECOELIDAE}

Pseudopecoelus umbrinae Manter and Van Cleave 1951. Southern

California. Intestine. Manter and Van Cleave (1951).

Genitocotyle acirra Park 1937. Southern Cal ifornia. Intestine.

Manter and Van CTeave (1951).

ISOPODA

\section{CYMOTHOIDAE}

Nerocila californica Schioedte and Meinert 1881. Southern California. Gills. Brusca (1977). Speculative host.

\section{MONOGENEA}

\section{DIPLECTANIDAE}

Rhamnocereus rhamnocereus Monaco, Wood and Miselle 1954. Southern California. Gills. Monaco et al. (1954). 


\section{KYPHOSIDAE -- Sea Chubs \\ Girel1a nigricans - Opaleye}

COPEPODA

CALIGIDAE

Caligus hobsoni Cressey 1969. Southern California. Hobson (1971).

PENNELLIDAE

Peniculus fissipes Wilson 1917. Southern California. External, fins. Wilson $(1935 b)$.

\section{DIGENEA}

HAPLOPORIDAE

Vitellibaculum girellicola Martin 1978. Southern California. Intestine. Martin (1978).

Vitellibaculum girellae Montgomery 1957. Southern California. Intestine. Montgomery, (1957).

HAPLOSPLANCHNIDAE

Schikhobalotrema girellae (Manter and Van Cleave 1951) Skrjabin and Guschanskaia 1955. Southern California. Intestine. Manter and Van Cleave (1951) as Haplosplanchus g.

HEMIURIDAE

Opisthadena cheni Martin 1978. Southern California. Stomach. Martin (1978).

\section{LEPOCREADI IDAE}

Opechona orientalis (Layman 1930) Ward and Fillingham 1934.

Southern California. Intestine. Manter and Van Cleave (1951).

OPECOELIDAE

Opegaster cameroni Caballero and Caballero 1969. Southern California. Intestine. Caballero and Caballero (1969b).

Opecoelus adsphaericus Manter and Van Cleave 1951. Southern

California. Intestine. Manter and Van Cleave (1951).

\section{MONOGENEA}

CAPSALIDAE

Neobenedenia girellae (Hargis 1955) Yamaguti 1963. Southern California. Skin. Hargis (1955a); Baja California. Bravo-Hollis (1957) as Benedenia $\mathrm{g}$. 
Girella nigricans (Continued)

\section{NEMATODA}

CAMALLANIDAE

Spirocamallanus pereirai (Annereaux 1946) 01 sen 1952. Southern California. Intestine. Noble and King (1960).

\section{PSEUDALI IDAE}

Parafilaroides decorus Dougherty and Herman 1947. Larva. Southern California. In mesentery, fat, under intestinal serosa. Dailey (1970).

PROTOZOA (CILIOPHORA)

OPHRYOGLENIDAE

Cryptocaryon irritans Brown 1951. Southern California. Tissues. Wilkie and Gordin (1969).

$$
\text { Kyphosus analogus - Striped Sea Chub }
$$

\section{DIGENEA}

\section{WARETREMATIDAE}

Megasolena kyphosi Sogandares-Bernal 1959. Gulf of Panama. Intestine. Sogandares-Bernal (1959).

$$
\text { Medialuna californiensis - Halfmoon }
$$

COPEPODA

\section{CALIGIDAE}

Caligus hobsoni Cressey 1969. Southern California. Cressey (1969a); body surface. Hobson (1971).

Lepeophtheirus sp. Southern California. Body surface. Hobson (1971).

\section{PENNELLIDAE}

Peniculus fissipes Wilson 1917. Southern California. Embedded in fins. Hobson (1971).

\section{DIGENEA}

\section{HEMIURIDAE}

Genolinea tanyopa Montgomery 1957. Southern California. Stomach. Montgomery (1957). 
Medialuna californiensis (Continued)

\title{
LEPOCREADI IDAE
}

Neolepidapedon medialunae Montgomery 1957. California. Intestine. Montgomery (1957).

\section{WARETREMATIDAE}

Scorpidicola cal iforniensis Montgomery 1957. Southern California. Intestine. Montgomery (1957).

Myodera medialunae Montgomery 1957. Southern California. Intestine. Montgomery (1957).

\author{
EMBIOTOCIDAEE -- Surfperches \\ Amphistichus argenteus - Barred Surfperch
}

COPEPODA

PHILICHTHYIDAE

Colobomatus embiotocae Noble, Collard and Wilkes 1969. Southern California. Under skin of cheek ridges, around eyes, in cephalic sensory canal system. Noble et al. (1969).

\section{DIGENEA}

\section{DIPLANGIDAE}

Diplangus triradiatus Manter and Van Cleave 1951. California. Arai (1967).

\section{MONORCHI IDAE}

Lasiotacus sp. California. Arai (1967).

Monorchis sp. California. Arai (1967).

Telolecithus pugetens is Lloyd and Guberlet 1932. Northern California. Rodella and Nahhas (1969).

\section{OPECOELIDAE}

Genitocotyle acirra Park 1937. California. Ara i (1967).

PseudopecoeTus umbrinae Manter and Van Cleave 1951. California. Arai (1967).

Helicometrina elongata Noble and Park 1937. Southern California. Intestine. Montgomery (1957).

Helicometrina nimia Linton 1910. Intestine. Arai (1962).

ISOPODA

CYMOTHOIDAE

Nerocila californica Schioedte and Meinert 1884. Southern California. Caudal fin. Valentine and Phelps (1977). 
Amphistichus argenteus (Continued)

\section{MONOGENEA}

\section{CAPSALIDAE}

Encotyllabe embiotocae Noble 1966. Southern California. Tongue. Noble $(1966 \mathrm{~b})$.

$$
\begin{gathered}
\frac{\text { Amphistichus koelzi - Calico Surfperch }}{\text { SYNONYM - Crossochir koelzi }} \\
\text { S }
\end{gathered}
$$

COPEPODA

\section{PHILICHTHYIDAE}

Colobomatus embiotocae Noble, Collard and Wilkes 1969. Southern California. Under the skin covering cheek ridge; around eyes, in cephalic sensory canal system. Noble et a1. (1969).

$$
\text { Amphistichus rhodoterus - Redtail Surfperch }
$$

\section{DIGENEA}

\section{OPECOEL IDAE}

Genitocotyle acirra Park 1937. Northern California. Stomach,

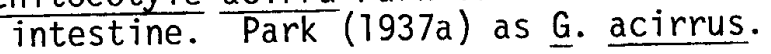

\section{MONOGENEA}

DICLIDOPHORIDAE

Diclidophora sp. Oregon. Gills. Hanson 26 .

$$
\text { Brachyistius frenatus - KeTp Perch }
$$

\section{DIGENEA}

\section{HEMIURIDAE}

Lecithaster gibbosus (Rudolphi 1802) Lühe 1901. British Columbia. Digestive tract. Arai (1969b).

$$
\text { Cymatogaster aggregata - Shiner Perch }
$$

\section{ACANTHOCEPHALA}

\section{ECHINORHYNCHIDAE}

Echinorhynchus gadi Zoega in Müller 1776. British Columbia.

$$
\text { Intestine. Arai (1969b). }
$$


Cymatogaster aggregata (Continued)

POLYMORPHIDAE

Corynosoma sp. Juvenile. British Columbia. Body cavity. Arai (1969b).

BRANCHIURA

ARGULIDAE

Argulus pugettensis Dana 1852. California. Wilson (1924); British Columbia. Beres:

CESTODA

AMPHICOTYLIDAE

Eubothrium sp. Larva. British Columbia. Arai (1967).

LACISTORHYNCHIDAE

Lacistorhynchus tenuis (Van Beneden 1858) Dollfus 1929. Plerocercoid. Southern California. Young (1954).

PHYLLOBOTHRI IDAE

Phyllobothrium sp. Larva. British Columbia. Digestive tract. Ärai (1̄969b).

\section{TETRAPHYLLIDEA}

Genera and species. Red pigmented pleurocercoid. Southern California. Intestine, pyloric caeca. Kunnenkeri (1962).

COPEPODA

BOMOLOCHIDAE

Bomolochus cuneatus Fraser 1920. British Columbia. Gills. Vervoort and Arai (1966) as Parabomolochus c.; Southern Cal ifornia. Hanan (1976).

Holobomolochus embiotocae Hanan 1976. Southern California. Nasa 7 cavities. Hanan (1976).

Taeniacanthodes haakeri Ho 1972. Southern California. Hanan 27.

CAL IGIDAE

Caligus quadratus Shiino 1954. Southern California. Mouth. Hanan27. Lepeophtheirus bifurcatus Wilson 1905. Southern California. External surface. Hanan 27.

Lepeophtheirus parvus Wilson 1908. Southern California. External surface. Hanan 27. 
ERGASILIDAE

Ergasilus turgidus Fraser 1920. British Columbia. Gīis. Ârai (1969b); British Columbia. Gill filaments. Fraser (1920). Ergasilus lizae Krøyer 1863. Southern California. Hanan (1976).

\section{PENNELLIDAE}

Peniculus fissipes Wilson 1917. Southern California. Pectoral fin. Hanan 27.

Haemobaphes diceraus Wilson 1917. British Columbia. Gill arches. Kabata ( $996 \mathrm{~b})$.

\section{PHILICHTHYIDAE}

Colobomatus embiotocae Noble, Collard and Wilkes 1969. Southern California. Under skin covering cheek ridges, around eyes, in cepha1ic sensory canal system. Noble et a1. (1969).

\section{DIGENEA}

\section{ACANTHOCOLPIDAE}

Stephanostomum sp. Immature. Northern California. Rodella and Nahhas (1969).

\section{DIPLANGIDAE}

Diplangus macrovitellus (Winter 1954) Winter 1960. Southern California. Intestine. Yamaguti (1971).

\section{HEMIURIDAE}

Lecithaster gibbosus (Rudolphi 1802) Lühe 1901. British Columbia. Digestive tract. Arai (1969b); Washington. Ching (1960a) as L. salmonis.

TubutovesicuTa lindbergi (Layman 1930) Yamaguti 1934. British Columbia. Digestive tract. Arai (1969b).

\section{HETEROPHYIDAE}

Galactosomum sp. Larva. British Columbia. Gills (encysted). Arai (1969b).

\section{MONORCHIDAE}

Telolecithus pugetensis Lloyd and Guberlet 1932. British Columbia. McFarlane (1936); Washington. Lloyd and Guberlet (1932); Northern California. Rodella and Nahhas (1969).

Postmonorchis donacis Young 1953. Southern California. Guts. Young (1953). 


\section{OPECOELIDAE}

Genitocotyle acirra Park 1937. California. Arai (1967) as

G. acirrus; Rode11a and Nahhas (1969) as G. acirrata.

Pséudopecoelus umbrinae Manter and Van Cleave 1951. California. Arai (1967).

\section{ZOOGONIDAE}

Neozoogonus californicus Arai 1954. California. Intestine. Arai (1954); British Columbia. Digestive tract. Arai (1969b).

ISOPODA

\section{CYMOTHOIDAE}

Lironeca californica Schioedte and Meinart 1884. Southern California. Gill cavity. Hanan 27; British Columbia. Arai (1969b). Lironeca vulgaris Stimpson 1857. Southern California. Gill cavity. Hanan 27.

Nerocila californica Schioedte and Meinert 1881. Southern California. Cauda 7 peduncle. Hanan 27 .

\section{MONOGENEA}

CAPSAL IDAE

Encotyllabe embiotocae Noble 1966. Southern California. Tongue. Nob7e $(1966 \mathrm{~b})$.

\section{GYRODACTYLIDAE}

Gyrodactylus aggregata Mizelle and Kritsky 1967. Northern California. Skin. Mize Tle and Kritsky (1967b).

Gyrodactylus sp. British Columbia. Arai (1967).

Archigyrodactylus archigyrodactylus Mizelle and Kritsky 1967. Northern California. Gills. Mizelle and Kritsky (1967b).

\section{GYRODACTYLOIDEA}

Genera and species. British Columbia. Gills, body surface. Arai (1969b).

\section{NEMATODA}

\section{ANISAKIDAE}

Anisakis sp. Larva. British Columbia. Arai (1967).

Contracaecum sp. Larva. British Columbia. Body cavity. Arai (1969b).

CUCULLANIDAE

Cucullanellus kanabus Walder and Arai 1974. British Columbia. Intestine. Walder and Arai (1974). 
Cymatogaster aggregata (Continued)

PROTOZOA (MYXOSPORIDA)

MYXIDIIDAE

Myxidium oviforme Parisi 1912. Central California. Gallbladder. Jameson (1929).

\author{
Damalichthys vacca - Pile Perch \\ SYNONYMS - Damalichthys argyrosomus, Rhacochilus vacca
}

\title{
BRANCHIURA
}

\section{ARGUL IDAE}

Argulus puqettensis Dana 1852. Southern California. Caudal fin.

\section{COPEPODA}

\section{BOMOLOCHIDAE}

Bomolochus cuneatus Fraser 1920. British Columbia. Kabata (1971). Holobomolochus embiotocae Hanan 1976. Southern California. Nasal cavity. Hanan (1976).

\section{CALIGIDAE}

Lepeophtheirus parvus Wilson 1908. Southern California. External surface. Hanan 27.

Lepeophtheirus sp. Oregon. Wares (1971).

Caligus hobsoni Cressey 1969. Southern California. Hobson (1971).

\section{LERNAEOPODIDAE}

Clavella sp. California. Gills. Harlan27 ;Oregon. Gill

$$
\text { filaments. Wares (1971). }
$$

\section{PHILICHTHY IDAE}

Colobomatus embiotocae Noble, Collard and Wilkes 1969. Southern California. Under skin covering cheek ridge, around eyes, in cephalic sensory canal system. Noble et al. (1969).

\section{DIGENEA}

\section{BUCEPHALIDAE}

Prosorhynchus sp. Metacercaria. Oregon. Internal organs. Wares (1971).

Rhipidocotyle sp. Metacercaria. Oregon. Internal organs. Wares (1971).

Prosorhynchoicies sp. Metacercaria. Oregon. Internal organs. Wares (1971) as Bucephalops is. 
Damalichthys vacca (Continued)

DIPLANGIDAE

Diplangus macrovitellus (Winter 1956) Winter 1960. California. Arai (1967) as Proctoeces $\mathrm{m}$.

HEMIURIDAE

Derogenoides sp. Immature. Oregon. Liver. Wares (1971). MONORCHI IDAE

Telolecithus pugetensis Lloyd and Guberlet 1932. California. Arai (1967); Oregon. Digestive tract. Wares (1971).

OPECOELIDAE

Genitocotyle acirra Park 1937. Southern California. Intestine. Manter and $\operatorname{Van}$ CTeave (1951) as G. acirrus.

ZOOGONIDAE

Neozoogonus californicus Arai 1954. Intestine. Arai (1962). ISOPODA

\section{CYMOTHOIDAE}

Lironeca vulgaris Stimpson 1857. Southern California. Gill cavity. Turner et al. (1969).

Nerocila californica Schioedte and Meinert 1881. Southern California. Caudal peduncle. Hanan 2 ?

MONOGENEA

HETERAXINIDAE

Allencotyle pricei Kritsky, Noble and Moser 1978. Southern California. Gi17s. Kritsky et a1. (1978).

NEMATODA

CUCULLANIDAE

Cucullanus sp. Oregon. Digestive tract. Wares (1971). PROTOZOA (MYXOSPORIDA)

MYXIDIIDAE

Myxidium oviforme Parisi 1912. Central California. Gallbladder. Jameson (1929).

Zschokkella embiotocidis Moser and Halderson 1976. Central California. Gallbiadder. Moser and Haldorson (1976). 
MYXOBOLIDAE

Henneguya sp. British Columbia. Hoskins et al 13

SINUOLINEIDAE

Davisia reginae Love and Moser 1976. Central California. Urinary bladder. Love and Moser (1976).

$$
\text { Embiotoca jacksoni - Black Perch }
$$

COPEPODA

BOMOLOCHIDAE

Holobomolochus embioticae Hanan 1976. Southern California. Nasal cavity. Hanan (1976).

Bomolochus cuneatus Fraser 1920. California. Hanan (1976).

\section{LERNAEOPODIDAE}

Clavella sp. Southern California. Gills. Hanan2?.

\section{PENNELLIDAE}

Peniculus fissipes Wilson 1917. Southern California. Robinson 28.

\section{PHILICHTHYIDAE}

Colobomatus embiotocae Noble, Collard and Wilkes 1969. Southern California. Cephalic canal. Hanan 2 ?

\section{DIGENEA}

\section{ACANTHOCOLPIDAE}

Stephanostomum dentatum (Linton 1900) Linton 1940. Southern and Central California; Baja California. Intestine. Arai (1962).

\section{DIPLANGIDAE}

Diplangus paxillus Linton 1910. California. Arai (1967).

Diplangus macrovitellus (Winter 1954) Winter 1960. California. Arai (1967) as Proctoeces $m$.

\section{MONORCHIIDAE}

Postmonorchis donacis Young 1953. Southern California. Intestine. Young (1953).

Telolecithus pugetensis Lloyd and Guberlet 1932. California. Arai (1967). 
Embiotoca jacksoni (Continued)

OPECOELIDAE

Helicometrina nimia Linton 1910. Southern California. 'Intestine. Montgomery (1957).

ZOOGONIDAE

Neozoogonus californicus Arai 1954. Southern and Central California. Intestine. Arai (1954); Northern California. Rodella and Nahhas (1969).

ISOPODA

CYMOTHOIDAE

Lironeca vulgaris Stimpson 1857. Southern California. Gill cavity. Hanan 2 ?.

Nerocila californica Schioedte and Meinert 1881. Southern California. Caudal peduncle. Hanan 2 ?.

\section{Embiotoca lateralis - Striped Seaperch}

SYNONYM - Taeniotoca lateral is

BRANCHIURA

ARGUL IDAE

Argulus pugettensis Dana 1852. British Columbia. External. Wilson $(1912 b)$.

COPEPODA

LERNAEOPODIDAE

Clavella parva Wilson 1912. British Columbia. Wilson (1912b).

\section{PHILICHTHYIDAE}

Colobomatus embiotocae Noble, Collard and Wilkes 1969. Central California. Under skin covering cheek ridges, around eyes, in cephalic sensory canal system. Noble et al. (1969).

\section{DIGENEA}

DIPLANGIDAE

Diplangus macrovitellus (Winter 1954) Winter 1960. Northern California. Rodella and Nahhas (1969).

HEMIURIDAE

Sterrhurus exodicus (McFarlane 1936) Yamaguti 1958. Northern California. Stomach. Chapa23as Separogermiductus e. 
Embiotoca lateralis (Continued)

MONORCHI IDAE

Telolecithus pugetensis Lloyd and Guberiet 1932. Oregon. Pratt and McCauley (1967); Cal ifornia. Arai (1967).

OPECOELIDAE

Genitocotyle acirra Park 1937. Oregon. Pratt (1970).

Helicometrina nimia Linton 1910. Northern California. Intestine. Chapa 23 .

\section{ZOOGONIDAE}

Neozoogonus californicus Arai 1954. Intestine. Arai (1962);

Northern California. Rodella and Nahhas (1969).

PROTOZOA (MYXOSPORIDA)

MYXIDIIDAE

Zschokkella embiotocidis Moser and Haldorson 1976. Central

California. Gallbladder. Moser and Haldorson (1976).

SINUOL INE IDAE

Davisia reginae Love and Moser 1976. Central California. Urinary bladder. Love and Moser (1976).

$$
\text { Hyperprosopon anale - Spotfin Surfperch }
$$

COPEPODA

\section{PHILICHTHYIDAE}

Colobomatus embiotocae Noble, Collard and Wilkes (1969). Northern California. Preopercular-mandibular canal. Iverson (1972).

\section{DIGENEA}

MONORCHI IDAE

Telolecithus pugetensis Lloyd and Guberlet 1932. California. Arai (1967).

OPECOELIDAE

Genitocotyle acirra Park 1937. California. Arai (1967). MONOGENEA

GYRODACTYLIDAE

Archigyrodactylus leibyi Mizelle and Kritsky 1967. Northern California. Skin. Mizelle and Kritsky (1967b). 
BRANCHIURA

ARGULLIDAE

Argulus pugettensis Dana 1852. California. Wilson (1924). COPEPODA

\section{PHILICHTHYIDAE}

Colobomatus embiotocae Noble, Collard and Wilkes 1969. Southern California. Under skin covering cheekridge, around eyes, in cephalic sensory canal system. Noble et al. (1969).

\section{DIGENEĀ}

\section{ACANTHOCOLPIDAE}

Stephanostomum sp. Immature. Northern California. Rodella and Nahhas (1969).

\section{CRYPTOGONIMIDAE}

Neochasmus sp. California. Arai (1967).

\section{HAPLOSPLANCHNIDAE}

Schikhobalotrema girellae (Manter and Van Cleave 1951) Skrjabin and Guschanskaja 1955. Cal ifornia. Arai (1967).

\section{MONORCHI IDAE}

Lasiotocus sp. California. Arai (1967).

Telolecithus pugetensis Lloyd and Guberlet 1932. Northern California. Rodel7a and Nahhas (1969).

OPECOELIDAE

Genitocotyle acirra Park 1937. California. Arai (1967). ISOPODA

CYMOTHOIDAE

Lironeca vulgaris Stimpson 1857. California. Gills. Richardson $(1905)$.

\section{MONOGENEA}

DICLIDOPHORIDAE

Diclidophora sp. Oregon. Gills. Hanson ${ }^{26}$. 
Hyperprosopon argenteum (Continued)

GYRODACTYLIDAE

Archigyrodactylus leibyi Mizelle and Kritsky 1967. Northern

California. Skin. Mizelle and Kritsky (1967b).

Hyperprosopon ellipticum - Silver Surfperch

DIGENEA

HEMIURIDAE

TubuTovesicula Tindbergi (Layman 1930) Yamaguti 1934. Northern California. Rodella and Nahhas (1969).

OPECOELIDAE

Genitocotyle acirra Park 1937. Northern California. Rodella and Nahhas (1969) as G. acirrata.

MONOGENEA

DICLIDOPHORIDAE

Diclidophora sp. Oregon. Gills. Hanson 26.

GYRODACTYLIDAE

Archigyrodactylus leibyi Mizelle and Kritsky 1967. Northern California. Skin. Mizelle and Kritsky (1967b).

$$
\text { Hypsurus caryi - Rainbow Seaperch }
$$

COPEPODA

PENNELLIDAE

Peniculus fissipes Wilson 1917. Southern California. Embedded in fin. Hobson (1971).

\section{PHILICHTHYIDAE}

Colobomatus embiotocae Noble, Collard and Wilkes 1969. Southern California. Under skin covering cheek ridge, around eyes, in the cephalic sensory canal system. Noble et al. (1969).

\section{DIGENEA}

ZOOGONIDAE

Neozoogonus californicus Arai 1954. California and Mexican Pacific. Intestine. Arai (1962). 
Hypsurus caryi (Continued)

PROTOZOA (MYXOSPORIDA)

MYXIDIIDAE

Myxidium oviforme Parisi 1912. Central California. Gallbladder. Jameson (1929).

$$
\text { Micrometrus minimus - Dwarf Perch }
$$

COPEPODA

\section{PHILICHTHYIDAE}

Colobomatus embiotocae Noble, Collard and Wilkes 1969. Southern California. Under skin covering cheek ridges, around eyes, in the cephalic sensory canal system. Noble et a1. (1969).

\section{DIGENEA}

HAPLOSPLANCHNIDAE

Schikhobalotrema girellae (Manter and Van Cleave 1951) Skrjabin and Guschanskaja 1955. California. Arai (1967).

MONORCHI IDAE

Postmonorchis donacis Young 1953. Southern California. Intestine. Young (1953).

OPECOELIDAE

Genitocotyle acirra Park 1937. California. Arai (1967).

ZOOGONIDAE

Neozoogonus californicus Arai 1954. California. Intestine. Arai (7954).

ISOPODA

CYMOTHOIDAE

Lironeca californica Schioedte and Meinert 1884. Baja California. GiT1s. Iverson (1974).

Nerocila californica Schioedte and Meinert 1881. Southern

California. Brusca (1977). 
COPEPODA

\section{CALIGIDAE}

Caligus hobsoni Cressey 1969. Southern California. Hobson (1971).

\section{Phanerodon furcatus - White Seaperch}

\section{BRANCHIURA}

ARGULIDAE

Argulus pugettensis Dana 1852. Southern California. Montgomery (1957); British Columbia. Fraser (1920).

\section{COPEPODA}

\section{BOMOLOCHIDAE}

Holobomolochus embiotocae Hanan 1976. Southern California. Nasal cavities. Hanan (1976).

Bomolochus cuneatus Fraser 1920. Southern California. Hanan (1976).

\section{CALIGIDAE}

Lepeophtheirus bifurcatus Wilson 1905. Southern California.

$$
\text { External. Hanan27. }
$$

\section{LERNAEOPODIDAE}

Clavella parva Wilson 1912. British Columbia. Bere ${ }^{5}$.

\section{PENNELLIDAE}

Peniculus fissipes Wilson 1917. Southern California. Embedded in fins. Hobson (1971).

\section{DIGENEA}

\section{DIPLANGIDAE}

Diplangus paxillus Linton 1910. California. Arai (1967).

Diplangus macrovitellus (Winter 1954) Winter 1960. Arai (1967). as Proctoeces macrovitellosus.

\section{MONORCHI IDAE}

Telolecithus pugetensis Lloyd and Guberlet 1932. California. Arai (1967).

OPECOELIDAE

Genitocotyle acirra Park 1937. California. Arai (1967). 
Phanerodon furcatus (Continued)

ZOOGONIDAE

Neozoogonus californicus Arai 1954. California. Intestine. Arai (1962).

ISOPODA

CYMOTHOIDAE

Lironeca vulgaris Stimpson 1857. California. Gill cavity. Turner et a1. (1969).

Nerocila californica Schioedte and Meinert 1881. Southern California. Cauda1 peduncle. Hanan 27.

MONOGENEA

DICLIDOPHORIDAE

Diclidophora sp. Oregon. Gills. Hanson ${ }^{26}$. PROTOZOA (MYXOSPORIDA)

MYXIDI IDAE

Myxidium oviforme Parisi 1912. Central California. Gallbladder. Jameson (1929).

$$
\text { Rhacochilus toxotes - Rubberl ip Seaperch }
$$

COPEPODA

CALIGIDAE

Caligus hobsoni Cressey 1969. Southern California. Cressey (1969a).

\section{PHILICHTHYIDAE}

Colobomatus embiotocae Noble, Collard and Wilkes 1969. California. Under skin covering cheek ridges, around eyes, in the cephalic sensory canal system. Noble et a1. (1969).

DIGENEA

ACANTHOCOLPIDAE

Stephanostomum sp. Immature. Central California. Chapa ${ }^{23}$. MONORCHI IDAE

Telolecithus pugetensis Lioyd and Guberlet 1932. California. Arai (1967). 
Rhacochilus toxotes (Continued)

ZOOGONIDAE

Zoogonus dextrocirrus Aldrich 1961. Central California. Intestine. Chapa 3 .

Neozoogonus californicus Arai 1954. California. Intestine. Arai (1962).

PROTOZOA (MYXOSPORIDA)

CERATOMYXIDAE

Leptotheca fisheri (Jameson 1929) Kudo 1933. California. GaTTbladder. Jameson (1931) as Ceratomyxa f.

Zalembius rosaceus - Pink Seaperch

COPEPODA

\section{PHILICHTHYIDAE}

Colobomatus embiotocae Noble, Collard and Wilkes 1969. Central and Northern California. Preopercular-mandibular canals. Iverson (1972).

\section{DIGENEA}

LEPOCREADI IDAE

Labrifer balli Arai 1971. Southern California. Intestine. Arai (1971). ZOOGONIDAE

Neozoogonus californicus Arai 1954. California and Mexico. Intestine. Arai (1962).

MONOGENEA

CAPSALIDAE

Entobdella rosaceus Crane 1972. Southern California. Skin. Crane (1972).

MICROCOTYLIDAE

Microcotyle zalembius Crane 1972. Southern California. Skin. Crane (1972). 
POMACENTRIDAE - Damselfishes

Chromis punctipinnis - Blacksmith

COPEPODA

CALIGIDAE

Caligus hobsoni Cressey 1969. Southern California. Cressey (1969a).

\section{LERNAEOPODIDAE}

Clavellopsis flexicervica Shiino 1956. Southern California. Gill arches. Hobson (1971).

ISOPODA

Genera and species. Gnathiid larva. Southern California. Body surface. Hobson (1971).

MONOGENEA

MICROCOTYLIDAE

Bivagina punctipinnis Crane 1972. Southern California. Gills. Crane (1972).

PROTOZOA (MYXOSPORIDA)

CERATOMYXIDAE

Leptotheca fisheri (Jameson 1929) Kudo 1933. Southern California. GaTlbladder. Jameson (1929) as Ceratomyxa f.

Hypsypops rubicunda - Garibaldi

COPEPODA

BOMOLOCHIDAE

Holobomolochus ardeole (Krøyer 1864) Vervoort 1969. Southern California. Branchial cavity. Hobson (1971) as Bomolochus a ; gill filaments. Wilson (1908a) as Artacolax a.

CALIGIDAE

Caligus hobsoni Cressey 1969. Southern California. Cressey (1969a). Lepeophtheirus sp. Southern California. Body surface. Hobson (1977).

PENNELLIDAE

Peniculus fissipes Wilson 1917. Southern California. External on fins. WiTson (1935b). 
Hypsypops rubicunda (Continued)

\section{DIGENEA}

\section{HEMIURIDAE}

Genolinea tanyopa Montgomery 1957. Southern California. Stomach. Montgomery (1957).

\section{ISOPODA}

Genera and species. Gnathiid larva. Southern California. On body. Hobson (1971).

$$
\text { LABRIDAE -- Wrasses }
$$

Halichoeres semicinctus - Rock Wrasse

COPEPODA

\section{CALIGIDAE}

Lepeophtheirus parviventris Wilson 1905. California. Kabata (1973); exterior. Wilson (1924).

PROTOZOA (MYXOSPORIDA)

\section{CERATOMYXIDAE}

Leptotheca fisheri (Jameson 1929) Kudo 1933. California. Gallbladder. Jameson (1937) as Ceratomyxa $f$.

$$
\text { Oxyjulis californica - Senorita }
$$

COPEPODA

\section{CALIGIDAE}

Caligus hobsoni Cressey 1969. Southern California. Body surface. Hobson (7971).

Caligus serratus Shiino 1965. Southern California. Body surface. Hobson (1971).

Lepeophtheirus sp. Southern California. Body surface. Hobson (7971).

\section{CHONDRACANTHIDAE}

Heterochondria atypica Ho 1972. Southern California. Gill

$$
\text { filaments. Ho (1972b). }
$$


SYNONYM - Pimelometopon pulcher

COPEPODA

CALIGIDAE

Caligus hobsoni Cressey 1969. Southern California. Hobson (1971). Lepeophtheirus parvus Wilson 1908. Baja California. Shiino (1959c); Southern California. Wilson (1908a).

\section{DIGENEA}

GORGODERIDAE

Phyllodistomum scrippsi Brooks and Mayes 1975. Southern California. Urinary bladder. Brooks and Mayes (1975).

\section{LEPOCREADIIDAE}

Lepocreadium bimarinum Manter 1940. California; Pacific Mexico. Intestine. Manter (1940a).

Labrifer secundus Manter 1940. Mexico; California. Intestine. Manter (1940a).

Neolabrifer bravoae Pritchard 1972. Southern California; Mexico.

Intestine. Pritchard (1972).

ISOPODA

Genera and species. Gnathiid larva. Southern California. Body surface. Hobson (1971).

MONOGENEA

CAPSALIDAE

Neobenedenia girellae (Hargis 1955) Yamaguti 1963. Southern

California. Skin. Brooks and Mayes (1975).

$$
\begin{gathered}
\text { MUGILIDAE -- Mullets } \\
\text { Mugil cephalus - Striped Mullet }
\end{gathered}
$$

\section{ACANTHOCEPHALA}

\section{DIPLOSENTIDAE}

Pararhadinorhynchus mugil is Johnston and Edmonds 1947. Australia. Intestine. Johnston and Edmonds (1947). 
Mugil cephalus (Continued)

\section{NEOECHINORHYNCHIDAE}

Neoechinorhynchus chilkaense Podder 1937. India. Intestine. Podder (1937).

Neoechinorhynchus agilis (Rudolphi 1819). Japan. Smal1 intestine. Yamaguti (1939e); Yugoslavia, USSR. Reshetnikova (1955).

Floridosentis elongatus Ward 1953. Florida. Intestine. Ward (1953); GuTf of California. Intestine. Bravo-Hollis (1969).

\section{BRANCHIURA}

\section{ARGUL IDAE}

Argulus chesapeakensis Cressey. Georgia. Skinner (1975).

Argulus funduli Krøyer 1863. Georgia. Rawson (1977).

\section{CESTODA}

\section{OTOBOTHRI IDAE}

Otobothrium mugilis Hiscook 1954. Larva. Australia. Hiscock (1954).

\section{TETRAPHYLLIDEA}

Scolex pleuronectis Müller 1788. Israel. Intestine. Pyloric caeca. Paperna (1964b).

Scolex polymorphus Rudolphi 1819. Massachusetts. Linton (1924).

\section{COPEPODA}

\section{BOMOLOCHIDAE}

Bomolochus mugilis. Florida. Skinner (1975).

Bomolochus Concinnus Wilson 1911. North Carolina. Gills. Wilson

(1911); Mexico. Causey (1960) as B. nitidus.

Bomolochus exilipes Wilson 1911. Gulf of Mexico. Gills. Wilson (1971).

Nothobomolochus teres (Wilson 1911). Texas. Gills. Causey (1953b) as Bomolochus teres.

\section{CALIGIDAE}

Caligus praetextus Bere 1936. Florida. Skinner (1975).

Caligus schistonyx Wilson 1905. Florida. Skinner (1975).

Caligus irritans Heller 1868. Florida. Mouth. Skinner (1975).

Caligus platytarsis Bassett - Smith 1898. India. Body surface. Rangnekar (1955) as C. bombayens is.

Caligus orientalis Gussev 1951. China. Body surface. Shen (1957) as C. communis.

Caligús rufimáculatus Wilson 1905. Massachusetts. Wilson (1905b). Caligus curtus Müller 1785. North Carolina. Parker et a1. (1968). Caligus epidemicus Hewitt 1971. Australia. Body surface. Hewitt (1971a). 
Caligus minimus 0tto 1821. Italy. Carli and Bruzzone (1973).

Caligus bonito Wi1son 1905. Gulf of Mexico. Body surface. Bere (1936).

Caligus mutabilis Wilson 1905. Mexico. Causey (1960).

Lepeophtheirus hospitalis Fraser 1920. China. Kabata (1973).

* Pseudocaligus apodus Brian 1924. Israel. Paperna and Lahav (1974).

ERGASILIDAE

Dermoergasilus amplectens (Dogiel and Akhmerov 1952). USSR. Gills. Dogiel and Akhmerov (1952).

Ergasilus longimanus Krøyer 1863. Florida. Gills. Skinner (1975). Ergasilus versicolor Wilson 1911. Georgia. Gi17s. Rawson (1977).

Ergasilus funduli Krøyer 1863. Georgia. Rawson (1977).

Ergasilus mugilis Vogt 1877. Mexico. Gills. Causey (1960).

Ergasilus lizae Krøyer 1863. Georgia, USA. Roberts (1970); Mexico. Causey (1960); Mediterranean. Gills. Delamare-Deboutteville and Nunes (1952) as E. nanus.

Ergasilus fryeri Paperna T964. Israel. Gills. Paperna (1964a). Ergasilus cyanopictus de Paiva Carvalho 1962. Brazil. Gills. de Paiva Carvalho (1962).

Ergasilus sp. Israel. Gi7ls. Paperna (1964b).

Nipergasilus bora (Yamaguti 1939) Yin 1956. Japan. Gills. Yamaguti (1939a) as Ergasilus b.

\section{LERNAEOPODIDAE}

*Clavella inversa Wilson 1913. North Carolina. Wilson (1913).

* Neobrachiella oblonga (Valle 1880) Kabata 1979. North Carolina. Pectoral fins. Wilson (1908b) as Brachiella o.

Clavellops is longimana Bere 1936. Gulf of Mexico. Pectoral fins. Bere (1936).

\section{LERNANTHROPIDAE}

Lernanthropus mugili Shishido 1898. Japan. Gills. Yamaguti (1936) NAOBRANCHIIDAE

Naobranchia lizae (Krøyer 1864). Texas. Gills. Causey (1953b). PENNELLIDAE

Lernaeenicus longiventris Wilson 1917. Western North Atlantic. Flesh. Wilson (1917). Lernaeenicus gracilis Heller 1865. Richiardi (1877). Lernaeenicus vorax Richiardi 1877. Yamaguti (1963a) as L. neglectus. 
Mugil cephalus (Continued)

\section{DIGENEA}

\section{ACANTHOCOLPIDAE}

Stephanostomum sp. Metacercaria. Florida. Hutton (1964).

\section{BIVESICULIDAE}

Paucivitellosus fragilis Coil, Read and Kuntz 1965. Australia. Intestine. Pearson (1968).

BUCEPHALIDAE

Rhipidocotyle lepisostei Hopkins 1954. Metacercaria. Texas, Louisiana, Oklahoma. Hopkins (1954).

\section{CRYPTOGONIMIDAE}

Exorchis oviformis Kobayashi 1915. Metacercaria. Japan. Komiya and Suzuki (1966).

\section{CYATHOCOTYLIDAE}

Mesostephanus appendiculatoides (Price 1934) Lutz 1935. Metacercaria. Florida. Hutton (1964).

\section{DIOYMOZOIDAEE}

Epithelionematobothrium fragile Skinner 1975. Florida. Body cavity. Skinner (1975).

HAPLOPORIDAE

Paraunisaccoides lobolecithus Martin 1973. Australia. Intestine. Martin (1973a).

Dicrogaster fastigatus Thatcher and Sparks 1958. Louisiana. Upper intestinal tract. Thatcher and Sparks (1958).

Lecithobotrys sprenti Martin 1973. Australia. Small intestine. Martin (1973d).

Lecithobotrys putrescens Looss 1902. Israel. Intestine. Paperna (1964b).

Paralecithobotrys brisbanensis Martin 1974. Australia. Sma11 intestine. Mart in (1974).

Isorchis manteri Martin 1973. Australia. Small intestine. Martin $(7973 \mathrm{c})$.

Unisaccoides vitellosus Martin 1973. Australia. Small intestine. Martin (1973b).

Unisaccus brisbanensis Martin 1973. Australia. Small intestine. Martin (1973b).

Haploporus benedeni (Stossich 1887) Looss 1902. Gulf of Naples. Intestine. Looss (1902); Israel. Paperna (1964b).

Haploporus longibollum (Wlassenko 1931). Black Sea. Intestine. Yamaguti (1971). 
Haploporus lateralis Looss 1902. Israel. Intestine. Paperna (1964b).

Carassotrema mugilicola Shireman 1964. Louisiana. Intestine. Shireman (1964).

Saccacoelium tensum Looss 1902. Israe1. Intestine. Paperna (1964b); BTack Sea. KovaT and Otsupok (1964).

Saccacoelium obesum Looss 1902. Adriatic; Black Sea; Egypt. Looss

(7902); Egypt. Smal1 intestine. Fischthal and Kuntz (1963).

Saccacoelioides beauforti (Hunter and Thomas 1961) Overstreet 1971. Mississippi, ATabama, Georgia. Intestine. Overstreet (1971b). Saccacoelioides pearsoni Martin 1973. Australia. Small intestine. Martin (1973d).

\section{HAPLOSPLANCHNIDAE}

Haplosplanchnus pachysomus (Eysenhardt 1829) Looss 1902. Egypt.

Fischthal and Kuntz (1963).

Haplosplanchnus mugil is Nahhas and Cable 1964. Florida. Intestine. Skinner (1975).

Schikhobalotrema magnum Skinner 1975. Florida. Intestine. Skinner (1975).

Schikhobalotrema sparisomae (Manter 1937) Skrjabin and Guschanskaia 1955. BTack Sea. Intestine. Koval and Otsupok (1964).

Schikhobalotrema sp. Florida. Intestine. Nahhas and Short (1965).

Schikhobalotrema elongatum Nahhas and Cable 1964. Curacao.

Intestine. Nahhas and Cable (1964); Florida. Skinner (1975).

Hymenocottoides manteri (Overstreet 1969) Yamaguti 1971. Florida, Georgia. Intestine. Overstreet (1969) as Hymenocotta m.

Hymenocotta mulli Manter 1961. New Caledonia; Fiji. Intestine. Durio and Manter (1968).

\section{HEMIURIDAE}

Bunocotyle mugilis Yamaguti 1970. Hawai i. Stomach. Yamaguti (1970). Saturnius segmentatus Manter 1969. Austra1ia. Stomach. Manter (1969).

Lecithaster helodes Overstreet 1973. Mississippi. Intestine. Overstreet (1973).

Lecithaster mugilis Yamaguti 1970. Hawaii. Intestine. Yamaguti 1970.

Aphanurus sp. Japan. Stomach. Machida et a1. (1970).

Aphanurus stossichi (Monticel1i 1891) Looss 1907. Japan. Ichihara (1974).

Dichadena galeata (Looss 1907) Skrjabina and Guschanskaja 1954. USSR. Reshetnikova (1955) as Lecithaster galeatus.

Hysterolecitha elongata Manter 1931. Mississippi. Stomach. Overstreet (1973); North Carolina. Manter (1931).

HETEROPHYIDAE

Stellantchasmus falcatus Onji and Nishio 1915. Metacercaria. Japan. Yamaguti (1971); Hawaii. Pearson (1964). 
Adleriella minutissima (Wittenberg 1929) Wittenberg 1930.

Metacercaria. Israe7. Paperna (1964b).

Heterophyes heterophyes (Siebold 1853) Stiles and Hassall 1900.

Metacercaria. Israet. Muscles. Paperna (1964b).

Heterophyes nocens Onji and Nishio 1915. Metacercaria. Japan. Komiya and Suzuki (1966).

Heterophyes katsuradai Ozaki and Asada 1926. Metacercaria. Japan. Komiya and Suzuki (1966).

Heterophyopsis continua (Onji and Nishio 1916) Price 1940.

Metacercaria Japan. Komiya and Suzuki (1966).

Haplorchis yokogawai (Katsuta 1932) Chen 1936. Metacercaria. Japan. Komiya and Suzuki (1966).

Haplorchis microrchis (Katsuta 1932) Chen 1936. Metacercaria. Japan. Komiya and Suzuki (1966).

Pygidiopsis summa Onji and Nishio 1916. Metacercaria. Japan. Komiya and Suzuki (1966).

Procerovum varium Onji and Nishio 1916. Metacercaria. Japan. Komiya and Suzuki (1966).

Stictodora perpendicula (Onji and Nishio 1916) Yamaguti 1958.

Metacercaria. Japan. Komiya and Suzuki (1966).

Stictodora fuscata (Onji and Nishio 1916). Metacercaria. Japan. Komiya and Suzuki (1966).

Pseudogalactosoma macrostoma Yamaguti 1942. Metacercaria. Japan. Komiya and Suzuki (1966).

Centrocestus asadai Mishima 1959 . Metacercaria. Japan. Komiya and Suzuki $(1966)$.

Metagonimus minutus Katsuta 1932. Metacercaria. Japan. Komiya and Suzuki (1966).

Metagonimus yokogawai (Katsurada 1912) Katsurada 1912. Metacercaria. Japan. Komiya and Suzuki (1966).

Phagicola longa (Ransom 1920) Price 1932. Metacercaria. Florida. Hutton (1964); Israel. Paperna (1964b) as Parascocotyle l.

Galactosomum sanaensis Kobayasi 1942. Metacercaria. China. Pearson (1973).

\section{MONORCHI IDAE}

Lasiotocus mugilis Overstreet 1969. Hawaii. Yamaguti (1970); Florida. Intestine. Overstreet (1969).

Lasiotocus giebulentus Overstreet 1971. Mississippi, Alabama. Intestine. Overstreet (1971b).

\section{SANGUINICOLIDAE}

Cardicola mugilis Yamaguti 1970. Hawaii. Vascular system. Yamaguti (1970).

Plethorchis acanthus Martin 1975. Australia. Coelom, blood vessels of mesentery, intestine and liver. Martin (1975).

\section{STRIGEIDAE}

Cardiocephalus medioconiger Dubois and Perez-Vigueras 1949. North Carolina. Skinner (1975). 
Mugil cephalus (Continued)

HIRUDINOIDEA

GLOSSIPHONIIDAE

Genera and species. Florida. Gills. Baker22.

\section{PISCICOLIDAE}

Myzobdella lugubris Leidy 1851. Georgia, Texas. External surface. Sawyer et a1. (1975).

Piscicola sp. Florida. Body surface, nostrals, mouth. Skinner (1975).

\section{ISOPODA}

\section{CYMOTHOIDAE}

Codonophilus gilberti (Richardson 1904) Hale 1926. Baja California. Gills. Brusca (1977).

Nerocila californica Schioedte and Meinert 1881. Gulf of California. Gi11s. Brusca (1977).

\section{MONOGENEA}

\section{CAPSALIDAE}

Neobenedenia pacifica Bravo-Holi is 1971. Baja California. Gills. Bravo-Hol1 is (1971).

\section{DACTYLOGYRIDAE}

Pseudohal iotrema mugilinus Hargis 1955. Florida. Gills. Hargis (1955b).

Ancyrocephalus vanbenedeni (Parona and Perugia 1890) Johnston and Tiegs 1922. Black Sea. Gills. Pogoreltseva (1964); Israel. Paperna (1964b); Georgia. Rawson (1973).

Cleidodiscus sp. Florida. Gills. Baker 22.

\section{GYRODACTYLIDAE}

Gyrodactylus mugil is Rawson 1973. Georgia. Skin. Rawson (1973). Polyclithrum mugilini Rogers 1967. Georgia. Fins, body. Rogers (1967).

Micropolyclithrum parvum Skinner 1975. Florida. Skin. Skinner (1975).

\section{MICROCOTYLIDAE}

Solostamenides pseudomugilis (Hargis 1956) Unnithan 1971. Florida. Gills. Hargis (1956a) as Microcotyle p.

Solostamenides mugilis (Vogt 1878) Unnithan 1971. Japan. Gills. Yamaguti (1938); France. Euzet and Combes (1969) both as Microcotyle $\mathrm{m}$. 
Metamicrocotyla macracantha (Alexander 1954) Korath 1955. Baja California. Gills. Alexander (1954) as Microcotyle m.; Florida. Gilis. Hargis (1956a); Australia. Young (1970). Metamicrocotyla bora Yamaguti 1953. Macassar. Gi11s. Yamaguti (1953a).

Metamicrocotyla cephalus (Azim 1939) Hargis 1954. Sète, France. Gills. Euzet and Combes (1969).

Metamicrocotyla filiformis Yamaguti 1953. Macasser. Yamaguti (1953a).

Metamicrocotyla mugilis Yamaguti 1968. Hawaii. Gills. Yamaguti (1968b).

\section{UDONELLIDAE}

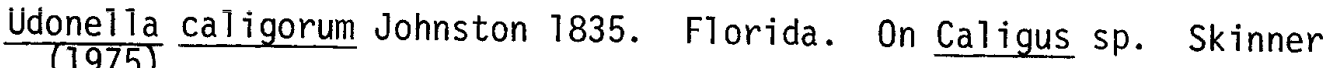

\section{NEMATODA}

\section{ANISAKIDAE}

Contracaecum spiculigerum (Rudolphi 1809) Railliet and Henry 1912. Larva. Caspian Sea. Petrushevski (1955). Contracaecum robustum Chandler 1935. Larva. Texas. Kidneys. Chandler (1935).

Contracaecum sp. Juvenile. Florida. Hutton (1964); Israel. Intestine. Paperna (1964b).

Raphidascaris? adelinae Cond.-Franc. 1898. Rome. Yamaguti (1961).

Thynnascaris sp. Larva. Florida. Cysts in liver. Skinner (1975).

CAPILLARI IDAE

Capillaria sp. Georgia. Rawson (1973).

\section{CUCULLANIDAE}

Cucullanus sp. New Zealand. Intestine. Hewitt and Hine (1972). Cucul1anus dodsworthi Barreto 1922. Florida. Intestine. Skinner (1975).

\section{PHILOMETRIDAE}

Philometra sp. Israel. Intestine. Paperna (1964b). Philometra cephalus Ramachandran 1975. India. Gonads. Ramachandran (1975).

PROTOZOA (CILIOPHORA)

EPISTYLIDAE

Epistylis sp. Florida. Hutton (1964). 
Mugil cephalus (Continued)

URCEOLARI IDAE

Trichodina sp. Florida. Gills. Skinner (1975).

Trichodina puytoraci Lom 1962. Black Sea. Zaika (1968).

Tripartiel1a obliqua Lom 1962. Black Sea. Zaika (1968).

PROTOZOA (MYXOSPORIDA)

MYXIDI IDAE

Zschokkella dogieli Pogorel 1964. Black Sea. Zaika (1968).

Zschokkella nova Klokacawa 1914. USSR. Galibladder. Reshetnikova (1955).

Myxidium incurvatum Thélohan 1892. Gallbladder. Kudo (1920).

MYXOBOLIDAE

Myxobolus mülleri Bütschli 1882. Black Sea. Zaika (1968).

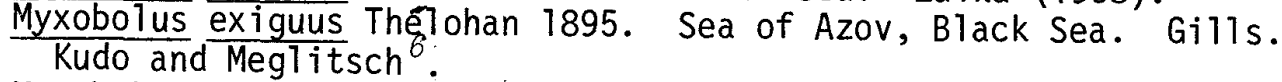

Myxobolus parvus Shulman 1962. China. Gills. Kudo and Meglitsch6.

Myxobolus cheni Shuiman 1962. China. Muscles. Kudo and Meglitsch6.

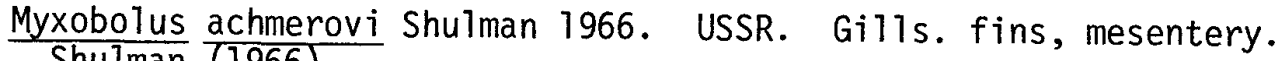
Shu7man (1966).

\section{MYXOSOMATIDAE}

Myxosoma dermatobia (Ishij 1916) Hoshina 1952. Florida. Surface Myxosoma cephal is Iverson, Chitty, Van Meter 1971. South Florida. Cysts in brain, gill arches, buccal cavity, jaw bone. Iverson et a1. (1971).

Myxosoma branchialis (Markevich 1932) Lom 1961. USSR. Gills, muscies. Shutman (1966).

TETRACAPSUL IDAE

Kudoa bora (Fujita 1930) Meglitsch 1947. Formosa. Muscles. Kudo
and
Meglitsch 6.

PROTOZOA (SARCOMASTIGOPHORA)

TRYPANOSOMATIDAE

Trypanosoma sp. Florida. Blood. Baker 22 .

PROTOZOA (SPOROZOA)

HAEMOGREGARINIDAE

Haemogregarina mugili. Florida. Blood. Saunders (1964). 


\section{SPHYRAENIDAE -- Barracudas \\ Sphyraena argentea - California Barracuda}

CESTODA

DASYRHYNCHIDAE

Callitetrarhynchus gracilis (Rudolphi 1819) Pintner 1931. Larva. Southern California. Viscera. Jensen 18.

TRYPANORHYNCHA

Genera and species. Larva. Southern California. Jensen18. COPEPODA

BOMOLOCHIDAE

* Bomolochus solae Claus 1864. Baja California. Causey (1960). CALIGIDAE

$\frac{\text { Lepeophtheirus dissimulatus Wilson 1905. Baja California. Causey }}{(1960) \text {. }}$ Caligus productus Dana 1953. West coast of Mexico. Causey (1960). Caligodes laciniatus (Krøyer 1863) Heller 1865. West coast of

MONOGENEA

AXINIDAE

Heteraxine argentea (Crane 1972) Crane 1974. Southern California. Gi17s. Crane (1972) as Pseudochauhanea a.

$$
\begin{gathered}
\text { POLYNEMIDAE -- Threadfins } \\
\frac{\text { POIydactyTuS approximans }}{\text { SYNONYM - BTue Bobo }} \text { - Polynemus approximans }
\end{gathered}
$$

DIGENEA

OPECOELIDAE

Opecoeloides eucinostomi (Manter 1940) Von Wicklen 1946. Columbia, Panama. Intestine. Manter (1940a) as Anisoporus e.

MONOGENEA

MICROCOTYLIDAE

Microcotyloides impudicus Caballero, Bravo-Hollis, and Grocott 1955. Panama. Gills. Caballero et a1. (1955). 


\title{
Polydactylus opercularis - Yellow Bobo \\ SYNONYM - Polynemus opercularis
}

COPEPODA

\section{CALIGIDAE}

Caligus coryphaenae Steenstrup and Lütken 1861. East Pacific. Body surface. Shitno (1959c).

\section{DIGENEA}

\section{BUCEPHALIDAE}

Prosorhynchus crucibulum (Rudolphi 1819) Odhner 1905. Panama. Kidneys. Cabal1ero et a1. (1953).

\author{
BATHYMASTERIDAE -- Ronquils \\ Ronquilus jordani - Northern Ronquil
}

DIGENEA

HEMIURIDAE

Derogenes varicus (Mü1ler 1784) Looss 1901. Alaska. Kruse (1977). Lecithaster gibbosus (Rudolphi 1802) Lühe 1901. British Columbia. Intestine. Arai (1969b).

NEMATODA

ANISAKIDAE

Anisakis sp. Larva. British Columbia. Body cavity. Arai (1969b).

CUCULLANIDAE

Cucullanus sp. British Columbia. Intestine. Arai (1969b).

$$
\text { CLINIDAE - - Clinids }
$$

Gibbonsia $\mathrm{sp.}$

DIGENEA

HEMIURIDAE

Genolinea laticauda Manter 1925. Northern California. Stomach. Chapa 23 . 
Sterrhurus exodicus (McFarlane 1936) Yamaguti 1958. Northern

California. Stomach. Chapa ${ }^{23}$ as Separogermiductus e.

\section{OPECOELIDAE}

Helicometrina elongata Noble and Park 1937. Northern California.

Intestine. Chapa23.

Podocotyle sp. Northern California. Intestine, caeca. Edmiston ${ }^{29}$.

$$
\text { Gibbonsia elegans - Spotted Kelpfish }
$$

\section{DIGENEA}

\section{OPECOELIDAE}

Helicometrina elongata Noble and Park 1937. Southern California.

Intestine. Manter and Van Cleave (1951).

Helicometrina nimia Linton 1910. Intestine. Arai (1962).

Podocotyle gibbonsiae Johnson 1949. California. Intestine. Johnson (1949).

PROTOZOA (MYXOSPORIDA)

\section{CERATOMYXIDAE}

Leptotheca sphaerula Noble 1939. Central California. Gallbladder. Noble (1947).

Leptotheca elegans Noble 1938. Southern California. Gallbladder. Noble (1938).

Ceratomyxa blennius Noble 1938. California. Gallbladder.

Noble $(1941)$.

MYXIDIIDAE

Sphaeromyxa gibbonsia Noble 1939. Southern California. Gallbladder. Nob7e (7939).

TRILOSPORIDAE

Trilospora californica Noble 1939. Southern California.

GaTTbTadder. NobTe (1939).

$$
\begin{aligned}
& \text { Gibbonsia metzi - Striped Kelpfish } \\
& \text { SYNONYMS - Gibbonsia evides, G. mitzi }
\end{aligned}
$$

\section{DIGENEA}

GORGODERIDAE

Phyllodistomum borisbychowski Caballero and Caballero 1969. Southern CaTifornia. Intestine. Caballero and Caballero (1969a). 
OPECOELIDAE

Pseudopecoelus gibbonsiae Manter and Van Cleave 1951. Southern California. Intestine. Manter and Van Cleave (1951).

Helicometrina elongata Noble and Park 1937. Southern California. Intestine. Manter and Van Cleave (1951).

Genitocotyle acirra Park 1937. Central California. Intestine. Chapa2 3 as G. acirrata.

PROTOZOA (MYXOSPORIDA)

\section{CERATOMYXIDAE}

Leptotheca sphaerula Noble 1939. Southern California. Urinary bladder. Noble (1939).

Leptotheca elegans Noble 1938. Southern California. Gallbladder. Nob7e (1947).

Ceratomyxa blennius Noble 1938. Central California. Gallbladder. Noble (1947).

Ceratomyxa gracilis Jameson 1929. Central California. Gallbladder. Jameson (1929).

MYXIDIIDAE

Sphaeromyxa gibbonsia Noble 1939. Southern California. Gallbladder. NobTe (1939).

TRILOSPORIDAE

Trilospora californica Noble 1939. Southern California. Gallbladder. Noble (1941).

$$
\text { Heterostichus rostratus - Giant Kelpfish }
$$

COPEPODA

CALIGIDAE

Lepeophtheirus parviventris Wilson 1905. Southern California. Outer surface. WiTson (1935b).

\section{CHONDRACANTHIDAE}

Chondracanthus heterostichi Ho 1972. Southern California. Gill cavity. Ho (1972b).

Chondracanthus horridus Heller 1865. Central California. Gills. Wilson (1935b).

\section{DIGENEA}

OPECOELIDAE

Genitocotyle heterostichi Montgomery 1957. Southern California. Intestine. Montgomery (1957). 
Neoclinus blanchardi - Sarcastic Fringehead

CESTODA

\title{
TETRÃPHYLLIDEA
}

Genera and species. Red pigmented plerocercoid. Southern California.

Pyloric caeca, intestine. Kunnenkeri and Martin (1962).

ISOPODA

CYMOTHOIDAE

Lironeca vulgaris Stimpson 1857. Southern California. Gills.

Turner et a1. (1969).

$$
\text { Neoclinus uninotatus - Onespot Fringehead }
$$

DIGENEA

OPECOELIDAE

Helicometrina elongata Noble and Park 1937. Southern California. Intestine. Montgomery (1957).

\section{ZOOGONIDAE}

Diphtherostomum macrosaccum Montgamery 1957. Southern California. Stomach. Mont gomery (1957).

$$
\begin{aligned}
& \text { Paraclinus integripinnis - Reef Finspot } \\
& \text { SYNONYM - Auchenopterus integripinnis }
\end{aligned}
$$

PROTOZOA (MYXOSPORIDA)

CERATOMYXIDAE

Leptotheca elegans Noble 1938. Southern California. Gallbladder. Nob7e (1939).

\author{
BLENNIIDAE -- Combtooth Blennies \\ Hypsoblennius gilberti - Rockpool Blenny
}

PROTOZOA (MYXOSPORIDA)

\section{CERATOMYXIDAE}

Ceratomyxa blennius Noble 1938. Southern California. Gallbladder. Noble (1938). 


\section{STICHAEIDAE -- Pricklebacks \\ Anoplarchus purpurescens - High Cockscomb}

DIGENEA

HEMIURIDAE

Lecithaster gibbosus (Rudolphi 1802) Lühe 1901. British Columbia. Robinson et a1.30

Tubulovesicula lindbergi (Layman 1930) Yamaguti 1934. Oregon.

Stomach. Mccautey (1960).

OPECOELIDAE

Podocotyle californica Park 1937. Northern California. Intestine. Nahhas and Krupin (1977).

NEMATODA

ANISAKIDAE

Anisakis sp. Larva. British Columbia. Body cavity. Arai (1969b).

Contracaecum sp. Larva. British Columbia. Body cavity. Arai (1969b).

Phocanema sp. Larva. British Columbia. Body cavity. Arai (1969b) as Porrocaecum sp.

CYSTIDICOLIDAE

Ascarophis sebastodis 01 sen 1952. British Columbia. Intestine. Arai (1969b).

PROTOZOA (MYXOSPORIDA)

MYXIDIIDAE

Zschokkella sp. Washington. Gallbladder. Kuris ${ }^{20}$.

\section{Cebidichthys sp.}

DIGENEA

OPECOELIDAE

Opecoelus adsphaericus Manter and Van Cleave 1951. California.

Intestine. Banerjee (1965). Likely $\underline{\mathrm{C}}$. violaceus

$$
\text { Cebidichthys violaceus - Monkeyface Prickleback }
$$

\section{DIGENEA}

HEMIURIDAE

Opisthadena bodegensis Johnson and Copsey 1953. Northern California. Gastro-intestinal tract. Johnson and Copsey (1953). 
DIGENEA

ZOOGONIDAE

Zoogonus dextrocirrus Aldrich 1961. Washington. Intestine. Aldrich (1961).

Lumpenus sagitta - Snake Prickleback

COPEPODA

CALIGIDAE

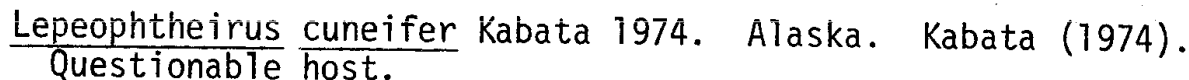

DIGENEA

HEMIURIDAE

Genolinea manteri Lloyd 1938. Oregon. McCauley (1960).

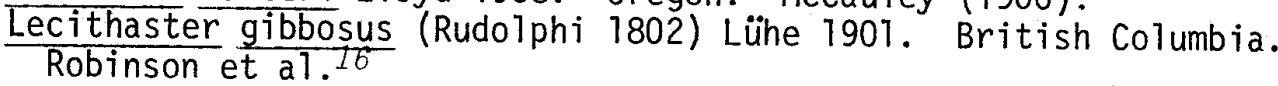

Xiphister atropurpureus - Black Prickleback

SYNONYMS - Anoplarchis atropurpureus, Cebidichthys atropurpureus, Epigeichthys atropurpureus

COPEPODA

CALIGIDAE

Lepeophtheirus parviventris Wilson 1905. British Columbia. Fraser (1920).

\section{CHONDRACANTHIDAE}

Chondracanthus pinguis Wilson 1912. British Columbia. Wilson (1912b). DIGENEA

OPECOELIDAE

Podocotyle atomon (Rudolphi 1802) Odhner 1905. British Columbia. Intestine. McFarlane (1936).

Podocotyle californica Park 1937. Northern California. Intestine. Nahhas and Krupin (1977).

Podocotyle reflexa (Creplin 1825) Odhner 1905. British Columbia. Intestine. McFarlane (1936). 
Xiphister atropurpureus (Continued)

NEMATODA

PHILOMETRIDAE

Philometra americana Kuitunen-Ekbaum 1933. British Columbia. Subcutaneous. Kuitunen-Ekbaum (1933a).

$$
\text { Xiphister mucosus - Rock Prickleback }
$$

DIGENEA

OPECOELIDAE

Podocotyle californica Park 1937. Northern California. Intestine. Nahhas and Krupin (1977).

\author{
PHOLIDAE -- Gunnels \\ Apodichthys flavidus - Penpoint Gunnel
}

CESTODA

BOTHRIOCEPHALIDAE

Bothriocephalus scorpij (Mül ler 1776) Rudolphi 1808. British Columbia. Margolis's.

COPEPODA

CHONDRACANTHIDAE

Chondracanthus pusillus Kabata 1968. British Columbia. Gill cavities. Kabata (1968).

\title{
DIGENEA
}

OPECOELIDAE

Podocotyle apodichthysi Park 1937. California. Intestine. Park (1937b). NEMATODA

ANISAKIDAE

Anisakis sp. Larva. British Columbia. Body cavity. Arai (1969b).

* Thynnascaris adunca (Rudolphi 1802) Hartwich 1957. British Columbia. Smedley (T934) as Contracaecum magnum. 
Apodichthys flavidus (Continued)

CAPILLARI IDAE

Capillaria sp. British Columbia. Stomach. Arai (1969b).

PROTOZOA (MYXOSPORIDA)

CERATOMYXIDAE

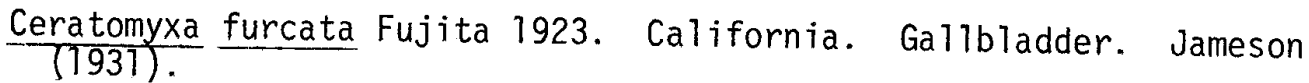

$$
\text { Phol is laeta - Crescent Gunnel }
$$

CESTODA

BOTHRIOCEPHALIDAE

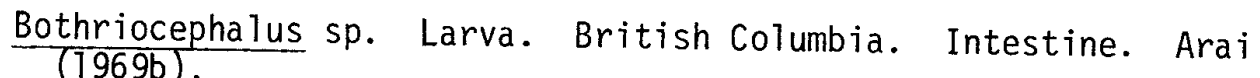

\section{DIGENEA}

OPECOEL IDAE

Podocotyle sp. Immature. British Columbia. Intestine. Arai (1969b). NEMATODA

\section{ANISAKIDAE}

Anisakis sp, Larva. British Columbia. Body cavity. Arai (1969b).

Contracaecum sp. Larva. British Columbia. Body cavity. Arai (1969b). PHILOMETRIDAE

Philometra americana Kuitunen-Ekbaum 1933. British Columbia. Intestine. Arai (1969b).

$$
\text { Phol is ornata - Saddleback Gunnel }
$$

\section{DIGENEA}

HEMIURIDAE

Lecithaster gibbosus (Rudolphi 1802) Lühe 1901. British Columbia. Intestine. Arai (1969b).

\section{OPECOELIDAE}

Podocotyle pacifica Park 1937. British Columbia. Intestine. Arai (1969b) as Neopodocotyloides p. 
Pholis ornata (Continued)

MONOGENEA

GYRODACTYLOIDEA

Genera and species. British Columbia. Gills. Arai (1969b).

NEMATODA

ANISAKIDAE

Anisakis sp. Larva. British Columbia. Body cavity. Arai (1969b).

PHILOMETRIDAE

Philometra americana Kuitunen-Ekbaum 1933. British Columbia.

Subcutaneous. Kuitunen-Ekbaum (1933a).

$$
\begin{gathered}
\text { ANARHICHADIDAE -- Wolffishes } \\
\text { Anarrhichthys ocellatus - Wolf-eel }
\end{gathered}
$$

MONOGENEA

GYRODACTYLIDAE

Gyrodactylus corti Mizelle and Kritsky 1967. Steinhart Aquarium, California. On skin. Mizelle and Kritsky (1967d).

$$
\begin{aligned}
& \text { CRYPTACANTHODIDAE -- Wrymouths } \\
& \text { Delolepis gigantea - Giant Wrymouth }
\end{aligned}
$$

CESTODA

BOTHRIOCEPHALIDAE

Bothriocephalus scorpii (Müller 1776) Rudolphi 1808. British Columbia. Pyloric caeca, intestine. Margolis ${ }^{3}$.

\section{NEMATODA}

ANISAKIDAE

* Thynnascaris adunca (Rudolphi 1802) Hartwich 1957. British Columbia. Stomach, intestine. Margol is ${ }^{3}$ as Contracaecum a. Anisakis sp. Larva. British Columbia. Throughout body cavity. MargoT is ${ }^{3}$. 
DIGENEA

HEMIURIDAE

Lecithaster gibbosus (Rudoiphi 1802) Lühe 1901. British Columbia. Robinson et a. 30

\author{
AMMODYTIDAE -- Sand Lances \\ Ammodytes hexapterus - Pacific Sandlance \\ SYNONYMS - Ammodytes tobianus personatus, Ammodytes personatus
}

ACANTHOCEPHALA

ECHINORHYNCHIDAE

Echinorhynchus gadi Zoega in Mü1ler 1776. British Columbia. Intestine. Arai (1969b).

\title{
CESTODA
}

PHYLLOBOTHRIIDAE

Phyllobothrium sp. Larva. British Columbia. Intestine. Arai (1969b).

COPEPODA

CALIGIDAE

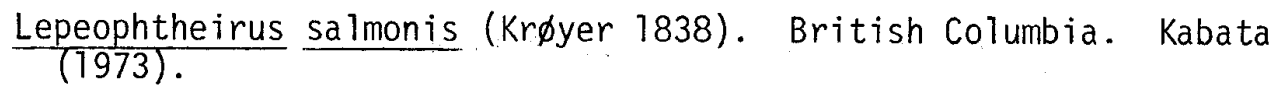

Lepeophtheirus sp. British Columbia. Body surface. Arai (1969b).

PENNELLIDAE

Haemobaphes sp. British Columbia. Kabata (1967b).

\section{DIGENEA}

HEMIURIDAE

Lecithaster gibbosus (Rudolphi 1802) Lühe 1901. British Columbia. Intestine. Arai (1969b); Japan. Yamaguti (1951) as L. Salmonis. HETEROPHYIDAE

Galactosomum sp. Larva. British Columbia. Gills (encysted). Arai (1969b). 
Ammodytes hexapterus (Continued)

MONOGENEA

GYRODACTYLOIDEA

Genera and species. British Columbia. Gills. Arai (1969b).

GYRODACTYLIDAE

Gyrodactylus ammodyti Zhukov 1960. Bering Sea. Gills. Zhukov $(1960 \mathrm{~b})$.

Gyrodactylus harengi Malmberg 1956. Bering Sea. Gills. Zhukov $(1960 \mathrm{~b})$.

NEMATODA

ANISAKIDAE

Anisakis sp. Larva. British Columbia. Body cavity. Arai (1969b).

Contracaecum sp. Larva. British Columbia. Mesentery, liver, stomach Wa17. Margol is 3 .

GOBIIDAE -- Gobies

Acanthogobius flavimanus - Yellowfin Goby

COPEPODA

CALIGIDAE

Caligus punctatus Shiino 1955. Japan. Exterior. Shiino (1955b). CHONDRACANTHIDAE

Acanthochondria yui Shiino 1964. Japan. Operculums, gill arches, roof of buccal cavity. Shiino (1964).

ERGASILIDAE

Ergasilus oriental is Yamaguti 1939. Japan. Gills. Yamaguti (1939a).

\section{DIGENEA}

BUCEPHALIDAE

Prosorhynchus uniporus Ozaki 1924. Metacercaria. Japan. Cysts in fins. Yamaguti (1971).

CRYPTOGONIMIDAE

Cryptogonimus sp. Metacercaria. Japan. Cysts in fins. Yamaguti (7938). 
Acanthogobius flavimanus (Continued)

FELLODISTOMATIDAE

Tergestia acanthogobii Yamaguti 1938. Japan. Intestine. Yamaguti 24 .

HEMIURIDAE

Lecithaster stellatus Looss 1907. Japan. Intestine. Yamaguti (1940). HETEROPHYIDAE

Procerovum varium Onji and Nishio 1916. Metacercaria. Pearson $(1964)$.

Metagonimus yokogawai (Katsurada 1912) Katsurada 1912.

Metacercaria. Japan. Komiya and Suzuki (1966).

Centrocestus asadai Mishima 1959. Metacercaria. Japan. Komiya and Suzuki $(1966)$.

Heterophyes nocens Onji and Nishio 1915. Metacercaria. Japan. Komiya and Suzuki (1966).

Stellantchasmas falcatus Onji and Nishio 1915. Metacercaria. Japan. Komiya and Suzuki (1966).

Pygidiopsis summa Onji and Nishio 1916. Metacercaria. Japan. Komiya and Suzuki (1966).

Stictodora perpendicula (Onji and Nishio 1916) Yamaguti 1958.

Metacercaria. Japan. Komiya and Suzuki (1966).

Stictodora fuscata (Onji and Nishio 1916). Metacercaria. Japan. Komiya and Suzuki (1966).

Pseudogalactosoma macrostoma Yamaguti 1942. Metacercaria. Japan. Komiya and Suzuki (1966).

OPECOEL IDAE

Ozakia orthorchis (0zaki . 1926) Wisniewski 1934. Japan. Intestine, ga17bTadder. Yamaguti 24 as Coitocaecum orthorchis.

\section{ZOOGONIDAE}

Zoogonoides acanthogobii Yamaguti 1938. Japan. Intestine. Yamaguti24. MONOGENEA

MICROCOTYLIDAE

Aspinatrium acanthogobii (Yamaguti 1940) Yamaguti 1963. Japan. Gills. Yamaguti (1940) as Microcotyle a.

PROTOZOA (CILIOPHORA)

URCEOLARI IDAE

Tríchodina sp. Japan. Gills. Noble $\mathrm{e}^{31}$. 
Acanthogobius flavimanus (Continued)

PROTOZOA (MYXOSPORIDA)

CERATOMYXIDAE

Ceratomyxa sp. Japan. Gallbladder. Noble31.

MYXIDI IDAE

Myxidium sp. Japan. Gallbladder. Noble 31.

MYXOBOLIDAE

Myxobolus acanthogobi i Hoshina 1952. Japan. Eyes, brain, spinal nerves. Hoshina (1952).

Henneguya tridentigeri Ozaki and Ishizaki 1941. Japan. Gi11s. Hoshina (1952).

TRIGONOSPORIDAE

Trigonosporus acanthogobii Hoshina 1952. Japan. Gills. Hoshina (1952).

$$
\text { Clevelandia ios - Arrow Goby }
$$

\section{CESTODA}

DASYRHYNCHIDAE

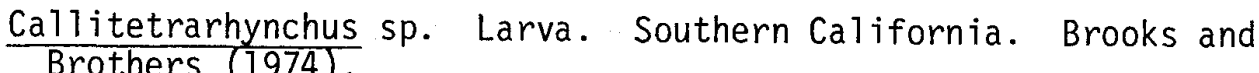

\section{TETRAPHYLLIDEA}

Genera and species. Larva. California. Lumen. Martin and Multani (1970).

\section{DIGENEA}

HEMIURIDAE

Lecithaster minimus Martin and Multani 1970. Southern California. Brooks and Brothers (1974).

MONOGENEA

GYRODACTYLIDAE

Gyrodactylus perforatus Mizelle and Kritsky 1967. California. Skin. Mizizelle and Kritsky (1967c). 
Clevelandia ios (Continued)

NEMATODA

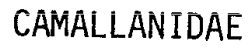

Spirocamallanus sp. Southern California. Brooks and Brothers (1974).

$$
\text { Coryphopterus nicholsi - Blackeye Goby }
$$

CESTODA

PHYLLOBOTHRI IDAE

Phyllobothrium sp. Larva. British Columbia. Intestine. Arai (1969b). DIGENEA

HEMIURIDAE

Lecithaster gibbosus (Rudolphi 1802) Lühe 1901. British Columbia. Intestine. Arai (1969b).

NEMATODA

ANISAKIDAE

Contracaecum sp. Larva. British Columbia. Body cavity. Arai (1969b). Gillichthys mirabilis - Longjaw Mudsucker

ACANTHOCEPHALA

NEOECHINORHYNCHIDAE

Microsentis wardae Martin and Multani 1966. Baja California. Intestine. Martin and Multani (1966).

COPEPODA

ERGASILIDAE

Ergasilus auritus Markevich 1940. Southern California. Gills. Roberts (1970).

\section{DIGENEA}

CYATHOCOTYLIDAE

Mesostephanus appendiculatus (Ciurea 1916) Lutz 1935. Metacercaria. Southern California. Muscles. Martin (1961).

DIDYMOZOIDAE

Genera and species. Larva. Southern California. Gills. Noble et al. (1963) as Torticaecum fenestratum. 
HEMIURIDAE

Hysterolecitha trilocalis King and Noble 1967. Southern California. Gills, stomach, esophagus. King and Noble (1961).

Lecithaster minimus Martin and Multani 1970. Baja California. Intestine (posterior). Martin and Multani (1970).

\section{HETEROPHYIDAE}

Stictodora hancocki (Martin 1950) Witenberg 1953. Metacercaria. Southern California. Muscles, brain case, etc. Martin (1950) as Parastictodora $h$.

Ascocotyle sexidigita Martin and Steele 1970. Baja California. Intestine. Martin and MuTtani (1970).

Ascocotyle angrense Travassos 1916. Metacercaria. Southern California. Gills. Baker ${ }^{22}$.

\section{RENICOLIDAE}

Renicola buchanani (Martin and Gregory 1951). Metacercaria. Southern California. Liver. Martin (1971).

\section{MONOGENEA}

\section{GYRODACTYLIDAE}

Gyrodactylus imperialis Mizelle and Kritsky 1967. Southern California. Skin. Mizelle and Kritsky (1967b).

Gyrodactylus olsoni Mizelle and Kritsky 1967. Southern California. Skin. Mizelle and Kritsky (1967b).

Gyrodactylus elegans Nordmann 1832. Southern California. Gills. Noble $(1961)$.

\section{NEMATODA}

\section{CAMALLANIDAE}

Spirocamallanus pereirai (Annereaux 1946) 01 sen 1952. Southern California. Intestine. Noble and King (1960).

\section{THELAZIIDAE}

Vasorhabdochona cablei Martin and Zam 1967. Baja California. Mesenteric blood vessels near bile duct. Martin and Zam (1967). PROTOZOA (CILIOPHORA)

URCEOLARI IDAE

Trichodina sp. Southern California. Gills. Noble (1961). 
Ilypnus gilberti - Cheekspot Goby

CESTODA

DASYRHYNCHIDAE

Callitetrarhynchus sp. Larva. Southern California. Brooks and Brothers (1974).

TETRAPHYLLIDEA

Scolex pleuronectis Müller 1788. Southern California. Gallbladder, intestine, mesentery. Brooks and Brothers (1974).

DIGENEA

HEMIURIDAE

Lecithaster minimus Martin and Multani 1970. Southern California. Brooks and Brothers (1974).

NEMATODA

CAMALLANIDAE

Spirocamallanus sp. Southern California. Brooks and Brothers (1974).

Lythrypnus dalli = Bluebanded Goby

DIGENEA

ACANTHOCOLPIDAE

Stephenostomum sp. California. Urethra. Haaker ${ }^{32}$.

OPECOELIDAE

Manteriella sp. California. Stomach and intestine. Haaker ${ }^{32}$.

Quietula y-cauda - Shadow Goby

CESTODA

DASYRHYNCHIDAE

Callitetrarhynchus sp. Larva. Southern California. Brooks and Brothers (1974).

TETRAPHYLLIDEA

Scolex pleuronectis Mũller 1788. Southern California. Galibladder, intestine, mesentery. Brooks and Brothers (1974). 
Quietula y-cauda (Continued)

DIGENEA

HEMIURIDAE

Lecithaster minimus Martin and Multani 1970. Southern California. Brooks and Brothers (1974).

NEMATODA

CAMALLANIDAE

Spirocamallanus sp. Southern California. Brooks and Brothers (1974).

Typhlogobius californiensis - BIind Goby

PROTOZOA (MYXOSPORIDA)

CERATOMYXIDAE

Leptotheca elegans Noble 1938. Southern California. Gallbladder. Noble (1947).

Ceratomyxa vepallida Meglitsch 1960. Southern California.

GalTb7adder. NobTe 31 .

TRILOSPORIDAE

Trilospora californica Noble 1939. Southern California. Gallbladder. Noble $(19 \overline{39})$.

$$
\begin{gathered}
\text { GEMPYLIDAE -- Snake Mackerels } \\
\text { Gempylus serpens - Snake Mackerel }
\end{gathered}
$$

ACANTHOCEPHALA

RHADINORHYNCHIDAE

Gorgorhynchus robertdollfusi Golvan 1956. Mauritania. Intestine. GoTvan (1956).

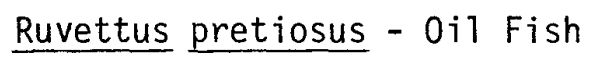

COPEPODA

\section{DISSONIDAE}

Dissonus ruvetti Nunes-Ruivo and Fourmanoir 1956. Madagascar. Ana 7 fins. Nunes-Ruivo and Fourmanoir (1956). 
Ruvettus pretiosus (Continued)

DIGENEA

DIDYMOZOIDAE

Neounitubulotestis orbitarium (Do17fus 1956) Yamaguti 1971. Morocco. Yamaguti (1971).

SANGUINICOLIDAE

Aporocotyle pacifica Yamaguti 1970. Hawaii. Yamaguti (1970).

SCOMBRIDAE - Mackerels and Tunas

Auxis rochei - Bullet Mackerel

\section{DIGENEA}

BUCEPHALIDAE

Rhipidocotyle capitata (Linton 1940) Manter 1947. Massachusetts. Linton (1940).

\section{DIDYMOZOIDAE}

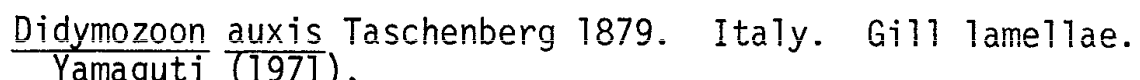

Didymozoon sp. Gi11s. Linton (1940).

FELLODISTOMATIDAE

Tergestia pectinata (Linton 1905) Manter 1940. Massachusetts. Intestine. Linton (1940).

\section{GASTEROSTOMIDAE}

Gasterostomum capitatum Linton 1940. Massachusetts. Linton (1940).

$$
\text { Auxis thazard - Frigate Mackerel }
$$

\section{ACANTHOCEPHALA}

\section{ECHINORHYNCHIDAE}

Neorhadinorhynchus nudus (Harada 1938) Yamaguti 1939. South China Sea. Intestine. Mamaev (1968b) as Echinorhynchus n.

RHADINORHYNCHIDAE

Rhadinorhynchus selkirki Van Cleave 1921. Japan. Yamaguti (1963c). 


\section{CESTODA}

\section{DASYRHYNCHIDAE}

Callitetrarhynchus gracilis (Rudolphi 1819) Pintner 1931. South China Sea. Intestine, Tiver. Mamaev (1968b).

\section{TRYPANORHYNCHA}

Genera and species. South China Sea. Intestine, Tiver. Mamaev (1968b). Scolex sp. South China Sea. Intestine. Mamaev (1968b).

\section{COPEPODA}

\section{BOMOLOCHIDAE}

Unicolax mycterobius (Vervoort 1965) Cressey and Cressey 1980. Gulf of Guinea. Nasal fossae. Vervoort (1965) as Parabomolochus m.

\section{CALIGIDAE}

Caligus productus Dana 1853. Eastern Pacific. Body surface. Shitino (1959d).

Caligus auxisi Pillai 1963. India. Operculums. Pillai (1963).

\section{DIGENEA}

\section{BUCEPHALIDAE}

Rhipidocotyle capitata (Linton 1940) Manter 1947. Hawaii. Intestine. Manter (1947). Rhipidocotyle pentagonum (Ozaki 1924) Ekmann 1932. South China Sea. Stomach. Mamaev (1968b). Prosorhynchus sp. South China Sea. Stomach. Mamaev (1968b). Prosorhynchoides gracilescens (Rudolphi 1819) n. comb. Adriatic Sea. Intestíne. Sey (T970 a) as Bucephalopsis $g$.

\section{DIDYMOZOIDAE}

Didymozoon auxis Taschenberg 1879. Japan. Gills. Yamaguti (1951). Colocyntotrema auxis Yamaguti 1951. Japan. Pyloric caeca. Yamaguti (1957).

Phacelotrema claviforme Yamaguti 1951. Japan. Pyloric caeca. Yamaguti (1957).

Opepherotrema planum Yamaguti 1951. Japan. Pyloric caeca. Yamaguti (1951).

Oesophagocystis dissimulis (Yamaguti 1938) Yamaguti 1970. Pacific. Mamaev (1968b) as Didymocystis dissimilis. ["Mamaev's description and figures do not agree with mine" - Yamaguti (1971).]

Annulocystis auxis Yamaguti 1970. Hawaii. Gills. Yamaguti (1970). Metanematobothrium bivitellatum Mamaev 1968. Pacific. Giils. Mamaev (1968b).

Didymosphaera mirabilis Mamaev 1968. Viet Nam Gulf. Mamaev (1968a).

Didymocystis dissimilis Yamaguti 1938. South China Sea. Mamaev (1968b). Lobatozoum multisacculatum Ishii 1935. South China Sea. Gills. Mamaev (1968b). 
FELLODISTOMATIDAE

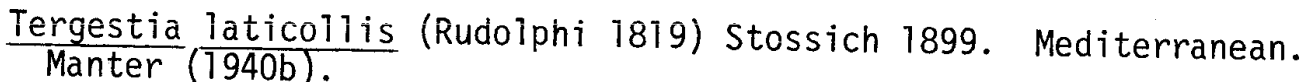

Tergestia acanthogobii Yamaguti 1938. Japan. Yamaguti (1971).

GORGODERIDAE

Phyllodistomum lancea Mamaev 1968. South China Sea. Urinary bladder. Mamaev (7968b).

\section{HEMIURIDAE}

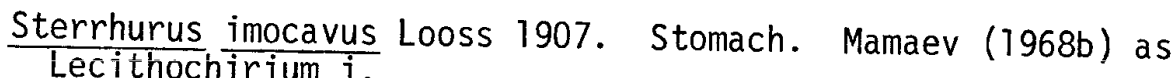

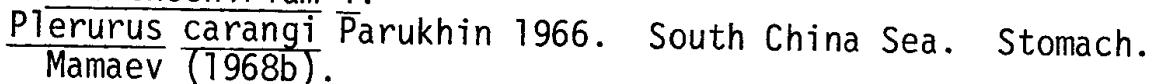

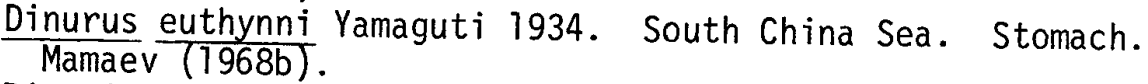

Dinurinae. Genera and species. Gulf of Mexico. Nikolaeva and Parukhin (1968).

Brachyphallus parvus (Manter 1947) Skrjabin and Guschanskaja 1955. Gulf of Mexico. Nikolaeva and Parukhin (1968).

$\frac{\text { Lecithochirium keokea Yamaguti 1970. Hawaii. Stomach. Yamaguti }}{(1970) \text {. }}$

\section{HIRUDINELLIDÁE}

Hirudinella sp. Japan. Stomach. Machida et al. (1970).

\section{MONOGENEA}

\section{CAPSALIDAE}

Caballerocotyla manteri affinis Mamaev 1968. South China Sea. Gil1s. Mamaev (1968b).

\section{GASTROCOTYLIDAE}

Pseudaxine sp. South China Sea. Gills. Mamaev (1968b).

Allopseudaxine macrova (Unnithan 1957) Yamaguti 1963. South China Sea. Gills. Mamaev (7968b).

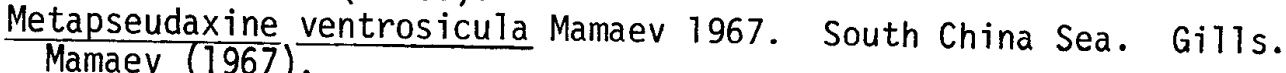

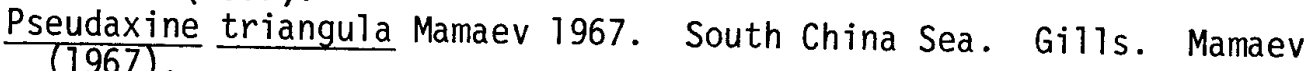

\section{HEXOSTOMATIDAE}

Hexostoma auxisi Palombi 1943. Italy. Gills. Price (1961); South China Sea. Mamaev (1968b).

Hexostoma keokeo Yamaguti 1968. Hawaii. Gills. Yamaguti (1968b). 
Auxis thazard (Continued)

NEMATODA

ANISAKIDAE

Contracaecum sp. Larva. South China Sea. Body cavity. Mamaev (1968b).

Anisakis sp. Larva. South China Sea. Body cavity. Mamaev (1968b).

CAMALLANIDAE

Oncophora melanocephala (Rudolphi 1819) Baudin-Laurencin 1970. Adriatic Sea. Intestine. Sey (1970'b) as Camallanus $\mathrm{m}$.

CYSTIDICOLIDAE

Ctenascarophis gastricus Mamaev 1968. South China Sea. Stomach. Mamaev (1968b).

Spinitectus ? mollis Mamaev 1968. South China Sea. Stomach. Mamaev (1968ठ).

Spinitectus sp. South China Sea. Stomach. Mamaev (1968b).

PROTOZOA (SPOROZOA)

HAEMOGREGARINIDAE

Haemogregarina bigemina Laveran and Mesni1 1901. Puerto Rico. B1ood. Saunders (1966).

\author{
Euthynnus affinis - Kawakawa \\ SYNONYMS - Thunnus thynnina, Thynnus thynnina
}

Three references below, marked by asterisks, refer to references to Thunnus or Thynnus thunnina. Hubbs (pers. comm.) states that

T. thynnina is now two species of Euthynnus, E. affinis and E. 1ineatus. However, the three references are all from the Red Sea, where only

E. affinis is found.

\title{
ACANTHOCEPHALA
}

\section{ECHINORHYNCHIDAE}

Neorhadinorhynchus nudus (Harada 1938) Yamaguti 1939. South China Sea. Intestine. Mamaev (1968b) as Echinorhynchus nudus.

\section{RHADINORHYNCHIDAE}

Serrasentis sagittifer (Linton 1889) Linton 1932. South China Sea.

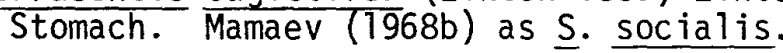


Euthynnus affinis (Continued)

CESTODA

DASYRHYNCHIDAE

Callitetrarhynchus gracilis (Rudolphi 1819) Pintner 1931. South China Sea. Intestine, liver. Mamaev (1968b).

COPEPODA

\section{CAL IGIDAE}

Caligus asymmetricus Kabata 1965. South India. Pillai (1963) as C. thynni.

CaTigus productus Dana 1853. Indian Ocean. Lewis (1967).

caligus bonito WiTson 1905 . India. Gills.

Thomas (T967) as $\underline{C}$. krishnai.

\section{PSEUDOCYCNIDAE}

Pseudocycnus appendiculatus Heller 1865. South India. Gills. PiTlai (1964).

\section{DIGENEA}

\section{BUCEPHALIDAE}

*Rhipidocotyle septpapillata Krul1 1934. Red Sea. Nagaty (1958).

*Rhipidocotyle heptathelata Stunkard 1974. Red Sea. Stunkard (1974). Rhipidocotyle pentagonum (Ozaki 1924) Ekmann 1932. South China Sea. Stomach. Mamaev (1968b). DIDYMOZOIDAE

Oesophagocystis dissimilis (Yamaguti 1938) Yamaguti 1970. South China Sea. Inner wall of esophagus. Mamaev (1968b). Yamaguti (1971) states that Mamaev's figures of $\underline{0}$. dissimilis do not agree with his.

Metanematobothrium bivitellatum Mamaev 1968. South China Sea. Gills. Mamaev (1968b).

Nematobothrium sp. South China Sea. Gills, liver, body cavity. Mamaev (1968b).

\section{GORGODERIDAE}

Phyllodistomum lancea Mamaev 1968. South China Sea. Urinary bladder? Mamaev (1968b).

\section{HEMIURIDAE}

*Sterrhurus imocavus Looss 1907. Egypt. Looss (1907a); South China Sea. Stomach. Mamaev (1968b) as Lecithochirium $i$.

Plerurus carangi Parukhin 1966. South China Sea. Stomach. Mamaev (19685).

Dinurus euthynni Yamaguti 1934. South China Sea. Stomach. Mamaev 
Euthynnus affinis (Continued)

HIRUDINELLIDAE

Hirudinella spinulosa Yamaguti 1938. South China Sea. Stomach. Mamaev (1968b).

SANGUINICOLIDAE

Cardicola congruenta Lebedev and Mamaev 1968. South China Sea. Vascular system. Lebedev and Mamaev (1968).

MONOGENEA

AXINIDAE

Allopseudaxine macrova (Unnithan 1957) Yamaguti 1963. India. Unnithan (1957) as Uraxine chura $\mathrm{m}$.

CAPSALIDAE

Caba17erocotyla paucispinosa Mamaev 1968. South China Sea. Gi17s, stomach. Mamaev (1968b).

Caballerocotyla notosinense Mamaev 1968. South China Sea. Gills. Mamaev (1968b).

Caballerocotyla manteri affinis Mamaev 1968. South China Sea. Gi11s. Mamaev (1968b).

GASTROCOTYLIDAE

Metapseudaxine ventrosicula Mamaev 1967. South China Sea. Gills. Mamaev (1968b).

Churavera macrova Unnithan 1968. India. Gil1s. Unnithan (1968).

HEXOSTOMAT IDAE

Homostoma chura Unnithan 1965. India. Gills. Unnithan (1965). Neohexostoma euthynni (Meserve 1938) Price 1961. South China Sea. Gī7s. Mamaev (1968b).

\section{NEMATODA}

ANISAKIDAE

Contracaecum sp. Larva. South China Sea. Body cavity. Mamaev (1968b).

Anisakis sp. Larva. South China Sea. Body cavity. Mamaev (1968b).

CYSTIDICOLIDAE

Ctenascarophis gastricus Mamaev 1968. South China Sea. Stomach. Mamaev (7968b).

PHILOMETRIDAE

Philometra sp. South China Sea. Mamaev (1968b). 
COPEPODA

CALIGIDAE

Caligus coryphaenae Steenstrup and Lütken 1861. Gulf of California. Shiino (1959d).

Caligus macarovi Gussev 1951. Mexico. Exterior. Shiino (1959c).

\section{DIGENEA}

HIRUDINELLIDAE

Hirudinella ventricosa (Pallas 1774) Baird 1853. Baja California. Stomach. Caballero and Caballero (1971a) as $\underline{H}$. marina.

MONOGENEA

HEXOSTOMATIDAE

Neohexostoma euthynni (Meserve 1938) Price 1961. Baja California. Gills. Mitlemann (1956) as Hexostoma e.

\section{Euthynnus pelamis - Skipjack Tuna}

SYNONYMS - Gymnosarda pelamys, Katsuwonus pelamys, Thynnus pelamys, Katsuwonus vagans

\section{ACANTHOCEPHALA}

\section{RHADINORHYNCHIDAE}

Rhadinorhynchus pristis (Rudolphi 1802) Luhe 1911. Bermuda. Intestine. Rees (1970) as Nipporhynchus ornatus; Japan. Yamaguti (1963c) as Nipporhynchus katsuwonis.

Raorhynchus terebra (Rudo7phi 1819) Tripathi 1959. Golvan (1969). Raorhynchus meyeri (Heinze 1934 ) Golvan 1969. Indian Ocean. Golvan (1969).

\section{CESTODA}

\section{TENTACULARIIDAE}

Tentacularia coryphaenae Bosc 1802. Post larva. Atlantic, Japan. Muscle, abdominal cavity. Dollfus (1942).

COPEPODA

\section{CALIGIDAE}

Caligus bonito Wilson 1905. Pacific Cabo San Lucas, Baja California. Gi17s. Shitno (1963a); Hawaii. Lewis (1967); Indian Ocean. Pillai (1963); Massachusetts. Wilson (1905b). 
Caligus productus Dana 1853. Pacific Costa Rica. Mouth. Shi ino (1965b); Indian Ocean. Body surface, buccal cavity, gill cavities. Lewis et a T. (1969); Hawaii. Lewis (1967).

Caligus coryphaenae Steenstrup and Lütken 1861. Indian Ocean. Lewis et a1. (1969); Hawaii. Lewis (1967); Japan. Shi ino (1960b). Caligus pelamydis Krøyer 1863. Massachusetts. Gill cavity. Wilson (1905b); South Africa. Barnard (1955).

Caligus quadratus Shiino 1954. Japan. Inner surface of operculum. Shino (1959e).

Caligus mutabilis Wilson 1905. West Coast of Mexico. Causey (1960). Lepeophtheirus dissimulatus Wilson 1905. Bermuda. Heegaard (1943). Heniochophilus branchialis (Rangnekar 1953) Pillai and Mohan 1965. India. PilTa and Mohan (1965).

Dentigryps curtus. Bermuda. Heegaard (1943) as Homoiotes bermudensis.

\section{PSEUDOCYCNIDAE}

Pseudocycnus appendiculatus Heller 1865. Angola. Gills. NunesRuivo (1956); Mediterranean. Hewitt (1969b).

\section{DIGENEA}

\section{DIDYMOZOIDAE}

Didymozoon filicolle Ishii 1935. Pacific coast of Japan. Gi11s. Ishii (1935).

Didymozoon longicolle Ishij 1935. Japan. Gills. Ishii (1935).

Didymozoon minus Yamaguti 1934. Japan. Gi11s. Yamaguti (1934a) as D. minor.

Didymocylindrus filiformis Ishii 1935. Japan, Hawaii. Gills. Ishii (1935).

Didymocylindrus simplex (Ishi i 1935) Yamaguti 1970. Japan, Hawaii. Yamaguti (1970).

Didymocystis bilobata Ishii 1935. Pacific Japan. Gills. Ishii (1935).

Didymocystis ovata Ishi i 1935. Japan. Mouths. Ishii (1935).

Didymocystis reniformis Ariola 1902. Japan. Encysted in esophagus and stomach. Yamaguti (1934a).

Didymocystis soleiformis Ishi i 1935. Pacific Japan. Gills. Ishii (1935).

Didymocystis wedli Ariola 1902. Japan. Yamaguti (1934a).

Didymoproblema fusiforme Ishi i 1935. Pacific Japan, Hawai i. GiTls. Ishii (1935).

Lobatozoum multisacculatum Ishii 1935. Pacific Japan, Hawaii. Gills. Ishii (1935).

Koellikeria globosa Ishii 1935. Tokyo Fish Market. Mouth, esophagus. Ishii (1935).

Koellikeria orientalis (Yamaguti 1934) Ishii 1935. Japan. Stomach, esophagus. Ishij (7935).

Koel1ikeria reniformis Ishii 1935. Tokyo Fish Market. Gi11s. Ishi i (1935). 
Neodiplotrema pelamydis (Yamaguti 1938) Yamaguti 1938. Pacific Japan. Gi17 arches. Yamaguti (1938) as Diplotrema p.

Oesophagocystis dissimilis (Yamaguti 1938) Yamaguti 1970. Hawaii, Pacific Japan. Esophagus, stomach. Yamaguti (1970).

Coeliodidymocystis kamegaii Yamaguti 1970. Hawaii. Caeca, stomach Yamaguti (1970).

Coeliodidymocystis abdominal is (Yamaguti 1938) Yamaguti 1970. Japan. Caeca, stomach. Yamaguti (1938) as Didymocystis a.

Didymocystoides intestinomuscularis Yamaguti 1970. Hawaii. Muscle layer of intestine. Yamaguti (1970).

Didymocystoides pinnicola Yamaguti 1970. Hawaii. Dorsal fins. Yamaguti (1970).

Didymocystoides submental is (Yamaguti 1938) Yamaguti 1970. Japan. Submenta T region. Yamaguti (1938) as Didymocystis $s$.

Lagenocystis katsuwoni Yamaguti 1970. Hawaii. Intestinal wal1. Yamaguti (1970).

Adenodidymocystis intestinal is Yamaguti 1970. Hawaij. Intestinal wa11. Yamaguti (1970).

Univitellannulocystis katsuwoni Yamaguti 1970. Hawaii. Intestine. Yamaguti (1970).

\section{FELLODISTOMATIDAE}

Tergestia laticollis (Rudolphi 1819) Stossich 1899. Mediterranean. Manter (1940b).

\section{HEMIURIDAE}

Dinurus euthynni Yamaguti 1934. Pacific Coast of Japan. Stomach. Yamaguti (1934a).

Lecithochirium microstomum Chandler 1935. Mexico. Stomach.

$$
\text { Lamothe-Argumedo (1966). }
$$

HIRUDINELLIDAE

Hirudinella ventricosa (Pallas 1774) Baird 1853. Atlantic. Manter (1940b).

\section{SYNCOELIIDAE}

Syncoelium filiferum (Leuckart in Sars 1885) Odhner 1911. Pacific Coast of Japan. Branchial cavity. Yamaguti (1938) as $\underline{\mathbf{s}}$. katuwo.

\section{MONOGENEA}

\section{AXINIDAE}

Allopseudaxine katsuwonis (Ishii 1936) Yamaguti 1943. Hawaii. Gi11s. Yamaguti (1968b).

Allopseudaxine vagans (Ishij 1936) Price 1962. Japan. Gills. Ishii (1936) as Pseudaxine v. [=Al lopseudoaxinoides vagansYamaguti (1970).J 
CAPSALIDAE

Capsala lintoni Price 1939. Massachusetts. Linton (1898) as Tristoma laeve; Massachusetts. Gills. Price (1939).

Capsala interrupta (Montice11i 1891) Price 1938. Mediterranean. Gil7s. Silas (1967).

Capsala laevis (Verri11 1874) Johnston 1929. Massachusetts. Gi11s. Silas (1967).

Capsala nozawae (Goto 1894) Price 1938. Japan. Fins. Silas (1967).

Caballerocotyla katsuwoni (Ishii 1936) Price 1960. Pacific. Ishii (1936) as Tristoma katsuwonum.

\section{GASTROCOTYLIDAE}

Pricea minimae Chauhan 1945. India. Chauhan (1945).

HEXOSTOMATIDAE

Hexostoma grossum (Goto 1894) Sproston 1946. Japan. Gills. Silas (1967).

\section{NEMATODA}

\section{ANISAKIDAE}

Contracaecum sp. Larva. Japan. Kagei et a1. (1970).

Anisakis sp. Larva. Japan. Gut. Otsuru et a1. (1965); larva. New Zealand. Viscera. Hewitt and Hine (1972).

\section{PHILOMETRIDAE}

Philometra sp. Atlantic. Ovaries. Simmons (1969).

SPIRUROIDEA

Genera and species. At7antic. Ovaries. Simmons (1969).

$$
\text { Lepidocybium flavobrunneum - Escolar }
$$

CESTODA

AMPHICOTYLIDAE

Pseudeubothrioides lepidocybii Yamaguti 1968. Hawaii. Intestine. Yamaguti (1968a).

\section{DIGENEA}

DIDYMOZOIDAE

Allometanematobothrioides lepidocybii Yamaguti 1965. Hawai Tissues. Yamaguti (1965a). 
Lepidocybium flavobrunneum (Continued)

HEMIURIDAE

Ectenurus lepidocybii Yamaguti 1970. Hawaii. Stomach. Yamaguti (1970).

MONOGENEA

DISCOCOTYLIDAE

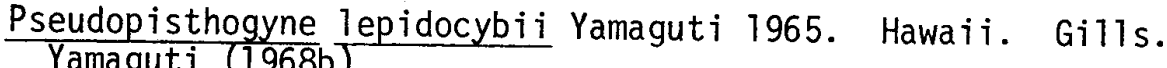

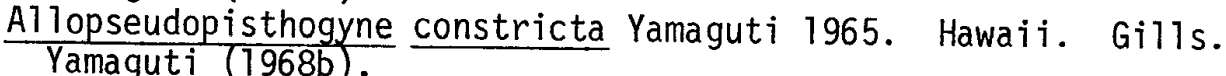

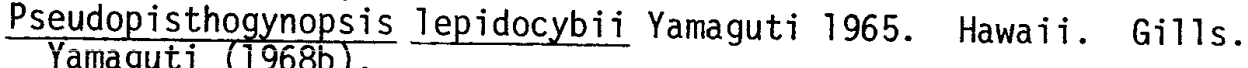

Sarda chiliensis - Pacific Bonito

SYNONYM - Sarda lineolata

CESTODA

DASYRHYNCHIDAE

Callitetrarhynchus gracilis (Rudolphi 1819) Pintner 1931. Larva. Southern California. Viscera. Jensen 18 .

DIPHYLLOBOTHRIIDAE

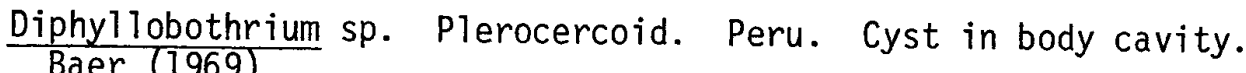
COPEPODA

CALIGIDAE

Caligus bonito Wilson 1905. Panama Bay. Exterior. Wilson (1937a); Southern CaTifornia. Shiino (1960c); Mexico. Mouths. Causey (1960).

Caligus mutabilis Wilson 1905. West Coast of Mexico. Causey (1960). PSEUDOCYCNIDAE

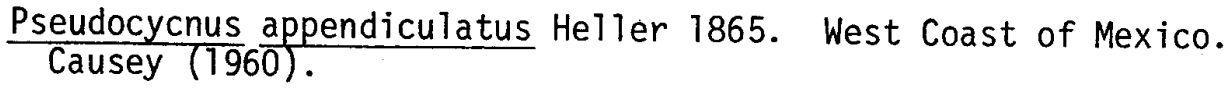

\section{DIGENEA}

DIDYMOZOIDAE

Unitubulotestis maris Caballero and Caballero 1971. Baja California. Gills. Caba17ero and Caballero (1971b). 
Sarda chiliensis (Continued)

MONOGENEA

CAPSALIDAE

Caballerocotyla gregalis Wagner and Carter 1967. Southern California. Gi17s. Wagner and Carter (1967).

HEXOSTOMATIDAE

Hexostoma sp. Eastern Pacific. Kunnenkeri and Martin (1963).

MAZOCRAEIDAE

Grubea sp. West Coast of Baja California. Gi11s. Wagner (1975). NEMATODA

ANISAKIDAE

Anisakis sp. Larva. Southern California. Dailey et al. (in press).

Scomber japonicus - Chub Mackere]

SYNONYMS - Pneumatophorus japonicus, Pneumatophorus grex, Scomber colias, Scomber diego

\section{ACANTHOCEPHALA}

POLYMORPHIDAE

Bolbosoma sp. Japan. Intestine. Ichihara et al. (1968).

\section{RHADINORHYNCHIDAE}

Rhadinorhynchus Tintoni Cable and Linderoth 1963. Europe. Golvan (1969).

Rhadinorhynchus cadenati (Golvan and Houin 1964) Golvan 1969. Senega7. GoTvan (1969).

Rhadinorhynchus pristis (Rudolphi 1802) Lühe 1911. Sea of Japan. Intestine. Zhukov (1960a).

Rhadinorhynchus japonicus Fujita 1920. Japan. Intestine. Fujita (1920).

Rhadinorhynchus seriolae (Yamaguti 1963) Golvan 1969. Japan.

Intestine. Ichihara et al. (1968) as Nipporhynchus $\underline{s}$.

CESTODA

BOTHRIOCEPHAL IDAE

Bothriocephalus sp. Plerocercoid. Massachusetts. Intestine. Linton (1947). 


\section{DASYRHYNCHIDAE}

Callitetrarhynchus gracilis (Rudolphi 1819) Pintner 1931. Larva. Southern California. Viscera. Jensen ${ }^{18}$.

\section{PHYLLOBOTHRI IDAE}

Rhinebothrium flexile Linton 1891 . Larva. Massachusetts. Viscera. Linton (1924).

\section{PSEUDOPHYLLIDEA}

Genera and species. Plerocercoid larva. California. Mesentery. Dailey (1969a).

\section{TENTACULARI IDAE}

Nybel inia surmenicola Okada 1929. Larva. Sea of Japan. Mesentery. Zhukov (1960a).

Nybel inia sp. Larva. South Atlantic. Body cavity. Solonchenko (1968).

Tentacularia corphaenae Bosc 1802. Postlarva. Japan. Muscles, sides of stomach. Dollfus (1942)

\section{TETRAPHYLLIDEA}

Scolex pleuronectis Müller 1788. South Atlantic. Intestinal tract. Solonchenko (1968); Sea of Japan. Zhukov (1960a).

\section{TRYPANORHYNCHA}

Genera and species. Plerocercus Tarva. California. Stomach, intestine, caeca, mesentery, abdominal cavity. Dailey (1969a); South Atlantic. Intestine. Solonchenko (1968).

COPEPODA

\section{CALIGIDAE} $\frac{\text { Caligus pelamydis Krøyer 1863. Pacific. Gills, Kazachenko et al. }}{(1972) .}$

\section{LERNAEOPODIDAE}

Clavellops is saba Yamaguti 1939. Japan. Gills. Yamaguti (1939c). Clavellisa scombri (Kurz 1877) Wilson 1915. Japan. Gills. Yamaguti (1939c); South Atlantic. Gills. Solonchenko (1968) as Clavella s.

\section{PENNELLIDAE}

Sarcotretes inflexus (Steenstrup and Lütken 1861) Richiardi (.877) as Lernaeanicus.

Lernaeocera branchialis (Linnaeus 1767) Wilson 1917. Juvenile. Pacific. Kabata (1960). 
Scomber japonicus (Continued)

\section{DIGENEA}

\section{ACANTHOCOLPIDAE}

Stephanostomum tristephanum McFarlane 1936. Sea of Japan. Intestine. Zhukov (1960a).

Acanthocolpoides pauloi Travassos, Freitas and Bührnheim 1965. Brazil. Intestine. Travassos et a7. (1967).

\section{COITOCAECIDAE}

Nicolla extrema Travassos, Freitas and Bührnheim 1965. Brazil. Intestine. Travassos et a1. (1969).

\section{DIDYMOZOIDAE}

Didymozoon Tongicolle Ishii 1935. Pacific Coast of Japan. Gills. Ishii (7935).

Didymozoon sp. Massachusetts. Gills. Linton (1940).

Nematobothrium filliforme Yamaguti 1934. Japan, Hawaii. Gills. Yamaguti (1934a); South Atlantic. Solonchenko (1968).

Nematobothrium robustum Yamaguti 1970. Hawaii. Inner surface of operculums. Yamaguti (1970).

Nematobothrium scombrii (Taschenberg 1879) Ishii 1935. Japan.

Gills. Ishii (1935).

Allonematobothrioides scombri Yamaguti 1970. Hawaî. Esophagus, gill arches. Yamaguti (1970).

\section{FELLODISTOMATIDAE}

Tergestia laticollis (Rudolphi 1819) Stossich 1899. Florida. Yamaguti (1971); Japan. Sma11 intestine. Yamaguti (1934a).

Tergestia acanthocephala (Stossich 1887) Stossich 1899. Yamaguti (1971).

\section{HEMIURIDAE}

Lecithocladium excisum (Rudolphi 1819) Lühe 1901. Japan. Stomach. Yamaguti (1934a); Italy. Looss (1907a); South Atlantic. Solonchenko (1968).

Ectenurus lepidus Looss 1907. Medjterranean. Looss (1907a). Lecithaster gibbosus (Rudolphi 1802) Lühe 1901. Sea of Japan. Intestine. Zhukov (1960a).

Dinurus scombri Yamaguti 1934. Japan. Stomach. Yamaguti (1934a).

Lecithochirium magnaporum Manter 1940. Southern California. Stomach. Montgomery (7957).

Lecithocladium harpodontis Srivastava 1937. Brazil. Stomach, intestine. Travassos et al. (1969).

Genera and species. California. Intestine. Dailey (1969a).

\section{LEPOCREADI IDAE}

Lepocreadium scombri (Yamaguti 1938) Ching 1960. Japan. Intestine. Yamaguti24 as Opechona s. 
Scomber japonicus (Continued)

Lepocreadium ghanensis Fischthal and Thomas 1970. Ghana. Small intestine. Fischthal and Thomas (1970a).

Neolepidapedon retrusum (Linton 1940) Sogandares-Bernal and Hutton 1960. Massachusetts. Linton (1940) as Lepocreadium $r$.

Cepha lolepidapedon saba Yamaguti 1970. Hawaîi. Intestine. Yamaguti (1970).

Opechona olssoni (Yamaguti 1934) Yamaguti 1938. Japan. Stomach, pyloric caeca, intestine. Ichihara et al. (1968).

Opechona orientalis (Layman 1930) Ward and Fillingham 1934. Central California. Chapa2z̧ South Atlantic. Solonchenko (1968); Immature. Japan. Stomach, intestine, pyloric caeca. Ichihara et al. (1968).

Opechona bacillaris (Mol in 1859) Looss 1907. Ghana. Small intestine. Fischthal and Thomas (1970a); Sea of Japan. Zhukov (1960a).

MONOGENEA

DACTYLOGYRIDAE

Microncotrematoides inversum (Goto and Kikuchi 1917) Yamaguti 1963. Japan. Gills. Ishij and Sawada (1938) as Dactylogyrus $\mathbf{i}$.

GASTROCOTYLIDAE

Gastrocotyle japonica Ishii and Sawada 1938. Japan. Ishii and Sawada (1938).

\section{MAZOCRAEIDAE}

Kuhnia minor (Goto 1894) Sproston 1946. Mediterranean, Massachusetts, Japan, USSR. Gills. Sproston (1946).

Kuhnia scombri (Kuhn 1829) Sproston 1945, Japan. Gills. Ichihara et a7. (1968); Hawaii. Yamaguti (1968b); South Atlantic. Solonchenko (1968).

Grubea pneumatophori Price 1961. Massachusetts. Gi11s. Linton (1940) as Pleurocotyle scombri.

Grubea cochlear Diesing 1858. South Atlantic. Gills. Solonchenko $(1968)$.

NEMATODA

ANISAKIDAE

*Thynnascaris adunca (Rudolphi 1802) Hartwich 1957. Sea of Japan. Intestine. Zhukov (1960a) as Contracaecum a.

*ontracaecum fabri (Rudolphi 1819). Sea of Japan. Intestine. Zhukov (1960a)

*Contracaecum saba Yamaguti 1941. Japan. Yamaguti (1941).

Contracaecum sp. Larva. California. Mesentery. Dailey (1969b); Japan. Kagei et a1. (1970); South Atlantic. Solonchenko (1968).

Anisakis sp. Larva. California. All parts of body. Dailey (1969a); Japan. Saito et al. (1970); Mediterranean. Nikolaeva and

Naidenova (1964); South Atlantic. Solonchenko (1968). 
Scomber japonicus (Continued)

Phocanema sp. Larva. California. Intestinal wall, abdominal cavity. Dailey (1969a); South Atlantic. Solonchenko (1968) as Porrocaecum sp.

Raphidascaris sp. Immature. California. Mesenteric tissue surrounding stomach. Dailey (1969a).

\section{CAMALLANIDAE}

Oncophora melanocephala (Rudolphi 1819) Baudin-Laurencin 1970. South Atlantic. Intestine. Solonchenko (1968) as Camallanus $\underline{\mathrm{m}}$.

\section{CAPILLARI IDAE}

Capillaria sp. Eggs. Japan. Liver. Margolis (1970a).

PROTOZOA (MYXOSPORIDA)

\section{CERATOMYXIDAE}

Ceratomyxa inconstans Jameson 1929. Central California. Gallbladder.

PROTOZOA (SPOROZOA)

\section{EIMERI IDAE}

Eimeria pneumatophori Dogiei 194̄8. Peter the Great Gulf, USSR. Liver. Pellerdy (7965).

$$
\text { Scomberomorus sierra - Sierra }
$$

COPEPODA

CALIGIDAE

Caligus mutabilis Wilson 1905. West Coast of Mexico. Causey (1960). Caligus productus Dana 1853. Gulf of California. Causey (1960).

\section{PSEUDOCYCNIDAE}

Pseudocycnus buccata Wilson 1922. Gulf of California. Causey (1960).

\section{DIGENEA}

\section{BUCEPHAL IDAE}

Bucephalus heterotentaculatus Bravo-Holl is and Sogandares-Bernal

1956. Mexico. Intestine. Bravo-Holl is and Sogandares-Bernàl (1956).

Prosorhynchoides cybii (Park 1939) n. comb. Mexico. Intestine. Yamaguti (1971). 
Scomberomorus sierra (Continued)

HEMIURIDAE

Lecithochirium microstomum Chandler 1935. Mexico. Stomach. Lamothe-Argumedo (1966).

Thunnus alalunga - Albacore

SYNONYMS - Germo alalunga, Orcyncnus alalunga, Thynnus alalunga

ACANTHOCEPHALA

GORGORHYNCHIDAE

Gorgorhynchus sp. California-Oregon. Intestine. Margolis 3 .

POLYMORPHIDAE

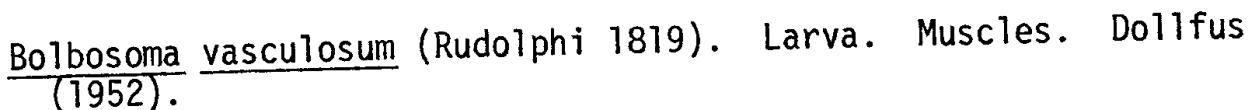

RHADINORHYNCHIDAE

Rhadinorhynchus pristis (Rudolphi 1802) Lühe 1911. Stomach. Do17fus (1952).

CESTODA

HEPATOXYLIDAE

Hepatoxylon trichiuri (Holten 1802) Dollfus 1942. Postlarva. Mediterranean. Sides of outside and inside of stomach. Dollfus (1942).

SPHYRIOCEPHALIDAE

Sphyriocephalus tergestinus Pintner 1913. Postlarva. Stomach. Dollfus (1952).

COPEPODA

CAL IGIDAE

Caligus productus Dana 1853. Indian Ocean. Buccal cavity, external surface. Lewis et a1. (1969); Japan. Shiino (1959e).

Caligus germoi Pearse 1951. Bahama Island. Pearse (1951).

EURYPHORIDAE

Euryphorus brachypterus (Gerstaecker 1853) Heegaard 1972. Indian Ocean. Gill cavity, external surface. Lewis et al. (1969); California, Oregon. Margolis ${ }^{3}$ both as Elytrophora b. ; Pacific Ocean. Shiino (1965C) as E. hemiptera. 
Euryphorus nordmanni Edwards 1840. Japan. Inner surface of operculums. Shiino (1959e); New Zealand. Hewitt (1968b) both as E. nympha.

\section{LERNAEOPODIDAE}

Brachiella thynni Cuvier 1829. Indian Ocean. External surface. Lewis et a1. (1969).

\section{LERNANTHROPIDAE}

Lernanthropus hiatus Pearse 1951. Bahama Is lands. Pearse (1951). PENNELLIDAE

Pennella filosa (Linnaeus 1758). Brittany. Embedded in skin. Leigh-Sharpe (1931) as P. germonia.

Pennella orthagorisci Wright 1870 . Southern California. Flesh. Wilson (1924); South Africa. Barnard (1955).

\section{PSEUDOCYCNIDAE}

Pseudocycnus appendiculatus Heller 1865. Indian Ocean. Gill cavity. Lewis et al. (1969); Massachusetts. Wilson (1922); New Zealand. Gills. Hewitt (1969b).

\section{DIGENEA}

\section{DIDYMOZOIDAE}

Didymocystis lanceolata Guiart 1938. Atlantic. Wide spread infection. Guiart (1938a).

Didymocystis macrorchis Guiart 1938. Atlantic. Wide spread infection. Guiart (1938a).

Didymocystis thynni (Taschenberg 1879) Dollfus 1926. Gills. Dorlfus (1952).

Didymocystis wedli Ariola 1902. Gills. Dollfus (1952).

Didymocystis phiTobranchia Yamaguti 1970. Hawaii. Gills. Yamaguti (7970).

Platocystis alalongae Yamaguti 1938. Pacific, Japan. Skin. Yamaguti (1938); Oregon-California. Margol is 3 .

Nematobothrium latum Guiart 1938. Atlantic. Stomachal peritoneum. Guiart (7938a).

Metanematobothrium guernei (Moniez 1891) Yamaguti 1938. France, Japan. Gills, muscles. Yamaguti (1938); California-Oregon. Muscles. Margolis ${ }^{3}$.

Didymocystoides alalongae (Yamaguti 1938) Yamaguti 1970. Pacific Japan. Gi11 arches. Yamaguti (1938) as Didymocystis a.

Didymocystoides buccal is Yamaguti 1970. Hawaij. Oral cavities. Yamaguti (1970).

Didymocystoides opercularis (Yamaguti 1938) Yamaguti 1970. Japan. Operculums. Yamaguti (1938) as Didymocystis 0.

Koellikeria bipartita (Wedl 1855) Ishij 1935. Stomach. Dollfus (1952). Koellikeria orientalis (Yamaguti 1934) Ishii 1935. Yamaguti (1971). 


\section{HIRUDINELLIDAE}

Hirudinella fusca (Bosc 1802) Manter 1926. Stomach. Dollfus (1952). Hirudinella oxysoma Guiart 1938. Atlantic. Stomach. Guiart (1938a). Hirudinella poirieri (Moniez 1891) Dollfus 1935. Atlantic. Stomach. Dollfus (1935b).

Hirudinella spinulosa Yamaguti 1938. Japan. Stomach. Yamaguti (1938); GuTf of Mexico. Nikolaeva and Parukhin (1968) as Uroproctinella s.

SYNCOELIDAE

Syncoel ium filiferum (Leuckart in Sars 1885) Odhner 1911. CalifornịaOregon. Branchial chambers. Margol is ${ }^{3}$.

ISOPODA

\section{AEGI IDAE}

Rocinela signata Schioedte and Meinert 1905. Gills. Richardson (1905).

\section{MONOGENEA}

CAPSALIDAE

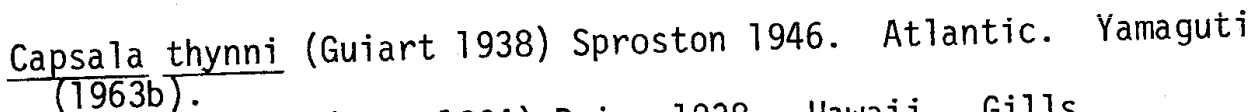
Capsala nozawae (Goto 1894) Price 1938. Hawaii. Gills. Yamaguti (1968b).

\section{GASTROCOTYLIDAE}

Areotestis sibi Yamaguti 1965. Hawaii. Gills. Yamaguti (1965b).

\section{HEXOSTOMATIDAE}

Hexostoma sibi Yamaguti 1968. Hawaii. Gills. Yamaguti (1968b).

\section{NEMATODA}

\section{ANISAKIDAE}

Anisakis $\mathrm{sp}_{3}$ Larva. California-Oregon. Many parts of body infected. Margolis 3 .

*Thynnascaris legendrei Dollfus 1933. Atlantic. Dollfus (1933); Pacific. Stomach. Yamaguti (1941).

Contracaecum sp. Larva. New Zealand. Body cavity. Hewitt and Hine (1972). 
SYNONYMS - Neothunnus albacores, Neothunnus macropterus, Neothynnus albacores, Thynnus macropterus, Thunnus macropterus, Thynnus a ibacora

\section{ACANTHOCEPHALA}

POLYMORPHIDAE

Bolbosoma vasculosum (Rudolphi 1819). Larva. Gulf of Guinea. Visceral wal1. Baudin-Laurencin (1971a).

\section{RHADINORHYNCHIDAE}

Rhadinorhynchus pristis (Rudolphi 1802) Lühe 1901. Gulf of Mexico. Intestine. Nikolaeva (1968b) as Nipporhynchus $k$. Rhadinorhynchus cadenati (Golvan and Houin 1964) Golvan 1969. Senegal. GoTvan (1969).

CESTODA

\section{DASYRHYNCHIDAE}

Callitetrarhynchus gracilis? (Rudolphi 1819) Pintner 1931. Ghana. Stomach. Bane (1969) "BTadder-like cysts similar to C. gracilis". Genera and species. Gulf of Mexico. Viscera. Ward (1962). Dasyrhynchus talismani Dollfus 1935. Postlarva. Gulf of Guinea. Branchial vessels, hepatic mesenteries. Baudin-Laurencin (1971a).

\section{GYMNORHYNCHIDAE}

Gymnorhynchus gigas (Cuvier 1817). Larva. Gulf of Mexico. Blood vessels. Nikolaeva (1968b).

HEPATOXYLIDAE

Hepatoxylon sp. Gulf of Mexico. Viscera. Ward (1962).

\section{LACISTORHYNCHIDAE}

Grillotia sp. Larva. Gulf of Mexico. Intestine. Nikolaeva (1968b). PHYLLOBOTHRI IDAE $\frac{\text { Echeneibothrium sp. Larva. Gulf of Mexico. Intestine. Nikolaeva }}{(1968 \mathrm{~b}) .}$

Phyllobothrium caudatum (Zshokke and Heitz 1914). Larva. Gulf of Mexico. Viscera. Ward (1962) as Pelichnibothrium speciosum.

\section{SPHYRIOCEPHALIDAE}

Sphyriocephalus sp. Larva. Gulf of Mexico. Viscera. Ward (1962). 
Thunnus albacares (Continued)

\section{TENTACULARI IDAE}

Tentacularia coryphaenae Bosc 1802. Post larva. Guif of Guinea. Abdominal cavity. Baudin-Laurencin (1971a).

Tentacularia sp. Gulf of Mexico. Viscera. Ward (1962).

Nybelinia sp. Larva. Gulf of Mexico. Intestine. Nikolaeva $(1968 b)$.

\section{TETRAPHYLLIDEA}

Scolex pleuronectis Müller 1788. Gulf of Mexico. Intestine. Nikolaeva (1968b).

\section{TRYPANORHYNCA}

Genera and species. Larva. Gulf of Mexico. Stomach. Nikolaeva $(1968 b)$.

COPEPODA

\section{CALIGIDAE}

Caligus productus Dana 1853. Indian Ocean. Body surface, gill cavity, buccal cavity. Lewis et al. (1969); East Pacific. Shiino (1959d); Angola. Gills. Nunes-Ruivo (1956) as C. katuwo. Caligus coryphaenae Steenstrup and Lütken 1861. Indian Ocean. Body surface. Lewis et a 1. (1969); Japan. Shi ino (1959d). Caligus quadratus Shi ino 1954. Japan. Inner surface of operculums. Shino (1954f).

\section{EURYPHORIDAE}

Euryphorus nordmanni Edwards 1840. Japan. Inner surface of gill operculums, body surface. Shiino (1954c) as E. nympha.

Euryphorus brachypterus (Gerstaecker 1853) Heegaard 1972. Indian Ocean. Lewis et al. (1969); Japan. Inside gill operculums. Shiino (1959e) as E. hemiptera.

\section{LERNAEOPODIDAE}

Brachiella thynni Cuvier 1829. Hawaii. Axis of pectoral fins. Shiino (1963a); Indian Ocean. Kirtisinghe (1935).

\section{PENNELLIDAE}

Pennella sp. Hawaii. Stomach. Lewis (1967); Gulf of Guinea. Branchial arches. Bane (1969).

Pennella crassicornis Steenstrup and Lütken 1861. Gulf of Guinea. Externa 1 surface. Baudin-Laurencin (1971a).

Pennella filosa (Linnaeus 1758). South Africa. External surface. Baudin-Laurencin (1971a). 
Thunnus albacares (Continued)

\section{PSEUDOCYCNIDAE}

Pseudocycnus appendiculatus Heller 1865. Pacific. Gill filaments. Shiino (1963a); Massachusetts, Mediterranean, Aden, Spain, etc. Witson (1932); Indian Ocean. Gill cavity. Lewis et a7. (1969); Angola. Nunes-Ruivo (1956).

\section{DIGENEA}

\section{DIDYMOZOIDAE}

Didymozoon longicolle Ishij 1935. Gulf of Mexico. Skin. Nikolaeva (7968b); Hawaij. Yamaguti (1971).

Didymocystis coatesi Nigrelli 1939. Gulf of Mexico. Nikolaeva (1968b) [ $=$ D. acanthocybi i - Yamaguti (1971)].

Didymocystis wedli Ariola 1902. Gulf of Mexico. Nikolaeva (1968b).

Didymocystis sp. Central Pacific. Gills. Iverson and Hoven (1958).

Platocystis sp. Gulf of Mexico. Connective tissues. Nikolaeva $(1968 \mathrm{~b})$.

Koellikeria globosa? Ishii 1935. Gulf of Guinea. Caecal mass. Bane (1969); Gulf of Mexico. Intestine. Nikolaeva (1968b). Koellikeria abdominal is Yamaguti 1970. Hawaii. Body cavity. Yamaguti (1970).

Koellikeria bipartita (Wed1 1855) Ishii 1935. Gulf of Mexico. Nikolaeva (1968b) as Wedlia bipartita.

Koellikeria orientalis (Yamaguti 1934) Ishii 1935. Gulf of Mexico. Gills. Nikolaeva (1968b) as Wedlia o.

Atalostrophion sardae MacCallum 1915. Gulf of Mexico. Connective tissue. Nikolaeva (1968b).

\section{GORGODERIDAE}

Phyllodistomum thunni Baudin-Laurencin 1971. Gulf of Guinea. Kidneys, ureters, urinary bladder. Baudin-Laurencin (1971a).

\section{HEMIURIDAE}

Brachyphallus parvus (Manter 1947) Skrjabin and Guschanskaja 1955. Gulf of Mexico. Stomach. Nikolaeva (1968b). Gonocerca sp. Gulf of Mexico. Nikolaeva (1968b).

\section{HIRUDINELLIDAE}

Hirudinella ventricosa (Pallas 1774) Baird 1853. Atlantic Ocean. Stomach. Watertor (1973); India. Raju (1962);

Central Pacific. Iverson and Hoven (1958) all as $\underline{H}$. marina.

Hirudinella fusca (Bosc 1802) Manter 1926. Gulf of Mexico. Stomach. Nikolaeva (1968b).

Hirudinella spinulosa Yamaguti 1938. Gulf of Mexico. Stomach. Nikolaeva (1968b) as Uroproctinella s.

Uroproctinella attenuata Hafeesullah 1971. Arabian Sea.

Stomach. Hafeezullah (1971). 
Thunnus albacares (Continued)

MONOGENEA

CAPSALIDAE

Capsala thynni (Guiart 1938) Sproston 1946. Atlantic. Gulf of Mexico. Gil ls. Nikolaeva (1968b).

Capsala gotoi Yamaguti 1968. On carapace of caligoid copepod. Hawaii. Buccal cavity. Yamaguti (1968b).

Capsala neothunni Yamaguti 1968. Hawaii. Gills. Yamaguti (1968b).

CapsaTa nozawae (Goto 1894) Price 1938. Hawaii. On caligold copepod. Buccal cavity. Yamaguti (1968b).

Caballerocotyla klawei Stunkard 1962. Mexico. Nasal capsules. Stunkard (1962); GuTf of Guinea, Peru, New York, Venezuela. Bane (1969); Hawaii. Yamaguti (1968b) as Nasicola k.

Caballerocotyla abidjani Bussieras and Baudin-Laurenc in 1970. Gulf of Guinea. Inside branchial gill covers. Bussieras and Baudin-Laurencin (1970).

Caballerocotyla biparasitica (Goto 1894) Price 1960. Christmas Island; Japan. Copepod carapaces. Lamothe-Argumedo (1968); Hawaii. Yamaguti (1968b) as Capsala b.

Caballerocotyla verrucosa Bussieras $797 \overline{2}$. Tropical eastern Atlantic. Tongues and palates. Bussieras (1972).

\section{GASTROCOTYLIDAE}

Areotestis sibi Yamaguti 1965. Hawaii. Gills. Yamaguti (1968b). HEXOSTOMATIDAE

Hexostoma sibi Yamaguti 1968. Hawaii. Gil1s. Yamaguti (1968b). Neohexostoma kawakawa Yamaguti 1968. Hawaii. Gills. Yamaguti (1968b).

\section{NEMATODA}

\section{ANISAKIDAE}

Contracaecum sp. Larva. Gulf of Mexico. Stomach, intestine. NikoTaeva (1968b).

\section{CAMALLANIDAE}

Oncophora melanocephala (Rudolphi 1819) Baudin-Laurencin 1970. Gulf of Mexico. Intestine. Nikolaeva (1968b) as Camallanus m.

Oncophora albacarensis Baudin-Laurencin 1972. Atlantic. BaudinLaurencin (1972).

\section{ICHTHYOSTRONGYLIDAE}

Ichthyostrongylus thunni Nikolaeva 1968. Gulf of Mexico. Nikolaeva $(1968 \mathrm{~b})$. 
SYNONYMS - Parathunnus sibi, Parathunnus obesus

CESTODA

SPHYRIOCEPHAL IDAE

Sphyriocephalus dollfusi Bussieras and Aldrin 1968. Atlantic. Bussieras and ATdrin (1968).

COPEPODA

CALIGIDAE

Caligus coryphaenae Steenstrup and Lütken 1861 . Indian Ocean. Body surface. Lewis et a 1. (1969).

Caligus productus Dana 1853. Indian Ocean. Buccal cavity. Lewis et a1. (1969); Hawai i. Lewis (1967).

EURYPHORIDAE

Euryphorus brachypterus (Gerstaecker 1853) Heegaard 1972. Indian Ocean. Body surface. Lewis et al. (1969); Japan. Inner surface of gill operculums. Shiino (1954c) all as Elytrophora b.

Gloiopotes huttoni (Thomson 1890) Bassett-Smith 1899. Western Pacific. Lewis (1967).

\section{LERNAAEOPODIDAE}

Brachiella thynni Cuvier 1829. Hawaii. Axis of pectoral fins. Shiino (1963a); Angola. Nunes-Ruivo (1956); Indian Ocean. Lewis et al. (1969).

\section{PSEUDOCYCNIDAE}

Pseudocycnus appendiculatus Heller 1865. Indian Ocean. Gill cavity. Lewis et a1. (1969); Angola. Nunes-Ruivo (1956).

\section{DIGENEA}

\section{DIDYMOZOIDAE}

Didymozoon longicolle Ishi i 1935. Hawait. Gills. Yamaguti (1970). Dermatodidymocystis viviparoides Yamaguti 1970. Hawaii. Skin. Yamaguti (1970).

Dermatodidymocystis vivipara Yamaguti 1970. Hawaii. Skin. Yamaguti (1970).

Didymocystis bifurcata Yamaguti 1970. Hawaii. Gills. Yamaguti (1970).

Didymocystis nasalis Yamaguti 1970. Hawaii. Nasal cavities. Yamaguti $(1970)$.

Didymocystis orbitalis Yamaguti 1970. Hawaii. Orbits. Yamaguti (1970).

Didymocystis philobranchia Yamaguti 1970. Hawaii. Forebody. Yamaguti (1970).

Didymocystis philobranchiarca Yamaguti 1970. Hawaii. Gi11 arches. Yamaguti (1970). 
Didymocystis poonui Yamaguti 1970. Hawaii. Palatal teeth. Yamaguti (1970).

Didymocystoides bifasciatus Yamaguti 1970. Hawaii. Forebody. Yamaguti (7970).

Didymocystoides pectoral is Yamaguti 1970. Hawaii. Pectoral fins. Yamaguti (1970).

Univitellodidymocystis neothunni Yamaguti 1970. Hawaii. Forebody. Yamaguti (1970).

Koellikeria pylorica Yamaguti 1970. Hawaii. Pyloric caeca. Yamaguti (1970).

Koellikeria retrorbital is Yamaguti 1970. Hawaii. Retroorbital adipose tissues. Yamaguti (1970).

Koellikeria submaxillaris Yamaguti 1970. Hawaii. Submaxillary region. Yamaguti (1970).

Koellikerioides internogastricus Yamaguti 1970. Hawaii. Muscular Tayer of stomach. Yamaguti (1970).

Koellikerioides apicalis Yamaguti 1970. Hawaii. Gill rakers. Yamaguti (1970).

Koellikerioides externogastricus Yamaguti 1970. Hawaii. Muscle Tayers of stomach. Yamaguti (1970).

Koellikerioides intestinalis Yamaguti 1970. Hawaii. Surface of smal1 intestine. Yamaguti (1970).

Neonematobothrioides poonui Yamaguti 1970. Hawaii. Connective tissues. Yamaguti (1970).

Opisthorchinematobothrium parathunni Yamaguti 1970. Hawaii. Connective tissues. Yamaguti (1970).

Orbitonematobothrium perioculare Yamaguti 1970. Hawaii. Eyes. Yamaguti (1970).

\section{SANGUINICOLIDAE}

Cardicola ahi Yamaguti 1970. Hawaii. Vascular system. Yamaguti (1970).

\section{MONOGENEA}

\section{AXINIDAE}

Sibitrema poonui Yamaguti 1966. Hawaii. Gi1ls. Yamaguti (1966).

CAPSALIDAE

Capsala onchidiocotyle (Setti 1899) Price 1938. Angola. Silas (1967). Capsala gotoi Yamaguti 1968. Hawaii. Gills. Yamaguti (1968b).

Capsala nozawae (Goto 1894) Price 1938. Hawaii. Gills. Yamaguti $(1968 \mathrm{~b})$.

Caballerocotyla biparasitica (Goto 1894) Price 1960. Hawaii. On caligoid copepod. Yamaguti (1968b) as Capsala b.

Caballerocotyla klawei Stunkard 1962. Hawai i. NasaT cavities. Yamaguti (1968b) as Nasicola k; Tropical east Atlantic. Bussieras (1972).

Caballerocotyla verrucosa Bussieras 1972. Eastern tropical

Atlantic. Buccal cavity, tongue, gi11s, etc. Bussieras (1972). 
Caballerocotyla pseudomagronum Bussieras 1972. Tropical eastern Atlantic. Buccal cavity. Bussieras (1972).

GASTROCOTYLIDAE

Areotestis sibi Yamaguti 1965. Hawaii. Gi1ls. Yamaguti (1968b). HEXOSTOMATIDAE

Hexostoma grossum (Goto 1894) Sproston 1946. Central Pacific. Gills. Iverson and Hoven (1958); Japan. Ishii and Sawada (1938). Hexostoma acutum (Goto 1894) Sproston 1946. Japan. Gills.

Price (1967a).

Hexostoma sibi Yamaguti 1968. Hawaii. Yamaguti (1968b).

Neohexostoma robustum Price 1961. Tropical Pacific. Gills. Price (7961a).

$$
\begin{aligned}
& \text { Thunnus thynnus - Bluef in Tuna } \\
& \text { SYNONYMS - Orcynchus thunnus, Scomber thunnus, } \\
& \text { Thunnus orientalis, Thunnus saliens }
\end{aligned}
$$

ACANTHOCEPHALA

ECHINORHYNCHIDAE

Neorhadinorhynchus nudus (Harada 1938) Yamaguti 1939. South China Sea. Intestine. Mamaev (1968b) as Echinorhynchus $\underline{n}$.

POLYMORPHIDAE

Bolbosoma thunni Harada 1935. Japan. Intestine. Harada (1935).

\section{CESTODA}

DASYRHYNCHIDAE

Callitetrarhynchus gracilis (Rudolphi 1819) Pintner 1931.

PTerocercoid. South China Sea. Intestine, liver. Mamaev (1968b); Mediterranean. Dollfus (1942); Massachusetts. Linton (1924) as Rhynchobothrium speciosum.

\section{LACISTORHYNCHIDAE}

Lacistorhynchus tenuis (Van Beneden 1858) Dollfus 1929. Larva. Massachusetts. Intestine. Linton (1924) as Rhynchobothrium b.

PHYLLOBOTHRIIDAE

Phyllobothrium caudatum (Zschokke and Heitz 1914). Larva. Pacific coast of Japan. Yamaguti (1959b) as Pelichnibothrium speciosum. 
Thunnus thynnus (Continued)

TENTACULARI IDAE

Tentacularia coryphaenae Bosc 1802. Post 1arvae. Gulf of Guinea. Abdominal cavity. Baudin-Laurencin (1971a).

COPEPODA

\section{CALIGIDAE}

Caligus bonito Wilson 1905. Japan. Exterior. Shiino (1959a) as C. kuroshio. Caligus corphaenae Steenstrup and Lütken 1861. Japan. Exterior. Shiino (1959e). Caligus productus Dana 1853. Japan. Exterior. Shiino (1959e)

\section{CECROPIDAE}

Cecrops 1atreil1ii Leach 1816. Mediterranean. Hewitt (1968a).

\section{EURYPHORIDAE}

Euryphorus brachypterus (Gerstaecker 1853) Heegaard 1972. North Sea. Gills. Mann (1970) as Elytrophora b.; Japan. Shiino (1959e) as Elytrophora hemiptera; Massachusetts. Gills. Wilson (1932) as ilytrophora atlantica.

\section{LERNAEOPODIDAE}

Brachiella thynni Cuvier 1829. North Atlantic. Lewis (1967);

GilTs. England. Wilson (1915).

\section{PENNELLIDAE}

Pennella filosa (Linneus 1758). Africa. Barnard $(1955)$.

\section{PSEUDOCYCNIDAE}

Pseudocycnus appendiculatus Heller 1865. Mediterranean. DelamareDeboutteville and Nunes-Ruivo (1958); North Sea. Gills. Brandes (1955) as Pseudocycnus thynnus.

\section{DIGENEA}

BUCEPHALIDAE $\frac{\text { Prosorhynchoides sibi (Yamaguti 1940) n. comb. Japan. Intestine. Fischthal }}{\text { and Kuntz (1964) as Bucephaloides }}$ and Kuntz (1964) as Bucephaloides
Rhipidocotyle pentagonum (Ozaki 1924) Ekmann 1932. Japan.
Intestine. Yamaguti ${ }^{24}$. 
Thunnus thynnus (Continued)

\section{DIDYMOZOIDAE}

Koellikeria bipartita (Wedi i855) Ishii 1935. Atlantic. Wide spread infection in body. Ishii (1935).

Koellikeria orientalis (Yamaguti 1934) Ishii 1935. Japan. Gills, stomach. Yamaguti (1971).

Koellikeria globosa Ishii 1935. Japan. Gills. Ishii (1935).

Koel1ikeria reniformis Ishi i 1935. Japan. Gills. Ishij (1935).

Coeliotrema thynni Yamaguti 1938. Japan. Outside pyloric caeca. Yamaguti (1938).

Didymocystis reniformis Ariola 1902. Yamaguti (1971).

Didymocystis wedli (Ariola 1902). Japan. Gills. Yamaguti (1934a); Mediterranean. Do11fus (1935b).

Didymocystis soleiformis Ishii 1935. Japan. Gills. Ishii (1935).

Didymocystis ovata Ishii 1935 . Japan. Gills. Ishii (1935).

Didymocystis crassa Ishii 1935. Japan. Gi11s. Ishii (1935).

Didymozoon pretiosus Ariola 1902.

Mediterranean. Gills, gill arches, skins. Dawes (1947).

Didymozoon filicolle Ishii 1935. Japan. Gills. Ishij (1935).

Didymozoon TongicolTe Ishii 1935. Japan. Gills. Ishii (1935).

Bidymocylindrus filiformis Ishii 1935. Japan. Gills. Ishii (1935). Didymoproblema fusiforme Ishi i 1935. Japan. Gills. Ishi i (1935). Lobatozoum multisacculatum Ishi i 1935. Japan. Gills. Ishii (1935). Didymocystoides semiglobuTaris (Ishii 1935) Yamaguti 1970. Japan. GiT7s. Ishii (1935).

Nematobothrium sp. South China Sea. Gills, liver, body cavity. Mamaev (1968b).

Oesophagocystis dissimilis (Yamaguti 1938) Yamaguti 1970. South

China Sea. Stomach. Mamaev (1968b) as Didymocystis d.

"[Mamaev's] description and figures do not agree with mine"

Yamaguti (1971).

\section{HEMIURIDAE}

Aponurus lagunculus Looss 1907 . South China Sea. Digestive tract. Mamaev (1968b).

Sterrhurus imocavus Looss 1907. South China Sea. Stomach. Mamaev (1968b) as Lecithochirium i.

\section{HIRUDINELLIDAE}

Hirudinella spinulosa Yamaguti 1938. South China Sea. Stomach. Mamaev (1968b).

Hirudinella ventricosa (Pallas 1774) Baird 1853. Atlantic. Stomach. Watertor (1973) as $\underline{H}$. marina.

\section{MONOGENEA}

\section{CAPSALIDAE}

Capsaia onchidiocotyle (Setti 1899) Price 1938. Dawes (1947). CapsaTa interrupta (Montice11 i 1891) Price 1938. Mediterranean. Yamaguti (1963b).

Capsala nozawae (Goto 1894) Price 1938. North Sea. British Isles. Yamaguti (1963b). 
Caballerocotyla albsmithi Dollfus 1962. California. Gills. Dollfus (1962).

Caballerocotyla paucispinosa Mamaev 1968. South China Sea. GiTls, stomach. Mamaev (1968b).

Caballerocotyla magronum (Ishii 1936) Price 1960. Japan. Gills. Ishi $i$ and Sawada (1938) as Tristoma $\mathrm{m}$.

\section{GASTROCOTYLIDAE}

Metapseudaxine ventrosicula Mamaev 1967. South China Sea. Gills. Mamaev (1968b).

\section{HEXOSTOMATIDAE}

Hexostoma grossum (Goto 1894) Sproston 1946. Japan. Gills. Ishii and Sawada (1938) as Hexacotyle g.

Hexostoma albsmithi Dollfus 1962. California. Gills. Dollfus (1962).

Hexostoma thynni (Delaroche 1811) Rafinesque 1815. Naples, North American Atlantic. Gills. Dawes (1947).

Hexostoma acutum (Goto 1894) Sproston 1946. Japan. Yamaguti $(1963 b)$.

Hexostoma dissimile (Yamaguti 1937) Sproston 1946. Japan.

Gills. Yamaguti 33 as Hexacotyle d.

Neohexostoma thunninas (Parona and Perugia 1889) Price 1961. Genoa. Gills. Dawes (1947) as Hexostoma t.

Neohexostoma extensicaudum (Dawes 1940) Price 1961. North Sea. Gi17s. Dawes (1940) as Hexostoma e.

\section{MAZOCRAEIDAE}

Kuhnia thunni (Ishii, in Ishii and Sawada 1936) Sproston 1946. Japan. Gi7ls. Ishii and Sawada (1938) as Dactylocotyle t.

\section{NEMATODA}

\section{ANISAKIDAE}

*Contracaecum cornutum (Stossich 1904). Norway. Beriand (1961)

Contracaecum sp. Larva. South Chına Sea. Intestine. Mamaev (1968b).

*Thynnascaris adunca (Rudolphi 1802) Hartwich 1957. Adult, juvenile. Norway. Berland (1961) as Contracaecum a.

Anisakis sp. Larva. South China Sea. Intestine. Mamaev (1968b).

\section{CAMALLANIDAE}

Oncophora melanocephala (Rudolphi 1819) Baudin-Laurencin 1970. Yamaguti (1961) as Camallanus $\mathrm{m}$.

\section{THELAZIIDAE}

Heptachona caudata (Mamaev 1968) Chabaud 1975. South China Sea. Intestine. Mamaev (1968c) as Pontochona $\underline{c}$. 
Thunnus thynnus (Continued)

PROTOZOA (MYXOSPORIDA)

TETRACAPSULIDAE

Kudoa clupeidae (Hahn 1917) Meglitsch 1947. Morocco. Muscles. Do77fus (1955).

$$
\begin{gathered}
\text { XIPHIIDAE -- Swordfishes } \\
\underline{\text { Xiphias gladius }- \text { Swordfish }} \\
\text { SYNONYMS - } \frac{\text { Histiophorus gladius }}{\text { Istiophorus gladius }} \text { Xiphias zeugopteri, }
\end{gathered}
$$

\section{CESTODA}

\section{AMPHICOTYLIDAE}

Pseudeubothrium xiphiados Yamaguti 1968. Hawaii. Intestine. Yamaguti (7968a).

BOTHRIOCEPHAL IDAE

Bothriocephalus manubriformis (Linton 1889) Ariola 1900. Hawaii. Intestine. Yamaguti (1968a); Rhode Island. Linton (1890) as Dibothrium $\underline{m}$.

DASYRHYNCHIDAE

Floriceps saccatus Cuvier 1817. Larva. France. Joyeux and Baer $(1936)$.

\section{GYMNORHYNCHIDAE}

Molicola uncinatus (Linton 1924). Larva. Massachusetts. Linton (1924) as Rhynchobothrium $\underline{u}$.

HEPATOXYLIDAE

Hepatoxylon trichuri (Holten 1802) Dollfus 1942. Postlarva. Baltic and Mediterranean Sea, Atlantic Ocean. Gills. Dollfus (1942).

\section{LACISTORHYNCHIDAE}

Grillotia erinacea (Van Beneden 1858) Guiart 1927. Larva. Massachusetts. Viscera, peritoneum. Linton (1924) as Rhynchobothrium imparispine. 
Xiphias gladius (Continued)

\section{OTOBOTHR I IDAE}

Otobothrium dipsacum Linton 1897. Larva. Massachusetts. Viscera. Linton (1924).

Otobothrium crenacolle Linton 1891. Larva. Massachusetts. On pyloric caeca. Linton (1924).

\section{TENTACULARIIDAE}

Tentacularia coryphaenae Bosc 1802. Nova Scotia. Nigrelli (1938). Nybelinia bisulcata (Linton 1889) Dollfus 1929. Larva. Massachusetts. Viscera. Dollfus (1942).

Nybelinia lingual is (Cuvier 1817) Dollfus 1929. Larva. Massachusetts. Viscera. Linton (1889) as Tetrarhynchus bisulcatus;

Mediterranean. Dollfus (1935b).

Nybelinia lamonteae Nigrelli 1938. Nova Scotia. Nigrelli (1938).

TETRAPHYLLIDEA

Scolex polymorphus Rudolphi 1819. Massachusetts. Linton (1924).

\section{TRIAENOPHORIDAE}

Fistulicola plicatus (Rudolphi 1819) Lühe 1899. Massachusetts. Rectum. Linton (1947).

\section{TRYPANORHYNCHA}

Dibothriorhynchus xiphiae MacCallum 1921. Massachusetts. Yamaguti (1959b) [=D. attenuatus - Nigrel1i (1938)]; [Species Incertae sedis - Yamaguti (1959b)].

COPEPODA

\section{CALIGIDAE}

Caligus elongatus Nordmann 1832. New England. Exterior. Wilson (7905b) as C. rapax.

Caligus chelifer WiTson 1905. Massachusetts. External surface. WiTson (1905b).

Caligus coryphaenae Steenstrup and Lütkin 1861. Massachusetts. Gills. Wilson (1924) as C. thynni.

\section{EURYPHORIDAE}

GToiopotes watsoni Kirtisinghe 1934. India. Body surface. Kurian (1955); Ceylon. Kirtisinghe (1934). Gloiopotes huttoni (Thomson 1890) Bassett-Smith 1899. Peru. Skin. Shiino (1963a) as G. longicaudatus; India. Body surface. Rao (1951) as G. zeugopterí; Southern CaTifornia. Wilson (1919) as $G$. costatus.

Gloiopotes ornatus Wilson 1905. Massachusetts. Exterior. Wilson (1905a). 
Xiphias gladius (Continued)

LERNAEOPODIDAE

Thysanote ramosa (Richiardi 1880) Yamaguti 1963. Mediterranean. Yamaguti (1963a).

\section{PENNELLIDAE}

Lernaeolophus sultanus (Edwards 1840) Heller 1865. Ceylon. Kirtisinghe (1935).

Pennella sp. Balakrishnan (1969).

Pennella instructa Wilson 1917. Massachusetts. In flesh. Wilson (1932); Japan. Yamaguti (1939b); Massachusetts. Wilson (1917).

Pennella filosa (Linnaeus 1758). Massachusetts. Wilson (1917); Africa. Barnard (1955).

* Pennella costai Richiardi 1880. Mediterranean. Yamaguti (1963a).

Pennella crassicornis Steenstrup and Lütken 1861. Africa. Barnard (1955).

\section{PHILICHTHYIDAE}

Philichthys xiphiae Steenstrup 1864. Massachusetts. Canals of head. Wilson (1932); New Zealand. Hewitt and Hine (1972). Southern California. Schultze 25 .

\section{CRUSTACEA}

\section{LEPADIDAE}

Conchoderma virgatum. On Pennella sp. Balakrishnan (1969).

\section{DIGENEA}

\section{DIDYMOZOIDAE}

Maccallumtrema xiphiados (MacCallum and MacCallum 1916) Yamaguti 1970. Massachusetts. Linton (1940) as Wedlia xiphiados;

Atlantic and Pacific. Abdominal muscle. Yamaguti (1970).

Reniforma multilobularis Yamaguti 1970. Hawaii. Gills. Yamaguti (1970).

\section{HIRUDINELL IDAE}

Hirudinella ventricosa (Pallas 1774) Baird 1853. Atlantic. Manter (1940b) as H. Clavata.

Hirudinella fusca (Bosc 1802) Manter 1926. Massachusetts. Linton $(1940)$.

\section{MONOGENEA}

\section{CAPSALIDAE}

Tristoma coccineum Cuvier 1817. Northwest Atlantic. Gill arches, inner surfaces of operculums, between filaments. Iles (1971);

Norway. Brinkman (1952); France. Euzet and Quignard (1961). 
Xiphias gladius (Continued)

Tristoma integrum Diesing 1850. Northwest Atlantic. Between gi11 filaments. ITes (1971); Norway. Brinkman (1952); France. Euzet and Quignard (1961): Massachusetts. Linton (1940) as $\mathbf{I}$. coccineum.

Tristoma papillosum Diesing 1836. Massachusetts. Gills. Linton (1940). [=T. coccineum - Price (1939)].

Tristoma adcoccineum Yamaguti 1968. Hawaii. Gills. Yamaguti (1968b).

Tristoma adintegrum Yamaguti 1968. Hawaii. Gills and buccal cavity. Yamaguti (1968b).

Capsala laevis (Verri11 1874) Johnston 1929. Massachusetts. Gi11s. Linton (7940).

\title{
NEMATODA
}

\section{ANISAKIDAE}

*Thynnascaris incurva (Rudolphi 1819) Hartwich 1957. Norway. Berland (1961); Japan. Machida (1971) both as Contracaecum i.

PROTOZOA (MYXOSPORIDA)

TETRACAPSAL IDAE

Kudoa maculoliquifaciens (Matsumoto 1954) Kudo and Meglitsch in preparation. Japan. Muscles. Matsumoto (1954) as Chloromyxum $\underline{m}$.

\author{
LUVARIDAE -- Louvars \\ Luvarus imperialis - Louvar
}

CESTODA

PHYLLOBOTHRI IDAE

Phyllobothrium caudatum (Zschokke and Heitz 1914). Plerocercoid. Norway. Intestine. Brinkman (1962) as Pelichnibothrium speciosum.

COPEPODA

\section{CECROPIDAE}

Luetkenia elongata Shi ino 1963. Baja California and California. Body surface. Shiino (1963b).

Luetkenia asterodermi Claus 1864. Gulf of Mexico. Gills. Topp and Girardin (1971)

DIGENEA

ACCACOELIIDAE

Tetrochetus rayneri (Nardo 1833) Looss 1912. Mediterranean. Intestine. Dol1fus (1935b). 


\section{ISTIOPHOR IDAE \\ Istiophorus platypterus - Sailfish}

SYNONYMS - Histiophorus americanus, Histiophorus orientalis, Istiophorus americanus, Istiophorus greyi, Istiophorus orientalis

ACANTHOCEPHALA

RHADINORHYNCHIDAE

Rhadinorhynchus pristis (Rudolphi 1802) Lühe 1911. Gulf of Mexico. Nikolaeva (1968b) as Nipporhynchus katsuwonus.

CESTODA

BOTHRIOCEPHALIDAE

Bothriocephalus manubriformis (Linton 1889) Ariola 1900. Gulf of Mexico. Nikolaeva (1968b); Hawaii. Intestine. Yamaguti (1968a); Massachusetts. Linton (1947).

\section{TENTACULARI IDAE}

Tentacularia sp. Larva. Gulf of Mexico. Nikolaeva (1968b). COPEPODA

\section{CALIGIDAE}

Caligus quadratus Shiino 1954. Japan. Body surface. Shiino (1959d).

\section{EURYPHORIDAE}

Gloiopotes huttoni (Thompson 1890) Bassett-Smith 1899. Indian Ocean. Cressey (1967b); Panama Bay. Wilson (1937a); Mexico. Causey (1960) previous two as G. costatus.

Gloiopotes watsoni Kirtisinghe $1 \overline{9} 34$. Pacific coast of Mexico. Cressey (1967b).

Gloiopotes americanus Cressey 1967. GuTf of Mexico. Cressey (1967b).

\section{PENNELLIDAE}

Pennella filosa (Linnaeus 1758). Guaymas, Mexico. Causey (1960).

\section{DIGENEA}

DIDYMOZOIDAE

Colocyntotrema sp. Gulf of Mexico. Nikolaeva (1968b). HEMIURIDAE

Parahemiurus sardiniae Yamaguti 1934. Japan. Stomach. Yamaguti (1934a). Dinurus scombri Yamaguti 1934. Gulf of Mexico. Nikolaeva (1968b). 


\section{HIRUDINELLIDAE}

Hirudinelia ampul lacea (Buttel-Reepen 1900) Skrjabin and Guschanska 1957. Gulf of Mexico. Nikolaeva (1968b).

Hirudinella spinulosa Yamaguti 1938. Gulf of Mexico. Nikolaeva

(T968b) as Uroproctinella s.

Hirudinella ventricosa (PalTas 1774) Baird 1853. Florida. Stomach. Ward (1954) as $\underline{H}$. marina.

\section{ISOPODA}

\section{CYMOTHOIDAE}

Nerocila californica Schioedte and Meinert 1881. West coast of Mexico. Gills. Brusca (1977). Speculative host.

\section{MONOGENEA}

\section{CAPSAL IDAE}

Tristomella pricei (Hidalgo 1959) Price 1960. Panama Bay. Pritchard (7961).

Caballerocotyla marielenae Lamothe-Argumedo 1968. Oaxaca, Mexico. Gills. Lamothe-Argumedo (1968).

Capsala ovalis (Goto 1894) Price 1938. Hawaii. Gill plates. Yamaguti (1968b).

Capsala sp. Gulf of Mexico. Nikolaeva (1968b).

Benedenia sp. Gulf of Mexico. Nikolaeva (1968b).

Capsa10ides istiophori Yamaguti 1968. Hawaii. Gi11s. Yamaguti $(1968 \mathrm{~b})$.

Capsaloides sinuatus (Goto 1894) Price 1938. Japan. Gi11s. Silas $(1967)$.

\section{NEMATODA}

\section{ANISAKIDAE}

* Thynnascaris histiophori (Yamaguti 1935). Miami, Florida. Stomach. Ward (1954); Japan. Yamaguti (1961).

* Thynnascaris incurva (Rudolphi 1819) Hartwich 1957. Gulf of Mexico. Nikolaeva (1968b) as Contracaecum i.

Makaira indica - Black Marl in

\section{COPEPODA}

EURYPHORIDAE

Gloiopotes huttoni (Thomson 1890) Bassett-Smith 1899. Socorro Island, Mexico. Skin. Shiino (1963a) as G. Iongicaudatus; Indian Ocean. Cressey (1967b). 
Makaira indica (Continued)

MONOGENEA

CAPSALIDAE

Capsala laevis (Verrill 1874) Johnston 1929. India. Silas (1967).

Makaira nigricans - Blue Marlin

SYNONYMS - Makaira ampla, Makaira ampla marlina, Makaira marlina

CESTODA

TENTACULARIIDAE

Nybelinia sp. Larva. Gulf of Mexico. Nikolaeva (1968b).

Tentacularia sp. Larva. Gulf of Mexico. Nikolaeva (1968b).

TRYPANORHYNCHA

Genera and species. Larva. Gulf of Mexico. Nikolaeva (1968b). COPEPODA

CALIGIDAE

Lepeophtheirus eminens Wilson 1944. Baja California. Exterior. Wilson (1944).

EURYPHORIDAE

GToiopotes ornatus WiTson 1905. Western North Atlantic, Caribbean. Cressey (1967b).

Gloiopotes watsoni Kirtisinghe 1934. Indian Ocean, Peru, Hawaii. Cressey (1967b).

GToiopotes huttoni (Thomson 1890) Bassett-Smith 1899. New Zealand. Body surface. Hewitt (1964b).

\section{DIGENEÁ}

DIDYMOZOIDAE

Colocyntotrema sp. Gulf of Mexico. Nikolaeva (1968b).

\section{HIRUDINELLIDAE}

Hirudinella fusca (Bosc 1802) Manter 1926. Gulf of Mexico. Nikolaeva (7968b).

Hirudinella spinulosa Yamaguti 1938. Gulf of Mexico. Nikolaeva (1968b) as Uroproctinella s. 
Makaira nigricans (Continued)

MONOGENEA

CĀPSĀLIIDAEE

Capsala poeyi (Vigueras 1935) Price 1938. Gulf of Mexico. Nikolaeva (1968b); Central Pacific - external surface. Iverson and Hoven (1958).

Capsala ovalis (Goto 1894) Price 1938. Hawaii. Gill operculums. Yamaguti (1968b).

Capsaloides sinuatus (Goto 1894) Price 1938. Hawaii. Gills. Yamaguti (1968b).

\section{Tetrapturus angustirostris - Shortbill Spearfish}

CESTODA

BOTHRIOCEPHALIDAE

Bothriocephalus manubriformis (Linton 1889) Ariola 1900. Hawaii. Intestine. Yamaguti (1968a).

MONOGENEA

CAPSALIDAE

Capsala ovalis (Goto 1894) Price 1938. Hawait. Gill plates.

Yamaguti (1968b).

Capsaloides cristatus Yamaguti 1968. Hawai i. Gil1s. Yamaguti (1968b). Capsaloides nairagi Yamaguti 1968. Hawaii. Gills. Yamaguti (1968b). Capsaloides tetrapteri Yamaguti 1968. Hawaii. Gills. Yamaguti (1968b).

Tetrapturus audax - Striped Marlin

SYNONYMS - Makaira audax, Makaira mitsukurii

CESTODA

BOTHR IOCEPHALIDAE

Bothriocephalus manubriformis (Linton 1899) Ariola 1900. Hawaii. Intestine. Yamaguti (1968a).

COPEPODA

EURYPHORIDAE Gloiopotes huttoni (Thomson 1890) Bassett-Smith 1899. Indian Ocean,
Peru. Gloiopotes watsoni Kirtisinghe 1934. Indian Ocean. Cressey (1967b). Gloiopotes ornatus Wilson 1905. Brazil. Yamaguti (1963a). 
Tetrapturus audax (Continued)

PENNELLIDAE

Pennella sp. Balakrishnan (1969).

CRUSTACEA

LEPADIDAE

Conchoderma virgatum. Balakrishnan (1969).

MONOGENEA

CAPSALIDAE

Capsala ovalis (Goto 1894) Price 1938. Hawaji. Gill plates. Yamaguti (7968b).

Capsala pricei Hidalgo Escalente 1958. West coast of Mexico. Exterior. Hidalgo Escalente (1958).

Capsaloides istiophori Yamaguti 1968. Hawaii. Gills. Yamaguti (1968b).

Capsaloides sinuatus (Goto 1894) Price 1938. Hawaii. Gills. Yamaguti $(1968 \mathrm{~b})$.

NEMATODA

ANISAKIDAE

Contracaecum sp. Central Pacific. Yoshihara and Nagasaki (1973).

$$
\begin{gathered}
\text { ICOSTEIDAE -- Ragfishes } \\
\frac{\text { Icosteus aegnigraticus - Ragfish }}{\text { SYNONYM - Acrotus willoughbyi. }}
\end{gathered}
$$

NEMATODA

ANISAKIDAE

Anisakis sp. Larva. British Columbia. Margolis ${ }^{3}$.

SCORPAENIDAE -- Scorpionfishes

Scorpaena guttata - Cal ifornia Scorpionfish

COPEPODA

BOMOLOCHIDAE

Holobomolochus spinulus (Cressey 1969) Kabata 1971. Southern California. Gil1 cavity. Cressey (1969b) as Bomolochus s. 
Holobomolochus attenuatus (Wilson 1913) Vervoort 1969. Puerto Penasco, Mexico. Causey (1960) as Bomolochus a.

\section{CALIGIDAE}

Lepeophtheirus pravipes Wilson 1912. Central California. Exterior. Wilson $(1935 \mathrm{~b})$.

Lepeophtheirus brachyurus Heller 1865. Southern California. Exterior. Wilson (1908a).

\section{CHONDRACANTHIDAE}

Pseudodiocus scorpaenus Ho 1972. Southern California. Gill cavity. Ho (1972b).

Chondracanthus gracilis Fraser 1920. Central California. Gills. Wilson $(1935 b)$.

\section{DIGENEA}

OPECOELIDAE

Helicometrina elongata Noble and Park 1937. Southern California. Intestine. Montgomery (1957).

HIRUDINOIDEA

PISCICOLIDAE

Australobdella californiana Burreson 1977. Southern California. Body surface. Burreson (1977a).

\section{ISOPODA}

\section{CYMOTHOIDAE}

Lironeca vulgaris Stimpson 1857. California. Turner et al. (1969). Nerocila californica Schioedte and Meinert 1881. California.

Fins. Richardson (1905).

\section{MONOGENEA}

CAPSALIDAE

Trochopus sprostoni Arai and Koski 1964. Southern California.

Gi77s. Arai and Koski (1964).

\section{TROCHOPODINAE}

Genera ard species. Eastern Pacific Ocean. Kunnenkeri and Martin (1963).

\section{NEMATODA}

\section{ANISAKIDAE}

Anisakis sp. Larva. Southern California. Dailey et al. (in press). 
Scorpaena guttata (Continued)

PROTOZOA (MYXOSPORIDA)

CERATOMYXIDAE

Ceratomyxa hopkinsi Jameson 1929. Southern California. Gallbladder. Jensen18.

MYXIDIIDAE

Myxidium incurvatum Thélohan 1892. Southern California. Gallbladder. Jensen 18 .

\author{
Sebastes $\mathrm{sp}$. \\ SYNONYMS - Sebastodes $s p$. Sebastodis $s p$.
}

CESTODA

BOTHRIOCEPHALIDAE

Bothriocephalus occidentalis (Linton 1898) Lühe 1899. British. Columbia. Pyloric caeca. Wardle (1932).

COPEPODA

CALIGIDAE

Caligus clemensi Parker and Margolis 1964. British Columbia. Fraser (1920) as $\underline{C}$. gurnardi.

\title{
DIGENEA
}

ACANTHOCOLPIDAE

Stephanostomum casum (Linton 1910) McFarlane 1934. British Columbia.

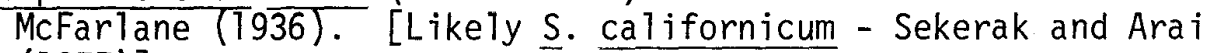
(1977)].

HEMIURIDAE

Sterrhurus monticel1i (Linton 1898) Linton 1910. Central California. Stomach. Nahhas 10 .

\section{LEPOCREADIIDAE}

Opechona orientalis (Layman 1930) Ward and Fillingham 1934. Central California. Intestine. Chapa23.

Opechona bacillaris (Molin 1859) Looss 1907. British Columbia. McFarTane (1936) as Pharyngora b.

Opechona occidental is Montgomery T957. British Columbia. Intestine. McFarlane (1936); Central California. Chapa23. 
Sebastes sp. (Continued)

MONORCHI IDAE

Telolecithus pugetensis Lloyd and Guberlet 1932. Northern California.

OPECOELIDAE

Podocotyle abitionis McFarlane 1936. British Columbia. Intestine. McFarlane (1936).
Opecoelus noblei Banerjee 1965. Central California. Intestine.
Banerjee (1965)

\section{SANGUINICOLIDAE} $\frac{\text { Aporocotyle macfarloni Holmes 1971. British Columbia. McFarlane }}{(1936) \text { as }} \frac{\text { A. simplex. }}{\text { s. }}$

\section{ZOOGONIDAE}

Deretrema pooli Annereaux 1947. Southern California. Intestine, Deretrema cholaeum McFarlane 1936. British Columbia. Gallbladder. McFarlane (1936).

$$
\text { Sebastes aleutianus - Rougheye Rockfish }
$$

\section{ACANTHOCEPHALA}

\section{ECHINORHYNCHIDAE}

Echinorhynchus gadi Zoega in Müller 1776. Bering Sea. Mamaev (1965); Northeastern Pacific. Sekerak and Arai (1977).

POLYMORPHIDAE

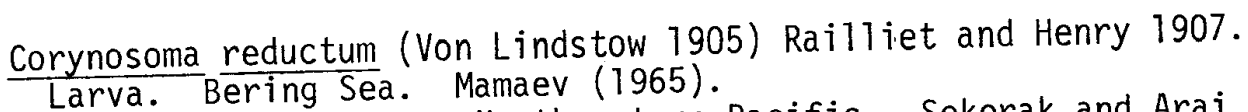

Corynosoma sp. Larva. Northeastern Pacific. Sekerak and Arai (1977).

\section{CESTODA}

\section{LACISTORHYNCHIDAE} $\frac{\text { Grillotia erinacea }}{\text { Mamaev }}\left(\frac{1965)}{1965}\right.$ Beneden 1858) Guiart 1927. Larva. Bering Sea.

PSEUDOPHYLLIDEA

Genera and species. Larva. Bering Sea. Mamaev (1965).

\section{TENTACULARI IDAE} Nybelinia surmenicola 0kada 1929. Larva. Northeastern Pacific.
Sekerak and Arai (1977). 
Sebastes aleutianus (Continued)

TETRAPHYLLIDEA

Scolex pleuronectis Müller 1788. Larva. Bering Sea. Mamaev (1965). COPEPODA

\section{CHONDRACANTHIDAE}

Chondracanthus pinguis Wilson 1912. Northeastern Pacific.

Sekerak and Arai (1977).

Chondracanthus triventricosus Sekerak 1970. Northeastern Pacific. Sekerak and Arai (1977).

\section{LERNAEOPODIDAE}

Neobrachiella robusta (Wilson 1912) Kabata 1979. Northeastern Pacific. Sekerak and Arai (1977) as Brachielia r.

Clavella parva Wilson 1912. Northeastern Pacific. Sekerak and Arai (1977).

\section{NAOBRANCHI IDAE}

Naobranchia occidentalis Wilson 1915. Northeastern Pacific. Sekerak and Arai (1977).

\section{PHILICHTHYIDAE}

Sarcotaces arcticus Collett 1874. Northeastern Pacific. Sekerak and Arai (1977).

Colobomatus kyphosus Sekerak 1970. Northeastern Pacific. Sekerak and Arai (1977).

\section{DIGENEA}

ACANTHOCOLPIDAE

Stephanostomum dentatum (Linton 1900) Linton 1940. Northeastern Pacific. Sekerak and Arai (1977).

\section{ALLOCREADIIDAE}

Anisorchis opisthorchis Polyansky 1955. Bering Sea. Mamaev (1965). BUCEPHALIDAE

Prosorhynchus crucibuTum (Rudolphi 1819) Odhner 1905. Northeastern Pacific. Sekerak and Arai (1977).

\section{FELLODISTOMATIDAE}

Fellodistomum furcigerum (01sson 1867) Yamaguti 1954. Bering Sea. Mamaev (1965) as Steringophorus $\mathrm{f}$. 
Sebastes aleutianus (Continued)

HEMIURIDAE

Derogenes varicus (Mü1ler 1784) Looss 1901. Northeastern Pacific. Stomach. Sekerak and Arai (1977).

Lecithophyllum botryophorum (01sson 1886) Odhner 1905 . Northeastern Pacific. Stomach. Sekerak and Arai (1977).

Lecithophyllum sphaerolecithum (Manter 1925) Odhner 1927. Bering Sea. Mamaev (7965).

Dinosoma tortum Yamaguti 1938. Bering Sea. Mamaev (1965).

Lecithaster gibbosus (Rudolphi 1802) Lühe 1901. Bering Sea. Mamaev (1965).

Lecithochirium platessae Mamaev, Parukhin and Baeva. 1963. Bering Sea. Mamaev (1965).

\section{LEPOCREADIIDAE}

Opechona alaskensis Ward and Fillingham 1934. Bering Sea. Mamaev (1965).

Neolepidapedon sebastisci (Yamaguti 1938) Manter 1954. Northeastern Pacific. Sekerak and Arai (1977).

OPECOELIDAE

Neohelicometra sebastis Sekerak and Arai 1974. Northeastern Pacific. Pyloric caeca, intestine. Sekerak and Arai (1974). Podocotyle sp. Northeastern Pacific. Sekerak and Arai (1977). Podocotyle reflexa (Crepl in 1825) Odhner 1905. Bering Sea. Mamaev (1965).

Podocotyle atomon (Rudolphi 1802) Odhner 1905. Bering Sea. Mamaev (1965).

\section{SANGUINICOLIDAE}

Psettarium sebastodorum Holmes 1971. Northeastern Pacific. Sekerak and Arai (1977).

\section{ZOOGONIDAE}

Steganoderma formosum Stafford 1904. Northeastern Pacific. Sekerak and Arai (1977).

\section{MONOGENEA}

\section{CAPSALIDAE}

Trochopus spp. Northeast Pacific. Gills. Sekerak and Arai (1977). Benedenia derzhavini (Layman 1930) Meserve 1938. Northeast Pacific. Gi11s. Sekerak and Arai (1977)

\section{NEMATODA}

\section{ANISAKIDAE}

Contracaecum sp. Larva. Northeast Pacific. Digestive tract. Sekerak and Arai (1977). 
* Thynnascaris adunca (Rudolphi 1802) Hartwich 1957 . Northeast Pacific. Sekerak and Arai (1977). Anisakis sp. Larva. Bering Sea. Mamaev (1965).

CUCULLANIDAE

Cucullanus sp. Northeastern Pacific. Sekerak and Arai (1977).

$$
\text { Sebastes alutus - Pacific Ocean Perch }
$$$$
\text { SYNONYM - Sebastodes alutus }
$$

\section{ACANTHOCEPHALA}

\section{ECHINORHYNCHIDAE}

Echinorhynchus gadi Zoega in Mü1ler 1776. Northeastern Pacific. Sekerak and Arai (1977).

\section{POLYMORPHIDAE}

Corynosoma villosum Van Cleave 1953. Cystacanth. Northeastern Pacific. Mesentery. Sekerak and Arai (1973). Corynosoma reductum (Von Lindstow 1905) Railiiet and Henry 1907. Bering Sea. Mamaev (1965).

\section{CESTODA}

\section{BOTHRIOCEPHALIDAE}

Bothriocephalus sp. Larva. British Columbia. Araj (1969b). Bothriocephalus scorpii (Müller 1776) Rudolphi 1808. Northeastern Pacific. Pyloric caeca. Sekerak and Arai (1973).

\section{PHYLLOBOTHRI IDAE}

Phyllobothrium sp. Larva. Northeastern Pacific. Gallbladder, intestine. Sekerak and Arai (1973).

\section{TENTACULARI IDAE}

Nybel inia surmenicola Okada 1929. Larva. Northeastern Pacific. Stomach wa11, mesentery. Sekerak and Arai (1973).

\section{TETRAPHYLLIDEA}

Scolex pleuronectis Müller 1788. Bering Sea. Mamaev (1965). COPEPODA

\section{CALIGIDAE}

Lepeophtheirus oblitus Kabata 1973. British Columbia. External surface. Lee (1975). 
Sebastes alutus (Continued)

CHONDRACANTHIDAE

Chondracanthus pinguis Wilson 1912. British Columbia. Gill cavity. Kabata (1968); Oregon. Sekerak (1970).

Chorldracanthus triventricosus Sekerak 1970. British Columbia. Nasal cavities. Sekerak (1970).

Chondracanthus sp. Oregon. Gill cavity, nostrils. Millemann ${ }^{34}$.

\section{LERNAEOPODIDAE}

Clavella parva Wilson 1912. British Columbia. Fins. Sekerak (1970).

Neobrachiella robusta (Wilson 1912) Kabata 1979. British Columbia. Kabata (1970c) as Brachiella $r$.

Clavellopsis sp. Oregon. Gills. Millemann ${ }^{34}$.

NAOBRANCHI IDAE

Naobranchia occidental is Wilson 1915. Northeastern Pacific. Sekerak and Arai (1977).

PENNELLIDAE

Peniculus sp. Northeastern Pacific. Sekerak and Arai (1977). * Haemobaphes theragrae Yamaguti 1939. Northeastern Pacific.

Gi]1s. Sekerak and Arai (1977).

PHILICHTHYIDAE

Colobomatus kyphosus Sekerak 1970. British Columbia. Cephalic sensory canal systems. Sekerak (1970).

Sarcotaces arcticus Collett 1874. British Columbia. Cysts in anal region of abdominal cavity. Sekerak (1970).

\section{DIGENEA}

\section{BUCEPHALIDAE}

Prosorhynchus crucibulum (Rudolphi 1819) Odhner 1905. Metacercaria. Northeastern Pacific. Pyloric caeca, intestine. Sekerak and Arai (1973).

Prosorhynchus sp. Larva. Northeastern Pacific. Cyst in muscles. Liston et a 1. (1960).

FELLODISTOMATIDAE

Fellodistomum sebastodis Yamaguti and Matumara 1942. Northeastern Pacific. Gallbladder. Sekerak and Arai (1973).

\section{HEMIURIDAE}

Lecithophyllum sphaerolecithum (Manter 1925) Odhner 1927. Bering Sea. Mamaev (1965).

Lecithophyllum botryophorum (01sson 1886) Odhner 1905. Northeastern Pacific. Stomach. Sekerak and Arai (1973). 
Lecithaster gibbosus (Rudolphi 1802) Lühe 1901. Northeastern Pacific. Sekerak and Arai (1977). Derogenes varicus (Müiler 1784) Looss 1901. Northeastern Pacific. Stomach. Sekerak and Arai (1973). TubuTovesicula 1indbergi (Layman 1930) Yamaguti 1934. Northeastern Pacific. Stomach. Sekerak and Arai (1973).

\section{LEPOCREADI IDAE}

Opechona alaskensis Ward and Fillingham 1934. Northeastern Pacific. Pyloric caeca, intestine. Sekerak and Arai (1973).

Opechona occidental is Montgomery 1957. Northeastern Pacific. Intestine. Sekerak and Arai (1977).

Neolepidapedon sebastisci (Yamaguti 1938) Manter 1954. Northeastern Pacific. Sekerak and Ärai (1977).

Lepidapedon gadi (Yamaguti 1934) Acena 1947. Bering Sea. Mamaev (1965).

\section{OPECOELIDAE}

Neohelicometra sebastis Sekerak and Arai 1974. Northeastern Pacific.

Pyloric caeca, intestine. Sekerak and Arai (1974).

Podocotyle sp. Immature. British Columbia. Arai (1969b).

Helicometrina sp. Northeastern Pacific. Pyloric caeca, intestine. Sekerak and Arai (1973).

Podocotyle atomon (Rudolphi 1802) Odhner 1905. Bering Sea. Mamaev (1965).

Podocotyle reflexa (Crepl in 1825) Odhner 1905. Bering Sea. Mamaev (1965).

\section{SANGUINICOLIDAE}

Psettarium sebastodorum Holmes 1971. Northeast Pacific. Heart. Sekerak and Arai (1973).

\section{SYNCOELIDAE}

Syncoelium filiferum (Leuckart in Sars 1885) Odhner 1911. Northeastern Pacific. Sekerak and Arai (1977).

\section{ZOOGON IDAE}

Steganoderma formosum Stafford 1904. Bering Sea. Mamaev (1965).

\section{MONOGENEA}

ACANTHOCOTYLIDAE

Pseudacanthocotyla williamsi (Price 1938) Yamaguti 1963. Northeastern Pacific. Gi17s. Sekerak and Arai (1973).

\section{CAPSALIDAE}

Trochopus trituba (Pratt and Aldrich 1953) Bravo-Hollis 1958. Oregon. Pratt and Aldrich (1953) as Megalocotyle $t$. 


\section{Sebastes auriculatus - Brown Rockfish}

\section{SYNONYM - Sebastodes auriculatus}

COPEPODA

\section{CHONDRACANTHIDAE}

Chondracanthus pinguis Wilson 1912. British Columbia. Gill cavity. Kabata (1968).

\section{LERNAEOPODIDAE}

Neobrachiella robusta (Wilson 1912) Kabata 1979 . British Columbia. Rabata (1970c); Fraser (1920) as Clavellopsis $r$.

Clavella parva Wilson 1912. British Columbia. Dorsal fin. Wi 1 son $(1912 b)$.

\section{DIGENEA}

\section{SANGUINICOLIDAE}

Psettarium sebastodorum Holmes 1971. Southern California. Holmes ${ }^{35}$. MONOGENEA

CAPSALIDAE

Trochopus marginata (Folda 1928) Price 1936. Southern California. Holmes 35 .

\section{MICROCOTYLIDAE}

Microcotyle sebastis Goto 1894. Northern California. Gills. Crane (1972); Southern California. Holmes ${ }^{35}$.

\section{NEMATODA}

\section{ANISAKIDAE}

Anisakis sp. Larva. Southern California. Dailey et a1. (in press); Phocanema sp. Larva. Southern California. Dailey et al. (in press).

$$
\text { Sebastes aurora - Aurora Rockfish }
$$

$$
\text { SYNONYM - Sebastodes aurora }
$$

\section{COPEPODA}

\section{LERNAEOPODIDAE}

Brachiella robusta (Wilson 1912) Kabata 1970 . British Columbia. Kabata (1970c). 
DIGENEA

\section{SANGUINICOLIDAE}

Psettarium sebastodorum Holmes 1971. Washington, British Columbia. Heart, buibus arteriosus, ventral aorta. Holmes (1971b).

\section{Sebastes babcocki - Redbanded Rockfish}

\section{ACANTHOCEPHALA}

\section{ECHINORHYNCHIDAE}

Echinorhynchus gadi Zoega in Müller 1776. Northeastern Pacific.

$$
\text { Sekerak and Ara } \dot{i} \text { (1977). }
$$

CESTODA

BOTHRIOCEPHALIDAE

Bothriocephalus sp. Northeastern Pacific. Sekerak and Arai (1977). COPEPODA

\section{CHONDRACANTHIDAE}

Chondracanthus pinguis Wilson 1912. Northeastern Pacific. Sekerak Chondracanthus triventricosus Sekerak 1970. Northeastern Pacific. Sekerak and Arai (1977).

\section{LERNAEOPODIDAE}

Neobrachiella robusta (Wilson 1912) Kabata 1979. Northeastern Pacific. Sekerak and Arai (1977) as Brachiella r.

Clavella parva Wilson 1912. Northeastern Pacific. Fins. Sekerak and Arai (1977).

\section{PENNELLIDAE}

Peniculus sp. Northeastern Pacific. Sekerak and Arai (1977).

\section{PHILICHTHYIDAE}

Colobomatus kyphosus Sekerak 1970. Northeastern Pacific. Sekerak and Arai (1977).

\section{DIGENEA}

\section{ACANTHOCOLPIDAE}

Stephanostomum dentatum (Linton 1900) Linton 1940. Northeastern Pacific. Sekerak and Arai (1977). 
Sebastes babcocki (Continued)

BUCEPHALIDAE

Prosorhynchus crucibulum (Rudo1phi 1819) Odhner 1905. Northeastern Pacific. Sekerak and Arai (1977).

HEMIURIDAE

Derogenes varicus (Mü1ler 1784) Looss 1901. Northeastern Pacific. Sekerak and Arai (1977).

\section{LEPOCREADIIDAE}

Neolepidapedon sebastisci (Yamaguti 1938) Manter 1954. Northeastern Pacific. Sekerak and Arai (1977).

OPECOELIDAE

Neohelicometra sebastis Sekerak and Araj 1974. Northeastern Pacific. Sekerak and Arai (1977).

Podocotyle sp. Northeastern Pacific. Sekerak and Arai (1977).

\section{SANGUINICOLIDAE}

Psettarium sebastodorum Holmes 1971. Northeastern Pacific. Sekerak and Arai (1977).

\section{MONOGENEA}

\section{CAPSALIDAE}

Trochopus sp. Northeastern Pacific. Sekerak and Arai (1977). NEMATODA

\section{ANISAKIDAE}

*Thynnascaris adunca (Rudolphi 1802) Hartwich 1957. Northeastern Pacific. Sekerak and Arai (1977).

Contracaecum spp. Larva. Northeastern Pacific. Sekerak and Arai (1977).

Anisakis sp. Larva. Southern California. Viscera. Jensen 18.

\section{CAPILLARI IDAE}

Capillaria sp. Northeastern Pacific. Sekerak and Arai (1977). CUCULLANIDAE

Cucullanus sp. Northeastern Pacific. Sekerak and Arai (1977). 
Sebastes borealis - Shortraker Rockfish

SYNONYM - Sebastes introniger

ACANTHOCEPHALA

ECHINORHYNCHIDAE

Echinorhynchus gadi Zoega in Mü1ler 1776. Bering Sea. Mamaev (1965); Northeastern Pacific. Sekerak and Arai (1977).

POLYMORPHIDAE

Corynosoma sp. Northeastern Pacific. Sekerak and Arai (1977).

Corynosoma reductum (Von Lindstow 1905) Railliet and Henry 1907. Larva. Bering Sea. Mamaev (1965).

CESTODA

BOTHRIOCEPHALIDAE

Bothriocephalus sp. Northeastern Pacific. Sekerak and Arai (1977). Bothriocephalus scorpii (Müller 1776) Rudolphi 1808. Bering Sea. Mamaev (1965).

\section{LACISTORHYNCHIDAE}

Grillotia erinacea (Van Beneden 1858) Guiart 1927. Larva. Bering Sea. Mamaev (1965).

PHYLLOBOTHRI IDAE

Phyllobothrium sp. Northeastern Pacific. Sekerak and Arai (1977).

\section{TENTACULARI IDAE}

Nybelinia surmenicola Okada 1929. Larva. Bering Sea. Mamaev (1965); Northeastern Pacific. Sekerak and Arai (1977).

TETRAPHYLLIDEA

Scolex pleuronectis Mü1ler 1788. Bering Sea. Mamaev (1965). COPEPODA

CHONDRACANTHIDAE

Chondracanthus pinguis Wilson 1912. Northeastern Pacific. Sekerak and Arai (7977).

\section{LERNAEOPODIDAE}

Neobrachielia robusta (Wilson 1912) Kabata 1979. Northeastern Pacific. Sekerak and Arai (1977) as Brachiella r. 
Sebastes borealis (Continued)

\section{NAOBRANCHI IDAE}

Naobranchia occidentalis Wilson 1915. Northeastern Pacific. Sekerak and Arai (1977).

\section{PHILICHTHYIDAE}

Colobomatus kyphosus Sekerak 1970. Northeastern Pacific. Sekerak and Arai (1977).

\section{DIGENEA}

\section{ACANTHOCOLPIDAE}

Stephanostomum baccatum (Nicoll 1907) Manter 1934. Bering Sea. Mamaev (1965).

Stephanostomum dentatum (Linton 1900) Linton 1940. Northeastern Pacific. Sekerak and Arai (1977).

BUCEPHALIDAE

Prosorhynchus crucibulum (Rudolphi 1819) Odhner 1905. Bering Sea. Mamaev (1965); Northeastern Pacific. Sekerak and Arai (1977).

\section{FELLODISTOMATIDAE}

Fellodistomum furcigerum (01sson 1867) Yamaguti 1954. Bering Sea. Mamaev (1965) as Steringophorus e.

\section{HEMIURIDAE}

Brachyphallus crenatus (Rudolphi 1802) Odhner 1905. Bering Sea. Mamaev (1965).

Derogenes varicus (Müler 1784) Looss 1901. Bering Sea. Mamaev (1965); Northeastern Pacific. Sekerak and Arai (1977).

Dinosoma tortum Yamaguti 1938. Bering Sea. Mamaev (1965).

Lecithaster gibbosus (Rudolphi 1802) Lühe 1901. Bering Sea. Mamaev (1965). Lecithophy17um botryophorum (01son 1886) Odhner 1905. Northeastern Pacific. Sekerak and Arai (1977).

Lecithophyllum sphaerolecithum (Manter 1925) Odhner 1927. Bering Sea. Mamaev (1965).

Tubulovesicula lindbergi (Layman 1930) Yamaguti 1934. Northeastern Pacific. Sekerak and Arai (1977).

\section{LEPOCREADI IDAE}

Lepidapedon gadi (Yamaguti 1934) Acena 1947. Bering Sea. Mamaev (1965).

Neolepidapedon sebastisci (Yamaguti 1938) Manter 1954. Northeastern Pacific. Sekerak and Arai (1977).

Opechona alaskensis Ward and Fi17ingham 1934. Bering Sea. Mamaev (1965). 
Sebastes borealis (Continued)

OPECOELIDAE

Podocotyle atomon (Rudolphi 1802) Odhner 1905. Bering Sea. Mamaev (1965).

Podocotyle reflexa (Creplin 1825) Odhner 1905. Bering Sea. Mamaev (1965).

HIRUDINOIDEA

PISCICOLIDAE

Piscicola sp. Northeastern Pacific. Sekerak and Arai (1977). MONOGENEA

CAPSALIDAE

Benedenia derzhavini (Layman 1930) Meserve 1938. Bering Sea. Mamaev (1965).

Trochopus spp. Northeastern Pacific. Sekerak and Arai (1977).

NEMATODA

ANISAKIDAE

Anisakis sp. Larva. Bering Sea. Mamaev (1965).

Contracaecum sp. Northeastern Pacific. Sekerak and Arai (1977).

Phocanema decipiens (Krabbe 1878) Myers 1959. Larva. Bering Sea. Mamaev (1965) as Terranova d.

CAPILLARIIDAE

Capillaria sp. Northeastern Pacific. Sekerak and Arai (1977).

CUCULLANIDAE

Cucullanus sp. Northeastern Pacific. Sekerak and Arai (1977).

Cucul7anus heterochrous RudoTphi 1802. Bering Sea. Mamaev (1965).

CYSTIDICOLIDAE

Ascarophis sebastodis 01 sen 1952. Northeastern Pacific. Sekerak and Arai (1977).

Sebastes brevispinis - Silvergray Rockfish

SYNONYM - Sebastodes brevispinis

ACANTHOCEPHALA

POLYMORPHIDAE

Corynosoma sp. Northeastern Pacific. Sekerak and Arai (1977). 
Sebastes brevispinis (Continued)

CESTODA

TENTĀCULĀÁRIIDÁE

Nybelinia surmenicola Okada 1929. Larva. Northeastern Pacific. Sekerak and Arai (1977).

COPEPODA

CHONDRACANTHIDAE

Chondracanthus pinguis Wi1son 1912. Northeastern Pacific. Sekerak and Arai (1977).

Chondracanthus triventricosus Sekerak 1970. Northeastern Pacific. Sekerak and Arai (1977).

LERNAEOPODIDAE

Neobrachiella robusta (Wilson 1912) Kabata 1979. Northeastern Pacific. Sekerak and Arai (1977) as Brachiella r.

PENNELLIDAE

Peniculus sp. Northeastern Pacific. Sekerak and Arai (1977).

Peniculus asinus Kabata and Wilkes 1977. British Columbia. Caudal fin. Kabata and Wilkes (1977).

\section{PHILICHTHY IDAE}

Colobomatus kyphosus Sekerak $1970 . \quad$ Northeastern Pacific. Sekerak and Arai (1977).

Sarcotaces arcticus Collett 1874. Northeastern Pacific. Sekerak and Arai (1977).

\section{DIGENEA}

BUCEPHALIDAE

Prosorhynchus crucibulum (Rudolphi 1819) Odhner 1905. Northeastern Pacific. Sekerak and Arai (1977).

HEMIURIDAE

TubuTovesicula lindbergi (Layman 1930) Yamaguti 1934. Northeastern Pacific. Sekerak and Arai (1977).

Derogenes varicus (Mü1ler 1784) Looss 1901. Northeastern Pacific. Sekerak and Arai (1977).

OPECOELIDAE

Podocotyle sp. Northeastern Pacific. Sekerak and Arai (1977). 
Sebastes brevispinis (Continued)

SANGUINICOLIDAE

Psettarium sebastodorum Hoimes 1971. British Columbia. Heart. Holmes (1971a).

SYNCOELIIDAE

Syncoelium filiferum (Leuckart in Sars 1885) Odhner 1911. Northeastern Pacific. Sekerak and Arai (1977).

MONOGENEA

CAPSALIDAE

Trochopus spp. Northeastern Pacific. Sekerak and Arai (1977).

MICROCOTYLIDAE

Microcotyle sebastis Goto 1894. Northeastern Pacific. Sekerak and Arai (1977).

NEMATODA

ANISAKIDAE

Contracaecum spp. Larva. Northeastern Pacific. Sekerak and Arai (1977).

* Thynnascaris adunca (Rudolphi 1802) Hartwich 1957. Northeastern Pacific. Sekerak and Arai (1977).

Anisakis sp. Larva. British Columbia. Throughout body. Margotis 3 .

\section{Sebastes carnatus - Gopher Rockfish}

SYNONYM - Sebastodes carnatus

COPEPODA

CALIGIDAE

Caligus hobsoni Cressey 1969. Southern California. Hobson (1971). NAOBRANCHI IDAE

Naobranchia occidentalis Wilson 1915. Southern California. Gills. Moser 19 .

DIGENEA

SANGUINICOLIDAE

Psettarium sebastodorum Holmes 1971. Southern California. Holmes ${ }^{35}$. 
Sebastes carnatus (Continued)

ISOPODA

Genera and species. Gnathiid larva. Southern California. Body surface. Hobson (1971).

MONOGENEA

CAPSALIDAE

Trochopus marginata (Folda 1928) Price 1936. Southern California. Holmes 35 .

MICROCOTYLIDAE

Microcotyle sebastis Goto 1894. Southern California. Holmes 35 . PROTOZOA (MYXOSPORIDA)

\section{CERATOMYXIDAE}

Ceratomyxa sebasta Moser, Love and Jensen 1976. Central California. Gallbladder. Moser et a1. (1976).

Ceratomyxa vepallida Meglitsch 1960. Northern California. Gallbladder. Moser et a . (1976).

MYXIDIIDAE

Myxidium incurvatum Thélohan 1892. Southern California. Gallbladder. Jameson (1931).

$$
\begin{gathered}
\frac{\text { Sebastes caurinus - Copper Rockfish }}{\text { SYNONYM - Sebastodes caurinuS }}
\end{gathered}
$$

\section{ACANTHOCEPHALA}

\section{ECHINORHYNCHIDAE}

Echinorhynchus gadi Zoega in Müller 1776. Northeastern Pacific.

$$
\text { Sekerak and Arai (1977). }
$$

POLYMORPHIDAE

Corynosoma sp. Larva. Northeastern Pacific. Sekerak and Arai (1977). BRANCHIURA

\section{ARGUL IDAE}

Argulus pugettensis Dana 1852. British Columbia. Bere ${ }^{5}$.

Argulus borealis Wi1son 1912. British Columbia. Bere ${ }^{5}$. 
Sebastes caurinus (Continued)

CESTODA

BOTHRIOCEPHALIDAE

Bothriocephalus sp. Northeastern Pacific. Sekerak and Arai (1977). PHYLLOBOTHRI IDAE

Phyllobothrium sp. Larva. Northeastern Pacific. Sekerak and Arai (1977).

\section{TENTACULARIIDAE}

Nybel inia surmenicola Okada 1929. Larva. Northeastern Pacific. Sekerak and Arai (1977).

COPEPODA

BOMOLOCHIDAE

Holobomolochus venustus Kabata 1971. British Columbia. Nasal cavities. Kabata (1971).

CALIGIDAE

Caligus clemensi Parker and Margol is 1964. Northeastern Pacific. Sekerak and Ara i (1977).

Lepeophtheirus oblitus Kabata 1973. British Columbia. Kabata (1973).

CHONDRACANTHIDAE

Chondracanthus triventricosus Sekerak 1970. Northeastern Pacific. Sekerak and Arai (1977).

Chondracanthus pinguis Wilson 1912. British Columbia. Bere ${ }^{5}$.

LERNAEOPODIDAE

Clavella parva Wilson 1912. Northeastern Pacific. Sekerak and Arai (1977).

Neobrachiella robusta (Wilson 1912) Kabata 1979. British Columbia. Kabata (1970c) as Brachiella r.

NAOBRANCHI IDAE

Naobranchia occidentalis Wilson 1915. British Columbia. Bere ${ }^{5}$.

PHILICHTHYIDAE

Colobomatus kyphosus Sekerak 1970. Northeastern Pacific. Sekerak and Arai (1977). 
DIGENEA

\section{ACANTHOCOLPIDAE}

Stephanostomum californicum Manter and Van Cleave 1951. Northeastern Pacific. Sekerak and Arai (1977).

GORGODERIDAE

Phyllodistomum sp. Northern Pacific. Sekerak and Arai (1977).

HEMIURIDAE

Lecithaster gibbosus (Rudolphi 1802) Lühe 1901. Northeastern Pacific. Sekerak and Arai (1977).

Tubulovesicula lindbergi (Layman 1930) Yamaguti 1934. Northeastern

Pacific. Sekerak and Arai (1977).

Derogenes varicus (Müller 1784) Looss 1901. Northeastern Pacific. Sekerak and Arai (1977).

Sterrhurus exodicus (McFarlane 1936) Yamaguti 1958. Northeastern

Pacific. Sekerak and Arai (1977).

Hemiurus levinseni Odhner 1905. Washington. Lloyd (1938).

\section{LEPOCREADI IDAE}

Neolepidapedon pugetense (Acena 1947) Yamaguti 1971. Northeastern Pacific. Sekerak and Arai (1977).

opechona occidentalis Montgomery 1957. British Columbia. Intestine. Arai $(1 \overline{969 b) .}$

OPECOELIDAE

Neohelicometra sebastis Sekerak and Arai 1974. Northeastern Pacific. Pyloric caeca and intestine. Sekerak and Arai (1974).

\section{SANGUINICOLIDAE}

Aporocotyle macfarlani Holmes 1971. Washington, British Columbia. Afferent branchial artery, ventral aorta, ventricle, bulbus arteriosus. Holmes (1971b).

Psettarium sebastodorum Holmes 1971. Washington, British Columbia. Heart, bulbus arteriosus, ventral aorta. Holmes (1971b).

\section{ZOOGONIDAE}

Deretrema cholaeum McFarlane 1936. Northeastern Pacific. Sekerak and Arai (1977).

HIRUD INOIDEA

PISCICOLIDAE

Piscicola sp. Northeastern Pacific. Sekerak and Arai (1977). 
Sebastes caurinus (Continued)

MONOGENEA

CÄPSALIDAE

Trochopus marginata (Folda 1928) Price 1936. Washington. Gills. Bonham (1950); British Columbia. Arai (1969b) as Megalocotyle m. Trochopus trituba (Pratt and Aldrich 1953) Bravo-Hollis 1958. British Columbia. Gills. Hoskins et a1.13

MICROCOTYLIDAE

Microcotyle sebastis Goto 1894. Washington. Guberlet (1936a); British Columbia. Gills. Arai (1969b).

NEMATODA

ANISAKIDAE

Contracaecum sp. Larva. British Columbia. Body cavity. Arai (1969b).

Phocanema sp. Larva. Southern California. Dailey et al. (in press).

* Thynnascaris adunca (Rudolphi 1802) Hartwich 1957. Washington. $01 \mathrm{sen}(1952)$ as Contracaecum a.

CAPILLARI IDAE

Capillaria sp. Northeastern Pacific. Sekerak and Arai (1977).

CUCULLANIDAE

Cucullanus elongatus Smedley 1933. Northeastern Pacific. Sekerak and Arai (7977).

CYSTIDICOLIDAE

Ascarophis sebastodis 01 sen 1952. Washington. Stomach. 01 sen (1952); British Columbia. Intestine. Arai (1969b).

PROTOZOA (MYXOSPORIDA)

MYXIDI IDAE

Myxidium incurvatum Thélohan 1892. Central California. Gallbladder. Jameson (1929).

Sebastes chlorostictus - Greenspotted Rockfish

SYNONYM - Sebastodes chlorostictus

CESTODA

BOTHRIOCEPHAL IDAE

Bothriocephalus scorpii (Müller 1776) Rudolphi 1808. Southern California. Jensen ${ }^{1}$. 
Sebastes chlorostictus (Continued)

COPEPODA

PHILICHTHY IDAE

Sarcotaces sp. Southern California. Abdominal cavity. Schultze 25 . MONOGENEA

MICROCOTYLIDAE

Microcotyle sebastis Goto 1894. Southern California. Gills. Crane (1972).

NEMATODA

ANISAKIDAE

Anisakis sp. Larva. Southern California. Dailey et al. (in press).

Phocanema sp. Larva. Southern California. Dailey et al. (in press). PROTOZOA (MYXOSPORIDA)

CERATOMYXIDAE

Leptotheca informis Auerbach 1910. Southern California. Gallbladder. Moser et a1. (1976).

MYXOBOLIDAE

Henneguya sebasta Moser and Love 1975. Central California.

Bulbus, truncus arteriosus. Moser and Love (1975); Southern

California. Gallbladder, urinary bladder. Moser et al. (1976).

Sebastes chrysomelas - Black and Yellow Rockfish

SYNONYM - Sebastodes chrysomelas

\section{DIGENEA}

LEPOCREADI IDAE

Opechona occidentalis Montgomery 1957. Central California. Intestine. Chapa23.

OPECOELIDAE

Helicometrina nimia Linton 1910. Central California. Intestine. Chapa23. Opecoelus noblei Banerjee 1965. Central California. Intestine. Chapaz? 
Sebastes chrysomelas (Continued)

SANGUINICOLIDAE

Psettarium sebastodorum Holmes 1971. Southern California. Holmes 35. Aporocotyle macfarlani Holmes 1971. Southern California. Holmes 35 . ISOPODA

Genera and species. Gnathiid 1arva. Southern California. Body surface. Hobson (1971).

CYMOTHOIDAE

$\frac{\text { Lironeca vulgaris Stimpson 1857. Southern California. Gills. }}{\text { Robinson } 28}$

$$
\text { Sebastes constellatus - Starry Rockfish }
$$$$
\text { SYNONYM - Sebastodes constellatus }
$$

DIGENEA

HEMIURIDAE

Sterrhurus exodicus (McFarlane 1936) Yamaguti 1958. Central California. Stomach. Nahhas ${ }^{70}$.

\section{ISOPODA}

Genera and species. Gnathiid 1arva. Southern California. Body surface. Hobson (1971).

NEMATODA

\section{ANISAKIDAE}

Anisakis sp. Larva. Southern California. Dailey et al. (in press).

$$
\text { Sebastes crameri - Darkblotched Rockfish }
$$

$$
\text { SYNONYM - Sebastodes crameri }
$$

\section{CESTODA}

\section{PHYLLOBOTHRI IDAE}

Phyllobothrium sp. Larva. Northeastern Pacific. Sekerak and Arai (1977).

\section{TENTACULARIIDAE}

Nybel inia surmenicola Okada 1929. Larva. Northeastern Pacific. Sekerak and Arai (1977). 
COPEPODA

CHONDRACANTHIDAE

Chondracanthus pinguis Wilson 1912. Northeastern Pacific. Sekerak and Arai (1977).

Chondracanthus triventricosus Sekerak 1970. Northeastern Pacific. Sekerak and Arai (1977).

\section{LERNAEOPODIDAE}

Neobrachiella robusta (Wilson 1912) Kabata 1979. British Columbia. Kabata (1970c) as Brachiella r.

PENNELLIDAE

Peniculus sp. Northeastern Pacific. Sekerak and Arai (1977).

PHILICHTHYIDAE

Sarcotaces arcticus Collett 1874. British Columbia. Body cavity. Kabata (1970a).

Colobomatus kyphosus Sekerak 1970. Northeastern Pacific. Sekerak and Arai $i(1977)$.

DIGENEA

BUCEPHALIDAE

Prosorhynchus crucibulum (Rudolphi 1819) Odhner 1905. Northeastern Pacific. Sekerak and Arai (1977).

HEMIURIDAE

Derogenes varicus (Mü1ler 1784) Looss 1901. Northeastern Pacific. Sekerak and Arai (1977).

Lecithophyl 1um botryophorum (01sson 1886) Odhner 1905. Northeastern Pacific. Sekerak and Arai (1977).

Tubulovesicula lindbergi (Layman 1930) Yamaguti 1934. Northeastern Pacific. Sekerak and Arai (1977).

\section{SANGUINICOLIDAE}

Psettarium sebastodorum Holmes 1971. Washington. British Columbia. Heart, buibus arteriosus, ventral aorta. Holmes (1971b).

\section{MONOGENEA}

\section{CAPSALIDAE}

Benedenia derzhavini (Layman 1930) Meserve 1938. Northeastern Pacific. Sekerak and Arai (1977).

Trochopus sp. Northeastern Pacific. Sekerak and Arai (1977). 
Sebastes crameri (Continued)

NEMATODA

ANISAKIDAE

Contracaecum spp. Larva. Northeastern Pacific. Sekerak and Arai (1977).

$\frac{\text { *Thynnascaris }}{(1977) \text { adunca }}$ (Rudolphi 1802) Hartwich 1957. Sekerak and Arai

CUCULLANIDAE

Cucullanus sp. Northeastern Pacific. Sekerak and Arai (1977).

$$
\text { Sebastes dalli - Calico Rockfish }
$$

NEMATODA

ANISAKIDAE

Anisakis sp. Larva. Southern California. Viscera. Jensen ${ }^{18}$.

Sebastes diploproa - Splitnose Rockfish

SYNONYM - Sebastodes diploproa

ACANTHOCEPHALA

ECHINORHYNCHIDAE

Echinorhynchus gadi Zoega in Müller 1776. Northeastern Pacific. Sekerak and Arai (1977).

CESTODA

PHYLLOBOTHRI IDAE

Phyllobothrium sp. Larva. British Columbia. Intestine. Arai (1969b).

TENTACULARI IDAE

Nybel inia surmenicola Okada 1929. Larva. Northeastern Pacific.

COPEPODA

CALIGIDAE

Lepeophtheirus paulus Cressey 1969. Northeastern Pacific. Sekerak and Arai (T977). 
Sebastes diploproa (Continued)

CHONDRACANTHIDAE

Chondracanthus pinguis wilson 1912. Northeastern Pacific. Sekerak and Arai (1977).

Chondracanthus triventricosus Sekerak 1970. Northeastern Pacific. Sekerak and Arai (1977).

\section{LERNAEOPODIDAE}

Neobrachiella robusta (Wilson 1912) Kabata 1979. Northeastern

Pacific. Sekerak and Arai (1977) as Brachiella $r$.

Clavella parva Wilson 1912. British CoTumbia. Fin rays. Kabata $(1970 \mathrm{c})$.

\section{NAOBRANCHI IDAE}

Naobranchia occidentalis Wilson 1915. Northeastern Pacific. Sekerak and Arai (1977).

PHILICHTHYIDAE

Colobomatus kyphosus Sekerak 1970. Northeastern Pacific. Sekerak and Arai (1977).

\section{DIGENEA}

BUCEPHALIDAE

Prosorhynchus crucibulum (Rudolphi 1819) Odhner 1905. Northeastern Pacific. Sekerak and Arai (1977).

GORGODERIDAE

Phyllodistomum sp. Northeastern Pacific. Sekerak and Arai (1977). HEMIURIDAE

Derogenes varicus (Müller 1784) Looss 1901. Northeastern Pacific. Sekerak and Arai (1977).

\section{OPECOELIDAE}

Podocotyle sp. Northeastern Pacific. Sekerak and Arai (1977). Neohelicometra sebastis Sekerak and Arai 1974. Northeastern

Pacific. Pyloric caeca, intestine. Sekerak and Arai (1974).

\section{SANGUINICOLIDAE}

Psettarium sebastodorum Holmes 1971. Washington, British Columbia. Heart, bulbus arteriosus, ventral aorta. Holmes (1971b). 
Sebastes diploproa (Continued)

MONOGENEA

CAPSÁLIDÁE

Trochopus trituba (Pratt and Aldrich 1953) Bravo-Hollis 1958.

Oregon. Pratt and Aldrich (1953) as Megalocotyle $t$.

Trochopus sp. Northeastern Pacific. Sekerak and Aräi (1977).

MICROCOTYLIDAE

Microcotyle sebastis Goto 1894. Southern California. Gills. Crane (1972); British Columbia. Arai (1969b).

NEMATODA

ANISAKIDAE

Anisakis sp. Larva. Southern California. Dailey et a1. (in press).

Contracaecum spp. Larva. Northeastern Pacific. Sekerak and Arai (1977).

*Thynnascaris adunca (Rudolphi 1802) Hartwich 1957. Northeastern

Pacific. Sekerak and Arai (1977).

CYSTIDICOL IDAE

Ascarophis sebastodis 01 sen 1952. Northeastern Pacific. Sekerak and Arai (1977).

PROTOZOA (MYXOSPORIDA)

MYXOBOL IDAE

Henneguya sebasta Moser and Love 1975. Southern California. Bulbus, truncus arteriosus. Moser and Love (1975).

Sebastes elongatus - Greenstriped Rockfish

SYNONYM - Sebastodes elongatus

ACANTHOCEPHALA .

POLYMORPHIDAE

Corynosoma sp. Northeastern Pacific. Sekerak and Arai (1977). CESTODA

PHYLLOBOTHRI IDAE

Phyllobothrium sp. Northeastern Pacific. Sekerak and Arai (1977). TENTACULARIIDAE

Nybelinia surmenicola Okada 1929. Larva. Northeastern Pacific. Sekerak and Arai (1977). 
Sebastes elongatus (Continued)

COPEPODA

BOMOLOCHIDAE

Holobomolochus venustus Kabata 1971. Northeastern Pacific. Sekerak and Arai (1977).

\section{LERNAEOPODIDAE}

Clavella parva Wilson 1912. Northeastern Pacific. Sekerak and Arai (1977).

\section{PHILICHTHYIDAE}

Colobomatus kyphosus Sekerak 1970. Northeastern Pacific. Sekerak and Arai (1977).

\section{DIGENEA}

\section{BUCEPHALIDAE}

Prosorhynchus crucibulum (Rudolphi 1819) Odhner 1905. Northeastern Pacific. Sekerak and Arai (1977).

\section{FELLODISTOMATIDAE}

Feilodistomum sebastodis Yamaguti and Matumura 1942. Northeastern Pacific. Sekerak and Arai (1977).

HEMIURIDAE

Sterrhurus exodicus (McFarlane 1936) Yamaguti 1958. Northeastern Pacific. Sekerak and Arai (1977). Derogenes varicus (Mü1 ler 1784) Looss 1901. Northeastern Pacific. Sekerak and Arai (1977).

\section{LEPOCREADI IDAE}

Opechona occidentalis Montgomery 1957. British Columbia. Intestine. Arai (1969b).

OPECOELIDAE

Podocotyle sp. Northeastern Pacific. Sekerak and Arai (1977). Opecoelina radifistuli (Acena 1941) Manter 1947. Washington. Intestine. Acena (1941) as Dideutosaccus $r$.

ZOOGONIDAE

Deretrema cholaeum McFarlane 1936. Northeastern Pacific. Sekerak and Arai (1977). 
Sebastes elongatus (Continued)

MONOGENEA

CAPSALIDAE

Trochopus sp. Northeastern Pacific. Sekerak and Arai (1977).

MICROCOTYLIDAE

Microcotyle sebastis Goto 1894. California. Gills.

Crane (1972); British Columbia. Gills. Arai (1969b).

NEMATODA

ANISAKIDAE

Anisakis sp. Larva. Southern California. Dailey et al. (in press). Contracaecum sp. Larva. Northeastern Pacific. Sekerak and Arai (1977).

* Thynnascaris adunca (Rudolphi 1802) Hartwich 1957. Northeastern Pacific. Sekerak and Arai (1977).

CYSTIDICOL IDAE

Ascarophis sebastodis 01 sen 1952. British Columbia. Intestine. Arai (1969b).

PROTOZOA (MYXOSPORIDA)

TETRACAPSUL IDAE

Kudoa clupeidae (Hahn 1917) Meglitsch 1947. Southern California. Muscies. Moser et a1. (1976).

\section{Sebastes ensifer - Swordspine Rockfish}

PROTOZOA (MYXOSPORIDA)

CERATOMYXIDAE

Ceratomyxa vepallida Meglitsch 1960. Southern California. Gallbladder. Moser et a1. (1976).

Ceratomyxa sebasta Moser, Love and Jensen 1976. Southern California.

Gallbladder. Moser et al. (1976).

MYXIDIIDAE

Myxidium incurvatum Thélohan 1892. Southern California. Gallbladder. Moser et al. (1976). 


\section{CESTODA}

PHYLLOBOTHRIIDAE

Phyllobothrium sp. Larva. Northeastern Pacific. Sekerak and Arai (1977).

\section{TENTACULARI IDAE}

Nybel inia surmenicola Okada 1929. Larva. Northeastern Pacific.

$$
\text { Sekerak and Arai (1977). }
$$

COPEPODA

\section{CHONDRACANTHIDAE}

Chondracanthus pinguis Wilson 1912. Northeastern Pacific. Sekerak and Arai (1977).

\section{LERNAEOPODIDAE}

Neobrachiella robusta (Wilson 1912) Kabata 1979. Northeastern Pacific. Sekerak and Arai (1977) as Brachiella r.

\section{PHILICHTHYIDAE}

Colobomatus kyphosus Sekerāk 1970. Northeastern Pácific. Sekerak and Arai (1977).

\section{DIGENEA}

BUCEPHALIDAE

Prosorhynchus crucibulum (Rudolphi 1819) Odhner 1905. Northeastern Pacific. Sekerak and Arai (1977).

HEMIURIDAE

Derogenes varicus (Müiler 1784) Looss 1901. Northeastern Pacific. Sekerak and Arai (1977).

\section{MONOGENEA}

CAPSALIDAE

Trochopus sp. Northeastern Pacific. Sekerak and Arai (1977).

\section{NEMATODA}

\section{ANISAKIDAE}

Contracaecum sp. Larva. Northeastern Pacific. Sekerak and Arai (1977).

* Thynnascaris adunca (Rudolphi 1802) Hartwich 1957. Sekerak and Arai (1977). 


\section{Sebastes eos - Pink Rockfish}

ACANTHOCEPHALA

ECHINORHYNCHIDAE

Echinorhynchus sp. Southern California. Jensen ${ }^{18}$.

$$
\begin{aligned}
& \frac{\text { Sebastes flavidus - Yellowtail Rockfish }}{\text { SYNONYM - Sebastodes flavidus }} \\
&
\end{aligned}
$$

ACANTHOCEPHALA

ECHINORHYNCHIDAE

Echinorhynchus gadi Zoega in Mü1ler 1776. Northeastern Pacific. Sekerak and Arai (1977).

\section{CESTODA}

\section{TENTACULARI IDAE}

Nybelinia surmenicola Okada 1929. Larva. Northeastern Pacific. Sekerak and Arai (1977).

\section{COPEPOOAA}

\section{CALIGIDAE}

Lepeophtheirus paulus Cressey 1969. British Columbia. Kabata (1973). CHONDRACANTHIDAE

Chondracanthus pinguis Wilson 1912. Northeastern Pacific. Sekerak and Arai (1977).

Chondracanthus triventricosus Sekerak 1970. Northeastern Pacific. Sekerak and Arai (1977).

\section{LERNAEOPODIDAE}

Neobrachiella robusta (Wilson 1912) Kabata 1979. British Columbia. Kabata (1970c) as Brachiella r.

Clavella parva Wilson 1912. Northeastern Pacific. Sekerak and Arai (1977).

\section{PENNELLIDAE}

Peniculus asinus Kabata and Wilkes 1977. British Columbia. Caudal fin. Kabata and Wilkes (1977).

\section{PHILICHTHYIDAE}

Colobomatus kyphosus Sekerak 1970. Northeastern Pacific. Sekerak and Arai (1977).

Sarcotaces arcticus Collett 1874. Alaska. Internal. Avdeev and Avdeev (1975). 


\section{DIGENEA}

BUCEPHALIDAE

Prosorhynchus crucibulum (Rudolphi 1819) Odhner 1905. Northeastern Pacific. Sekerak and Arai (1977).

\section{HEMIURIDAE}

Sterrhurus exodicus (McFarlane 1936) Yamaguti 1958. Northeastern Pacific. Sekerak and Arai (1977).

Derogenes varicus (Mü11er 1784) Looss 1901. Northeastern Pacific. Sekerak and Arai (1977).

Lecithophyllum botryophorum (01sson 1886) Odhner 1905. Northeastern Pacific. Sekerak and Arai (1977).

\section{LEPOCREADI IDAE}

Opechona alaskensis Ward and Fillingham 1934. Northeastern Pacific. Sekerak and Arai (1977).

Opechona occidentalis Montgomery 1957. Northeastern Pacific. Sekerak and Arai (T977).

\section{OPECOELIDAE}

Neohelicometra sebastis Sekerak and Arai 1974. Northeastern Pacific. Pyloric caeca, intestine. Sekerak and Arai (1974).

Podocotyle sp. Northeastern Pacific. Sekerak and Arai (1977).

\section{SANGUINICOLIDAE}

Aporocotyle macfarlani Holmes 1971. Washington. British Columbia. Branchial arteries, ventral aorta, bulbus arteriosus, ventricle. Holmes (1971b).

Psettarium sebastodorum Holmes 1971. Washington, British Columbia. Heart, bulbus arteriosus, ventral aorta. Holmes (1971b).

\section{SYNCOELIDAE}

Syncoelium filiferum (Leuckart in Sars 1885) Odhner 1911. Northeastern Pacific. Sekerak and Arai (1977).

\section{MONOGENEA}

\section{CAPSALIDAE}

Trochopus sp. Northeastern Pacific. Sekerak and Arai (1977).

\section{NEMATODA}

\section{ÂNISÁKIDÁE}

Contracaecum spp. Larva. Northeastern Pacific. Sekerak and Arai (1977).

* Thynnascaris adunca (Rudoiphi 1802) Hartwich 1957. Sekerak and Arai (1977). 
Sebastes flavidus (Continued)

CAPILLARI IDAE

Capillaria sp. Northeastern Pacific. Sekerak and Arai (1977).

CUCULLANIDAE

Cucullanus sp. Northeastern Pacific. Sekerak and Arai (1977).

CYSTIDICOLIDAE

Ascarophis sebastodis 01sen 1952. Northeastern Pacific. Sekerak and Arai (1977).

PROTOZOA (MYXOSPORIDA)

MYXOBOLIDAE

Henneguya sebasta Moser and Love 1975. Northern California. Heart, gallbladder. Moser et a1. (1976).

\section{Sebastes gilli - Bronzespotted Rockfish}

NEMATODA

ÂNISÂKIDÂE

Phocanema sp. Larva. Southern Californja. Dailey et al. (In press). Anisakis sp. Larva. Southern California. Dailey et al. (In press).

\section{Sebastes goodei - Chilipepper}

DIGENEA

HEMIURIDAE

Derogenes varicus (Müiler 1784) Looss 1901. Northeastern Pacific. Sekerak and Arai (1977).

OPECOEL IDAE

Podocotyle sp. Northeastern Pacific. Sekerak and Arai (1977). MONOGENEA

CAPSALIDAE

Trochopus sp. Northeastern Pacific. Sekerak and Arai (1977). NEMATODA

ANISAKIDAE

Contracaecum sp. Larva. Northeastern Pacific. Sekerak and Arai (1977). 
Sebastes goodei (Continued)

Anisakis sp. Larva. Southern California. Dailey et al. (in press). PROTOZOA (MYXOSPORIDA)

\section{CERATOMYXIDAE}

Ceratomyxa hopkinsi Jameson 1929. Northern California. Gallbladder. Moser et a1. (1976).

Leptotheca informis Auerbach 1910. Northern California. Gallbladder. Moser et a1. (1976).

MYXOBOLIDAE

Henneguya sebasta Moser and Love 1975. Southern California. Heart, urinary bladder. Moser et a1. (1976).

Sebastes helvomaculatus - Rosethorn Rockfish

SYNONYM - Sebastodes helvomaculatus

ACANTHOCEPHALA

ECHINORHYNCHIDAE

Echinorhynchus gadi Zoega in Mü1ler 1776. Northeastern Pacific. Sekerak and Arai (1977).

POLYMORPHIDAE

Corynosoma sp. Larva. Northeastern Pacific. Sekerak and Arai (1977). COPEPODA

CALIGIDAE

Lepeophtheirus oblitus Kabata 1973. Northeastern Pacific. Sekerak and Arai (1977).

CHONDRACANTHIDAE

Chondracanthus triventricosus Sekerak 1970. Northeastern Pacific. Sekerak and Arai (1977).

DIGENEA

HEMIURIDAE

Derogenes varicus (Mü17er 1784) Looss 1901. Northeastern Pacific. Sekerak and Arai (1977).

LEPOCREADI IDAE

Neolepidapedon sebastisci (Yamaguti 1938) Manter 1954. Northeastern Pacific. Sekerak and Arai (1977). 
OPECOEL IDAE

Neohelicometra sebastis Sekerak and Arai 1974. Northeastern

Pacific. Pyloric caeca, intestine. Sekerak and Arai (1974). Podocotyle sp. Northeastern Pacific. Sekerak and Arai (1977).

SANGUINICOLIDAE

Psettarium sebastodorum Holmes 1971. Washington, British Columbia. Heart region. Holmes (1971b).

MONOGENEA

MICROCOTYLIDAE

Microcotyle sebastis Goto 1894. Northeastern Pacific. Sekerak and Arai (1977).

NEMATODA

ANISAKIDAE

Contracaecum spp. Larva. Northeastern Pacific. Sekerak and Arai $(1977)$.

* Thynnascaris adunca (Rudolphi 1802) Hartwich 1957. Larva.

Northeastern Pacific. Sekerak and Arai (1977).

Sebastes hopkinsi - Squarespot Rockfish

NEMATODA

ANISAKIDAE

Anisakis sp. Larva. Southern California. Dailey et al. (in press).

Sebastes jordani - Shortbelly Rockfish

PROTOZOA (MYXOSPORIDA)

MYXOBOLIDAE

Henneguya sebasta Moser and Love 1975. Southern California.

Bulbus, trunchus arteriosus. Moser and Love (1975).

\section{Sebastes levis - Cow Rockfish}

\section{ACANTHOCEPHALA}

ECHINORHYNCHIDAE

Echinorhynchus sp. Southern California. Jensen ${ }^{18}$. 
Sebastes levis (Continued)

\section{CESTODA}

TRYPANORHYNCHA

Genera and species. Larva. Southern California. Jensen ${ }^{18}$. NEMATODA

ANISAKIDAE

Anisakis sp. Larva. Southern California. Dailey et al. (in press). PROTOZOA (MYXOSPORIDA)

MYXOBOLIDAE

Henneguya sebasta Moser and Love 1975. Southern California. Heart. Moser et a1. (T976).

PROTOZOA (SPOROZOA)

EIMERI IDAE

Genera and species. Southern California. Jensen ${ }^{18}$.

Sebastes macdonaldi - Mexican Rockfish

NEMATODA

ANISAKIDAE

Anisakis sp. Larva. Southern California. Dailey et al. (in press).

Sebastes maliger - Quillback Rockfish

SYNONYM - Sebastodes maliger

ACANTHOCEPHALA

POLYMORPHIDAE

Corynosoma sp. Larva. Northeastern Pacific. Sekerak and Arai (1977). CESTODA

PHYLLOBOTHRIIDAE

Phyllobothrium sp. Larva. British Columbia. Intestine. Arai (1969b). TENTACULARI IDAE

Nybelinia surmenicola Okada 1929. Larva. Northeastern Pacific. Sekerak and Arai (1977). 
Sebastes maliger (Continued)

COPEPODA

BOMOLOCHIDAE

Holobomolochus venustus Kabata 1971. Northeastern Pacific. Sekerak and Arai (1977).

CALIGIDAE

Lepeophtheirus paulus Cressey 1969. British Columbia. Kabata (1973). Lepeophtheirus oblitus Kabata 1973. British Columbia. Kabata (1973). Lepeophtheirus longipes Wilson 1975. Washington. Skin. Nichols (1975).

\section{CHONDRACANTHIDAE}

Chondracanthus triventricosus Sekerak 1970. Northeastern Pacific. Sekerak and Arai (1977).

Chondracanthus pinguis Wilson 1912. Northeastern Pacific. Sekerak and Arai (1977).

\section{LERNAEOPODIDAE}

Clavella parva Wilson 1912. British Columbia. Bere ${ }^{5}$.

Neobrachiella robusta (Wilson 1912) Kabata 7979. British Columbia. Kabata (1970c) as Bracheilla r.

\section{NAOBRANCHI IDAE}

Naobranchia occidentalis Wilson 1915. British Columbia. Bere ${ }^{5}$.

\section{PHILICHTHYIDAE}

Colobomatus kyphosus Sekerak 1970. Northeastern Pacific.

$$
\text { Sekerak and Araj (1977). }
$$

\section{DIGENEA}

\section{ACANTHOCOLPIDAE}

Stephanostomum californicum Manter and Van Cleave 1961. Northeastern Pacific. Sekerak and Arai (1977).

HEMIURIDAE

Derogenes varicus (Mü1ler 1784) Looss 1901. Washington. Lloyd (7938).

Lecithaster gibbosus (Rudolphi 1802) Lühe 1901. Washington. Ching (1960a) as L. salmonis.

Sterrhurus exodicus (McFarlane 1936) Yamaguti 1958. Washington. Stomach. Lloyd (1938) as Lecithochirium e.

Tubulovesicula lindergi (Layman 1930) Yamaguti 1934. Northeastern

Pacific. Sekerak and Arai (1977). 
Sebastes maliger (Continued)

\section{LEPOCREADI IDAE}

Opechona occidentāi is Montgomery 1957. Washington. Ching (1960a).

Opechona theragrae LToyd 1938. Washington. Ching (1960a).

Opechona alaskensis Ward and Fillingham 1934. Northeastern Pacific. Sekerak and Arai (1977).

\section{OPECOELIDAE}

Podocotyle sp. Northeastern Pacific. Sekerak and Arai (1977).

Podocotyle abitionis McFarlane 1936. Washington. Ching (1960a).

Podocotyle sinusacca Ching 1960. British Columbia. Intestine. Arai (1969b) as Neopodocotyloides s.

Neohelicometra sebastis Sekerak and Ärai 1974. Northeastern Pacific. Pyloric caeca, intestine. Sekerak and Arai (1974).

Helicometra sp. British Columbia. Intestine. Arai (1969b).

\section{SANGUINICOLIDAE}

Aporocotyle macfarlani Holmes 1971. Washington. Ching (1960a) as A. simplex; British Columbia. Afferent branchial arteries, ventral aorta, bulbus arteriosus, ventricle. Holmes (1971b).

Psettarium sebastodorum Holmes 1971. Washington, British Columbia. Hearts bulbus arteriosus, ventral aorta. Holmes (1971b).

\section{ZOOGONIDAE}

Deretrema cholaeum McFarlane 1936. British Columbia. Gallbladder. Arai (1969b).

\section{ISOPODA}

\section{AEGIIDAE}

Rocinela belliceps (Stimpson 1864) Richardson 1899. British Columbia. Gi11 cavity. Fee (1927).

Rocinela propodialis Richardson 1905. British Columbia. Gill cavity. Fee $(1927)$.

\section{MONOGENEA}

\section{CAPSALIDAE}

Trochopus marginata (Folda 1928) Price 1936. Washington. Gills. Bonham (1950) as Megalocotyle m.

\section{MICROCOTYLIDAE}

Microcotyle sebastis Goto 1894. Washington. Bonham and Guberlet (1937). 
Sebastes maliger (Continued)

NEMATODA

ANISAKIDAE

Contracaecum spp. Larva. Northeastern Pacific. Sekerak and Arai (1977).

* Thynnascaris adunca (Rudolphi 1802) Hartwich 1957. Northeastern Pacific. Sekerak and Arai (1977).

CAPILLARI IDAE

Capillaria sp. Northeastern Pacific. Sekerak and Arai (1977).

CUCULLANIDAE

Cucullanus elongatus Smedley 1933. Northeastern Pacific. Sekerak and Arai $(1977)$.

CYSTIDICOLIDAE

Ascarophis sebastodis 01 sen 1952. Northeastern Pacific. Sekerak and Arai (1977).

PROTOZOA (MYXOSPORIDA)

SINUOLINEIDAE

Davisia reginae Love and Moser 1976. Northern California. Urinary bladder. Love and Moser (1976).

$$
\text { Sebastes melanops - Black Rockfish }
$$

SYNONYM - Sebastodes melanops

COPEPODA

CHONDRACANTHIDAE

Acanthochondria deltoideus (Fraser 1920). British Columbia. Bere ${ }^{5}$ as Chondracanthus d.

Chondracanthus sp. Oregon. Gill cavity. Millemann ${ }^{34}$.

Chondracanthus pinguis Wilson 1912. British Columbia. Beres.

\section{LERNAEOPODIDAE}

Neobrachiella robusta (Wilson 1912) Kabata 1979. British Columbia. Kabata (1970c) as Brachiella r.; Fraser (1920) as Clavellopsis r. Clavella parva Wilson 1912. Alaska. Dorsa1, caudal fins. Wilson (1915) as $\underline{\mathrm{C}}$ recta.

\section{DIGENEA}

BUCEPHALIDAE

Prosorhynchus sp. Metacercaria. Northeast Pacific. Liston et a1. (1960). 
Sebastes melanops (Continued)

HEMIURIDAE

Lecithaster gibbosus (Rudolphi 1802) Lühe 1901. Washington. Ching (1960a) as L. Salmonis.

Tubulovesicula lindbergi (Layman 1930) Yamaguti 1934. Washington. Ching (1960a).

LEPOCREADI IDAE

Opechona parvasoma Ching 1960. Washington. Intestine. Ching (1960a).

SANGUINICOLIDAE

Aporocotyle macfarlani Holmes 1971. Washington, British Columbia. Afferent branchial arteries, ventral aorta, bulbus arteriosus, ventricle. Holmes (1971b).

Psettarium sebastodorum Holmes 1971. Washington, British Columbia. Heart, bulbus arteriosus, ventral aorta. Holmes (1971b).

HIRUDINOIDEA

PISCICOLIDAE

Ma lmiana diminuta Burreson 1977. Oregon. Skin, fins. Burreson (1977c). Ostreobde 11a papi11ata Burreson 1977. Oregon. Burreson (1977b).

MONOGENEA

CAPSALIDAE

Trochopus marginata (Folda 1928) Price 1936. Washington. Gills. Bonham (1950) as Megalocotyle $\mathbf{m}$.

MICROCOTYLIDAE

Microcotyle sebastis Goto 1894. Washington. Bonham and Guberlet (1937); Oregon. Gi11s. Pratt and McCauley (1961).

PROTOZOA (MYXOSPORIDA)

MYXOBOLIDAE

Henneguya sebasta Moser and Love 1975. Central California. Heart. Moser et al. (1976).

\section{Sebastes miniatus - Vermilion Rockfish}

SYNONYM - Sebastodes minatus

CESTODA

BOTHRIOCEPHAL IDAE

Bothriocephalus scorpii (Müller 1776) Rudolphi 1808. Southern California. Jensen 18 . 
Sebastes miniatus (Continued)

TRYPANORHYNCHA

Genera and species. Larva. Southern California. Jensen ${ }^{18}$. COPEPODA

NAOBRANCHI IDAE

$\frac{\text { Naobranchig occidentalis Wilson 1915. Southern California. Gills. }}{\text { Jensen }}{ }^{8}$. MONOGENEA

MICROCOTYLIDAE

Microcotyle sebastis Goto 1894. California. Crane (1972). NEMATODA

ANISAKIDAE

Anisakis sp. Larva. Southern California. Viscera. Dailey et al. (in press).

Phocanema sp. Larva. Southern California. Viscera. Dailey et al. (in press).

PROTOZOA (MYXOSPORIDA)

MYXIDI IDAE

Myxidium calcariferi Chakravarty 1943. Southern California. Ga17bTadder. Moser et a1. (1976).

MYXOBOLIDAE

Henneguya sebasta Moser and Love 1975. Central California.

Bulbus, truncus arteriosus. Moser and Love (1975).

Sebastes mystinus - Blue Rockfish

SYNONYM - Sebastodes mystinus

COPEPODA

BOMOLOCHIDAE

Holobomolochus spinulus (Cressey 1969) Kabata 1971. Southern

California. Gill cavity. Cressey (1969b).

\section{CALIGIDAE}

Caligus hobsoni Cressey 1969. Southern California. Hobson (1971). 
Sebastes mystinus (Continued)

\section{DIGENEA}

HEMIURIDAE

Sterrhurus exodicus (McFarlane 1936) Yamaguti 1958. Northern California. Stomach. Chapa 23 as Separogermiductus e.

\section{LEPOCREADI IDAE}

Lepidapedon elongatum (Lebour 1908) Nicol1 1915. Northern California. Intestine. Chapa 23.

ISOPODA

CYMOTHOIDAE

Lironeca vulgaris Stimpson 1857. Southern California. Gills. Robinson 28 .

Sebastes nebulosus - China Rockfish

SYNONYM - Sebastodes nebulosus

DIGENEA

HEMIURIDAE

Sterrhurus exodicus (McFarlane 1936) Yamaguti 1958. Northern California. Stomach. Chapa 23 as Separogermiductus e.

\section{LEPOCREADI IDAE}

Opechona occidental is Montgomery 1957. Northeastern Pacific. Sekerak and Arai (1977).

Lepidapedon elongatum (Lebour 1908) Nicol1 1915. Washington. Intestine. Ching (1961).

Neolepidapedon pugetense (Acena 1947) Yamaguti 1971. Washington. Intestine. Acena (1947) as Lepidapedon p.

OPECOELIDAE

Opecoelina pharynmagna Annereaux 1943. Northern California. Stomach, intestine. Annereaux (1943).

Neohelicometra sebastis Sekerak and Arai 1974. Northeastern Pacific. Sekerak and Arai (1977).

\section{MONOGENEA}

\section{CAPSALIDAE}

Trochopus marginata (Folda 1928) Price 1936. Washington. Gills. Folda (1928); Southern California. Gills. Crane (1972) both as Megalocotyle $\mathrm{m}$. 
Sebastes nebulosus (Continued)

NEMATODA

\section{ANISAKIDAE}

Contracaecum sp. Larva. Northeastern Pacific. Sekerak and Arai (1977).

* Thynnascaris adunca (RudoTphi 1802) Hartwich 1957. Northeastern Pacific. Sekerak and Arai (1977).

\section{Sebastes nigrocinctus - Tiger Rockfish}

$$
\text { SYNONYM - Sebastodes nigrocinctus }
$$

CESTODA

TENTACULARIIDAE

Nybelinia surmenicola Okada 1929. Larva. Northeastern Pacific. Sekerak and Arai (1977).

COPEPODA

CALIGIDAE

Lepeophtheirus paulus Cressey 1969. British Columbia. Kabata (1973). NAOBRANCHI IDAE

Naobranchia occidental is Wilson 1915. Northeastern Pacific. Sekerak and Arai (1977).

\section{PHILICHTHYIDAE}

Colobomatus kyphosus Sekerak 1970. Northeastern Pacific. Sekerak and Arai (1977).

\section{DIGENEA}

HEMIURIDAE

Tubulovesicula lindbergi (Layman 1930) Yamaguti 1934. Washington. Ching (1960a).

\section{OPECOELIDAE}

Neohelicometra sebastis Sekerak and Arai 1974. Northeastern Pacific. Pyloric caeca, intestine. Sekerak and Arai (1974).

\section{SANGUINICOLIDAE}

Psettarium sebastodorum Holmes 1971. Many sites. Washington. British Columbia. Heart, bulbus arteriosus, ventral aorta. Holmes (1971b). 
Sebastes nigrocinctus (Continued)

ZOOGONIDAE

Deretrema cholaeum McFarlane 1936. Northeastern Pacific.

Sekerak and Arai (1977).

MONOGENEA

MICROCOTYLIDAE

Microcotyle sebastis Goto 1894. Northeastern Pacific. Sekerak and Arai (1977).

NEMATODA

ANISAKIDAE

Contracaecum sp. Larva. Northeastern Pacific. Sekerak and Arai (1977).

CAP ILLARI IDAE

Capillaria sp. Northeastern Pacific. Sekerak and Arai (1977).

Sebastes ovalis - Speckled Rockfish

NEMATODA

ANISAKIDAE

Anisakis sp. Larva. Southern California. Dailey et al. (in press).

Sebastes paucispinis - Boccacio

SYNONYMS - Sebastodes paucispinis, Sebastodes paucispirius

ACANTHOCEPHALA

ECHINORHYNCHIDAE

Echinorhynchus sp. Southern California. Jensen and Heckmann (1977b). POLYMORPHIDAE

Corynosoma sp. Southern California. Jensen and Heckmann (1977b). CESTODA

BOTHRIOCEPHALIDAE

Bothriocephalus sp. Northeastern Pacific. Sekerak and Arai (1977). 


\section{LACISTORHYNCHIDAE}

Lacistorhynchus tenuis (van Beneden 1858) Dollfus 1929. Larva. Southern California. Jensen and Heckmann (1977b). Grillotia smarisgora (Wagener 1854) Dollfus 1946. Larva.

Southern California. Mesentery. Jensen 18.

Grillotia sp. Larva. Southern California. Jensen 18.

ONCOBOTHRIIDAE

Acanthobothrium sp. Larva. Southern California. Intestine. Jensen 18 .

PARABOTHRIOCEPHALIDAE

Parabothriocephalus sagitticeps (S1eggs 1927) Jensen 1976. Southern California. Intestine. Jensen (1976).

TETRAPHYLLIDEA

Genera and species. Larva. Southern California. Jensen ${ }^{18}$. COPEPODA

CALIGIDAE

Lepeophtheirus sp. Southern California. Jensen and Heckmann (1977b). NAOBRANCHI IDAE

Naobranchia occidentalis Wilson 1915. Northeastern Pacific. Gill filaments. Sekerak and Arai (1977); Southern California. Jensen and Heckmann (1977b).

\section{DIGENEA}

BUCEPHALIDAE

Prosorhynchus crucibulum (Rudolphi 1819) Odhner 1905. Northeastern Pacific. Sekerak and Arai (1977).

HEMIURIDAE

Derogenes varicus (Mu17er 1784) Looss 1901. Northeastern Pacific. Sekerak and Arai (1977); Southern California. Jensen and Heckmann (1977b).

Derogenes crassus Manter 1934. Oregon. McCauley (1960).

Lecithophyllum botryophorum (01sson 1886) Odhner 1905. Northeastern Pacific. Sekerak and Arai (1977).

Sterrhurus exodicus (McFarlane 1936) Yamaguti 1958. Northeastern Pacific. Sekerak and Arai (1977); Southern California. Jensen and Heckmann (1977b).

Tubulovesicula lindbergi (Layman 1930) Yamaguti 1934.

Northeastern Pacific. Sekerak and Arai (1977). 
Sebastes paucispinis (Continued)

\section{LEPOCREADI IDAE}

Neoiepidapedon sebastisci (Yamaguti 1938) Manter 1954. Northeastern Pacific. Sekerak and Arai (1977).

\section{OPECOEL IDAE}

Podocotyle sp. Northeastern Pacific. Sekerak and Arai (1977).

\section{SANGUIN ICOL IDAE}

Psettarium sebastodorum Holmes 1971. British Columbia. Heart. Holmes (1971a); Southern California. Jensen and Heckmann (1977b).

\section{ZOOGONIDAE}

Deretrema cholaeum McFarlane 1936. Southern California. Gallbladder. Jensen 18 .

\section{MONOGENEA}

\section{CAPSAL IDAE}

Trochopus trituba (Pratt and Aldrich 1953) Bravo-Hollis 1958.

Oregon. Gills. Pratt and Aldrich (1953) as Megalocotyle t.

Pseudobenedenia noblei (Menzies 1946) Yamaguti 1963. Centra1

California. Gills. Menzies (1946) as Benedenia noblei.

\section{MICROCOTYLIDAE}

Microcotyle sebastis Goto 1894. Southern California. Gills. Crane (1972); Northeastern Pacific. Sekerak and Arai (1977).

\section{NEMATODA}

\section{ANISAKIDAE}

Contracaecum spp. Larva. Northeastern Pacific. Sekerak and Arai (1977); Southern California. Jensen and Heckmann (1977b). Anisakis sp. Larva. British Columbia. Many places in body. Margolis 3; Southern Cal ifornia.

* Thynnascaris adunca (Rudolphi 1802) Hartwich 1957. Northeastern Pacific. Sekerak and Arai (1977); Southern California. Jensen and Heckmann (1977b).

Phocanema sp. Larva. Southern California. Viscera. Dailey et al. (in press).

\section{CAPILLARI IDAE}

Capillaria sp. Northeastern Pacific. Sekerak and Arai (1977).

\section{CUCULLANIDAE}

Cucullanus sp. Northeastern Pacific. Sekerak and Arai (1977); Southern California. Jensen and Heckmann (1977b). 
Sebastes paucispinis (Continued)

PROTOZOA (MYXOSPORIDA)

CERATOMYXIDAE

Leptotheca informis Auerbach 1910. Southern California. Ga7lbladder. Moser et a1. (1976).

Ceratomyxa vepallida Meglitsch 1960. Southern California. Gal1b7adder. Moser et al. (1976).

Ceratomyxa californica Jameson 1929. Southern California. GalTbladder. Moser et al. (1976).

Ceratomyxa sebasta Moser, Love and Jensen 1976. Northern California. GaT7bladder. Moser et a . (1976).

Ceratomyxa elegans Jameson 1929. Southern California. Jensen and Heckmann $(1977 \mathrm{~b})$.

Ceratomyxa hopkinsi Jameson 1929. Southern California. Jensen and Heckman (1977b).

MYXIDIIDAE

Myxidium incurvatum Thélohan 1892. Southern California. Gallbladder. Moser et a 1. (1976).

Myxidium calcariferi Chakravarty 1943. Southern California. Ga17bTadder. Moser et a1. (1976).

MYXOBOLIDAE

Henneguya sebasta Moser and Love 1975. Central and Southern California. Bulbus, truncus arteriosus. Moser and Love (1975).

\section{TETRACAPSULIDAE}

Kudoa clupeidae (Hahn 1917) Meglitsch 1947. Southern California. Muscles. Moser et al. (1976).

Sebastes phillipsi - Chameleon Rockfish

NEMATODA

ANISAKIDAE

Anisakis sp. Larva. Southern California. Dailey et al. (in press).

$$
\text { Sebastes pinniger - Canary Rockfish }
$$

SYNONYM - Sebastodes pinniger

CESTODA

BOTHRIOCEPHALIDAE

Bothriocephalus sp. Immature. Northeastern Pacific. Sekerak and Arai (1977). 
Sebastes pinniger (Continued)

PHYLLOBOTHRIIDAE

Phyllobothrium sp. Larva. Northeastern Pacific. Sekerak and Arai (1977).

TENTACULARI IDAE

Nybel inia surmenicola Okada 1929. Larva. Northeastern Pacific. Sekerak and Arai (7977).

COPEPODA

BOMOLOCHIDAE

Homobomolochus venustus Kabata 1971. Northeastern Pacific. Sekerak and Arai (1977).

CALIGIDAE

Lepeophtheirus parviventris Wilson 1905. British Columbia. Kabata (1973). CHONDRACANTHIDAE

Chondracanthus pinguis Wilson 1912. British Columbia. Gill cavity. Kabata (1968).

LERNAEOPODIDAE

Neobrachiella robusta (Wilson 1912) Kabata 1979. British Columbia.

Kabata (1970c) as Brachieila $r$.

Clavella parva Wi1son 1912. Northeastern Pacific. Sekerak and Arai (1977).

NAOBRANCHI IDAE

Naobranchia occidentalis Wilson 1915. Northeastern Pacific. Sekerak and Arai (1977).

PHILICHTHYIDAE

Colobomatus kyphosus Sekerak 1970. Northeastern Pacific. Sekerak and Arai (1977).

DIGENEA

BUCEPHAL IDAE

Prosorhynchus sp. Pacific Northwest. Liston et al. (1960).

Prosorhynchus crucibulum (Rudolphi 1819) Odhner 1905. Northeastern Pacific. Sekerak and Arai (1977)

HEMIURIDAE

Tubulovesicula lindbergi (Layman 1930) Yamaguti 1934. Northeastern Pacific. Sekerak and Arai (1977). 
Sebastes pinniger (Continued)

Derogenes varicus (Müller 1784) Looss 1901. Northeastern Pacific. Sekerak and Arai (1977).

Lecithaster gibbosus (Rudolphi 1802) Lühe 1901. Northeastern Pacific. Sekerak and Arai (1977).

\section{LEPOCREADI IDAE}

Neolepidapedon sebastisci (Yamaguti 1938) Manter 1954. Northeastern Pacific. Sekerak and Arai (1977).

Opechona alaskens is Ward and Fillingham 1934. Northeastern Pacific. Sekerak and Arai (1977).

OPECOELIDAE

Podocotyle sp. Northeastern Pacific. Sekerak and Arai (1977).

SANGUINICOLIDAE

Aporocotyle macfarlani Holmes 1971. Washington, British Columbia. Heart region and branchial arteries. Holmes (1971b).

Psettarium sebastodorum Holmes 1971. Washington, British Columbia. Heart region. Holmes (1971b).

\section{SYNCOEL IDAE}

Syncoelium filiferum (Leuckart, in Sars 1885) Odhner 1911. Northeastern Pacific. Sekerak and Arai (1977).

MONOGENEA

\section{CAPSALIDAE}

Trochopus trituba (Pratt and Aldrich 1953) Bravo-Hollis 1958. Oregon. Pratt and Aldrich (1953) as Megalocotyle t. Trochopus sp. Northeastern Pacific. Sekeräk and Arai (1977).

NEMATODA

ANISAKIDAE

Contracaecum sp. Larva. Northeastern Pacific. Sekerak and Arai (1977).

* Thynnascaris adunca (Rudolphi 1802) Hartwich 1957. Northeastern Pacific. Sekerak and Arai (1977).

Phocanema decipiens (Krabbe 1878) Myers 1959. Pacific northwest. Liston et al. (1960) as Porrocaecum d.

Anisakis sp. Larva. Southern California. Liver. Jensen ${ }^{18}$.

PROTOZOA (MYXOSPORIDA)

CERATOMYXIDAE

Ceratomyxa elegans Jameson 1929. Southern California. Gallbladder. Moser et a7. (1976).

Ceratomyxa sebasta Moser, Love and Jensen 1976. Northern California. Gallbladder. Moser et al. (1976). 
Sebastes proriger - Redstripe Rockfish

SYNONYM - Sebastodes proriger

ACANTHOCEPHALA

ECHINORHYNCHIDAE

Echinorhynchus gadi Zoega in Müller 1776. Northeastern Pacific. Sekerak and Arai (1977).

\section{CESTODA}

BOTHRIOCEPHAL IDAE

Bothriocephalus sp. Northeastern Pacific. Sekerak and Arai (1977).

\section{TENTACULARI IDAE}

Nybel inia surmenicola Okada 1929. Larva. Northeastern Pacific. Sekerak and Arai (1977).

COPEPODA

CHONDRACANTHIDAE

Chondracanthus pinguis Wilson 1912. Northeastern Pacific. Sekerak and Arai (1977).

Chondracanthus triventricosus Sekerak 1970. Northeastern Pacific. Sekerak and Arai (1977).

\section{LERNAEOPODIDAE}

Neobractiella robusta (Wilson 1912) Kabata 1979. British Columbia. Kabata (1970c) as Brachiella $r$.

NAOBRANCHI IDAE

Naobranchia occidentalis Wilson 1915. Northeastern Pacific. Sekerak and Arai $(1977)$.

\section{PENNELL IDAE}

Peniculus sp. Northeastern Pacific. Sekerak and Arai (1977).

Peniculus asinus Kabata and Wilkes 1977. British Columbia. Caudal fin. Kabata and Witkes (1977).

\section{PHILICHTHYIDAE}

Colobomatus kyphosus Sekerak 1970. Northeastern Pacific. Sekerak and Arai $(1977)$. 
Sebastes proriger (Continued)

\section{DIGENEA}

HEMIURIDĀE

Derogenes varicus (Mü1ler 1784) Looss 1901. Northeastern Pacific. Sekerak and Arai (1977).

Lecithaster gibbosus (Rudolphi 1802) Lühe 1901. Northeastern Pacific. Sekerak and Arai (1977).

OPECOELIDAE

Podocotyle sp. Northeastern Pacific. Sekerak and Arai (1977). SANGUINICOLIDAE

Psettarium sebastodorum Holmes 1971. British Columbia. Heart. Holmes (1971a).

\section{SYNCOELIDAE}

Syncoelium filiferum (Leuckart, in Sars 1885) Odhner 1911. Northeastern Pacific. Sekerak and Arai (1977).

MONOGENEA

CAPSALIDAE

Benedenia derzhavini (Layman 1930) Meserve 1938. Northeastern Pacific. Sekerak and Arai (1977).

Trochopus spp. Northeastern Pacific. Sekerak and Arai (1977).

MICROCOTYLIDAE

Microcotyle sebastis Goto 1894. Northeastern Pacific. Sekerak and Arai (1977).

\section{NEMATODA}

\section{ANISAKIDAE}

Contracaecum sp. Larva. Northeastern Pacific. Sekerak and Arai (1977).

* Thynnascaris adunca (Rudolphi 1802) Hartwich 1957. Northeastern Pacific. Sekerak and Arai (1977).

CYSTIDICOLIDAE

Ascarophis sebastodis 01sen 1952. Northeastern Pacific. Sekerak and Arai (1977). 
Sebastes rastrelliger - Grass Rockfish

SYNONYM - Sebastodes rastrelliger

CESTODA

LACISTORHYNCHIDAE

Lacistorhynchus tenuis (Van Beneden 1858) Dollfus 1929. Larva. Southern California. Viscera. Jensen ${ }^{18}$.

NEMATODA

ANISAKIDAE

Anisakis sp. Larva. Southern California. Dajley et al. (in press).

Phocanema sp. Larva. Southern California. Dailey et al. (in press).

Sebastes reedi - Yellowmouth Rockfish

CESTODA

BOTHRIOCEPHALIDAE

Bothriocephalus sp. Immature. Northeastern Pacific. Sekerak and Arai (1977).

TENTACULARI IDAE

Nybel inia surmenicola Okada 1929. Larva. Northeastern Pacific. Sekerak and Arai (1977).

COPEPODA

CHONDRACANTHIDAE

Chondracanthus pinguis Wilson 1912. Northeastern Pacific. Sekerak and Arai (1977).

Chondracanthus triventricosus Sekerak 1970. Northeastern Pacific. Sekerak and Arai (1977).

LERNAEOPODIDAE

Neobrachiel.1a robusta (Wilson 1912) Kabata 1979. Northeastern Pacific. Sekerak and Arai (1977)as Brachiella r.

PENNELLIDAE

Peniculus sp. Northeastern Pacific. Sekerak and Arai (1977).

PHILICHTHYIDAE

Colobomatus kyphosus Sekerak 1970. Northeastern Pacific. Sekerak and Arai (1977). 
Sebastes reedi (Continued)

DIGENEA

BUCEPHALIDAE

Prosorhynchus crucibulum (Rudolphi 1819) Odhner 1905. Northeastern Pacific. Sekerak and Arai (1977).

HEMIURIDAE

Derogenes varicus (Müller 1784) Looss 1901. Northeastern Pacific. Sekerak and Arai (1977).

Lecithophyl7um botryophorum (01sson 1886) Odhner 1905. Northeastern

Pacific. Sekerak and Arai (1977).

\section{OPECOELIDAE}

Neohelicometra sebastis Sekerak and Arai 1974. Northeastern Pacific.

Pyloric caeca, intestine. Sekerak and Arai (1974).

Podocotyle sp. Northeastern Pacific. Sekerak and Arai (1977).

SANGUINICOLIDAE

Psettarium sebastodorum Holmes 1971. Northeastern Pacific. Sekerak and Arai (1977).

MONOGENEA

CAPSAL IDAE

Benedenia derzhavini (Layman 1930) Meserve 1938. Northeastern Pacific. Sekerak and Arai (1977).

Trochopus sp. Northeastern Pacific. Sekerak and Arai (1977).

MICROCOTYLIDAE

Microcotyle sebastis Goto 1894. Northeastern Pacific. Sekerak and Arai (1977).

NEMATODA

ANISAKIDAE

Contracaecum spp. Larva. Northeastern Pacific. Sekerak and Arai (1977).

* Thynnascaris adunca (Rudolphi 1802) Hartwich 1957. Northeastern Pacific. Sekerak and Arai (1977).

Sebastes rosaceus - Rosy Rockfish

SYNONYM - Sebastodes rosaceus

NEMATODA

ANISAKIDAE

Anisakis sp. Larva. Southern California. Dailey et al. (in press). 
Sebastes rosaceus (Continued)

PROTOZOA (MYXOSPORIDA)

\section{CERATOMYXIDAE} $\frac{\text { Ceratomyxa }}{\text { Jameson }} \frac{\text { starksi Jameson 1929. Central California. Gallbladder. }}{1929)}$.

Ceratomyxa elegans Jameson 1929. Southern California. Gallbladder. Moser et a1. (1976).

Leptotheca informis Auerbach 1910. Southern California. Gallbladder. Moser et a1. (1976).

\section{Sebastes rosenblatti - Greenblotched Rockfish}

NEMATODA

ANISAKIDAE

Anisakis sp. Larva. Southern California. Dailey et al. (in press).

Sebastes ruberrimus - Yelloweye Rockfish

SYNONYM - Sebastodes ruberrimus

ACANTHOCEPHALA

ECHINORHYNCHIDAE

Echinorhynchus gadi Zoega in Müller 1776. Northeastern Pacific. Sekerak and Arai (1977).

POLYMORPHIDAE

Corynosoma sp. Larva. Northeastern Pacific. Sekerak and Arai (1977). CESTODA

BOTHRIOCEPHALIDAE

Bothriocephalus sp. Immature. Northeastern Pacific. Sekerak and Arai (1977). PHYLLOBOTHRI IDAE

Phyllobothrium sp. Larva. Northeastern Pacific. Sekerak and Arai (1977). TENTACULARI IDAE

Nybel inia surmenicola Okada 1929. Larva. Northeastern Pacific. Sekerak and Arai (1977). 
Sebastes ruberrimus (Continued)

COPEPODA

BOMOLOCHIDAE

Holobomolochus venustus Kabata 1971. Northeastern Pacific. Sekerak and Arai (1977).

CALIGIDAE

Lepeophtheirus paulus Cressey 1969. British Columbia. Kabata (1973). CHONDRACANTHIDAE

Chondracanthus pinguis Nilson 1912. Northeastern Pacific. Sekerak and Arai (1977).

Chondracanthus triventricosus Sekerak 1970. Northeastern Pacific. Sekerak and Arai (1977).

\section{LERNAEOPODIDAE}

Neobrachiella robusta (Wilson 1912) Kabata 1979. British Columbia. Kabata (1970c) as Brachiella r.

\section{PHILICHTHYIDAE}

Sarcotaces arcticus Collett 1874. British Columbia. Cysts in abdominal cavity, muscles. Kuitunen-Ekbaum (1949).

Colobomatus kyphosus Sekerak 1970. Northeastern Pacific. Sekerak and Arai (1977).

\section{DIGENEA}

BUCEPHALIDAE

Prosorhynchus crucibulum (Rudolphi 1819) Odhner 1905. Northeastern Pacific. Sekerak and Arai (1977).

\section{FELLODISTOMATIDAE}

Fellodistomum sebastodis Yamaguti and Matumura 1942. Northeastern Pacific. Sekerak and Arai (1977).

GORGODERIDAE

Phyllodistomum sp. Northeastern Pacific. Sekerak and Arai (1977). HEMIURIDAE

Derogenes varicus (Müller 1784) Looss 1901. Northeastern Pacific. Sekerak and Arai (1977).

Hemiurus levinseni Odhner 1905 . Washington. Lloyd (1938). Lecithochirium medium Acena 1941. Washington. Acena (1941). 
Sebastes ruberrimus (Continued)

\section{LEPOCREADI IDAE}

Opechona alaskensis Ward and Fillingham 1934. Washington. Ching (1960a). Neolepidapedon sebastisci (Yamaguti 1938) Manter 1954. Northeastern

Pacific. Sekerak and Arai (1977).

OPECOELIDAE

Neohelicometra sebastis Sekerak and Arai 1974. Northeastern Pacific. Pyloric caeca, intestine. Sekerak and Arai (1974).

Podocotyle sp. Northeastern Pacific. Sekerak and Arai (1977).

ZOOGONIDAE

Deretrema cholaeum McFarlane 1936. Northeastern Pacific. Sekerak and Arai (1977).

MONOGENEA

CAPSALIDAE

Trochopus trituba (Pratt and Aldrich 1953) Bravo-Hollis 1958. Oregon. Pratt and Aldrich (1953) as Megacotyle t.

Trochopus marginata (Folda 1928) Price 19 $\overline{3} 6$. Alaska. Gills. Yamaguti

(7963b) as Megalocotyle m.

Trochopus sp. Northeasterñ Pacific. Sekerak and Arai (1977).

NEMATODA

ANISAKIDAE

Contracaecum spp. Larva. Northeastern Pacific. Sekerak and Arai (1977).

* Thynnascaris adunca (Rudolphi 1802) Hartwich 1957. Northeastern Pacific. Sekerak and Arai (1977).

CAPILLARI IDAE

Capillaria sp. Northeastern Pacific. Sekerak and Arai (1977).

CUCULLANIDAE

Cucullanus elongatus Smedley 1933. Northeastern Pacific. Sekerak and Arai (1977).

CYSTIDICOLIDAE

Ascarophis sebastodis 01sen 1952. Northeastern Pacific. Sekerak and Arai (1977). 


\section{Sebastes rubrivinctus - Flag Rockfish \\ SYNONYM - Sebastodes rubrivinctus}

COPEPODA

CALIGIDAE

Lepeophtheirus parviventris Wilson 1905. California. Wilson (1908a). Lepeophtheirus salmonis (Kroyer 1838) Pacific coast, United States. Kazachenko et a1. (1972).

\section{LERNAEOPODIDAE}

Neobrachiella robusta (Wilson 1912) Kabata 1979. British Columbia. Kabata $(1970 \mathrm{c})$ as Brachiel 1a $r$.

NEMATODA

\section{ANISAKIDAE}

Anisakis sp. Larva. Southern California. Dailey et al. (in press).

* Thynnascaris sp. Southern California. Stomach, intestine. Jensen ${ }^{1} \dot{8}$. PROTOZOA (MYXOSPORIDA)

MYXOBOLIDAE

Henneguya sebasta Moser and Love 1975. California. Heart. Jensen ${ }^{18}$.

$$
\text { Sebastes rufus - Bank Rockfish }
$$

NEMATODA

\section{ANISAKIDAE}

Anisakis sp. Larva. Southern California. Dailey et al. (in press). PROTOZOA (MYXOSPORIDA)

MYXOBOLIDAE

Henneguya sebasta Moser and Love 1975. Southern California. Heart. Moser et al. (1976).

\section{Sebastes saxicola - Stripetail Rockfish}

MONOGENEA

MICROCOTYLIDAE

Microcotyle sebastis Goto 1894. Southern California. Gills. Crane (1972). 
Sebastes serranoides - 01ive Rockfish

SYNONYM - Sebastodes serranoides

COPEPODA

BOMOLOCHIDAE

Holobomolochus spinulus (Cressey 1969) Kabata 1971. Southern California. Gi11 cavity. Cressey (1969b).

\section{CALIGIDAE}

Caligus hobsoni Cressey 1969. Southern California. Hobson (1971). ISOPODA

Genera and species. Gnathiid larva. Southern California. Body surface. Hobson (1971).

CYMOTHOIDAE

Lironeca vulgaris Stimpson 1857. Southern California. Turner et al. (1969). NEMATODA

\section{ANISAKIDAE}

Anisakis sp. Larva. Southern California. Dailey et al. (in press). Phocanema sp. Larva. Southern California. Dailey et al. (in press). PROTOZOA (MYXOSPORIDA)

CERATOMYXIDAE

Ceratomyxa laxa Meglitsch 1960. Southern California. Gallbladder. Moser et a1. (1976).

Ceratomyxa sebasta Moser, Love and Jensen 1976. Central California. Gallbladder. Moser et al. (1976).

Ceratomyxa vepallida Meglitsch 1960. Southern California. Gallbladder. Moser et al. (1976).

Leptotheca informis Auerbach 1910. Northern, Central, Southern California. Gallbladder. Moser et al. (1976).

Leptotheca longipes Auerbach 1910. Central California. Gallbladder. Moser et al. (1976).

Leptotheca macrospora Auerbach 1909. Southern, Central California. Gallbladder. Moser et al. (1976).

Leptotheca sebasta Moser, Love and Jensen 1976. Southern California. Gallbladder. Moser et a 1. (1976).

MYXIDIIDAE

Myxidium incurvatum Thélohan 1892. Central California. Gallbladder. Moser et al. (1976).

Zschokkella ilishae Chakravarty 1941. Central California. Gallbladder. Moser et a T. (1976). 
Sebastes serranoides (Continued)

MYXOBOLIDAE

Henneguya sebasta Moser and Love 1975. Northern, Central, and Southern

California. Bulbus, truncus arteriosus. Moser and Love (1975).

SINUOLINEIDAE

Davisia reginae Love and Moser 1976. Central California. Urinary bladder. Love and Moser (1976).

$$
\begin{aligned}
& \text { Sebastes serriceps - Treefish } \\
& \text { SYNONYM - Sebastodes serriceps }
\end{aligned}
$$

COPEPODA

CALIGIDAE

Lepeophtheirus paulus Cressey 1969. Southern California. Roof of mouth. Cressey (1969b).

Lepeophtheirus longipes Wilson 1905. Southern California. Body surface. Hobson (1971).

DIGENEA

OPECOELIDAE

Pellamyzon sebastodis Montgomery 1957. Southern California. Intestine. Montgomery (1957).

Helicometrinia nimia Linton 1910. Southern California. Intestine. Montgomery (1957).

SANGUINICOLIDAE

Psettarium sebastodorum Holmes 1971. Southern California. Holmes ${ }^{35}$. ISOPODA

Genera and species. Gnathiid larva. Southern California. Body surface. Hobson (1971).

MONOGENEA

CAPSALIDAE

Trochopus marginata (Folda 1928) Price 1936. Southern California. Holmes 35. NEMATODA

\section{ANISAKIDAE}

Anisakis sp. Larva. Southern California. Dailey et al. (in press). 
Sebastes umbrosus - Honeycomb Rockfish

SYNONYM - Sebastodes umbrosus

MONOGENEA

CAPSALIDAE

Trochopus marginata (Folda 1928) Price 1936. Southern California. Holmes ${ }^{35}$. MICROCOTYLIDAE

Microcotyle sebastis Goto 1894. Northern California. Gills. Crane (1972).

Sebastes vexillaris - Whitebelly Rockfish

SYNONYM - Sebastodes vexillaris

DIGENEA

OPECOELIDAE

Helicometrina nimia Linton 1910. Intestine. Arai (1962).

PROTOZOA (MYXOSPORIDA)

MYXOBOLIDAE

Henneguya sebasta Moser and Love 1975. Central California. Bulbus, truncus arteriosus. Moser and Love (1975).

Sebastes wilsoni - Pygmy Rockfish

SYNONYM - Sebastodes wilsoni

DIGENEA

OPECOELIDAE

Podocotyle atomon (Rudolphi 1802) Odhner 1905. Washington. Aldrich12.

Podocotyle sp. Northeastern Pacific. Sekerak and Arai (1977).

MONOGENEA

MICROCOTYLIDAE

Microcotyle sebastis Goto 1894. Northeastern Pacific. Sekerak and Arai (1977).

NEMATODA

ANISAKIDAE

Contracaecum sp. Larva. Northeastern Pacific. Sekerak and Arai (1977). 


\section{Sebastes zacentrus - Sharpchin Rockfish}

\section{SYNONYM - Sebastodes zacentrus}

ACANTHOCEPHALA

ECHINORHYNCHIDAE

Echinorhynchus gadi Zoega in Müller 1776. Northeastern Pacific. Sekerak and Arai (1977).

POLYMORPHIDAE

Corynosoma sp. Larva. Northeastern Pacific. Sekerak and Arai (1977). CESTODA

BOTHRIOCEPHALIDAE

Bothriocephalus sp. Immature. Northeastern Pacific. Sekerak and Arai (1977).

PHYLLOBOTHRI IDAE

Phyllobothrium sp. Larva. Northeastern Pacific. Gallbladder. Sekerak and Arai (7977).

TENTACULARI IDAE

Nybelinia surmenicola Okada 1929. Larva. Northeastern Pacific. Sekerak and Arai (1977).

COPEPODA

CHONDRACANTHIDAE

Chondracanthus pinguis Wilson 1912. Northeastern Pacific. Sekerak and Arai (1977).

Chondracanthus triventricosus Sekerak 1970. Northeastern Pacific.

Sekerak and Arai (1977).

LERNAEOPODIDAE

Neobrachie1la robusta (Wilson 1912) Kabata 1979. British Columbia. Kabata (7970c) as Brachiella r.

PENNELLIDAE

* Haemobaphes theragrae Yamaguti 1939. Northeastern Pacific. Sekerak and Arai (1977).

PHILICHTHYIDAE

Colobomatus kyphosus Sekerak 1970. Northeastern Pacific. Sekerak and Arai (1977). 
Sebastes zacentrus (Continued)

DIGENEA

BUCEPHALIDAE

Prosorhynchus crucibulum (Rudolphi 1819) Odhner 1905. Northeastern Pacific. Sekerak and Arai (1977).

\section{FELLODISTOMAT IDAE}

Fellodistomum sebastodis Yamaguti and Matumura 1942. Northeastern Pacific. Sekerak and Arai (1977).

\section{HEMIURIDAE}

Lecithaster gibbosus (Rudolphi 1802) Lühe 1901. Northeastern Pacific. Sekerak and Arai (1977).

Derogenes varicus (Muiller 1784) Looss 1901. Northeastern Pacific.

Sekerak and Arai (1977).

\section{LEPOCREADIIDAE}

Opechona alaskensis Ward and Fillingham 1934. Northeastern Pacific. Sekerak and Arai (1977).

Neolepidapedon sebastisci (Yamaguti 1938) Manter 1954. Northeastern

Pacific. Sekerak and Arai (1977).

\section{OPECOELIDAE}

Podocotyle sp. Northeastern Pacific. Sekerak and Arai (1977).

Neohelicometra sebastis Sekerak and Arai 1974. Northeastern Pacific. Pyloric caeca, intestine. Sekerak and Arai (1974).

\section{SANGUINICOLIDAE}

Psettarium sebastodorum Holmes 1971. Washington and British Columbia. Heart, buTbus arteriosus, ventral aorta. Holmes (1971b).

\section{MONOGENEA}

\section{CAPSALIDAE}

Trochopus spp. Northeastern Pacific. Sekerak and Arai (1977).

Benedenia derzhavini (Layman 1930) Meserve 1938. Northeastern Pacific. Sekerak and Arai (1977).

MICROCOTYLIDAE

Microcotyle sebastis Goto 1894. Northeastern Pacific. Sekerak and Arai (1977). 
Sebastes zacentrus (Continued)

NEMATODA

ANISAKIDAE

Contracaecum spp. Larva. Northeastern Pacific. Sekerak and Arai (1977). * Thynnascaris adunca (Rudolphi 1802) Hartwich 1957. Northeastern Pacific. Sekerak and Arai (1977).

CYSTIDICOLIDAE

Ascarophis sebastodis 01 sen 1952. Northeastern Pacific. Sekerak and Arai (1977).

Sebastolobus sp. - Thornyhead

PROTOZOA (MYXOSPORIDA)

Genera and species. Southern California. Gallbladder. Noble (1966a).

$$
\begin{aligned}
& \text { ANOPLOPOMATIDAE -- Sablefishes } \\
& \text { Anoplopoma fimbria - Sablefish }
\end{aligned}
$$

ACANTHOCEPHALA

ECHINORHYNCHIDAE

Echinorhynchus gadi Zoega in Müller 1776. Bering Sea. Mamaev (1965).

POLYMORPHIDAE

Corynosoma reductum (Von Lindstow 1905) Railliet and Henry 1907.

Larva. Bering Sea. Mamaev (1965).

Corynosoma sp. Larva. Northeast Pacific. Kovalenko (1970).

CESTODA

TENTACULARI IDAE

Nybelinia surmenicola Okada 1929. Northeast Pacific. Kovalenko (1970).

\section{TETRAPHYLLIDEA}

Scolex sp. Northeast Pacific. Kovalenko (1970).

Scolex pleuronectis Muiller 1788. Bering Sea. Mamaev (1965).

TRYPANORHYNCHA

Genera and species. Larva. Northeast Pacific. Kovalenko (1970); Southern California. Jensen ${ }^{18}$. 
Anoplopoma fimbria (Continued)

COPEPODA

CALIGIDAE

Lepeophtheirus parviventris Wilson 1905. British Columbia, Pacific coast of United States. Kabata (1973).

NAOBRANCHIIDAE

Naobranchia occidentalis Wilson 1915. Southern California. Gills. Moser ${ }^{19}$.

\section{DIGENEA}

\section{ACANTHOCOLPIDAE}

Stephanostomum baccatum (Nicoll 1907) Manter 1934. Bering Sea. Mamaev $(1965)$.

Stephanostomum californicum Manter and Van Cleave 1951. Northeast Pacific. Kovalenko (1970).

\section{FELLODISTOMATIDAE}

Fellodistomum furcigerum(01sson 1867) Yamaguti 1954. Bering Sea. Mamaev (1965) as Sterinogophorus $f$.

\section{HEMIURIDAE}

Derogenes varicus (Müller 1784)Looss 1901. Bering Sea. Mamaev (1965). Dinosoma tortum Yamaguti 1938. Bering Sea. Mamaev (1965).

$$
\text { OPECOELIDAE }
$$

Podocotyle reflexa (Creplin 1825) Odhner 1905. Bering Sea. Mamaev (1965). Pseudopecoelus vulgaris (Manter 1934) Von Wicklen 1946. Central California. Intestine. Chapa 23.

\section{ISOPODA}

CYMOTHOIDAE

Lironeca vulgaris Stimpson 1857. Southern California. Gills. Jensen ${ }^{18}$. NEMATODA

\section{ANISAKIDAE}

Phoconema decipiens (Krabbe 1878) Myers 1959. Larva. British Columbia. Musculature. Hoskins et a1. 12.

Phocanema sp. Larva. British Columbia. Cysts in musculature. Margol is 3 as Porrocaecum sp.; Southern California. Dailey et al. (in press). Anisakis sp. Larva. British Columbia. Many places in body. Margolis 3 ; Southern California. Dailey et al. (in press). Contracaecum sp. Larva. Northeast Pacific. Kovalenko (1970). 
Anoplopoma fimbria (Continued)

PROTOZOA (MYXOSPORIDA)

CERATTOMYXIDÁE

Ceratomyxa anoplopoma Moser 1976. Southern California. Gallbladder. Moser (1976).

Leptotheca informis Auerbach 1910. Southern California. Gallbladder. Moser and Noble (1976b).

MYXIDI IDAE

Myxidium sp. Noble and Collard (1970).

SINUOL INEI DAE

Davisia anoplopoma Moser and Noble 1975. Southern and Central California. Urinary bladder, kidney tubules. Moser and Noble (1975).

\author{
HEXAGRAMMIDAE -- Green 1 ings
}

Hexagrammos $\mathrm{sp}$.

COPEPODA

CÁLIGIDÁE

Caligus clemensi Parker and Margolis 1964. British Columbia. Parker and Margolis (1964).

Hexagrammos decagrammus - Kelp Greenling

CESTODA

BOTHRIOCEPHALIDAE

Bothriocephalus scorpii (Müller 1776) Rudolphi 1808. British Columbia. Pyloric caeca. Ward7e (1932).

COPEPODA

CALIGIDAE

Lepeophtheirus parviventris Wilson 1905. British Columbia. Fraser (1920). Lepeophtheirus oblitus Kabata 1973. British Columbia. Kabata (1973). CHONDRACANTHIDAE

Acanthochondria deltoideus (Fraser 1920). British Columbia.

Gi11 cavity. Fraser (1920) as Chondracanthus del toideus. 
Hexagrammos decagrammus (Continued)

Chondracanthus pinguis Wilson 1912. British Columbia. Wilson (1912b). Chondracanthus gracilis Fraser 1920. British Columbia. Bere ${ }^{5}$.

\section{PENNELLIDAE}

Haemobaphes sp. Juvenile. British Columbia. Gills. Arai (1969b). DIGENEA

FELLODISTOMATIDAE

Hexagrammia longitestis Schel1 1973. Washington. Gastric caeca. Sche11 (1973b).

HEMIURIDAE

Genolinea laticauda Manter 1925. Central California. Stomach. Chapa23. OPECOELIDAE

Neohel icometra pleurogrammi (Baeva 1968) Sekerak and Arai 1974. Washington. Intestine. Sche11 (1973b) as Helicometra p.

MONOGENEA

MICROCOTYLIDAE

Microcotyle chiri Goto 1894. Washington. Bonham (1950). TURBELLARIA

HYPOTRICHINIDAE

Ichthyophaga subcutanea Syromjatnikova. Sea of Japan. Gill filaments. Menitskii (1963).

Hexagrammos lagocephalus and Hexagrammos superciliosus - Rock Greenling SYNONYM - Lebius superciliosus

Quast (1964) synonymized Hexagrammos lagocephalus and $\underline{H}$. superciliosus under the name $\underline{H}$. Tagocephalus. Mil ter and Lea (1972) rejected this synonomy, listing $H$. superciliosus as the California species. We have included both species, primarily because if, in the future, the species are generally recognized as synonymous, the pertinent parasite information will be available. Listings with an asterisk were listed as $\underline{H}$, superciliosus in the original papers.

ACANTHOCEPHALA

ECHINORHYNCHIDAE

Echinorhynchus gadi Zoega in Müller 1776. Sea of Japan. Intestine. 
Hexagrammos lagocephalus (Continued)

POLYMORPHIDAE

Corynosoma strumosum (Rudolphi 1802) Lühe 1904. Larva. Sea of Japan. Mesentery. Zhukov (1960a).

Corynosoma sp. Alaska. Schiller (1954).

CESTODA

BOTHRIOCEPHALIDAE

Bothriocephalus scorpii (Mül ler 1776) Rudolphi 1808. Larva. British Columbia. Intestine.* Arai (1969b); adult. Sea of Japan. Intestine. Zhukov (1960a).

PHYLLOBOTHRI IDAE

*Phyllobothrium sp. Larva. British Columbia. Intestine. Arai (1969b).

TETRAPHYLLIDEA

Scolex pleuronectis Müller 1788. Sea of Japan. Intestine. Zhukov (1960a). COPEPODA

\section{CALIGIDAE}

Lepeophtheirus cuneifer Kabata 1974. Alaska. Kabata (1974). Questionable host.

Lepeophtheirus hexagrammi Gussev 1951. Japan. Body surface. Shiino (1959e).

CHONDRACANTHIDAE

Acanthochondria solida Gussev 1951. Japan. Yamaguti (1963b).

\section{DIGENEA}

\section{ALLOCREADI IDAE}

Neophasis oculata (Levinsen 1881) Miller 1941. Japan. Smal1 intestine. Machida et a1. (1972).

*Anisorchis opisthorchis Polyanski 1955. British Columbia. Intestine. Arai (1969b).

Anisorchis zhukovi nom. nov. Sakhalein. Yamaguti (1971).

BUCEPHALIDAE

Prosorhynchus sp. Immature. Japan. Muscles. Machida et al. (1972).

FELLODISTOMATIDAE

Pseudopentagramma petrowi (Layman 1930) Yamaguti 1971. USSR Pacific. Margolis and Ching (1965) as Pentagramma $p$. 
Hexagrammos lagocephalus (Continued)

HEMIURIDAE

Genolinea anura (Layman 1930) Yamaguti 1954. Sea of Japan. Stomach. Zhukov (1960a).

Genolinea laticauda Manter 1925. British Columbia. Stomach. Arai (1969b). Lecithaster gibbosus (Rudolphi 1802) Lühe 1901. British Columbia. Intestine. Arai (1969b).

Lecithophyllum botryophorum (01sson 1868) Odhner 1905. British Columbia. Intestine. Arai (1969b) as L. anteroporum.

Derogenes varicus (Müller 1786) Looss 1901. Sea of Japan. Stomach. Zhukov (1960a).

Genarchopsis muelleri (Levinsen 1881) Yamaguti 1954. Japan. Stomach. Machida et al. (1972).

HETEROPHYIDAE

*Galactosomum sp. Larva. British Columbia. Gills (encysted). Arai $(1969 b)$.

OPECOELIDAE

*Podocotyle sp. Northern California. Intestine, caeca. Edmiston ${ }^{29}$. Podocotyle atomon (Rudolphi 1802) Odhner 1905. Sea of Japan. Intestine. Zhukov (1960a).

Podocotyle reflexa (Creplin 1825) Odhner 1905. Sea of Japan. Zhukov (1960a).

Podocotyle sinusacca Ching 1960. British Columbia. Intestine. Arai (1969b) as Neopodocotyloides s.

\section{ZOOGONIDAE}

Urinatrema hirudinaceum Zhukov 1957. Sea of Japan. Urinary bladder. Zhukov (1960a).

Urinatrema aspinosum Schiller 1956. Alaska. Urinary bladder. Schiller $(1956)$.

\section{MONOGENA}

GYRODACTYLIDAE

*Gyrodactylus vancleavi Mizelle and Kritsky 1967. Northern California. Skin. Mizelie and Kritsky (1967d).

\section{MICROCOTYLIDAE}

Microcotyle sebastis Goto 1894. British Columbia. Gills. Arai (1969b). Prosomicrocotyla gotoi (Yamaguti 1934) Yamaguti 1958. USSR. Bykhovsky (1957) as Microcotyle $\mathrm{g}$. 
Hexagrammos lagocephalus (Continued)

NEMATODA

ANISAKIDAE

Anisakis sp. Larva. British Columbia. Body cavity.* Arai (1969b); Sea of Japan. Mesentery. Zhukov (1960a).

Phocanema decipiens (Krabbe 1878) Myers 1959. Larva. Alaska. Schiller (1954) as Porrocaecum d.

* Thynnascaris adunca (Rudōphi 1802) Hartwich 1957. Sea of Japan.

Stomach, intestine. Zhukov (1960a) as Contracaecum a.

Contracaecum sp. Larva. British Columbia. Body cavity, stomach. Arai (1969b).

\section{CYSTIDICOLIDAE}

${ }^{*}$ Ascarophis Arai sebastodis 01 sen 1952. British Columbia. Intestine.

Ascarophis curvicauda Zhukov 1960. Sea of Japan. Stomach. Zhukov (1960a).

Hexagrammos stelleri - Whitespotted Greenling

ACANTHOCEPHALA

ECHINORHYNCHIDAE

Echinorhynchus gadi Zoega in Müiier i776. Kamchatka, USSR. Zhukov (1963). POLYMORPHIDAE

Corynosoma strumosum (Rudolphi 1802) Lühe 1904. Kamchataka, USSR. Zhukov (1963).

\section{CESTODA}

BOTHRIOCEPHALIDAE

Bothriocephalus scorpii (Müller 1776) Rudolphi 1808. British Columbia.

Intestine. Boyce (1976); Kamchatka, USSR. Zhukov (1963).

PHYLLOBOTHRI IDAE

Phyllobothrium sp. Larva. British Columbia. Intestine. Arai (1969b).

TENTACULARI IDAE

Nybel inia surmenicola Okada 1929. Larva. Kamchatka, USSR. Strelkov (1960).

TETRAPHYLLIDEA

Scolex pleuronectis Müller 1788. Kamchatka, USSR. Zhukov (1963). 
Hexagrammos stelleri (Continued)

COPEPODA

CALIGIDAE

Lepeophtheirus oblitus Kabata 1973. British Columbia. Kabata (1973). DIGENEA

ALLOCREADIIDAE

Anisorchis opisthorchis Polyanski 1955. British Columbia. Intestine. Arai (1969b).

Neophasis oculata (Levinsen 1881) Miller 1941. Kamchatka, USSR. Zhukov (1963).

BUCEPHALIDAE

Prosorhynchus sp. Japan. Muscles. Machida et al. (1972). HEMIURIDAE

Derogenes varicus (Müil ler 1784) Looss 1901. Kamchatka, USSR. Zhukov (1963). Genarchopsis mueTleri (Levinsen 1881) Yamaguti 1954. Kamchatka, USSR. Zhukov $(1963)$.

Genolinea anura (Layman 1930) Yamaguti 1954. Kamchatka, USSR. Zhukov (1963).

Hemiurus levinseni Odhner 1905. Kamchatka, USSR. Zhukov (1963). Lecithaster gibbosus (Rudolphi 1802) Lühe 1901. British Columbia. Intestine. Arai (1969b).

Lecithophyllum botryophorum (01sson 1868) Odhner 1905. British Columbia. Intestine. Arai (1969b) as L. anteroporum.

Parahemiurus merus (Linton 1910) Woolcock 1935. British Columbia. Intestine. Arai (1969b).

OPECOELIDAE

Anoma lotrema putjatini Zhukov 1957. Kamchatka, USSR. Zhukov (1963). Podocotyle sp. Immature. British Columbia. Intestine. Arai (1969b). Podocotyle atomon (Rudolphi 1802) Odhner 1905. British Columbia. Intestine. McFarlane (1936); Kamchatka, USSR. Zhukov (1963).

Podocotyle reflexa (Crepi in 1825) Odhner 1925. Kamchatka, USSR. Zhukov (1963).

Podocotyle sinusacca Ching 1960. British Columbia. Intestine. Arai (1969b) as Neopodocotyloides s.

MONOGENEA

MICROCOTYLIDAE

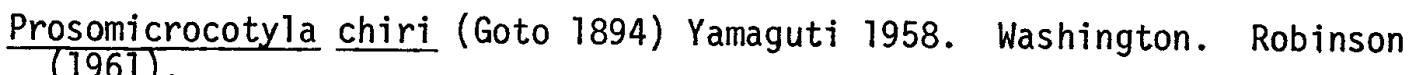


Hexagrammos stelleri (Continued)

NEMATODA

\section{ANISAKIDAE}

* Thynnascaris adunca (Rudolphi 1802) Hartwich 1957. Kamchatka, USSR. Strelkov (1960) as Contracaecum a.

PROTOZOA (CILIOPHORA)

URCEOLARI IDAE

Trichodina hexagrammi Zhukov 1964. Kamchatka, USSR. Zhukov (1964).

$$
\text { Ophiodon elongatus - Lingcod }
$$

CESTODA

PHYLLOBOTHRIIDAE

Phyllobothrium sp. Larva. British Columbia. Intestine. Arai (1969b).

TENTACULARI IDAE

Nybelinia surmenicola Okada 1929. British Columbia. Intestine, stomach. Arai (1969b).

Tentacularia sp. Larva. Washington. Region of vertebra. Hart (1936a).

COPEPODA

CALIGIDAE

Lepeophtheirus breviventris Fraser 1920. British Columbia. Buccal cavity. Fraser (1920).

Lepeophtheirus pravipes Wilson 1912. British Columbia. Wilson (1912b); California. Shino (1965d) as L. trifidus.

Lepeophtheirus salmonis (Kroyer 1838). British Columbia. Bere ${ }^{5}$.

CHONDRACANTHIDAE

Chondracanthus narium Kabata 1969. British Columbia. Nasal cavity. Kabata (1969a).

\section{DIGENEA}

\section{ACANTHOCOLPIDAE}

Stephanostomum casum (Linton 1910) McFarlane 1934. British Columbia. McFarlane (1936) [Likely S. californicum - Sekerak and Arai (1977)]. Stephanos tomum tristephanum McFarlane 1936. McFarlane (1936); Northern and CentraT California. Chapa 23 . 


\section{BUCEPHALIDAE}

Rhipidocotyle elongata McFarlane 1936. British Columbia. McFarlane (1936); Washington. Ching (1960a).

Prosorhynchus facilis (Ozaki 1924) Ekmann 1933. British Columbia. McFarlane (1936); Washington. Ching (1960a).

Prosorhynchus apertus McFarlane 1936. Washington. Ching (1960a); British Columbia. McFarlane (1936).

Bucephalus sp. California. Giles (1962).

\section{HEMIURIDAE}

Derogenes crassus Manter 1934. Oregon. McCauley (1960).

Derogenes varicus (Mü1ler 1784) Looss 1901. Washington. Lloyd (1938).

Genolinea laticauda Manter 1925. Washington. Lloyd (1938).

Hemiurus levinseni Odhner 1905. Washington. Lloyd (1938).

Sterrhurus exodicus (McFarlane 1936) Yamaguti 1958. Central California. Stomach. Nahhas 10, British Columbia. McFarlane (1936); Washington. Lloyd (1938); Oregon. McCauley (1960) all as Lecithochirium e.

Tubulovesicula lindbergi (Layman 1930) Yamaguti 1934. Oregon. Stomach. McCauley $(1960)$; Central Cal ifornia. Nahhas ${ }^{10}$; Washington. Ching (1960a); British Columbia. Arai (1969b).

Parahemiurus merus (Linton 1910) Woolcock 1935. British Columbia. Intestine. Arai (1969b).

Lecithaster gibbosus (Rudolphi 1802) Lühe 1901. British Columbia. Intestine. Arai (1969b).

HIRUDINOIDEA

PISCICOLIDAE

Malmiana sp. Oregon. Exterior. Burreson?.

ISOPODA

CYMOTHOIDAE

Lironeca vulgaris Stimpson 1857. California. Gills. Richardson (1905). MONOGENEA

GYRODACTYLIDAE

Gyrodactylus elegans Nordmann 1832. Washington. Guberlet (1936a).

MICROCOTYLIDAE

Microcotyle sebastis Goto 1894. Washington. Guberlet (1936a). UDONELLIDAE

Udonella ophiodontis (Kay 1945) Winter 1955. Washington. On Lepeophtheirus sp. attached to oral cavity. Kay (1945). 
Ophiodon elongatus (Continued)

NEMATODA

\section{ÂNISAKKIDAE}

*Thynnascaris adunca (Rudolphi 1802) Hartwich 1957. British Columbia. Stomach, intestine. Margol is ${ }^{3}$ as Contracaecum a.

Anisakis sp. Larva. British Columbia. Stomach. Arai (1969b); Southern California. Dailey et al. (in press).

Phocanema sp. Larva. British Columbia. Body cavity, stomach. Arai (1969b).

Phocanema decipiens (Krabbe 1878) Myers 1959. Larva. British Columbia. Margolis (1956b) as Terranova d.

CUCULLANIDAE

Cucullanus elongatus Smedley 1933. British Columbia. Intestine. Smedley (1933).

PROTOZOA (CILIOPHORA)

URCEOLARIIDAE

Genera and species. British Columbia. Gills. Bell (1962).

$$
\text { Oxylebius pictus - Painted Greenling }
$$

COPEPODA

BOMOLOCHIDAE

Holobomolochus spinulus (Cressey 1969) Kabata 1971. Southern California. Gill cavity. Cressey (1969b).

CHONDRACANTHIDAE

Heterochondria atypica Ho 1972. Southern California. Gills. Ho (1972b). ISOPODA

Genera and species. Gnathiid larva. Southern California. Body surface. Hobson (1971).

Pleurogrammus monopterygius - Atka Mackerel

ACANTHOCEPHALA

ECHINORHYNCHIDAE

Echinorhynchus gadi Zoega in Mü17er 1776. Kamchatka, USSR. Intestine. Strelkov (1960). 
Pleurogramm us monopterygius (Continued)

POLYMORPHIDAE

Corynosoma strumosum (Rudolphi 1802) Lühe 1904. USSR. Baeva (1965b). CESTODA

TENTACULARIIDAE

Nybel inia surmenicola Okada 1929. Larva. Kamchatka, USSR. Strelkov (1960).

TETRAPHYLLIDEA

Scolex pleuronectis MU1ler 1788. Kamchatka, USSR. Intestine. Strelkov (1960).

COPEPODA

CALIGIDAE

Lepeophtheirus parviventris Wilson 1905. Alaska. Exterior. Kabata (1973); Siberia. Wilson (1905b).

DIGENEA

BUCEPHALIDAE

Prosorhynchus crucibu7um (Rudolphi 1819) Odhner 1905. USSR. Baeva $(1965 b)$.

FELLODISTOMATIDAE

Hexagrammia zhukovi Baeva 1965. USSR. Intestine. Baeva (1965b). HEMIURIDAE

Genolinea aburame Yamaguti 1934. USSR. Baeva (1965b). OPECOELIDAE

Podocotyle reflexa (Creplin 1825) Odhner 1905. USSR. Baeva (1965b). NEMATODA

\section{ANISAKIDAE}

Phocanema sp. Larva. USSR. Baeva (1965b) as Terranova sp.

Anisakis sp. Larva. Kamchatka, USSR. Strelkov (1960). 
COTTIDAE -- Sculpins

Artedius fenestralis - Padded Sculpin

\section{DIGENEA}

HEMIURIDAE

Genolinea laticauda Manter 1925. British Columbia. Stomach. Arai (1969b).

PROTOZOA (CILIOPHORA)

URCEOLARI IDAE

Trichodina vancouverense Laird 1961. British Columbia. Gills. Laird (1967).

Trichodina lairdi Lom 1970. British Columbia. Gills. Laird (1961) as T. decipiens.

Trichōina rectuncinata Raabe 1958. British Columbia. Gills. Lom (1970b).

PROTOZOA (SPOROZOA)

HAEMOGREGARINIDAE

Haemogregarina bigemina Laveran and Mesnil 1901. British Columbia. Blood. Laird (1961).

Artedius harringtoni - Scalyhead Sculpin

SYNONYM - Axyrias harringtoni

CESTODA

BOTHRIOCEPHALIDAE

Bothriocephalus scorpii (Mü1ler 1776) Rudolphi 1808. Larva. British Columbia. Intestine. Arai (1969b).

COPEPODA

LERNAEOPODIDAE

Clavella parva Wilson 1912. British Columbia. Fraser (1920). PENNELLIDAE

Haemobaphes intermedius Kabata 1967. British Columbia. Gill arches. Kabata (1967b). 
Artedius harringtoni (Continued)

DIGENEA

ACANTHOCOLPIDAE

Stephanostomum californicum Manter and Van Cleave 1951. British

Columbia. Intestine. Arai (1969b) as $\underline{S}$. casum.

NEMATODA

ANISAKIDAE

Contracaecum sp. Larva. British Columbia. Body cavity. Arai (1969b). CYSTIDICOLIDAE

Ascarophis sebastodis 01 sen 1952. British Columbia. Intestine. Arai $(1969 \mathrm{~b})$.

\section{Artedius lateralis - Smoothhead Sculpin}

PROTOZOA (MYXOSPORIDA)

CERATOMYXIDAE

Leptotheca elegans Noble 1938. Southern California. Gallbladder. Noble (1947).

MYXIDIIDAE

Sphaeromyxa lateral is Noble 1941. Southern California. Gallbladder. Noble (1947).

$$
\text { Artedius notospilotus - Bonyhead Sculpin }
$$

DIGENEA

OPECOELIDAE

Podocotyle enophrysi Park 1937. Northern California. Intestine. Nahhas and Krupin (1977).

$$
\text { Ascel ichthys rhodorus - Rosylip Sculpin }
$$

DIGENEA

OPECOELIDAE

Podocotyle sp. Northern California. Intestine. Edmiston 29. Podocotyle californica Park 1937. Northern California. Intestine. Nahhas and Krupin (1977).

Podocotyle enophrysi Park 1937. Northern California. Intestine. Nahhas and Krupin (1977). 
Ascelichthys rhodorus (Continued)

PROTOZOA (MYXOSPORIDA)

MYXIDIIDAE

Myxidium incurvatum Thélohan 1892. Washington. Gallbladder. Kuris20.

$$
\text { Blepsias cirrhosus - Silverspotted Sculpin }
$$

\section{ACANTHOCEPHALA}

\section{ECHINORHYNCHIDAE}

Echinorhynchus gadi Zoega in Müller 1776. Sea of Japan. Intestine. Zhukov (1960a).

CESTODA

BOTHRIOCEPHALIDAE

Bothriocephalus scorpii (Müller 1776) Rudolphi 1808. Sea of Japan. Intestine. Zhukov (1960a).

\section{PHYLLOBOTHRI IDAE}

Phyllobothrium sp. Larva. British Columbia. Intestine. Arai (1969b); Sea of Japan. Mesentery. Zhukov (1960a).

\section{TETRAPHYLLIDEA}

Scolex pleuronectis Müller 1788. Sea of Japan. Intestine. Zhukov (1960a).

COPEPODA

\section{PENNELLIDAE}

Haemobaphes sp. Immature. British Columbia. Kabata (1967b).

\section{DIGENEA}

\section{ALLOCREADI IDAE}

Liliatrema skrjabini Gubanov 1954. Larva. Sea of Japan. Muscle. Zhukov (1960a).

\section{HEMIURIDAE}

Lecithaster gibbosus (Rudolphi 1802) Lühe 1901. British Columbia. Intestine. Arai (1969b); Sea of Japan. Zhukov (1960a).

Genolinea laticauda Manter 1925. Oregon. McCauley (1960); British Columbia. Stomach. Arai (1969b); Kamchatka, USSR. Skryabina (1963). Genolinea anura (Layman 1930) Yamaguti 1954. Sea of Japan. Stomach. Zhukov (1960a). 
Blepsias cirrhosus (Continued)

Brachyphallus crenatus (Rudolphi 1802) Odhner 1905. Sea of Japan. Intestine. Zhukov (1960a). Derogenes varicus (Múl1er 1784) Looss 1901. Sea of Japan. Stomach. Zhukov (1960)a).

\section{OPECOELIDAE}

Podocotyle sinusacca Ching 1960. British Columbia. Intestine. Arai (1969b) as Neopodocotyloides s.

Podocotyle sp. Immature. British Cólumbia. Intestine. Arai (1969b). Podocotyle atomon (Rudolphi 1802) Odhner 1905. Sea of Japan. Intestine. Zhukov (1960a).

Podocotyle reflexa (Crepl in 1825) Odhner 1905. Sea of Japan. Intestine. Zhukov (1960a).

\section{MONOGENEA}

GYRODACTYLIDAE

Gyrodactylus gröenlandicus pacificus Bykhovsky and Polyanski 1953. Sea of Japan. Gills. Bykhovsky and Polyanski (1953).

\section{NEMATODA}

\section{ANISAKIDAE}

Anisakis sp. Larva. British Columbia. Body cavity. Arai (1969b). *Thynnascaris adunca (Rudolphi 1802) Hartwich 1957. Sea of Japan. Intestine. Zhukov (1960a) as Contracaecum a.

\section{1) Chitonotus pugetensis - Roughback Sculpin}

COPEPODA

ERGASILIDAE

Ergasilus turgidus Fraser 1920. British Columbia. Bere ${ }^{5}$.

\section{LERNAEOPODIDAE}

Clavella parva Wilson 1912. British Columbia. Bere ${ }^{5}$. NEMATODA

\section{ANISAKIDAE}

Contracaecum sp. Larva. British Columbia. Body cavity. Arai (1969b).

\section{CAP ILLARI IDAE}

Capillaria sp. British Columbia. Stomach. Arai (1969b). 


\section{DIGENEA}

\section{HEMIURIDAE}

Lecithaster gibbosus (Rudolphi 1802) Lühe 1901. Oregon. Hall and Pratt (1969) as L. salmonis.

\section{MONOGENEA}

GYRODACTYLIDAE

Gyrodactylus bodegensis Mizelle and Kritsky 1967. Northern

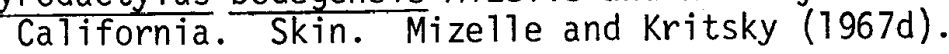

$$
\text { Clinocottus analis - Wooly Sculpin }
$$

\section{DIGENEA}

\section{HEMIURIDAE}

Genolinea montereyensis Annereaux 1947. California. Stomach. Annereaux (1947).

Genolinea laticauda Manter 1925. Central California. Stomach.

\section{OPECOELIDAE}

Helicometrina elongata Noble and Park 1937. Southern California.

Intestine. Yamaguti (1971).

Helicometrina nimia Linton 1910. Intestine. Arai (1962); Central

California. Chapa 23.

Opecoelus adsphaericus Manter and Van Cleave 1951. Southern California and Baja California. Intestine. Manter and Van Cleave (1951).

Opegaster cameroni Caballero and Caballero 1969. Southern California. Intestine. Caballero and Caballero (1969b).

Podocotyle sp. Northern California. Intestine, caeca. Edmiston29.

Podocotyle californica Park 1937. Northern California. Intestine. Niahhas and Krupin (1977).

PROTOZOA (MYXOSPORIDA)

\section{CERATOMYXIDAE}

Ceratomyxa obesa Jameson 1929. Central California. Gallbiadder. Jameson (1929).

$$
\text { Clinocottus embryum - Calico Sculpin }
$$

DIGENEA 


\section{Clinocottus embryum (Continued)}

Lecithaster gibbosus (Rudolphi 1802) Lühe 1901. Oregon. Hal1 and Pratt (1969); Washington. Ching (1960a) as L. salmonis.

$$
\begin{gathered}
\frac{\text { Clinocottus globiceps }}{\text { SYNONYM - Mlennicottus globiceps }} \\
\text { SYlead Sculpin }
\end{gathered}
$$

DIGENEA

HEMIURIDAE

Lecithaster gibbosus (Rudolphi 1802) Lühe 1901. Oregon. Ha11 and Pratt (1969) as L. Salmonis. Genol inea laticauda Manter 1925. Oregon. Hall and Pratt (1969).

OPECOELIDAE

Podocotyle atomon (Rudolphi 1802) Odhner 1905. Oregon. Hall and Pratt (1969). Podocotyle californica Park 1937. Northern California. Lower intestine. Park (1937b) as P. elongata and P. kofoidi.

Podocotyle enophrysi Park 1937. Oregon. Hall and Pratt (1969); Northern California. Intestine. Park (1937b) as P. blennicottusi and $P$. pacifica.

Podocotyle reflexa (Creplin 1825) Odhner 1905. Oregon. Hall and Pratt (1969).

\section{ZOOGONIDAE}

Zoogonoides viviparus (01sson 1868) Odhner 1902. Oregon. Hall and Pratt (1969).

$$
\text { Dasycottus setiger - Spinyhead Sculpin }
$$

\section{CESTODA}

PHYLLOBOTHRI IDAE

Phyllobothrium sp. Larva. British Columbia. Intestine. Arai (1969b).

\section{DIGENEA}

\section{ACANTHOCOLPIDAE}

Stephanostomum dentatum (Linton 1900) Linton 1940. British Columbia. Intestine. Arai (1969b).

\section{HEMIURIDAE}

Lecithaster gibbosus (Rudolphi 1802) Lühe 1901. British Columbia. Intestine. Arai (7969b).

Lecithophyllum botryophorum (01sson 1868) Odhner 1905. British Columbia. Intestine. Arai (1969b) as L. anteroporum.

Genol inea manteri Lloyd 1938. Washington. Ching (1960a). 
Dasycottus setiger (Continued)

PROTOZOA (CILIOPHORA)

URCEOLARIIDAE

Trichodina ovonucleata Raabe 1958. Washington. Gi11s. Lom (1970b).

Trichodina parvula Lom 1970. Washington. Gills. Lom (1970b).

$$
\begin{aligned}
& \text { Enophrys bison - Buffalo Sculpin } \\
& \text { SYNONYM - Aspicottus bison, Endophrys bison }
\end{aligned}
$$

CESTODA

BOTHRIOCEPHALIDAE

Bothriocephalus scorpii (Müller 1776) Rudolphi 1808. British Columbia. Pyloric caeca. Wardle (1932).

\section{COPEPODA}

\section{CALIGIDAE}

Lepeophtheirus parviventris Wilson 1905. British Columbia. Fraser (1920).

\section{CHONDRACANTHIDAE}

Chondracanthus irregularis Fraser 1920. British Columbia.. Gill cavity. Fraser (1920).

\section{DIGENEA}

\section{BUCEPHALIDAE}

Prosorhynchus squamatus Odhner 1905. Oregon. Pratt and McCauley (1967).

HEMIURIDAE

Genolinea 1aticauda Manter 1925. Oregon. McCauley (1960).

Genolinea manteri Lloyd 1938. Oregon. McCauley (1960).

Intuscirrus aspicotti Acena 1947. Washington. Stomach. Acena (1947).

TubuTovesicuTa lindbergi (Layman 1930) Yamaguti 1934. Oregon.

Stomach. Mccauley (1960); California. Park (1936) as I. californica.

\section{OPECOELIDAE}

Podocotyle enophrysi Park 1937. Northern California. Intestine, stomach. Park (1937b) as P. endophrysi.

Podocotyle californica Park 1937 . Northern Caifornia. Intestine, caeca. Edmiston 29 . 
Enophrys bison (Continued)

HIRUDINOIDEA

PISCICOLIDAE

Malmiana virida Burreson 1977. Oregon. Pectoral fin. Burreson (1977c).

PROTOZOA (CILIOPHORA)

URCEOLARI IDAE

Trichodina domerguei subsp. domerguei Haider 1964. Washington.

GilTs. Lom (1970b).

\section{Gilbertidia sigalutes - Soft Sculpin}

NEMATODA

ANISAKIDAE

Anisakis sp. Larva. British Columbia. Body cavity. Arai (1969b).

Hemi lepidotus hemilepidotus - Red Irish Lord

ACANTHOCEPHALA

ECHINORHYNCHIDAE

Echinorhynchus gadi Zoega in Müller 1776. British Columbia. Intestine. Arai (1969b).

POLYMORPHIDAE

Corynosoma sp. Juvenile. Schiller (1954).

COPEPODA

CHONDRACANTHIDAE

Chondracanthus sp. Oregon. Gill cavity. Millemann ${ }^{34}$.

\section{DIGENEA}

\section{ALLOCREADI IDAE}

Anisorchis opisthorchis Polyansky 1955. British Columbia. Intestine: Arai (1969b).

HEMIURIDAE

Tubulovesicula lindbergi (Layman 1930) Yamaguti 1934. Washington. Ching (1960a). 
Hemilepidotus hemilepidotus (Continued)

Derogenes varicus (Muller 1784) Looss 1901. British Columbia. Intestine. Arai (1969b).

Genolinea laticauda Manter 1925. British Columbia. Stomach. Arai (7969a).

Lecithaster gibbosus (Rudolphi 1802) Lühe 1901. British Columbia. Intestine. Arai (1969b).

OPECOELIDAE

Podocotyle sinusacca Ching 1960. British Columbia. Intestine. Arai $(1969 \mathrm{~b})$ as Neopodocotyloides s.

ZOOGONIDAE

Lepidophyllum cameroni Arai 1969. British Columbia. Urinary bladder, rectal gland. Arai (1969b).

Steganoderma formosum Stafford 1904. British Columbia. Intestine.

NEMATODA

ANISAKIDAE

Phocanema decipiens (Krabbe 1878) Myers 1959. Larva. Alaska. Schiller (1954) as Porrocaecum d.

CYSTIDICOLIDAE

Ascarophis sebastodis 01 sen 1952. British Columbia. Intestine. Arai (1969b).

$$
\text { Hemilepidotus spinosus - Brown Irish Lord }
$$

DIGENEA

OPECOELIDAE

Podocotyle sp. Northern California. Intestine. Edmiston29. Podocotyle californica Park 1937. Northern California. Intestine. Nahhas and Krupin (1977).

$$
\text { Icelinus filamentosus - Threadfin Sculpin }
$$

ACANTHOCEPHALA

ECHINORHYNCHIDAE

Echinorhynchus gadi Zoega in Müller 1776. British Columbia. Intestine. Arai (1969b). 
Icelinus filamentosus (Continued)

CESTODA

PHYLLOBOTHRIIDAE

Phyllobothrium sp. Larva. British Columbia. Intestine. Arai (1969b).

NEMATODA

ANISAKIDAE

Contracaecum sp. Larva. British Columbia. Body cavity. Arai (1969b).

$$
\text { Icelinus tenuis - Spotfin Sculpin }
$$

CESTODA

PHYLLOBOTHRI IDAE

Phyllobothrium sp. Larva. British Columbia. Intestine. Arai (1969b). DIGENEA

HEMIURIDAE

Genera and species. Immature. British Columbia. Stomach. Arai (1969b).

$$
\text { Jordania zonope - Longfin ScuTpin }
$$

NEMATODA

ANISAKIDAE

Contracaecum sp. Larva. British Columbia. Body cavity. Arai (1969b).

Leptocottus armatus - Pacific Staghorn Sculpin

ACANTHOCEPHALA

ECHINORHYNCHIDAE

Echinorhynchus gadi Zoega in Müller 1776. British Columbia. Intestine. Arai (1969b).

POLYMORPHIDAE

Corynosoma strumosum (Rudolphi 1802) Lühe 1904. Larva. Washington. Van Cleave (1953).

Corynosoma sp. Juvenile. British Columbia. Intestine. Arai (1969b). 
CESTODA

BOTHRIOCEPHALIDAE

Bothriocephalus scorpii (Müller 1776) Rudolphi 1808. British Columbia. Pyloric caeca. Wardle (1932).

Bothriocephalus occidental is (Linton 1898) Lühe 1899. British Columbia. Pyloric caeca. Wardle (1932).

COPEPODA

\section{BOMOLOCHIDAE}

HoIobomolochus spinulus (Cressey 1969) Kabata 1971. British Columbia. Gi77ts, Kabata (1971).

Holobomolochus prolixus (Cressey 1969) Kabata 1971. Southern California. Gill cavity. Ho (1975).

\section{CALIGIDAE}

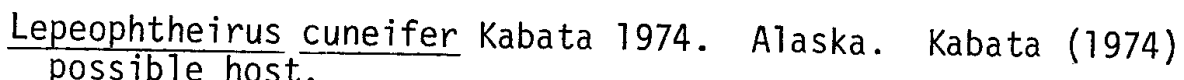

\section{CHONDRACANTHIDAE}

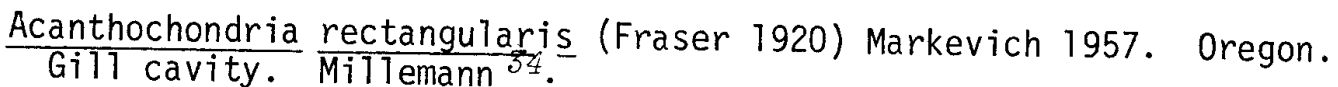

\section{ERGASILIDAE}

Ergasilus sp. Southern California. Body surface. Baker 22. DIGENEA

\section{ACANTHOCOLPIDAE}

$\frac{\text { Stephanostomum } \mathrm{sp} \text {. Northern California. Intestine. Chapa } 23 \text { as }}{\underline{\text { S. casum. }}}$

\section{BUCEPHALIDAE}

Prosorhynchoides ozakii (Ozaki 1928) Margolis and Arthur 1979. Oregon. Pratt and McCauley (1961) as Bucephalopsis o.

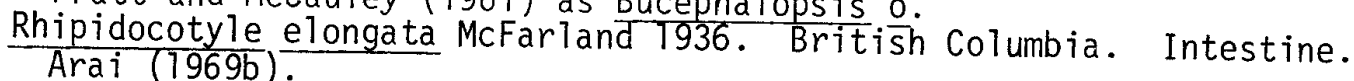

\section{HEMIURIDAE}

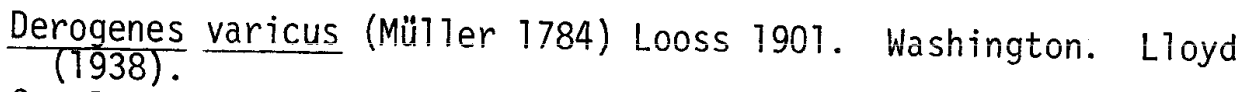
Genolinea laticauda Manter 1925. Oregon. McCauley (1960); British Columbia. Stomach. Araj (1969b); Northern California. Chapa23. Genolinea manteri Lloyd 1938. Washington. Stomach. Lloyd (1938). Genolinea montereyensis Annereaux 1947. Oregon. McCauley (1960). 
Lecithaster gibbosus (Rudolphi 1802) Lühe 1901. Washington. Ching (1960a) as L. salmonis.

Tubulovesicula 1 indbergi (Layman 1930) Yamaguti 1934. Oregon. Stomachs. McCauley (1960); Washington. Ching (1960a); British Columbia. Arai (1969b); Northern California. Chapđ23 Baja California. King (1962).

\section{OPECOELIDAE}

Podocotyle atomon (Rudolphi 1802) Odhner 1905. British Columbia. Intestine. McFarlane (1936).

Podocotyle olssoni Odhner 1905. Oregon. Pratt and McCauley (1961). Podocotyle reflexa (Creplin 1825) Odhner 1905. British Columbia. McFarlane (1936); Oregon. Pratt and McCauley (1961).

Podocotyle enophrysi Park 1937. Washington. Intestine. Aldrich ${ }^{2}$ as P. pacifica.

Podocotyle sp. Northern California. Intestine. Edmiston 29.

Podocotyle californica Park 1937. Washington. Intestine. Ching (1960a) as P. pedunculata.

Podocotyle sinusacca Ching 1960. British Columbia. Intestine. Arai (1969b); Washington. Intestine. Ching (1960a).

Helicometrina nimia Linton 1910. Southern California. Intestine. Montgomery (1957).

HIRUDINOIDEA

GLOSSIPHONIIDÁE

Genera and species. Southern California. External surface. Tasto (1975).

\section{PISCICOLIDAE}

Aetabdella abditovesiculata (Moore 1952) Burreson 1976. Oregon, California. External surface. Burreson (1976a).

\section{ISOPODA}

\section{CYMOTHOIDAE}

Lironeca vulgaris Stimpson 1857. Oregon. Gill cavity. Burreson7. MONOGENEA

CAPSALIDAE

Neobenedenia girellae (Hargis 1955) Yamaguti 1963. California. Hargis (1955a) as Benedenia $g$.

GYRODACTYLIDAE

Gyrodactylus armatus Crane and Mizelle 1967. Northern California. Skin. Crane and Mizelle (1967). 
Gyrodactylus sculpinus Crane and Mizelle 1967. Northern California. Skin. Crane and Mizelle (1967).

Gyrodactylus sp. Southern California. Gills. Baker ${ }^{22}$.

\section{NEMATODA}

\section{ANISAKIDAE}

Anisakis sp. Larva. British Columbia. Body cavity. Arai (1969b).

Contracaecum sp. Larva. British Columbia. Body cavity. Arai (1969b). * Thynnascaris adunca (Rudolphi 1802) Hartwich 1957. British Columbia. Smedley (1934) as Contracaecum magnum.

\section{CAMALLANIDAE}

Spirocamallanus pereirai (Annereaux 1946) 01 sen 1952. California. Intestine. Noble and King (1960).

PROTOZOA (CILIOPHORA)

\section{SCYPHIDIIDAE}

Scyphidia sp. Oregon. Gills. Burreson?

Ambipherya sp. Southern California. Gilis. Baker ${ }^{22}$.

\section{URCEOLARI IDAE}

Trichodina sp. Southern California. Gills. Baker 22. PROTOZOA (MYXOSPORIDA)

\section{CERATOMYXIDAE}

Ceratomyxa tenuis Fujita 1923. Central California. Gallbladder. Jameson (1929).

Ceratomyxa crassa Jameson 1929. Central California. Gallbladder. Jameson (1929).

$$
\text { Malacottus kincaidi - Blackfin Sculpin }
$$

NEMATODA

\section{ANISAKIDAE}

Contracaecum sp. Larva. British Columbia. Body cavity. Arai

\section{CAPILLARI IDAE}

Capillaria sp. British Columbia. Stomach. Arai (1969b). 
ACANTHOCEPHALA

ECHINORHYNCHIDAE

Echinorhynchus gadi Zoega in Müller 1776. British Columbia.

Intestine. Arai (1969b); Kamchatka, USSR. Zhukov (1963).

POLYMORPHIDAE

Corynosoma sp. Juvenile. British Columbia. Intestine. Arai (1969b). Corynosoma strumosum (Rudolphi 1802) Lahe 1904. Larva. Kamchatka, USSR. Zhukov (1963).

\section{CESTODA}

BOTHRIOCEPHALIDAE

Bothriocephalus scorpii (Mü17er 1776) Rudolphi 1808. British Columbia. Intestine. Arai (1969b).

PHYLLOBOTHRI IDAE

Phyllobothrium sp. Larva. British Columbia. Intestine. Arai $(1969 b)$.

TETRAPHYLLIDAE

Scolex pleuronectis Müller 1788. Kamchatka, USSR. Zhukov (1963). COPEPODA

\section{CALIGIDAE}

Lepeophtheirus sp. British Columbia. Body surface. Arai (1969b). Lepeophtheirus parviventris Wilson 1905. British Columbia. Kabata (1973).

\section{CHONDRACANTHIDAE}

Chondracanthus irregularis Fraser 1920. British Columbia. Gills. Arai (1969b).

Chondracanthus lotellae Wilson 1935. Alaska. Gills. Wilson (1935b).

\section{NAOBRANCHI IDAE}

Naobranchia occidentalis Wilson 1915. British Columbia. Gills. Arai $(1969 \mathrm{~b})$.

\section{DIGENEA}

\section{ÂLLOCTREẪİ İAAE}

Neophasis oculata (Levinsen 1881) Mi1ler 1941. Kamchatka, USSR. Zhukov (1963). 
Myoxocephalus polyacanthocephaTus (Continued)

BUCEPHALIDAE

Prosorhynchus crucibulum (Rudolphi 1819) Odhner 1905. Kamchatka, USSR. Zhukov (1963).

\section{FELLODISTOMATIDAE}

Stenakron problematicum (Issaitschikov 1928) Yamaguti 1971. Kamchatka, USSR. Zhukov (1963) as Rhodotrema p.

\section{HEMIURIDAE}

Genolinea laticauda Manter 1925. British Columbia. Stomach. Arai (1969b).

Genolinea anura (Layman 1930) Yamaguti 1954. Kamchatka, USSR. Stomach. Zhukov (1963).

Tubulovesicula lindbergi (Layman 1930) Yamaguti 1934. British Columbia. Stomach. Arai (1969b).

Hemiurus levinseni Odhner 1905. Kamchatka, USSR. Zhukov (1963). Derogenes varicus (Müller 1784) Looss 1901. Kamchatka, USSR. Zhukov (1963).

Genarchops is muelleri (Levinsen 1881) Yamaguti 1954. Kamchatka, USSR. Zhukov (1963).

Lecithaster gibbosus (Rudolphi 1802) Lühe 1901. Kamchatka, USSR. Zhukov (1963).

\section{OPECOEL IDAE}

Podocotyle enophrysi Park 1937. British Columbia. Intestine. Arai $(196 \overline{9 b})$.

Podocotyle atomon (Rudolphi 1802) Odhner 1905. Kamchatka, USSR. Zhukov (1963).

Podocotyle reflexa (Creplin 1825) Odhner 1905. Kamchatka, USSR. Zhukov (1963).

\section{NEMATODA}

\section{ANISAKIDAE}

Anisakis sp. Larva. British Columbia. Intestine. Arai (1969b). Contracaecum sp. Larva. British Columbia. Intestine, body cavity. Arai (1969b).

Phocanema sp. Larva. British Columbia. Intestine. Arai (1969b).

CYSTIDICOLIDAE

Ascarophis sebastodis 01 sen 1952. British Columbia. Intestine. Arai (1969b).

Ascarophis pacificus Zhukov 1960. Kamchatka, USSR. Zhukov (1963). 
Myoxocephalus polyacanthocephalus (Continued)

PROTOZOA (MYXOSPORIDA)

CERATOMYXIDAE

Ceratomyxa porrecta Dogiel 1948. Kamchatka, USSR. Zhukov (1964). MYXIDI IDAE

Myxidium japonicum Dogiel 1948. Kamchatka, USSR. Zhukov (1964). PROTOZOA (SPOROZOA)

EIMERIIDAE

Eimeria myoxocephali Fitzgerald 1975. Washington. Fitzgerald (1975).

$$
\text { Nautichthys oculofasciatus - Sailfin Sculpin }
$$

CESTODA

BOTHRIOCEPHALIDAE

Bothriocephalus scorpii (Müller 1776) Rudolphi 1808. British Columbia. Intestine. Arai (1969b).

DIGENEA

HEMIURIDAE

Genolinea laticauda Manter 1925. British Columbia. Stomach. Arai (1969b).

Lecithaster gibbosus (Rudolphi 1802) Lühe 1901. British Columbia. Intestine. Arai (1969b).

NEMATODA

CYSTIDICOLIDAE

Ascarophis sebastodis 01 sen 1952. British Columbia. Intestine. Arai (1969b).

\section{1igocottus maculosus - Tidepool Sculpin}

COPEPODA

BOMOLOCHIDAE

Holobomolochus spinu7us (Cressey 1969) Kabata 1971. British Columbia. GiTis. Kabata (1971). 


\section{PENNELLIDAE}

Haemobaphes intermedius Kabata 1967. British Columbia. Gill arches. Kabata (1967b).

Haemobaphes sp. Juvenile. British Columbia. Gills. Arai (1969b).

\section{DIGENEA}

HEMIURIDAE

Genolinea laticauda Manter 1925. Oregon. Hal1 and Pratt (1969); British Columbia. Stomach. Arai (1969b).

Lecithaster gibbosus (Rudolphi 1802) Lühe 1901. British Columbia. Intestine. Arai (1969b); Oregon. Hal1 and Pratt (1969) as L. salmonis.

TubulovesicuTa lindbergi (Layman 1930) Yamaguti 1934. British Columbia. Stomach. Arai (1969b).

OPECOELIDAE

Podocotyle californica Park 1937. Northern California. Intestine. Park (1937b) as P. pedunculata; Oregon. Hall and Pratt (1969).

\section{MONOGENEA}

GYRODACTYLOIDEA

Genera and species. British Columbia. Gills. Arai (1969b).

\section{NEMATODA}

\section{ANISAKIDAE}

Anisakis sp. Larva. British Columbia. Body cavity. Arai (1969b). Contracaecum sp. Larva. British Columbia. Body cavity. Arai (1969b). PROTOZOA (CILIOPHORA)

URCEOLARI IDAE

Trichodina lairdi Lom 1970. Washington. Gills. Lom (1970b).

Trichodina cottidarum subsp. cottidarum Lom 1970. Washington. Gi11s. Lom (1970b).

$$
\begin{gathered}
\frac{01 \text { igocottus }}{\text { snyderi }}-\text { Fluffy Sculpin } \\
\text { SYNONYM - Dialarchus snyderi }
\end{gathered}
$$

\section{DIGENEA}

\section{HEMIURIDAE}

Lecithaster gibbosus (Rudolphi 1802) Lühe 1902. Oregon. Hal1 and Pratt (1969) as L. salmonis. 
OPECOELIDAE

Podocotyle cal ifornica Park 1937. Oregon. Hâl and Pratt (1969); Northern California. Intestine, caeca. Nahhas and Krupin (1977).

ZOOGONIDAE

Zoogonoides viviparous (01sson 1868) Odhner 1902. Oregon. Hall and Pratt (1969).

PROTOZOA (MYXOSPORIDA)

MYXIDI IDAE

Myxidium incurvatum Thélohan 1892. Southern California. Gallbiadder. Noble (1947).

$$
\text { Radul inus asprel1us - S1 im Sculpin }
$$

MONOGENEA

MICROCOTYLIDAE

Microcotyle sp. Washington. Bonham (1950).

PROTOZOA (CILIOPHORA)

SCYPHIDIIDAE

Scyphidia arctica Zhukov 1960. Washington. Gills. Lom and Laird (1969).

URCEOLARI IDAE

Trichondina ovonucleata Raabe 1958. Washington. Lom (1970b).

$$
\text { Rhamphocottus richardsoni - Grunt Sculpin }
$$

NEMATODA

ANISAKIDAE

Phocanema sp. Larva. British Columbia. Body cavity. Arai (1969b) as Porrocaecum sp.

CYSTIDICOLIDAE

Ascarophis sebastodis 01 sen 1952. British Columbia. Intestine. Árai (1969b). 
COPEPODA

BOMOLOCHIDAE

Holobomolochus venustus Kabata 1971. British Columbia. Nasal cavities. Kabata (1971).

\section{CALIGIDAE}

Caligus hobsoni Cressey 1969. Southern California. Hobson (1971). Lepeophtheirus parviventris Wilson 1905. British Columbia. Fraser (1920).

\section{CHONDRACANTHIDAE}

Chondracanthus pinguis Wilson 1912. British Columbia. Fraser (1920). Chondracanthus gracilis Fraser 1920. British Columbia. Inner surface of operculums. Kabata (1968).

\section{DIGENEA}

\section{BUCEPHALIDAE}

Prosorhynchus scapellus McFarlane 1936. British Columbia. Pyloric caeca. McFarlane (1936); Central California. Stomach, intestine. Chapa 23.

\section{HEMIURIDAE}

Tubulovesicula lindbergi (Layman 1930) Yamaguti 1934. British Columbia. Intestine. McFarlane (1936) as Dinurus nanaimoensis. Genolinea laticauda Manter 1925. British Columbia. McFarlane (1936); Washington. Stomach. Lloyd (1938) as G. robusta; Central California. Nahhas ${ }^{10}$.

\section{OPECOEL IDAE}

Podocotyle enophrysi Park 1937. Northern California. Intestine. Nahhas and Krupin (1977).

Podocotyle sp. Northern California. Intestine. Edmiston ${ }^{29}$.

Podocotyle californica Park 1937. Northern California. Intestine. Nahhas and Krupin (1977).

Genitocotyle acirra Park 1937. Oregon. Pratt (1970).

HIRUDINOIDEA

\section{PISCICOLIDAE}

Trachelobde11a oregonensis Burreson 1976. Oregon. Fins. Burreson $(1976 b)$.

Malmiana diminuta Burreson 1977. Oregon. Skin, fins. Burreson

$(1977 \mathrm{c})$. 
Scorpaenichthys marmoratus (Continued)

ISOPODA

Genera and species. Gnathiid larva. Southern California. Body surface. Hobson (1971).

CYMOTHOIDAE

$\frac{\text { Lironeca vulgaris Stimpson } 1857 . \text { Central California. Gill cavity. }}{\text { Moser } 19}$.

NEMATODA

CYSTIDICOLIDAE

Caballeronema wardlei (Smedley 1934) Margol is 1977. Canada.

Intestine. Smediey (1934) as Metabronema w.

PROTOZOA (SARCOMASTIGOPHORA)

CRYPTOBI IDAE

Cryptobia sp. Oregon. Blood. Burreson?

PROTOZOA (SPOROZOA)

EIMERI IDAE

Eimeria sp. Central California. Intestine. Moser 19.

Synchirus gilli - Manacled Sculpin

DIGENEA

HEMIURIDAE

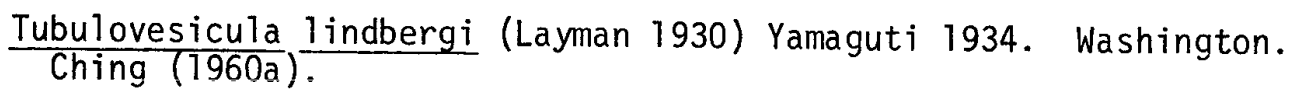

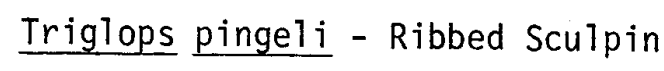

CESTODA

PHYLLOBOTHRI IDAE

Phyllobothrium sp. Larva. British Columbia. Intestine. Arai (1969b). TENTACULARI IDAE

Nybelinia surmenicola Okada 1929. Larva. British Columbia. Intestine. Arai (1969b). 
Triglops pingeli (Continued)

COPEPODA

\section{PENNELLIDAE}

Haemobaphes sp. Juvenile. British Columbia. Gills. Arai (1969b).

Peniculus clavatus (Müiler 1779) Nordman 1832. Atlantic.

Prefontaine and Brune1 (1962).

\section{DIGENEA}

\section{OPECOELIDAE}

Podocotyle enophrysi Park 1937. British Columbia. Intestine. Arai (1969b) as P. blennicottusi.

NEMATODA

\section{ANISAKIDAE}

Anisakis sp. Larva. British Columbia. Body cavity. Arai (1969b).

Contracaecum sp. Larva. British Columbia. Body cavity. Arai (1969b).

$$
\begin{gathered}
\text { AGONIDAE -- Poachers and Alligatorfishes } \\
\frac{\text { Agonopsis emmelane - Northern Spearnose Poacher }}{\text { SYNONYM - Agonopsis averuncus }}
\end{gathered}
$$

\section{DIGENEA}

OPECOELIDAE

Podocotyle gibbonsiae Johnson 1949. Washington. Intestine. A7drich 22 . Podocotyle enophrysi Park 1937. Washington. Intestine. Aldrich ${ }^{12}$ as $\underline{\text { P. pacifica. }}$

PROTOZOA (MYXOSPORIDA)

MYXIDIIDAE

Myxidium sp. Washington, Gallbladder. Kuris 20.

Sphaeromyxa balbianii Thélohan 1895. Washington. Gallbladder. Kuris 20.

$$
\text { Agonus acipenserinus - Sturgeon Poacher }
$$

\section{ACANTHOCEPHALA}

\section{ECHINORHYNCHIDAE}

Echinorhynchus gadi Zoega in Müller 1776. British Columbia. 
Agonus acipenserinus (Continued)

CESTODA

PHYLLOBOTHRIIDAE

Phyllobothrium sp. Larva. British Columbia. Intestine. Arai (1969b). COPEPODA

\section{PENNELLIDAE}

Haemobaphes sp. Immature. British Columbia. Kabata (1967b);

British Columbia. Gills. Arai (1969b).

\section{DIGENEA}

OPECOELIDAE

Podocotyle reflexa (Creplin 1825) Odhner 1905. Washington. Intestine. Aldrich 12 .

Podocotyle sp. Immature. British Columbia. Intestine. Arai (1969b). NEMATODA

\section{ANISAKIDAE}

* Thynnascaris adunca (Rudolphi 1802) Hartwich 1957. Larva. British Columbia. Intestine. Ârai (1969) as Contracaecum a.

$$
\text { Asterotheca infraspinata - Spinycheek Starsnout }
$$

PROTOZOA (CILIOPHORA)

\section{SCYPHIDIIDAE}

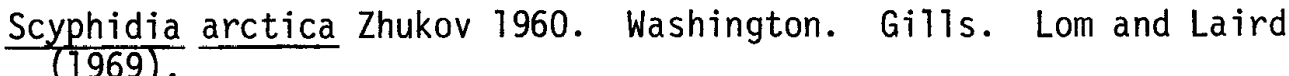
URCEOLARI IDAE

Trichodina ovonucleata Raabe 1958. Washington. Lom (1970b).

$$
\text { Pallasina barbata - Tubenose Poacher }
$$

\section{MONOGENEA}

\section{GYRODACTYLIDAE}

Gyrodactylus gröenlandicus Levinsen 1881. Okhotsk Sea, USSR. Anal, pectoral fins. Bykhovsky and Polyanski (1953). Gyrodactylus proximus Bykhovsky and Polyanski 1953. USSR. Bykhovsky (1957). 


\section{CESTODA}

PHYLLOBOTHRIIDAE

Phyllobothrium sp. Larva. British Columbia. Intestine. Arai (1969b).

$$
\begin{aligned}
& \text { CYCLOPTERIDAE - Lumpfishes and Snailfishes } \\
& \text { Liparis callyodon - Spotted Snailfish }
\end{aligned}
$$

CESTODA

SPATHEBOTHRI IDAE

Spathebothrium simplex Linton 1922. Northern California. Intestine. Nahhas and Krupin (7977).

\section{DIGENEA}

BUCEPHALIDAE

Prosorhynchus scalpelus McFarlane 1936. Northern California. Intestine. Nahhas and Krupin (1977).

CRYPTOGONOMIDAE

Metadena caballeroi Nahhas and Krupin 1977. Northern California. Intestine. Nahhas and Krupin (1977).

HEMIURIDAE

Genolinea laticauda Manter 1925. Northern California. Intestine. Nahhas and Krupin (1977).

OPECOELIDAE

Podocotyle sinusacca Ching 1960. Northern California. Intestine. Nahhas and Krupin (1977).

$$
\text { Liparis cyclopus - Ribbon Snailfish }
$$

PROTOZOA (MYXOSPORIDA)

MYXIDIIDAE

Sphaeromyxa magna Zhukov 1964. Washington. Gallbladder. Lom (1969). 


\title{
Liparis fucensis - Slipskin Snailfish
}

CESTODA

SPATHEBOTHRI IDAE

$\frac{\text { Spathebothrium simplex Linton 1922. Washington. Hart and Guberlet }}{(1936) \text {. }}$ DIGENEA

OPECOELIDAE

$\frac{\text { Allopodocotyle }}{\text { Intestine. }} \frac{\text { lepomis }}{\text { Tdricht2 }}$ (Dobrovolny 1939) Pritchard 1966. Washington. Podocotyle atomon (Rudolphi 1802) Odhner 1905. Washington.

Podocotyle enophrysi Park 1937. Washington. Intestine. Aldrich ${ }^{12}$ also as $P$. pacifica and $P$. blennicotusi.

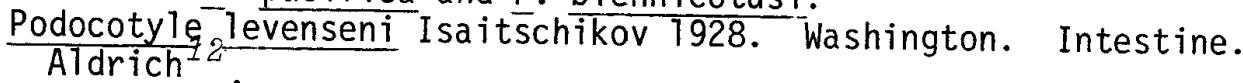

\section{Liparis pulchellus - Showy Snailfish}

DIGENEA

HEMIUR IDAE

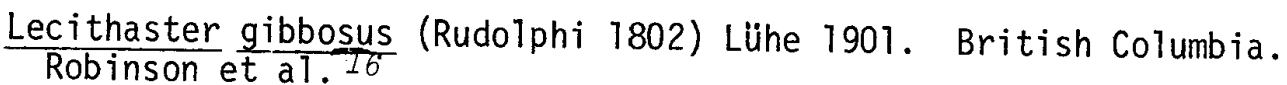
OPECOEL IDAE $\frac{\text { Podocotyle enophrysi }}{\text { as } \text { P. pack } \text { pacica. }}$ 1937. Washington. Intestine. Aldrich 12

\author{
BOTHIDAE -- Lefteye Flounders \\ Citharichthys sordidus - Pacific Sanddab \\ SYNONYM - Orthopsetta sordida
}

COPEPODA

LERNAEOPODIDAE

Neobrachiella nitida (Wilson 1915) Kabata 1978. Central California. Buccal cavity. Wilson (1935b) as Brachiella n.

\section{PENNELLIDAE}

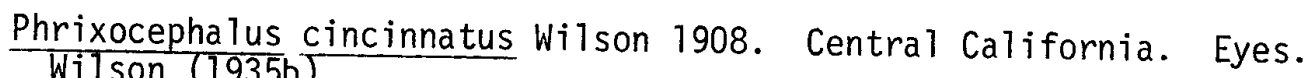


Citharichthys sordidus (Continued)

\section{DIGENEA}

\section{AZZYGIIDAE}

Otodistomum veliporum (Criplin 1837) Stafford 1904. Metacercaria. British Columbia. Margolis ${ }^{3}$.

\section{HEMIURIDAE}

Sterrhurus magnatestis Park 1936. Northern California. Stomach. Park $(1936)$.

Derogenes varicus (Müller 1784) Looss 1901. British Columbia. Stomach. Margol is 3 .

Tubulovesicula lindbergi (Layman 1930) Yamaguti 1934. Oregon. Stomach. McCauley (1960); British Columbia. Arai (1969b).

NEMATODA

\section{ANISAKIDAE}

Anisakis sp. Larva. British Columbia. Many places in body. Margolis ${ }^{3}$; Southern California. Dailey et a1. (in press).

\section{PROTOZOA (SARCOMASTIGOPHORA)}

\section{TRYPANOSOMATIDAE}

Trypanosoma pacifica Burreson and Pratt 1972. Oregon. Blood.

$$
\text { Citharichthys stigmaeus - Speckled Sanddab }
$$

CESTODA

\section{GILQUINIIDAE}

Gilquinia squali (Fabricius 1794) Dollfus 1930. Larva. British Columbia. Yamaguti (1959b).

\section{DIGENEA}

\section{HEMIURIDAE}

TubuTovesicula lindbergi (Layman 1930) Yamaguti 1934. Oregon. Stomach. Mccauley (1960); British Columbia. Stomach. Arai (1969b). Sterrhurus magnatestis Park 1936. Northern California. Chapa23as Separogermiductus $\underline{m}$.

\section{NEMATODA}

\section{ANISAKIDAE}

Anisakis sp. Larva. British Columbia. Body cavity. Arai (1969b). 
PROTOZOA (MYXOSPORIDA)

CERATOMYXIDAE

Ceratomyxa hopkinsi Jameson 1929. Southern California. Gallbladder. Jameson (1929).

$$
\text { Hippoglossina stomata - Bigmouth Sole }
$$

HIRUDINOIDEA

PISCICOLIDAE

Australobdella californiana Burreson 1977. Southern California. Body surface. Burreson (1977a).

NEMATODA

ANISAKIDAE

Anisakis sp. Larva. Southern California. Dailey et al. (in press). Paralichthys californicus - California Halibut

CESTODA

DASYRHYNCHIDAE

Callitetrarhynchus gracilis (Rudolphi 1819) Pintner 1931. Larva. Southern California. Viscera. Jensen ${ }^{18}$.

ECHENEIBOTHRI IDAE

Echeneibothrium sp. Larva. Southern California. Mesentery. Haaker (1975).

LACISTORHYNCHIDAE

Lacistorhynchus tenuis (Van Beneden 1858) Dollfus 1929. Larva. Southern California. Viscera. Jensen ${ }^{18}$.

TRYPANORHYNCHA

Genera and species. Larva. Southern California. Jensen ${ }^{18}$. COPEPODA

BOMOLOCHIDAE

Holobomolochus prolixus (Cressey 1969) Kabata 1971. Southern California. Inner surfaces of operculums. Ho (1972a).

Taeniacanthodes haakeri Ho 1972. Southern California. Between rays of dorsal and anal fins. Ho (1972a). 
Paralichthys californicus (Continued)

* Bomolochus solae Claus 1864. Baja California. Causey (1960).

\section{CALIGIDAE}

Lepeophtheirus bifidus Fraser 1920. Southern California. Ho (1972a). Lepeophtheirus dissimulatus Wilson 1905. Baja California. Causey (1960).

\section{CHONDRACANTHIDAE}

Acanthochondria sp. California. Ho (1972a).

\section{TREB I IDAE}

Trebius latifurcatus Wilson 1921. Southern California. Exterior. Wilson (1921).

DIGENEA

ACANTHOCOLIDAE

Stephanostomum dentatum (Linton 1900) Linton 1940. California. Intestine. Manter and Van Cleave (1951).

\section{BUCEPHALIDAE}

Bucephalus heterotentaculatus Bravo-HoiT is and Sogandares-Bernal 1956. Baja CaTifornia. Intestine. Arai (1962). Prosorhyncinoides labiatus (Manter and Van Cleave 1951) $n$. comb. Southern California. Intestine. Manter and Van Cleave (1951) as Bucephalops is 1. CRYPTOGONIMIDAE

Metadena magdalenae Arai 1962. Baja California. Intestine. Arai $(1962)$.

\section{HEMIURIDAE}

Tubulovesicula lindbergi (Layman 1930) Yamaguti 1934. Baja California. Stomach. King (1962); Southern California. Digestive tract. Haaker (1975).

ISOPODA

CYMOTHOIDAE

Lironeca vulgaris Stimpson 1857. California. Gill cavity. Ho (1975). MONOGENEA

\section{CAPSAL IDAE}

Entobdella squamula (Heath 1902) Johnston 1929. Central California. Body surface, gi17 chamber. Price (1939). 
Paralichthys californicus (Continued)

NEMATODA

ANISAKIDAE

Contracaecum sp. Larva. Southern California. Dailey et al. (in press). Anisakis sp. Larva. Southern California. Dailey et al. (in press). Phocanema sp. Larva. Southern California. Dailey et al. (in press).

\title{
CAMALLANIDAE
}

Spirocamallanus pereirai (Annereaux 1946) 01 sen 1952. Southern California. Stomach. Haaker (1975).

\author{
PLEURONECTIDAE - Righteye Flounders \\ Atheresthes stomias - Arrowtooth Flounder
}

ACANTHOCEPHALA

ECHINORHYNCHIDAE

Echinorhynchus gadi Zoega in Mülier 1776. Bering Sea, USSR. Mamaev (1965).

POLYMORPHIDAE

Corynosoma reductum (Von Lindstow 1905) Railliet and Henry 1907. Larva. Bering Sea. Intestine. Mamaev (1965).

Corynosoma strumosum (Rudo7phi 1802) Lühe 1904. Larva. Bering Sea, USSR. Mamaev (1965).

\section{CESTODA}

\section{LACISTORHYNCHIDAE}

Grillotia erinacea (Van Beneden 1858) Guiart 1927. Larva. Bering Sea, USSR. Body cavity, stomach wal1. Mamaev (1965).

\section{TENTACULARI IDAE}

Nybel inia surmenicola Okada 1929. Larva. Bering Sea, USSR. Body cavity, stomach wa17, intestine. Mamaev (1965).

\section{TETRAPHYLLIDEA}

Scolex pleuronectis Müller 1788. Bering Sea, USSR. Intestine, ga71b Tadder. Mamaev (1965).

CŌPEETŌDÁ

\section{PENNELL IDAE}

Phrixocephalus cincinnatus Wilson 1908. Northern California. Eyes. Wilson (1935b); British Columbia. Eyes. Kabata (1969b). 


\section{DIGENEA}

\section{ACANTHOCOLPIDIAEE}

Stephanostomum sp. Metacercaria. Alaska. Kovalenko (1969).

\section{BUCEPHALIDAE}

Prosorhynchus crucibulum (Rudolphi 1819) Odhner 1905. Bering Sea, USSR. Mamaev (1965).

\section{HEMIURIDAE}

Lecithaster gibbosus (Rudolphi 1802) Lühe 1901. Bering Sea, USSR. Intestine. Mamaev (1965).

\section{ZOOGONIDAE}

Steganoderma formosum Stafford 1904. Bering Sea, USSR. Intestine. Mamaev (1965).

Brachyenteron doderleiniae Yamaguti 1938. Alaska to California. Kovalenko (1969).

\section{MONOGENEA}

\section{CAPSALIDAE}

Entobdella pugetensis Robinson 1961. Washington. Gi11s. Robinson (1961).

Entobdelia hippoglossi (Müller 1776) Johnston 1856. Bering Sea, USSR. GiT1s. Mamaev (1965).

\section{DICLIDOPHORIDAE}

Diclidophora pugetensis Robinson 1961. Washington. Gills. Robinson (1961).

Heterobothrium affinis (Linton 1898) Price 1936. Bering Sea, USSR. Mamaev (1965).

NEMATODA

\section{AN ISAKIDAE}

Anisakis sp. Larva. British Columbia. Many places in body. Margolis ${ }^{3}$; Bering Sea. Mamaev (1965).

Phocanema decipiens (Krabbe 1878) Myers 1959. Larva. Bering Sea, USSR. Musculature, body cavity. Mamaev (1965).

* Thynnascaris adunca (Rudolphi 1802) Hartwich 1957. Bering Sea, USSR. Mamaev (1965).

\section{PROTOZOA (MYXOSPORIDA)}

\section{TETRACAPSULIDAE}

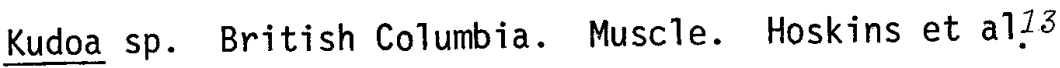


ACANTHOCEPHALA

\section{ECHINORHYNCHIDAE}

Echinorhynchus gadi Zoega in Mü1ler 1776. British Columbia. Intestine. Margol is 3 .

POLYMORPHIDAE

Corynosoma strumosum (Rudolphi 1802) Lühe 1904. Juvenile. British Columbia. Encysted in liver. Margolis ${ }^{3}$.

\section{CESTODA}

\section{PHYLLOBOTHRI IDAE}

Phyllobothrium caudatum (Zschokke and Heitz 1914). Larva. British Columbia. Liver. Margol is ${ }^{3}$ as $\underline{\text { P. Ketae. }}$

\section{TENTACULARI IDAE}

Nybelinia surmenicola Okada 1929. Postlarve. British Columbia. Stomach wa11. Margol is 3.

\section{COPEPODA}

\section{CALIGIDAE}

Lepeophtheirus parviventris Wilson 1905. British Columbia. Kabata (7973).

Caligus pectinatus Shiino 1965. Southern California. External body surface. Shiino (1965b).

\section{DIGENEA}

\section{AZYGIIDAE}

Otodistomum veliporum (Creplin 1837) Stafford 1904. Metacercaria. British Columbia. Cysts in connective tissue of stomach wall. Margolis ${ }^{3}$.

\section{HEMIURIDAE}

Tubulovesicula lindbergi (Layman 1930) Yamaguti 1934. British Columbia. Stomach. Margol is and Boyce (1969). Sterrhurus exodicus (McFarlane 1936) Yamaguti 1958. Central California. Stomach. Nahhas 10 ; British Columbia. Margol is 3 as Lecithochirium exodicum; Oregon. Gregoire and Pratt (1952).

\section{OPECOELIDAE}

Podocotyle gibbonsiae Johnson 1949. Washington. Intestine. A7drich12.

Podocotyle sp. British Columbia. Pyloric caeca. Margolis 3 as P. wilsoni. 
MONOGENEA

CAPSALIDAE

Entobdella squamula (Heath 1902) Johnston 1929. British Columbia. Fin, skin. Margol is 3 .

NEMATODA

ANISAKIDAE

* Thynnascaris adunca (Rudolphi 1802) Hartwich 1957. British Columbia. Stomach, intestine. Margol is ${ }^{3}$.

Contracaecum sp. Larva. British Columbia. Mesentery, liver, stomach. Margol is ${ }^{3}$.

Anisakis sp. Larva. British Columbia. Many places in body.

Margolis 3 ; Southern California. Dailey et al. (in press).

PROTOZOA (MYXOSPORIDA)

TETRACAPSULIDAE

Kudoa sp. Washington. Muscle. Patashnik and Groninger (1964).

PROTOZOA (SARCOMASTIGOPHORA)

CRYPTOBIIDAE

Cryptobia sp. Oregon. Blood. Burreson?

TRYPANOSOMATIDAE

Trypanosoma sp. Oregon. Blood. Burreson?

$$
\text { Glyptocephalus zachirus - Rex Sole }
$$

CESTODA

TETRAPHYLLIDEA

Genera and species. Red pigmented plerocercoid. Southern California. Intestine, pyloric caeca. Kunnenkeri and Martin (1962).

DIGENEA

HEMIURIDAE

Dissosaccus laevis (Linton 1898) Manter 1947. Central California. Stomach. Nahhas 10 . 
NEMATODA

\section{ANISAKIDAE}

* Thynnascaris adunca (Rudolphi 1802) Hartwich 1957. British Columbia. Stomach, intestine. Margol is ${ }^{3}$.

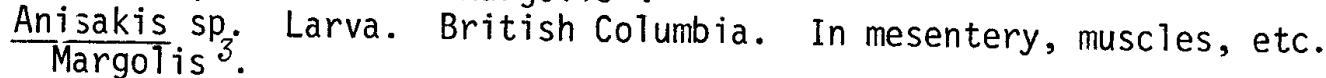

\section{PROTOZOA (SARCOMASTIGOPHORA)}

TRYPANOSOMATIDAE

Trypanosoma sp. Oregon. Blood. Burreson?

$$
\text { Hippoglossoides elassodon - Flathead Sole }
$$

\section{ACANTHOCEPHALA}

\section{ECHINORHYNCHIDAE}

Echinorhynchus gadi Zoega in Müller 1776. Kamchatka, USSR. Skryabina (1963).

\section{POLYMORPHIDAE}

Corynosoma strumosum (Rudolphi 1802) Lühe 1904. Larva. Kamchatka, USSR. Strelkov (1960); Sea of Japan. Intestine. Zhukov (1960a). Corynosoma reductum (Von Lindstow 1905) Railliet and Henry 1907. Bering Sea. Intestine. Mamaev (1965).

\section{BRANCHIURA}

\section{ARGUL IDAE}

Argulus borealis Wilson 1912. British Columbia. Fraser (1920). CESTODA

AMPHICOTYLIDAE

Eubothrium sp. Okhotsk Sea, USSR. Intestine. Mamaev et a 1. (1963). BOTHRIOCEPHALIDAE

Bothriocephalus sp. Okhotsk Sea, USSR. Mamaev et al. (1963). DIPHYLLOBOTHRI IDAE

Diphyllobothrium sp. Larva. Kamchatka, USSR. On digestive tract. Tsimbalyuk and Semeshko (1971).

Pyramicocepha7us phocarum (Fabricius 1780). Larva. Pacific Ocean, Okhotsk Sea, USSR. Mamaev et al. (1963). 
Hippoglossoides elassodon (Continued)

PHYLLOBOTHRI IDAE

Phyllobothrium sp. Larva. British Columbia. Intestine. Margolis ${ }^{3}$. PSEUDOPHYLLIDEA

Genera and species. Pacific Ocean, Okhotsk Sea, USSR. Stomach, intestine. Mamaev et a1. (1963).

\section{TENTACULARIIDAE}

Nybel inia surmenicola Okada 1929. Larva. Kamchatka, USSR. Body cavity, abdominaT walls. Skryabina (1963); postlarva. British Columbia. Margolis ${ }^{3}$.

TETRAPHYLLIDEA

Scolex pleuronectis Muller 1788. Sea of Japan. Intestine. Zhukov (1960a).

COPEPODA

BOMOLOCHIDAE

Holobomolochus occultus Kabata 1971. British Columbia. Gills and gill cavity. Kabata (1971).

NAOBRANCHI IDAE

Naobranchia occidentalis Wilson 1915. Washington. Gills. Villadolid (1927).

DIGENEA

ACANTHOCOLPIDAE

Stephanostomum baccatum (Nicol1 1907) Manter 1934. Sea of Japan. Intestine. Zhukov (7960a).

\section{ALLOCREADIIDAE}

Pseudozoogonoides microacetabulum (Schulman-Albowa 1952) Zhukov 1957. Kamchatka, USSR. Intestine. Skryabina (1963).

BUCEPHALIDAE

Prosrhynchoides gracilescens (Rudolphi 1819) n. comb. Sea of Japan. Intestine. Zhukov (1960a).

FELLODISTOMATIDAE

Stenakron quinquelobatum (Layman 1930) Mamaev, Parukhin and Baeva. 1963. Sea of Japan. Intestine. Zhukov (1960a) as Rhodotrema $q$. 
Hippoglossoides elassodon (Continued)

Stenakron vestustum Stafford 1904. Kamchatka, USSR. Intestine. Strelkov (1960).

Steringotrema ovacutum (Lebour 1908) Odhner 1911. Sea of Japan. Intestine. Zhukov (1960a) as Rhodotrema o.

Fellodistomum furcigerum (01sson 1867) Yamaguti 1954. Kamchatka, USSR. Strelkov (1960) as Steringophorus f.

HEMIURIDAE

Brachyphallus crenatus (Rudolphi 1802) Odhner 1905. Sea of Japan. Intestine. Zhukov (1960a).

Genarchopsis mulleri (Levinson 1881) Yamaguti 1954. Kamchatka, USSR. Intestine. Strelkov (1960) as Genarches $\mathrm{m}$.

Hemiurus levinseni Odhner 1905. Kamchatka, USSR. Stomach, pyloric caeca. Skryabina (1963).

Lecithaster gibbosus (Rudoiphi 1802) Lühe 1901. Sea of Japan. Intestine. Zhukov (1960a).

Derogenes varicus (Müller 1784) Looss 1901. Sea of Japan. Stomach. Zhukov (1960a).

Parahemiurus merus (Linton 1910) Woolcock 1935. Sea of Okhotsk, USSR. Mamaev et a1. (1963).

\section{OPECOELJDAE}

Decemtestis azumae (Layman 1930) Yamaguti 1934. Sea of Japan. Intestine. Zhukov (1960a) as Helicometrina a.

Podocotyle atomon (Rudolphi 1802) Odhner 1905. Pacific Ocean. Mamaev et al. (1963).

SANGUINCOLIDAE

Aporocotyle simplex Odhner 1900. Bering Sea. Mamaev (1965).

\section{ZOOGONIDAE}

Diphtherostomum microacetabulum Schulman-Albova 1952. Sea of Okhotsk, USSR. Intestine. Mamaev et al. (1963).

Lepidophy 71 um pleuronectini Zhukov 1957. Sea of Japan. Urinary b Tadder. Zhukov (1960a).

Steganoderma formosum Stafford 1904. Kamchatka, USSR. Intestine. Skryabina (1963).

Zoogonoides viviparus (01sson 1868) Odhner 1902. Sea of Japan. Intestine. Zhukov (1960a).

NEMATODA

ANISAKIDAE

Phocanema sp. Larva. Kamchatka, USSR. Muscles, body cavity. Skryabina (1963) as Porrocaecum sp.

Contracaecum sp. Lârvà. Kamchatka, USSR. Kidneys, intestine, liver, body cavity. Skryabina (1963).

* Thynnascaris adunca (Rudolphi 1802) Hartwich 1957. Bering Sea. Mamaev $(1965)$; British Columbia. Stomach, intestine. Margol is 3 both as Contracaecum a. 
Hippoglossoides elassodon (Continued)

Anisakis sp. Larva. Sea of Japan. Mesentery. Zhukov (1960a);

British Columbia. Margolis 3 .

CUCULLANIDAE

Cucullanus heterochrous Rudolphi 1802. Pacific Ocean. Intestine. Mamaev et al. (1963).

\author{
Hippoglossus stenolepis - Pacific Halibut \\ SYNONYM - Hippoglossus hippoglossus stenolepis
}

ACANTHOCEPHALA

ECHINORHYNCHIDAE

Echinorhynchus gadi Zoega in Mü1ler 1776. Kamchatka, USSR. Skryabina (1963).

POLYMORPHIDAE

Corynosoma strumosum (Rudolphi 1802) Lühe 1904. Kamchatka, USSR. Intestine. Strelkov (1960).

Corynosoma reductum (Von Lindstow 1905) Railliet and Henry 1907. Larva. Bering Sea. Intestine. Mamaev (1965).

CESTODA

TENTACULARI IDAE

Nybelinia surmenicola Okada 1929. Larva. Kamchatka, USSR. Strelkov (1960).

\title{
TETRAPHYLLIDEA
}

Scolex pleuronectis Müller 1788. Kamchatka, USSR. Gallbladder, intestine. Skryabina (1963).

COPEPODA

CALIGIDAE

Lepeophtheirus parviventris Wilson 1905. Alaska. Kabata (1973); Body surface. Wilson (1905b) from "Halibut".

Lepeophtheirus pravipes Wilson 1912. British Columbia. Bere5.

Lepeophtheirus appendiculatus Krøyer 1863. Alaska. Gills. WiTson $(1935 b)$.

Lepeophtheirus cuneifer Kabata 1974. Alaska. Kabata (1974). Questionable host. 
Hippoglossus stenolepis (Continued)

\section{CHONDRACANTHIDAE}

Acanthochondria cornuta (Müller 1777) Oakley 1927. Alaska. Gill cavity. Wilson (1935b).

\section{DIGENEA}

\section{FELLODISTOMATIDAE}

Stenakron vetustum Stafford 1904. Kamchatka, USSR. Strelkov (1960). HEMIURIDAE

Brachyphallus crenatus (Rudolphi 1802) Odhner 1905. Japan. Stomach. Machida et a1. (1972).

Hemiurus levinseni Odhner 1905. Japan. Stomach. Machida et al. (1972).

Tubulovesicula lindbergi (Layman 1930) Yamaguti 1934. Japan. Stomach. Machida et al. (1972).

Derogenes varicus (Müller 1784) Looss 1901. Kamchatka, USSR. Stomach, intestine. Skryabina (1963).

Genarchops is muelleri (Levinsen 1881) Yamaguti 1954. Kamchatka, USSR. Intestine. Strelkov (1960) as Genarches m.

Parahemiurus merus (Linton 1910) Wollcock $1 \overline{9} 35$. Bering Sea. Stomach. Mamaev (1965).

\section{LEPOCREADIIDAE}

Lepidapedon gadi (Yamaguti 1934) Acena 1947. Kamchatka, USSR. Intestine. Skryabina (1963).

\section{OPECOEL IDAE}

Podocotyle reflexa (Crepl in 1825) Odhner 1905. Kamchatka, USSR. Stomach, intestine, caeca. Skryabina (1963).

Podocotyle atomon (Rudolphi 1802) Odhner 1905. Kamchatka, USSR. Intestine. Strelkov (1960).

Pseudopecoelus nossamani Kruse 1977. Alaska. Kruse (1977).

\section{ZOOGONIDAE}

Steganoderma formosum Stafford 1904. Kamchatka, USSR. Intestine. Skryabina (1963).

\section{MONOGENEA}

\section{CAPSALIDAE}

Entobdella squamula (Heath 1902) Johnson 1929. Alaska to California. Guberlet (1936a); Bering Sea. Gills. Mamaev (1965).

Entobdella hippoglossi (Múiler 1776) Jonnston 1856. British Columbia. Skin. Margolis ${ }^{3}$. 
Hippoglossus stenolepis (Continued)

DICLIDOPHORIDAE

Heterobothrium affinis (Linton 1898) Price 1936. Bering Sea. Mamaev (1965).

NEMATODA

ANISAKIDAE

Anisakis sp. Larva. Kamchatka, USSR. Strelkov (1960).

Contracaecum sp. Larva. Kamchatka, USSR. Liver, kidneys, body cavity, intestine. Skryabina (1963).

Phocanema decipiens (Krabbe 1878) Myers 1959. Larva. British Columbia. Margolis (1956b); Bering Sea. Mamaev (1965) as Terranova d.

CUCULLANIDAE

Cucullanus heterochrous Rudolphi 1802. Kamchatka, USSR. Strelkov (1960).

PROTOZOA (MYXOSPORIDA)

SPHAEROSPORIDAE

Unicapsula muscularis Davis 1924. Muscles. Guberlet (1933).

TETRACAPSULIDAE

Kudoa sp. Washington to Alaska. Muscles. Patashnik and Groninger (1964).

$$
\text { Hypsopsetta guttulata - Diamond Turbot }
$$

COPEPODA

CALIGIDAE

Lepeophtheirus dissimulatus Wilson 1905. Baja California. Causey (1960). Lepeophtheirus bifidus Fraser 1920. Southern California. Body surface. Ho (1975).

DIGENEA

HEMIURIDAE

Tubulovesicula lindbergi (Layman 1930) Yamaguti 1934. Baja

California. Stomach. King (1962).

HIRUDI NOIDEA

PISCICOLIDAE

Aestabdella abditovesiculata (Moore 1952) Burreson 1976. California. Burreson (7976a). 
Hypsopsetta guttulata (Continued)

ISOPODA

CYMOTHOIDAE

Braga sp. Southern California. Gill cavity. Ho (1975).

$$
\text { Isopsetta isolepis - Butter Sole }
$$

COPEPODA

CALIGIDAE

Lepeophtheirus cuneifer Kabata 1974. Alaska. Kabata (1974). Questionable host.

\section{DIGENEA}

HEMIURIDAE

Derogenes varicus (Mü1ler 1784) Looss 1901. Washington. Ching (1960a). Genolinea laticauda Manter 1925. Central California. Intestine. Nahhas 10

Tubulovesicula lindbergi (Layman 1930) Yamaguti 1934. Washington. Ching (1960a).

ZOOGONIDAE

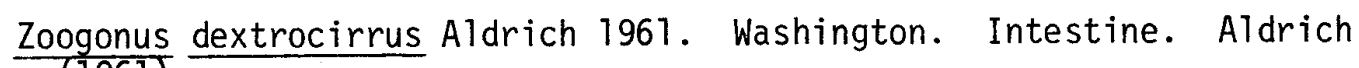
(1961).

NEMATODA

ANISAKIDAE

Anisakis sp. Larva. British Columbia. Mesentery, muscle, etc. Margolis ${ }^{3}$.

PROTOZOA (CILIOPHORA)

URCEOLARI IDAE

Genera and species. British Columbia. Gills. Bell (1962).

$$
\text { Lepidopsetta bilineata - Rock Sole }
$$

\section{ACANTHOCEPHALA}

ECHINORHYNCHIDAE

Metechinorhynchus lageniformis (Ekbaum 1938) Petrotchenko 1956. British Columbia. Intestine. Ekbaum (1938) as Echinorhynchus 1. 
Echinorhynchus gadi Zoegga in Müller 1776. British Columbia. Intestine. Margol is ${ }^{3}$; Kamchatka, USSR. Strelkov (1960).

\section{POLYMORPHIDAE}

Corynosoma strumosum (Rudo1phi 1802) Lühe 1904. Juveniles. British Columbia. Liver, mesentery, intestinal walls. Ekbaum (1938); Kamchatka, USSR. Strelkov (1960).

\section{BRANCHIURA}

\section{ARGULIDAE}

Argulus borealis Wilson 1912. British Columbia. Beneath pectoral fins. Wilson (1912b).

\section{CESTODA}

\section{AMPHICOTYLIDAE}

Eubothrium sp. Pacific Ocean. Intestine. Mamaev et al. (1963).

BOTHRIOCEPHALIDAE

Bothriocephalus sp. Larva. British Columbia. Intestine. Arai (1969b).

\section{PHYLLOBOTHRIIDAE}

Phyllobothrium sp. Larva. British Columbia. Intestine. Arai (1969b).

\section{PSEUDOPHYLLIDEA}

Genera and species. Plerocercoid. British Columbia. Cyst in stomach wall. Margolis ${ }^{3}$.

Genera and species. Pacific Ocean. Stomach, intestine. Mamaev et

$$
\text { a. (1963). }
$$

\section{TENTACULARI IDAE}

Nybelinia surmenicola Okada 1929. Postlarval. British Columbia.

$$
\text { Stomach wa77. Margol is } 3 \text {. }
$$

\section{TETRAPHYLLIDEA}

Scolex pleuronectis Mü1ler 1788. Sea of Japan. Zhukov (1960a). COPEPODA

\section{CALIGIDAE}

Lepeophtheirus bifidus Fraser 1920. British Columbia. Body surfaces. Fraser (1920).

Lepeophtheirus hospitalis Fraser 1920. British Columbia. Kabata (1973). 
Lepidopsetta bilineata (Continued)

Lepeophtheirus parviventris Wilson 1905. British Columbia. Kabata (1973).

\section{LERNAEOPODIDAE}

Nectobranchia indivisa Fraser 1920. British Columbia. Gills. Kabata (1970c).

\section{NAOBRANCHI IDAE}

Naobranchia occidentalis Wilson 1915. British Columbia. Bere ${ }^{5}$. PENNELLIDAE

Haemobaphes sp. Juveniles. British Columbia. Gills. Arai (1969b). DIGENEA

ACANTHOCOLPIDAE

Stephanostomum baccatum (Nicol 1907) Manter 1934. Kamchatka, USSR. Intestine. strelkov (1960).

\section{AZYGIIDAE}

Otodistomum veliporum (Crepl in 1837) Stafford 1904. Metacercaria. British Coimbia. Cysts in connective tissue of stomach wail. Margolis ${ }^{3}$.

BUCEPHALIDAE

Prosorhynchoides gracilescens (Rudolphi 1819) n. comb, Kamchatka, USSR. Intestine. Strelkov (1960) as Bucephalopsis g.

\section{FELLODISTOMATIDAE}

Fellodistomum furcigerum (01sson 1867) Yamaguti 1954. Sea of Japan. Intestine. Zhukov (1960a) as Steringophorus f.

Stenakron vetustum Stafford 1904. Kamchatka, USSR. Strelkov (1960); Sea of Japan. Zhukov (1960a) as Rhodotrema quinquelobota. Steringotrema ovacutum (LeBour 1908) Odhner 1971. Pacific Ocean. Mamaev et a 1 . (1963) as Rhodotrema o.

HEMIURIDAE

Derogenes varicus (Mü1ler 1784) Looss 1901. Alaska. Kruse (1977). TubuTovesicuTa lindbergi (Layman 1930) Yamaguti 1934. Oregon. Stomach. McCauley (1960).

Lecithaster gibbosus (Rudolphi 1802) Luhe 1901. British Columbia. Intestine. Arai (1969b); Kamchatka, USSR. Strelkov (1960).

Hemiurus levinseni Odhner 1905. Kamchatka, USSR. Intestine. Strelkov (1960).

Brachypha1lus crenatus (Rudolphi 1802) Odhner 1905. Kamchatka, USSR. Strelkov (1960).

Genarchops is muelleri (Levinsen 1881) Yamaguti 1954. Kamchatka, USSR. Intestine. Strelkov (1960) as Genarches m. 


\section{LEPOCREADIIDAE}

Lepidapedon gadi (Yamaguti 1934) Acena 1947. Kamchatka, USSR. Stre1kov (1960).

\section{OPECOEL IDAE}

Podocotyle atomon (Rudolphi 1802) Odhner 1905. Kamchatka, USSR. Intestine. Strelkov (1960).

Podocotyle gibbonsiae Johnson 1949. Washington. Intestine. ATdrich 12 .

\section{ZOOGONIDAE}

Pseudozoogonoides microacetabulum (Schulman-Albova 1952) Zhukov 1957. Kamchatka, USSR. Intestine. Strelkov (7960).

Zoogonus dextrocirrus A1drich 1961. Washington. Intestine. ATdrich (1967)

Zoogonoides viviparus (01sson 1868) Odhner 1902. Sea of Japan. Zhukov (1960a).

Lepidophyilum pleuronectini Zhukov 1958. Pacific Ocean. Urinary bladder. Mamaev et a1. (1963).

Diphtherostomum microacetabulum Schulman-Albova 1952. Pacific Ocean. Mamaev et a 7 . (1963).

Steganoderma formosum Stafford 1904. Kamchatka, USSR. Intestine. Strelkov (1960).

\section{NEMATODA}

\section{ANISAKIDAE}

* Thynnascaris adunca (Rudolphi 1802) Hartwich 1957. Kamchatka, USSR. Intestine. Strelkov (1960) as Contracaecum a.

Phocanema sp. Larva. Kamchatka, USSR. Strelkov (1960) as Porrocaecum sp.

Anisakis sp. Larva. British Columbia. Muscles, mesentery. Margolis ${ }^{3}$; Kamchatka, USSR. Strelkov (1960).

\section{CAPILLARIIDAE}

Capillaria sp. British Columbia. Stomachs. Arai (1969b).

\section{CUCULLANIDAE}

Cucullanus heterochous Rudolphi 1802. Sea of Japan. Intestine. Zhukov (1960a).

Cucullanus sp. British Columbia. Intestine. Arai (1969b).

\section{CYSTIDICOLIDAE}

Ascarophis pacificus Zhukov 1960. Pacific Ocean. Mamaev et al. (1963). 
Lepidopsetta bilineata (Continued)

\section{PHILOMETRIDAE}

Philometra americana Kuitunen-Ekbaum 1933. British Columbia.

Tissues, body cavity. Kuitunen-Ekbaum (1933a).

PROTOZOA (CILIOPHORA)

URCEOLARI IDAE

Genera and species. British Columbia. Gills. Bell (1962).

Trichodina borealis Shulman and Shulman-Albova 1953. Sea of Japan. Lom and Laird (1969).

PROTOZOA (MYXOSPORIDA)

TETRACAPSULIDAE

Kudoa sp. British Columbia. Muscles. Hoskins et al.$^{13}$

$$
\text { Lyopsetta exilis - Slender Sole }
$$

\section{CESTODA}

\section{TENTACULARI IDAE}

Nybelinia surmenicola Okada 1929. British Columbia. Stomach wall. Margolis 3 .

COPEPODA

BOMOLOCHIDAE

Holobomolochus occultus Kabata 1971. British Columbia. Gills, gill cavity. Kabata (1971).

\section{DIGENEA}

\section{HEMIURIDAE}

Parahemiurus merus (Ljnton 1910) Woolcock 1935. British Columbia. Stomach. Margolis 3 .

Derogenes varicus (Müller 1784) Looss 1901. British Columbia.

$$
\text { Stomach. Margol is }{ }^{3} \text {. }
$$

MONOGENEA

\section{DICLIDOPHORIDAE}

Choricotyle exilis Crane 1972. Southern California. Mouth. Crane (1972). 
Lyopsetta exilis (Continued)

NEMATODA

\section{ANISAKIDAE}

Contracaecum sp. Larva. British Columbia. Stomachs, mesentery,

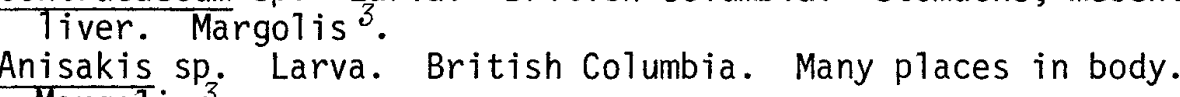
Margolis ${ }^{\circ}$.

PROTOZOA (SARCOMASTIGOPHORA)

CRYPTOBI IDAE

Cryptobia sp. Oregon. Blood. Burreson?.

\section{TRYPANOSOMATIDAE}

Trypanosoma pacifica Burreson and Pratt 1972. Oregon. B1ood. Burreson?

$$
\text { Microstomus pacificus - Dover Sole }
$$

CESTODA

PSEUDOPHYLLIDEA

Genera and species. Plerocercoid. British Columbia. Body cavity. Margol is 3 .

\section{TENTACULARIIDAE}

Nybelinia surmenicola Okada 1929. Post larvae. British Columbia. Stomach wa11. Margol is ${ }^{3}$.

\section{DIGENEA}

\section{FELLODISTOMATIDAE}

Fellodistomum brevum Ching 1960. Washington. Intestine. Ching (1960a).

\section{LEPOCREADI IDAE}

Lepidapedon calli Acena 1947. Washington. Acena (1947). OPECOELIDAE

Eurycreadium vitellosum Manter 1934. Washington. Intestine. Ching (1961). 
Microstomus pacificus (Continued)

ZOOGONIDAE

Zoogonoides viviparus (01sson 1868) 0dhner 1902. Washington. Intestine. Ching (1960a).

Zoogonus dextrocirrus Aldrich 1961. Washington. Intestine. Aldrich (1961).

NEMATODA

ANISAKIDAE

$\frac{\text { Anisakis }}{\text { Margolis }} \mathrm{sp}_{3}$. Larva. British Columbia. Muscles, mesentery, etc.

PROTOZOA (MYXOSPORIDA)

CERATOMYXIDAE

Ceratomyxa hopkinsi Jameson 1929. Central California. Gallbladder. Jameson (1929).

TETRACAPSULIDAE

Kudoa sp. Washington. Muscles. Patashnik and Groninger (1964). PROTOZOA (SARCOMASTIGOPHORA)

TRYPANOSO MATIDAE

Trypanosoma sp. Oregon. Blood. Burreson?

$$
\text { Parophrys vetulus - English Sole }
$$

ACANTHOCEPHALA

ECHINORHYNCHIDAE

Metechinorhynchus lageniformis (Ekbaum 1938) Petrotchenko 1956. Oregon. Intestine. 01son and Pratt (1973) as Echinorhynchus 1.

\section{CESTODA}

PHYLLOBOTHRI IDAE

Phyllobothrium sp. Larva. Oregon. Intestine. 01son (1978b).

\section{TENTACULARI IDAE}

Nybelinia surmenicola Okada 1929. Postlarva. British Columbia. Stomach wall. Margolis. 
Parophrys vetulus (Continued)

COPEPODA

CALIGIDAE

Lepeophtheirus bifidus Fraser 1920. British Columbia. Kábata (1973).

Lepeophtheirus hospitalis Fraser 1920. British Columbia. Kabata (1973).

CHONDRACANTHIDAE

Acanthochondria rectangularis (Fraser 1920) Markevich 1957.

British Columbia. Beres.

NAOBRANCHI IDAE

Naobranchia occidentalis Wilson 1915. British Columbia. Bere ${ }^{5}$. PENNELLIDAE

Phrixocephalus concinnatus Wilson 1908. Southern California. Eyes. SchuTtze 25 .

DIGENEA

AZYGIIDAE

Otodistomum veliporum (Crepl in 1837) Stafford 1904. Metacercaria. Oregon. OTson and Pratt (1973).

FELLODISTOMATIDAE

Fellodistomum phrissovum Aldrich 1961. Washington. Intestine. A]drich (1967).

HEMIURIDAE

Genolinea laticauda Manter 1925. Oregon. Stomach. 01son (1978b). TubuTovesicula lindbergi (Layman 1930) Yamaguti 1934. British Columbia. McFarlane (1936) as Dinurus nanaimoensis; Washington. Ching (1960a).

LEPOCREADI IDAE

Lepidapedon calli Acena 1947. Washington. Stomach, intestine. Acena (1947).

OPECOELIDAE

Podocotyle sp. Oregon. Intestine. 01 son (1978b).

Podocotyle sinusacca Ching 1960. British Columbia. Intestine. Arai (1969) as Neopodocotyloides s.

Genitocotyle acirra Park 1937. Oregon. Pratt (1970). 
Parophrys vetulus (Continued)

ZOOGONIDAE

Steganoderma formosum Stafford 1904. Washington. Ching (1960a).

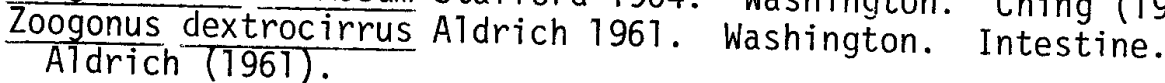

HIRUDINOIDEA

PISCICOLIDAE

Oceanobdella sp. Oregon. Exteriors. Burreson? .

ISOPODA

CYMOTHOIDAE

Lironeca vulgaris Stimpson 1857. Oregon. Gill cavity. 01son (1978b). MONOGENEA

GYRODACTYLIDAE

Gyrodactylus sp. Oregon. Fins. 01 son (1978b).

NEMATODA

ANISAKIDAE

* Thynnascaris sp. Oregon. Intestine. 01 son (1978b).

* Thynnascaris adunca (Rudolphi 1802) Hartwich 1957. British Columbia. Stomach, intestine. Margolis 3 as Contracaecum a. Contracaecum sp. Larva. British Columbia. Stomach, mesentery, Anisakis sp. Larva. British Columbia. Many places in body.

Margolis ${ }^{3}$; Oregon. 01 son (1978b).

CAPILLARI IDAE

$\frac{\text { Capillaria sp. British Columbia. Intestine. Margolis }}{3}$ as

\section{CUCULLANIDAE}

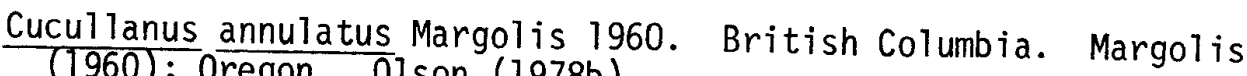
(1960); Oregon. 01 son (1978b).

\section{PHILOMETRIDAE}

Philometra americana Kuitunen-Ekbaum 1933. Oregon. Between fin rays, in muscles. 01 son and Pratt (1973); Northern California.
R. 0ison (1972). 
Parophrys vetulus (Continued)

PROTOZOA (CILIOPHORA)

URCEOLARI IDAE

Trichodina sp. Oregon. Gi11s. 01 son (1978b).

PROTOZOA (MICROSPORIDA)

NOSEMATI IDAE

Glugea stephani (Hagenmuller 1899) Woolcock 1904 . Oregon.

Stomach, intestine. Wellings et al. (1969) as $\underline{G}$. hertwigi.

PROTOZOA (MYXOSPORIDA)

CERATOMYXIDAE

Ceratomyxa hopkinsi Jameson 1929. Central California. Gallbladder. Jameson (1929).

TETRACAPSULIDAE

Kudoa sp. British Columbia. Muscles. Margolis ${ }^{3}$.

PROTOZOA (SARCOMASTIGOPHORA)

CRYPTOBI IDAE

Cryptobia sp. Oregon. Blood. Burreson?.

TRYPANOSOMAT IDAE

Trypanosoma pacifica Burreson and Pratt 1972. Oregon. Blood. Burreson and Pratt (1972).

$$
\begin{aligned}
& \text { Platichthys stellatus - Starry Flounder } \\
& \text { SYNONYM - Pleuronectes stellatus }
\end{aligned}
$$

ACANTHOCEPHALA

ECHINORHYNCHIDAE

Echinorhynchus gadi Zoega in Müller 1776. British Columbia. Intestine. Arai (1969b); Kamchatka, USSR. Skryabina (1963).

Metechinorhynchus lageniformis (Ekbaum 1938) Petrotchenko 1956. British Columbia. Intestine. Ekbaum (1938).

POLYMORPHIDAE

Corynosoma strumosum (Rudolphi 1802) Lühe 1904. Juvenile. British Columbia. Liver, mesentery, intestinal wa11. Ekbaum (1938); Sea of Japan. Zhukov (1960a); Washington. Van Cleave (1953). 
Platichthys stellatus (Continued)

CESTODA

\section{AMPHICOTYLIDAE}

Eubothrium crassum (B1och 1779) Nybe1 in 1922. Western Kamchatka, USSR. Intestine. Tsimbalyuk and Semeshko (1971).

\section{BOTHRIOCEPHALIDAE}

Bothriocephalus scorpi (Müller 1776) Rudolphi 1808. Western Kamchatka, USSR. Digestive tract. Tsimbalyuk and Semeshko (1971).

\section{DIPHYLLOBOTHRI IDAE}

Diphyl lobothrium sp. Larva. Western Kamchatka, USSR. Digestive tract. Tsimbalyuk and Semeshko (1971).

PHYLLOBOTHRI IDAE

Phyllobothrium sp. Larva. British Columbia. Intestine. Arai (1969b).

\section{TENTACULARI IDAE}

Nybel inia surmenicola Okada 1929. Larva. Kamchatka, USSR.

$$
\text { Strelkov (1960). }
$$

\section{TETRAPHYLLIDEA}

Scolex pleuronectis Müller 1788. Kamchatka, USSR. Intestine, gal1bladder. Skryabina (1963).

\section{COPEPODA}

\section{CALIGIDAE}

Lepeophtheirus parviventris Wi1son 1905. Alaska, Washington. Townsend (1938) as L. Septentriona 1 is: Kabata (1973). Lepeophtheirus hospitalis Fraser 1920. British Columbia. Fraser (1920); Oregon. Ventral surface. Millemann 34 .

Lepeophtheirus parvicruris Fraser 1920. British Columbia. Fraser (1920).

Pseudolepeophtheirus longicauda Markevich 1940. Kamchatka, USSR. Mouth. Bykhovskaya-Pavlovskaya et a1. (1962).

\section{CHONDRACANTHIDAE}

Acanthochondria rectangularis (Fraser 1920) Markevich 1957.

Oregon. Gill cavity. Millemann 34 ; British Columbia. Gilis. Fraser (1920) as Chondracanthus r. 
Platichthys stellatus (Continued)

\section{LERNAEOPODIDAE}

Nectobrachia indivisa Fraser 1920. British Columbia. Gills. Fraser (1920); USSR. Bykhovskaya-Pavlovskaya et al. (1962).

\section{NAOBRANCHI IDAE}

Naobranchia occidentalis Wilson 1915. Pacific coast, USA. Villadolid (1927).

\section{DIGENEA}

BUCEPHALIDAE

Prosorhynchoides basaringi (Layman 1930) Margol is and Arthur 1979. Sea of Japan. Intestine. Yamaguti (1971) as Bucephalops is $\underline{b}$. Prosorhynchoides gracilescens (Rudolphi 1819) n. comb.

Kamchatka, USSR. Intestine. Skryabina (1963) as Bucephalops is g.

FELLODISTOMATIDAE

Stenakron vetustum Stafford 1904. Kamchatka, USSR. Strelkov (1960). HEMIURIDAE

Lecithaster gibbosus (Rudolphi 1802) Lühe 1901. British Columbia. Intestine. Arai (1969b).

Derogenes varicus (Mül1er 1784) Looss 1901. Washington. Ching (1960a); Kamchatka, USSR. Stomach, intestine. Skryabina (1963). Sterrhurus exodicus (McFarlane 1936) Yamaguti 1958. Washington. Ching (1960a) as Lecithochirium e.

Parahemiurus merus (Linton 1910) Woolcock 1935. Washington. Stomach. Lloyd (1938) as P. platichthyi.

Tubulovesicula lindbergi (Layman 1930) Yamaguti 1934. Oregon. Stomach. McCauley (1960); Central California. Nahhas 10 ; Sea of Japan. Zhukov (1960a).

Genarchops is muelleri (Levinsen 1881) Yamaguti 1954. Kamchatka, USSR. Intestine. Strelkov (1960) as Genarches m.

\section{OPECOELIDAE}

Podocotyle atomon (Rudolphi 1802) Odhner 1905. British Columbia. Intestine. Arai (1969b); Sea of Japan. Zhukov (1960a). Decemtestis azumae (Layman 1930) Yamaguti 1934. Sea of Japan. Intestine. Zhukov (1960a) as Helicometrina a.

\section{ZOOGONIDAE}

Zoogonoides viviparus (01sson 1868) Odhner 1902. Sea of Japan. Intestine. Zhukov (1960a). 


\section{MONOGENEA}

\section{GYRODACTYLIDÂE}

Gyrodactylus ackerti Mizelle and Kritsky 1967. Northern California. Gills. Mizelle and Kritsky (1967d).

Gyrodactylus branchius Mizelle and Kritsky 1967. Northern California. Gills. Mizelle and Kritsky (1967d).

Gyrodactylus stellatus Crane and Mizelle 1967. Northern California, Pacific Ocean. Skins. Crane and Mizelle (1967).

Gyrodactylus gröenlandicus Levinsen 1881. Bering Sea. Gills. Zhukov (1960b).

Gyrodactylus robustus Malmberg 1956. Bering Sea. Zhukov (1960b).

Gyrodactylus unicopula Gluchowa 1955. Bering Sea. Gills. Zhukov (1960b).

\section{GYRODACTYLOIDEA}

Genera and species. British Columbia. Gills. Arai (1969b).

\section{NEMATODA}

\section{ANISAKIDAE}

* Thynnascaris adunca (Rudolphi 1802) Hartwich 1957. Kamchatka, USSR. Intestine. Skryabina (1963) as Contracaecum a. Contracaecum sp. Larva. British Columbia. Body cavity. Arai (1969b); Kamchatka, USSR. Intestine, body cavity, liver, kidneys. Skryabina (1963).

Anisakis sp. British Columbia. Intestine. Arai (1969b); Kamchatka, USSR. Body cavity, intestine, abdominal wall. Skryabina (1963).

Phocanema sp. Southern California. Dailey et al. (in press).

\section{CUCULLANIDAE}

Cucullanus annulatus Margol is 1960. British Columbia. Intestine. Arai (1969b).

Cucullanus heterochrous Rudolphi 1802. Kamchatka, USSR. Intestine, stomach. Skryabina (1963).

\section{PHILOMETRIDAE}

Philometra americana Kuitunen-Ekbaum 1933. British Columbia. Subcutaneous. Kuitunen-Ekbaum (1933a).

Philometra sp. British Columbia. Intestines. Arai (1969b) British Columbia. Between fin rays. Margolis ${ }^{3}$.

PROTOZOA (MICROSPORIDA)

\section{NOSEMATIDAE}

Glugea stephani (Hagenmuller 1894) Woolcock 1904. Northern California. Intestine. Jensen and Wellings (1972) - tentative identification. 
PROTOZOA (MYXOSPORIDA)

CERATTOMYXIDĀE

Ceratomyxa platichthytis (Fujita 1923) Dogiel 1948. Japan.

Gallbladder. Fujita (1923) as Leptotheca p.

MYXIDIIDAE

Myxidium oshoroense Fujita 1923. Japan. Gallbladder. Fujita (1923). TETRACAPSUL IDAE

Kudoa sp. Washington. Muscles. Patashnik and Groninger (1964).

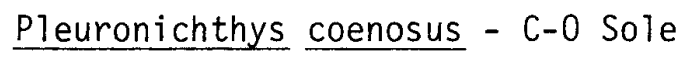

COPEPODA

BOMOLOCHIDAE

Holobomolochus prolixus (Cressey 1969) Kabata 1971. Southern California. Gil1 cavity. Cressey (1969b).

CALIGIDAE

Lepeophtheirus hospitalis Fraser 1920. British Columbia. Fraser (1920). CHONDRACANTHIDAE

Acanthochondria fraseri Ho 1972. Southern California. Gills. Ho $(7972 b)$.

Acanthochondria rectangularis (Fraser 1920) Markevich 1957.

British Columbia. Beres.

$$
\text { Pleuronichthys decurrens - Curlfin Sole }
$$

COPEPODA

PENNELLIDAE

Phrixocephalus cincinnatus Wilson 1908. Southern California. Eyes. Schuttze 25 .

\section{DIGENEA}

FELLODISTOMATIDAE

Fellodistomum furcigerum (01sson 1867) Yamaguti 1954. Washington. Intestine. Ching (1960a). 
Pleuronichthys decurrens (Continued)

HIRUDINOIDEA

PISCICOLIDAE

Australobde11a californiana Burreson 1977. Southern California. Body surface. Burreson (1977a).

MONOGENEA

UDONELLIDAE

Udonella cal igorum Johnston 1835. Northern California. Skin. Crane (1972).

Pleuronichthys vertical is - Hornyhead Turbot

HIRUDINEA

PISCICOLIDAE

Australobdella californiana Burreson 1977. Southern California. Body surface. Burreson (1977a).

PROTOZOA (MYXOSPORIDA)

SPHAEROSPORIDAE

Sphaerosora divergens Thélohan 1895. Southern California. Urinary biadder. Jameson (1931).

\section{Psettichthys melanostictus - Sand Sole}

\section{ACANTHOCEPHALA}

POL YMORPHIDAE

Corynosoma strumosum (Rudolphi 1802) Lühe 1904. Larva. British Columbia. Margolis (1956a).

COPEPODA

\section{CALIGIDAE}

Lepeophtheirus bifurcatus Wilson 1905. Northern California. Exterior. WiTson (1908a).

Lepeophtheirus sp. Oregon. Gills. Millemann?

\section{CHONDRACANTHIDAE}

Acanthochondria rectangularis (Fraser 1920) Markevich 1957. Oregon. Git7 cavity. MilTemann 34 . 
Psettichthys melanostictus (Continued)

\section{DIGENEA}

HEMIURIDAE

Tubulovesicula lindbergi (Layman 1930) Yamaguti 1934. Washington. Oregon. Stomach. McCauley (1960).

HIRUDINOIDEA

PISCICOLIDAE

Aestabde1la abditovesicula (Moore 1952) Burreson 1976. Oregon. Mouth. Burreson (7976a).

ISOPODA

CYMOTHOIDAE

Lironeca vulgaris Stimpson 1857. Northern California. Gill chambers. Crane (1972).

MONOGENEA

UDONELLIDAE

Udonella caligorum Johnston 1835. Northern California. Gill chambers. Crane (1972) on Lironeca vulgaris.

NEMATODA

ANISAKIDAE

Anisakis sp. Larva. British Columbia. Many places in body. Margolis ${ }^{3}$.

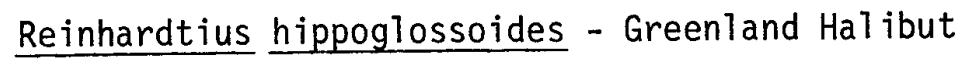

\section{ACANTHOCEPHALA}

ECHINORHYNCHIDAE

Echinorhynchus gadi Zoega in Mü7ler 1776. Pacific Ocean. Mamaev et al. $(1963)$.

POLYMORPHIDAE

Corynosoma strumosum (Rudolphi 1802) Llihe 1904. Pacific Ocean. Mamaev et al. (1963). Corynosoma reductum (Von Lindstow 1905) Railliet and Henry 1907. Larva. Bering Sea. Mamaev (1965). 
Reinhardtius hippoglossoides (Continued)

CESTODA

AMPHICOTYLIDĀE

Eubothrium sp. Pacific Ocean. Intestine. Mamaev et al. (1963).

BOTHRIOCEPHALIDAE

Bothriocephalus scorpii (Müller 1776) Rudolphi 1808. Pacific Ocean. Mamaev (1965).

LACISTORHYNCHIDAE

Grillotia erinacea (Van Beneden 1858) Guiart 1927. Larva. Bering Sea. Mamaev (1965).

PHYLLOBOTHRI IDAE

Phyllobothrium sp. Larva. Bering Sea. Mamaev (1965).

\section{PSEUDOPHYLLIDEA}

Genera and species. Larva. Pacific Ocean. Stomach, intestine. Mamaev et a1. (1963).

TENTACULARI IDAE

Nybelinia surmenicola Okada 1929. Pacific Ocean. Intestine. Mamaev et al. (1963).

\section{TETRAPHYLLIDEA}

Scolex polymorphus Rudolphi 1819. Barents Sea, USSR. Intestine. Polyanski (1955b).

Scolex pleuronectis Müller 1788. Pacific Ocean. Pyloric caeca, intestine. Mamaev et al. (1963).

\section{DIGENEA}

ACANTHOCOLPIDAE

Stephanostomum baccatum (Nicoll 1907) Manter 1934. Pacific Ocean. Intestine. Mamaev et al. (1963).

BUCEPHALIDAE

Prosorhynchoides gracilescens (Rudolphi 1819) n. comb. Pacific Ocean. Mamaev et a1. (1963) as Bucephalops is $g$.

FELLODISTOMIDAE

Fellodistomum furcigerum (01sson 1867) Yamaguti 1954. Pacific Ocean. Mamaev et al. (1963) as Steringophorus furciger.

Stenakron vetustum Stafford 1904. Pacific Ocean. Mamaev et a1. (1963). 
Reinhardtius hippoglossoides (Continued)

HEMIURIDAE

Derogenes varicus (Müijer 1784) Looss 1901. Barents Sea, USSR. Stomach. Polyanski (1955b).

Genarchopsis muel1eri (Levinsen 1881) Yamaguti 1954. Barents Sea, USSR. Stomach. Polyanski (1955b) as Genarches m.

Hemiurus levinseni Odhner 1905. Pacific Ocean. Mamaev et al. (1963).

Lecithaster gibbosus (Rudolphi 1802) Lühe 1901. Pacific Ocean. Mamaev et a1. (1963).

Brachypha77us crenatus (Rudolphi 1802) Odhner 1905. Pacific Ocean. Mamaev et aT. (1963).

Lecithophy17um sphaerolecithum (Manter 1925) Odhner 1927. Bering Sea. Mamaev (1965).

OPECOELIDAE

Podocotyle atomon (Rudolphi 1802) Odhner 1905. Pacific Ocean. Mamaev et al. (1963).

ZOOGONIDAE

Steganoderma formosum Stafford 1904. Pacific Ocean. Mamaev et al. (1963).

MONOGENEA

DICLIDOPHORIDAE

Heterobothrium affinis (Linton 1898) Price 1936. Bering Sea. Mamaev (1965).

NEMATODA

ANISAKIDAE

* Thynnascaris adunca (Rudolphi 1802) Hartwich 1957. Adult, larva. Barents Sea, USSR. Intestine, body cavity, mesentery. Polyanski (1955b) as Contracaecum a.

Anisakis sp. Larva. Barents Sea, USSR. Liver, intestine. Polyanski (1955b).

Phocanema sp. Larva. Pacific Ocean. Mamaev et a1. (1963) as Porrocaecum sp.

PROTOZOA (MYXOSPORIDA)

CERATOMYXIDAE

Ceratomyxa drepanosettae Awerinzew 1907. Barents Sea, USSR. Gallbladder. Polyanski (1955b). 
BALISTIDAE -- Triggerfishes and Filefishes

Balistes polylepis - Finescale Triggerfish

SYNONYM - Verruncuius poiyiepis

COPEPODA

CALIGIDAE

Caligus productus Dana 1853. Gulf of California. Causey (1960).

DIGENEA

ACANTHOCOLPIDAE

Stephanostomum provitellosum Sogandares-Bernal 1959. Galapagos Islands. Sogandares-Bernal (1959); Baja California. Intestine. Arai (1962).

ALLOCREADI IDAE

Pseudocreadium galapagoense Manter 1946. Baja California.

Intestine. Arai (1962) as P. Scaphosomum: Panama.

Caballero et al. (1953) as P. Scaphosomum.

HOMALOMETRIDAE

Homa lometron caballeroi Lamothe-Argumedo 1965. Gulf of California. Yamaguti (1971).

LEPOCREAD I IDAE

Lepocreadium bravoae Lamothe-Argumedo 1965. Gulf of California. Intestine. Lamothe-Argumedo (1965).

MONORCHI IDAE

Lasiotocus longicaecum (Manter 1940) Yamaguti 1954. Baja California. Intestine. Arai (1962).

MONOGENEA

CALCEOSTOMATIDAE

Paracalceostoma calceostomoides Caballero and Bravo-Holl is 1960. Baja California. Gills. Caballero and Bravo-Holl is (1962). DISCOCOTYLIDAE

Pseudomazocraes monsivaisae Caballero and Bravo-Holl is 1955. Mexico. Gills. Caballero and Bravo-Holl is (1955). 


$$
\begin{gathered}
\text { OSTRACIIDAE -.- Boxfishes } \\
\text { Ostracion diaphanum - Spiny Boxfish }
\end{gathered}
$$

\section{DIGENEA}

FELLODISTOMATIDAE

Discogasteroides ostracij (Yamaguti 1934) Strand 1935. Japan. Intestine. Yamaguti (1934a) as Discogaster.

Discogasteroides minor (Yamaguti 1934) Strand 1935 . Japan.

Yamaguti (1971).

$$
\begin{aligned}
& \text { TETRAODONTIDAE -- Puffers } \\
& \text { Sphoeroides sp. }
\end{aligned}
$$

COPEPODA

CHONDRACANTHIDAE

Pseudochondracanthus diceraus Wilson 1908. Southern California.

Gi17s and gill cavity. Wilson (1908a).

$$
\text { Sphoeroides annulatus - Bu11seye Puffer }
$$

SYNONYM - Cheilichthys annulatus

COPEPODA

\section{BOMOLOCHIDAE}

Holobomolochus attenuatus (Wilson 1913) Vervoort 1969. Ecuador. Gills. Wilson (1937a) as Bomolochus a.

\section{CALIGIDAE}

Caligus elongatus Nordmann 1832. West coast of Mexico. Causey (1960) as C. Tatifrons.

Lepeophtheirus dissimulatus Wilson 1905. Eastern Pacific. Lewis (1967).

\section{DIGENEA}

\section{ACCACOELIDAE}

Tetrochetus proctocolus Manter 1940. Galapagos Islands. Rectum. Manter (1940a).

\section{APOCREADIIDAE}

Apocreadium longisinosum Manter 1937. Panama. Intestine. Caballero et a1. (1952); Galapagos Islands. Rectum. Manter (1940a). 
Sphoeroides annulatus (Continued)

FELLODISTOMATIDAE

Lintonium srivastavi Lamothe-Argumedo 1969. West coast of Mexico. Intestine. Lamothe-Argumedo (1969a).

GORGODERIDAE

Phyllodistomum mirandai Lamothe-Argumedo 1969. West coast of Mexico. Urinary bladder. Lamothe-Argumedo (1969a).

\section{LEPOCREADI IDAE}

Bianium plicitum (Linton 1928) Stunkard 1930. Baja California. Arai (1962) as Diploproctodaeum p.; Galapagos Islands and Ecuador. Intestine. Manter (1940a).

\section{SANGUINICOLIDAE}

Cardicola tropicus (Manter 1940) Short 1953. Ecuador. Coelom, intestine. Manter (1940a) as Psettarium t.

\section{ISOPODA}

\section{CYMOTHOIDAE}

Nerocila californica Schioedte and Meinert 1881. Gulf of California. Gi17s. Brusca (1977).

\section{MONOGENEA}

CAPSALIDAE

Benedenia melleni (MacCullum 1927) Johnston 1929. New York Aquarium. Eyes. Yamaguti (1963b) as Neobenedenia $\mathrm{m}$.

\section{DISCOCOTYLIDAE}

Tagia ecuadori (Meserve 1938) Sproston 1946. Panama. Gills. Caballero, Bravo-Hollis and Grocott (1953); Galapagos Islands. Gills. Meserve (1938) as Heterobothrium e.; Mexico. Lamothe-Argumedo (1967b).

$$
\begin{gathered}
\text { DIODONTIDAE -- Porcupinefishes } \\
\text { Chilomycterus affinis - Pacific Burrfish }
\end{gathered}
$$

\section{DIGENEA}

OPISTHOLEBETIDAE

Opistholebes colylophorus Ozaki 1935. Japan. Intestine. Ozaki (1935). 
Heterolebes maculosus Ozaki 1935. Hawaii. Intestine. Ozaki (1935).

Maculifer chilomycteri Yamaguti 1959. Japan. Digestive tract. Yamaguti (1959a).

Pseudoheterolebes chilomycteri Yamaguti 1959. Japan. Intestine. Yamaguti (1959a).

\section{Diodon hystrix - Spotted Porcupinefish}

COPEPODA

NAOBRANCHIIDAE

Naobranchia wilsoni Nigrelli 1933. New York Aquarium. Gills. Nigre11i (1935).

TUCCIDAE

Tucca impressus Krøyer 1837. West Indies. Exterior. Wilson $(1932)$.

CRUSTACEA

LEPADIDAE

Conchoderma virgatum. India. External surface. Balakrishnan (1969).

\section{DIGENEA}

ACCOCOELIIDAE

Tetrochetus coryphaenae Yamaguti 1934. Jamaica. Intestine. Nahhas and Cable (1964).

FELLODISTOMATIDAE

Megalomyzon robustus Manter 1947. Puerto Rico. Stomach. Siddigi and Cable (1960).

\section{LEPOCREADI IDAE}

Bianium plicitum (Linton 1928) Stunkard 1930. Gulf of Panama. Intestine. Sogandares-Bernal (1959) as Diploprotodaeum.

Pseudocreadium diodontis (Nahhas and Cable 1964) Yamaguti 1971. Curacao and Jamaica. Intestine. Nahhas and Cable (1964) as Diploproctodaeum $\underline{\mathrm{d}}$.

\section{MICROPHALLIDAE}

Pseudomegalophallus diodontis (Siddigi and Cable 1960) Yamaguti 1971. Puerto Rico. Intestine. Siddigi and Cable (1960) as Mega lophal1us d. 
Diodon hystrix (Continued)

\title{
OPISTHOLEBETIDAE
}

Opistholebes diodontis Cable 1956. Puerto Rico. Intestine. Cable (1956); Hawaij. Intestine. Manter and Pritchard (1962). Opistholebes cotylophorus 0zaki 1935. Hawaii. Intestine. Manter and Pritchard (7962).

Heterolebes maculosus Ozaki 1935. Hawaii. Intestine. Manter and Pritchard (1962).

\section{SCLERODISTOMATIDAE}

Sclerodistomum bravoae Pritchard 1963. Hawaii. Pritchard (1963). ScTerodistomum diodontis Yamaguti 1942. Curacao. Stomach. Nahhas and Cable (1964).

Sclerodistomum sphoeroides Manter 1947. Curacao, Jamaica. Stomach. Nahhas and Cable (1964).

NEMATODA

\section{ANISAKIDAE}

Raphidascaroides diadonis (Thwaite 1927) 01 sen 1952. South India. Bayl is (1936) as Raphidascaris $\underline{d}$.

PROTOZOA (CILIOPHORA)

OPHRYOGLENIDAE

Cryptocaryon irritans Brown 1951. New York Aquarium. Body surface. Nigrelli and Ruggieri (1966).

\author{
MOL IDAE - - Molas \\ Mola mola - Ocean Sunfish \\ SYNONYM - Orthagoriscus mola
}

\section{ACANTHOCEPHALA}

ECHINORHYNCHIDAE

Echinorhynchus gadi Zoega in Mü1ler 1776. Massachusetts. Do11fus (1946) as E. acus.

\section{BRANCHIURA}

ARGULIDAE

Ârgui is scutiformis Thiele 1900. Japan. Yamaguti (1963a); France. Skin. Dollfus (1946). 
Mola mola (Continued)

CESTODA

BOTHRIOCEPHALIDAE

Bothriocephalus monorchis Linstow 1903. France. Intestine. Do17fus (1946).

\section{DASYRHYNCHIDAE}

Floriceps saccatus Cuvier 1817. France. Many places in body. Dol1fus (1946).

\section{GYMNORHYNCHIDAE}

Molicola horridus (Goodsir 1841). France. Dolifus (1946) ; New ZeaTand. Robinson (1959a).

\section{TRIAENOPHORIDAE}

Ancistrocephalus microcephalus (Rudolphi 1819) Monticelli 1890. Newfoundland. Intestine. Threlfall (1967); New Zealand. Robinson (1959a); France. Dollfus (1946).

Fisticula plicatus (Rudolphi 1819) Lühe 1899. Massachusetts. Linton (1941).

\section{TRYPANORHYNCHA}

Rhynchobothrium sp. Larva. Newfoundland. Intestine wall. Threlfa11 (1967).

Tetrarhynchus elongatus Wagener 1901. Larva. Newfoundland. Liver. Thre 1 fal1 (1967); Massachusetts. Linton (1924). [Species incertae sedis- Yamaguti (1959b)] [ = Molicola horridus Dollfus (1942)].

\section{COPEPODA}

\section{CALIGIDAE}

Caligus elongatus Nordmann 1832. Mediterranean, North Sea. Parker (1969).

Lepeophtheirus nordmanni (Edwards 1840) Baird 1850. France. Skin. Do11fus (1946); New Zealand. Hewitt (1964b) as $\underline{L}$. insignis; Southern California. Wilson (1908a); New Foundland. ThrelfaT1 (1967); Japan. Shiino (1957).

Lepeophtheirus hastatus Shiino 1960. Japan. Body surface. Shiino (1960a); New Zealand. Hewitt (1964b) as L. molae; Australia. Heegaard (1962) as L. molae.

\section{CECROPIDAE}

Cecrops exiguus Wilson 1923, Japan. Bucca1 cavity, gills. Shiino (1965a).

Cecrops latreil1 i Leach 1816. Newfoundland. Gills.

ThrelfalT (1967); Massachusetts. Wilson (1932); France.

Dollfus (1946); New Zealand. Hewitt (1968a). 
Mola mola (Continued)

Orthagoriscola muricatus (Krøyer 1837) Poche 1902. Massachusetts. Wilson (1907b); South Africa. Barnard (1955); France. Skin, gi115. Dōl1fus (1946).

Philorthagoriscus serratus (Krøyer 1863) Horst 1897 . Newfoundland. Body surface. Threlfal1 (1967); Netherlands, Massachusetts, English seas. Wilson (1932); South Africa. Barnard (1955); Japan. Shiino (1959C).

\section{DICHELESTHI IDAE}

Anthosoma crassum (Abildgaard 1794) Gould 1841. France. Inner face of opercuTums, fins. Dollfus (1946).

\section{LERNAEOPODIDAE}

Lernaeopodoa bidiscalis Kane 1892. France. Skin. Dollfus (1946).

\section{PANDARIDAE}

Pandarus bicolor Leach 1816. France. Skin. Dollfus (1946). Echthrogaleus coleoptratus (Guerin 1817) Wilson 1907. France. Skin. Dol1fus (1946).

\section{PENNELLIDAE}

Pennella crassicornis Steenstrup and Lütken 1861. Yamaguti (1963a). Pennelia filosa (Linnaeus 1758). France. Doiifus (1946) as P. rubra; Massachusetts. Flesh. Wilson (1932); South Africa. Barnard (1955); Pacific. Wilson (1932) as $\underline{P}$. orthagorisci.

TREBI IDAE

Trebius sp. France. Skin, gills. Dollfus (1946).

CRUSTACEA

LEPADIDAE

Conchoderma virgatum. Body surface. Balakrishnan (1969).

\section{DIGENEA}

ACANTHOCOLPIDAE

Stephanostomum baccatum (Nicoll 1907) Manter 1934. Immature. Massachusetts. Linton (1940) as $\underline{\mathrm{S}}$. valde-inflatum. 
Mola mola (Continued)

\section{ACCACOELIIDAE}

Âccacladocoelium macrocotyle (Diesing 1858) Odhner 1928. Oregon. Pratt and McCauTey (1961); Massachusetts. Linton (1940); Canada, Naples, Ireland. Intestine. Dawes (1947).

Accacladocoelium nigroflavum (Rudolphi 1819) Odhner 1928.

Europes, Mediterranean. Intestine. Dawes (1947).

Accacladocoelium petasiporum Odhner 1928. Mediterranean. Intestine. Dawes (1947); France. Do17fus (1946).

Accacladocoelium alveolatum Robinson 1934. Atlantic. Intestine. Robinson (1934); New Zealand. Manter (1960).

Accacladocoelium serpentulum Odhner 1928. Japan. Intestine. Dawes (1947); Massachusetts. Linton (1940); France. Dollfus (1946); Central California. Noble and Noble (1937) as Accacladium nematuium.

Accacoelium contortum (Rudolphi 1819) Looss 1899. Newfoundland, Massachusetts. Pharynx. Threlfal1 (1967); Gills. Linton (1940); France. Timon-David and Musso (1971).

Odhnerium calyptrocotyle (Montice11i 1893) Yamaguti 1934. British Columbia. Intestine. Lloyd (1938); Southern California. Montgomery (1957): Japan. Yamaguti (1934b); New Zealand. Manter (1954a); Massachusetts. Linton (1940) as Orophocotyle foliata.

Orophocotyle planci (Stassich 1899) Looss 1902. Trieste. Dawes (1947).

Rhyncopharynx paradoxa Odhner 1928. Japan. Intestine. Yamaguti (1934a); France. Dollfus (1935b); New Zealand. Manter (1960).

\section{DIDYMOZOIDAE}

Didymozoon molae (Rudolphi 1819) Dollfus 1946. Dorsal muscles. Dol7fus (1946).

Nematobothrium molae MacLaren 1903. Mediterranean. Gi1ls. Dawes (1947).

Koellikeria filicollis (Rudolphi 1819) Cobbold 1860. Intestine. Nicol1 (1975).

Nematobibothrioides histoidi Noble 1974. California. Connective tissue. Noble (1975).

\section{LEPOCREADIIDAE}

Dihemistephanus lydiae (Stossich 1896) Looss 1901. France. Digestive tract. Dollfus (1946); New Zealand. Manter (1960).

Dihemistephanus fragilis (Linton 1900) Stafford 1904. Massachusetts. Digestive tract. Stafford (1904); Newfoundland. Threlfall

(1967) as Distomum fragile.

ISOPODA

\section{CYMOTHOIDAE}

Nerocila orbignyi. France. Skin. Dollfus (1946). Nerocila macleayi White 1843. France. Skin. Dollfus (1946). 
Mola Mola (Continued)

MONOGENEA

CAPSALIDAE

Capsala martinieri Bosc 1811. Newfoundland. Body surface.

Threlfal1 (1967); British Columbia. Gills, body surface. Margolis ${ }^{3}$; Norway. Brinkmann (1952).

Capsala molae (Blanchard 1847) Johnston 1929. Massachusetts. Linton (1940); New Jersey. Skin. Price (1962a) as Tricotyla m.

Capsala cephala (Risso 1826) St. Remy 1898. Europe. Yamaguti $(1963 b)$.

Capsala cutanea (Guiart 1938) Price 1939. Dawes (1947). Capsa1a grimaTdi (Guiart 1938) Price 1939. Dawes (1947). Capsa1a pelamydis (Taschenberg 1878) Price 1939. Dawes (1947). Capsala maculata (Martiniere 1787). France. Skin. Dollfus (1946). Tristoma papillosum Diesing 1836. France. Skin. Dollfus (1946).

NEMATODA

ANISAKIDAE

Anisakis sp. Larva. New Zealand. Viscera, mesentery, under peritoneum. Hewitt and Hine (1972).

CUCULLANIDAE

Cucullanus orthagorisci (Rudolphi 1819). France. Dollfus (1946) as Ascaris 0.

PROTOZOA (SARCOMASTIGOPHORA)

ENTAMOEB IDAE

Entamoeba molae Noble and Noble 1966. Southern California. Hindgut. Noble and Noble (1966).

MONOCERCOMONIDAE

Monocercomonas molae Noble and Noble 1966. Southern California. Hindgut. Noble and Noble (1966).

\author{
Ranzania laevis - Slender Mola \\ SYNONYM - R. truncata
}

DIGENEA

ACCACOELIIDAE

Orophocotyle planci (Stossich 1899) Looss 1902. Trieste. Yamaguti (1971).

Orophocotyle divergens Looss 1902. Trieste. Looss (1902). 


\section{ACANTHOCEPHALA}

Genera and species - Argyropelecus pacificus, Stenobrachius leucopsarus DIPLOSENT IDAE

Pararhadinorhynchus mugilis - Mugil cephalus ECHINORHYNCHIDAE

Acanthocephalus anguillae - Salmo gairdneri Acanthocephalus echigoensis - Oncorhynchus keta, $\underline{0}$. nerka, Sa]mo gairdneri

Acanthocephalus jacksoni - Oncorhynchus nerka, Salmo gairdneri Acanthocephalus lucii - Clupea harengus, Gasterosteus aculeatus Acanthocephalus minor - Gasterosteus aculeatus, Salmo gairdneri, Salvelinus malma

Acanthocephalus opsariichthys - Salmo gairdneri Acanthocephalus parksidei - Salmo gairdneri

Echinorhynchus sp. - Sebastes eos, S. levis, $\underline{S}$. paucispinis

Echinorhynchus canyonensis - Maynea californica

Echinorhynchus gadi - Agonus acipenserinus, Ammodytes hexapterus, Anoplopoma fimbria, Aprodon cortezianus, Atheresthes stomias, Blepsias cirrhosus, Clupea harengus, Cololabis saira, Cymatogaster aggregata, Eopsetta jordani, Gadus macrocephalus, Gasterosteus aculeatus, Hemilepidotus hemilepidotus, Hexagrammos lagocephalus, H. stelleri, Hippoglossoides elassodon, Hippoglossus stenolepis, Icelinus filamentosus, Lepidopsetta bilineata, Leptocottus armatus, Microgadus proximus, Mola mola, Morone saxatilis, Myoxocephalus polyacanthocephalus, Oncorhynchus gorbuscha, $\underline{0}$. keta, $\underline{0}$. kisutch, $\underline{0}$. nerka, $\underline{0}$. tshawytscha, Platichthys stellatus, Pleurogrammus monopterygius, Reinhardtius hippoglossoides, Salvelinus malma, Sebastes aleutianus, $\underline{\mathrm{S}}$. alutus, $\underline{\mathrm{S}}$. babcocki,$\underline{\mathrm{S}}$. borealis, $\underline{\mathrm{S}}$. caurinus, $\underline{\text { S. diploproa, }} \underline{\mathrm{S}}$. flavidus, $\underline{\mathrm{S}}$. helvomaculatus, $\underline{\mathrm{S}}$. proriger, $\underline{\mathrm{S}}$. ruberrimus, $\underline{\text { S. zacentrus, Squalus acanthias, Theragra chalcogramma }}$ Metechinorhynchus lageniformis - Lepidopsetta bilineata, Parophrys vetulus, Platichthys stellatus 
ACANTHOCEPHALA (continued)

ECHINORHYNCHIDAE (continued)

Metechinorhynchus lateralis - Gasterosteus aculeatus, Oncorhynchus

kisutch, Salmo clarki, s. gairdneri

Metechinorhynchus leidyi - Microgadus proximus, Salmo gairdneri

Metechinorhynchus salmonis - Clupea harengus, Gasterosteus aculeatus, Oncorhynchus kisutch, $\underline{0}$. nerka, $\underline{0}$. tshawytscha, Salmo gairdneri

Metechinorhynchus truttae - Salmo gairdneri

Neorhadinorhynchus nudus - Auxis thazard, Euthynnus affinis, Thunnus thynnus

Pseudoechinorhynchus clavula - Clupea harengus, Gasterosteus aculeatus, Salmo gairdneri

Pseudorhadinorhynchus samegaiensis - Salmo gairdneri

GORGORHYNCHIDAE

Gorgorhynchus sp. - Thunnus alalunga

LEPTORHYNCHOIDIDAE

Leptorhynchoides thecatus - Morone saxatilis, Oncorhynchus nerka, Salmo gairdneri

NEOECHINORHYNCHIDAE

Floridosentis elongatus - Mugil cephalus

Gracilisentis gracilisentis - Dorosoma petenense

Microsentis wardae - Gillichthys mirabilis

Neoechinorhynchus agilis - Mugil cephalus

Neoechinorhynchus chilkaense - Mugil cephalus

Neoechinorhynchus crassus - Salmo clarki

Neoechinorhynchus cristatus - Gasterosteus aculeatus, Oncorhynchus kisutch, 0. tshawytscha

Neoechinorhynchus pungitius - Oncorhynchus nerka

Neoechinorhynchus rutili - Gasterosteus aculeatus, Oncorhynchus keta,

0. kisutch, $\underline{0}$. nerka, Salmo clarki, S. gairdneri, Salvelinus malma

Neoechinorhynchus tumidus - Oncorhynchus nerka

Tanaorhamphus longirostris - Dorosoma petenense 
POLYMORPHIDAE

Arhythmorhynchus macracanthus - Umbrina roncador Bolbosoma sp. - Scomber japonicus

Bolbosoma caenoforme - Cololabis saira, Oncorhynchus gorbuscha, $\underline{0}$. keta,

$\underline{0}$. kisutch, $\underline{0}$. nerka, $\underline{0}$. tshawytscha, Salvelinus malma

Bolbosoma thunni - Thunnus thynnus

Bolbosoma nipponicum - Theragra chalcogramma

Bolbosoma vasculosum - Thunnus alalunga, I. albacares

Corynosoma sp. - Anoplopoma fimbria, Cymatogaster aggregata,

Hemi lepidotus hemi lepidotus, Hexagrammos lagocephalus, Leptocottus

armatus, Myoxocephalus polyacanthocephalus, Oncorhynchus kisutch,

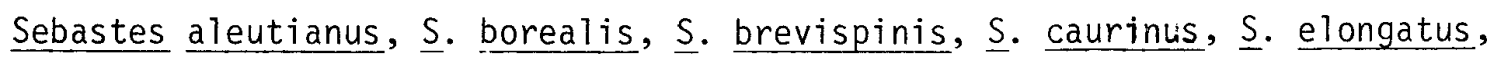

$\underline{\mathrm{S}}$. helvomaculatus, $\underline{\mathrm{S}}$. maliger, $\underline{\mathrm{S}}$. paucispinis, $\underline{\mathrm{S}}$. ruberrimus, $\underline{\mathrm{S}}$.

zacentrus

Corynosoma obtuscens - Umbrina roncador

Corynosoma osmeri - Umbrina roncador

Corynosoma reductum - Annoplopoma fimbria, Atheresthes stomias,

Hippoglossoides elassodon, Hippoglossus stenolepis, Reinhardtius

$\underline{\text { hippoglossoides, }}$ Sebastes aleutianus, $\underline{\text { S. alutus, }} \underline{\text { S. borealis }}$

Corynosoma semerme - Clupea harengus, Gasterosteus aculeatus,

Oncorhynchus nerka

Corynosoma strumosum - Atheresthes stomias, Clupea harengus, Eopsetta jordani, Gadus macrocephalus, Gasterosteus aculeatus, Hexagrammos lagocephalus, $\underline{\text { H. stelleri, Hippoglossoides elassodon, Hippoglossus }}$ stenolepis, Lepidopsetta bilineata, Leptocottus armatus, Myoxocephalus polyacanthocephalus, Oncorhynchus gorbuscha, $\underline{0}$ nerka, $\underline{0}$. tshawytscha, Platichthys stellatus, Pleurogrammus monopterygius, Psettichthys melanostictus, Reinhardtius hippoglossoides, Salvelinus malma, Theragra chalcogramma 
POLYMORPHIDAE (continued)

Corynosoma villosum - Oncorhynchus gorbuscha, $\underline{0}$. keta , Sebastes alutus Corynosoma wegeneri - Oncorhynchus nerka

POMPHORHYNCHIDAE

Pomphorynchus bulbocolli - Oncorhynchus kisutch, $\underline{0}$. nerka, Salmo clarki, S. gairdneri

Pomphorhynchus kostylewi - Clupea harengus

Pomphorhynchus laevis - Clupea harengus, Gasterosteus aculeatus, Morone saxatilis, Salmo gairdneri

Pomphorhynchus rocci - Morone saxatilis

RHADINORHYNCHIDAE

Gorgorhynchoides sp. - Caranx hippos

Gorgorhynchus medius - Echeneis naucrates

Gorgorhynchus robertdollfusi - Gempylus serpens

Illiosentis africanus - Albula vulpes

Il1iosentis cetratus - Menticirrhus undulatus, Roncador stearnsi, Umbrina roncador

Raorhynchus meyeri - Euthynnus pelamis

Raorhynchus terebra - Euthynnus pelamis

Rhadinorhynchus sp. - Salmo gairdneri, Trachurus symmetricus

Rhadinorhynchus cadenati - Scomber japonicus, Thunnus albacares

Rhadinorhynchus cololabis - Cololabis saira

Rhadinorhynchus japonicus - Scomber japonicus

Rhadinorhynchus lintoni - Scomber japonicus

Rhadinorhynchus pristis - Coryphaena hippurus, Euthynnus pelamis, Istiophorus platypterus, Scomber japonicus, Thunnus alalunga, I. albacares

Rhadinorhynchus selkirki - Auxis thazard, Cololabis saira

Rhadinorhynchus seriolae - Scomber japonicus

Rhadinorhynchus tenuicornis - Echeneis naucrates

Rhadinorhynchus trachuri - Cololabis saira, Oncorhynchus gorbuscha, o. keta, $\underline{0}$. kisutch, $\underline{0}$. nerka 
ACANTHOCEPHALA (continued)

RHADINORHYNCHIDAE (continued)

Serrasentis lamelliger - Naucrates ductor

Serrasentis sagittifer - Clupea harengus, Coryphaena hippurus, Echeneis naucrates, Euthynnus affinis

Telosentis exiquus - Gasterosteus aculeatus 
ARACHNOIDEA

Hydrachna sp. - Oncorhynchus nerka 
ASPIDOCOTYLEA

ASPIDOGASTERIDAE

Lobatostoma albulae - Albula vulpes

Multicalyx cristata - Cephaloscyllum ventriosum

Taeniocotyle elegans - Hydrolagus colliei

RUGOGASTRIDAE

Rugogaster hydrolagi - Hydrolagus colliei 
ARGUL IDAE

Argulus sp. - Oncorhynchus tshawytscha

Arguius alosae - Clupea harengus, Gasterosteus aculeatus

Argulus bicolor - Morone saxatilis

ArguTus boreal is - Hippoglossoides elassodon, Lepidopsetta bilineata,

Sebastes caurinus, Synodus lucioceps

Argulus canadensis - Alosa sapidissima, Gasterosteus aculeatus

Argulus chesapeakensis - Mugil cephalus

Arguius coregoni - Oncorhynchus gorbuscha, $\underline{0}$. keta

Argulus foliaceus - Gasterosteus aculeatus, Salmo gairdneri

Argulus funduli - Mugil cephalus

Argulus japonicus - Gasterosteus aculeatus

Argulus melanostictus - Leuresthes tenuis

Argulus pugettensis - Cymatogaster aggregata, Damalichthys vacca,

Embiotoca lateralis, Hyperprosopon argenteum, Oncorhynchus kisutch,

Phanerodon furcatus, Salmo gairdneri, Sebastes caurinus

Argulus scutiformis - Mola mola

Argulus varians - Echeneis naucrates 
Genera and species - Tarletonbeania crenularis

AMPHICOTYLIDAE

Abothrium gadi - Gadus macrocephalus, Theragra chal cogramma

Eubothrium sp. - Cymatogaster aggregata, Gasterosteus aculeatus,

Hippoglossoides elassodon, Lepidopsetta bilineata, Oncorhynchus

nerka, ‥ tshawytscha, Reinhardtius hippoglossoides, Salmo gairdneri

Eubothrium crassum - Clupea harengus, Gasterosteus aculeatus,

Oncorhynchus gorbuscha, $\underline{0}$. keta,$\underline{0}$. kisutch, $\underline{0}$. nerka, $\underline{0}$. tshawytscha, Platichthys stellatus, Salmo gairdneri, Salvelinus malma

Eubothrium salvelini - Oncorhynchus kisutch, $\underline{0}$. nerka,

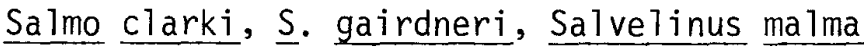

Pseudeubothrioides lepidocybii - Lepidocybium flavobrunneum

Pseudeubothrium xiphiados - Xiphias gladius

AMPHIL INIDAE

Amphilina bipunctata - Acipenser sp., A. transmontanus

Amphilina japonica - Acipenser medirostris

BALANOBOTHRI IDAE

Balanobothrium parvum - Galeocerdo cuvieri

BOTHRIOCEPHALIDAE

Anatrum histocephalum - Synodus lucioceps

Bothriocephalus sp. - Aulorhynchus flavidus, Gadus macrocephalus, Gasterosteus aculeatus, Hippoglossoides elassodon, Lepidopsetta bilineata, Merluccius productus, Oncorhynchus kisutch, $\underline{0}$. tshawytscha, Phol is laeta, Remora remora, Scomber japonicus, Sebastes alutus, s. babcocki, $\underline{S}$. borealis, $\underline{S}$. caurinus, $\underline{s}$. paucispinis, $\underline{S}$. pinniger, $\underline{S}$. proriger, $\underline{\mathrm{S}}$. reedi, $\underline{\mathrm{S}}$. ruberrimus, $\underline{\mathrm{S}}$. zacentrus

Bothriocephalus? claviceps - Gasterosteus aculeatus 
BOTHRIOCEPHALIDAE (continued)

Bothriocephalus manubriformis - Istiophorus platypterus, Tetrapturus angustirostris, I. audax, Xiphias gladius

Bothriocephalus monorchis - Mola mola

Bothriocephalus occidentalis - Leptocottus armatus, Sebastes sp.

Bothriocephalus ospariichthydis - Merluccius productus

Bothriocephalus scorpii-Apodichthys flavidus, Aprodon cortezianus,

Artedius harringtoni, Blepsias cirrhosus, Clupea harengus,

Delolepis giganteA, Enophrys bison, Gasterosteus aculeatus,

Hexagrammos decagrammus, $\underline{H}$ lagocephalus, $\underline{H}$. stelleri, Leptocottus

armatus, Myoxocephalus polyacanthocephalus, Nautichthys

oculofasciatus, 0ligoplites saurus, Platichthys stellatus,

Reinhardtius hippoglossoides, Sebastes alutus, $\underline{\text { S. borealis, }}$

$\underline{S}$. chlorostictus, $\underline{S}$. miniatus, Theragra chalcogramma

Parabothriocephalus sagitticeps - Sebastes paucispinis

\section{CATHETOCEPHALIDAE}

Cathetocephalus thatcheri - Carcharhinus leucas

CYATHOPHYLIDAE

Bothrimonus sturionis - Oncorhynchus gorbuscha, $\underline{0}$. nerka, Salvelinus malma

Cyathocephalus sp. - Salmo clarki

Cyathocephalus truncatus - Gasterosteus aculeatus, Oncorhynchus

kisutch, $\underline{0}$. nerka a, Salmo gairdneri

DASYRHYNCHIDAE

Russia - Russia $\frac{\text { Fance? }}{\text { Frand }}$

Genera and species - Thunnus al bacares

Callitetrarhynchus sp. - Clevelandia ios, Ilypnus gilberti,

Quietula y-cauda

Callitetrarhynchus gracilis - Auxis thazard, Carcharhinus obscurus,

Coryphaena hippurus, Cynoscion nobilis, Euthynnus affinis,

Morone saxatilis, Paralichthys californicus, Prionace glauca,

Remora remora, Sarda chiliensis, Scomber japonicus, Seriola

dorsalis, Sphyraena argentea, Stereolepis gigas, Synodus 
DASYRHYNCHIDAE (continued)

lucioceps, Thunnus albacares, Thunnus thynnus

Dasyrhynchus sp. - Trachurus symmetricus

Dasyrhynchus giganteus - Carcharhinus leucas

Dasyrhynchus talismani - Carcharhinus longimanus, Thunnus albacares Floriceps saccatus - Carcharhinus obscurus, Mola mola, Notorynchus maculatus, Prionace glauca, Xiphias gladius

\section{DIPHYLLOBOTHRIIDAE}

Diphyllobothrium sp. - Gasterosteus aculeatus, Hippoglossoides

elassodon, Merluccius productus, Oncorhynchus gorbuscha, 0 . keta, $\underline{0}$. kisutch, $\underline{0}$. nerka, $\underline{0}$. tshawytscha, Platichthys stellatus,

Salmo clarki, S. gairdneri, Sarda chiensis,

Theragra chal cogramma

Diphyllobothrium cordiceps - Oncorhynchus kisutch, $\underline{0}$. nerka, Salmo clarki, s. gairdneri, Salvelinus malma

Diphyllobothrium dallii - Salvelinus malma

Diphyllobothrium dendriticum - Gasterosteus aculeatus, Salmo gairdneri

Diphyl lobothrium ditremum - Gasterosteus aculeatus, Salmo gairdneri Diphyllobothrium latum - Oncorhynchus gorbuscha, $\underline{0}$. keta, $\underline{0}$. nerka, Salmo gairdneri, Salvelinus malma

Diphyllobothrium ursi - Oncorhynchus nerka

Diphyl 1obothrium vogeli - Gasterosteus aculeatus

Pyramicocephalus phocarum - Hippoglossoides elassodon, Theragra chal cogramma

DISCULICIPITIDAE

Disculiceps pileatus - Carcharhinus leucas, $\underline{\text { C. obscurus }}$

\section{ECHINOBOTHRIIDAE}

Caulobothrium opisthorchis - Myliobatis californica

Caulobothrium tetrascaphium - Myliobatis californica 
CESTODA (continued)

\section{EUTETRARHYNCHIDAE}

Christianella trygonbrucco - Urolophus halleri

Eutetrarhynchus litocephalus - Mustelus californicus, Triakis semifasciata

Eutetrarhynchus macrotrachelus - Mustelus californicus

Eutetrarhynchus ruficollis - Squalus acanthias

Eutetrarhynchus schmidti - Rhinobatos productus, Urolophus halleri

Mecistobothrium myliobati - Myliobatis californica, Urolophus halleri

Parachristianel la monomegacantha - Rhinobatos productus

Parachristianella trygonis - Urolophus halleri

Prochristianella fragilis - Rhinobatos productus

Prochristianella minima - Platyrhinoidis triseriata, Urolophus halleri

GILQUINI IDAE

Gilquinia squali - Citharichthys stigmeus, Squalus acanthias

GYMNORHYNCHIDAE

Gymnorhynchus gigas - Isurus oxyrinchus, Thunnus albacares

Gymnorhynchus isuri - Isurus oxyrinchus

Molicola horridus - Isurus oxyrinchus, Mola mola

Molicola uncinatus - Alopias vulpinus, Xiphias gladius 
HEPATOXYLIDAE

Hepatoxylon sp. - Thunnus albacares

Hepatoxylon squali - Alopias vulpinus, Prionace glauca

Hepatoxylon trichiuri - Carcharodon carcharias, Coryphaena hippurus,

Isurus oxyrinchus, Oncorhynchus keta, $\underline{0}$. tshawytscha, Prionace

glauca, Squalus acanthias, Thunnus alalunga, Xiphias gladius

\section{LACISTORHYNCHIDAE}

Grillotia sp. - Sebastes paucispinis, Thunnus albacares

Grillotia acanthoscolex - Hexanchus griseus

Grillotia erinacea - Atheresthes stomias, Carcharhinus obscurus, Clupea

harengus, Reinhardtius hippoglossoides, Sebastes aleutianus, $\underline{S}$.

borealis, Squalus acanthias, Theragra chalcogramma, Xiphias gladius

Grillotia heptanchii - Hexanchus griseus

Grillotia musculara - Hexanchus griseus, Raja rhina

Grillotia (Paragrillotia) rowei - Coryphaenoides armatus

Grillotia scolecina - Hexanchus griseus

Grillotia smarisgora - Cynoscion nobilis, Sebastes paucispinis,

Squatina californica, Synodus lucioceps

Lacistorhynchus tenuis - Alopias vulpinus, Cymatogaster aggregata,

Cynoscion nobilis, Clupea harengus, Genyonemus lineatus, Leuresthes

tenuis, Morone saxatilis, Mustelus californicus, M. henlei,

Paralichthys californicus, Rhinobatos productus, Sebastes

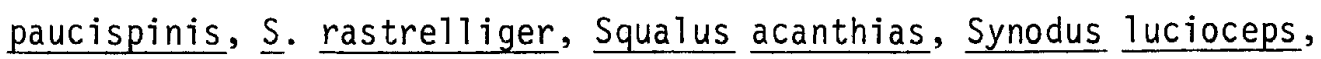

Thunnus thynnus, Triakis semifasciata, Zenops is nubulosa

\section{LECANICEPHALIDAE}

Discobothrium myliobatidis - Myliobatis californica

\section{LIGULI DAE}

Ligula intestinalis - Salmo gairdneri

Schistocephalus sp. - Gasterosteus aculeatus, Oncorhynchus nerka,

Salmo gairdneri

Schistocephalus solidus - Gasterosteus aculeatus, Salmo gairdneri 
CESTODA (continued)

\section{LITOBOTHRIDAE}

Litobothrium alopias - Alopias superciliosus

Litobothrium coniformis - Alopias superciliosus

Litobothrium daileyi - Alopias superciliosus

Litobothrium gracile - Odontaspis ferox

Renyxa amplifica - Alopias superciliosus

ONCOBOTHRI IDAE

Acanthobothrium sp. - Sebastes paucispinis, Synodus Iucioceps

Acanthobothrium bajaensis - Heterodontus francisci

Acanthobothrium benedeni - Dasyatis violacea

Acanthobothrium brachyacanthum - Raja binoculata,

R. inornata

Acanthobothrium coronatum - Alopias vulpinus, Prionace glauca,

Squalus acanthias

Acanthobothrium crassicolle - Urolophus halleri

Acanthobothrium dujardinii - Raja binoculata, Rhinobatos productus, Urolophus halleri

Acanthobothrium goldsteini - Platyrhinoidis triseriata

Acanthobothrium hispidum - Torpedo californica

Acanthobothrium holorhini - Myliobatis californica

Acanthobothrium maculatum - Myliobatis californica

Acanthobothrium magnum - Dasyatis violacea

Acanthobothrium microcephalum - Myliobatis californica

Acanthobothrium olseni - Rhinobatos productus, Urolophus halleri

Acanthobothrium parviuncinatum - Gymnura marmorata

Acanthobothrium paulum - Hexachus griseus

Acanthobothrium rhinobati - Rhinobatos productus

Acanthobothrium robustum - Rhinobatos productus

Acanthobothrium unilateralis - Myliobatis californica

Calliobothrium pellucidum - Mustelus californicus

Calliobothrium verticillatum - Hexanchus griseus, Prionace glauca, Squalus acanthias 
CESTODA (continued)

ONCOBOTHRIIDAE (continued)

Ceratobothrium xanthocephalum - Isurus oxyrinchus

Cylindrophorus posteroporus - Prionace glauca

Pedibothrium hutsoni - Galeocerdo cuvieri

Pedibothrium longispine - Galeocerdo cuvieri

Phoreiobothrium exceptum - Carcharhinus leucas, Sphyrna zygaena

Phoreiobothrium lasium - Alopias vulpinus, Carcharhinus leucas, $\underline{\mathrm{C}}$.

obscurus, Galeocerdo cuvieri, Sphyrna zygaena

Phoreiobothrium pectinatum - Sphyrna zygaena

Phoreiobothrium triloculatum - Carcharhinus obscurus

Pinguicollum pinguicollum - Raja binoculata, R. inornata, R. rhina

Thysanocephalum crispum - Galeocerdo cuvieri, Sphyrna zygaena

Thysanocephalum rugosum - Galeocerdo cuvieri

OTOBOTHRI I DAE

Otobothrium crenacolle - Carcharhinus leucas, $\underline{\text { C. obscurus, Coryphaena }}$

hippurus, Sphyrna zygaena, Squalus acanthias, Xiphias gladius

Otobothrium curtum - Galeocerdo cuvieri

Otobothrium dipsacum - Carcharhinus abscurus, Xiphias gladius

Otobothrium mugilis - Mugil cephalus

Otobothrium penetrans - Carcharhinus leucas, Sphyrna zygaena

Otobothrium pephrikos - Sphyrna zygaena

Otobothrium propecysticum - Sphyrna zygaena

Poecilancistrum caryophyllum - Carcharhinus leucas

PHORE IOBOTHRI IDAE

Trilocularia acanthiaevulgaris - Squalus acanthias

PHYLLOBOTHRI IDAE

Genera and species - Cololabis saira

Anthobothrium auriculatum - Hexanchus griseus, Prionace glauca

Anthobothrium cornucopia - Notorynchus maculatus, Prionace glauca

Anthobothrium exiquum - Alopias vulpinus

Anthobothrium laciniatum - Carcharinus leucas, $\underline{C}$. obscurus, Prionace

glauca, Sphyrna zygaena, Urolophus halleri

Anthobothrium minutum - Prionace glauca

Anthobothrium oligorchidum - Urolophus halleri

Anthobothrium parvum - Mustelus hentei, Triakis semifasciata 
CESTODA (continued)

PHYLLOBOTHRIIDAE (continued)

Anthobothrium pulvinatum - Squalus acanthias

Anthobothrium variabile - Cetorhinus maximus

Caulobothrium multorchidum - Urolophus halleri

Crossobothrium angustum - Alopias vulpinus, Carcharhinus obscurus, Prionace glau

Dinobothrium keilini - Prionace glauca

Dinobothrium planum - Triakis semifasciata

Dinobothrium plicitum - Carcharodon carcharias

Dinobothrium septaria - Cetorhinus maximus, Carcharodon carcharias,

Lamna ditropis, Prionace glauca

Dinobothrium simile - Lamna ditropis

Discobothrium (Echeneibothrium) fallax - Raja inornata, R. rhina

Echeneibothrium sp. - Paralichthys californicus, Thunnus albacares

Echeneibothrium dolichoophorum - Raja rhina

Echeneibothrium dubium abyssorum - Raja rhina

Echeneibothrium macrascum - Raja inornata

Echeneibothrium myzorhynchum - Myliobatis californica, Raja binoculata

Echeneibothrium octorchis - Raja binoculata, R. inornata, R. rhina

Gastrolecithus planus - Cetorhinus maximus, Mustelus henlei

Inermiphyllidium brachyascum - Myliobatis californica

Marsupiobothrium alopias - Alopias vulpinis

Marsupiobothrium forte - Sphyrna zygaena

Monorygma sp. - Squalus acanthias

Monorygma galeocerdonis - Galeocerdo cuvieri

Monorygma perfectum - Somniosus pacificus

Orygmatobothrium musteli - Galeorhinus zyopterus, Mustelus henlei,

Triakis semifasciata

Pelichnibothrium sp. - Oncorhynchus nerka, Salmo gairdneri,

Pelichnibothrium speciosum - Prionace glauca

Phyllobothrium sp. - Agonus acipenserinus, Ammodytes hexapterus, Aprodon cortezianus, Autorhynchus flavidus, Blepsias cirrhosus, Clupea harengus, Coryphopterus nicholsi, Cymatogaster aggregata, Dasycottus setiger, Gadus macrocephalus, Gasterosteus aculeatus, Hexagrammos lagocephalus, Hippoglossoides elassodon, Icelinus filamentosus, I. tenuis, Lampetra tridentata, Lepidopsetta bilineata, Myoxocephalus polyacanthocephalus, Oncorhynchus gorbuscha, $\underline{0}$. keta, $\underline{0}$. kisutch, $\underline{0}$. nerka, $\underline{0}$. tshawytscha, Ophiodon elongatus, Parophrys vetulus, Platichthys stellatus, Reinhardtius hippoglossoides, Salvel inus malma, Sebastes alutus, $\underline{\mathrm{s}}$. borealis, $\underline{\mathrm{s}}$. caurinus, 
PHYLLOBOTHRIIDAE (continued)

$\underline{\mathrm{S}}$. crameri, $\underline{\mathrm{S}}$. diploproa, $\underline{\mathrm{S}}$. elongatus, $\underline{\mathrm{S}}$. entomelas, $\underline{\mathrm{S}}$. maliger,

$\underline{\mathrm{S}}$. pinniger, $\underline{\mathrm{S}}$. ruberrimus, $\underline{\mathrm{S}}$. zacentrus, Squalus acanthias,

Sygnathus leptorhynchus, Thaleichthys pacificus, Theragra

chal cogramma, Triglops pingeli, Xeneretmus latifrons

Phyllobothrium caudatum - Alepisaurus ferox, Alosa sapidissima,

Eopsetta jordani, Lampris regius, Luvarus imperialis, Oncorhynchus gorbuscha, $\underline{0}$. keta,$\underline{0}$. kisutch, $\underline{0}$. nerka, $\underline{0}$. tshawytscha, Thunnus albacares, Thunnus thynnus

Phyllobothrium centrurum - Hexanchus griseus

Phyllobothrium dagnallium - Galeocerdo cuvieri, Prionace glauca

Phyllobothrium dohrnii - Hexanchus griseus

Phyllobothrium foliatum - Carcharhinus obscurus

Phyllobothrium gracile - Squalus acanthias

Phyllobothrium lactuca - Carcharodon carcharias, Galeocerdo cuvieri,

Hexanchus griseus, Squalus acanthias, Triakis semifasciata

Phyllobothrium magnum - Somniosus pacificus

Phyllobothrium musteli - Carcharhinus leucas, Galeocerdo cuvieri

Phyllobothrium prionacis - Prionace glauca

Phyllobothrium radioductum - Mustelus henlei, Raja binoculata, $\underline{R}$.

inornata, Raja rhina, Triakis semifasciata

Phyllobothrium rotundum - Hexanchus griseus

Phyllobothrium sinuosiceps - Hexanchus griseus

Phyllobothrium squali - Squalus acanthias

Phyllobothrium thridax - Squalus acanthias

Phyllobothrium triacis - Hexanchus griseus

Phyllobothrium tumidum - Carcharodon carcharias, Isurus oxyrinchus, Lamna ditropis

Pithophorus sp. - Mustelus henlei

Pithophorus vulpeculae - Alopias vulpinus

Reesium paciferum - Cetorhinus maximus

Rhinebothrium bilobatum - Urolophus halleri

Rhinebothrium ditesticulum - Urolophus halleri

Rhinebothrium flexile - Myliobatis californica, Scomber japonicus, Urolophus halleri 
CESTODA (continued)

PHYLLOBOTHRIIDAE (continued)

Rhinebothrium minimum - Urolophus halleri

Rhinebothrium palombi - Dasyatis violacea

Rhinebothrium tumidulum - Urolophus halleri

Rhinebothrium urobatidium - Urolophus halleri

Rhodobothrium pulvinatum - Squalus acanthias

Scyphophyllideum giganteum - Galeorhinus zyopterus, Squalus acanthias PROSOBOTHRI IDAE

Platybothrium auriculatum - Prionace glauca

Platybothrium baeri- Prionace glauca

Platybothrium cervinum - Carcharhinus obscurus, Prionace glauca

Platybothrium parvum - Isurus oxyrinchus, Prionace glauca, Sphyrna zygaena

Prosobothrium adhaerans - Prionace glauca, Sphyrna zygaena

Prosobothrium armigerum - Prionace glauca, Squalus acanthias

Prosobothrium japonicum - Prionace glauca

PROTEOCEPHALIDAE

Genera and species - Morone saxatilis

Gangesia parasiluri - Oncorhynchus gorbuscha, $\underline{0}$. keta

Proteocephalus sp. - Oncorhynchus kisutch, $\underline{0}$. nerka, Salmo gairderni

Proteocephalus ambloplitis - Oncorhynchus kisutch, Salmo clarki,

S. gairdneri

Proteocephalus arcticus - Oncorhynchus nerka, Salmo

clarki

Proteocephalus exiguus - Oncorhynchus keta, $\underline{0}$. kisutch, $\underline{0}$. nerka,

o. tshawytscha

Proteocephalus filicollis - Gasterosteus aculeatus

Proteocephalus laruei - Oncorhynchus nerka, Salmo clarki

Proteocephalus longicollis - Gasterosteus aculeatus, Salmo gairdneri

Proteocephalus percae - Gasterosteus aculeatus, Salmo gairaneri

Proteocephalus pinguis - Salmo gairdneri

Proteocephalus primaverus - Salmo clarki 
PROTEOCEPHALIDAE (continued)

Proteocephalus pugetensis - Gasterosteus aculeatus

Proteocephalus salmonidicola - Oncorhynchus kisutch, Salmo clarki,

S. gairdneri, Salvelinus malma

PSEUDOPHYLLIDEA

Genera and species - Ceratoscopelus townsendi, Clupea harengus, Diaphus

theta, Hippoglossoides elassodon, Lampanyctus ritteri, Lepidopsetta

bilineata, Microstomus pacificus, Reinhardtius hippoglossoides,

Sebastes aleutianus, Scomber japonicus, Stenobrachius leucopsarus,

Symbolophorus californiensis, Theragra chalcogramma, Triphoturus

mexicanus

PTEROBOTHRI IDAE

Pterobothrium heteracanthum - Carcharhinus obsurus, Galeocerdo

cuvieri

PTYCHOBOTHRI IDAE

Clestobothrium crassiceps - Merluccius productus, Squalus acanthias

SPATHEBOTHRI IDAE

Spathebothrium simplex - Liparis callyodon, L. fucensis

SPHYRIOCEPHALIDAE

Sphyriocephalus sp. - Euprotomicrus bispinatus, Thunnus albacares

Sphyriocephalus dollfusi - Thunnus obesus

Sphyriocephalus pelorosoma - Alopias superciliosus

Sphyriocephalus tergestinus - Alopias vulpinus, Euprotomicrus bispinatus,

Isurus oxyrinchus, Thunnus alalunga

Sphyriocephalus viridis - Alopias superciliosus

TENTACULARI IDAE

Nybelinia sp. - Engraul is mordax, Lamna ditropis, Makaira nigricans, Notorynchus maculatus, Oncorhynchus gorbuscha, $\underline{0}$. keta, $\underline{0}$. kisutch,

0. nerka, $\underline{0}$. tshawytscha, Scomber japonicus, Thunnus albacares Nybelinia anthicosum - Heterodontus francisci, Triakis semifasciata 
CESTODA (continued)

TENTACULARIIDAE (continued)

Nybelinia bisulcata - Caranx hippos, Carcharhinus obscurus, Coryphaena hippurus, Galeocerdo cuvieri, Remora remora, Sphyrna zygaena,

Squalus acanthias, Xiphias gladius

Nybelinia lamonteae - Xiphias gladius

Nybelinia lingualis - Isurus oxyrinchus, Naucrates ductor,

Prionace glauca, Xiphias gladius

Nybel inia palliata - Lamna ditropis, Sphyrna zygaena

Nybelinia pintneri - Isurus oxyrinchus, Prionace glauca

Nybelinia robusta - Carcharhinus obscurus, Echeneis naucrates, Isurus

oxyrinchus, Remora remora

Nybelinia sphyrnae - Sphyrna zygaena

ilybelinia surmenicola - Anoplopoma fimbria, Aprodon cortezianus,

Atheresthes stomias, Eopsetta jordani, Gadus macrocephalus,

Hexagrammos stelleri, Hippoglossoides elassodon, Hippoglossus

stenolepis, Lamna ditropis, Lepidopsetta bilineata, Lyopsetta

exilis, Merluccius productus, Microstomus pacificus, Oncorhynchus

gorbuscha, $\underline{0}$. keta, $\underline{0}$. kisutch, $\underline{0}$. tshawytscha, Ophiodon elongatus,

Parophrys vetulus, Platichthys stellatus, Pleurogrammus monopterygius,

Reinhardtius hippoglossoides, Scomber japonicus, Sebastes aleutianus,

$\underline{\text { s. alutus, }} \underline{\mathrm{s}}$. borealis, $\underline{\mathrm{S}}$. brevispinis, $\underline{\mathrm{S}}$. caurinus, $\underline{\mathrm{S}}$. crameri,

$\underline{\mathrm{S}}$. diploproa, $\underline{\mathrm{s}}$. elongatus, $\underline{\mathrm{S}}$. entomelas, $\underline{\mathrm{S}}$. flavidus, $\underline{\mathrm{S}}$. maliger,

$\underline{S}$.nigrocinctus, $\underline{S}$. pinniger, $\underline{S}$, proriger, $\underline{S}$. reedi, $\underline{S}$. ruberrimus,

S. zacentrus, Theragra chalcogramma, Triglops

pingeli

Nybelinia syngenes - Sphyrna zygaena

Tentacularia sp. - Istiophorus platypterus, Makaira nigricans,

Ophiodon elongatus, Thunnus albacares

Tentacularia coryphaenae - Alepisaurus ferox, Carcharhinus longimanus,

C. obscurus, Carcharodon carcharias, Coryphaena hippurus, Echeneis

naucrates, Euthynnus pelamis, Galeocerdo cuvieri, Oncorhynchus

tshawytscha, Prionace glauca, Scomber japonicus, Sphyrna zygaena,

Thunnus albacares, Thunnus thynnus, Xiphias gladius

Tentacularia macropora - Galeocerdo cuvieri

Tentacularia megalobothrida - Hexanchus griseus

Tetrarhynchus sp. - Hexanchus griseus 


\section{TETRABOTHRI I DAE}

Priapocephalus sp. - Cololabis saira

\section{TETRAGONOCEPHALIDAE}

Tetragonocephalum trygonis - Dasyatis violacea

\section{TETRAPHYLLIDEA}

Genera and species - Ceratoscopelus townsendi, Clevelandia ios, Diaphus theta, Lampanyctus ritteri, Lyopsetta exilis, Macroparalepis sp., Neoclinus blanchardi, Roncador stearnsi, Sebastes paucispinis, Stenobrachius leucopsarus, Sternoptyx diaphana, Stomias atriventer, Symbolophorus californiensis

Yellow pigmented plerocercoid - Atherinopsis californiensis Red pigmented plerocercoid - Alosa sapidissima, Cymatogaster aggregata, Engraul is mordax, Glyptocephalus zachirus, Rhinobatos productus, Sardinops sagax, Urolophus halleri

Scolex sp. - Anoplopoma fimbria, Auxis thazard Scolex pleuronectis - Anoplopoma fimbria, Atheresthes stomias, Blepsias cirrhosus, Clupea harengus, Cololabis saira, Gadus macrocephalus, Hexagrammos lagocephalus, $\underline{H}$. stelleri, Hippoglossoides elassodon, Hippoglossus stenolepis, Ilypnus gilberti, Lepidopsetta bilineata, Mallotus villosus, Morone saxatilis, Mugil cephalus, Myoxocephalus polyacanthocephalus, Oncorhynchus gorbuscha, $\underline{0}$. keta, $\underline{0}$. kisutch, $\underline{0}$. nerka, $\underline{0}$. tshawytscha, Platichthys stellatus, Pleurogrammus monopterygius, Quietula y-cauda, Reinhardtius hippoglossoides,

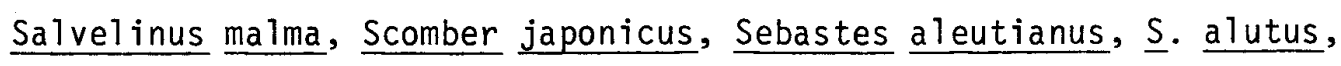
s. borealis, Theragra chal cogramma, Thunnus albacares, Trachurus symmetricus

Scolex polymorphus - Gasterosteus aculeatus, Mugil cephalus, Oncorhynchus gorbuscha, $\underline{0}$. kisutch, $\underline{0}$. nerka, $\underline{0}$. tshawytscha, Reinhardtius hippoglossoides, Remora remora, Trachurus symmetricus, Xiphias gladius 
CESTODA (continued)

TRIAENOPHORIDAE

Ancistrocephalus microcephalus - Mola mola

Eubothrioides Tamellatum - Zenopsis nebulosa

Fisticula plicatus - Mola mola, Xiphias gladius

Triaenophorus crassus - Gasterosteus aculeatus, 0ncorhynchus keta,

o. nerka

Triaenophorus lucii - Gasterosteus aculeatus, Salmo gairdneri

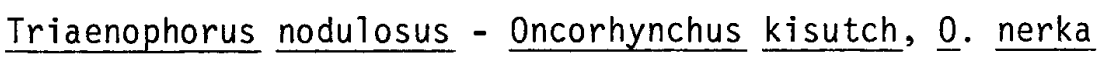

TRYPANORHYNCHA

Genera and species - Anoplopoma fimbria, Auxis thazard, Makaira nigricans,

Merluccius productus, Morone saxatilis, Paralichthys californicus,

Scomber japonicus, Sebastes levis, $\underline{S}$. miniatus, Sphyraena argentea,

Thunnus albacraes

Dibothriorhynchus maccallumi - Sphyrno tiburo

Dibothriorhynchus xiphiae - Xiphias gladius

Rhynchobothrium sp. - Mola mola

Rhynchobothrium exile - Galeocerdo cuvieri

Rhynchobothrium plicatum - Sphyrna tiburo

Rhynchobothrium tumidulum - Carcharhinus obscurus

Rhynochobothrius carangis - Caranx hippos

Tetrarhynchus bicolor - Carcharhinus obscurus

Tetrarhynchus elongatus - Mola mola

Tetrarhynchus papillosus - Coryphaena hippurus

Tetrarhynchus tetrabothrium - Squalus acanthias 
CESTODARIA

GYROCOTYLIDAE

Gyrocotyle fimbriata - Hydrolagus colliei

Gyrocotyle parvispinosa - Hydrolagus colliei

459 
Intermediate stage - Ceratoscopel us townsendi

BOMOLOCHIDAE

Bomolochus concinnus - Mugil cephalus

Bomolochus constrictus - Atherinops affinis

Bomolochus cuneatus - Clupea harengus, Cymatogaster aggregata,

Damalichthys vacca, Embiotoca jacksoni, Gasterosteus aculeatus,

Leuresthes tenuis, Oncorhynchus gorbuscha, Phanerodon furcatus

Bomolochus decapteri - Cololabis saira

Bomolochus exilipes - Mugil cephalus

Bomolochus mugilis - Mugil cephalus

*Bomolochus solae - Paralabrax clathratus, $\underline{\text { P. nebulifer, Paralichthys }}$

californicus, Menticirrhus undulatus, Sphyraena argentea

Holobomolochus ardeole - Hypsypops rubicunda

Holobomolochus attenuatus - Genyonemus lineatus, Roncador stearnsi,

Scorpaena guttata, Sphoeroides annulatus

Holobomolochus embiotocae - Cymatogaster aggregata, Damalichthys

vacca, Embiotoca jacksoni, Phanerodon furcatus

Holobomolochus longicaudus - Paralabrax clathratus, P. maculatofasciatus,

P. nebulifer

Holobomolochus occultus - Hippoglossoides elassodon, Lyopsetta exilis

Holobomolochus prolixus - Leptocottus armatus, Paralichthys

cal ifornicus, Pleuronichthys coenosus

Holobomolochus spinulus - Leptocottus armatus, 01 igocottus maculosus,

Oxylebius pictus, Scorpaena guttata, Sebastes mystinus, $\underline{\text { S. serranoides }}$ Holobomolochus venustus - Scorpaenichthys marmoratus, Sebastes caurinus,

$\underline{S}$. elongatus, $\underline{S}$. maliger, $\underline{S}$. pinniger, $\underline{S}$. ruberrimus

Nothobomolochus teres - Mugil cephalus

Taeniacanthus albidus - Sphyrna tiburo

Taeniacanthus flagellans - Sphyrna zygaena

Taeniacanthodes haakeri - Cymatogaster aggregata, Paralichthys

cal ifornicus 
COPEPODA (continued)

BOMOLOCHIDAE (continued)

Taeniastrotos californiensis - Paralabrax clathratus, P. maculatofasciatus,

P. nebulifer

Unicolax mycterobius - Auxis thazard

CALIGIDAE

Anuretes heckelii - Caranx hippos

Anuretes quadrilaterus - Zenopsis nebulosa

Caligodes laciniatus - Sphyraena argentea

Caligus sp. - Hydrolagus colliei, Merluccius productus, Morone saxatilis, Oncorhynchus tshawytscha

Caligus asymmetricus - Euthynnus affinis

Caligus auxisi - Auxis thazard

Caligus belones - Coryphaena hippurus

Caligus bonito - Coryphaena hippurus, Engraulis mordax, Euthynnus affinis,

E. pelamis, Mugil cephalus, 0ligoplites saurus, Sarda chiliensis,

Thunnus thynnus

Caligus chelifer - Xiphias gladius

Caligus chorinemi - Seriola dorsalis

Caligus clemensi - Clupea harengus, Gasterosteus aculeatus, Hexagrammos

sp., Hydrolagus colliei, Oncorhynchus gorbuscha, $\underline{0}$. keta, $\underline{0}$. kisutch,

Salmo gairdneri, Sebastes sp., S. caurinus, Theragra chalcogramma

Caligus confusus - Caranx hippos, Coryphaena hippurus

Caligus constrictus - Caranx hippos, Coryphaena hippurus

Caligus coryphaenae - Caranx hippos, Coryphaena hippurus, Euthynnus

lineatus, E. pelamis, Isurus oxyrinchus, Polydactylus opercularis,

Seriola dorsalis, Squalus acanthias, Thunnus albacares, I. obesus,

I. thynnus, $\underline{x}$. gladius

*Caligus curtus - Mugil cephalus, Squalus acanthias

Caligus diaphanus - Caranx hippos

Caligus elongatus - Alopias vulpinus, Alosa sapidissima, Caranx hippos,

Clupea harengus, Mola mola, Remora remora, Salmo gairdneri, Sphoeroides annulatus, Squalus acanthias, Xiphias gladius

Caligus epidemicus - Mugil cephalus 


\section{CALIGIDAE (continued)}

Caligus germoi - Thunnus alalunga

Caligus glandifer - Zenopsis nebulosa

Caligus hobsoni - Chromis punctipinnis, Damalichthys vacca, Girella nigricans, Hypsypops rubicunda, Medialuna californiensis, Oxyjulis californica, Phanerodon atripes, Pimelometopon pulchrum, Rhacochilus toxotes, Scorpaenichthys marmoratus, Sebastes atrovirens, $\underline{\text { s. carnatus, }}$

S. mystinus, $\underline{S}$. serranoides, synodus lucioceps

Caligus irritans - Mugil cephalus

Caligus klawei - Engraulis mordax

Caligus lacustris - Gasterosteus aculeatus

Caligus lunatus - Seriola dorsalis

Caligus macarovi - Cololabis saira, Euthynnus lineatus

Caligus minimus - Mugil cephalus

Caligus mutabilis - Calamus brachysomus, Euthynnus pelamis,

Menticirrhus undulatus, Mugil cephalus, Paralabrax clathratus,

P. maculatofasciatus, $\underline{\text { P. nebulifer, Sarda chiliensis, Scomberomorus }}$ sierra

Caligus olsoni - Leuresthes tenuis

Caligus orientalis - Mugil cephalus

Caligus patulus - Coryphaena hippurus

Caligus pectinatus - Eopsetta jordani

Caligus pelamydis - Euthynnus pelamis, Scomber japonicus, Trachurus symmetricus.

Caligus platytarsis - Mugil cephalus

Caligus praetextus - Caranx hippos, Echeneis naucrates, Mugil cephalus, Sphyrna tiburo

Caligus productus - Auxis thazard, Balistes polylepis, Calamus brachysomus, Coryphaena hippurus, Euthynnus affinis, E. pelamis, Naucrates ductor, Paralabrax clathratus, P. maculatofasciatus, Scomberomorus sierra, Seriola dorsalis, Sphyraena argentea, Thunnus alalunga, $I$. albacares, $I$. obesus, $I$. thynnus 
CALIGIDAE (continued)

Caligus punctatus - Acanthogobius flavimanus

Caligus quadratus - Coryphaena hippurus, Cymatogaster aggregata,

Euthynnus pelamis, Istiophorus platypterus, Thunnus albacares

Caligus rectus - Caranx hippos

Caligus robustus - Caranx hippos

Caligus rufimaculatus - Mugil cephalus

Caligus schistonyx - Mugil cephalus

Caligus serratus - Atherinops affinis, Atherinopsis californiensis, Oxyjul is californica

Caligus spinosurculus - Caranx hippos

Caligus tenax - Caranx hippos

Caligus tenuicaudatus - Seriola dorsalis

Caligus tenuifurcatus - Nematistius pectoral is

Dentigryps curtus - Euthynnus pelamis

Dentigryps longicauda - Caranx hippos

Heniochophilus branchialis - Euthynnus pelamis

Lepeophtheirus sp. - Ammodytes hexapterus, Damalichthys vacca,

Genyonemus lineatus, Hysypops rubicunda, Medialuna californiensis,

Menticirrhus undulatus, Myoxocephalus polyacanthocephalus, 0xyjul is

californica, Sebastes paucispinis, Psettichthys melanostictus

Lepeophtheirus appendiculatus - Hippoglossus stenolepis

Lepeophtheirus bifidus Hypsopsetta guttulata, Lepidopsetta bilineata,

Paralichthys californicus, Parophrys vetulus

Lepeophtheirus bifurcatus - Cymatogaster aggregata, Phanerodon

furcatus, Psettichthys melanostictus

Lepeophtheirus brachyurus - Scorpaena guttata

Lepeophtheirus breviventris - Ophiodon elongatus

Lepeophtheirus constrictus - Paralabrax clathratus, $\underline{\text { P. maculatofasciatus, }}$

P. nebulifer

Lepeophtheirus crassus - Remora australis, R. remora

Lepeophtheirus cuneifer - Gadus macrocephalus, Hexagrammos lagocephalus, 


\section{CALIGIDAE (continued)}

Hippoglossus stenolepis, Isopsetta isolepis, Leptocottus armatus, Lumpenus sagitta, Microgadus proximus, Raja binoculata, Squalus acanthias, Theragra chalcogramma

Lepeophtheirus dissimulatus - Euthynnus pelamis, Gadus macrocephalus, Hypsopsetta guttulata, Merluccius productus, Mycteroperca xenarcha, Paralabrax nebulifer, Paralichthys californicus, Sphoeroides annulatus, Sphyraena argentea

Lepeophtheirus edwardsi - Caranx hippos

Lepeophtheirus emiens - Makaira nigricans

Lepeophtheirus goniistii - Zenopsis nebulosa

Lepeophtheirus hastatus - Mola mola

Lepeophtheirus hexagrammi - Hexagrammos lagocephalus

Lepeophtheirus hospital is - Gadus macrocephalus, Lepidopsetta

bilineata, Mugil cephalus, Parophrys vetulus, Platichthys stellatus,

Pleuronichthys coenosus

Lepeophtheirus abdominis - Cynoscion nobilis

Lepeophtheirus longipes - Echeneis naucrates, Paralabrax clathratus,

Sebastes maliger, $S$. serriceps, Stereolepis gigas

Lepeophtheirus longispinosus - Sphyrna zygaena

Lepeophtheirus nordmanni - Mola mola

Lepeophtheirus oblitus - Hexagrammos decagrammus, $\underline{H}$. stelleri,

Sebastes alutus, $\underline{S}$. caurinus, $\underline{S}$. helvomaculatus, $\underline{S}$. maliger

Lepeophtheirus parvicruris - Platichthys stellatus

Lepeophtheirus parviventris - Anoplopoma fimbria, Enophrys bison,

Eopsetta jordani, Gadus macrocephalus, Halichoeres semicinctus, Heterostichus rostratus, Hexagrammos decagrammus, Hippoglossus

stenolepis, Lepidopsetta bilineata, Myoxocephalus polyacanthocephalus, Platichthys stellatus, Pleurogrammus monopterygius, Raja binoculata,

R. rhina, Scorpaenichthys marmoratus, S. pinniger, S. rubrivinctus,

Urolophus halleri, Theragra chalcogramma, Xiphister atropurpureus Lepeophtheirus parvus - Cymatogaster aggregata, Damalichthys vacca, 
CALIGIDAE (continued)

Pimelometopon pulchrum

Lepeophtheirus paulus - Sebastes diploproa, $\underline{\mathrm{s}}$. flavidus, $\underline{\mathrm{S}}$. maliger,

$\underline{\text { S. nigrocinctus, }} \underline{\mathrm{S}}$. ruberrimus, $\underline{\mathrm{s}}$. serriceps

Lepeophtherus pollachius - Clupea harengus

Lepeophtheirus pravipes - Hippoglossus stenolepis, Ophiodon elongatus,

Raja binocuiata, Scorpaena guttata

Lepeophtheirus salmonis - Acipenser transmontanus, Ammodytes

hexapterus, Oncorhynchus gorbuscha, $\underline{0}$. keta, $\underline{0}$. kisutch, $\underline{0}$. nerka,

0. tshawytscha, Ophiodon elongatus, Salmo clarki, s. gairdneri,

Sebastes rubrivinctus

Lepeophtheirus thompsoni - Cynoscion nobilis

*Pseudocaligus apodus - Mugil cephalus

Pseudolepeophtheirus longicauda - Platichthys stellatus

Pupulina brevicauda - Mobula lucasana

Pupulina minor - Mobula lucasana

Sciaenophilus bennetti - Paralabrax maculatofasciatus

Tuxophorus caligodes - Echeneis naucrates

CECROPIDAE

Cecrops exiguus - Mola mola

Cecrops latreillij-Mola mola, Thunnus thynnus

Luetkenia asterodermi - Luvarus imperialis

Luetkenia elongata - Luvarus imperialis

Orthagoriscola muricatus - Mola mola

Philorthagoriscus serratus - Mola mola, Squalus acanthias

\section{CHONDRACANTHIDAE}

Acanthochondria sp. - Merluccius productus, Paralichthys californicus Acanthochondria cornuta - Hippoglossus stenolepis, Maynea californica Acanthochondria del toideus - Hexagrammos decagrammus, Sebastes melanops Acanthochondria epachthes - Hydrolagus colliei.

Acanthochondria fraseri - Pleuronichthys coenosus 
CHONDRACANTHIDAE (continued)

Acanthochondria rectangularis - Hydrolagus colliei, Leptocottus armatus, Parophrys vetulus, Platichthys stellatus, Pleuronichthys coenosus, Psettichthys melanostictus

Acanthochondria solida - Hexagrammos lagocephalus

Acanthochondria yui - Acanthogobius flavimanus

Blias prionoti - Hemanthias peruanus

Chondracanthodes deflexus - Coryphaenoides armatus

Chondracanthus sp. - Hemilepidotus hemilepidotus, Sebastes alutus, S. melanops

Chondracanthus distortus - Zenopsis nebulosa

Chondracanthus gracilis - Hexagrammos decagrammus, Scorpaenichthys marmoratus, Scorpaena guttata

Chondracanthus heterostichi - Heterostichus rostratus

Chondracanthus horridus - Heterostichus rostratus

Chondracanthus irregularis - Enophrys bison, Myoxocephalus

polyacanthocephalus

Chondracanthus lotellae - Myoxocephalus polyacanthocephalus

Chondracanthus narium - Opiodon elongatus

Chondracanthus palpifer - Gadus macrocephalus, Merluccius productus Chondracanthus pinguis - Hexagrammos decagrammus, Scorpaenichthys marmoratus, Sebastes aleutianus, $\underline{S}$. alutus, $\underline{s}$. auriculatus, $\underline{S}$. babcocki, $\underline{\text { S. borealis, }}$ s. brevispinis, s. caurinus, $\underline{\mathrm{S}}$. crameri, $\underline{\mathrm{S}}$. diploproa, $\underline{\mathrm{S}}$. entomelas, $\underline{\mathrm{S}}$. flavidus, $\underline{\mathrm{S}}$. maliger, $\underline{\mathrm{S}}$. melanops, $\underline{\mathrm{S}}$. pinninger, $\underline{\mathrm{S}}$. proriger, $\underline{\mathrm{S}}$. reedi,$\underline{\mathrm{S}}$. ruberrimus, $\underline{\mathrm{S}}$. zacentrus, Xiphister atropurpureus

Chondracanthus pusillus - Apodichthys flavidus

Chondracanthus rickettsi - Coryphaenoides armatus

Chondracanthus theragrae - Theragra chalcogramma

Chondracanthus triventricosus - Sebastes aleutianus, S. alutus,

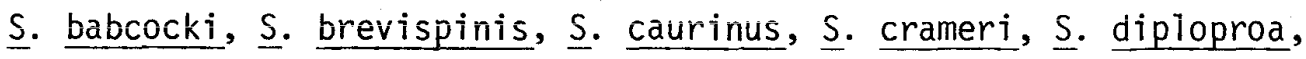

$\underline{\mathrm{S}}$. flavidus, $\underline{\mathrm{S}}$. helvomaculatus, $\underline{\mathrm{S}}$. maliger, $\underline{\mathrm{S}}$. proriger, $\underline{\mathrm{S}}$. reedi,

$\underline{\text { s. }}$ ruberrimus, $\underline{\mathrm{S}}$. zacentrus 
CHONDRACANTHIDAE (continued)

Heterochondria atypica - Oxyjulis californica, oxylebius pictus

Lateracanthus quadripedis - Coryphaenoides acrolepis

Pseudochondracanthus diceraus - Sphoeroides sp.

Pseudodiocus scorpaenus - Scorpaena guttata

DICHELESTHI IDAE

Anthosoma crassum - Carcharhinus obscurus, Carcharodon carcharias, Cetorhinus maximus, Isurus oxyrinchus, Lamna ditropis, Mola mola, Prionace glauca

DISSONIDAE

Dissonus ruvetti - Ruvettus pretiosus

ERGASILIDAE

Dermoergasilus amplectens - Mugil cephalus

Ergasilus sp. - Atherinops affinis, Fundulus parvipinnis, Leptocottus armatus, Mugil cephalus

Ergasilus auritus - Gasterosteus aculeatus, Gillichthys mirabilis, Oncorhynchus kisutch, $\underline{0}$. nerka

Ergasilus briani - Oncorhynchus gorbuscha, $\underline{0}$. keta

Ergasilus caeruleus - Oncorhynchus nerka

Ergasilus clupeidarum - Dorosoma petenense

Ergasilus cyanopictus - Mugil cephalus

Ergasilus fryeri - Mugil cephalus

Ergasilus funduli - Mugil cephalus

Ergasilus globosus - Gasterosteus aculeatus

Ergasilus labracis - Morone saxatilis

Ergasilus longimanus - Mugil cephalus

Ergasilus manicatus - Gasterosteus aculeatus

Ergasilus lizae - Cymatogaster aggregata, Morone saxatilis, Mugil cephalus

Ergasilus mugilis - Mugil cephalus

Ergasilus myctarothes - Sphyrna zygaena 


\section{ERGASILIDAE (continued)}

Ergasilus nerkae - Oncorhynchus kisutch, $\underline{0}$. nerka, Salmo gairdneri Ergasilus oriental is - Acanthogobius flavimanus Ergasilus sieboldi - Gasterosteus aculeatus, Salmo gairdneri Ergasilus turgidus - Chitonotus pugetensis, Cymatogaster aggregata, Gasterosteus aculeatus, Oncorhynchus nerka

Ergasilus versicolor - Mugil cephalus

Ergasilus wilsoni - Oncorhynchus gorbuscha

Nipergasilus bora - Mugil cephalus

Thersitina gasterostei - Gasterosteus aculeatus

EUDACTYLINIDAE

Bariaka alopiae - Alopias superciliosus

Bariaka pamelae - Hexanchus griseus

Eudactylina acanthii - Squalus acanthias

Eudactylina acuta - Squalus acanthias

Eudactylina longispina - Sphyrna tiburo

Eudactylina pusilla - Galeocerdo cuvieri

Eudactylina spinifera - Carcharhinus obscurus

Eudactylina valei - Squalus acanthias

Eudactylinodes nigra - Sphyrna tiburo

Eudactylinodes uncinata - Galeorhinus zyopterus

Nemesis aggregata - Alopias vulpinus

Nemesis atlantica - Alopias vulpinus, Carcharhinus leucas, $\underline{\mathrm{C}}$. obscurus

Nemesis carchariaeglauci - Prionace glauca, Triakis semifasciata

Nemesis lamna - Alopias vulpinus, Carcharodon carcharias, Cetorhinus maximus, Isurus oxyrinchus, Lamna ditropis, Odontaspis ferox, Prionace glauca

Nemesis robusta - Alopias vulpinus, Carcharhinus leucas, C. obscurus, Carcharodon carcharias, Galeocerdo cuvieri, Prionace glauca, Sphyrna tiburo, $\underline{S}$. zygaena

Nemesis spinulosus - Carcharhinus obscurus 
COPEPODA (continued)

EUDACTYLINIDAE (continued)

Nemesis versicolor - Sphyrna zygaena

EURYPHORIDAE

Alebion carchariae - Carcharinus leucas, C. obscurus, Galeocerdo cuvieri

Alebion crassus - Sphyrna lewini, S. zygaena

Alebion glaber - Squalus acanthias

Alebion gracilis - Carcharhinus leucas, $\underline{C}$. longimanus, $\underline{C}$. obscurus, Galeocerdo cuvieri, Squalus acanthias

Euryphorus brachypterus - Isurus oxyrinchus, Thunnus alalunga, I. albacares, I. obesus, I. thynnus

Euryphorus nordmanni - Coryphaena hippurus, Thunnus alalunga, I. albacares

Gloiopotes americanus - Istiophorus platypterus

Gloiopotes huttoni - Istiophorus platypterus, Makaira indica, M. nigricans, Tetrapturus audax, Thunnus obesus, Xiphias gladius Gloiopotes ornatus - Makaira nigricans, Tetrapturus audax, Xiphias gladius

Gloiopotes watsoni - Istiophorus platypterus, Makaira nigricans, Tetrapturus audax, Xiphias gladius

Paralebion elongatus - Carcharhinus leucas, $\underline{C}$. obscurus

HATSCHEKIIDAE

Hatschekia albirubra - Menticirrhus undulatus, Paralabrax clathratus Hatschekia oblonga - Caranx hippos

Hatschekia pacifica - Paralabrax clathratus, P. maculatofasciatus,

P. nebulifer

Hatschekia pinguis - Gymnothorax mordax 
COPEPODA (continued)

\section{KROYERI IDAE}

Kroyeria aculeata - Prionace glauca

Kroyeria dispar - Galeocerdo cuvieri

Kroyeria echinata - Sphyrna zygaena

Kroyeria gracilis - Carcharhinus leucas, c. longimanus, ․ obscurus,

Prionace glauca, Sphyrna zygaena

Kroyeria lineata - Sphyrna zygaena

Kroyeria papillipes - Galeocerdo cuvieri, Sphyrna zygaena

Kroyeria praelongacicula - Sphyrna lewini

Kroyeria sphyrnae - Sphyrna zygaena

* Kroyeria trecai - Sphyrna zygaena

Kroyerina elongata - Galeocerdo cuvieri, Prionace glauca

\section{LERNAIDAE}

Lernaea cyprinacea - Gasterosteus aculeatus, Oncorhynchus tshawytscha, Salmo gairdneri

\section{LERNAEOPODIDAE}

Genera and species - Oncorhynchus gorbuscha

Achtheres lacae - Morone saxatilis

Advena saba - Scomber japonicus

Brachiella thynni - Thunnus alalunga, I. albacares, I. obesus,

I. thynnus

Charopinopsis quaternius - Coryphaena hippurus

Clavella sp. - Damalichthys vacca, Embiotoca jacksoni

Clavella adunca - Gadus macrocephalus, Microgadus proximus, Theragra chalcogramma

Clavella canaliculata - Microgadus proximus

*Clavella inversa - Mugil cephalus

Clavella parva - Artedius harringtoni, Chitonotus pugetensis,

Embiotoca lateralis, Paralabrax clathratus, P. maculatofasciatus,

P. nebulifer, Phanerodon furcatus, Sebastes aleutianus, $\underline{S}$. alutus,

S. auriculatus, $\underline{\text { S. babcocki, }}$ S. caurinus, $\underline{\text { S. diploproa, }}$ s. elongatus,

S. flavidus, $\underline{\text { s. maliger, }}$ s. melanops, $\underline{\text { S. pinniger }}$ 


\section{LERNAEOPODIDAE (continued)}

Clavella perfida - Merluccius productus, Theragra chalcogramma

Clavellisa scombri - Scomber japonicus

Clavellistes shoyoae - Lampris regius

Clavellopsis sp. - Leuresthes tenuis, Sebastes alutus

Clavellopsis flexicervica - Chromis punctipinnis

Clavellopsis longimana - Mugil cephalus

Dendrata cameroni longiclavata - Raja inornata

Lernaeopoda bidiscalis - Mola mola

Lernaeopoda galei - Raja binoculata, Squalus acanthias, Triakis

semifasciata

Lernaeopodina pacifica - Raja rhina

Nectobranchia indivisa - Lepidopsetta bilineata, Platichthys stellatus

Neobrachiella sp. - Merluccius productus

Neobrachiella annulata - Coryphaenoides acrolepis

Neobrachiella gracilis - Caulolatilus princeps, Cynoscion nobilis,

Genyonemus lineatus

Neobrachiella gulosa - Roncador stearnsi

Neobrachiella nitida - Citharichthys sordidus, Coryphaenoides

pectoralis

* Neobrachiella oblonga - Mugil cephalus

Neobrachiella regia - Lampris regius

Neobrachiella robusta - Merluccius productus, Sebastes aleutianus, $\underline{\text { S. }}$

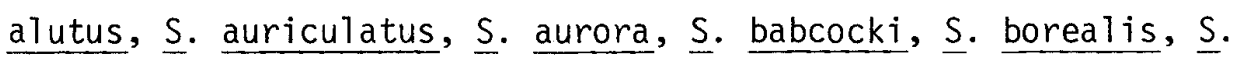

brevispinis, $\underline{S}$. caurinus, $S$. crameri, $\underline{S}$. diploproa, $\underline{S}$. entomelas, $\underline{S}$.

flavidus, $\underline{\text { S. maliger, }} \underline{\mathrm{S}}$. melanops, $\underline{\mathrm{S}}$. pinniger, $\underline{\mathrm{S}}$. proriger, $\underline{\mathrm{S}}$. reedi,

$\underline{\mathrm{S}}$. ruberrimus, $\underline{\mathrm{s}}$. rubrivinctus, $\underline{\mathrm{s}}$. zacentrus 
COPEPODA (continued)

\section{LERNAEOPODIDAE (continued)}

Neobrachiella sciaenophila - Roncador stearnsi

Pseudocharopinus bicaudatus - Mustelus californicus, Squalus acanthias

Pseudocharopinus dentatus - Raja binoculata, R. rhina

Salmincola californiensis - Oncorhynchus keta, $\underline{0}$. kisutch,

0. nerka, $\underline{0}$. tshawytscha, Salmo gairdneri, Salvelinus malma

Salmincola carpionis - Oncorhynchus nerka, Salvelinus malma

Salmincola edwardsi i - Oncorhynchus nerka, Salmo clarki, s. gairdneri,

Salvelinus malma

Salmincola salmoneus - Oncorhynchus keta

Schistobrachia tertia - Raja binoculata, R. rhina

Thysanote ramosa - Xiphias gladius

LERNANTHROPIDAE

Lernanthropus giganteus - Caranx hippos

Lernanthropus hiatus - Thunnus alalunga

Lernanthropus mugili - Mugil cephalus

NAOBRANCHI IDAE

Naobranchia lizae - Mugil cephalus

Naobranchia occidental is - Anoplopoma fimbria, Gadus macrocephalus,

Hippoglossoides elassodon, Lepidopsetta bilineata, Myoxocephalus

polyacanthocephalus, Parophrys vetulus, Platichthys stellatus,

Sebastes aleutianus, $\underline{S}$. alutus, $\underline{\mathrm{s}}$. boreal is, $\underline{\mathrm{s}}$. carnatus, $\underline{\mathrm{s}}$. caurinus,

$\underline{\mathrm{S}}$. diploproa, $\underline{\mathrm{S}}$. maliger, $\underline{\mathrm{S}}$. miniatus, $\underline{\mathrm{S}}$. nigrocinctus, $\underline{\mathrm{S}}$. paucispinis,

S. pinniger, $\underline{S}$. proriger

Naobranchia wilsoni - Diodon hystrix

PANDARIDAE

Demoleus heptapus- Hexanchus griseus, Squalus acanthias

Demoleus latus - Squalus acanthias

Dinemoura discrepans - Alopias superciliosus, A. vulpinus

Dinemoura latifolia - Alopias vulpinus, Carcharodon carcharias, Isurus oxyrinchus, Lamna ditropis, Prionace glauca 
PANDARIDAE (continued)

Dinemoura producta - Alopias vulpinus, Carcharodon carcharias, Cetorhinus maximus, Isurus oxyrinchus, Lamna ditropis, Prionace glauca Echthrogaleus coleoptratus - Isurus oxyrinchus, Lamna ditropis, Mola mola, Squalus acanthias, Triakis semifasciata, Sphyrna zygaena

Echthrogaleus denticulatus - Alopias vulpinus, Carcharodon carcharias, Isurus oxyrinchus, Sphyrna zygaena

Gangliopus pyriformis - Alopias vulpinus, Isurus oxyrinchus, Prionace glauca

Nesippus borealis - Isurus oxyrinchus

Nesippus crypturus - Carcharodon carcharias, Carcharhinus leucas,

C. Iongimanus, Galeocerdo cuvieri, Sphyrna zygaena Nesippus gracilis - Carcharhinus obscurus

Nesippus orientailis - Carcharhinus leucas, C. obscurus, Carcharodon

carcharias, Galeocerdo cuvieri, Sphyrna tiburo, s. zygaena Nesippus tirgis - Galeocerdo cuvieri Nogagus ambiguus - Squalus acanthias Pagina tunica - Alopias superciliosus.

Pandarus bicolor - Galeorhinus zyopterus, Isurus oxyrinchus, Mola mola, Mustelus henlei, Notorynchus maculatus, Prionace glauca, Squalus acanthias, Triakis semifasciata Pandarus carcharini - Carcharhinus leucas

Pandarus cranchii - Alopias vulpinus, Carcharhinus leucas, $\underline{\text {. }}$ longimanus, $\underline{C}$. obscurus, Carcharodon carcharias, Galeocerdo cuvieri, Galeorhinus zyopterus, Sphyrna lewini, Sphyrna zygaena, Triakis semifasciata

Pandarus floridanus - Carcharodon carcharias

Pandarus katoi - Isurus oxyrinchus 
PANDARIDAE (continued)

Pandarus satyrus - Carcharhinus obscurus, Isurus oxyrinchus, Prionace glauca, Sphyrna zygaena

Pandarus sinuatus - Caranx hippos, Carcharhinus leucas, C. obscurus, Carcharodon carcharias, Sphyrna tiburo

Pandarus smithii - Alopias vulpinus, Carcharhinus leucas, C. obscurus, Carcharodon carcharias, Isurus oxyrinchus, Sphyrna zygaena

Pandarus spinaciiacanthiae - Squalus acanthias

Pannosus japonicus - Sphyrna zygaena

Perissopus dentatus - Carcharhinus leucas, $\underline{\text { C. obscurus, Sphyrna }}$ tiburo, $\underline{S}$. zygaena

*Perissopus oblongatus - Alopias vulpinus, Mustelus californicus, M. henlei, M. lunulatus, Notorynchus maculatus, Raja binoculata,

Remora remora, Rhinobatos productus, Sphyrna zygaena, Squalus acanthias, Triakis semifasciata

Phyllosthyreus cornutus - Isurus oxyrinchus, Prionace glauca, Sphyrna zygaena

Prosaetes rhinodontis - Rhincodon typus

PENNELLIDAE

Cardiodectes medusaeus - Diaphus theta, Lampanyctus ritteri, Leuroglossus stilbius, Lycodapus mandibularis, Stenobrachius leucopsarus

Haemobaphes sp. - Agonus acipenserinus, Ammodytes hexapterus,

Blepsias cirrhosus, Hexagrammos decagrammus, Lepidopsetta bilineata, Merluccius productus, 0ligocottus maculosus,

Syngnathus leptorhynchus, Triglops pingeli

Haemobaphes diceraus - Cymatogaster aggregata, Thaleichthys pacificus, Theragra chalcogramma

Haemobaphes disphaerocephalus - Thaleichthys pacificus Haemobaphes enodis - Lycodapus fierasfer Haemobaphes intermedius - Artedius harringtoni, 01 igocottus maculosus 
PENNELLIDAE (continued)

* Haemobaphes theragrae - Sebastes alutus, $\underline{\mathrm{S}}$. zacentrus, Theragra chal cogramma

Lernaeenicus gracilis - Mugil cephalus

Lernaeenicus longiventris - Caranx hippos, Coryphaena hippurus, Mugil cephalus, Seriola dorsalis

Lernaeenicus vorax - Mugil cephalus

Lernaeenicus sprattae - Clupea harengus

Lernaeocera branchial is - Scomber japonicus

Lernaeolophus sultanus - Xiphias gladius

Peniculus sp. - Sebastes alutus, $\underline{\text { S. babcocki, }} \underline{\mathrm{S}}$. brevispinis, $\underline{\mathrm{S}}$. crameri, $\underline{\mathrm{s}}$. proriger, $\underline{\mathrm{S}}$. reedi

Peniculus asinus - Sebastes brevispinis, $\underline{S}$. flavidus, $\underline{S}$. proriger Peniculus clavatus - Triglops pingeli

Peniculus fissipes - Atherinops affinis, Cymatogaster aggregata, Embiotoca jacksoni, Girella nigricans, Hypsurus caryi, Hypsypops rubicunda, Medialuna californiensis, Paralabrax clathratus, . nebulifer, Phanerodon furcatus

Pennella sp. - Cololabis saira, Tetrapturus audax, Thunnus albacares, Xiphias gladius

* Pennella costai - Xiphias gladius

Pennella crassicornis - Mola mola, Thunnus albacares, Xiphias gladius Pennella filosa - Istiophorus platypterus, Mola mola, Thunnus alalunga, I. albacares, I. thynnus, Xiphias gladius

Pennella instructa - Xiphias gladius

Pennella remorae - Remora remora

Phrixocephalus cincinnatus - Atheresthes stomias, Citharichthys sordidus, Parophrys vetulus, Pleuronichthys decurrens 
COPEPODA (continued)

\section{PHILICHTHYIDAE}

Colobomatus embiotocae - Amphistichus argenteus, A. koelzi,

Cymatogaster aggregata, Damalichthys vacca, Embiotoca jacksoni,

E. lateralis, Hyperprosopon anale, $\underline{H}$. argenteum, Hypsurus caryi,

Micrometrus minimus, Rhacochilus toxotes, Zalembius rosaceus

Colobomatus kyphosus - Sebastes aleutianus, $\underline{S}$. alutus, $\underline{S}$. babcocki,

$\underline{\mathrm{S}}$. borealis, $\underline{\mathrm{S}}$. brevispinis, $\underline{\mathrm{S}}$. caurinus, $\underline{\mathrm{S}}$.

crameri, $\underline{S}$. diploproa, $\underline{S}$. elongatus, $\underline{S}$. entomelas, $\underline{S}$. flavidus,

$\underline{S}$. maliger, $\underline{\mathrm{S}}$ nigrocinctus, $\underline{\mathrm{S}}$. pinniger, $\underline{\mathrm{S}}$. proriger, $\underline{\mathrm{S}}$. reedi,

S. ruberrimus, $\underline{S}$. zacentrus

Philichthys xiphiae - Xiphias gladius

Sarcotaces sp. - $\underline{s}$. chlorostictus

Sarcotaces arcticus - Sebastes aleutianus, $\underline{\mathrm{s}}$. alutus, $\underline{\mathrm{s}}$. brevispinis,

S. crameri, $\underline{S}$. flavidus, $\underline{S}$. ruberrimus

Sarcotaces komaii - Antimora microlepis

Sarcotretes inflexus - Scomber japonicus

\section{PSEUDOCYCNIDAE}

Pseudocycnus appendiculatus - Euthynnus affinis, E. pelamis,

Paralabrax maculatofasciatus, Sarda chiliensis, Thunnus alalunga,

I. albacares, T. obesus, T. thynnus

Pseudocycnoides buccata - Scomberomorus sierra

SPHYRIIDAE

Opimia exilis - Carcharhinus obscurus, Galeorhinus zyopterus

Paeon elongatus - Carcharhinus obscurus, Triakis semifasciata

Paeon ferox - Sphyrna tiburo

Paeon vaissierei? - Sphyrna lewini

THERODAMASIDAE

Paeonodes nemaformis - Oncorhynchus tshawytscha

TREBIIDAE

Trebius sp. - Mola mola

Trebius caudatus - Myliobatis californica, Squalus acanthias

Trebius latifurcatus - Gymnura marmorata, Myliobatis californicus, 
COPEPODA (continued)

TREBIIDAE (continued)

Paralichthys californicus, Urolophus halleri

Trebius tenuifurcatus - Urolophus halleri

\section{TUCCIDAE}

Tucca impressus - Diodon hystrix 
CRUSTACEA

\section{LEPADIDAE}

Conchoderma auritum - Cetorhinus maximus

Conchoderma virgatum - Diodon hystrix, Mola mola, Tetrapturus audax, Xiphias gladius 


\section{DIGENEA}

ACANTHOCOLPIDAE

Acanthocolpoides pauloi - Scomber japonicus

Manteria brachyderus - Caranx hippos, 01igoplites saurus

Pleorchis californiensis - Cynoscion nobilis

Pleorchis magniporus - Cynoscion nobilis, c. parvipinnis

Stephanostomum sp. - Atheresthes stomias, Cymatogaster aggregata, Hyperprosopon argenteum, Leptocottus armatus, Lythrypnus dal1i, Mugil cephalus, 01 igoplites saurus, Rhacochilus toxotes

Stephanostomum baccatum - Anoplopoma fimbria, Gadus macrocephalus, Hippoglossoides elassodon, Lepidopsetta bilineata, Mola mola,

Reinhardtius hippoglossoides, Sebastes borealis

Stephanostomum californicum - Anoplopoma fimbria, Artedius harringtoni,

Genyonemus lineatus, Sebastes caurinus, S. maliger, Umbrina roncador

Stephanostomum casum - Ophiodon elongatus, Sebastes sp.

Stephanostomum coryphaenae - Coryphaena hippurus

Stephanostomum dentatum - Dasycottus setiger, Embiotoca jacksoni,

Paralichthys californicus, Sebastes aleutianus, s. babcocki, $\underline{\text { s. }}$ borealis

Stephanostomum ditrematis - Caranx hippos

Stephanostomum filiforme - Caranx caballus

Stephanostomum hispidum - Caranx hippos, Seriola dorsal is

Stephanostomum imparispine - Echeneis naucrates

Stephanostomum longisomum - Caranx hippos

Stephanostomum megacephalum - Caranx hippos

Stephanostomum microstephanum - Epinephelus niveatus

Stephanostomum naucrotis - Naucrates ductor

Stephanostomum provitellosum - Balistes polylepis

Stephanostomum tenue - Morone saxatilis

Stephanostomum tristephanum - Ophiodon elongatus, Scomber japonicus Tormopsolus echenei - Echeneis naucrates 


\section{ACCACOELIIDAE}

Genera and species - Naucrates ductor

Accacladocoel ium alveolatum - Mola mola

Accacladocoel ium macrocotyle - Mola mola

Accacladocoelium nigroflavum - Mola mola

Accacladocoelium petasiporum - Mola mola

Accacladocoel ium serpentulum - Mola mola

Accacoelium contortum - Mola mola

Odhnerium calyptrocotyle - Mola mola

Orophocotyle divergens - Ranzania laevis

Orophocotyle planci - Mola mola, Ranzania laevis

Rhyncopharynx paradoxa - Mola mola

Tetrochetus coryphaenae - Coryphaena hippurus, Diodon hystrix

Tetrochetus proctocolus - Sphoeroides annulatus, Trachinotus rhodopus

Tetrochetus rayneri = Luvarus imperialis

ALBULATREMATIDAE

Albulatrema ovale - Albula vulpes

\section{ALLOCREADI IDAE}

Allobunodera mediovitellata - Gasterosteus aculeatus

Allocreadium lobatum - Salmo clarki, s. gairdneri

Anisorchis opisthorchis - Hemilepidotus hemilepidotus, Hexagrammos

lagocephalus, $H$. stelleri, Sebastes aleutianus, Theragra chalcogramma

Anisorchis zhukovi - Hexagrammos lagocephalus

Bunodera luciopercae - Gasterosteus aculeatus, Salmo gairdneri,

Salvelinus malma

Bunoderina eucaliae - Gasterosteus aculeatus

Cainocreadium gasterostei - Gasterosteus aculeatus

Crepidostomum canadense - Salmo gairdneri

Crepidos tomum cooperi - Gasterosteus aculeatus, Salmo clarki, s. gairdneri

Crepidostomum cornutum - Gasterosteus aculeatus 
ALLOCREADIIDAE (continued)

Crepidostomum farionis - Gasterosteus aculeatus, Oncorhynchus kisutch, $\underline{0}$. nerka, Salmo clarki, $\underline{\text { S. gairdneri }}$ Salvel inus ma7ma

Crepidostomum metoecus - Salmo gairdneri, Salvelinus malma Crepidostomum salmonis - Oncorhynchus keta Crepidostomum transmarinum - Salmo clarki Crepidostomum ussuriensis - Oncorhynchus gorbuscha Liliatrema skrjabini - Blepsias cirrhosus

Neophasis oculata - Hexagrammos lagocephalus, $\underline{H}$. stelleri, Myoxocephalus polyacanthocephalus, Salvelinus malma

Pseudocreadium galapagoense - Balistes polylepis

Pseudozoogonoides microacetabulum - Hippoglossoides elassodon APOCREADI IDAE

Apocreadium longisinosum - Sphoeroides annulatus

\section{AZYGIIDAE}

Azygia lucii - Salmo gairdneri, Salvelinus malma Azygia robusta - Oncorhynchus tshawytscha Otodis tomum hydrolagi - Hydrolagus colliei Otodistomum plicatum - Hexanchus griseus Otodistomum scymni - Hexanchus griseus Otodistomum veliporum - Citharichthys sordidus, Eopsetta jordani, Hexanchus griseus, Lepidopsetta bilineata, Parophrys vetulus, Raja binoculata, R. rhina, Squalus acanthias, Squatina californica BATHYCOTYLIDAE

Bathycotyle branchialis - Coryphaena hippurus Bathycotyle coryphaenae - Coryphaena hippurus

\section{BIVESICULIDAE}

Paucivitellosus fragilis - Mugil cephalus 
BOTULIDAE

Botulus alepidosauri - Alepisaurus ferox

BOTUL ISACCIDAE

Botulisaccus pisceus - Albula vulpes

BUCEPHALIDAE

Bucephalus sp. - Ophiodon elongatus

Bucephalus heterotentaculatus - Mycteroperca xenarcha, Paralichthys

californicus, Scomberomorus sierra

Bucephalus introversus - Caranx hippos, Seriola dorsal is

Bucephalus polymorphus - Clupea harengus

Bucephalus varicus - Caranx hippos

Dollfus trema cal iforniae - Gymnothorax mordax

Prosorhynchoides sp. - Damalichthys vacca

Prosorhynchoides arcuata - Caranx hippos, Carcharhinus obscurus

Prosorhynchoides basargini - Oncorhynchus gorbuscha, Platichthys stellatus

Prosorhynchoides cybii - Scomberomorus sierra

Prosorhynchoides gracilescens - Auxis thazard, Caranx hippos, Clupea

harengus, Hippoglossoides elassodon, Lepidopsetta bilineata,

Oncorhynchus gorbuscha, $\underline{0}$. keta, $\underline{0}$. nerka, $\underline{0}$. tshawytscha, Platichthys stellatus, Reinhardtius hippoglossoides, Salvel inus malma, Theragra chal cogramma

Prosorhynchoides labiatus - Leuresthes tenuis, Paralichthys californicus

Prosorhynchoides ozakij - Leptocottus armatus, Salvelinus malma

Prosorhynchoides sibi - Thunnus thynnus

Prosorhynchus sp. - Auxis thazard, Damalichthys vacca, Hexagrammos

lagocephalus, $\underline{H}$. stelleri, Sebastes alutus, $\underline{\text { S. melanops, }} \underline{\mathrm{S}}$.

pinniger

Prosorhynchus apertus - Ophiodon elongatus

Prosorhynchus crucibulum - Atheresthes stomias, Gadus macrocephalus,

Menticirrhus undulatus, Myoxocephalus polyacanthocephalus,

Pleurogrammus monopterygius, Polydactylus opercularis, Salvelinus

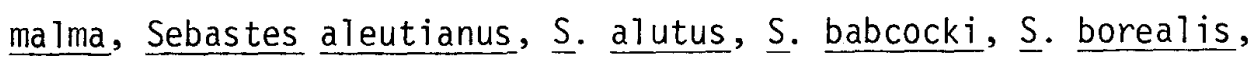


BUCEPHALIDAE (continued)

$\underline{\mathrm{S}}$. brevispinis, $\underline{\mathrm{S}}$. crameri, $\underline{\mathrm{S}}$. diploproa, $\underline{\mathrm{S}}$. elongatus, $\underline{\mathrm{S}}$.

entomelas, $\underline{\text { S. flavidus, }}$ S. paucispinis, $\underline{\text { S. pinniger, }}$

$\underline{\mathrm{S}}$. $\underline{\text { reedi }}, \underline{\mathrm{S}}$. ruberrimus, $\underline{\mathrm{S}}$. zacentrus, Theragra chalcogramma

Prosorhynchus facilis - Ophiodon elongatus

Prosorhynchus gonoderus - Epinephelus analogus

Prosorhynchus ozakii - Epinephelus analogus, E. niveatus, Mycteroperca xenarcha

Prosorhynchus pacificum - Mycteroperca xenarcha

Prosorhynchus scapellus - Liparis callyodon, Scorpaenichthys

marmoratus

Prosorhynchus squamatus - Enophrys bison, Squalus acanthias

Prosorhynchus uniporus - Acanthogobius flavimanus

Rhipidocotyle sp. - Damalichthys vacca

Rhipidocotyle capitata - Auxis rochei, A. thazard

Rhipidocotyle elongata - Leptocottus armatus, Ophiodon elongatus

Rhipidocotyle heptathelata - Euthynnus affinis

Rhipidocotyle lepisostei - Mugil cephalus

Rhipidocotyle pentagonum - Auxis thazard, Euthynnus affinis, Thunnus thynnus

Rhipidocotyle septpapillata - Euthynnus affinis

CEPHALOPORIDAE

Plectognathotrema hydrolagi - Hydrolagus colliei

CLINOSTOMATIDAE

Clinostomum marginatum - Alosa sapidissima, Dorosoma petenense,

Morone saxatilis, Salmo clarki, s. gairdneri

COITOCAECIDAE

Nicolla extrema - Scomber japonicus

CRYPTOGONIMIDAE

Claribulla longula - Albuta vulpes

Cryptogonimus sp. - Acanthogobius flavimanus 
CRYPTOGONIMIDAE (continued)

Exorchis oviformis - Mugil cephalus

Metadena caballeroi - Liparis callyodon

Metadena magdalenae - Paralichthys californicus

Neochasmus sp. - Hyperprosopon argenteum

Neochasmus sogandaresi - Morone saxatilis

CYATHOCOTYLIDAE

Holostephanus luhei - Gasterosteus aculeatus

Mesostophenus appendiculatoides - Mugil cephalus

Mesostephanus appendiculatus - Fundulus parvipinnis, Gillichthys mirabilis

DEROPROSTIDAE

Cestrahelmins rivularis - Acipenser transmontanus

DIDYMOZOIDAE

Genera and species - Argyropelecus pacificus, Cololabis saira, Coryphaena hippurus, Engraul is mordax, Gillichthys mirabil is, Leuresthes tenuis, Oncorhynchus kisutch, Remora remora, Stenobrachius leucopsarus

Adenodidymocystis intestinal is - Euthynnus pelamis

Al lometanema tobothrioides lepidocybii - Lepidocybium flavobrunneum

Allonema tobothrioides scombri - Scomber japonicus

Annulocystis auxis - Auxis thazard

Atalostrophion sardae - Thunnus albacares

Coeliodidymocystis abdominal is - Euthynnus pelamis

Coeliodidymocystis kamegaii - Euthynnus pelamis

Coeliotrema thynni - Thunnus thynnus

Colocyntotrema sp. - Istiophorus platypterus, Makaira nigricans

Colocyntotrema auxis - Auxis thazard

Dermatodidymocystis vivipara - Thunnus obesus

Derma todidymocystis viviparoides - Thunnus obesus

Didymocylindrus filiformis - Euthynnus pelamis, Thunnus thynnus 
DIDYMOZOIDAE (continued)

Didymocylindrus simplex - Euthynnus pelamis

Didymocystis sp. - Thunnus albacares

Didymocystis bifurcata - Thunnus obesus

Didymocystis bilobata - Euthynnus pelamis

Didymocystis coatesi - Thunnus albacares

Didymocystis crassa - Thunnus thynnus

Didymocystis dissimilis - Auxis thazard

Didymocystis lanceolata - Thunnus alalunga

Didymocystis macrorchis - Thunnus alalunga

Didymocystis nasalis - Thunnus obesus

Didymocystis orbitalis - Thunnus obesus

Didymocystis ovata - Euthynnus pelamis, Thunnus thynnus

Didymocystis philobranchia - Thunnus alalunga, I. obesus

Didymocystis philobranchiarca - Thunnus obesus

Didymocystis poonui - Thunnus obesus

Didymocystis reniformis - Euthynnus pelamis, Thunnus thynnus

Didymocystis soleiformis - Euthynnus pelamis, Thunnus thynnus

Didymocystis thynni - Thunnus alalunga

Didymocystis wedl $i$ - Euthynnus pelamis, Thunnus alalunga, I. albacares,

I. thynnus

Didymocystoides alalongae - Thunnus alalunga

Didymocystoides bifasciatus - Thunnus obesus

Didymocystoides buccalis - Thunnus alalunga

Didymocystoides intestinomuscularis - Euthynnus pelamis

Didymocys toides opercularis - Thunnus alalunga

Didymocys toides pectoralis - Thunnus obesus

Didymocys toides pinnicola - Euthynnus pelamis

Didymocys toides semiglobularis - Thunnus thynnus

Didymocystoides submentalis - Euthynnus pelamis

Didymoproblema fusiforme - Euthynnus pelamis, Thunnus thynnus

Didymosphaera mirabilis - Auxis thazard

Didymozoides sp. - Echeneis naucrates 
DIDYMOZOIDAE (continued)

Didymozoon sp. - Auxis rochei, Scomber japonicus

Didymozoon auxis - Auxis rochei, Auxis thazard

Didymozoon filicalle - Euthynnus pelamis, Thunnus thynnus

Didymozoon longicolle - Euthynnus pelamis, Scomber japonicus, Thunnus

albacares, I. obesus, I. thynnus

Didymozoon minus - Euthynnus pelamis

Didymozoon molae - Mola mola

Didymozoon pretiosus - Thunnus thynnus

Epithel ionematobothrium fragile - Mugil cephalus

Koellikeria abdominalis - Thunnus albacares

Koellikeria bipartita - Thunnus alalunga, I. albacares, I. thynnus

Koellikeria filicollis - Mola mola

Koellikeria globosa - Euthynnus pelamis, Thunnus albacares, I. thunnus

Koellikeria orientalis - Euthynnus pelamis, Thunnus alalunga, I.

al bacares, $I$. thynnus

Koellikeria pylorica - Thunnus obesus

Koellikeria reniformis - Euthynnus pelamis, Thunnus thynnus

Koellikeria retrorbital is - Thunnus obesus

Koellikeria submaxillaris - Thunnus obesus

Koellikerioides apical is - Thunnus obesus

Koellikerioides externogastricus - Thunnus obesus

Koellikerioides internogastricus - Thunnus obesus

Koellikerioides intestinalis - Thunnus obesus

Lagenocystis katsuwoni - Euthynnus pelamis

Lamprididymozoon lampridis - Lampris regius

Lobatozoum multisacculatum - Auxis thazard, Euthynnus pelamis,

Thunnus thynnus

Maccalumtrema xiphiados - Xiphias gladius

Metadidymocystis cymbiformis - Lampris regius

Metanema tobothrium bivitellatum - Auxis thazard, Euthynnus affinis

Metanema tobothrium guernei - Thunnus alalunga

Nema tobibothrioides histoidi - Mola mola

Nematobothrium sp. - Euthynnus affinis, Thunnus thynnus 
DIGENEA (continued)

DIDYMOZOIDAE (continued)

Nematobothrium filliforme - Scomber japonicus

Nema tobothrium latum - Thunnus alalunga

Nema tobothrium molae - Mola mola

Nematobothrium robustum - Scomber japonicus

Nematobothrium scombrii - Scomber japonicus

Neodiplotrema pelamydis - Euthynnus pelamis

Neonema tobothrioides poonui - Thunnus obesus

Neounitubulotestis orbitarium - Ruvettus pretiosus

Oesophagocystis dissimilis - Auxis thazard, Euthynnus affinis,

E. pelamis, Thunnus thynnus

Opepherotrema planum - Auxis thazard

Opisthorchinematobothrium parathunni - Thunnus obesus

Orbitonematobothrium perioculare - Thunnus obesus

Phacelotrema claviforme - Auxis thazard

Platocystis sp. - Thunnus albacares

Platocystis alalongae - Thunnus ala lunga

Reniforma multilobularis - Xiphias gladius

Unitubulotestis maris - Sarda chiliensis

Univitellannulocystis katsuwoni - Euthynnus pelamis

Univitellodidymocystis neothunni - Thunnus obesus

\section{DIPLANGIDAE}

Diplangus macrovitellus - Cymatogaster aggregata, Damalichthys vacca,

Embiotoca jacksoni, E. lateralis, Phanerodon furcatus

Diplangus mexicanus - Cynoscion parvipinnis

Diplangus paxillus - Embiotoca jacksoni, Phanerodon furcatus

Diplangus triradiatus - Amphistichus argenteus, Menticirrhus undulatus,

Roncador stearnsi, Umbrina roncador

DIPLOSTOMATIDAE

Diplostomum sp. - Gasterosteus aculeatus, Morone saxatilis, Oncorhynchus

kisutch, $\underline{0}$. nerka, Salmo clarki

Diplostomum clavatum - Gasterosteus aculeatus 
DIPLOSTOMATIDAE (continued)

Diplostomum gasterostei - Gasterosteus aculeatus

Diplos tomum gobiorum - Gasterosteus aculeatus

Diplostomum pungitius - Gasterosteus aculeatus

Diplostomum scudderi - Gasterosteus aculeatus

Diplostomum spathaceum - Gasterosteus aculeatus, Oncorhynchus nerka, Salmo gairdneri

Neascus sp. - Gasterosteus aculeatus, Morone saxatilis, Salmo clarki

Neascus brevicaudatus - Gasterosteus aculeatus

Posthodiplostomum brevicaudatum - Gasterosteus aculeatus

Posthodiplostomum cuticola - Gasterosteus aculeatus

Posthodiplostomum minimum - Fundulus parvipinnis, Gasterosteus

aculeatus, Salmo clarki

Spathaceum indistinctum - Oncorhynchus kisutch, Salmo gairdneri

Tetracotyle sp. - Gasterosteus aculeatus, Oncorhynchus nerka

Tylodelphys clavata - Gasterosteus aculeatus, Salmo gairdneri

Tylodelphys podicipina - Salmo gairdneri

ECHINOSTOMATIDAE

Echinochasmus milvi - Salmo gairdneri

Patasiger cf. pungens - Gasterosteus aculeatus

FELLODISTOMATIDAE

Genera and species - Ceratoscopelus townsendi

Discogasteroides minor - Ostracion diaphanum

Discogasteroides ostracii - Ostracion diaphanum

Faustula gasterostei - Gasterosteus aculeatus

Fellodistomum brevum - Microstomus pacificus

Fellodistomum furcigerum - Anoplopoma fimbria, Hippoglossoides

elassodon, Lepidopsetta bilineata, Merluccius productus,

Pleuronichthys decurrens, Reinhardtius hippoglossoides, Sebastes

aleutianus, $\underline{S}$. borealis, Theragra chalcogramma

Fellodistomum melanostigum - Melanostigma pammelas

Fellodistomum phrissovum - Parophrys vetulus 
FELLODISTOMATIDAE (continued)

Fellodistomum sebastodis - Sebastes alutus, $\underline{S}$. elongatus, $\underline{S}$. ruberrimus, $\underline{S}$. zacentrus

Hexagrammia Tongitestis - Hexagrammos decagrammus

Hexagrammia zhukovi - Pleurogrammus monopterygius

Lintonium novikovi - Cololabis saira

Lintonium srivastavi - Sphoeroides annulatus

Megalomyzon robustus - Diodon hystrix

Monascus typicus - Caranx hippos

Pseudopentagramma petrowi - Clupea harengus, Gadus macrocephalus, Hexagrammos lagocephalus, Hypomesus pretiosus, Mallotus villosus, Oncorhynchus gorbuscha, $\underline{0}$. nerka, Salvelinus malma, Spirinchus thaleichthys

Stenakron problematicum - Myoxocephalus polyacanthocephalus

Stenakron quinquelobatum - Hippoglossoides elassodon

Stenakron vetustum - Gadus macrocephalus, Hippoglossides elassodon,

Hippoglossus stenolepis, Lepidopsetta bilineata, Platichthys

stellatus, Reinhardtius hippoglossoides

Steringotrema ovacutum - Hippoglossoides elassodon, Lepidopsetta bilineata

Tergestia acanthocepha1a - Scomber japonicus

Tergestia acanthogobii - Acanthogobius flavimanus, Auxis thazard

Tergestia laticollis - Auxis thazard, Caranx caballus, Euthynnus

pelamis, Scomber japonicus

Tergestia pectinata - Auxis rochei, Caranx hippos, 01igoplites saurus

GASTEROSTOMIDAE

Gasterostomum capitatum - Auxis rochei

GORGODERIDAE

Phyllodistomum sp. - Fundulus parvipinnis, Sebastes caurinus, $\underline{\mathrm{s}}$.

diploproa, $\underline{\text { S. ruberrimus }}$

Phyllodistomum borisbychowski - Gibbonsia metzi 
DIGENEA (continued)

GORGODERIDAE (continued)

Phyllodistomum conos tomum - Oncorhynchus nerka

Phyllodistomum folium - Gasterosteus aculeatus

Phyllodistomum lachancei - Salmo gairdneri

Phyllodistomum lancea - Auxis thazard, Euthynnus affinis

Phyllodistomum mirandae - Sphoeroides annulatus

Phyllodistomum scrippsi - Pimelometopon pulchrum

Phyllodistomum thunni - Thunnus albacares

Probolitrema californiense - Myliobatis californica

Probolitrema richiardi - Mustelus lunulatus, Platyrhinoidis

triseriata, Rhinobatos productus, Squalus acanthias

HAPLOPORIDAE

Carassotrema mugilicola - Mugil cephalus

Dicrogaster fastigatus - Mugil cephalus

Haploporus benedeni - Mugil cephalus

Haploporus lateral is - Mugil cephalus

Haploporus longibollum - Mugil cephalus

Isorchis manteri - Mugil cephalus

Lecithobothrys putrescens - Mugi 1 cephalus

Lecithobothrys sprenti - Mugil cephalus

Paralecithobotrys brisbanensis - Mugil cephalus

Paraunisaccoides lobolecithus - Mugil cephalus

Saccacoelium obesum - Mugil cephalus

Saccacoelium tensum - Mugil cephalus

Saccacoelioides beauforti - Mugil cephalus

Saccacoelioides pearsoni - Mugil cephalus

Unisaccoides vitellosus - Mugil cephalus

Unisaccus brisbanensis - Mugil cephalus

Vitellibaculum girellicola - Girella nigricans

Vitellibaculum girellae - Girella nigricans

HAPLOSPLANCHNIDAE

Haplosplanchnus mugilis - Mugil cephalus 
HAPLOSPLANCHNIDAE (continued)

Hymenocotta mulli - Mugil cephalus

Hymenocottoides manteri - Mugil cephalus

Schikhobalotrema sp. - Mugil cephalus

Schikhobalotrema elongatum - Mugil cephalus

Schikhobalotrema girellae - Girella nigricans, Hyperprosopon argenteum,

Micrometrus minimus

Schikhobalotrema magnum - Mugil cephalus

Schikhobalotrema sparisomae - Mugil cephalus

HEMIURIDAE

Genera and species - Ceratoscopelus townsendi, Cololabis saira,

Icel inus tenuis, Melamphaes acanthomus, Scomber japonicus, Scopelogadus mizolepis bispinosus

Aphanurus sp. - Mugil cephalus

Aphanurus stossichi - Mugil cephalus

Aponurus sp. - Melanostigma pammelas, salmo gairdneri

Aponurus argentini - Merluccius productus

Aponurus californicus - Leuroglossus stilbius, Melanostigma pammelas

Aponurus lagunculus - Thunnus thynnus

Aponurus pacificus - Melanostigma pammelas

Aponurus trachinoti - Porichthys $\mathrm{sp}$.

Brachadena pyriformis - Anisotremus davidsoni, Porichthys sp.

Brachyphallus amuriensis - Clupea harengus, Oncorhynchus gorbuscha,

0. keta

Brachyphallus crenatus - Blepsias cirrhosus, Clupea harengus,

Cololabis saira, Gadus macrocephalus, Gasterosteus aculeatus,

Hippoglossoides elassodon, Hippoglossus stenolepis, Lepidopsetta

bilineata, Oncorhynchus gorbuscha, $\underline{0}$. keta, $\underline{0}$. kisutch, $\underline{0}$. nerka,

0. tshawytscha, Reinhardtius hippoglossoides, Remora remora,

Salvelinus malma, Sebastes boreal is, Theragra chalcogramma

Brachyphal Tus parvus - Auxis thazard, Thunnus albacares

Bunocotyle mugilis - Mugil cephalus

Derogenes sp. - Oncorhynchus kisutch, ‥tshawytscha, Salmo clarki,

S. gairdneri 
HEMIURIDAE (continued)

Derogenes crassus - Ophiodon elongatus, Sebastes paucispinis Derogenes infirmus - Oncorhynchus tshawytscha Derogenes macrostoma - Merluccius productus Derogenes varicus - Anoplopoma fimbria, Blepsias Cirrhosus, Citharichthys sordidus, Clinocottus embryum, Clupea harengus, Gadus macrocephalus, Gasterosteus aculeatus, Hemilepidotus hemilepidotus, Hexagrammos lagocephalus, $\underline{H}$. stelleri, Hippoglossoides elassodon, Hippoglossus stenolepis, Isopsetta isolepis, Lepidopsetta bilineata, Leptocottus armatus, Lyopsetta exilis, Merluccius productus, Microgadus proximus, Myoxocephalus polyacanthocephalus, Oncorhynchus gorbuscha, $\underline{0}$. nerka, Ophiodon elongatus, Platichthys stellatus, Porichthys notatus, Reinhardtius hippoglossoides, Ronquilus jordani, Salvelinus malma, Sebastes aleutianus, s. alutus, $\underline{\mathrm{S}}$. babcocki, $\underline{\mathrm{s}}$. borealis, $\underline{\mathrm{S}}$. brevispinis, $\underline{\mathrm{S}}$. caurinus, $\underline{\mathrm{s}}$. crameri, $\underline{\mathrm{S}}$. diploproa, $\underline{\mathrm{s}}$. elongatus, $\underline{\mathrm{S}}$. entomelas, $\underline{\mathrm{S}}$. flavidus, $\underline{\mathrm{s}}$. goodei, $\underline{\mathrm{S}}$. helvomaculatus, $\underline{\mathrm{S}}$. maliger, $\underline{\mathrm{S}}$. paucispinis, $\underline{\mathrm{S}}$. pinniger,

$\underline{\mathrm{S}}$. proriger, $\underline{\mathrm{S}}$. reedi, $\underline{\mathrm{S}}$. ruberrimus, $\underline{\mathrm{S}}$. zacentrus, squalus acanthias, Theragra chalcogramma

Derogenoides sp. - Damalichthys vacca

Deropegus aspina - Oncorhynchus kisutch, $\underline{0}$. tshawytscha, Salmo clarki, s. gairdneri

Dichadena galeata - Mugil cephalus

Dinosoma oregonensis - Coryphaenoides acrolepis, $\underline{C}$. pectoralis Dinosoma pectoral is - Coryphaenoides acrolepis, $\underline{C}$. pectoral is Dinosoma tortum - Anoplopoma fimbria, Sebastes aleutianus, S. borealis Dinurinae - Auxis thazard

Dinurus barbatus - Coryphaena hippurus, Paralabrax maculatofasciatus Dinurus breviductus - Coryphaena hippurus

Dinurus euthynni - Auxis thazard, Euthynnus affinis, E. pelamis

Dinurus longisinus - Coryphaena hippurus

Dinurus scombri - Istiophorus platypterus, Scomber japonicus Dinurus tornatus - Coryphaena hippurus 
HEMIURIDAE (continued)

Dissosaccus sp.? - Melanostigma pammelas

Dissosaccus laevis - Glyptocephalus zachirus

Ectenurus americanus - Caranx hippos

Ectenurus lepidocybii - Lepidocybium flavobrunneum

Ectenurus lepidus - 01 igoplites saurus, Scomber japonicus

Ectenurus virgulus - Caranx hippos

Ectenurus yamagutii - Caranx hippos

Elytorphallus mexicanus - Paralabrax clathratus

Genarchopsis muelleri - Hexagrammos lagocephalus, H. stelleri,

Hippoglossoides elassodon, Hippoglossus stenolepis, Lepidopsetta

bilineata, Myoxocephalus polyacanthocephalus, Oncorhynchus

gorbuscha, $\underline{0}$. keta, $\underline{0}$. nerka, Platichthys stellatus, Reinhardtius

hippoglossoides, Salvelinus malma, Squalus acanthias

Genolinea aburame - Pleurogrammus monopterygius

Genolinea anura - Blepsias cirrhosus, Hexagrammos lagocephalus, $\underline{H}$.

stelleri, Myoxocephalus polyacanthocephalus, Oncorhynchus

gorbuscha, Salvelinus malma, Theragra chalcogramma

Genolinea laticauda - Artedius fenestralis, Blepsias cirrhosus,

Clinocottus analis, C. globiceps, Enophrys bison, Gibbonsia sp.,

Hemilepidotus hemilepidotus, Hexagrammos decagrammus, $\underline{H}$. lagocephalus,

Isopsetta isolepis, Leptocottus armatus, Liparis callyodon,

Myoxocephalus polyacanthocephalus, Nautichthys oculofasciatus

01igocottus maculosus, Oncorhynchus nerka, Ophiodon elongatus,

Parophrys vetuTus, Scorpaenichthys marmoratus

Genolinea lintoni - Oncorhynchus tshawytscha

Genolinea manteri - Dasycottus setiger, Enophrys bison, Leptocottus

armatus, Lumpenus sagitta

Genolinea montereyensis - Clinocottus analis, Leptocottus armatus

Genolinea oncorhynchi - Oncorhynchus gorbuscha

Genolinea tanyopa - Hypsypops rubicunda, Medialuna californiensis

Glomericirrus ulmeri - Coryphaenoides armatus, $\underline{\text { C. }}$ leptolepis

Gonocerca sp. - Thunnus albacares

Gonocerca haedrichi - Coryphaenoides armatus 
HEMIURIDAE (continued)

Gonocerca oregonensis - Coryphaenoides filifer, $\underline{C}$. pectoral is,

Parabassogigas grandis

Gonocerca oshoro - Coryphaenoides pectoral is

Gonocerca phycidis - Coryphaenoides armatus

Gonocercella pacifica - Irachinotus rhodopus

Gonocercella trachinoti - Albula vulpes

Hemiurus communis - Theragra chalcogramma

Hemiurus levinseni - Clupea harengus, Gadus macrocephalus, Hexagrammos

stelleri, Hippoglossoides elassodon, Hippoglossus stenolepis,

Lepidopsetta bilineata, Microgadus proximus, Myoxocephalus

polyacanthocephalus, Oncorhynchus gorbuscha, $\underline{0}$. keta, $\underline{0}$. kisutch,

0. nerka, $\underline{0}$. tshawytscha, Ophiodon elongatus, Reinhardtius,

hippoglossoides, Salvel inus malma, Sebastes caurinus, $\underline{\text { s. ruberrimus, }}$

Squaius acanthias, Theragra chalcogramma

Hemiurus luehei - Clupea harengus

Hemiurus odhneri - Theragra chalcogramma

Hysterolecitha elongata - Mugil cephalus

Hysterolecitha trilocalis - Gillichthys mirabilis

Intuscirrus aspicotti - Enophrys bison

Lampritrema hawaiiense - Lampris regius

Lampritrema nipponicum - Lampris regius

Lecithaster confusus - Clupea harengus

Lecithaster gibbosus - Ammodytes hexapterus, Anoplarchus purpurescens,

Aprodon cortezianus, Atheresthes stomias, Aulorhynchus flavidus,

Blepsias cirrhosus, Brachyistius frenatus, Clinocottus acuticeps,

C. embryum, $\underline{\text {. }}$ globiceps, Clupea harengus, Cololabis saira,

Coryphopterus nicholsi, Cymatogaster aggregata, Dasycottus

setiger, Gadus macrocepha]us, Gasterosteus aculeatus, Hemilepidotus

hemilepidotus, Hexagrammos lagocephalus, $\underline{H}$. stelleri, Hippoglossoides

elassodon, Hypomesus pretiosus, Lepidopsetta bilineata, Leptocottus

armatus, Liparis pulchellus, Lumpenus sagitta, Lyconectes aleutens is

Mallotus villosus, Myoxocephalus polyacanthocephalus, Nautichthys 
HEMIURIDAE (continued)

oculofasciatus, 01igocottus maculosus, $\underline{0}$ snyderi, Oncorhynchus gorbuscha, $\underline{0}$. keta, $\underline{0}$. kisutch, $\underline{0}$. nerka, $\underline{0}$. tshawytscha, Ophiodon elongatus, Pholis ornata, Platichthys stellatus, Porichthys sp., P. notatus, Reinhardtius hippoglossoides, Ronquilus jordani, Salvelinus malma, Scomber japonicus, Sebastes aleutianus, $\underline{s}$. alutus, $\underline{\mathrm{S}}$. borealis, $\underline{\mathrm{S}}$. caurinus, $\underline{\mathrm{S}}$. maliger, $\underline{\mathrm{S}}$. melanops, $\underline{\mathrm{S}}$. pinniger, $\underline{\mathrm{S}}$. proriger, $\underline{\mathrm{S}}$. zacentrus, syngnathus leptorhynchus, Thaleichthys pacificus, Theragra chalcogramma

Lecithaster helodes - Mugil cephalus

Lecithaster minimus - Clevelandia ios, Gillichthys mirabilis, Ilypnus gilberti, Quietula y-cauda

Lecithaster mugilis - Mugil cephalus Lecithaster sayori - Cololabis saira

Lecithaster stellatus - Acanthogobius flavimanus, Oncorhynchus gorbuscha, $\underline{0}$. keta

Lecithochirium sp. - Merluccius productus

Lecithochirium albulae - Albula vulpes

Lecithochirium keokeo - Auxis thazard

Lecithochirium magnaporum - Scomber japonicus, Seriola dorsalis,

Trachurus symmetricus

Lecithochirium medium - Sebastes ruberrimus

Lecithochirium microstomum - Calamus brachysomus, Euthynnus pelamis, 0ligoplites saurus, Scomberomorus sierra

Lecithochirium monticellii - Echeneis naucrates, 0ligoplites saurus,

Remora remora

Lecithochirium parvum - Caranx hippos

Lecithochirium platessae - Sebastes aleutianus

Lecithocladium excisum - Scomber japonicus

Lecithocladium harpodontis - Scomber japonicus

Lecithophyllum botryophorum - Dasycottus setiger, Hexagrammos

lagocephalus, $\underline{H}$. stelleri, Merluccius productus, Oncorhynchus gorbuscha, $\underline{0}$. nerka, Sebastes aleutianus, s. alutus, 
DIGENEA (continued)

HEMIURIDAE (continued)

$\underline{S}$. borealis, $\underline{S}$ crameri, $\underline{S}$. flavidus, $\underline{S}$. paucispinis, $\underline{S}$. reedi Lecithophyllum intermedium - Gymnothorax mordax, Xenistius californiensis

Lecithophyllum sphaerolecithum - Reinhardtius hippoglossoides,

Sebastes aleutianus, $\underline{S}$. alutus, $\underline{S}$. boreal is, Theragra chalcogramma Lecithophyllum trachinoti - Trachinotus rhodopus

Magniscyphus taboganus - Gymnothorax mordax, Paralabrax maculatofasciatus

Mecoderus oligoplitus - Oligoplites saurus

Myosaccium ecaude - Sardinops sagax

Opisthadena bodegnesis - Cebidichthys violaceus

Opisthadena cheni - Girella nigricans

Parahemiurus merus - Aprodon cortezianus, Caranx hippos, Clupea

harengus, Echeneis naucrates, Engraul is mordax, Gadus macrocephalus,

Gasterosteus aculeatus, Hexagrammos stelleri, Hippoglossoides

elassodon, Hippogiossus stenolepis, Lyopsetta exilis, Oncorhynchus

gorbuscha, $\underline{0}$. keta, $\underline{0}$. kisutch, $\underline{0}$. nerka, Ophiodon elongatus,

Platichthys stellatus, Sardinops sagax, Syngnathus leptorhynchus

Parahemiurus noblei - Sardinops sagax

Parahemiurus sardiniae - Istiophorus platypterus

Plerurus carangi - Auxis thazard, Euthynnus affinis

Pronopyee ocreata - Clupea harengus

Saturnis segmentatus - Mugil cephalus

Sterrhurus sp. - Oncorhynchus nerka

Sterrhurus exodicus - Embiotoca lateral is, Eopsetta jordani, Gibbonsia

sp., Gobiesox maeandricus, Ophiodon elongatus, Platichthys stellatus,

Sebastes atrovirens, $\underline{\mathrm{S}}$. caurinus, $\underline{\mathrm{S}}$. constellatus, $\underline{\mathrm{S}}$. elongatus, $\underline{\mathrm{S}}$.

flavidus, $\underline{S}$. maliger, $\underline{S}$. mystinus, $\underline{S}$. nebulosus, $\underline{S}$. paucispinis

Sterrhurus floridensis - Echeneis naucrates

Sterrhurus imocavus - Auxis thazard, Euthynnus affinis, Thunnus thynnus

Sterrhurus magnatestis - Citharichthys sordidus, $\underline{C}$. stigmaeus

Sterrhurus monticelli - Sebastes $\mathrm{sp.}$ 
HEMIURIDAE (continued)

Sterrhurus musculus - Caranx hippos

Sterrhurus taboganus - Gymnothorax mordax

Sterrhurus zeloticus - Caranx hippos

Tubulovesicula lindbergi - Acipenser transmontanus, Anoplarchus purpurescens, Citharichthys sordidus, $\underline{\text { C. stigmaeus, Cymatogaster }}$ aggregata, Echeneis naucrates, Enophrys bison, Eopsetta jordani, Gadus macrocephalus, Gasterosteus aculeatus, Hemilepidotus hemilepidotus, Hippoglossus stenolepis, Hyperprosopon ellipticum, Hypsopsetta guttulata, Isopsetta isolepis, Lepidopsetta bilineata, Leptocottus armatus, Myoxocephalus polyacanthocephalus, 0ligottus maculosus, Oncorhynchus gorbuscha, $\underline{0}$. keta, $\underline{0}$. kisutch, $\underline{0}$. nerka, o. tshawytscha, Ophiodon elongatus, Paralichthys californicus, Parophrys vetulus, Platichthys stellatus, Porichthys notatus, Psettichthys melanostictus, Salvelinus malma, Scorpaenichthys marmoratus, Sebastes alutus, $\underline{\mathrm{S}}$. boreal is, $\underline{\mathrm{S}}$. brevispinis, $\underline{\mathrm{s}}$. caurinus, $\underline{\mathrm{s}}$. crameri, $\underline{\mathrm{s}}$. maliger, $\underline{\mathrm{S}}$. melanops, $\underline{\mathrm{S}}$. nigrocinctus, $\underline{\mathrm{s}}$. paucispinis, $\underline{\mathrm{s}}$. pinniger, synchirus gilli, Syngnathus leptorhynchus, Theragra chalcogramma

HETEROPHYIDAE

Adleriella minutissima - Mugil cephalus Apophailus sp. - Oncorhynchus gorbuscha, $\underline{0}$. keta, Salmo clarki Apophallus bacalloti - Gasterosteus aculeatus

Apophallus brevis - Gasterosteus aculeatus Apophallus donicus - Oncorhynchus kisutch, Salmo gairdneri Ascocotyle angrense - Atherinops affinis, Fundulus parvipinnis, Gillichthys mirabilis

Ascocotyle sexidigita - Fundulus parvipinnis, Gillichthys mirabilis Centrocestus asadai - Acanthogobius flavimanus, Mugil cephalus Cercarioides humbargari - Atherinops affinis, Leuresthes tenuis Cryptocotyle concavum - Gasterosteus aculeatus

Cryptocotyle lingua - Clupea harengus 
HETEROPHYIDAE (continued)

Euhaplorchis californiensis - Fundulus parvipinnis

Galactosomum sp. - Ammodytes hexapterus, Cymatogaster aggregata,

Hexagrammos lagocephalus, Oncorhynchus keta

Galactosomum phalacrocoracis - Oncorhynchus gorbuscha

Galactosomum sanaensis - Mugil cephalus

Haplorchis microrchis - Mugil cephalus

Haplorchis yokogawai - Mugil cephalus

Heterophyes heterophyes - Mugil cephalus

Heterophyes katsuradai - Mugil cephalus

Heterophyes nocens Acanthogobius flavimanus, Mugil cephalus

Heterophyopsis continua - Mugil cephalus

Metagonimus minutus - Mugil cephalus

Metagonimus yokogawai - Acanthogobius flavimanus, Mugil cephalus

Phagicola longa - Mugil cephalus

Phocitremoides ovale - Fundulus parvipinnis

Procerovum varium - Acanthogobius flavimanus, Mugil cephalus

Pseudogalactosoma macrostoma - Acanthobobius flavimanus, Mugi1 cephalus

Pygidiopsis summa - Acanthogobius flavimanus, Mugi1 cephalus

Pygidiopsoides spindalis - Fundulus parvipinnis

Stellantchasmas falcatus - Acanthogobius flavimanus, Mugil cephalus

Stictodora fuscata = Acanthogobius flavimanus, Mugil cephalus

Stictodora hancocki - Fundulus parvipinnis, Gillichthys mirabilis

Stictodora perpendicula - Acanthobobius flavimanus, Mugil cephalus

Stictodora sawakinesis - Mustelus californicus

HIRUDINELLIDAE

Hirudinella sp. - Auxis thazard

Hirudinella ampullacea - Istiophorus platypterus

Hirudinella fusca - Makaira nigricans, Thunnus alalunga, I. albacares,

Xiphias gladius

Hirudinella oxysoma - Thunnus alalunga 
HIRUDINELLIDAE (continued)

Hirudinella poirieri - Thunnus alalunga

Hirudinella spinulosa - Euthynnus affinis, Istiophorus platypterus,

Makaira nigricans, Thunnus alalunga, I. albacares, I. thynnus Hirudinella ventricosa - Coryphaena hippurus, Euthynnus lineatus,

E. pelamis, Istiphorus platypterus, Naucrates ductor,

I. albacares, I. thynnus, Xiphias gladius

Uroproctinella attenuata - Thunnus albacares

HOMALOMETRIDAE

Homalometron caballeroi - Balistes polylepis

Myzotus vitellosus - Caulolatilis princeps

ISOPARORCHI IDAE

Isoparorchis hypselobargi - Oncorhynchus keta

LAMPRITREMATIDAE

Lampritrema miescheri - Brama japonica, Oncorhynchus gorbuscha,

0. nerka

LEPOCREADIIDAE

Bianium plicitum - Diodon hystrix, Sphoeroides annulatus

Cephalolepidapedon saba - Scomber japonicus

Dihemistophenus fragilis - Mola mola

Dihemistophenus lydiae - Mola mola

Echeneidocoelium indicum - Echeneis naucrates, Remora remora

Labrifer balli - Zalembius rosaceus

Labrifer secundus - Pimelometopon pulchrum

Lepidapedon sp. - Mycteroperca sp.

Lepidapedon antimorae - Antimora microlepis

Lepidapedon calli - Microstomus pacificus, Parophrys vetulus

Lepidapedon cascadensis - Coryphaenoides filifer, $\underline{c}$. leptolepis

Lepidapedon elongatum - Sebastes mystinus, $\underline{S}$. nebulosus

Lepidapedon epinepheli - Epinephelus analogus 
DIGENEA (continued)

\section{LEPOCREADIIDAE (continued)}

Lepidapedon filiformis - Coryphaenoides filifer Lepidapedon gadi - Gadus macrocephalus, Hippoglossus stenolepis, Lepidopsetta bilineata, Sebastes alutus, $\underline{S}$. borealis, Theragra chal cogramma

Lepidapedon hancocki - Epinephelus analogus, Mycteroperca xenarcha Lepidapedon luteum abyssensis - Coryphaenoides sp., $\underline{C}$. acrolepis,

C. filifer, c. leptolepis

Lepidapedon microtyleum - Theragra chalcogramma

Lepidapedon nicolli - Epinephelus niveatus

Lepidapedon oregonensis - Coryphaenoides filifer, $\underline{C}$. leptolepis

Lepidapedon yaquina - Coryphaenoides filifer, $\underline{C}$. leptolepis

Lepocreadium sp. - Atherinops is californiensis

Lepocreadium areolatum - Morone saxatilis

Lepocreadium bimarinum - Pimelometopon pulchrum

Lepocreadium bravoae - Balistes polylepis

Lepocreadium californianum - Morone saxatilis

Lepocreadium ghanensis - Scomber japonicus

Lepocreadium manteri - Leuresthes tenuis

Lepocreadium scombri - Scomber japonicus

Lepocreadium setiferoides - Morone saxatilis

Neolabrifer bravoae - Pimelometopon pulchrum

Neolepidapedon medialunae - Medialuna californiensis

Neolepidapedon pugetense - Sebastes caurinus, $\underline{S}$. nebulosus

Neolepidapedon retrusum - Scomber japonicus

Neolepidapedon sebastisci - Sebastes aleutianus, $\underline{S}$. alutus, $\underline{S}$.

babcocki, $\underline{S}$. boreal is, $\underline{S}$. helvomaculatus, $\underline{S}$. paucispinis, $\underline{S}$.

pinniger, $\underline{S}$. ruberrimus, $\underline{S}$. zacentrus

Opechona alaskensis - Porichthys notatus, Sebastes aleutianus,

$\underline{\mathrm{S}}$. alutus, $\underline{\mathrm{S}}$. borealis, $\underline{\mathrm{S}}$. flavidus, $\underline{\mathrm{S}}$. maliger, $\underline{\mathrm{S}}$. pinniger,

$\underline{S}$. ruberrimus, $\underline{S}$. zacentrus, Theragra chal cogramma

Opechona bacillaris - Clupea harengus, Scomber japonicus, Sebastes sp. 


\section{LEPOCREADIIDAE (continued)}

Opechona occidental is - Sebastes sp., S. alutus, $\underline{\text { S. atrovirens, }}$

$\underline{\mathrm{s}}$. caurinus, $\underline{\mathrm{s}}$. chrysomelas, $\underline{\mathrm{s}}$. elongatus, $\underline{\mathrm{s}}$. flavidus, $\underline{\mathrm{s}}$. maliger,

S. nebulosus, Syngnathus leptorhynchus

Opechona olssoni - Scomber japonicus

Opechona orientalis - Girella nigricans, Scomber japonicus, Sebastes sp.

Opechona parvasoma - Sebastes meianops

Opechona pharyngodactyla - Trachinotus rhodopus

Opechona theragrae - Sebastes maliger

Pseudocreadium diodontis - Diodon hystrix

MICROPHALLIDAE

Pseudomegalophallus diodontis - Diodon hystrix

MONORCHI IDAE

Asymphylodora atherinopsidis - Atherinopsis californiensis, Leuresthes tenuis

Lasiotocus sp. - Amphistichus argenteus, Hyperprosopon argenteum Lasiotocus albulae - Albula vulpes

Lasiotocus glebulentus - Mugil cephalus

Lasiotocus longicaecum - Anisotremus davidsoni, Balistes polylepis

Lasiotocus mugilis - Mugil cephalus

Lasiotocus oculatus - Albula vulpes

Monorcheides alexanderi - Paralabrax clathratus

Monorchis sp. - Amphistichus argenteus

Postmonorchis donacis - Cymatogaster aggregata, Embiotoca jacksoni,

Menticirrhus undulatus, Micrometrus minimus, Roncador stearnsi

Telolecithus pugetensis - Amphistichus argenteus, Cymatogaster aggregata, Damalichthys vacca, Embiotoca jacksoni, E. lateral is, Hyperprosopon anale, Hyperprosopon argenteum, Phanerodon furcatus, Rhacochilus toxotes, Sebastes sp.

NANOPHYETIDAE

Nanophyetus salmincola - Lampetra tridentata, Oncorhynchus keta, 
DIGENEA (continued)

NANOPHYETIDAE (continued)

$\underline{0}$. kisutch, $\underline{0}$. tshawytscha, Salmo clarki, $\underline{\text { S. gairdneri }}$

OPECOELIDAE

Alloanomalotrema ghanensis - Caranx hippos

Allopodocotyle lepomis - Liparis fucensis

Anomalotrema putjatini - Hexagrammos stelleri

Crowcrocaecum testiobliqum - Salmo gairdneri

Crowcrocaecum wisniewskii - Salmo gairdneri

Dactylos tomum winteri - Paralabrax maculatofasciatus

Decemtestis azumae - Hippoglossoides elassodon, Platichthys stellatus

Eurycreadium vitellosum - Microstomus pacificus

Genitocotyle acirra - Amphistichus argenteus, A. rhodoterus,

Cymatogaster aggregata, Damalichthys vacca, Embiotoca lateral is,

Gibbonsia metzi, Hyperprosopon anale, ‥ argenteum, H. ellipticum,

Micrometrus minimus, Parophrys vetulus, Phanerodon furcatus,

Scorpaenichthys marmoratus, Umbrina roncador

Genitocotyle heterostichi - Heterostichus rostratus

Hamacreadium bolivari - Paralabrax maculatofasciatus

Hamacreadium mutabile - Mycteroperca xenarcha

Helicometra sp. - Sebastes maliger

Helicometra pretiosa - Paralabrax maculatofasciatus

Helicometrina sp. - Sebastes alutus

Helicometrina elongata - Amphistichus argenteus, Clinocottus analis,

Gibbonsia sp., G. elegans, G. metzi, Gobiesoxmaeandricus, Neoclinus uninotatus, Scorpaena guttata

Helicometrina fasciata - Paralabrax maculatofasciatus

Helicometrina nimia - Amphistichus argenteus, $\mathrm{Clinocottus}$ analis,

Coryphaena hippurus, Embiotoca jacksoni, E. lateral is, Epinephelus

analogus, Gibbonsia elegans, Leptocottus armatus, Menticirrhus

undulatus, Paralabrax clathratus, P. macuiatofasciatus, P. nebul ifer,

Sebastes atrovirens, $\underline{S}$. chrysomelas, $\underline{s}$. serriceps, $\underline{s}$. vexillaris,

Synodus lucioceps, Xenistius californiensis 
OPECOELIDAE (continued)

Manteriella sp. - Lythrypnus dalli

Neohel icometra pleurogrammi - Hexagrammos decagrammus

Neohelicometra sebastis - Sebastes aleutianus, S. alutus, S. babcocki,

$\underline{S}$. caurinus, $\underline{S}$. diploproa, $\underline{S}$. flavidus, $\underline{S}$. helvomaculatus, $\underline{S}$.

maliger, $\underline{S}$. nebulosus, $\underline{s}$. nigrocinctus, $\underline{S}$. reedi, $\underline{S}$. ruberrimus,

s. zacentrus

Opecoel ina pharynmagna - Sebastes nebulosus

Opecoelina radifistuli - Sebastes elongatus

Opecoelina theragrae - Theragra chalcogramma

Opecoeloides sp. - Coryphaena hippurus

Opecoeloides eucinostomi - Polydactylus approximans

Opecoelus adsphaericus - Cebidichthys sp., Clinocottus analis,

Girella nigricans

Opecoelus inimici - Paralabrax nebulifer

Opecoelus mexicanus - Paralabrax maculatofasciatus

Opecoelus minor - Sebastes atrovirens

Opecoelus noblei - Sebastes sp., $\underline{\mathrm{s}}$. chrysomelas

Opecoelus xenistij - Xenistius californiensis

Opegaster sp. - Trachurus symmetricus

Opegaster cameroni - Clinocottus analis, Girella nigricans

Opegaster parapristipomatis - Trachinotus rhodopus

Ozaki orthorchis - Acanthogobius flavimanus

Pellamyzon sebastodis - Sebastes atrovirens, $\underline{\text { S. serriceps }}$

Plagioporus angusticolle - Salmo gairdneri

Plagioporus issaitschikowi - Paralabrax clathratus

Plagioporus shawi - Oncorhynchus kisutch, $\underline{0}$. nerka, Salmo clarki,

S. gairdneri

Plagioporus siliculus - Salmo clarki

Podocotyle sp. - Agonus acipenserinus, Aprodon cortezianus,

Ascelichthys rhodorus, Blepsias cirrhosus, Clinocottus analis,

Eopsetta jordani, Gibbonsia sp., Hemilepidotus spinosus,

Hexagrammos lagocephalus, $\underline{H}$. stelleri, Leptocottus armatus, 
DIGENEA (continued)

OPECOELIDAE (continued)

Oncorhynchus kisutch, Parophrys vetulus, Phol is laeta, Scorpaenichthys marmoratus, Sebastes aleutianus, $\underline{\mathrm{s}}$. alutus, $\underline{\mathrm{s}}$. babcocki, $\underline{\mathrm{s}}$. brevispinis, $\underline{s}$. diploproa, $\underline{S}$. elongatus, $\underline{S}$. flavidus, $\underline{s}$. goodei, $\underline{S}$. helvomaculatus, $\underline{S}$. maliger, $\underline{S}$. paucispinis, $\underline{S}$. pinniger, $\underline{S}$. proriger, $\underline{S}$. reedi, $\underline{S}$. ruberrimus, $\underline{S}$. wilsoni, $\underline{S}$. zacentrus Podocotyle abitionis - Sebastes sp., s. maliger Podocotyle apodichthysi - Apodichthys flavidus, Gasterosteus aculeatus Podocotyle atomon - Aulorhynchus flavidus, Blepsias cirrhosus, Clinocottus globiceps, Gadus macrocephalus, Gasterosteus aculeatus, Hexagrammos lagocephalus, H. stelleri, Hippoglossoides elassodon, Hippoglossus stenolepis, Lepidopsetta bilineata, Leptocottus armatus, Liparis fucensis, Myoxocephalus polyacanthocephalus, Oncorhynchus gorbuscha, Platichthys stellatus, Porichthys notatus, Reinhardtius hippogiossoides, Salvelinus malma, Sebastes aleutianus,

$\underline{\mathrm{s}}$. alutus, $\underline{\mathrm{S}}$. borealis, $\underline{\mathrm{S}}$. wilsoni, syngnathus leptorhynchus, Xiphister atropurpureus

Podocotyle californica - Anoplarchus purpurescens, Ascelichthys rhodorus, Clinocottus analis, $\underline{C}$. globiceps, Enophrys bison, Hemilepidotus spinosus, Leptocottus armatus, 01igocottus maculosus, 0 . snyderi, Scorpaenichthys marmoratus, Xiphister atropurpureus, $\underline{x}$. mucosus

Podocotyle enophrysi - Agonops is emmelane, Artedius notospilotus, Ascelichthys rhodorus, Clinocottus globiceps, Enophrys bison, Gasterosteus aculeatus, Leptocottus armatus, Liparis fucensis, L. pulchelius, Lycodops is pacifica, Myoxocephalus polyacanthocephalus, Oncorhynchus gorbuscha, Scorpaenichthys marmoratus, Syngnathus leptorhynchus, Triglops pingeli Podocotyle gibbonsiae - Agonopsis emmelane, Eopsetta jordani, Gibbonsia elegans, Gobiesox maeandricus, Lepidopsetta bilineata, Lycodopsis pacifica, Oncorhynchus gorbuscha Podocotyle levenseni - Liparis fucensis Podocotyle olssoni - Leptocottus armatus 
DIGENEA (continued)

OPECOELIDAE (continued)

Podocotyle pacifica - Pholis ornata

Podocotyle reflexa - Agonus acipenserinus, Anoplopoma fimbria, Blepsias cirrhosus, Clinocottus globiceps, Clupea harengus, Gadus macrocephalus, Gasterosteus aculeatus, Hexagrammos lagocephalus, $\underline{\text { H. stelleri, }}$ Hippoglossus stenolepis, Leptocottus armatus, Myoxocephalus polyacanthocephalus, Pleurogrammus monopterygius, Salvel inus malma, Sebastes aleutianus, $\underline{S}$. alutus, $\underline{\mathrm{S}}$. boreal is, Syngnathus leptorhynchus, Theragra chalcogramma, xiphister atropurpureus

Podocotyle simplex - Gasterosteus aculeatus

Podocotyle sinusacca - Blepsias cirrhosus, Gasterosteus aculeatus,

Hemilepidotus hemilepidotus, Hexagrammos lagocephalus, $\underline{H}$. stelleri,

Leptocottus armatus, Liparis callyodon, Parophrys vetulus,

Sebastes maliger, Syngnathus leptorhynchus, Theragra chalcogramma

Pseudopecoeloides carangis - Caranx hippos

Pseudopecoelus gibbonsiae - Gibbonsia metzi

Pseudopecoelus japonicus - Zenopsis nebulosa

Pseudopecoelus nassamani - Hippoglossus stenolepis

Pseudopecoelus umbrinae - Amphistichus argenteus, Cymatogaster

aggregata, Roncador stearnsi, Umbrina roncador

Pseudopecoelus vulgaris - Anoplopoma fimbria, Lycodopsis pacifica

OPISTHOLEBETIDAE

Heterolebes maculosus - Chilomycterus affinis, Diodon hystrix Maculifer chilomycteri - Chilmycterus affinis Opistholebes cotylophorus - Chilomycterus affinis, Diodon hystrix Opistholebes diodontis - Diodon hystrix

Pachycreadium gastrocotylum - Calamus brachysomus

Pseudoheterolebes chilomycteri - Chilomycterus affinis

PTYCHOGONIMIDAE

Ptychogonimus megastomus - Prionace glauca

RENICOLIDAE

Renicola sp. - Clupea harengus

Renicola buchanani - Fundulus parvipinnis, Gillichthys mirabilis

Renicola cerithidicola - Fundulus parvipinnis 


\section{SANGUINICOLIDAE}

Aporocotyle macfarlani - Sebastes sp., s. atrovirens, $\underline{S}$. caurinus,

$\underline{\mathrm{S}}$. chrysomelas, $\underline{\mathrm{S}}$. flavidus, $\underline{\mathrm{s}}$. maliger, $\underline{\mathrm{S}}$. melanops, $\underline{\mathrm{s}}$. pinniger Aporocotyle margolisi - Merluccius productus

Aporocotyle pacifica - Ruvettus pretiosus

Aporocotyle simplex - Hippoglossoides elassodon, Theragra chalcogramma

Aporocotyle theragrae - Theragra chalcogramma

Cardicola ahi - Thunnus obesus

Cardicola alseae - Salmo clarki, s. gairdneri

Cardicola congruenta - Euthynnus affinis

Cardicola davisi - Salmo clarki, s. gairdneri

Cardicola klamathensis - Salmo clarki

Cardicola mugilis - Mugil cephalus

Cardicola tropicus - Sphoeroides annulatus

Piethorchis acanthus - Mugil cephajus

Psettarium sebastodorum - Sebastes aleutianus, $\underline{S}$. alutus, $\underline{S}$. atrovirens,

$\underline{\mathrm{S}}$. auriculatus, $\underline{\mathrm{S}}$. aurora, $\underline{\mathrm{S}}$. babcocki, $\underline{\mathrm{S}}$. brevispinis, $\underline{\mathrm{S}}$. carnatus,

$\underline{S}$. caurinus, $\underline{\mathrm{S}}$. chrysomelas, $\underline{\mathrm{s}}$. crameri, $\underline{\mathrm{S}}$. diploproa, $\underline{\mathrm{S}}$. flavidus,

$\underline{\mathrm{S}}$. helvomaculatus, $\underline{\mathrm{S}}$. maliger, $\underline{\mathrm{s}}$. melanops, $\underline{\mathrm{s}}$. nigrocinctus, $\underline{\mathrm{s}}$.

paucispinis, $\underline{S}$. pinniger, $\underline{S}$. proriger, $\underline{S}$. reedi, $\underline{S}$. serriceps,

S. zacentrus

Sanguinicola idahoensis - Salmo gairdneri

SCLERODISTOMATIDAE

Sclerodistomum bravoae - Diodon hystrix

Sclerodistomum diodontis - Diodon hystrix

Sclerodistomum sphoeroides - Diodon hystrix

SPIRORCHIDAE

Genera and species - Cetorhinus maximus

STEGANODERMATIDAE

Pseudochetosoma salmonicola - Salmo gairdneri 
DIGENEA (continued)

\section{STRIGEIDAE}

Apatemon gracilis - Gasterosteus aculeatus

Cardiocephalus medioconiger - Mugil cephaius

Cotylurus erraticus - Oncorhynchus kisutch, $\underline{0}$. nerka, Salmo gairdneri Cotylurus pileatus - Gasterosteus aculeatus

SYASCOELIIDAE

Capiatestes thyrsitae - Brama japonica

Syncoelium sp. - Brama japonica

Syncoelium filiferum - Euthynnus pelamis, Oncorhynchus gorbuscha,

$\underline{0}$. kisutch, $\underline{0}$. nerka, Sebastes alutus, $\underline{\mathrm{S}}$. brevispinis, $\underline{\mathrm{S}}$. flavidus,

$\underline{S}$. pinniger, $\underline{S}$. proriger, Thunnus alalunga

\section{WARETREMATIDAE}

Magasolena kyphosi - Kyphosus analogus

Myodera medialunae - Medialuna californiensis

Scorpidicola californiensis - Medialuna californiensis

Vitellibaculum girellae - Girella nigricans

\section{ZOOGONIDAE}

Brachyenteron doderleiniae - Atheresthes stomias

Deretrema cholaeum - Sebastes sp., $\underline{\mathrm{S}}$. caurinus, $\underline{\mathrm{s}}$. elongatus, $\underline{\mathrm{S}}$.

maliger, $\underline{S}$. nigrocinctus, $\underline{S}$. paucispinis, $\underline{\text { S. ruberrimus }}$

Deretrema pooli - Sebastes sp.

Diphtherostomum albulae - Albula vulpes

Diphtherostomum macrosaccum - Neoclinus uninotatus

Diphtherostomum microacetabulum - Hippoglossoides elassodon,

Lepidopsetta bilineata

Lepidophyllum cameroni - Hemilepidotus hemilepidotus

Lepidophyllum pleuronectini - Hippoglossoides elassodon, Lepidopsetta bilineata

Neozoogonus californicus - Cymatogaster aggregata, Damalichthys vacca,

Embiotoca jacksoni, E. lateralis, Hypsurus caryi, Micrometrus

minimus, Phanerodon furcatus, Rhacochilus toxotes, Zalembius rosaceus 
DIGENEA (continued)

ZOOGONIDAE (continued)

Pseudozoogonoides microacetabulum - Lepidopsetta bilineata

Steganoderma formosum - Atheresthes stomias, Gadus macrocephalus,

Hemilepidotus hemilepidotus, Hippoglossoides elassodon, Hippoglossus stenolepis, Lepidopsetta bilineata, Parophrys vetulus, Reinhardtius

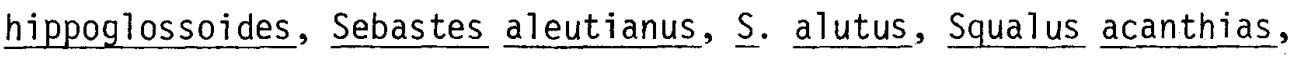
Theragra chalcogramma

Urinatrema aspinosum - Hexagrammos lagocephalus

Urinatrema hirudinaceum - Hexagrammos lagocephalus

Zoogonoides acanthogobii - Acanthogobius flavimanus

Zoogonoides viviparus - Clinocottus globiceps, Hippoglossoides

elassodon, Lepidopsetta bilineata, Microstomus pacificus, 0ligocottus snyderi, Platichthys stellatus

Zoogonus dextrocirrus - Isopsetta isolepis, Lepidopsetta bilineata,

Lumpenus anguillaris, Lycodes brevipes, Lycodopsis pacifica Microstomus pacificus, Parophrys vetulus, Rhacochilus toxotes 
GLOSSIPHONI IDAE

Genera and species - Leptocottus armatus, Mugil cephalus

Hemiclepsis marginata - Oncorhynchus gorbuscha

PISCICOLIDAE

Aestabdella abditovesiculata - Gymnothorax mordax, Hypsopsetta

guttulata, Leptocottus armatus, Psettichthys melanostictus

Australobdella californiana - Hippoglossina stomata, Pleuronichthys

decurrens, $\underline{\text { P. verticalis, Scorpaena guttata }}$

Beringobdella rectangulata - Gadus macrocephalus, Theragra chalcogramma

Branchellion lobata - Galeorhinus zyopterus, Hydrolagus colliei,

Mustelus henlei, Notorynchus maculatus, Raja binoculata, Squalus

acanthias, Squatina californica, Triakis semifasciata

Calliobdella vivida - Squalus acanthias

Malmiana sp. - Ophiodon elongatus

Malmiana diminuta - Scorpaenichthys marmoratus, Sebastes melanops

Malmiana virida - Enophrys bison

Myzobdella sp. - Salmo clarki, S. gairdneri

Myzobdella lugubris - Morone saxitilis, Mugil cephalus

Oceanobdella sp. - Parophrys vetulus

Orientobdella sp. - Raja binoculata

Ostreobdella papillata - Sebastes malanops

Piscicola sp. - Mugil cephalus, Sebastes borealis, S. caurinus

Piscicola geometra - Gasterosteus aculeatus, Salmo gairdneri

Piscicola salmositica - Oncorhynchus gorbuscha, $\underline{0}$. keta, $\underline{0}$. kisutch,

0. nerka, $\underline{0}$ tshawytscha, Salmo clarki, $\underline{\text { S. gairdneri }}$

Stibarobdella macrothela - Carcharhinus leucas, $\underline{\text {. Iongimanus, }}$

c. obscurus

Trachelobdella okae - Oncorhynchus keta

Trachelobdella oregonensis - Scorpaenichthys marmoratus 
Genera and species - Gnathiid larva - Chromis punctipinnis, Hypsypops rubicunda, Oxylebius pictus, Pimelometopon puichrum, Scorpaenichthys marmoratus, Sebastes atrovirens, S. carnatus,

$\underline{\text { S. chrysomelas, }} \underline{\mathrm{S}}$. constellatus, $\underline{\mathrm{S}}$. serranoides, $\underline{\text { S. serriceps }}$ AEGI IDAE

Rocinela belliceps - Hydrolagus colliei, Sebastes maliger Rocinela propodialis - Sebastes maliger Rocinela signata - Thunnus alalunga

CYMOTHOIDAE

Aegathoa cf. oculata - Morone saxatilis

Braga sp. - Hypsopsetta guttulata

Codonophilus gilberti - Mugil cephalus

Lironeca californica - Cymatogaster aggregata, Engraul is mordax, Leuresthes tenuis, Micrometrus minimus

Lironeca convexa - Chloroscombrus orqueta

Lironeca ovalis - Caranx hippos, Morone saxatilis, Trachurus symmetricus Lironeca vulgaris - Anoplopoma fimbria, Cymatogaster aggregata,

Damalichthys vacca, Embiotoca jacksoni, Hyperprosopon argenteum, Leptocottus armatus, Neoclinus blanchardi, Ophiodon elongatus, Paralabrax nebulifer, Paralichthys californicus, Parophrys vetulus, Phanerodon furcatus, Psettichthys melanostictus, Scorpaena guttata, Scorpaenichthys marmoratus, Sebastes chrysomelas, S. mystinus, $\underline{S}$. serranoides, Synodus lucioceps,

Nerocila californica - Amphistichus argenteus, Atherinops affinis, Atherinopsis californiensis, Cetengraul is mysticetus, Cymatogaster aggregata, Damalichthys vacca, Embiotoca jacksoni, Engraul is mordax, Heterodontus francisci, Istiophorus platypturus, Leuresthes tenuis, Micrometrus minimus, Mugil cephalus, Mycteroperca xenarcha, Myliobatis sp., Paralabrax clathratus,

Phanerodon furcatus, Scorpaena guttata, Sphoeroides annulatus, Stereolepis gigas, Triakis semifasciata, Umbrina roncador 
ISOPODA (continued)

CYMOTHOIDAE (continued)

Nerocila macleayi - Mola mola

Nerocila orbignyi - Mola mola 
Genera and species - Gasterosteus aculeatus, Morone saxatil is,

Oncorhynchus gorbuscha

UNIONIDAE

Anodonta sp. - Salmo gairdneri

Anodonta beringiana - Gasterosteus aculeatus, Oncorhynchus nerka,

0. tshawytscha

Margaritifera margaritifera - Oncorhynchus tshawytscha, Salmo clarki, S. gairdneri

Unio sp. - Oncorhynchus keta 
Genera and species - Cynoscion nobilis

ACANTHOCOTYLIDAE

Allacanthocotyla pugetensis - Raja binoculata

Pseudacanthocotyla pacifica - Raja binoculata, R. rhina, $\underline{\text { R. stellulata }}$

Pseudacanthocotyla williamsi - Sebastes alutus

ALLOPYRAEGRAPHORIDAE

Allopyragraphorus hippos - Caranx hippos

AXINIDAE

Allopseudaxine katsuwonis - Euthynnus pelamis

Allopseudaxine macrova - Euthynnus affinis

Allopseudaxine vagans - Euthynnus pelamis

Heteraxine argentea - Sphyraena argentea

Sibitrema poonui - Thunnus obesus

CALCEOSTOMATIDAE

Paracalceostoma calceostomoides - Balistes polylepis

CAPSALIDAE

Ancyrocotyle bartschi - Naucrates ductor

Ancyrocotyle vallei - Naucrates ductor

Benedenia sp. - Istiophorus platypterus

Benedenia derzhavini - Sebastes aleutianus, $\underline{\mathrm{s}}$. borealis, $\underline{\mathrm{S}}$. $\underline{\text { crameri, }}$

$\underline{\mathrm{S}}$. proriger, $\underline{\mathrm{S}}$. reedi, $\underline{\mathrm{S}}$. zacentrus

Benedenia hendorffi - Coryphaena hippurus

Benedenia melleni - Morone saxatilis, Naucrates ductor, Paralabrax

maculatofasciatus, Sphoeroides annulatus

Caballerocotyla abidjani - Thunnus albacares

Caballerocotyla albsmithi - Thunnus thynnus

Caballerocotyla biparasitica - Thunnus albacares, $I$. obesus

Caballerocotyla gregalis - Sarda chiliens is

Caballerocotyla katsuwoni - Euthynnus pelamis 
CAPSALIDAE (continued)

Caballerocotyla klawei - Thunnus albacares, I. obesus

Caballerocotyla magronum - Thunnus thynnus

Caballerocotyla manteri affinis - Auxis thazard, Euthynnus affinis

Caballerocotyla marielenae - Istiophorus platypterus

Caballerocotyla notosinense - Euthynnus affinis

Caballerocotyla paucispinosa - Euthynnus affinis, Thunnus thynnus

Caballerocotyla pseudomagronum - Thunnus obesus

Caballerocotyla verrucosa - Thunnus albacares, I. obesus

Capsala sp. - Istiophorus platypterus

Capsala cephala - Mola mola

Capsala cutanea - Mola mola

Capsala gotoi - Thunnus albacares, I. obesus

Capsala grimaldii - Mola mola

Capsala interrupta - Euthynnus pelamis, Thunnus thynnus

Capsala laevis - Coryphaena hippurus, Euthynnus affinis, Makaira

indica, Xiphias gladius

Capsala lintoni - Euthynnus pelamis

Capsala maculata - Mola mola

Capsala martinieri - Mola mola

Capsala molae - Mola mola

Capsala neothunni - Thunnus albacares

Capsala nozawae - Euthynnus pelamis, Thunnus alalunga, I. albacares,

I. obesus, I. thynnus

Capsala onchidiocotyle - Thunnus obesus, I. thynnus

Capsala ovalis - Istiophorus platypterus, Makaira nigricans, Tetrapturus

angustirostris, I. audax

Capsala pelamydis - Mola mola

Capsala poeyi - Makaira nigricans

Capsala pricei - Tetrapturus audax

Capsala thynni - Thunnus alalunga, I. albacares

Capsaloides cristatus - Tetrapturus angustirostris

Capsaloides istiophori - Istiophorus platypterus, Tetrapturus audax 
CAPSALIDAE (continued)

Capsaloides nairagi - Tetrapturus angustirostris

Capsaloides sinuatus - Istiophorus platypterus, Makaira nigricans,

Tetrapturus audax

Capsaloides tetrapteri - Tetrapturus angustirostris

Encotyllabe embiotocae - Amphistichus argenteus, Cymatogaster aggregata

Encotyllabe masu - Oncorhynchus kisutch

Entobdella diadema - Dasyatis violacea

Entobdella guberleti - Urolophus halleri

Entobdella hippoglossi - Atheresthes stomias, Hippoglossus stenolepis

Entobdella pugetensis - Atheresthes stomias

Entobdella rosaceus - Zalembius rosaceus

Entobdel la squamula - Eopsetta jordani, Hippoglossus stenolepis,

Paralichthys californicus

Neobenedenia girellae - Girella nigricans, Leptocottus armatus,

Pimelometopon pulchrum

Neobenedenia pacificia - Mugil cephalus

Nitzchia quadritestes - Acipenser medirostris, A. transmontanus

Pseudobenedenia noblei - Sebastes paucispinis

Pseudoentobdella pacifica - Myliobatis californica

Tristoma adcoccineum - Xiphias gladius

Tristoma adintegrum - Xiphias gladius

Tristoma coccineum - Sphyrna zygaena, Xiphias gladius

Tristoma integrum - Xiphias gladius

Tristoma papillosum - Mola mola, Xiphias gladius

Tristomella pricei - Istiophorus platypterus

Trochopus sp. - Sebastes aleutianus, $\underline{\text { s. alutus, }} \underline{\mathrm{s}}$. babcocki, $\underline{\mathrm{S}}$.

borealis, $\underline{S}$. brevispinis, $\underline{S}$. crameri, $\underline{S}$. diploproa, $\underline{\text { s. elongatus, }}$

$\underline{S}$. entomelas, $\underline{S}$. flavidus, $\underline{S}$. goodei, $\underline{S}$. pinniger, $\underline{S}$. proriger,

S. reedi, $\underline{S}$. ruberrimus, $\underline{S}$. zacentrus

Trochopus marginata - Sebastes alutus, $\underline{S}$. atrovirens, $\underline{S}$. auriculatus,

$\underline{S}$. carnatus, $\underline{S}$. caurinus, $\underline{S}$. maliger, $\underline{S}$. melanops, $\underline{S}$. nebulosus,

$\underline{S}$. ruberrimus, $\underline{S}$. serriceps, $\underline{S}$. umbrosus 
CAPSALIDAE (continued)

Trochopus pseudomarginatus - Epinephelus analogus

Trochopus sprostoni - Scorpaena guttata

Irochopus trituba - Sebastes a]utus, $\underline{\text { S. caurinus, }}$ S. diploproa,

$\underline{S}$. paucispinis, $\underline{\text { S. pinniger, }} \underline{\text { S. ruberrimus }}$

CHIMAERICOLIDAE

Chimaericola leptogaster - Hydrolagus colliei

DACTYLOGYRIDAE

Amphibdelloides maccallumi - Squalus acanthias, Torpedo cal ifornica

Ancyrocephalus vanbenedeni - Mugil cephalus

Aristocleidus hastatus - Morone saxatilis

Cleidodiscus sp. - Mugil cephalus

Cleidodiscus pricei - Morone saxatil is

Dactylogyrus sp. - Gasterosteus aculeatus

Microncotrematoides inversum - Scomber japonicus

Pseudohaliotrema mugilinus - Mugil cephalus

Urocleidus sp. - Fundulus parvipinnis

DICLIDOPHORIDAE

Choricotyle caulolatili - Caulolatilus princeps

Choricotyle exilis - Lyopsetta exil is

Choricotyle oregonensis - Antimora microlepis

Cyclobothrium sp. - Engraul is mordax

Cyclocotyloides pinguis - Coryphaenoides pectoralis

Diclidophora sp. - Amphistichus rhodoterus, Hyperprosopon argenteum,

A. ellipticum, Phanerodon furcatus

Diclidophora pugetensis - Atheresthes stomias

Heterobothrium affinis - Atheresthes stomias, Hippoglossus stenolepis,

Reinhardtius hippoglossoides

DICLYBOTHRI IDAE

Paradiclybothrium pacificum - Acipenser medirostris 
MONOGENEA (continued)

\section{DIONCIDAE}

Dioncus agassizi - Echeneis naucrates, Remora brachyptera, R. remora Dioncus remorae - Caranx hippos, Echeneis naucrates

DIPLECTANIDAE

Diplectanum americanum - Stereolepis gigas

Diplectanum amplidiscatum - Paralabrax maculatofasciatus

Diplectanum collinsi - Morone saxatilis

Rhamnocereus rhamnocereus - Umbrina roncador

DISCOCOTYLIDAE

Al lodiscocotyla mexicana - Caranx hippos

Allopseudopisthogyne constricta - Lepidocybium flavobrunneum

Anthocotyle americana - Merluccius productus

Anthocotyle merlucci - Merluccius productus

Cyciocotyloides pinguis - Coryphaenoides acrolepis, $\underline{\text { C. filifer }}$

Diplozoon paradoxum - Gasterosteus aculeatus

Discocotyle sagittata - Salmo gairdneri, Salvelinus malma

Discocotyle salmonis - Salmo gairdneri

Pseudomazocraes monsivaisae - Balistes polylepis

Pseudopisthogyne lepidocybii - Lepidocybium flavobrunneum

Pseudopis thogynopsis lepidocybii - Lepidocybium flavobrunneum

Tagia ecuadori - Sphoeroides annulatus

Vallisia oligoplites - oligoplites saurus

Vallisia riojai - Caranx hippos

GASTROCOTYLIDAE

Allopseudaxine macrova - Auxis thazard

Areotestis sibi - Thunnus alalunga, I. albacares, I. obesus

Churavera macrova - Euthynnus affinis

Gastrocotyle japonica - Scomber japonicus

Lithidocotyle acanthophallus - Morone saxatilis

Metapseudaxine ventrosicula - Auxis thazard, Euthynnus affinis,

Thunnus thynnus 


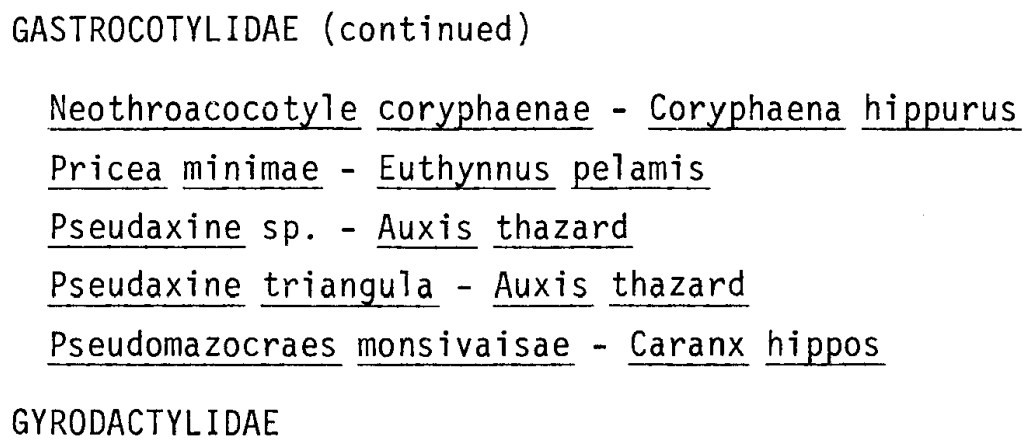

Archigyrodactylus archigyrodactylus - Cymatogaster aggregata

Archigyrodactylus atherinops - Atherinops affinis

Archigyrodactylus leibyi - Hyperprosopon anale, $\underline{H}$. argenteum, $\underline{H}$. ellipticum

Gyrodactyloides baueri - Clupea harengus

Gyrodactyloides petruschewskii - Clupea harengus

Gyrodactyloides strelkowi - Oncorhynchus gorbuscha, $\underline{0}$. keta , $\underline{0}$. kisutch,

o. nerka

Gyrodactylus sp. - Atherinops affinis, Cymatogaster aggregata,

Fundulus parvipinnis, Leptocottus armatus, Morone saxatilis,

Parophrys vetulus

Gyrodactylus ackerti - Platichthys stellatus

Gyrodactylus aculeati - Gasterosteus aculeatus

Gyrodactylus aggregata - Cymatogaster aggregata

Gyrodactylus al exanderi - Gasterosteus aculeatus

Gyrodactylus ammodyti - Ammodytes hexapterus

Gyrodactylus arcuatus - Gasterosteus aculeatus

Gyrodactylus armatus - Leptocottus armatus

Gyrodactylus avalonia - Gasterosteus aculeatus

Gyrodactylus bodegensis - Clinocottus acuticeps

Gyrodactylus branchius - Platichthys stellatus

Gyrodactylus brevis - Salmo gairdneri

Gyrodactyius bychowskyi - Gasterosteus aculeatus

Gyrodactylus californiensis - Microgadus proximus

Gyrodactylus canadens is - Gasterosteus aculeatus 
GYRODACTYLIDAE (continued)

Gyrodactylus colemanensis - Salmo gairdneri

Gyrodactylus corti - Anarrhichthys ocellatus

Gyrodactylus cranei - Microgadus proximus

Gyrodactylus doglieli - Clupea harengus

Gyrodactylus elegans - Gasterosteus aculeatus, Gillichthys mirabilis,

Ophiodon elongatus, Salmo clarki, $\underline{S}$. gairdneri

Gyrodactylus elongatus - Microgadus proximus

Gyrodactylus groenlandicus - Pallasina barbata, Platichthys stellatus

Gyrodactylus groenlandicus pacificus - Blepsias cirrhosus

Gyrodactylus harengi - Ammodytes hexapterus, Clupea harengus

Gyrodactylus imperialis - Gillichthys mirabilis

Gyrodactylus lairdi - Gasterosteus aculeatus

Gyrodactylus lenoki - Oncorhynchus keta

Gyrodactylus marinus - Gadus macrocephalus, Theragra chalcogramma

Gyrodactylus memorial is - Gasterosteus aculeatus

Gyrodactylus mugilis - Mugil cephalus

Gyrodactylus olsoni - Gillichthys mirabilis

Gyrodactylus pacificus - Microgadus proximus

Gyrodactylus perforatus - Clevelandia ios

Gyrodactylus proximus - Pallasina barbata

Gyrodactylus pterigial is - Theragra chalcogramma

Gyrodactylus pungiti - Gasterosteus aculeatus

Gyrodactylus rarus - Gasterosteus aculeatus

Gyrodactylus robustus - Platichthys stellatus

Gyrodactylus rogersi - Microgadus proximus

Gyrodactylus salaris - Salmo gairdneri

Gyrodactylus sculpinus - Leptocottus armatus

Gyrodactylus stellatus - Platichthys stellatus

Gyrodactylus unicopula - Platichthys stellatus

Gyrodactylus vancleavi - Hexagrammos lagocephalus

Micropolyclithrum parvum - Mugil cephalus

Polyclithrum mugilini - Mugil cephalus 
MONOGENEA (continued)

GYRODACTYLOIDEA

Genera and species - Ammodytes hexapterus, Clupea harengus, Cymatogaster aggregata, Gasterosteus aculeatus, 01 igocottus maculosus, Oncorhynchus kisutch, Phol is ornata, Platichthys stellatus, Syngnathus leptorhynchus

HETERAXINIDAE

Allencotyle pricei - Damalichthys vacca

Cemocotyle borinquenensis - Caranx caballus

Cemocotyle noveborancensis - Caranx hippos

Hel ixaxine winteri - Caranx hippos

Heteraxinoides oligoplitis- Oligoplites saurus

Leuresthicola olsoni - Leuresthes tenuis

Zeuxapta seriolae - Caranx hippos, Seriola dorsal is

HEXABOTHRI IDAE

Genera and species - Triakis semifasciata

Erpocotyle abbreviata - Squalus acanthias

Erpocotyle grisea - Hexanchus griseus, Sphyrna zygaena

Erpocotyle microstoma - Sphyrna zygaena

Erpocotyle somniosi - Somniosus pacificus

Erpocotyle sphyrnae - Sphyrna lewini, s. zygaena

Erpocotyle squali - Galeorhinus zyopterus, Mustelus henlei, Squalus acanthias, Triakis semifasciata

Erpocotyle striata - Squalus acanthias

Erpocotyle taschenbergi - Hexanchus griseus

Erpocotyle tiburonis - Sphyrna tiburo

Heteroncocotyle leucas - Carcharhinus leucas

Hexabothrium appendiculata - Squalus acanthias

Neoerpocotyle platensis - Sphyrna zygaena

Pseudohexabothrium rajae - Mustelus henlei

Rajonchocotyle sp. - Raja binoculata

Rajonchocotyle batis - Raja binoculata, R. stellulata

Rhinobatonchocotyle cyclovaginatus - Rhinobatos productus 


\section{HEXOSTOMATIDAE}

Hexostoma $s p$. - Sarda chiliensis

Hexostoma acutum - Thunnus obesus, Thunnus thynnus

Hexostoma albsmithi - Thunnus thynnus

Hexostoma auxisi - Auxis thazard

Hexostoma dissimile - Thunnus thynnus

Hexostoma grossum - Euthynnus pelamis, Thunnus obesus, I. thynnus

Hexostoma keokeo - Auxis thazard

Hexostoma sibi - Thunnus alalunga, I. albacares, I. obesus

Hexostoma thynni - Thunnus thynnus

Homostoma chura - Euthynnus affinis

Neohexostoma euthynni - Euthynnus affinis, E. lineatus

Neohexostoma extensicaudum - Thunnus thynnus

Neohexostoma kawakawa - Thunnus albacares

Neohexostoma robustum - Thunnus obesus

Neohexostoma thunninas - Thunnus thynnus

\section{LOIMOIDAE}

Loimosina wilsoni - Sphyrna zygaena

MACROVALVITREMATIDAE

Genera and species - Lampanyctus ritteri

Pseudotagia clathratus - Paralabrax clathratus

MAZOCRAEIDAE

Grubea sp. - Sarda chiliensis

Grubea cochlear - Scomber japonicus

Grubea pnematophori - Scomber japonicus

Kuhnia minor - Scomber japonicus

Kuhnia scombri - Scomber japonicus

Kuhnia thunni - Thunnus thynnus

Mazocraeoides georgei - Clupea harengus

Mazocraes harengi - Clupea harengus 


\section{MICROBOTHRI I DAE}

Microbothrium apiculatum - Squalus acanthias

MICROCOTYLIDAE

Aspinatrium acanthogobii - Acanthogobius flavimanus

Bivagina punctipinnis - Chromis punctipinnis

Metamicrocotyla bora - Mugil cephalus

Metamicrocotyla cephalus - Mugil cephalus

Metamicrocotyla filiformis - Mugil cephalus

Metamicrocotyla macracantha - Mugil cephalus

Metamicrocotyla mugil is - Mugil cephalus

Microcotyle sp. - Radulinus asprellus, Sebastes

alutus

Microcotyle chiri - Hexagrammos decagrammus

Microcotyle eueides - Morone saxatil is

Microcotyle macroura - Morone saxatilis

Microcotyle pacifica - Lycodopsis pacifica

Microcotyle sebastis - Hexagrammos lagocephalus, Ophiodon elongatus,

$\underline{\text { Sebastes alutus, }} \underline{\mathrm{s}}$. atrovirens, $\underline{\mathrm{S}}$. auriculatus, $\underline{\mathrm{s}}$. brevispinis,

$\underline{\mathrm{S}}$. carnatus, $\underline{\mathrm{S}}$. caurinus, $\underline{\mathrm{S}}$. chlorostictus, $\underline{\mathrm{S}}$. diploproa, $\underline{\mathrm{S}}$.

elongatus, $\underline{S}$. helvomaculatus, $\underline{S}$. maliger, $\underline{S}$. melanops, $\underline{S}$. miniatus,

$\underline{\mathrm{S}}$. nigrocinctus, $\underline{\mathrm{S}}$. paucispinis, $\underline{\mathrm{S}}$. proriger, $\underline{\mathrm{S}}$. reedi, $\underline{\mathrm{s}}$.

saxicola, $\underline{\text { S. umbrosus, }}$ S. wilsoni, $\underline{\text { S. zacentrus }}$

Microcotyle zalembius - Zalembius rosaceus

Microcotyloides impudicus - Polydactylus approximans

Prosomicrocotyla chiri - Hexagrammos stelleri

Prosomicrocotyla gotoi - Hexagrammos lagocephalus

Pyragraphorus caballeroi - Caranx hippos

Solostamenides mugilis - Mugil cephalus

Solostamenides pseudomugilis - Mugil cephalus

MONOCOTYLIDAE

Genera and species - Rhinobatos productus

Anoplocotyloides papillata - Rhinobatos productus 
MONOGENEA (continued)

MONOCOTYLIDAE (continued)

Calicotyle ramsayi - Squalus acanthias

Cathariotrema selachii - Carcharhinus obscurus, Sphyrna zygaena

Heterocotyle minima - Squalus acanthias

Merizocotyle pugetensis - Raja binoculata

Spinuris lophosoma - Rhinobatos productus

POLYOPISTHOCOTYLEA

Genera and species - Atherinops affinis

PROTOMICROCOTYLIDAE

Abortipedia indica - Caranx hippos

Protomicrocotyle manteri - Caranx hippos

Protomicrocotyle mirabilis - Caranx hippos

PTERINOTREMATIDAE

Pseudopterinotrema albulae - Albula vulpes

Pterinotrema macrostomon - Albula vulpes

Pterinotrema mirabilis - Albula vulpes

TETRAONCHIDAE

Tetraonchus alaskensis - Oncorhynchus nerka, Salmo

clarki Limalma, Q. kusutch - alsha

TROCHOPODINAE

Genera and species - Scorpaena guttata

UDONELLIDAE

Udonella caligorum - Mugil cephalus, Myliobatis californica,

Pleuronichthys decurrens, Psettichthys melanostictus

Udonella ophiodontis - Ophiodon elongatus 


\section{NEMATODA}

\section{ACUARI IDAE}

Synhimantus brevicaudatus - Gasterosteus aculeatus

\section{ACANTHOCHEILIDAE}

Acanthocheilus bicuspis - Squalus acanthias

Acanthocheilus nudiflex - Galeocerdo cuvieri, Sphyrna zygaena

Acanthocheilus quadridentatus - Apristurus brunneus, Mustelus henlei, squalus acanthias

Pseudanisakis tricupula - Lampetra tridentata, Squalus acanthias

\section{ANISAKIDAE}

Anisakis sp. - Acipenser transmontanus, Ammodytes hexapterus, Anoplarchus purpurescens, Anoplopoma fimbria, Apodichthys flavidus, Atheresthes stomias, Atherinopsis californiensis, Aulorhynchus flavidus, Auxis thazard, Blepsias cirrhosus, Ceratoscopelus townsendi, Cheilotrema saturnum, Citharichthys sordidus, $\underline{C}$. stigmaeus, Clupea harengus, Cololabis saira, Cymatogaster aggregata, Cynoscion nobilis, Delolepis gigantea, Diaphus theta, Engraul is mordax, Eopsetta jordani, Euthynnus affinis, E. pelamis, Gadus macrocephalus, Gasterosteus aculeatus, Genyonemus lineatus, Gilbertidia sigalutes, Glyptocephalus zachirus, Hexagrammos lagocephalus, Hippoglossina stomata, Hippoglossoides elassodon, Hippoglossus stenolepis, Hydrolagus colliei, Hypomesus pretiosus, Icosteus aenigmaticus, Isopsetta isolepis, Isurus oxyrinchus, Lampanyctus regalis, L. ritteri, Lampris regius, Lepidopsetta bilineata, Leptocottus armatus, Lycodes brevipes, Lyopsetta exilis, Macroparalepis sp., Mallotus villosus, Melanostigma pammelas, Merluccius productus, Microstomus pacificus, Mola mola, Myoxocephalus polyacanthocephalus, 0ligocottus maculosus, Oncorhynchus gorbuscha, $\underline{0}$. keta, $\underline{0}$. kisutch, $\underline{0}$. nerka, $\underline{0}$. tshawytscha, Ophiodon elongatus, Paralabrax clathratus, P. nebulifer, Paralichthys californicus, Parophrys vetulus, Parvilux ingens, Phol is laeta, 
ANISAKIDAE (continued)

P. ornata, Platichthys stellatus, Pleurogrammus monopterygius, Prionace glauca, Psettichthys melanostictus, Raja rhina, Reinhardtius hippoglossoides, Ronquilus jordani, Salmo gairdneri, Salvelinus malma, Sarda chiliensis, Sardinops sagax, Scomber japonicus, Scorpaena guttata, Sebastes aleutianus, $\underline{\mathrm{S}}$. alutus, $\underline{\mathrm{S}}$. atrovirens, $\underline{\mathrm{S}}$. auriculatus, $\underline{\mathrm{S}}$ babcocki, $\underline{\mathrm{S}}$. borealis, $\underline{\mathrm{s}}$. brevispinis, $\underline{\mathrm{s}}$. chlorostictus, $\underline{\mathrm{s}}$. constellatus, $\underline{\mathrm{s}}$. dalli,$\underline{\mathrm{s}}$. diploproa, $\underline{\mathrm{S}}$. elongatus, $\underline{S}$. gilli, $\underline{\mathrm{S}}$. goodei, $\underline{\mathrm{S}}$. hopkinsi, $\underline{\mathrm{S}}$. levis, $\underline{\mathrm{S}}$. macdonaldi, $\underline{S}$. miniatus, $\underline{S}$. ovalis, $\underline{S}$. paucispinis, $\underline{S}$. phillipsi, $\underline{\mathrm{S}}$. pinniger, $\underline{\mathrm{S}}$. rastrelliger, $\underline{\mathrm{S}}$. rosaceus, $\underline{\mathrm{S}}$. rosenblatti, $\underline{\mathrm{S}}$. rubrivinctus, $\underline{S}$. rufus, $\underline{s}$. serranoides, $\underline{s}$. serriceps, Seriphus politus, Serrivomer sector, Squalus acanthias, Stenobrachius leucopsarus, Symbolophorus californiensis, Syngnathus leptorhynchus, Synodus lucioceps, Tarletonbeania crenularis, Theragra chalcogramma, Thunnus alalunga, I. thynnus, Trachurus symmetricus, Triglops pingeli, Triphoturus mexicanus, Zenopsis nebulosa Contracaecum sp. - Ammodytes hexapterus, Anoplarchus purpurescens, Anoplopoma fimbria, Aprodon cortezianus, Artedius harringtoni, Atherinops affinis, Atherinopsis californiensis, Auxis thazard, Ceratoscopelus townsendi, Chitonotus pugetensis, Clupea harengus, Coryphaena hippurus, Coryphopterus nicholsi, Cymatogaster aggregata, Diaphus theta, Dorosoma petenense, Engraul is mordax, Eopsetta jordani, Euthynnus affinis, E. pelamis, Gadus macrocephalus, Gasterosteus aculeatus, Hexagrammos lagocephalus, Hippoglossoides elassodon, Hippoglossus stenolepis, Hypomesus pretiosus, Icelinus filamentosus, Jordania zonope, Lampanyctus ritteri, Leptocottus armatus, Leuresthes tenuis, Lyopsetta exilis, Macroparalepis sp., Malacottus kincaidii, Melamphaes acanthomus, Melanostigma pammelas, Merluccius productus, Mugil cephalus, Myoxocephalus polyacanthocephalus,

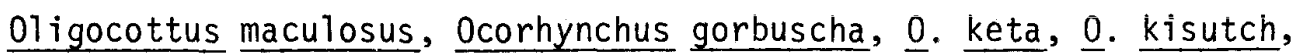
$\underline{0}$. nerka, $\underline{0}$. tshawytscha, Paralichthys cal ifornicus, Parophrys vetulus, Pholis laeta, Platichthys stellatus, 


\section{ANISAKIDAE (continued)}

S. gairdneri, Salvelinus malma, Sardinops sagax, Scomber japonicus, Sebastes aleutianus, $\underline{S}$. alutus, $\underline{S}$. babcocki, s. brevispinis, $\underline{S}$. borealis,

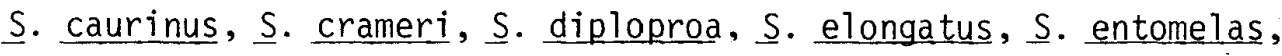

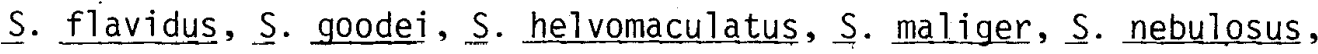

$\underline{S}$. nigrocinctus, $\underline{S}$. paucispinis, $\underline{S}$. pinniger, $\underline{S}$. proriger, $\underline{S}$. reedi,

S. ruberrimus, S. wilsoni, $\underline{S}$. zacentrus, Stenobrachius leucopsarus,

Syngnathus leptorhynchus, Synodus lucioceps, Tarletonbeania

crenularis, Tetrapturus audax, Thaleichthys pacificus. Theragra

chalcogramma, Thunnus alalunga, I. albacares, I. thynnus, Irachurus symmetricus, Triglops pingeli, Triphoturus mexicanus, Zenopsis nebulosa

Contracaecum benimasu - Oncorhynchus keta, $\underline{0}$. nerka Contracaecum brachyurum - Alosa sapidissima, Morone saxatilis

*Contracaecum cornutum - Thunnus thyminus

Contracaecum crassicaudatum - Oncorhynchus kisutch

Contracaecum elongatum - Oncorhynchus nerka

*Contracaecum fabri - Cololabis saira, Scomber japonicus

Contracaecum hippoglossi - Oncorhynchus kisutch, $Q$. nerka

Contracaecum hypomesi - Clupea harengus, Oncorhynchus gorbuscha,

0. keta, $\underline{0}$. nerka

* Contracaecum longispiculum - Oncorhynchus keta

Contracaecum ochotense - Oncorhynchus nerka

Contracaecum oshoroensis - Oncorhynchus keta

Contracaecum plagiostomorum - Cetorhinus maximus

Contracaecum robustum - Mugil cephalus, Oncorhynchus gorbuscha

*Contracaecum saba - Scomber japonicus

Contracaecum spiculigerum - Mugil cephalus, Oncorhynchus nerka,

0. tshawytscha, Salmo gairdneri

Contracaecum tridentatum - Oncorhynchus keta

Contracaecum unidentatum - Oncorhynchus keta

*Contracaecum zenopsis - Zenopsis nebulosa

Goezia sp. - Morone saxatilis 
NEMATODA (continued)

\section{ANISAKIDAE (continued)}

Goezia annulata - Morone saxatilis

Goezia ascaroides - Salmo gairdneri

Goezia oncorhynchi - Oncorhynchus keta

Paranisakis sp. - Cyclothone acclinidens, Lampanyctus ritteri

Phocanema sp. - Anoplarchus purpurescens, Anoplopoma fimbria,

Ceratoscopelus townsendi, Clupea harengus, Gadus macrocephalus,

Hippoglossoides elassodon, Lepidopsetta bilineata, Macroparalepis sp.,

Myoxocephalus polyacanthocephalus, Ophiodon elongatus, Paralabrax

clathratus, P. nebulifer, Paralichthys californicus, Platichthys stellatus, Pleurogrammus monopterygius, Reinhardtius hippoglossoides, Rhamphocottus richardsoni, Scomber japonicus, Sebastes auriculatus,

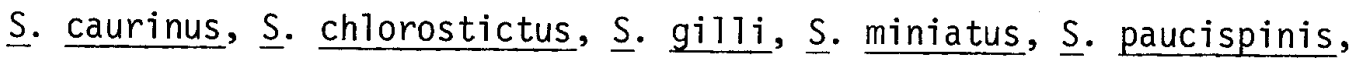
$\underline{s}$. rastrelliger, $\underline{\mathrm{s}}$. serranoides, Serrivomer sector, Squalus acanthias, Stenobrachius leucopsarus, Synodus lucioceps, Theragra chalcogramma, Trachurus symmetricus

Phocanema decipiens - Ânopiopoma fimbria, Âtheresthes stomias, Ciupea

harengus, Gadus macrocephalus, Hemilepidotus hemilepidotus,

Hexagrammos lagocephalus, Hippoglossus stenolepis, Ophiodon

elongatus, Sebastes alutus, $\underline{s}$. boreal is, $\underline{s}$. pinniger, Theragra chalcogramma

Raphidascaris sp. - Alosa sapidissima, Echeneis naucrates, Engraulis mordax, Gasterosteus aculeatus, $\underline{0}$. kisutch, $\underline{0}$. nerka, $\underline{0}$. tshawytscha, Scomber japonicus

Raphidascaris acus - Gasterosteus aculeatus, Salmo gairdneri

Raphidascaris? adelinae - Mugil cephalus

Raphidascaris cristata - Gasterosteus aculeatus

Raphidascaris gracillima - Gasterosteus aculeatus

Raphidascaroides diadonis - Diodon hystrix

Terranova brevicapitata - Carcharhinus obscurus, Galeocerdo cuvieri

Terranova galeocerdonis - Galeocerdo cuvieri, Sphryna lewini, S. zygaena *Thynnascaris sp. - Mugil cephalus, Oncorhynchus kisutch, $\underline{0}$. tshawytscha, Parophrys vetulus, Sebastes rubrivinctus 
NEMATODA (continued)

ANISAKIDAE (continued)

*Thynnascaris adunca - Agonus acipenserinus, Apodichthys flavidus, Atheresthes stomias, Aulorhynchus flavidus, Blepsias cirrhosus, Clupea harengus, Cololabis saira, Delolepis gigantea, Eopsetta jordani, Gadus macrocephalus, Gasterosteus aculeatus, Glyptocephalus zachirus, Hexagrammos lagocephalus, $\underline{H}$. stelleri, Hippoglossoides elassodon, Hypomesus pretiosus, Lepidopsetta bilineata, Leptocottus armatus, Mallotus villosus, Merluccius productus, Oncorhynchus gorbuscha, $\underline{0}$. keta, $\underline{0}$. kisutch, $\underline{0}$. nerka, $\underline{0}$. tshawytscha, Ophiodon elongatus, Parophrys vetulus, Platichthys stellatus, Reinhardtius hippoglossoides, Salvelinus malma, Scomber japonicus, Sebastes aleutianus, $\underline{\text { s. alutus, }} \underline{\mathrm{s}}$. babcocki, $\underline{\mathrm{s}}$. brevispinis, $\underline{\mathrm{s}}$. caurinus, $\underline{\mathrm{S}}$. crameri, $\underline{\mathrm{S}}$. diploproa, $\underline{\mathrm{s}}$. elongatus, $\underline{\mathrm{s}}$. entomelas, $\underline{\mathrm{s}}$. flavidus, $\underline{\mathrm{S}}$. helvomaculatus, $\underline{\mathrm{S}}$. maliger, $\underline{\mathrm{S}}$. nebuTosus, $\underline{\mathrm{S}}$. paucispinis, $\underline{\mathrm{S}}$. pinniger, $\underline{s}$. proriger, $\underline{S}$. reedi, $\underline{S}$. ruberrimus, $\underline{s}$. serranoides, s. zacentrus, Synodus Tucioceps, Theragra chalcogramma, Thunnus thynnus

* Thynnascaris clavata - Squalus acanthias

* Thynnascaris histiophori - Istiophorus platypterus

* Thynnascaris incurva - Istiophorus platypterus, Xiphias gladius

* Thynnascaris legendrei - Thunnus alalunga

* Thynnascaris melanogrammi - Merluccius productus

CAMALLANIDAE

Camailanus sp. - Dorosoma petenense

Camallanus lacustris - Gasterosteus aculeatus, Salmo gairdneri

Camallanus oxycephalus - Dorosoma petense

Camallanus truncatus - Gasterosteus aculeatus

Oncophora albacarensis - Thunnus albacares

Oncophora melanocephala - Auxis thazard, Scomber japonicus, Thunnus

albacares, I. thynnus

Spirocamallanus sp. - Clevelandia ios, Ilypnus gilberti, Quietula y-cauda

Spirocamallanus olseni - Echeneis naucrates 
CAMALLANIDAE (continued)

Spirocamallanus pereirai - Atherinops affinis, Atherinopsis

californiensis, Fundulus parvipinnis, Gillichthys mirabilis, Girel1a nigricans, Leptocottus armatus, Leuresthes tenuis, Paralichthys californicus

\section{CAPILLARI IDAE}

Capillaria sp. - Apodichthys flavidus, Aprodon cortezianus, Chitonotus pugetensis, Lepidopsetta bilineata, Lycodes brevipes, Malacottus kincaidi i, Mugil cephalus, Oncorhynchus kisutch, $\underline{0}$. nerka, $\underline{0}$. tshawytscha, Parophrys vetulus, Salmo gairdneri, Salvelinus malma, Scomber japonicus, Sebastes alutus, $\underline{\text { s. babcocki, }} \underline{\mathrm{s}}$. borealis, $\underline{\mathrm{s}}$. caurinus, $\underline{S}$. flavidus, $\underline{S}$. maliger, $\underline{S}$. nigrocinctus, $\underline{S}$. paucispinis, S. ruberrimus

Capillaria echenei - Echeneis naucrates

Capillaria salvelini - Salmo gairdneri

Hepaticola petruschewskii - Gasterosteus aculeatus

Skrjabinocapillaria bakeri - Oncorhynchus kisutch, Salmo clarki, s. gairdneri

Thominx catenata - Oncorhynchus nerka, Salmo clarki

\section{CUCULLANIDAE}

Bulbodacnitis ampullastoma - Salmo gairdneri

Cucullanellus kanabus - Cymatogaster aggregata

Cucullanus sp. - Damalichthys vacca, Lepidopsetta bilineata, Leuresthes tenuis, Morone saxatilis, Mugil cephalus, Oncorhynchus kisutch, Ronquilus jordani, Salvelinus malma, Sebastes

aleutianus, $\underline{\text { s. alutus, }} \underline{\mathrm{s}}$. babcocki, $\underline{\mathrm{s}}$ borealis, $\underline{\mathrm{s}}$. $\underline{\text { crameri }}, \underline{\mathrm{s}}$. flavidus, $\underline{\mathrm{s}}$. paucispinis, $\underline{S}$. ruberrimus

Cucullanus annulatus - Parophrys vetulus, Platichthys stellatus

Cucullanus carangis - Caranx hippos

Cucullanus dodsworthi - Mugil cephalus

Cucullanus elongatus - Ophiodon elongatus, Sebastes caurinus, $\underline{\text { S. maliger, }}$ S. ruberrimus 
CUCULLANIDAE (continued)

Cucullanus heterochrous - Hippoglossoides elassodon, Hippoglossus stenolepis, Lepidopsetta bilineata, Platichthys stellatus, Sebastes alutus, $\underline{\text { s. boreal is }}$

Cucullanus orthagorisci - Mola mola

Truttaedacnitis trutta - Oncorhynchus gorbuscha, $\underline{0}$. kisutch,

$\underline{0}$. nerka, $\underline{0}$. tshawytscha, Salmo clarki, s. gairdneri, Salvel inus $\underline{\text { malma }}$

CYSTIDICOLIDAE

Genera and species - Salmo clarki

Ascarophis sp. - Echeneis naucrates, Serrivomer sector, Stenobrachius leucopsarus

Ascarophis curvicauda - Hexagrammos lagocephalus

Ascarophis filiformis - Gadus macrocephalus

Ascarophis morrhuae - Gasterosteus aculeatus

Ascarophis pacificus - Gadus macrocephalus, Clupea harengus, Lepidopsetta

bilineata, Myoxocephalus polyacanthocephalus, Salvel inus malma,

Theragra chal cogramma

Ascarophis sebastodis - Anoplarchus purpurescens, Artedius harringtoni,

Aulorhynchus flavidus, Hemilepidotus hemilepidotus, Hexagrammos

lagocephalus, Myoxocephalus polyacanthocephalus, Nautichthys

oculofasciatus, Oncorhynchus kisutch, $\underline{0}$. nerka, Rhamphocottus

richardsoni , Sebastes alutus, $\underline{\mathrm{S}}$. borealis, $\underline{\mathrm{S}}$. caurinus, $\underline{\mathrm{S}}$. diploproa,

$\underline{S}$. elongatus, $\underline{S}$. flavidus, $\underline{S}$. maliger, $\underline{S}$. proriger, $\underline{S}$. ruberrimus,

S. zacentrus

Ascarophis skrjabini - Oncorhynchus gorbuscha, $\underline{0}$. keta, Salmo gairdneri, Theragra chalcogramma

Caballeronema wardlei - Scorpaenichthys marmoratus

Ctenascarophis gastricus - Auxis thazard, Euthynnus affinis

Cystidicola sp. - Salvelinus malma

Cystidicola farionis - Gasterosteus aculeatus, Oncorhynchus gorbuscha,

$\underline{0}$. kisutch, $\underline{0}$. nerka, $\quad \underline{\text { s. gairdneri, Salvelinus malma }}$ Cystidicola stigmatura - Oncorhynchus nerka 
NEMATODA (continued)

CYSTIDICOLIDAE (continued)

Cystidicoloides sp. - Salmo clarki

Cystidicoloides tenuissima - Gasterosteus aculeatus, Oncorhynchus

kisutch, $\underline{0}$. nerka, Salmo clarki, s. gairdneri, Salvelinus malma

Metabronema laticauda - Oncorhynchus nerka

Parascarophis galeata - Sphyrna tiburo

Salvelinema ishii - Oncorhynchus keta

Salvelinema salmonicola - Oncorhynchus keta, $\underline{0}$. nerka

Salvelinema walkeri - Oncorhynchus kisutch, $\underline{0}$. tshawytscha, Salmo

clarki, s. gairdneri

Spinitectus sp. - Auxis thazard, Cololabis saira, Morone saxatilis

Spinitectus beaveri - Albula vulpes

Spinitectus echenei - Echeneis naucrates

Spinitectus gordani - Salmo gairdneri

Spinitectus gracilis - Oncorhynchus nerka, Salmo gairdneri

Spinitectus? mol1is - Auxis thazard

Spinitectus pacificus - Cololabis saira

CYSTOOPSIDAE

Cystoopsis acipenseri - Acipenser transmontanus

\section{DIOCTOPHYMATIDAE}

Eustrongylides sp. - Gasterosteus aculeatus, Lampetra tridentata, Oncorhynchus kisutch, Salmo clarki, s. gairdneri, Salvelinus malma

GNATHOSTOMATIDAE

Echinocephalus pseudouncinatus - Heterodontus francisci, Myliobatis californica

Spiroxys sp. - Morone saxatilis

ICHTHYOSTRONGYLIDAE

Ichthyostrongylus thunni - Thunnus albacares

PHILOMETRIDAE

Philometra sp. - Euthynnus affinis, E. pelamis, Mugil cephalus, Morone saxatilis, Platichthys stellatus, Salmo clarki, s. gairdneri

Philometra americana - Gobiesox maeandricus, Lepidopsetta bilineata,

Parophrys vetulus, Phol is laeta, Phol is ornata, Platichthys

stellatus, Xiphister atropurpureus 
NEMATODA (continued)

PHILOMETRIDAE (continued)

Philometra cephalus - Mugil cephalus

Philometra rubra - Morone saxatil is

Philonema sp. - Oncorhynchus kisutch, ‥ tshawytscha, Salmo gairdneri,

Philonema agubernaculum - Gasterosteus aculeatus, Oncorhynchus

kisutch, Salmo gairdneri

Philonema kondai - Oncorhynchus keta

Philonema oncorhynchi - Oncorhynchus gorbuscha, $\underline{0}$. keta, $\underline{0}$. kisutch,

0. nerka, $\underline{0}$. tshawytscha, Salmo clarki, s. gairdneri, Salvelinus malma

Phlyctainophora squali - Squalus acanthias

PHYSALOPTERIDAE

Proleptus acutus - Heterodontus francisci, Mustelus californicus,

Mustelus henlei, Platyrhinoidis triseriata, Squalus acanthias

Proleptus austral is - Galeocerdo cuvieri

Proleptus elegans - Hexanchus griseus

Proleptus obtusus - Mustelus henlei, Squalus acanthias, Triakis semifasciata

PSEUDALIIDAE

Parafilaroides decorus - Girella nigricans

THELAZI IDAE

Heptachona caudata - Thunnus thynnus

Rhabdochona sp. - Oncorhynchus nerka, Salmo clarki

Rhabdochona cascadilla - Oncorhynchus nerka, Salmo clarki, S. gairdneri

Rhabdochona denudata - Salmo gairdneri

Rhabdochona fujii - Oncorhynchus nerka

Rhabdochona kisutchi - Oncorhynchus kisutch

Rhabdochona milleri - Salvelinus malma 
NEMATODA (continued)

Rhabdochona oncorhynchi - Oncorhynchus keta

Sterliadochona pedispicula - Salmo gairdneri

Vasorhabdochona cablei - Gillichthys mirabilis

SPIRURIDAE

Dollfusnema piscicola - Paralabrax clathratus

SPIRUROIDEA

Genera and species - Euthynnus pelamis 
AMPHILEPTIDAE

Litonotus branchiarum - Gasterosteus acuieatus

CHLAMYDODONTIDAE

Chilodonella cyprini - Oncorhynchus tshawytscha, Salmo gairdneri

DENDROSOMATIDAE

Trichophyra sp. - Morone saxatilis, Oncorhynchus kisutch, Salmo clarki, s. gairdneri

Trichophyra intermedia - Gasterosteus aculeatus, Oncorhynchus keta

Trichophyra piscium - Oncorhynchus nerka, Salmo gairdneri

EPISTYLIDAE

Epistylis sp. - Morone saxatilis, Mugil cephalus, Salmo gairdneri Epistylis Iwoffi - Gasterosteus aculeatus

OPHRYOGLENIDAE

Cryptocaryon irritans - Diodon hystrix, Girella nigricans, Paralabrax nebul i fer

Ichthyophthirius sp. - Gasterosteus aculeatus

Ichthyophthirius multifillis - Gasterosteus aculeatus, Oncorhynchus gorbuscha, $\underline{0}$. keta, Salmo gairdneri

SCYPHIDI IDAE

Ambipherya sp. - Leptocottus armatus

Apiosoma sp. - Gastereus aculeatus

Glossatella sp. - Morone saxatilis, Salmo gairdneri

Glossatella amebae - Gasterosteus aculeatus

Glossatella conica - Oncorhynchus keta

Scyphidia sp. - Leptocottus armatus, Morone saxatilis, Salmo gairdneri

Scyphidia arctica - Asterotheca infraspinata, Oncorhynchus keta, Radulinus asprellus 
PROTOZOA (CILIOPHORA) (continued)

\section{URCEOLARI IDAE}

Genera and species - Isopsetta isolepis, Lepidopsetta bilineata, Ophiodon elongatus

Trichodina sp. - Acanthogobius flavimanus, Atherinops affinis,

Fundulus parvipinnis, Gasterosteus aculeatus, Gillichthys mirabilis, Leptocottus armatus, Mugil cephalus, Oncorhynchus kisutch, Parophrys vetulus, Salmo clarki

Trichodina boreal is - Lepidopsetta bilineata

Trichodina cottidarum subsp. cottidarum - 0ligocottus maculosus

Trichodina davisi - Morone saxatilis

Trichodina domerguei - Salmo gairdneri

Trichodina domerguei subsp. domerguei - Enophrys bison, Gasterosteus aculeatus

Trichodina domerguei latispina - Oncorhynchus keta

Trichodina fultani - Salmo gairdneri

Trichodina gracil is - Gasterosteus aculeatus, Oncorhynchus gorbuscha, 0. keta

Trichodina hexagrammi - Hexagrammos stelleri

Trichodina lairdi - Artedius fenestralis, 01igocottus maculosus

Trichodina megamicronucleata - Gasterosteus aculeatus

Trichodina microdenticulata - Dorosoma petenense

Trichodina murmanica - Gadus macrocephalus

Trichodina nigra kamchatka - Oncorhynchus nerka

Trichodina ovonucleata - Asterotheca infraspinata, Dasycottus setiger,

Radul inus asprellus

Trichodina parvula - Dasycottus setiger

Trichodina ploveri - Clupea harengus

Trichodina puytoraci - Mugil cephalus

Trichodina rectuncinata - Artedius fenestral is

Trichodina salmincola - Salmo gairdneri

Trichodina tenuidens - Gasterosteus aculeatus

Trichodina truttae - Oncorhynchus gorbuscha, $\underline{0}$. keta, Salmo clarki

Trichodina vancouverense - Artedius fenestral is 
PROTOZOA (CILIOPHORA) (continued)

URCEOLARI IDAE (continued)

Trichodinella sp. - Morone saxatil is

Tripartiella californica - Oncorhynchus gorbuscha, $\underline{0}$ keta, $\underline{0}$. tshawytscha

Tripartiella obliqua - Mugil cephalus 


\section{PROTOZOA (HAPLOSPORIDA)}

Genera and species - Oncorhynchus gorbuscha, $\underline{0}$ kisutch Dermocystidium gasterostei - Gasterosteus aculeatus

Dermocystidium salmonis - Oncorhynchus tshawytscha, Salmo gairdneri 
Genera and species - Coryphaenoides filifer, Lycodopsis pacificA, Oncorhynchus gorbuscha

NOSEMATIDAE

Glugea anomala - Gasterosteus aculeatus

Glugea punctifera - Theragra chal cogramma

Glugea stephani - Parophrys vetulus, Platichthys stellatus

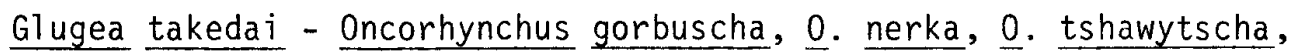

Salmo gairdneri, Salvelinus malma

Nosema sp. - Morone saxatilis

Plistophora sp. - Oncorhynchus keta, 0 . nerka, Salmo gairdneri

Plistophora salmonae - Oncorhynchus kisutch, ‥ tshawytscha, Sa7mo gairdneri 


\section{PROTOZOA (MYXOSPORIDA)}

Genera and species - Apristurus brunneus, Sebastolobus sp., Serrivomer sector, Stenobrachius leucopsarus, Triphoturus mexicanus

\section{CERATOMYXIDAE}

Ceratomyxa sp. - Acanthogobius flavimanus, Caulolatilus princeps, Coryphaenoides filifer, Gasterosteus aculeatus, Synodus lucioceps Ceratomyxa acadiensis - Clupea harengus

Ceratomyxa anoplopoma - Anoplopoma fimbria

Ceratomyxa auerbachi - Clupea harengus

Ceratomyxa blennius - Gibbonsia elegans, G. metzi, Hysoblennius gilberti

Ceratomyxa californica - Eptatretus stouti, Sebastes paucispinis Ceratomyxa crassa - Leptocottus armatus Ceratomyxa drepanosettae - Reinhardtius hippoglossoides Ceratomyxa elegans - Porichthys notatus, Sebastes paucispinis, $\underline{\text { S. }}$ pinniger, $\underline{S}$. rosaceus

Ceratomyxa filiformis - Sardinops sagax

Ceratomyxa furcata - Apodichthys flavidus

Ceratomyxa gracilis - Gibbonsia metzi

Ceratomyxa hokarari - Coryphaenoides acrolepis

Ceratomyxa hopkinsi - Citharichthys xanthostigma, Microstomus

pacificus, Nezumia stelgidolepis, Parophrys vetulus, Scorpaena guttata, Sebastes goodei, $\underline{S}$. paucispinis

Ceratomyxa inconstans - Scomber japonicus

Ceratomyxa jamesoni - Triakis semifasciata

Ceratomyxa laxa - Sebastes serranoides

Ceratomyxa lunata - Galeocerdo cuvieri

Ceratomyxa mesospora - Sphyrna tiburo, $\underline{\text { S. zygaena }}$ 
PROTOZOA (MYXOSPORIDA) (continued)

CERATOMYXIDAE (continued)

Ceratomyxa obesa - clinocottus anal is

Ceratomyxa orientalis - Clupea harengus, Sardinops sagax

Ceratomyxa platichthytis - Platichthys stellatus

Ceratomyxa porrecta - Myoxocephalus polyacanthocephalus

Ceratomyxa recurvata - Sphyrna zygaena

Ceratomyxa sebasta - Sebastes carnatus, $\underline{\text { S. ensifer, }} \underline{\mathbf{S}}$. paucispinis,

$\underline{\mathrm{S}}$. pinniger, $\underline{\mathrm{S}}$. serranoides

Ceratomyxa shasta - Oncorhynchus ketà, 0 . kisutch, $\underline{0}$. tshawytscha,

Salmo clarki, $\underline{\text { s. gairdneri }}$

Ceratomyxa starski - Sebastes rosaceus

Ceratomyxa tenuis - Leptocottus armatus

Ceratomyxa venusta - Cynoscion nobilis

Ceratomyxa vepallida - Sebastes carnatus, Sebastes ensifer, $\underline{S}$.

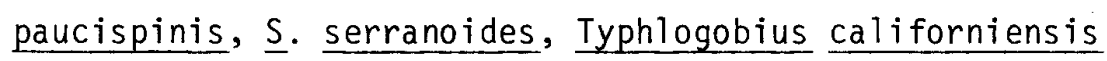

Leptotheca armatura - Coryphaenoides acrolepis, $\underline{\text { C.armatus, }} \underline{\mathrm{C}}$.

leptolepis, $\underline{C}$. pectoral is

Leptotheca coelorhynchus - Coryphaenoides armatus

Leptotheca compressa - Rimicola eigenmanni

Leptotheca elegans - Artedius lateralis, Gibbonsia elegans, G. metzi,

Paraclinus integripinnis, Typhlogobius californiensis

Leptotheca fisheri - Chromis punctipinnis, Halichoeres semicinctus Hydrolagus colliei, Rhacochilus toxotes

Leptotheca fusiformis - Sphyrna zygaena

Leptotheca galeata - Eptatretus stouti

Leptotheca informis - Anoplopoma fimbria, Coelorinchus scaphopsis,

Coryphaenoides acrolepis, $\underline{C}$. filifer, $\underline{\text { C. pectoral is, Sebastes }}$

chlorostictus, $\underline{S}$. goodei, $\underline{S}$. paucispinis, $\underline{\text { s. rosaceus, }} \underline{\mathrm{S}}$.

serranoides

Leptotheca krogiusi - Oncorhynchus gorbuscha, $\underline{0}$. keta, $\underline{0}$. kisutch,

0. nerka, $\underline{0}$. tshawytscha

Leptotheca longipes - Sebastes serranoides

Leptotheca macrospora - Sebastes serranoides

Leptotheca minima - Coryphaenoides acrolepis, $\underline{\text { c. armatus }}$

Leptotheca sebasta - Sebastes serranoides

Leptotheca sphaerula - Gibbonsia elegans, G. metzi 


\section{MYXIDI IDAE}

Myxidium sp. - Acanthogobius flavimanus, Agonopsis emmelane, Anoplopoma fimbria

Myxidium bajacalifornium - Bajacalifornia burragei

Myxidium calcariferi - Sebastes carnatus, $\underline{\text { S. miniatus, }} \underline{\mathrm{S}}$. paucispinis Myxidium coryphaenoidium - Coryphaenoides armatus, $\underline{C}$. filifer, $\underline{C}$.

leptolepis, Nezumia liolepis, N. stelgiodolepis

Myxidium gasterostei - Gasterosteus aculeatus

Myxidium incurvatum - Ascelichthys rhodorus, Mugil cephalus,

01igocottus snyderi, Scorpaena guttata, $\underline{\text { S. carnatus, }} \underline{\mathrm{S}}$. caurinus,

$\underline{S}$. ensifer, $\underline{S}$. paucispinis, $\underline{S}$. serranoides

Myxidium japonicum - Myoxocephalus polyacanthocephalus

Myxidium melanostigmum - Melanostigma pammelas, Sagamichthys abei

Myxidium minteri - Oncorhynchus keta, ‥ kisutch, ‥ tshawytscha,

Salmo clarki, s. gairdneri

Myxidium obscurum - Oncorhynchus gorbuscha, $\underline{0}$. keta, $\underline{0}$. nerka

Myxidium ochotense - Gadus macrocephalus

Myxidium oriental is - Coryphaenoides leptolepis

Myxidium oshoroense - Platichthys stellatus

Myxidium oviforme - Cymatogaster aggregata, Damalichthys vacca,

Hypsurus caryi, Oncorhynchus gorbuscha, $\underline{0}$. keta, $\underline{0}$ nerka, $\underline{0}$.

tshawytscha, Phanerodon furcatus, Salmo gairdneri, Salvelinus malma

Myxidium pearcyi - Coryphaenoides armatus, $\underline{C}$. leptolepis

Myxidium theragrae - Theragra chalcogramma

Sphaeromyxa balbianii - Agonopsis emmelane, Gobiesox maeandricus

Sphaeromyxa gibbonsia - Gibbonsia elegans, G. metzi

Sphaeromyxa lateralis - Artedius lateralis

Sphaeromyxa magna - Liparis cyclopus

Sphaeromyxa maiyai - Microgadus proximus

Sphaeromyxa ovula - Gobiesox rhessodon

Sphaeromyxa parva - Cololabis saira

Sphaeromyxa reinhardti - Engraul is mordax

Zschokkella sp. - Anoplarchus purpurescens, Gobiesox maeandricus 
MYXIDIIDAE (continued)

Zschokkella dogieli - Mugil cephalus

Zschokkella embiotocidis - Damalichthys vacca, Embiotoca lateralis

Zschokkella globulosa - Coryphaenoides acrolepis, ․ armatus, $\underline{\mathrm{C}}$.

leptolepis, Nezumia liolepis, N. stelgidolepis

Zschokkella ilishae - Sebastes serranoides

Zschokkella meglitschi - Coryphaenoides armatus, $\underline{\text { C. filifer, }}$

C. pectoralis, Nezumia stelgidolepis

Zschokkella nova - Mugil cephalus, Salmo gairdneri

Zschokkella oriental is - Oncorhynchus gorbuscha, $\underline{0}$. kisutch, $\underline{0}$.

tshawytscha

Zschokkella rovignensis - Coelorinchus scaphops is

Zschokkella russelli - Coryphaenoides armatus, ․ filifer

MYXOBOLIDAE

Henneguya $\mathrm{sp.} \mathrm{-} \mathrm{Damalichthys}$ vacca, Gasterosteus aculeatus

Henneguya salminicola - Oncorhynchus gorbuscha, $\underline{0}$. keta, $\underline{0}$. kisutch,

$\underline{0}$. nerka, $\underline{0}$. tshawytscha

Henneguya sebasta - Sebastes chlorostictus, $\underline{S}$. diploproa, $\underline{S}$. flavidus,

$\underline{\text { S. goodei, }}$ S. jordani, S. levis, $\underline{\text { S. melanops, }}$ S. miniatus, $\underline{\mathrm{S}}$. paucispinis,

$\underline{\mathrm{S}}$. rubrivinctus, $S$. rufus, $\underline{\mathrm{S}}$. serranoides, $\underline{\mathrm{S}}$. vexillaris

Henneguya tridentigeri - Acanthogobius flavimanus

Myxobilatus sp. - Gasterosteus aculeatus

Myxobilatus gasterostei - Gasterosteus aculeatus

Myxobilatus medius - Gasterosteus aculeatus

Myxobolus sp. - Leuroglossus stilbius

Myxobolus acanthogobii - Acanthogobius flavimanus

Myxobolus achmerovi - Mugil cephalus

Myxobolus cheni - Mugil cephalus

Myxobolus exiguus - Mugil cephalus

Myxobolus insidiosus - Oncorhynchus kisutch, $\underline{0}$. tshawytscha

Myxobolus kisutchi - Oncorhynchus kisutch, $\underline{0}$ tshawytscha, Salmo

gairdneri

Myxobolus krokhini - Oncorhynchus nerka 
PROTOZOA (MYXOSPORIDA) (continued)

MYXOBOLIDAE (continued)

Myxobolus mexicanus - Coelorinchus scaphopsis, Coryphaenoides armatus

Myxobolus mulleri - Mugil cephalus

Myxobolus neurobius - Oncorhynchus kisutch, $\underline{0}$ nerka

Myxobolus parvus - Mugil cephalus

Myxobolus physophilus - Gasterosteus aculeatus

Myxobolus squamae - Oncorhynchus kisutch

MYXOSOMATIDAE

Myxosoma sp. - Morone saxatilis

Myxosoma branchialis - Mugil cephalus

Myxosoma cephalus - Mugil cephalus

Myxosoma cerebralis - Oncorhynchus gorbuscha, $\underline{0}$. keta, $\underline{0}$. nerka,

Salmo clarki, s. gairdneri, Salvelinus malma

Myxosoma dermatobia - Mugil cephalus, Oncorhynchus keta, $\underline{0}$. kisutch

Myxosoma salmonis - Oncorhynchus keta

Myxosoma soldatovi - Oncorhynchus keta

Myxosoma squamalis - Oncorhynchus keta, $\underline{0}$. kisutch, Salmo gairdneri

SINUOL INEIDAE

Conispora renalis - Merluccius productus

Davisia sp. - Gobiesox maeandricus, Porichthys notatus

Davisia anoplopoma - Anoplopoma fimbria

Davisia bidens - Porichthys notatus

Davisia cella - Porichthys notatus

Davisia coryphaenoidia - Coryphaenoides acrolepis, $\underline{\text { C. pectoral is }}$

Davisia pectoralis - Coryphaenoides acrolepis, C. pectoral is

Davisia reginae - Damalichthys vacca, Embiotoca lateralis, Sebastes

maliger, s. serranoides

Myxoproteus abyssus - Coryphaenoides acrolepis, c. armatus, $\underline{\text { C. filifer }}$

Myxoproteus californicus - Coryphaenoides acrolepis, $C$. pectoralis

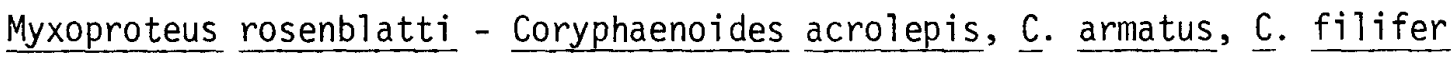

Sinuolinea dimorpha - Porichthys notatus

Sinuolinea magna - Coryphaenoides acrolepis, $c$. pectoralis

Sinuolinea triangulata - Coryphaenoides acrolepis, $\underline{C}$. armatus, $\underline{C}$.

filifer, $\underline{\text { C. leptolepis, }}$ C. pectoralis 
PROTOZOA (MYXOSPORIDA) (Continued)

SPHAEROSPORIDAE

Auerbachia pulchra - Coryphaenoides acrolepis, $c$ filifer, c. pectoralis

Chloromyxum sp. - Sphyrna zygaena

Chloromyxum chitosense - Oncorhynchus keta

Chloromyxum corregoni - Oncorhynchus nerka

Chloromyxum giganteum - Oncorhynchus gorbuscha

Chloromyxum kabatai - Coryphaenoides acrolepis

Chloromyxum levigatum - Squatina californica

Chloromyxum leydigi - Sphyrna tiburo, s. zygaena, Squalus acanthias, Torpedo californica

Chloromyxum majori - Oncorhynchus tshawytscha, Salmo gairdneri

Chloromyxum orientalis - Oncorhynchus gorbuscha, $\underline{0}$. keta

Chloromyxum ovatum - Galeorhinus zyopterus, Mustelus californicus,

M. henlei, Squalus acanthias, Torpedo californica, Urolophus halleri

Chloromyxum quadriformé - Oncorhycnhus gorbuscha, $\underline{0}$. keta

Chloromyxum sphyrnae - Sphyrna tiburo

Chloromyxum subsphaericum - Coryphaenoides armatus

Chioromyxum tanakai - Oncorhynchus keta

Chloromyxum truttae - Salmo gairdneri

Chloromyxum wardi - Oncorhynchus nerka

Sphaerospora divergens - Pleuronichthys verticalis

Sphaerospora elegans - Gasterosteus aculeatus

Sphaerospora orientalis - Clupea harengus

Unicapsula muscularis - Hippoglossus stenolepis

Unicauda brevis - Gasterosteus aculeatus

TETRACAPSULIDAE

Kudoa sp. - Atheresthes stomias, Coryphaena hippurus, Eopsetta jordani, Gadus macrocephalus, Hippoglossus stenolepis, Lepidopsetta bilineata, Microstomus pacificus, Parophrys vetulus, Platichthys stellatus

Kudoa bora - Mugil cephalus

Kudoa cerebralis - Morone saxatilis

Kudoa clupeidae - Clupea harengus, Cynoscion nobilis, Sebastes

elongatus, s. paucispinis, Thunnus thynnus

Kudoa maculoliquifaciens - Xiphias gladius 
PROTOZOA (MYXOSPORIDA)

TRILOSPORIDAE

Trilospora californica - Gibbonsia elegans, G. metzi, Typhlogobius californiensis

TRIGONOSPORIDAE

Trigonosporus acanthogobii - Acanthogobius flavimanus 
BLASTODINIIDAE

Oodinium sp. - Morone saxatilis

BODONIDAE

Colponema sp. - Morone saxatilis

CRYPTOBIIDAE

Cryptobia sp. - Bathylagus ochotensis, B. wesethi, Coryphaenoides filifer, Dorosoma petenense, Eopsetta jordani, Lyopsetta exilis, Parophrys vetulus, Scorpaenichthys marmoratus

Cryptobia borreli - Oncorhynchus kisutch, $\underline{0}$. tshawytscha, Salmo gairdneri

Cryptobia branchialis - Gasterosteus aculeatus, Oncorhychus nerka

Cryptobia coryphaenoideana - Coryphaenoides acrolepis

Cryptobia makeevi - Oncorhynchus gorbuscha, $\underline{0}$. keta

Cryptobia salmositica - Gasterosteus aculeatus, Oncorhynchus gorbuscha,

$\underline{0}$. keta, $\underline{0}$. kisutch, $\underline{0}$ nerka, $\underline{0}$. tshawytscha, Salmo gairdneri

Cryptobia stilbia - Leuroglossus stilbius

ENTAMOEBIDAE

Entamoeba molae - Mola mola

HEXAMITIDAE

Hexamita salmonis - Clupea harengus, Gasterosteus aculeatus,

Oncorhynchus nerka, $\underline{0}$. tshawytscha, Salmo clarki, s. gairdneri

Octomitus truttae - Salmo gairdneri

MONOCERCOMONIDAE

Monocercomonas molae - Mola mola

TETRAMITIDAE

Costia pyriformis - Salmo clarki, $\underline{\text { s. gairdneri }}$

Ichthyobodo necatrix - Gasterosteus aculeatus, Oncorhynchus gorbuscha,

0. keta, ‥ nerka, Salmo clarki, S. gairdneri 
PROTOZOA (SARCOMASTIGOPHORA) (continued)

\section{THECAMOEBIDAE}

Schizamoeba salmonis - Salmo gairdneri

Thecamoeba hoffmani - Oncorhynchus kisutch, $\underline{0}$. tshawytscha, Salmo gairdneri

TRYPANOSOMATIDAE

Trypanosoma sp. - Eopsetta jordani, Glyptocephalus zachirus, Microstomus pacificus, Mugil cephalus

Trypanosoma gargantua - Raja binoculata

Trypanosoma occidentalis - Gasterosteus aculeatus

Trypanosoma pacifica - Citharichthys sordidus, Lyopsetta exilis,

Parophrys vetulus 
HAEMOGREGARINIDAE

Haemogregarina sp. - Salmo clarki

Haemogregarina bigemina - Artedius fenestralis, Auxis thazard, Caranx hippos, Coryphaena hippurus

Haemogregarina delagei - Squalus acanthias

Haemogregarina mugili - Mugil cephalus

\section{EIMERI IDAE}

Genera and species - Sebastes levis

Eimeria sp. - Scorpaenichthys marmoratus

Eimeria auxidis - Cololabis saira

Eimeria clupearum - Clupea harengus, Engraul is mordax

Eimeria gasterostei - Gasterosteus aculeatus

Eimeria myoxocephali - Myoxocephalus polyacanthocephalus

Eimeria lucida - Squalus acanthias

Eimeria nishin - Clupea harengus

Eimeria pneumatophori - Scomber japonicus

Eimeria sardinae - Clupea harengus

Eimeria squali - Squalus acanthias 
TURBELLARIA

HYPOTRICHINIDAE

Ichthyophaga subcutanea - Hexagrammos decagrammus 


\section{ACKNOWLEDGMENTS}

A large number of people have given us information and assistance and we would like to thank: Gary Alderson, Palomar College; Hisao Arai, University of Calgary; Richard Arthur, Z. Kabata, Leo Margolis, and Tom McDonald, Pacific Biological Station, Nanaimo; Robert Baker, Alaska Department of Fish and Game; Wilbur Bullock, University of New Hampshire; Eugene Burreson, Virginia Institute of Marine Science; Bruce Collette, Smithsonian Institution; Murray Dailey and Ju-Shey Ho, Long Beach State University; Peter Haaker, D. L. Hanan, and Donald Schultze, California Department of Fish and Game; Fred C. Hochberg, Santa Barbara Museum of Natural History; Glenn L. Hoffman, U.S. Fish and Wildlife Service; John Holmes, University of Alberta; Carl Hubbs and Richard Rosenblatt, Scripps Institution of Oceanography; Barbara Javor, University of Oregon; Lauritz Jensen, Los Angeles, Calif.; Jiri Lom, Czechoslovak Academy; Walter Martin, University of Southern California; James McCauley and Robert Olson, Oregon State University; Paul Meglitsch, Drake University; F. M. Nahhas, University of the Pacific; Judy Moser, University of California, Santa Cruz; Ross Nigrelli, New York Zoological Society; Andrew Olson, Jr., San Diego State University; Mary Pritchard, University of Nebraska; Robert Profant, Santa Barbara City College; Gerald Schmidt, Northern Colorado University; A. D. Sekerak, Environmental Research Associates, West Toronto, Ontario; and Timothy Yoshino, Lehigh University.

We are particularly grateful to the Marine Science Institute, University of California, Santa Barbara, and in particular to Henry Offen and Fran Ciluaga, for assisting and supporting us in the funding, typing, and reproduction of the first edition of this checklist.

We would like to thank Mary Ankeny, Kathy Courtney, Regina Love, Susan Penn, Jessica Schultz, Susan Smith, and Carol Schuler for typing various revisions of this work. The typing was an unmitigated bore and we appreciate their efforts.

This work is a result of research sponsored by NOAA Office of Sea Grant, Department of Commerce, under Grants \#SGP 04-6-158-44021 and 04-6-158-44110.

Additional funding for typing the final draft came from the Center for Coastal Marine Studies, University of California, Santa Cruz; Occidental College; and the Marine and Freshwater Biomedical Center, University of Southern California.

\section{REFERENCES}

ACENA, S. P.

1941. Preliminary notes on a trematode with two yolk reservoirs and a new species of Lecithochirium. Philipp. J. Sci. 75:285-289.

1947. New trematodes from Puget Sound fishes. Trans. Am. Microsc. Soc. 66:127-139.

AKHMEROV, A. Kh.

1951. Some data on the parasites of Alaska pollock. [In Russ.] Izv. Tikhookean. Nauchno-Issled. Inst. Rȳbn. Khoz. Okeanogr. 34:99-104.

1954a. On the parasite fauna of the fish in the Kamchatka River. [In Russ.] Trudy Probl. Temat. Soveshch. Zool. Inst. (4):89-98.

1954b. On the parasite fauna and the species relationships of the Kamchatka azabach, Oncorhynchus nerka infresp. Asabatch Berg, 1932. Dokl. Akad. Nauk SSSR 94:969-971. (Fish. Res. Board Can. Transl. Ser. 283.)

1955. The parasite fauna of Kamchatka River fishes. Izv. Tikhookean. Nauchno-Issled. Inst. Rybn. Khoz. Okeanogr. 43:99-137. (Fish. Res. Board Can. Transl. Ser. 105.)
1959. Acanthocephala of fishes of Amur trout. [In Russ.] Tr. Gel'mintol. Lab. 9:23-44.

1960. Myxosporidia of Amur River Basin fish. [In Russ.] Rȳb. Khoz. Vnut. Vod. Latv. SSR 5:239-308.

1961. The trematode fauna of fish in the Amur Basin. [In Russ.] Tr. Akad. Nauk SSSR Hel'mintologicheskaia Lab. 11:22-31.

ALDRICH, L. E., Jr.

1961. Two new digenetic trematodes from marine fishes of Puget Sound, Washington. J. Parasitol. 47:77-80.

ALEXANDER, C. G.

1951. A new species of Proteocephalus (Cestoda) from Oregon trout. J. Parasitol. 37:160-164.

1953. Five new species of Acanthobothrium (Cestoda: Tetraphyllidea) from southern California rays. J. Parasitol. 39:481-486.

1954. Microcotyle macracantha n. sp., a monogenetic trematode from the Gulf of California, with a redescription of Amphibdelloides maccallumi (Johnston and Tiegs, 1922) Price, 1937. J. Parasitol. 40:279-283.

1963. Tetraphyllidean and Diphyllidean cestodes of New Zealand selachians. Trans. R. Soc. N.Z. 3:117-142.

ALPERIN, I.

1966. A new parasite of striped bass. N.Y. Fish Game J. 13:12I-123. AMIN, O. M

1975. Acanthocephalus parksidei sp. n. (Acanthocephala: Echinorhynchidae) from Wisconsin fishes. J. Parasitol. 61:301-306.

AMIN, O. M., and J. M. BURROWS

1977. Host and seasonal associations of Echinorhynchus salmonis (Acanthocephala: Echinorhynchidae) in Lake Michigan fishes. J. Fish. Res. Board Can. 34:325-331.

ANNEREAUX, R. F.

1943. Opecoelina pharynmagna n. sp. (Trematoda) from the China rockfish. J. Parasitol. 29:155-156.

1946. A new nematode, Procamallanus pereirai, with a key to the genus. Trans. Am. Microsc. Soc. 65:299-303.

1947. Three new trematodes from marine fishes of California. Trans. Am. Microsc. Soc. 66:249-255.

APPY, R. G. and M. D. DAILEY.

1973. Two new species of Acanthobothrium (Cestoda: Tetraphyllidea) from elasmobranchs of the eastern Pacific. J. Parasitol. 59:817-820.

1977. A new species of Rhinebothrium (Cestoda: Tetraphyllidea) and redescription of three rhinebothriate species from the round stingray, Urolophus halleri Cooper in southern California. Bull. South. Calif. Acad. Sci. 76:116-127.

ARAI, H. P.

1954. Helminth parasites of embiotocid fishes. I. A new genus of the trematode family Zoogonidae. J. Parasitol. 40:356-359.

1962. Trematodos digeneos de peces marinos de Baja California, Mexico. An. Inst. Biol. Univ. Nac. Auton. Mex. 33:113-130.

1967. Ecological specificity of parasites of some embiotocid fishes. J. Fish. Res. Board Can. 24:2161-2168.

1969a. A new trematode of the genus Lepidophyllum (Digenea: Steganodermatidae) from a cottid fish, Hemilepidorus hemilepidotus. J. Fish. Res. Board Can. 26:799-803.

1969h. Preliminary report on the parasites of certain marine fishes of British Columbia. J. Fish. Res. Board Can. 26:2319-2337.

1971. Helminth parasites of embiotocid fishes. II. A new species of Labrifer (Digenea: Lepocreadiidae) from Zalembius rosaceus from California. J. Fish. Res. Board Can. 28:767-769.

ARAI, H. P. and C. H. KOSKI

1964. A new species of Trochopus (Monogenea: Capsalidae) from Scorpaena guttata Girard. Can. J. Zool. 42:1007-1010.

ARNOLD, J. G., Jr., H. E. SCHAEFER, D. GEAGAN, and R. L. VULLIET

1966. The parasites of the freshwater fishes of Louisiana. Proc. Annu. Conf. Southeast. Assoc. Game Fish Comm. 20:462-468.

AVDEEV, G. V., and V. V. AVDEEV.

1975. Parasitic crustacea of the genus Sarcotaces Olsson, 1872 (Copepoda) from fishes of the Pacific Ocean. [In Russ.] lzv. Tikhookean. NauchnoIssled. Inst. Rȳbn. Khoz. Okeanogr.

AWAKURA, T.

1965. Studies on the Plistophora disease of salmonid fishes-l. Observations on the state of the occurrence and the cause in Chitose River in Hokkaido. Sci. Rep. Hokkaido Fish. Exp. Stn. 20:1-27.

1972. Studies on acanthocephaliasis of the salmonid fishes infected by Acanthocephalus minor Yamaguti, 1935. Sci. Rep. Hokkaido Fish. Exp. Stn. 27. 
1974. Studies on the microsporidian infection in salmonid fishes. Sci. Rep. Hokkaido Fish. Exp. Stn. 29:1-95.

BAER, J

1969. Diphyllobothrium pacificum, a tapeworm from sea lions endemic in man along the coastal area of Peru. J. Fish. Res. Board Can. 26:717-723.

BAEVA, O. M.

1965a. Helminth fauna of Cololabis saira from the Pacific Ocean. [In Russ.] Paraziticheskie Chervi Domashnikh i Dikikh Zhivotnykh. Rabot $\vec{y}$ Po Gel'mintologii, p. 33-42.

1965b. Helminth fauna of far eastern fish, Pleurogrammus. [In Russ.] Paraziticheskje Chervi Domashnikh i Dikikh Zhivotnykh. Raboty Po Gel'mintologii, p. 23-32.

1970. Some features of infection of Pacific saury, Cololabis saira, with parasites. [In Russ.] "Naukova Dumka" Kiev:3-5.

BAILEY, R. M., J. E. FITCH, E. S. HERALD, E. A. LACHNER, C. C. LINDSEY, C. R. ROBINS, and W. B. SCOTT.

1970. A list of common and scientific names of fishes from the United States and Canada. 3d ed. Am. Fish. Soc., Spec. Publ. 6, 149 p.

BAJLJOZOV, D., D. SAVOV, and G. YANKOV.

1964. Fish parasites in some fisheries. [In Russ.] Veterinarnomedicinski Nauki 1:49-59.

BALAKRISHNAN, K. P

1969. Observations on the occurrence of Conchoderma virgatum (Spengler) (Cirripedia) on Diodon hystrix Linnaeus (Pisces). Crustaceana 16 (Part 1):101-103.

BANE, G. W.

1969. Parasites of the yellowfin tuna, Thunnus albacores, in the Atlantic Ocean (Pisces: Scombridae). Wasmann J. Biol. 27:163-175.

BANERJEE, A. C.

1965. Some opecoelid trematodes from California with a description of Opecoelus noblei, sp. nov. Am. Midl. Nat. 74:451-456.

BANGHAM, R. V.

1944. Parasites of northern Wisconsin fish. Trans. Wisc. Acad. Sci Arts Lett. 36:291-325.

1951. Parasites of fish in the upper Snake River drainage and in Yellowstone Lake, Wyoming. Zoologica (N.Y.) 36:213-217.

BANGHAM, R. V., and J. R. ADAMS

1954. A survey of the parasites of freshwater fishes from the mainland of British Columbia. J. Fish. Res. Board Can. 11:673-708.

BARNARD, K. H.

1955. South African parasitic Copepoda. Ann. S. Afr. Mus. 41:223-312.

BASSETT-SMITH, P. W.

1896. A list of the parasitic Copepoda of fish obtained at Plymouth. J Mar. Biol. Assoc. U.K. 4:155-163.

BAUDIN-LAURENCIN, F.

1971a. Crustaces et helminthes parasites de l'albacore (Thunnus albacores) du Golfe de Guinee - note preliminaire. Doc. Sci. Centre Rech. Oceanogr. Abidjan 2:11-30.

1971b. Oncophora melanocephala (Rud, 1819) n. comb., Nématode Camallanidae, parasite du thon albacore Neothunnus albacores. Bull. Mus. Natl. Hist. Nat. $2^{\mathrm{e}}$ Sér. 42:984-988.

1972. Oncophora albacarensis n. sp. (Nématode, Camallanidae) parasite du thon albacore Thunnus albacores. Bull. Mus. Natl. Hist. Nat. Paris Sér. $3: 1329-1331$.

BAYLIS, H. A

1933. On the nematode genus Proleptus. Ann. Mag. Nat. Hist. Ser. 10, 12:325-335.

1936. Fauna of British India, including Ceylon and Burma. I. Ascaroidea and Strongyloidea. Lond., $408 \mathrm{p}$.

1950. A review of the species of Dinobothrium (Cestoda) with a description of a new species. Parasitology 40:96-104

BECKER, C. D.

1967a. The parasite fauna of teleosts in six Washington Lakes. Northwest Sci. 41:160-168.

1967b. Trypanosoma occidentalis $\mathrm{sp}$. $\mathrm{n}$. from fresh water teleosts in Washington State. J. Protozool. 14:153-156.

1970a. Haematozoa of fishes, with emphasis on North American records. In S. F. Snieszko (editor), A symposium of diseases of fishes and shellfishes, p. 82-100. Am. Fish. Soc. Spec. Publ. 5.

1970b. Marine trematode Tubulovesicula lindbergi (Digenea: Hemiuridae) from resident white sturgeon in the Columbia River. J. Fish. Res. Board Can. 27:1313-1316.

1971. Cestrahelmins rivularis sp. n. (Digenea: Deropristiidae) from white sturgeon, Acipenser transmontanus, in the Columbia River, Washington Proc. Helminthol. Soc. Wash. 38:23-26.
BECKER, C. D., and W. D. BRUNSON.

1967. Diphyllobothrium (Cestoda) infections in salmonids from three Washington lakes. J. Wildl. Manage. 31:813-824.

BECKER, C. D., and M. KATZ.

1965a. Distribution, ecology and biology of the salmonid leech, Piscicola salmositica (Rhynchobdellae: Piscicolidae). J. Fish. Res. Board Can. 22:1175-1195.

1965b. Infections of the hemoflagellate, Cryptobia salmositica Katz, 1951, in freshwater teleosts of the Pacific Coast. Trans. Am. Fish. Soc. 94:327-333.

BELL, G.

1962. Trichodinids from the gills of a new host, the lingcod Ophiodon elongatus. J. Fish. Res. Board Can. 19:515-516.

BERE, R.

1930. The parasitic copepods of the fish of the Passamaquoddy region. Contrib. Can. Biol. Fish., New Ser. 5:423-430.

1936. Parasitic copepods from Gulf of Mexico fish. Am. Midl. Nat. $17: 577-625$

BERLAND, $B$.

1961. Nematodes from some Norwegian marine fishes. Sarsia 2:1-50.

BIER, J. W., W. L. PAYNE, and G. J. JACKSON.

1974. Seasonal periodicity of a Philometra sp. (Nematoda) infection in the rockfish, Morone saxatilis. Proc. 3rd Int. Congr. Parasitol. 3(sect. 02): 1631 .

BOGDANOVA, E, A.

1960a. An endemic disease of salmonids in Sakhalin Island. Dokl. Akad. Nauk. SSSR 134:1501-1503.

1960b. Parasitic diseases of young Pacific salmon in fish hatcheries of the Far East. Nauchno-Teknicheskii Biulleten' GosNIORKh 11:49-52. (Fish. Res. Board Can. Transl. Ser. 391.)

1963. Alterations of parasites of Pacific salmon during spawning. [In Russ.] Izv. Gos. Nauchno-Issled. Inst. Ozern. Rech. Rȳbn. Khoz. 54:58-63.

1967. Parasite fauna of Atlantic salmon (Salmo salar L.), chum saimon (Oncorhynchus keta W.) and pink salmon (Oncorhynchus gorbuscha W.) in hatcheries of the Kola Peninsula. [In Russ.] Izv. Gos. NauchnoIssled. Inst. Ozern. Rech. Rȳbn. Khoz. 63:173-188.

BOGGDANOVA, E. A., and G. A. STEIN.

1963. Infusoria fam. Urceolaridae, infecting the fingerlings of the salmonid fishes. [In Russ.] Izv. Gos. Nauchno-Issled. Inst. Ozern. Rech. Rȳbn. Khoz. 54:48-57.

BONHAM, $\mathrm{K}$.

1950. Some monogenetic trematodes of Puget Sound fishes. In Studies Honoring Trevor Kincaid, p. 85-103. University of Washington Press, Seattle.

BONHAM, K., and J. E. GUBERLET.

1937. Notes on Microcotyle sebastis Goto from Puget Sound. J. Parasitol. 23:281-290.

1938. Ectoparasitic trematodes of Puget Sound fishes - Acanthocotyle. Am. Midl. Nat. 20:590-602.

BOYCE, N. P. J

1969. Parasite fauna of pink salmon (Oncorhynchus gorbuscha) of the Bella Coola River, central British Columbia, during their early sea life. J. Fish. Res. Board Can. 26:8ิ 13-820.

1976. A new organ in cestode surface ultrastructure. Can. J. Zool. 54:610-613.

BRANDES, C. H.

1955. Uber eine neue art der parasitischen Copepoden: Pseudocycnus thynnus n. sp. Veröeff. Inst. Meeresforsch. Bremerhaven 3:190-198.

BRAVO-HOLLIS, $M$.

1954a. Diplectanum amplidiscatum $\mathbf{n}$. sp. trématodes monogénetique des branchies d'un poisson marin. Ann. Parasitol. Hum. Comp. 29:37-41. 1954b. Trematodos de peces marinos de aguas Mexicanas VII. An. Inst. Biol. Univ. Nac. Auton. Méx. 25:219-252.

1956. Trematodos de peces marinos de aguas Mexicanas. XI. Estudio de 17 digeneos de las costas del Pacifico, incluyendo seis especies nuevas y un genero nuevo. An. Inst. Biol. Univ. Nac. Auton. Méx. 27:245-277.

1957. Trematodos de peces marinos de aguas Mexicanas. XIV.-Cuatro monogeneos de la familia Capsalidae Baird, 1853, de las costas del Pacifico, incluyendo una especie nueva. An. Inst. Biol. Univ. Nac. Auton. Méx. 28:195-217.

1969. Helmintos de peces del Pacífico Mexicano. XXVIII. Sobre dos especies del genero Floridosentis Ward, 1953, Acantocefalos de la familia Neoechinorhynchidae Van Cleave, 1919. An Inst. Biol. Univ. Nac. Auton. Méx. Sér. Zool. 40:1-14. 
1971. Helmintos de peces del Pacifico Mexicano. XXIX. Descripción de dos monogéneos nuevos de la familia Capsalidae Baird, 1853, subfamilia Benedeniinae Johnston, 1931, de Baja California. Rev. Biol. Trop. 18:155-171.

BRAVO-HOLLIS, M., and H. W. MANTER.

1957. Trematodes of marine fishes of Mexican waters. X. Thirteen Digenea, including nine new species and two new genera from the Pacific coast. Proc. Helminthol. Soc. Wash. 24:35-48.

BRAVO-HOLLIS, M., and F. SOGANDARES-BERNAL.

1956. Trematodes of marine fishes of Mexican waters. IX. Four gasterostomes from the Pacific coast. J. Parasitol. 42:536-539.

BRIAN, A. G. G.

1906. Copepoda parassiti dei pesci d'Italia. Genova, $191 \mathrm{p}$

BRIGGS, P. T

1970. Records of ectoparasitic isopods from Great South Bay, New York. N.Y. Fish Game J. 17:55-57.

BRINKMAN, A., Jr.

1952. Fish trematodes from Norwegian waters. Univ. Bergen Arb. (now Sarsia) 1:1-134.

1962. A plerocercoid (Cestoda: Tetraphyllidae) from Luvarus imperialis Raf., captured in Norwegian waters. Sarsia 7:1-5.

BROOKS, D. R., and E. B. BROTHERS.

1974. Helminths of three species of goby (Pisces: Gobiidae) from Mission Bay, San Diego. J. Parasitol. 60:1062-1063.

BROOKS, D. R., and M. A. MAYES.

1975. Phyllodistomum scrippsi sp. n. (Digenea: Gorgoderidae) and Neobenedenia girellae (Hargis, 1955) Yamaguti, 1963 (Monogenea: Capsalidae) from the California sheephead, Pimelometopon pulchrum (Ayres) (Pisces: Labridae). J. Parasitol. 61:407-408.

BROOKS, G. L.

1934. Some new ectoparasitic trematodes (Onchocotylinae) from the gills of American sharks. Parasitology 26:259-267.

BRUSCA, R. C.

1977. Range extensions and new host records of cymothoid isopods (Isopoda: Cymothoidae) in the eastern Pacific Ocean. Bull. South. Calif. Acad. Sci. 76:128-131.

BULLOCK, W. L.

1962. A new species of Acanihocephatus from Nêw England fisheses, with observations on variability. J. Parasitol. 48:442-451.

BURNETT-HERKES, J.

1974. Parasites of the gills and buccal cavity of the dolphin, Coryphaena hippurus, from the Straits of Florida. Trans. Am. Fish. Soc. 103:101-106.

BURRESON, E. M.

1976a. Aestabdella gen. n. (Hirudinea: Piscicolidae) for Johnanssonia $a b$ ditovesiculata Moore 1952 and Icthyobdella platycephali Ingram 1957. J. Parasitol. 62:789-792.

1976b. Trachelobdella oregonensis sp. n. (Hirudinea: Piscicolidae), parasitic on the cabezon, Scorpaenichthys marmoratus (Ayres), in Oregon. J. Parasitol. 62:793-798.

1977a. A new marine leech Austrobdella californiana n. sp. (Hirudinea: Piscicolidae) from southern California flatfishes. Trans. Am. Microsc. Soc. 96:263-267.

1977b. Two new marine leeches (Hirudinea: Piscicolidae) from the west coast of the United States. Publ. Inst. Biol. Especiales 4:503-512.

1977c. Two new species of Malmiana (Hirudinea: Piscicolidae) from Oregon coastal waters. J. Parasitol. 63:130-136.

BURRESON, E. M., and I. PRATT.

1972. Trypanosoma pacifica sp. n. from the English sole Parophrys vetulus Girard from Oregon. J. Protozool. 19:555-556.

BURT, M. D. B., and I. M. SANDEMANN.

1969. Biology of Bothrimonus (= Diplocotyle) (Pseudophyllidea: Cestoda). Part I. History, description, synonymy, and systematics. J. Fish. Res. Board Can. 26:975-996.

BUSSIERAS, J.

1972. Les monogènes Capsalinae parasites des thons de l'Atlantique tropical oriental. Ann. Parasitol. Hum. Comp. 47:29-49.

BUSSIERAS, j., and J. F. ALDRIN.

1968. Sphyriocephalus dollfusi n. sp., cestode Trypanorhyncha parasite de l'estomac du thon patudo, Thunnus obesus. Ann. Parasitol. Hum. Comp. 43:645-653.

BUSSIERAS, J., and F. BAUDIN-LAURENCIN.

1970. Caballerocotyla abidjani n. sp. (Monogenea, Capsalidae), parasite des opercules du thon albacore, Thunnus albacares. Cah. O.R.S.T.O.M., ser. Océanogr. 8:47-51.
BYKHOVSKAYA-PAVLOVSKAYA, 1 . E., A. V. GUSSEV, M. N. DUBININA, N. A. IZYUMOVA, P. F. SMIRNOVA, I. L. SOKOLOVSKAYA, G. A. SHTEIN, S. S. SHULMAN, and V. M. EPSHTEIN.

1962. Key to parasites of fresh water fish of the USSR. [In Russ.] Izv. Akad. Nauk SSSR, Moscow and Leningrad, 776 p. (Transl, from Russian by $A$. Birron and Z. S. Cole, Israel Program for Scientific Translations, Jerusalem, No. 1136.)

BYKHOVSKY, B. E.

1957. Monogenetic trematodes their systematics and phylogeny. (Engl. Ed. W. J. Hargis, Jr.) Am. Inst. Biol. Sci., Wash. (1961

BYKHOVSKY, B. E., and A. V. GUSSEV.

1950. The family Diclybothriidae and its systematic position. [In Russ.] Parazitol. Sb. 12:275-299.

BYKHOVSKY, B. E., and L. F. NAGABINA.

1959. On new representatives of monogenetic trematodes from the South China Seas. [In Chin. and Russ.] Acta Zool. Sin. 11:211-236.

BYKHOVSKY, B. E., and Y U. I. POLYANSKI.

1953. Contribution to the knowledge of marine monogenetic trematodes of the family Gyrodactylidae Cobb. [In Russ.] Tr. Zool. Inst. Akad. Nauk SSSR 13:91-126.

CABALLERO, E

1950. Un nuevo genero de trematodo de peces marinos perteneciente a la familia Acanthocolpidae Lühe, 1909. An. Inst. Biol. Univ. Nac. Auton. Méx. 21:95-102.

CABALLERO, E., and M. BRAVO-HOLLIS

1955. Trematodos de peces marinos de aguas Mexicanas de Oceano Pacifico. VIII. Descripcion de tres nuevos generos de trematodos monogeneos. An. Inst. Biol. Univ, Nac. Auton. Méx. 26:89-115.

1961. Trematodos de peces de aguas Mexicanas del Pacifico. XX. Tres especies de monogenoidea Bychowsky, 1937. An. Inst. Biol. Univ. Nac. Auton. Méx. 32:201-217.

1962. Trematodos de peces de aguas Mexicanas del Pacifico. XXII. Algunos monogenoideos de la costa Sonorense del Golfo de California. An. Inst. Biol. Univ. Nac. Auton. Méx. 33:57-76.

1963. Helmintos de peces de aguas Mexicanas del Pacifico. XXIII. Descipcion de cuatro nuevos monogeneos y una breve consideracion sobre nomenclatura de esta claste. An. Inst. Biol. Univ. Nac. Auton. Méx. 34:163-203.

1967. Monogenea (Van Beneden, 1858) Carus, 1863, de peces marinos del litoral Mexicano del Golfo de Mexico y del Mar Caribe III. An. Inst. Biol. Univ. Nac. Auton Méx. 38:27-34.

CABALLERO, E., M. BRAVO-HOLLIS, and R. GROCOTT.

1952. Helmintos de la Republica de Panama. III. Tres trematodos de peces marinos condescripcion de una nueva especie. An. Inst. Biol. Univ. Nac. Auton. Méx. 23:167-180.

1953. Helmintos de la Republica de Panama. VII. Descripcion de algunos trematodos de peces marinos. An. Inst. Biol. Univ. Nac. Auton. Méx. 24:97-136.

1954. Helmintos de la Republica de Panama. XIII. Descripción de dos nuevos tremátodos monogéneos, parásitos de peces marinos comestibles del Oceano Pacifico del Norte. Ciencia 14:81-86.

1955. Helmintos de la Republica de Panama. XIV. An. Inst. Biol. Univ. Nac. Auton. Méx. 26:117-147.

CABAllero, E., and G. R. CABALlero.

1969a. Etudes de la collection de trematodes collectés par Howard A. Winter chez les poissons marins de l'Océan Pacifique du Mexique et des Etats Unis d'Amerique du Nord. I. Parazitol. Sb. 24:70-73.

1969b. Etudes des trématodes récoltes par Howard Winter chez les poissons marins de l'Ocean Pacifique du Mexique et des Etats Unis. II. J. Fish. Res. Board Can. 26:957-963.

1970. Estudios de la colección de tremátodos colectados por Howard A Winter en peces marinos del Oceano Pacífico de México y de los Estados Unidos de Norte America. III. An. Esc. Nac. Cienc. Biol. Méx. 18:265-275.

1971a. Estudios de la colección de tremátodos colectados por Howard A. Winter en peces del Océano Pacífico de México y de los Estados Unidos de Norte America. IV. Rev. Biol. Trop. 18:139-147.

1971b. Presencia de una nueva especie de trematoda de la familia Didymozoidae que parasita a peces marinos de Ensenada, Baja California, Mexico. An. Inst. Biol. Univ. Nac. Auton. Mex. 42. Ser. Cienc. Mar. Limnol. 1:57-64.

CABALLERO, R. G.

1974. Contribucion al conocimiento de los nematodos do peces de los literales de Mexico. III. Dos nuevas formas. Publ. Biol. Inst. Invest. Cient. U.A.N.L. 1:33-40 
CABLE, R. M

1956. Opistholebes diodontis n. sp., its development in the final host, the affinities of some amphistomatous trematodes from marine fishes and the allocreadioid problem. Parasitology 46:1-13.

CABLE, R. M., and J. LINDEROTH.

1963. Taxonomy of some Acanthocephala from marine fishes with reference to species from Curacao, N.A., and Jamaica, W.I. J. Parasitol. 49:706-716.

CAMPANA-ROUGET, Y.

1955. Parasites de poissons de mer ouest-africains récoltés par J. Cadenat. IV. Nematodes (lre note). Parasites de sélaciens. Bull. Inst. Fr. Afr. Noire Sér. A, Sc. Nat. 17:818-839.

CAMPANA-ROUGET, Y., and M. RAZARIHELISSOA.

1965. Spirocamallanus olseni n. sp. (Nematoda, Camallanidae), parasite de poissons de mer de Nossi-Bé. Ann. Parasitol. Hum. Comp. 40:171176.

CAMPBELL, R. A

1977. New tetraphyllidean and trypanorhynch cestodes from deep-sea skates in the western North Atlantic. Proc. Helminthol. Soc. Wash. 44:191-197.

CAMPBELL, R. A., and T. A. MUNROE.

1977. New hemiurid trematodes from deep-sea benthic fishes in the western North Atlantic. J. Parasitol. 63:285-294.

CANAVAN, W. P.

1928. A new species of Phyllobothrium van Ben. from an Alaska dog salmon with a note on the occurrence of Crossobothrium angustum Linton, in the thresher shark. J. Helminthol. 6:51-55.

CAPART, A.

1953. Quelques copepodes parasites de poissons marine de la région de Dakar. Bull. Inst. Fr. Afr. Noire Sér. A, 15:647-671.

CARLI, A., and C. BRUZZONE.

1973. Liste de copépodes parasites (nouveaux genres, nouvelles especes, nouvelles variétés) décrits par Alexandre Brian. Crustaceana 25:129-132.

CARVAJAL, J.

1971. Grillotia dollfusi sp. n. (Cestoda: Trypanorhyncha) from the skate, Raja chilensis, from Chile, and a note on G. heptanchi. J. Parasitol. 57:1269-1271.

1974. Records of cestodes from Chilean sharks. I. Parasitol 60:29-34

CAUSEY, D.

1953a. Parasitic Copepoda from Grand Isle, Louisiana. Occas. Pap. Mar. Lab. La. State Univ. 7:1-18

1953b. Parasitic copepoda of Texas coastal fishes. Publ. Inst. Mar. Sci. Univ. Tex. 3:7-16.

1955. Parasitic Copepoda from Gulf of Mexico fish. Occas. Pap. Mar. Lab. La. State Univ. 9:1-19.

1960. Parasitic copepoda from Mexican coastal fishes. Bull. Mar. Sci. Gulf Caribb. 10:323-337.

CHANDLER, A. C.

1935. Parasites of fishes in Galveston Bay. Proc. U.S. Natl. Mus. 83(2977):123-157.

1954. Cestoda. U.S. Fish Wildl. Serv., Fish. Bull. 55:351-353.

CHAPPELL, L. H.

1969. The parasites of the three-spined stickleback Gasterosteus aculeatus L. from a Yorkshire pond. I. Seasonal variation of parasite fauna. J. Fish Biol. 1:137-152.

CHAPPELL, L. H., and R. W. OWEN.

1969. A reference list of parasite species recorded in fresh water fish from Great Britain and Ireland. J. Nat. Hist. 3:197-216.

CHAUHAN, B. S.

1945. Trematodes from Indian marine fishes. Part I. On some new monogenetic trematodes of the sub-orders Monopisthocotyla Odhner, 1912 and Polyopisthocotylea Odhner, 1912. Proc. Indian Acad. Sci. sect. B 21:129-159.

1953. Studies on the trematode fauna of India. Part I. Subclass Monogenea. Rec. Indian Mus. 51 (Part II):113-208.

CHIA-JUI, $\mathrm{S}$.

1957. Parasitic copepods from fishes of China. Part II. Caligoida, Caligidae (1). Acta Zool. Sin. 9:351-377.

CHING, H. L.

1960a. Some digenetic trematodes of fishes of Friday Harbor, Washington. J. Parasitol. 46:241-250.

1960b. Some digenetic trematodes of shore birds at Friday Harbor, Washington. Proc. Helminthol. Soc. Wash. 27:53-62.

1961. Redescription of the digenetic trematodes Lepidapedon calli and $L$. pugetensis Acena, and new host records for L. calli and Eurycreadium vitellosum Manter from fishes of Washington State. Can. J. Zool. 39:615-621.

CHUBB, J. C

1970a. The parasite fauna of British fresh water fish. In A. E. R. Taylor and R. Muller (editors), Symposia of the British Society for Parasitology. Vol. 8. Aspects of fish parasitology, p. 119-144. Blackwell Sci. Publ., Oxf.

1970b. The parasites of the three-spined stickleback Gasterosteus aculeatus L., in an oligotrophic lake, Llyn Padarn, North Wales. [Abstr.] J. Parasitol. 56 (4, sect. 2) (Int. Congr. Parasitol. (2nd), Wash., D.C. Sept. 6-12, 1970, Proc. Part I), p. 56.

COHN, L.

1902. Zur Kenntnis des Genus Wageneria Monticelli und anderer Cestoden. Zentralbl. Bakteriol. 33:53-60.

COLLINS, J. J., and A. O. DECHTIAR.

1974. Parasite fauna of kokanee salmon (Oncorhynchus nerka) introduced into Lake Huron. J. Fish. Res. Board Can. 31:1818-1821.

COOPER, A. R.

1915. Trematodes from marine and freshwater fishes, including one species of ectoparasitic turbellarian. Trans. R. Soc. Can. Ser. 3, 9(4):181-205.

1921. Trematodes and cestodes of the Canadian Arctic Expedition, 1913-18. Rep. Can. Arct. Exped. 1913-18, 9, (G-H):1-27.

COPE, O. B.

1959. New parasite records from stickleback and salmon in an Alaska stream. Trans. Am. Microsc. Soc. 78:157-162.

CORDERO-DEL-CAMPILLO, M., and M. P. ALVAREZ-PELLITERO.

1976. Spinitectus gordoni n. sp. (Nematoda: Spiruroidea) from brown and rainbow trouts in West Spain. Rev. Iber. Parasitol. 36:35-66.

CORDONNIER, L. M., and H. L. WARD.

1967. Pomphorhynchus roccisp. n. (Acanthocephala) from the rock bass, Roccus saxatilis. J. Parasitol. 53:1295-1297.

CRANE, J. W.

1972. Systematics and new species of marine monogenea from California. Wasmann J. Biol. 30:109-166.

CRANE, J. W., and J. D. MIZELLE.

1967. Studies on monogenetic trematodes. XXX1. Five new species of Gyrodactylus from California fishes. J. Parasitol. 53:270-273.

CRESSEY, R.

1963. A new genus of copepods (Caligoida, Pandaridae) from a thresher shark in Madagascar. Cah. O.R.S.T.O.M. Ser. Océanogr. 1:285-297.

1966. A new species of Dentigryps Wilson (Copepoda, Caligoida) from Madagascar. Proc. Biol. Soc. Wash. 79:91-94.

1967a. Caligoid copepods parasitic on sharks of the Indian Ocean. Proc. U.S. Natl. Mus. 121(3572):1-21.

1967b. Genus Gloiopotes and a new species with notes on host specificity and intraspecific variation (Copepoda: Caligoida). Proc. U.S. Natl. Mus. 122:1-22.

1967c. Revision of the family Pandaridae (Copepoda: Caligoida). Proc. U.S. Natl. Mus. 121(3570):1-133.

1968. Caligoid copepods parasitic on Isurus oxyrinchus with an example of habitat shift. Proc. U.S. Natl. Mus. 125:1-26.

1969a. Caligus hobsoni, a new species of parasitic copepod from California. J. Parasitol. 55:431-434.

1969b. Five new parasitic copepods from California inshore fish. Proc. Biol. Soc. Wash. 82:409-427.

1970. Copepods parasitic on sharks from the west coast of Florida. Smithson. Contrib. Zool. 38:1-30.

1972. Revision of the genus Alebion (Copepoda: Caligoida). Smithson. Contrib. Zool. 123:1-29.

CUNHA, A. M. DA, and O. DA FONSECA.

1918. Sobre os Myxosporidios dos peixes do Brazil. (2a nota previa). Bras.-Med. 32:385.

DAILEY, M. D.

1969a. A survey of helminth parasites in the squid, Loligo opalescens, smelt, Osmerus mordax, jack mackerel, Trachurus symmetricus, and Pacific mackerel, Scomber japonicus. Calif. Fish Game 55:221-226.

1969b. Litobothrium alopias and $L$. coniformis, two new cestodes representing a new order from elasmobranch fishes. Proc. Helminthol. Soc. Wash. 36:218-224.

1970. The transmission of Parafilaroides decorus (Nematoda: Metastrongyloidea) in the California sea lion (Zalophus californianus). Proc. Helminthol. Soc. Wash. 37:215-222.

1971. Litobothrium gracile sp. n. (Eucestoda: Litobothridea) from the sand shark (Odontaspis ferox). J. Parasitol. 57:94-96.

DAILEY, M., L. JENSEN, and B. W. HILL.

In press. Larval ascaridaid roundworms of marine fishes from southern 
California with comments on public health implications. Calif. Fish Game.

DAILEY, M. D., and D. R. MUDRY.

1968. Two new species of cestoda from California rays. J. Parasitol. $54: 1141-1143$.

DAILEY, M., and R. M. OVERSTREET.

1973. Cathetocephalus thatcheri gen. et. sp. n. (Tetraphyllidea: Cathetocephalidae fam. n.) from the bull shark: A species demonstrating multistrobilization. J. Parasitol. 59:469-473.

DAVIS, H. S.

1917. Myxosporidia of the Beaufort region, a systematic and biologic study. Bull. U.S. Bur. Fish. 35:203-243.

1923. Observations on an intestinal flagellate of trout. J. Parasitol. 9:153-160.

1925. Schizamoeba salmonis, a new ameba parasitic in salmonid fishes. Bull. U.S. Bur. Fish. 42:1-8.

1943. A new polymastigine flagellate, Costia pyriformis, parasitic on trout J. Parasitol. 29:385.

1947. Studies of the protozoan parasites of fresh water fishes. U.S. Fish Wildl. Serv, Zool. Bull. 51.

1953. Culture and diseases of game fishes. Univ. Calif. Press, Berkeley, $332 \mathrm{p}$.

DAVIS, H. S., G. HOFFMAN, and E. W. SURBER.

1961. Notes on Sanguinicola davisi (Trematoda: Sanguinicolidae) in the gills of trout. J. Parasitol. 47:512-514.

DAVIS, W. S.

1956. American shad, Alosa sapidissima, parasitized by Argulus canadensis in the Connecticut River. J. Parasitol. 42:315.

DAVISON, R. C., and W. BREESE.

1954. The hemoflagellate, Cryptobia salmositica, in Oregon salmon. J Parasitol. 40:703.

DAWES, B.

1940. Hexacotyle extensicauda $\mathrm{n}$. sp., a monogenetic trematode from the gills of the tunny (Thunnus thynnus L.). Parasitology 32:271-286.

1947. The Trematoda of British fishes. Ray Soc., Lond., 364 p.

DEARDORFF, T. L., and R. M. OVERSTREET.

1980. Review of Hysterothylacium and Iheringascaris (both previously = Thynnascaris) (Nematoda: Anisakidae) from the northern Gulf of Mexico. Proc. Biol. Soc. Wash. 93:1035-1079.

DELAMARE-DEBOUTTEVILLE, C.

1948. Sur un Conchoderma auritum (Crust. Cirripede) parasite branchial de squale pélerin (Cetorhinus maximus Gun.) à Banyuls. Bull. Mus. Natl. Hist. Nat.-2e serie, 20:448-449.

1962. Prodrome d'une faune d'Europe des copépods parasites de poissons. Bull. Inst. Océanogr. Monaco. 1249:3-44.

DELAMARE-DEBOUTTEVILLE, C., and L. P. NUNES.

1952. Copépods parasites des poissons de Banyuls (2me serie). Vie Milieu 3:292-300.

DELAMARE-DEBOUTTEVILLE, C., and L. NUNES-RUIVO.

1953. Copépodes parasites des poissons Méditerranéens. Vie Milieu 4:201-218.

1954. Parasites de poissons de mer ouest-Africains récoltés par M. J. Cadenat. Bull. Inst. Fr. Afr. Noire Ser. A, 16:139-166.

1958. Copépodes parasites des poissons Méditerranées (4e série). Vie Milieu 9:215-235.

DEL CAMPILLO, M. CORDERO, and A. M. FERNANDEZ.

1971. Crepidostomum farionis (Muller, 1784) Luhe, 1909, en Salmo trutta de Leon. Con notas sobre sinonimia, lista de hospedadores y distribución geográfica. Anal. Fac, Vet. Leon 17:55-68.

DE PAIVA CARVALHO, J.

1962. Ergasilus cyanopictus sp. nov., parasito da tainha-Mugil cephalus

(L.) Arch. Mus. Nac. Rio de Janeiro 52:31-36.

DILLON, W. A., and W. J. HARGIS, Jr.

1968. Monogenetic trematodes from the southern Pacific Ocean. Part IV. Polyopisthocotyleids from New Zealand fishes. The families Mazocraeidae, Diclidophoridae and Hexabothriidae. Proc. Biol. Soc. Wash. $81: 351-366$.

DOGIEL, V. A.

1948. Parasitic Protozoa of fish in Peter the Great Bay. [In Russ.] Izv. Gos. Nauchno-Issled. Inst. Ozern. Rech. Rȳbn. Khoz. 27:17-66. (From Ind.-Cat. Med. Vet. Zool. Suppl. 16, p. 79.)

1955. The general character of the parasitic fauna of animals inhabiting Far Eastern seas. [In Russ.] Tr. Zool. Inst. Leningr. 21:53-61.

DOGIEL, V. A., and A. AKHMEROV.

1952. Parasitic copepods of Amur fishes. Uchen. Zapiski Leningr. Gosudarstv. Univ. Ser. Biol. Nauk 28:268-294.
1959. New species of parasitic protozoa in Amur River fishes. [In Czek]. Cesk. Parasitol. 6:15-25.

DOLLFUS, R. P.

1933. Thynnascaris legendrei n. gen., n. sp, de l'estomac du germon, Germo alalonga (Gmel.). Bull. Soc. Zool. Fr. 58:7-13.

1935a. Nématode du genre Goezia chez une truite arc-en-ciel (Salmo irideus W. Gibbons) d'élevage. Bull. Soc. Zool. Fr. 60: $244-\overline{2} 6 \overline{5}$.

1935b. Sur quelques parasites de poissons récoltés a Castiglione (Algére). Bull. d. Travaux Publiés par la Station d'Agriculture et Pêche de Castiglione, Ann. 1933. 2:199-279.

1937. Les trématodes Digenea des sélaciens (Plagiostomes) catalogue par hotes. Distribution geographique. Ann. Parasitol. Hum. Comp. 15:57-73.

1942. Études critiques sur les tétrarhynques du Muséum de Paris. Arch. Mus. Natl. Hist. Nat. (Paris) 19:1-466.

1946. Essaj de catalogue des parasites poisson-lune. Ann. Soc. Sci. Natl. Charante-Maritime. 3:69-76.

1952. Parasites du Germo alalunga (J. F. Gmelin 1788). J. Cons. Perm. Int. Explor. Mer 18:42-44.

1955. Cnidosporidie chez un thon, Thunnus thynnus (L.) de l'Atlantique Marocain. Soc. Sci. Nat. Phys. Maroc. 5:92-95.

1956. Liste des parasites animaux du hareng de l'Atlantique Nord et de la Baltique. J. Cons. Perm. Int. Explor. Mer 22:58-65.

1961. Station experimentale de parasitologie de Richelieu (Indre-et-Loire). Contribution a la Faune parasitaire regionale. Chapter 1. Liste des parasites par hôtes. Ann. Parasitol. Hum. Comp. 36:174-265.

1962. Deux espèces de trématodes monogénétiques parasite du "bluefin tuna" de Californie. Ann. Parasitol. Hum. Comp. 37:517-529.

1969a. De quelques cestodes tétraryhnques (Hétéracantes et Pécilacanthes) récoltés chez des poissons de la Méditerranée. Vie Milieu, Ser. A, 20:491-542.

1969b. Quelques espéces de cestodes tétrarhynques de la côte Atlantique des Etats Unis, dont l'une n'etait pas connue a l'état adulte. J. Fish. Res. Board Can. 26:1037-1061.

DOMBROSKI, E.

1955. Cestode and nematode infection of sockeye smolts from Babine Lake, British Columbia. J. Fish. Res. Board Can. 12:93-96.

DORAN, D. J

1953. New monogenetic trematodes from the shovelnose guitarfish, Rhinobatos productus (Ayres). J. Parasitol. 39:145-151.

DUBININA, M. N.

1971. Cestodes of fish in Amur Basin. [In Russ.] Parazitol. Sb. 25:77-119.

DUFF, D. C. B.

1934. Octomitus. Biol. Board Can. Annu. Rep. for 1933, p. 76.

DUNKERLY, J. S.

1920. Fish Myxosporidia from Plymouth. Parasitology 12:328-333.

DURIO, W. O., and H. W. MANTER.

1968. Some digenetic trematodes of marine fishes of New Caledonia. Part I. Bucephalidae, Monorchidae, and some smaller families. Proc. Helminthol. Soc. Wash. 35:143-153.

EDWARDS, S. R., and F. M. NAHHAS.

1968. Some endoparasites of fishes from the Sacramento-San Joaquin Delta, California. Calif. Fish Game 54:247-256.

EGUCHI, S.

1932. Studies on some parasites of Oncorhynchus in Japan. II. On the secondary intermediate host of Diphyllobothrium latum in Japan, with special reference to the fishes of genus Oncorhynchus. Osakakoto-IgakuSemon-Gakko Zasshi 1:130-136.

EKBAUM, E. K.

1935. Über eine neue Cystidicola in der Schwimmblase von Oncorhynchus kisutch Walbaum. Z. Parasitenkd. 7:515.

1938. Notes on the occurrence of Acanthocephala in Pacific fishes. I. Echinorhynchus gadi (Zoega) Müller in salmon and E. lageniformis sp. nov. and Corynosoma strumosum (Rudolphi) in two species of flounder. Parasitology 30:267-274.

ELKAN, E.

1962. Dermocystidium gasterostei n. sp., a parasite of Gasterosteus aculeatus L. and Gasterosteus pungitius L. Nature(Lond.) 196:958-960.

ELLIS, M. F.

1930. Investigations on the protozoan fish parasites of the St. Andrew's Region. Trans. Nova Scotian Inst. Sci. 17:268-275.

EPSHTEIN, V.M

1964. Towards a zoogeographical characterization of the fish leeches of the Amur Basin. [In Russ.] Dokl. Akad. Nauk SSSR 159:1179-1182. 
ERASMUS, D. A.

1962. Studies on the adult and metacercaria of Holostephanus Iuhei Szidat, 1936. Parasitology 52:353-374.

EUZET, L.

1956. Recherches sur les cestodes tétraphyllides des sélaciens des côtes de France. Theses Fac. Sci. de Montpellier, 266 p.

EUZET, L., and C. COMBES.

1969. Contribution a l'etude des Microcotylidae (Monogenea), parasites de Mugil cephalus L. (Teleostei). Parazitol. Sb. 24:91-105.

EUZET, L., and J. P. QUIGNARD.

1961. Sur deux parasites de Xiphias gladius L. Rapp. P.-V. Réun. Cons. Perm. Int. Explor. Mer New Ser. 16:321-323.

FEE, A. R.

1927. The Isopoda of Departure Bay and vicinity, with descriptions of new species, variations, and color notes. Contrib. Can. Biol. Fish. New Ser. 3:13-47.

FILIMONOVA, L. V.

1964. Occurrence of new intermediate and additional hosts of the trematode Nanophyetus schikhobalowi. [In Russ.] Tr. Gel'mintol. Lab. 14:246-251.

FISCHTHAL, J. H.

1972. Zoogeography of digenetic trematodes from West African marine fishes. Proc. Helminthol. Soc. Wash. 39:192-203.

FISCHTHAL, J. H., and R. E. KUNTZ.

1963. Trematode parasites of fishes from Egypt. Part V. Annotated record of some previously described forms. J. Parasitol. 49:91-98.

1964. Digenetic trematodes of fishes from Palawan Isiand, Philippines. IV. Some immature Didymozoidae, a bucephalid; a new hemiurid genus and subfamily. J. Parasitol. 50:253-260.

FISCHTHAL, J., and I. D. THOMAS.

1970a. Digenetic trematodes of marine fishes from Ghana: family Lepocreadiidae. J. Helminthol. 44:365-386.

1970b. Digenetic trematodes of marine fishes from Ghana: family Opecoelidae. Proc. Helminthol. Soc. Wash. 37:129-144.

FISH, F. F.

1939. Observations on Henneguya salminicola Ward, a myxosporidian parasitic in Pacific salmon. J. Parasitol. 25:169-172.

FITZGERALD, P. R.

1975. New coccidia from the spiny dogfish shark (Squalus acanthias) and great sculpin (Myoxocephalus polyacanthocephalus). J. Fish. Res. Board Can. 32:649-651.

FOERSTER, R. E.

1929. An investigation of the life history and propagation of the sockeye salmon (Oncorhynchus nerka) at Cultus Lake, British Columbia, III. The down-stream migration of the young in 1926 and 1927. Contrib. Can. Biol. Fish. New Ser, 5:55-82.

FOLDA, F.

1928. Megalocotyle marginata, a new genus of ectoparasitic trematodes from the rock fish. Publ. Puget Sound Biol. Stn. 6:195-206.

FOX, A. C.

1965. Some effects of strigeid metacercariae on rainbow trout (Salmo gairdneri) (Abstract of report before 80th Annu. Meet., Boulder, Colo., Aug. 24-25). Trans. Am. Micros. Soc. 84:153.

FRASER, C.

1920. Copepods parasitic on fish from the Vancouver Island region. Trans. R. Soc. Can. Sect. 5, Ser. 3, 13:45-68.

FREZE, V. I.

1965. Proteocephalata-tapeworms of fish, amphibians and reptiles. In K. I. Skrjabin (editor), Principles of cestodology, Vol. 5, 538 p. Izdat. "Nauka", Moscow. (Transl. from Russian by Israel Program for Sci. Transl. Jerusalem, No. 1853, 1969.)

FRIT'TS, D. H.

1959. Helminth parasites of the fishes of Northern Idaho. Trans. Am. Microsc. Soc. 78:194-202.

FRYER, J. L., and J. F. CONRAD.

1965. Some observations on furunculosis in adult Pacific salmon and steelhead trout. Prog. Fish-Cult. 27:99-100.

FUJITA, T.

1920. On the parasite of Japanese fishes. I. Acanthocephala. Zool. Mag. Tokyo 32:275-283.

1921a. On parasites of Japanese fishes. IV. 3 Nematoda. Zool. Mag. Tokyo 33:292-300.

1921b. Parasites of fishes. 1l. Trematoda. Zool. Mag. Tokyo 33:137141.
1923. Studies on Myxosporidia of Japan. J. Fac. Agric. Hokkaido Imp. Univ. 10:191-248.

1932. On new nematodes (Contracaecum) in fishes of Japan. Bull. Sch. Fish. Hokkaido Imp. Univ. 2:24-30.

1936. Notes on some Myxosporidia in freshwater fishes. Zool. Mag. Tokyo 48:595-601.

1939. On the nematode parasites of the Pacific salmon. J. Fac. Agric. Hokkaido Imp. Univ. 42 (part 3):239-266.

1940. Further notes on nematodes of salmonoid fishes in Japan. Jpn. J. Zool. 8:377-394.

1941. New names for Metabronema salvelini Fujita and Cystidicola minuta Fujita. J. Parasitol. 27:542.

FUKUI, T.

1958. Studies on the parasites of salmonid fishes in Japan. I. Collection of theses, Yokohama University. 10:581-634. (Transl. Fish. Res. Board Can. 1962.)

GEBHARDT, G. A., R. E. MILLEMANN, S. E. KNAPP, and P. A. NYBERG.

1966. "Salmon poisoning" disease. II. Second intermediate host susceptibility studies. J. Parasitol. 52:54-59.

GETSEVICHIUTE, S.

1958. Parasite fauna of fish in Kursiu Maru Bay. [In Russ.] Liet TSR Mokslu Akad. Biol. Inst. Darb. 3:101-139. (From Ind.-Cat. Med. Vet. Zool. Suppl. 15.)

GIBSON, D. I.

1976. Monogenea and Digenea from fishes. Discovery Rep. 36:176-266.

GLLES, D. E.

1962. New bucephalid cercaria from the mussel, Mytilus californianus. J. Parasitol. 48: 293-295.

GINETSINSKAYA, T. A.

1955. The life cycles of fish helminths and the biology of their larval stages. In V. A. Dogiel, G. K. Petruschevski, and Yu. I. Polyansky (editors), Parasitology of fishes, p. 140-179. (Engl. ed. 1961). Oliver and Boyd, Lond. Russian ed. Leningrad Univ. Press.

GOHAR, N.

1934. Les trematodes parasites du milan Egyptien Milvus migrans avec description d'une nouvelle espece et remarques sur les genres Haplorchus Looss 1899 et Monorchotrema Nishigori 1924. Ann. Parasitol. Hum. Comp. 12:218-227.

GOLDSTEIN, R. J.

1963. A note on the genus Poecilancistrium Dollfus, 1929 (Cestoda: Trypanorhyncha). J. Parasitol. 49:301-304.

1967. The genus Acanthobothrium Van Beneden, 1849 (Cestoda: Tetraphyllidea). J. Parasitol. 53:455-483.

GOLVAN, Y. J.

1956. Parasites de poissons de mer Ouest-Africains recoltes par J. Cadenat. Bull. Inst. Fr. Afr. Noire 18:461-481.

1969. Systematique des Acanthocephales. Premiere Partie. L'ordre des Palaeacanthocephala Meyer 1931. Premier fascicule. La super-famille des Echinorhynchoidea (Cobbold 1876) Golvan et Houin 1963. Mem. Mus. Natl. Hist. Nat. Ser. A Zool. 57:1-373.

GRABDA, J,

1963. On the invasionology of Ergasilus sieboldi Nordm. in Poland. Acta Hydrobiol. $5: \bar{z} 45-2 \overline{5} 4$.

1975. Observations on the localization and pathogenicity of Haemobaphes diceraeus Wilson, 1917 (Copepoda: Lernaeoceridae) in the gills of Theragra chalcogramma (Pallas). Acta Ichthyologica Piscatoria 5:13-23.

1976. Haemobaphes disphaerocephalus sp. n. (Copepoda: Lernaeoceridae) from the gill cavity of Thaleichthys pacificus (Richardson, 1836) (Osmeridae). Acta Ichthyologica Piscatoria 6:23-33.

GREGOIRE, E., and I. PRATT.

1952. Helminth parasites of the petrale sole. J. Pr.asitol. 38:84.

GRIFFITH, R. E.

1953. Preliminary survey of the parasites of fish of the Palouse area. Trans. Am. Microse. Soc. 73:51-57.

GROZDILOVA, T. I.

1968. Parasite fauna of pink salmon acclimatized in the White Sea. (Proceedings of the Seventh Session of the Scientific Council on Problems of "The Biological Resources of the White Sea and Internal Waters of Karelia"). Fish. Res. Board Can., Transl. Ser. 1858, p. 131-132.

GUBERLET, J, E.

1932. Parasitism in northwestern United States of North America in relationship to public health. C. R. Congr. Int. Med. Trop. Hyg. (LeCaire, Dec. 1928) 4:41-47. 
1933. Recent advances in our knowledge of the parasites of the marine fishes of the Pacific. Proc. 5th Pac. Sci. Congr. 5:4165-4169.

1936a. Trematodos ectoparasitos de los peces de la costas del Pacifico. An. Inst. Biol. Univ. Nac. Auton. Mex. 7:457-467.

1936b. Two new ectoparasitic trematodes from the stingray, Myliobalus colifornicus: Am. Midl Nat 17:954-964

GUBERLET, J., H. A. HANSEN, and J. A. KAVANAUGH.

1927. Studies on the control of Gyrodactylus. Univ. Wash. Publ. Fish., New Ser. 2:17-29.

GUIART, J

1938a. Trematode parasites provenant des campagnes scientifiques de Prince Albert $1^{\mathrm{cr}}$ de Monaco. Res. Cap. Scient. Albert $1 \mathrm{cr}$ Prince Monaco, fasc. C 75:76-84.

1938b. Trematodes parasites provenant des campagnes scientifiques de S.A.S. le Prince Albert $1^{\text {er }}$ de Monaco (1886-1912) (Monogenea), p. 1-16. GUSSEV, A. B

1951. Parasitic Copepoda of some marine fishes. [In Russ.] Parazitol. Sb. 13:394-463.

HAAKER, R. L.

1975. The biology of the California halibut, Paralichthys californicus (Ayres) in Anaheim Bay. In E. D. Lane and C. W. Hill (editors), The marine resources of Anaheim Bay, p. 137-151. Calif. Fish Game, Fish Bull. 165.

HADERLIE, E. C.

1953. Parasites of the freshwater fishes of Northern California. Univ. Calif. Publ. Zool. 57:303-440.

HAFEEZULLAH, M.

1971. On some new and known digenetic trematodes from marine fishes of India. J. Helminthol. 45:73-88.

HALL, J. R., and I. PRATT.

1969. Some digenetic trematodes of Oregon tidepool cottid fishes. J. Parasitol. 55:207.

HALLIDAY, M. M.

1976. The biology of Myxosoma cerebralis: the causative organism of whirling disease of salmonids. J. Fish Biol. 9:339-357.

HANAN, D. A.

1976. A new species of cyclopoid copepod, parasitic on shiner surfperch, Cymatogaster aggregata Gibbons, in Anaheim Bay and Huntington Harbor, California, with notes on Bomolochus cuneatus Fraser and Ergasilus lizae Krфyer. Bull. South. Calif, Acad. Sci. 75:22-28.

HANEK, G., and K. MOLNAR.

1974. Parasites of freshwater and anadromous fishes from Matamek River systems, Quebec. J. Fish. Res. Board Can. 31:1135-1139.

HANEK, G., and W. THRELFALL.

1969a. Digenetic trematodes from Newfoundland, Canada. I. Three species from Gasterosteus aculeatus Linnaeus, 1758. Can. J. Zool. 47:793794.

1969b. Digenetic trematodes from Newfoundland, Canada. 2. Two species from Gasterosteus aculeatus Linnaeus, 1758. Can. J. Zool. 47:1086-1087.

1969c. Monogenetic trematodes from Newfoundland, Canada. 1. New species of the genus Gyrodactylus Nordmann, 1832. Can. J. Zool. 47:951.955.

1970. Parasites of the threespine stickleback (Gasterosteus aculeatus) in Newfoundland and Labrador. J. Fish. Res. Board Can. 27:901-907.

HARADA, I.

1935. Zur Acanthocephalen fauna von Japan. Mem. Fac. Sci. Agric. Taihoku Imp. Univ. 14:7-23.

HARGIS, W. J., Jr.

1955a. A new species of Benedenia (Trematoda: Monogenea) from Girella nigricans, the opaleye. J. Parasitol. 41:48-50.

1955b. Monogenetic trematodes of Gulf of Mexico fishes. Part III. The superfamily Gyrodactyloidea (Continued), Q.J. Fla. Acad. Sci. 18: 33-47.

1955c. Monogenetic trematodes of Gulf of Mexico fishes. Part V. The superfamily Capsaloidea. Trans. Am. Microsc. Soc. 74:203-225.

1956a. Monogenetic trematodes of Gulf of Mexico fishes. Part X. The family Microcotylidae Taschenberg, 1879. Trans. Am. Microsc. Soc. 74:436-453.

1956b. Monogenetic trematodes of Gulf of Mexico fishes. Part XI. The family Microcotylidae Taschenberg, 1879. (Continued.) Proc. Helminthol. Soc. Wash. 23:153-162.

1956c. Monogenetic trematodes of Gulf of Mexico fishes. Part XII. The family Gastrocotylidae Price, 1943. Bull. Mar. Sci. Gulf Caribb. $6: 28-43$.
1957. Monogenetic trematodes of (julf of Mexico fishes. Part X1II. The family Gastrocolylidae Price, 1943. (Continued.) Trans. Am. Microsc. Soc. $76: 1-12$.

HART, J. F.

1936a. Cestoda from fishes of Puget Sound. II. Tetrarhynchoidea. Trans. Am. Microsc. Soc, 55:369-387.

1936b. Cestoda from fishes of Puget Sound. III. Phyllobothrioidea. Trans. Am. Microse. Soc. 55:488-496.

HART, J. F., and J. E. GUBERLET.

1936. Cestoda from fishes of Puget Sound. I. Spathebothrioidea, a new superfamily. Trans. Am. Microsc. Soc. 55:199-207.

HATAI, K., S. EGUSA, and T. NOMURA.

1977. Saprolegnia australis Elliot isolated from body surface lesions of rainbow trout fingerlings. Fish Pathol. 11:201-206.

HECKMANN, $R$.

1971. Parasites of cutthroat trout from Yellowstone Lake, Wyoming. Prog. Fish-Cult. 33:103-106.

HEEGAARD, $P$.

1943. Parasitic copepods mainly from tropical and Antarctic seas. Ark. Zool. 34A:1-37.

1962. Parasitic Copepoda from Australian waters. Rec. Aust. Mus. $25: 149-234$

HEINZ, M. L., and M. D. DAILEY.

1974. The Trypanorhyncha (Cestoda) of elasmobranch fishes from southern California and northern Mexico. Proc. Helminthol. Soc. Wash. 41:161-169.

HENDRIX, S. S., and R. M. OVERSTREET.

1977. Marine aspidogastrids (Trematoda) from fishes in the northern Gulf of Mexico. J. Parasitol. 63:810-817.

HENSLEY, G. H., and F. M. NAHHAS.

1975. Parasites of fishes from the Sacramento-San Joaquin Delta, California. Calif. Fish Game 61:201-208.

HEWITT, G. C.

1964a. A redescription of Gloiopotes huttoni (Thomson, 1889) with a key to the species of the genus. Trans. R. Soc. N.Z. Zool. 5:85-96.

1964b. The occurrence of Lepeophtheirts insignis Wilson (Copepoda parasitica) in New Zealand waters and its relationship to $L$. molae Heegaard. Trans. R. Soc. N.Z. Zool. 4:153-155.

1967. Some New Zealand parasitic Copepoda of the family Pandaridae. N.Z. J. Mar. Freshwater Res. 1:180-264.

1968a. Cecrops tatreilli Leach (Cecropidae, Copepoda) on Mola mola in New Zealand waters. Rec. Dom. Mus. Wellington 6:49-59.

1968b. Elytrophora brachyptera Gerstaeker (Euryphoridae, Caligoida) from New Zealand waters with a tentative revision of the genus. Trans. R. Soc. N.Z. Zool. 10:117-126.

1968c. Some New Zealand parasitic Copepoda of the family Anthosomidae. Zool. Publ. Victoria Univ. Wellington 47:1-31.

1969a. A new species of Paeonodes (Therodamasidae, Cyclopoida, Copepoda) parasitic on New Zealand freshwater fish, with a re-examination of Paeonodes exiguus Wilson. Zool. Publ. Victoria Univ. Wellington 50:32-39.

1969b. Pseudocycnus appendiculatus Heller, 1868 (Copepoda, Dichelesthiidae) in New Zealand waters. N.Z. J. Mar. Freshwater Res. 3:169176.

1969c. Some New Zealand parasitic Copepoda of the family Eudactylinidae. Zool. Publ. Victoria Univ. Wellington 49:1-31.

1971a. Species of Lepeophtheirus (Copepoda, Caligidae) recorded from the ocean sunfish (Mola mola) and their implications for the caligid genus Dentigryps. J. Fish. Res. Board Can. 28:323-334.

1971b. Two species of Caligus (Copepoda, Caligidae) from Australian waters, with a description of some developmental stages. Pac. Sci. 25:145-164.

HEWITT, G. C., and P. M. HINE.

1972. Checklist of parasites of New Zealand fishes and of their hosts. N.Z. J. Mar. Freshwater Res. 6:69-114.

HIDALGO, ESCALENTE, E.

1958. Hallazgo de una nueva especie de Capsala, Capsala pricei $\mathrm{n}$. sp. (Trematoda, Monogenea) en un pez marino del Puerto de Mazatlan Sinaloa, Mexico. An. Inst. Biol. Univ. Nac. Auton. Méx. 29:209-217.

HISCOCK, I. D.

1954. A new species of Otobothrium (Cestoda, Trypanorhyncha) from Australian fishes. Parasitology 44:65-70.

HNATH, J. C.

1969. Transfer of an adult Acanthocephalan from one fish host to another. Trans. Am. Fish. Soc. 98:332 
HO, J.

1963. On five species of Formosan parasitic copepods belonging to the suborder Caligoida. Crustaceana 5:81-98.

1970. Revision of the genera of the Chondracanthidae, a copepod family parasitic on marine fishes. Beaufortia 17:105-218.

1972a. Copepod parasites of California halibut, Paralichthys californicus

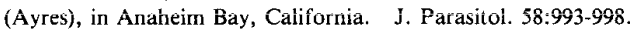

1972b. Four new parasitic copepods of the family Chondracanthidae from California inshore fishes. Proc. Biol. Soc. Wash. 85:523-539.

1975. Parasitic crustacea. In E. D. Lane and C. W. Hill (editors), The marine resources of Anaheim Bay, p. 69-72. Calif. Fish Game, Fish Bull. 165.

HOBSON, E.

1971. Cleaning symbiosis among California inshore fishes. Fish. Bull., U.S. $69: 491-523$.

HOFFMAN, G. L.

1967. Parasites of North American freshwater fishes. Univ. Calif. Press, Berkeley, $486 \mathrm{p}$.

HOLLIS, E. H., and C. M. COKER.

1948. A trematode parasite of the genus Clinostomum new to the shad, Alosa sapidissima. J. Parasitol. 34:493-495.

HOLMES, J. C.

1971a. Habitat segregation in sanguinicolid blood flukes (Digenea) of scorpaenid rockfishes (Perciformes) on the Pacific Coast of North America. J. Fish. Res. Board Can. 28:903-909.

1971b. Two new sanguinicolid blood flukes (Digenea) from scorpaenid rockfishes (Perciformes) of the Pacific Coast of North America. J. Parasitol. 57:209-216.

HOPKINS, S. H.

1954. The American species of trematode confused with Bucephalus (Bucephalopsis) haimeanus. Parasitology 44:353-370.

1966. A comparison of the parasites of two freshwater shads, Dorosoma (Dorosoma) cepedianum and Dorosoma (Signalosa) petenense, in Lake Texoma. J. Parasitol. 52:843.

HOSHINA, T.

1949. On a new myxosporidian parasite of the genus Myxosoma, M. salmonis n. sp., infecting the scales of the dog salmon. BioScience (Tokyo) 4:106-109.

1952. Notes on some myxosporidian parasites on fishes of Japan. J. Tokyo Univ. Fish. 39:69-89.

HUBBS, C. L., T. IWAI, and K. MATSUBARA.

1967. External and internal characters, horizontal and vertical distribution, luminescence, and food of the dwarf pelagic shark, Euprotomicrus bispinatus. Bull. Scripps Inst. Oceanogr. 10, $81 \mathrm{p}$.

HUFFMAN, D. G., and R. G. KLIEVER.

1977. Echinorhynchus canyonensis sp. n. (Acanthocephala) from Maynea californica (Osteichthyes: Zoarcidae) from the Monterey Submarine Canyon, California. Proc. Helminthol. Soc. Wash. 44:171-178.

HUGHES, S. E.

1973. Some metazoan parasites of the eastern Pacific saury, Cololabis saira. Fish. Bull., U.S. 71:943-953.

HUNNINEN, A. V., and G. W. HUNTER III.

1933. On the species of Crepidostomum in trout. Trans. Am. Microsc. Soc. 52:150-157.

HUTTON, R. F.

1964. A second list of parasites from marine and coastal animals of Florida. Trans. Am. Microsc. Soc. 83:439-447.

ICHIHARA, A

1970. A new blood fluke, Aporocotyle theragrae n. sp. (Aporocotylidae) from a marine fish, Theragra chalcogramma. Res. Bull. Meguro Parasitol. Mus. 3:1-4.

ICHIHARA, I.

1974. Hemiurida trematodes from marine fishes near the Tsushima Islands in the Sea of Japan. Proc. 3d Int. Congr. Parasitol. 3 (sect. G2): 1614-1615

ICHIHARA, I., K. KATO, S. KAMEGAI, and M. MACHIDA

1968. On the parasites of fishes and shell-fishes in Sagami Bay. (No. 4) Parasitic helminths of mackerel Pneumatophorus japonicus (Houttouyn). Res. Bull. Meguro Parasitol. Mus. 2:45-60.

ILES, C.

1971. Fistulicola plicatus (Cestoda) and Tristoma spp. (Trematoda) on swordfish from the northwest Atlantic. J. Fish. Res. Board Can. 28:31-34.

INGHAM, L., and C. ARME.

1973. Intestinal helminths in rainbow trout, Salmo gairdneri (Richardson):
Absence of effect on nutrient absorption and fish growth. J. Fish Biol. 5:309-313.

ISHII, N.

1935. Studies on the family Didymozoidae (Monticelli, 1888). Jpn. J. Zool. 6:279-335.

1936. Some new ectoparasitic trematodes of marine fishes. Zool. Mag. (Tokyo) 48:781-790.

ISHII, N., and T. SAWADA.

1938. Studies on the ectoparasitic trematodes. Liv. Jub. Prof. Travassos 1913-1938, p. 231-244.

IVERSEN, E. S.

1954. A new myxosporidian, Myxosoma squamalis, parasite of some salmonoid fishes. J. Parasitol. 40:397-404.

IVERSEN, E. S., N. CHITTY, and N. van METER.

1971. Some Myxosporida from marine fishes in south Florida. J. Protozool. 18:82-86.

IVERSEN, E. S., and E. E. HOVEN.

1958. Some trematodes of fishes from the central equatorial Pacific. Pac. Sci. 12:131-134.

IVERSON, E. W.

1972. New hosts and bathymetric range extension for Colobomatus embiotocae (Crustacea, Copepoda). Calif. Fish Game 58:323-325.

1974. Range extensions for some California marine isopod crustaceans. Bull. South. Calif. Acad. Sci. 73:164-169.

JAMESON, A. P.

1929. Myxosporidia from California fishes. J. Parasitol. 16:59-68.

1931. Notes on Californian Myxosporidia. J. Parasitol. 18:59-68.

JENSEN, H. M., and S. R. WELLINGS.

1972. Development of the polar filament-polaroplast complex in a microsporidian parasite. J. Protozool. 19: 297-305.

JENSEN, L. A.

1976. Parabothriocephalus sagitticeps (Sleggs 1927) comb. n. (Cestoda: Parabothriocephalidae) from Sebastes paucispinis of southern and central California. J. Parasitol. 62:560-562.

JENSEN, L. A., and R. A. HECKMANN.

1977a. Anantrum histocephalum sp. n. (Cestoda: Bothriocephalidae) from Synodus lucioceps (Synodontidae) of southern California. J Parasitol. 63:471-472.

1977b. Parasites of Sebastes paucispinis, with special reference to the histopathology of Kudoa clupeidae and Henneguya sebasta. Abstr. 52nd Annu. Meet., Am. Soc. Parasitol., Las Vegas, Nev., Aug. 14-19, 1977, p. 61

JOHNSON, S. K., and W. A. ROGERS.

1972. Ergasilus clupeidarum sp. n. (Copepoda: Cyclopoida) from clupeid fishes of the southeastern U.S. with a synopsis of the North American Ergasilus species with a two-jointed first endopod. J. Parasitol. 58:385-392.

JOHNSON, W. F.

1949. A new species of trematode, Podocotyle gibbonsia, from tide pool fishes of Monterey Bay, California. Trans. Am. Microsc. Soc. 68:107-109.

JOHNSON, W. F., and J. E. COPSEY.

1953. Opisthadena bodegensis n. sp. (Trematoda) from Dillon Beach California. Trans. Am. Microsc. Soc. 72:78-81.

JOHNSTON, T. H., and S. J. EDMONDS.

1947. Australian Acanthocephala. Trans. R. Soc. S. Aust. 71:13-19.

JOHNSTON, T. H., and O. W. TIEGS

1922. New gyrodactylid trematodes from Australian fishes, together with a reclassification of the superfamily Gyrodactyloidea. Proc. Linn. Soc. N.S.W. 47: 83-131.

JOYEUX, C. H., and J. G. BAER.

1936. Cestodes. Faune Fr. 30, $613 \mathrm{p}$.

KABATA, Z

1960. On the specificity of Lernaeocera (Copepoda Parasitica). Ann. Mag. Nat. Hist., Ser. 3, 13:133-139.

1962. Five new species of Myxosporidia from marine fishes. Parasitology 52:177-186.

1967a. Morphology of Phrixocephalus cincinnatus Wilson, 1908 (Copepoda: Lernaeoceridae). J. Fish. Res. Board Can. 24:515-526.

1967b. The genus Haemobaphes (Copepoda: Lernaeoceridae) in the waters of British Columbia. Can. J. Zool. 45:853-875.

1968. Some Chondracanthidae (Copepoda) from fishes of British Columbia. J. Fish. Res. Board Can. 25:321-345.

1969a. Chondracanthus narium sp. n. (Copepoda: Chondracanthidae), a 
parasite of nasal cavities of Ophiodon elongatus (Pisces: Teleostei) in British Columbia. J. Fish. Res. Board Can. 26:3043-3047.

1969b. Phrixocephalus cincinnatus Wilson, 1908 (Copepoda: Lernaeoceridae): morphology, metamorphosis, and host-parasite relationship. J. Fish. Res. Board Can. 26:921-934.

1969c. Revision of the genus Salminicola Wilson, 1915 (Copepoda:

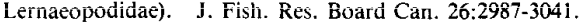

1970a. Diseases of fishes. Book I. Crustacea as enemies of fishes. T.F.H. Publ., Jersey City, N.J.

1970b. Discovery of Brachiella lageniformis (Copepoda: Lernaeopodidae) in the Canadian Pacific and its significance to zoogeography of the genus Merluccius (Pisces: Teleostei). J. Fish. Res. Board Can. 27:2159-2165.

1970c. Some Lernaeopodidae (Copepoda) from fishes of British Columbia. J. Fish. Res. Board Can. 27:865-885.

1971. Four Bomolochidae (Copepoda) from fishes of British Columbia. J. Fish Res. Board Can. 28:1563-1572.

1972. Caligus chelifer Wilson, 1905 (Copepoda: Caligidae), with a description of the male. Proc. Biol. Soc. Wash. 85:389-398.

1973. The species of Lepeophtheirus (Copepoda: Caligidae) from fishes of British Columbia. J. Fish. Res. Board Can. 30:729-759.

1974. Lepeophtheirus cuneifer sp. nov. (Copepoda: Caligidae), a parasite of fishes from the Pacific Coast of North America. J. Fish. Res. Board Can, 31:43-47.

KABATA, Z., and A. V. GUSEV.

1966. Parasitic Copepoda of fishes from the collection of the Zoological Institute in Leningrad. J. Linn. Soc. Lond. Zool. 46:155-207.

KABATA, Z., and S. N. WILKES.

1977. Peniculus asinus (Copepoda: Pennellidae), a new species of copepod parasitic on fishes of the genus Sebastes along the west coast of North America. Can. J. Zool. 55:1988-1991.

KAGEI, N., Y. SAKAGUCHI, D. KATAMINE, and Y. IKEDA.

1970. Studies on anisakid Nematoda (Anisakinne). II. Contracaecum sp. (type V of Yamaguti) found in marine fishes (Appendix: list and main features of the larvae of Contracaecum spp. recorded from marine fishes and squids caught off the Japan and its offshore islands). Bull. Inst. Publ. Health Jap. 19:243-251.

KAKATCHEVA-AVRAMOVA, D.

1966. Pseudochetosoma salmonicola Dollfus 1951 et Pseudochetsoma leucisci Ergens $196 \overline{3}$ sont-elles des espèces différentes? Ann. Parasitol. Hum. Comp. 41:307-311.

KANE, M. B.

1966. Parasites of Irish fishes. Proc. R. Soc. Sci. Dubl. Ser. B, 1:205-221.

KAY, M. W.

1942a. A new species of Phyllobothrium Van Beneden from Raja binoculata (Girard). Trans. Am. Microsc. Soc. 61:261-266.

1942b. Notes on the genus Merizocotyle Cerfontaine with a description of a new species. Trans. Am. Microsc. Soc. 61:254-260.

1945. A description of Calinella ophiodontis n. sp. (Trematoda, Monogenea) from the ling cod Ophiodon elongatus Girard. Ohio J. Sci. 45:111-114.

1947. Otodistomum plicatum n. sp. (Trematoda, Digenea) from Hexanchus griseus (Bonnaterre). Ohio J. Sci. 47:79-83.

KAZACHENKO, V. N, , V. D. KOROTAEVA, and J. V. KUROCHKIN.

1972. Parasitic crustacea of some fishes of the Pacific Ocean. [In Russ.] TINRO 81: 224-238.

KENNEDY, C. R.

1974. A checklist of British and Irish freshwater fish parasites with notes on their distribution. J. Fish Biol. 6:613-644.

1975. The distribution of some crustacean fish parasites in Britain in relation to the introduction and movement of freshwater fish. J. Inst. Fish. Manage. 6:36-41.

KHALIL, L. F.

1969. Larval nematodes in the herring (Clupea harengus) from British coastal waters and adjacent territories. J. Mar. Biol. Assoc. U.K. 49:641-659.

KILAMBI, R. V., and A. C. DeLACY.

1967. Heterogeneity of surf smeit, Hypomesus pretiosus (Girard), in the State of Washington, as judged by incidence of larval Anisakis (Nematoda). J. Fish. Res. Board Can. 24:629-633.

KING, R, E.

1962. A new species of Parahemiurus and notes on Tubulovesicula lindbergi (Trematoda: Hemiuridae) from fishes of Bahia de San Quintin, Baja California. Cont. Beaudette Found. Biol. Res. 3:330-336.

$\mathrm{KING}, \mathrm{R}$. E., and E. R. NOBLE

1961. A new species of Hysterolecitha (Trematoda: Hemiuridae) from the mudsucker Gillichthys mirabilis Cooper. J. Parasitol. 47:465-468.

KIRTISINGHE, $\mathrm{P}$.

1934. Gloiopotes watsoni n. sp. and Lernaeenicus seeri n. sp. parasitic copepods of fish from Ceylon. Parasitology 26:167-175.

1935. Parasitic copepods of fish from Ceylon. Parasitology 27:332-344.

1964. A review of the parasitic copepods of fish recorded from Ceylon with descriptions of additional forms. Bull. Fish. Res. Stin. Ceylon 17:45-132.

KNISKERN, V. B.

1952. Studies on the trematode family Bucephalidae Poche, 1907. Part I. A systematic review of the family Bucephalidae. Trans. Am. Microsc. Sóc. 71:253-266.

KO, R. C., and R. C. ANDERSON.

1969. A revision of the genus Cystidicola Fischer, 1798 (Nematoda: Spiruroidea) of the swim bladder of fishes. J. Fish. Res. Board Can. 26:849-864.

KOMIYA, Y., and N. SUZUKI.

1966. The metacercariae of trematodes belonging to the family Heterophyidae from Japan and adjacent countries. Jpn. J. Parasitol. 15:208-214.

KONOVALOV, S. M

1971. Differentiation of local populations of sockeye salmon Oncorhynchus nerka (Walbaum). Leningrad:"Nauka"' Publishing House, 1971, 229 p. (Transl. Coll. Fish., Univ. Wash., 1975, New Ser. 6, 290 p.)

KONOVALOV, S. M., A. G. SHEVLIAKOV, aITd V. K. KRASIN.

1970. Parasite fauna of various groups of the young of Oncorhynchus nerka (Walb.) from the local stock of Lake Azabachje. [In Russ.] Parazitol. 4:547-556.

KORATHA, K. J.

1955. Studies on the monogenetic trematodes of the Texas coast. II. Descriptions of species from marine fishes from Port Aransas. Publ. Inst. Mar. Sci. Univ. Tex. 4:251-273.

KOVAL, V. P., and N. D. OTSUPOK.

1964. Trematodes of some commercial fish of the Black Sea in the region of Evpatoria. [In Russ.] Tr. Ukrainsk. Respub. Nauch. Obshchest. Parazitol. 3:48-52. (From Ind.-Cat. Med. Vet. Zool. Supp1. 17, Part 7, p. 302.)

KOVALENKO, L. M.

1969. Helminth infections in Atheresthes stomias [Abstr.] In Voprosky Morskoi Biologii Tezisy II. Vsesoyuznogo Symposium Molodylch Uchenylch, Sevastopol, 1969. Kiev, USSR “Naukova Dumka," p. 61-62.

1970. Parasite infection of hake and sablefish of the northeastern part of the Pacific Ocean. [In Russ.] "Naukova Dumka”' Kiev, p. 41-43.

KOZIKOWSKA, S.

1965. Crustacés, parasites des poissons de la Pologne. III. Résultats des explorations sur les poissons de la côte méridionale de la mer Baltique. Pol. Archiwum Hydrobiol. 13:97-104.

KRITSKY, D. C., E. R. NOBLE, and M. MOSER.

1978. Allencotyla pricei $\mathrm{sp}$. n. (Microcotyloidea: Heteraxinidae) from the gills of the pile surfperch, Damalichthys vacca (Girard), in southern California. J. Parasitol. 64:45-48.

KRUSE, G. O. W

1977. Some digenetic trematodes from fishes of the Bering Sea with the descriptions of Prosorhynchus mizellei sp. n. (Bucephalidae) and Pseudopecoelus nossamani sp. n. (Opecoelidae). Helminthol. Soc. Wash. Proc. 44:73-76.

KUDO, R.

1920. Studies on Myxosporidia. A synopsis of genera and species of Myxosporidia. Ill. Biol. Monogr. 5(3,4):5-265.

KUITUNEN-EKBAUM, E.

1933a. A case of dracontiasis in Pacific coastal fishes. Contrib. Can. Biol. Fish. New Ser, 8:161-168.

1933b. Philonema oncorhynchi nov, gen. et spec, Contrib. Can. Biol. Fish. New Ser. 8:71-75.

1949. The occurrence of Sarcotaces in Canada. J. Fish. Res. Board Can. 7:505-512

KULACHKOVA, V. G.

1970. Gyrodactyloides baueri sp. n. (Monogenoidea: Gyrodactylidae) from nasal cavities of White Sea herrings. [In Russ.] Parazitol. (Leningr.) 4:544-546.

KUNNENKERI, J. K.

1962. Preliminary report on the parasites of the California sardine and the parasitic distribution in Clupeidae. J. Parasitol. 48:149.

KUNNENKERI, J. K., and W. E. MARTIN.

1962. Pigmented cestode larvae, mainly tetraphyllideans and tetrarhynchids, from fishes of the Eastern Indo-Pacific. J. Parasitol. 48:148. 
KUNNENKERI, K., and W. E. MARTIN.

1963. Further studies on the life history of Monogenea mainly from eastern Pacific fishes. J. Parasitol. 49 (Sect. 2):27.

KURIAN, C. V.

1955. Parasitic copepods of Travancore-Cochin. Bull. Cent. Res. Inst. Univ. Kerala, Ser. C, 4:103-116

KUROCHKIN, Yu. V., and A. Ya. SLANKIS

1973. New representation and the composition of the order Litobothridea Dailey, 1969 (Eucestoda). [In Russ.] Parazitol. (Leningr.) 7:502-508.

\section{KURTZ, $\mathrm{H}$.}

1924. Parasitische Copepoden aus der sammlung des naturhistorischen museums in Wien 1. Pholidopus (Achteinus) intermedius und Dissonus glaber, zwi neue arten aus der familie der Caligidae. Sber. Akad. Wiss. Wien. Math-Naturw. Abt. 1, 133:613-624.

LAIRD, M.

1961. Trichodinids and other parasitic protozoa from the intertidal zone at Nanaimo, Vancouver Island. Can. J. Zool. 39:833-844.

LAIRD, M., and W. L. BULLOCK.

1969. Marine fish haematozoa from New Brunswick and New England. J. Fish. Res. Board Can. 26:1075-1102.

LAMOTHE-ARGUMEDO, R.

1965. Descripcion de dos especiés nuevas de Lepocreadiidae. Parasitas de Verrunculus polylepis de Bahia Kino (Mexico). Rev. Iber. Parasitol. $25: 29-40$.

1966. Trematodos de peces (II). Presencia de los trematodos Bianium plicitum (Linton, 1928) Stunkard, 1931 y Lecithochirium microstomum Chandler, 1935, en peces del Pacifico Mexicano. An. Inst. Biol. Univ. Nac. Auton. Mex. 36:147-157.

1967a. Monogenos de peces. IV. Descripcion de Bravocotyle sanblasensis gen. nov., sp. nov. (Diclidophoridae) parasito de las branquias de Cynoscion xanthulus (Sciaenidae) de la costa Pacifica Mexicana. An. Inst. Biol. Univ. Nac. Auton. Mex. Ser. Zool. 38:47-58.

1967b. Monogeneos de peces. V. Redescripcion de Tagia ecuadori (Meserve, 1938) Sprosion, 1946. An. Inst. Biol. Univ. Nac. Auton. Mex. Ser. Zool. 38:35-46.

1968. Monogeno de peces. VI. Caballerocotyla marielenae sp. nov. (Monogenea: Capsalinae), parasito de las branquias de Istiophorus greyi Jordan and Hill, de Puerto Angel, Oaxaca, Mexico. Riv. Parassitol. 29:171-184.

1969a. Tremátodos de peces. III. Cuatro especies nuevas de trematodos parasitos de peces del Pacifico Mexicano. An. Inst. Biol. Univ. Nac. Auton. Mex. Ser. Zool. 40:21-42.

1969b. Trematodos de peces. IV. Registro de cuatro especies de trematodos de peces marinos de la costa del Pacifico Mexicano. An. Inst. Biol. Univ. Nac. Auton. Mex. Ser. Zool. 40:179-194.

1970. Monogéneos de peces. II. Reporte de tres especies de Monogenea parasitas de las branquias de Caranx hippos del Pacifico mexicano y redescripción de Zeuxapta seriolae (Meserve, 1938) Price, 1962. Rev. Biol. Trop. 16:153-169.

LAUBIER, L., C. MAILLARD, and G. OLIVER.

1966. Contribution a l'étude des parasites du "griset": Hexanchus griseus (Bonnaterre, 1788). Vie Mileu, Ser. A, 17:1197-1233.

LAURS, R. M., and J. E. McCAULEY.

1964. A new acanthocephalan from the Pacific saury. J. Parasitol. 50: 569-571.

LEBEDEV, B. I

1968. Morphology and taxonomy of trematodes of Syncoelinae Looss, 1899. [In Russ.] Gelminty Zhivotnykh Tikhogo Okeans, p. 65-71.

LEBEDEV, B. I., and Y. L. MAMAEV.

1968. Two new species of trematode genus Cardicola Short, 1953 (Sanguinicolidae) from fishes of South China Sea. [In Russ.] Gelminty Zhivotnykh Tikhogo Okeans, p. 72-75.

LEE, R. F.

1975. Lipids of parasitic copepods associated with marine fish. Comp. Biochem. Physiol. 52B:363-364.

LEIGH-SHARPE, W. H.

1931. Pennella germonia n. sp. a parasitic copepod of Germo alatunga. Parasitology 23:109-111.

1933. A list of British fishes with their characteristic parasitic Copepoda. Parasitology 25:109-112.

1936. Some rare and new parasitic Copepoda, etc. from Brighton and elsewhere. Parasitology 28:410-413.

LEITRITZ, E., and R. C. LEWIS.

1976. Trout and salmon culture (hatchery methods). Calif. Dep. Fish Game, Fish Bull. 164, 197 p.

LEONG, T. S., and J. C. HOLMES

1974. Acquisition of helminths by coho salmon, Oncorhynchus kisutch introduced into Cold Lake, Alberta: a comparison with helminths of native salmonid fish. [Abstr.] Proc. Third Int. Congr. Parasitol. 3:1639.

LESTER, R. J. G.

1974. Parasites of Gasterosteus aculeatus near Vancouver, British Columbia. Syesis 7:195-200.

LEWIS, A. G.

1966. Copepod crustaceans parasitic on elasmobranch fishes of the Hawaiian Islands. Proc. U.S. Natl. Mus. 118:57-154.

1967. Copepod crustaceans parasitic on teleost fishes of the Hawaiian Islands. Proc. U.S. Natl. Mus, 121(3574):1-204.

1968. Copepod crustaceans parasitic on fishes of Eniwetok Atoll. Proc. U.S. Natl. Mus. 125(3656):1-78.

LEWIS, A. G., J. DEAN, and E. GILFILLAN III.

1969. Taxonomy and host associations of some parasitic copepods (Crustacea) from pelagic teleost fishes. Pac. Sci. 23:414-437.

LINTON, E.

1889. Notes on Entozoa of marine fishes of New England, with descriptions of several new species. Rep. U.S. Fish. Comm. 1886:453-511.

1891. Notes on Entozoa of marine fishes of New England, with descriptions of several new species. Rep. U.S. Fish. Comm. 1887:719-899.

1892. Notes on Entozoa of marine fishes, with descriptions of new species. Part III. Acanthocephala. Rep. U.S. Fish. Comm. 1888:523-542.

1898. Notes on trematode parasites of fishes. Proc. U.S. Natl. Mus. 20:507-548.

1901. Fish parasites collected at Woods Hole in 1898. Bull. U.S. Fish. Comm. 19:267-304.

1905. Parasites of fishes of Beaufort, North Carolina. Bull. Bur. Fish. 24:321-428.

1910. Helminth fauna of the Dry Tortugas. II. Trematodes. Carnegie Inst. Wash. Publ. 4(133):11-98.

1924. Notes on cestode parasites of sharks and skates. Proc. U.S. Natl. Mus. 64(21):1-111.

1940. Trematodes from fishes mainly from the Woods Hole Region, Massachusetts. Proc. U.S. Natl. Mus. 88:1-171.

1941. Cestode parasites of teleost fishes of the Woods Hole Region, Massachusetts. Proc. U.S. Natl. Mus. 90:417-442.

LISTON, J., J. PETERS, and J. A. STERN.

1960. Parasites in summer-caught Pacific rockfishes. U.S. Fish Wildl. Serv., Spec. Sci. Rep. Fish. 352, 10 p.

LLOYD, L. C.

1938. Some digenetic trematodes from Puget Sound Fish. J. Parasitol. 24:103-133.

LLOYD, L. C., and J. E. GUBERLET.

1932. A new genus and species of Monorchidae. J. Parasitol. 18:232-239.

1936. Syncoelium filiferum (Sars) from the Pacific salmon. Trans. Am. Microsc. Soc. 55:44-48

LOM. J.

1966. Sessiline peritrichs from the surface of some freshwater fishes. Folia Parasitol. 13:36-56.

1969. Notes on the ultrastructure and sporoblast development in fish parasitizing myxosporidian of the genus Sphaeromyxa. Z. Zellforsch. Mikrosk. Anat. 97:416-437.

1970a. Protozoa causing diseases in marine fishes. In S. F. Snieszko (edi tor), A symposium on diseases of fishes and shellfishes, p. 101-123. Am. Fish. Soc. Spec. Publ. 5.

1970b. Trichodinid ciliates (Peritrichida: Urceolariidae) from some marine fishes. Folia Parasitol. 17:113-125.

LOM, J., and M. LAIRD.

1969. Parasitic protozoa from marine and euryhaline fish of Newfoundland and New Brunswick. I. Peritrichous ciliates. Can. J. Zool. 47: 1367-1380.

LOOSS, A.

1902. Zur kenntnis der trematodenfauna des triester hafens. 1. Ueber die gattung Orophocotyle n. g. Zentralbl. Bakterial Parasitenkd. Infek tionskr. Hyg. Abt. I Orig. Part 1, Ser, 0, 31:637-644.

1907a. Beitrage zur systematik der Distomen. Zur kenntnis der familie Hemiuridae. Zool. Jahrb. 26:63-180.

1907b. Wissenschaftliche mitteilungen. I. Zur kenntnis der distomenfamilie Hemiuridae. Zool. Anz. 31:585-620.

LOPEZ, G

1976. Redescription and ontogeny of Lepeophtheirus kareii Yamaguti, 1936 (Copepoda, Caligoida). Crustaceana 31:203-207.

LOVE, M. S., and M. MOSER.

1976. Davisia reginae sp. n. (Protozoa: Myxosporida) from four California marine fishes. J. Parasitol. 62:982-983. 
LUKEN, E. T.

1962. Characteristics of the leech fauna of the Amur Basin. [In Russ.] Izv. Tikhookean. Nauchno-Issled. Inst. Rȳbn. Khoz. Okeanogr. 48: 195-202.

LYNCH, J. E.

1945. Redescription of the species of Gyrocotyle from the ratfish, Hydrolagus colliei (Lay and Bennet), with notes on the morphology and taxonomy of the genus. J. Parasitol. 31:418-448.

MacCALLUM, G. A.

1916. Some new species of parasitic trematodes of marine fishes. Zoopathologica 1:1-37.

MACHIDA, J.

1971. Contracaecum incurvum collected from Xiphias gladius. Jpn. J. Parasitol. 20:305.

MACHIDA, M., J. ARAKI, H. KAMIYA, and M. OHBAYASHI.

1972. Trematodes collected from sea fish of the Hidaka District, Hokkaido. Mem. Natl. Sci. Mus. (Tokyo) 5:1-9.

MACHIDA, M., A. ICHIHARA, and S. KAMEGAI.

1970. Digenetic trematodes collected from the fishes in the sea north of the Tsushima Islands. Mem. Natl. Sci. Mus. (Tokyo) 3:101-112.

MacKENZIE, K.

1975. Renicola metacercariae (Digenea: Renicolidae) in clupeoid fish: New host records. J. Fish Biol. 7:359-360

MADHAVI, $\mathbf{R}$.

1970. Redescription and systematic position of Echeneidocoelium indicum Simha and Pershad, 1964 (Trematoda: Digenea) from the sucker fish Echeneis naucrates. J. Parasitol. 56:317-320.

MAGGENTI, A. R.

1971. A review of the family Cucullanidae Cobbold 1864 and the genus Bulbodacnitis Lane, 1916 with a description of Bulbodacnitis ampullastoma sp. n. (Nematoda: Cucullanidae) from Salmo gairdnerii Richardson. Proc. Helminthol. Soc. Wash. 38:80-85.

MAGGENTI, A. R., and G. A. PAXMAN.

1971. Sterliadochona pedispicula sp. n. (Nematoda: Spirurinae) from Salmo gairdnerii Richardson, and a discussion of the genera Steriadochona Skrjabin, 1946 and Cystidicoloides Skinker, 1931. Proc. Helminthol. Soc. Wash. 38:210-214.

MAMAEV, Y, L.

1965. Helminths of fish from the Bering Sea. [In Russ.] Paraziticheskie Chervi Domaschnikh i Dikikh Zhivotnykh. Raboty Po Gel'mintologii. Akad. Nauk, SSSR, Vladivostok, p. 168-188.

1967. Pseudaxine triangula sp. n. and Metapseudaxine ventrosicula gen. et sp. n. and their position in the system Monogenoidea. [In Russ.] Zool. Zh. 46:993-998.

1968a. Didymosphaera mirabilis gen. et sp. nov. (Didymozoidae) paired trematode from the body of tuna. [In Russ.] Parazitol. 2:159-166.

1968b. Helminths of tuna fish in the South China Sea. In K. I. Skryabin and Y. L. Mamaev (editors), Helminths of animals of the Pacific Ocean, p. 5-27. [In Russ.] "Nauka":Moscow.

1968c. Pontochona gen nov. (Rhabdochonidae, Spiruata) a nematode genus from fish in South China Sea. [In Russ.] "Nauka":Moscow, p. 30-35.

MAMAEV, Y. L., and O. M. BAEVA.

1963. Helminth fauna of Theragra chalcogramma from Kamchatka waters. [In Russ.] Helminthologia 4:318-331.

MAMAEV, Y. L., A. M. PARUKHIN, and O. M. BAEVA.

1963. Parasitic worms of flatfish of Far Eastern seas. Parazit. Chervi Zhivotnykh Primor. i Tikh. Okeana (Sborn. Rabot.), p. 82-113. (From Ind.-Cat. Med. Vet. Zool. Suppl. 17, pt. 7, p. 203-204.)

MAMAEV, Y. L., A. M. PARUKHIN, O. M. BAEVA, and P. G. OSCHMARIN

1959. Helminthofauna of far eastern Salmonidae and their relation to the phenomena of local schooling and ways of migration of these fishes. [In Russ.] Izd. Dal'nevost. Fil. ANSSSR Vladivostok, 74 p.

MAÑE-GAR'ZÓN, F., and B. HOFFMAN-SPECTOR.

1968. Monogenea de peces marinos del Uraguay, I. Comun. Zool. Mus. Hist. Nat. Montev. 9:1-9.

MANGER, B. R.

1972. Some cestode parasites of the elasmobranchs Raja batis and Squalus acanthias from Iceland. Bull. Br. Mus. Nat. Hist. Zool. 24: 161-181.

MANN, H.

1970. Copepoda and Isopoda as parasites of marine fishes, In S. F. Snieszko (editor), A symposium on diseases of fishes and shellfishes, $p$. 177-189. Am. Fish. Soc. Spec. Publ. 5
MANTER, H. W

1926. Some North American Fish trematodes. III. Biol. Monogr. 10: $7-138$.

1931. Some digenetic trematodes of marine fishes of Beaufort, North Carolina. Parasitology 23:396-411.

1934. Some digenetic trematodes from decp-water fish of Tortugas, Florida. Tortugas Lab. Pap. 28:261-345.

1940a. Digenetic trematodes of fishes from the Galapagos Islands and the neighboring Pacific. Allan Hancock Found. Pac. Exped. 2:329-496

1940b. The geographical distribution of digenetic trematodes of marine fishes of the tropical American Pacific. Allan Hancock Found. Pac. Exped. 2:531-557.

1944. Notes on the trematode subfamily Loimoinae (Monogenea), with a description of a new genus. J. Wash. Acad. Sci. 34:86-89.

1947. The digenetic trematodes of marine fishes of Tortugas, Florida. Am. Midl. Nat. 38:257-416.

1954a. Some digenetic trematodes from fishes of New Zealand. Trans. R. Soc. N.Z, $82: 475-568$.

1954b. Trematoda of the Gulf of Mexico. U.S. Fish Wildl. Serv., Fish. Bull. 55:335-350.

1960. Some additional Digenea (Trematoda) from New Zealand fishes. Caballero y Caballero, Eduardo, Jubileo, 1930-60, Mexico, p. 197-201.

1969. Threc now genera of hemiurid trematodes from Ausiraian fishes. J. Fish. Res. Board Can. 26:787-792.

MANTER, H. W., and M. H. PRITCHARD.

1961. Studies on digenetic trematodes of Hawaiian fishes: families Monorchiidae and Haploporidae. J. Parasitol. 47:483-490.

1962. Studies on digenetic trematodes of Hawaiian fishes: Families Fellodistomatidae, Opistholebetidae and Gyliauchenidae. Trans. Am. Microsc. Soc. 81:113-123.

MANTER, H. W., and H. J. van CLEAVE.

1951. Some digenetic trematodes, including eight new species, from marine fishes of La Jolla, Calif. Proc. U.S. Natl. Mus. 101:315-340.

MARGOLIS, L.

1953. Milkiness in lemon sole fillets. Fish. Res. Board Can. Annu. Rep. Pac. Biol. Stn. for 1953, p. 158.

1956a. Anomalous development of vitellaria in Hemiurus levinseni (Trematoda). Can. J. Zool. 34:207-208.

1956b. Parasitic helminths and arthropods from pinnipedia of the Canadian Pacific coast. J. Fish. Res. Board Can. 13:489-505.

1958a. A new species of Lecithophyllum from North Pacific fishes with a consideration of the taxonomy of the genera Lecithophyllum, Aponurus, and Brachadena (Trematoda: Hemiuridae). Can. J. Zool. 36:893-904.

1958b. The occurrence of juvenile Corynosoma (Acanthocephala) in Pacific salmon (Oncorhynchus spp.). J. Fish. Res. Board Can. 15:983-990.

1958c. The identity of the species of Lepeophtheirus (Copepoda) parasitic on Pacific salmon (genus Oncorhynchus) and Atlantic salmon (Salmo salar). Can. J. Zool. 36:889-892.

1960. A new nematode of the genus Cucullanus (Camallanata: Cucullanidae) from a flounder, Parophrys vetulus Girard, 1854, with notes on the species from Pleuronectiformes. Can. J. Zool. 38:839-849.

1962. Lampritrema nipponicum Yamaguti (Trematoda) from new hosts in the North Pacific Ocean, the relationship of Distomun miescheri Zschokke, and the status of the family Lampritromatidace. Caìi. J. Zool. 40:941-950.

1963. Parasites as indicators of the geographical origin of sockeye salmon, Oncorhynchus nerka (Walbaum), occurring in the North Pacific Ocean and adjacent seas. Int. North Pac. Fish. Comm. Bull. 11:101-156.

1965. Parasites as an auxiliary source of information about the biology of Pacific salmons (genus Oncoryhnchus). J. Fish. Res. Board Can. 22: 1387-1395.

1967a. The swimbladder nematodes (Cystidicolinae) of Pacific salmon (genus Oncorhynchus). Can. J. Zool. 45:1183-1199.

1967b. Triaenophorus crassus pleroceroids in sockeye salmon smolts from the Kvichak River system, Alaska. I. Fish. Res. Board Can. 24:893-894.

1968. Review of the Japenese species of Cystidicola, Metabronema and Rhabdochona (Nematoda) from salmonid fishes. Res. Bull. Meguro Parasitol. Mus. 2:23-44.

1970a. Nematode diseases of marine fishes. In S. F. Snieszko (editor), A symposium on diseases of fishes and shellfishes, p. 190-208. Am. Fish. Soc. Spec. Publ. 5.

1970b. The trematode Plagioporus shawi (Mclntosh, 1939) Margolis, 1970. n. comb. (Opecoelidae: Plagioporinae) from the North Pacific sockeye salmon, Oncorhynchus nerka. An. Inst. Biol. Univ. Nac. Auton. Méx. Ser. Zool. 41:99-108. 
1974. Cephalic structure of some spiruroid nematodes from fish as revealed by scanning electron microscopy. Proc. Third Int. Congr. Parasitol., p. 1629.

1977. Caballeronema gen. nov. for Metabronema wardlei Smedley, 1934 (Nematoda, Spiruroidea) from the marine fish Scorpaenichthys marmoratus from the Pacific coast of Canada. Excerta Parasitológica en Memoria del Dóctor Euduardo Cáballero y Cáballerô. Univ. Nac. Auton. Mex. Inst. Biol. Publ. Espec. 4:447-454.

MARGOLIS, L., and J. R. ADAMS.

1956. Description of Genolinea oncorhynchi n. sp. (Trematoda: Hemiuridae) from Oncorhynchus gorbuscha in British Columbia with notes on the genus. Can. J. Zool. 34:573-577.

MARGOLIS, L., and N. P. BOYCE.

1969. Life span, maturation, and growth of two hemiurid trematodes, Tubulovesicula lindbergi and Lecisthaster gibbosus, in Pacific salmon (genus Oncorhynchus). J. Fish. Res. Board Can. 26:893-907.

MARGOLIS, L., and H. L. CHING.

1965. Review of the trematode genera Bacciger and Pentragramma (Fellodistomatidae) and description of $P$. petrowi (Layman, 1930) n. comb. from marine fishes from the Pacific coast of Canada. Can. J. Zool. 43: 381-405.

MARGOLIS, L., and T. P. T. EVELYN.

1975. Ceratomyxa shasta (Myxosporida) disease in chum salmon (Onchorynchus keta) in British Columbia. J. Fish. Res. Board Can. 32: 1640-1643.

MARGOLIS, L., F. MORAVEC, and T. E. McDONALD.

1975. Rhabdochona kisutchi sp. nov. (Nematoda: Spiruroidea) from coho salmon, Oncorhynchus kisutch (Walbaum), of western Canada. Can. J. Zool. 53:960-966.

MARKELL, E. K.

1956. Probolitrema mexicana, n. sp., an anaporrhutine trematode from elasmobranchs of Baja California. J. Parasitol. 42:56-59.

MARKEVICH, A. P.

1957. Parasitic copepoda of fish of SSSR. [In Russ.] Parazit. Veslonogie Ry̆b, SSSR, 259 p.

MARTIN, W. E.

1950. Parasitictodora hancocki n. gen., n. sp. (Trematoda: Heterophyidae), with observations on its life cycle. J. Parasitol. 36:360-370.

1951. Pygidiopsoides spindalis n. gen., n. sp., (Heterophyidae; Trematoda), and its second intermediate host. J. Parasitol. 37:297-300.

1961. Life cycle of Mesostephanus appendiculatus (Ciurea, 1916) Lutz, 1935 (Trematoda: Cyathocotylidae). Pac. Sci. 15:278-281.

1971. Larval stages of renicolid trematodes. Trans. Am. Microsc. Soc. 90: $188-194$

1973a. A new genus and species of haploporid trematode (Haploporidae: Trematoda) from Australia mullet. Bull. South. Calif. Acad. Sci. 72: 166-168.

1973b. A new subfamily, two new genera, and three new species of haploporid trematodes. Proc. Helminthol. Soc. Wash. 40:112-117.

1973c. Isorchis manteri sp. $\mathrm{n}$. from Australian mullet and a key to haploporid trematode genera with two testes. Proc. Helminthol. Soc. Wash. 40:237-240.

1973d. Life history of Saccocoelioides pearsoni $\mathrm{n}$. sp. and the description of Lecithobotrys sprenti n. sp. (Trematoda: Haploporidae). Trans. Am. Microsc. Soc. 92:80-95.

1974. Paralecithobotrys brisbanensis sp. n. from Australian mullet (Trematoda: Haploporidae). Proc. Helminthol. Soc. Wash. 41:16-18.

1975. Plethorchis acanthus gen. et sp. n. (Trematoda: Sanguinicolidae) in mullet, Mugil cephalus L., from Queensland, Australia. Proc. Helminthol. Soc. Wash. 42:79-82.

1978. Digenetic trematodes of the marine fish, Girella nigricans (Ayres), from southern California with the description of two new species. Proc. Helminthol. Soc. Wash. 45:175-181.

MARTIN, W. E., and S. MULTANI.

1966. Microsentis wardae n. g., n. sp. (Acanthocephala) in the marine fish Gillichthys mirabilis Cooper. Trans. Am. Microsc. Soc. 85:536-540.

1970. Some helminths of the mudsucker fish Gillichthys mirabilis Cooper. Bull. South. Calif. Acad. Sci. 69:161-168.

MARTIN, W. E., and D. F. STEELE.

1970. Ascocotyle sexidigita sp. n. (Trematoda: Heterophyidae) with notes on its life cycle. Proc. Helminthol. Soc. Wash. 37:101-104.

MARTIN, W. E., and S. G. ZAM.

1967. Vasorhabdochona cablei, gen. et. sp. n. (Nematoda) from blood vessels of the marine fish, Gillichthys mirabilis Cooper. J. Parasitol. 53: 389-391.
MASMARK, K. E.

1937. A revision of the trematode family Paramphistomidae. Zool. Bidr. Upps. (1935-1937) 16:301-365.

MATSUMOTO, $\mathrm{K}$.

1954. On the two new Myxosporidia, Chloromyxum musculoliquefaciens $s p$. nov. and Neochloromyxum cruciformum gen. et sp. nov, from the jellied muscie of swordíish, Xiphias giadius Linné, and common Japanese sea-bass Lateolabrax japonicus (Temmink et Schlegel). Bull. Jpn. Soc. Sci. Fish. 20:469-478.

McCAULEY, J, E.

1960. Some hemiurid trematodes of Oregon marine fishes. J. Parasitol. 46:84-89.

1964. A deep-sea digenetic trematode. J. Parasitol. 50:112-114.

1960. Six species of Lepidapedon Stafford, 1904 (Trematoda: Lepocreadiidae) from deep-sea fishes. J. Parasitol. 54:496-505.

McCAULEY, J. E., and J. E. PEQUEGNAT.

1968. Two new species of Dinosoma Manter, 1934 (Trematoda: Hemiuridae) from deep-water macrourid fishes off the coast of Oregon. J. Parasitol. 54:931-934.

McCAULEY, J. E., J. E. PEQUEGNAT, and C. L. BROWNELL.

1970. A new species of Gonocerca Manter, 1925 (Trematoda: Hemiuridae) from the eastern Pacific. Proc. Helminthol. Soc. Wash. 37:169-171.

McCAUleY, J. E., and I. PRATT.

1961. A new genus Deropegus with a redescription of D. aspina (Ingles, 1936) nov. comb. Trans. Am. Microsc. Soc. 80:373-377.

McCAULEY, J. E., and W. W. SMOKER.

1969. Two diclidophoran trematodes (Monogenea) from deep-sea fishes. J. Parasitol. 55:742-746.

McFARLANE, S. H.

1936. A study of the endoparasitic trematodes from marine fishes of Departure Bay, B.C. J. Biol. Board Can. 2:335-347.

McINTOSH, A.

1939. A new allocreadiid trematode Podocotyle shawi n. sp., from the silver salmon. J. Wash. Acad. Sci. 29:379-381.

MEADE, T. G., and I. PRATT.

1965. Description and life history of Cardicola alseae sp. n. (Trematoda: Sanguinicolidae). J. Parasitol. 51:575-578.

MEGLITSCH, P. A.

1952. The myxosporidian fauna of some freshwater and marine fishes. Proc. Iowa Acad. Sci. 59:480-486.

MENITSKII, Yu. L.

1963. The structure and systematic position of Ichthyophaga subcutanea. Parazitol. Sb. 21:245-258.

MENZIES, R. J.

1946. Benedenia noblei, a new monogenetic trematode of the Pacific coast rock fish. J. Parasitol. 32:428-430.

MENZIES, R. J., T. E. BOWMAN, and F. G. ALVERSON.

1955. Studies of the biology of the fish parasite Livoneca convexa Richardson (Crustacea, Isopoda, Cymothoidae), Wasmann J. Biol. 13: 277-295.

MERRELL, T. R., Jr.

1964. Ecological studies of sockeye salmon and related limnological and climatological investigations, Brooks Lake, Alaska, 1957. U.S. Fish Wildl. Serv., Spec. Sci. Rep. Fish. 456, 66 p.

MERRITT, S. V., and I. PRATT.

1964. The life history of Neoechinorhynchus rutili and its development in the intermediate host (Acanthocephala: Neoechinorhynchidae). J. Parasitol. 50:394-400.

MESERVE, F. G.

1938. Some monogenetic trematodes from the Galapagos Islands and the neighboring Pacific. Allan Hancock Found. Pac. Exped. 2:31-88.

MEYER, $M$.

1946. A new leech, Piscicola salmositica n. sp. (Piscicolidae), from steelhead trout (Salmo gairdneri gairdneri Richardson, 1838). J. Parasitol. 32:467-476.

1960. Notes on Philonema agubernaculum and other related dracunculoids infecting salmonids. Caballero y Caballero, Eduardo, Jubileo, 1930-60, Mexico:487-492.

MILLEMANN, R. E.

1956. Notes on the genus Hexostoma (Monogenea: Hexostomatidae) with a rcdescription of $H$. euthynni Meserve, 1938. J. Parasitol. 42:316-319.

1963. Studies on the taxonomy and life history of echinocephalid worms (Nematoda: Spiruroidea) with a complete description of Echinocephalus pseudouncinatus Millemann, 1951. J. Parasitol. 49:754-764. 
MILLEMANN, R. E., G. A. GEBHARDT, and S. E. KNAPP.

1964. "Salmon poisoning" disease. I. Infection in a dog from marine salmonids. J. Parasitol. 50:588-589.

MILIER, D. J., and R. N. LEA.

1972. Guide to the coastal marine fishes of California. Calif. Dep. Fish Game, Fish Bull. 157, 235 p.

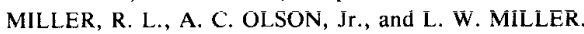

1973. Fish parasites occurring in thirteen southern California reservoirs. Calif. Fish Game 59:196-206.

MIZELLE, J. D., and D. C. KRITSKY.

1967a. Studies on monogenetic trematodes. XXX. Five new species of Gyrodactylus from the Pacific tomcod, Microgadus proximus (Girard). J. Parasitol. 53:263-269.

1967b. Studies on monogenetic trematodes. XXX11. New species of marine Gyrodactylinae from California with the proposal of Archigyrodactylus gen. n. Proc. Helminthol. Soc. Wash. 34:130-136.

1967c. Studies on monogenetic trematodes. XXX111. New species of Gyrodactylus and a key to the North American species. Trans. Am. Microsc. Soc. 86:390-401

1967d. Studies on monogenetic trematodes. XXXVI. Gyrodactylid parasites of importance to California fishes. Calif. Fish Game 53:264-272.

MONACO, L. H., R. A. WOOD, and J. D. MIZELLE.

1954. Studies on monogenetic trematodes. XVI. Rhamnocercinae, a new subfamily of Dactylogyridae. Am. Midl. Nat. 52:129-132.

MONTGOMERY, W. R.

1957. Studies on digenetic trematodes from marine fishes of La Jolla, California. Trans. Am. Microsc. Soc. 76:13-36.

MOORE, J. P.

1952. New Piscicolidae (Leeches) from the Pacific and their anatomy. Occas. Pap. Bernice P. Bishop Mus. 21:17-44.

MOORE, J. P., and M. C. MEYER.

1951. Leeches (Hirudinea) from Alaskan and adjacent waters. Wasmann J. Biol, 9:11-77.

MORRISON, N. D., and I, PRATT

1973. Sphaeromyxa maiyai sp. n. (Protozoa: Myxosporidea), coelozoic parasite of the Pacific tomcod, Microgadus proximus. J. Protozool. 20: 214-217

MOSER, M

1976. Ceratomyxa anoplopoma sp. n. (Protozoa, Myxosporida) from the sablefish, Anoplopoma fimbria. Can. J. Zool. 54:1405-1406.

MOSER, M., and S. ANDERSON.

1977. An intrauterine leech infection: Branchellion lobata Moore 1952 (Piscicolidae) in the Pacific angel shark (Squatina californica) from California. Can. J. Zool. 54:759-760.

MOSER, M., and L. HALDORSON.

1976. Zschokkella embiotocidis sp. n. (Protozoa, Myxosporida) from California pile perch, Damalichthys vacca, and striped perch, Embiotoca lateralis. Can. J. Zool. 54:1403-1405.

MOSER, M., and M. S. LOVE.

1975. Henneguya sebasta sp. n. (Protozoa, Myxosporida) from California rockfish, Sebastes spp. J. Parasitol. 61:481-483.

MOSER, M., M. S. LOVE, and L. A. JENSEN.

1976. Myxosporida (Protozoa) in California rockfish, Sebastes spp. J. Parasitol. 62:690-692.

MOSER, M., and E. R. NOBLE.

1975. The myxosporidan genus Davisia in two deep-sea fishes. J. Parasitol. 61:691-694.

1976a. The genus Ceratomyxa (Protozoa: Myxosporida) in macrourid fishes. Can. J. Zool. 54:1535-1537.

1976b. The genus Leptotheca (Protozoa: Myxosporida) in macrourid fishes and sablefish, Anoplopoma fimbria. J. Protozool, 23:490-492.

1977a. Myxosporidan genera Auerbachia, Sphaerospora, Davisia and Chloromyxum in macrourid fishes and the sablefish, Anoplopoma fim bria. Z. Parasitenkd. 51:159-163.

1977b. The genus Myxoproteus (Protozoa: Myxosporida) in macrourid fishes. Int. J. Parasitol. 7:253-255.

$1977 \mathrm{c}$. Three genera of myxosporida (Protozoa) in macrourid fishes. Int. J. Parasitol. 7:93-96.

1977d. Zschokkella (Protozoa: Myxosporida) in macrourid fishes. Int. J. Parasitol. 7:97-100.

MOSER, M., E. R. NOBLE, and R. S. LEA.

1976. The genus Myxidium (Protozoa: Myxosporida) in macrourid fishes. J. Parasitol. 62:685-689.

MOZGOVI, A. A., T. I. POPOVA, and M. K. SEMENOVA.

1965. An elucidation of the life cycle of the nematode Synhimantus brevi- caudatus (Dujardin, 1845) - A parasite of wading birds and freshwater fish. Dokl. Acad. Nauk. SSSR 162:719-720 Russian, 380-384 English.

MUDRY, D. R., and R. S. ANDERSON.

1977. Helminth and arthropod parasites of fishes in the mountain national parks of Canada. J. Fish Biol. 11:21-33.

MUDRY, D. R., and M. D. DAILEY.

1969. Pihiyctainophora squail sp. nov. (Nematoda, Philometridae) from the spiny dogfish, Squalis acanthias. Proc. Helminthol. Soc. Wash. 36: 280-284.

MURAV'EV, V. I.

1970. On the biology of parasitic nematodes of herring and blue whiting in North Atlantic. Tr. Polyarn. Nauchno-Issled Procktn Inst. Morsk. Rybn. Khoz. Okeanogr. 14:86-95. (Fish. Res. Board Can. 1971. Transl. Ser. 1888.)

MYERS, B. J.

1959. Parasites from elasmobranch hosts from the Magdalen Islands region of the Gulf of St. Lawrence. Can. J. Zool. 37:245-246.

MYERS, B. J., and R. E. KUNTZ.

1962. Nematode parasites from vertebrates taken on Lan Yi, Formosa. II. Nematodes from fish, amphibians, reptiles, birds. Can. J. Zool. 40:135-136.

NAGATY, H. F.

1957. Trematodes of fishes from the Red Sea. Part 8. Five species in the families Schistorchidae, Acanthocolpidae, and Heterophyidae. J. Parasitol. 43:217-220.

1958. List of trematodes of fishes and their hosts, so far recorded by the author from the Red Sea. J. Egypt. Med. Assoc. 41:449-460.

NAHHAS, F. M., and R. M. CABLE.

1964. Digenetic and aspidogastrid trematodes from marine fishes of Curacao and Jamaica. Tulane Stud. Zool. 11:168-228.

NAHHAS, F. M., and R. KRUPIN.

1977. Parasites of Liparis callyodon (Pallas) with a description of a new species, Metadena caballeroi. Inst. Biol. Publ. Espec. 4:261-266.

NAHHAS, F. M., and E. C. POWELL.

1971. Digenetic trematodes of marine fishes from the Floridian northern Gulf of Mexico. Tulane Stud. Zool. 17:1-9.

NAHHAS, F. M., and R. B. SHORT.

1965. Digenetic trematodes of marine fishes from A palachee Bay, Gulf of Mexico. Tulane Stud. Zool. 12:39-50.

NAKAJIMA, K., and S. EGUSA.

1975a. Acanthocephalus opsarichthys Yamaguti, 1935, from cultured rainbow trout, Salmo gairdnerii iridens, in Japan. [In Jpn.] Fish Pathol. 10:53-57.

1975b. Pseudorhadinorhynchus samegaiensis n. sp. (Acanthocephala), from cultured rainbow trout, Salmo gairdnerii, in Japan. [In Jpn. and Engl.] Fish Pathol. 10:58-68.

NEEDHAM, P. R., and R. J. BEHNKE.

1965. The effect of nematode (Philonema) and cestode (Diphyllobothrium) parasites in rainbow trout of Tebay Lake, Alaska. Trans. Am. Fish. Soc. 94:184-186.

NEILAND, K, A.

1952. A new species of Proteocephalus Weinland, 1858 (Cestoda), with notes on its life history. J. Parasitol. 38:540-545.

NICHOLS, K. C.

1975. Observations on lesser-known flatworms: Udonella. Int. J. Parasitol. 5:475-482.

NICOLL, W.

1907. A contribution towards a knowledge of the entozoa of British marine fishes. Pt. I. Ann. Mag. Nat. Hist., Ser. 7, 19:66-94.

1915. A list of trematode parasites of British marine fishes. Parasitology 7:339-378.

NIEMI, D. R., and R. W. MACY.

1974. The life cycle and infectivity to man of Apophallus donicus (Skrjabin and Lindtrop, 1919) (Trematoda: Heterophyidae) in Oregon. Proc. Helminthol. Soc. Wash. 41:223-229.

NIGRELLI, R. F.

1935. Naobranchia wilsoni, a parasitic copepod from the gills of the porcupine fish, Diodon hystrix Linn. Trans. Am. Microsc. Soc. 54:52-56

1938. Parasites of the swordfish, Xiphias gladius Linnaeus. Am. Mus. Novit. 996:1-16.

NIGRELLI, R. F., and G. D. RUGGIERI.

1966. Enzootics in the New York Aquarium caused by Cryptocaryon irritans Brown, 1951 ( = Ichthyophthirius marinus Sikama, 1961), a histophagous ciliate in the skin, eyes and gills of marine fishes. Zoologica (N.Y.) 51:97-102 
NIGRELLI, R. F., and H. W. STUNKARD.

1947. Studies on the genus Hirudinella, giant trematodes of scombriform fishes. Zoologica (N.Y.) 31:185-196.

NIKOLAEVA, V. M.

1965. On the developmental cycle of trematodes belonging to the family Didymozoidae (Monticelli, 1888) Poche, 1907. [In Russ.] Zool. Zh. 44: $1317-1327$.

1968a. On finding Accacoeliidae larvae in the fishes and invertebrates. In v. A. Bodyanitski (editor), Biology of the seas (Biolgiia Moriia), p. 83-89. [In Russ.] "Naukova Dumka" Kiev 14.

1968b. To the studies of helminthofauna of Thunnus albacores and Histiophoridae in the Gulf of Mexico. In Z. B. Yankovskaya (editor), Studies of Central American Seas, p. 150-157. [In Russ.] "Naukova Dumka" Kiev 2.

NIKOLAEVA, V. M., and N. N. NAIDENOVA.

1963. Nematodes of pelagic and littoral fish of the Mediterranean Basin. [In Russ.] Trudy 4. Nauch. Konf. Parazitol. USSR, p. 469-470. (From Ind.-Cat. Med. Vet. Zool. Suppl. 18, Pt. 7:168.)

1964. Nematodes of pelagic and benthopelagic fishes of seas of the Mediterranean Basin. [In Russ.] Trudy Sevastopol'. Biol. Stn. 17:125-158.

NIKOLAEVA, V. M., and A. M. PARUKHIN.

1968. To the study of fish helminths in the Gulf of Mexico. In Z. B. Yankovskaya (editor), Studies of Central American Seas, p. 126-149. [In Russ.] "Naukova Dumka" Kiev 2.

NOBLE, A. E., and G. A. NOBLE.

1937. Accacladium nematulum n. sp., a trematode from the sunfish Mola mola. Trans. Am. Microsc. Soc. 56:55-60.

NOBLE, A. E., and J. T. PARK.

1937. Helicometrina elongata n. sp. from the gobiesocid fish Caularchus meandricus, with an emended diagnosis of the trematode genus Helicometrina. Trans. Am. Microse. Soc. 56:344-347.

NOBLE, E. $\mathbf{R}$.

1938. Two new Myxosporidia from tide pool fishes of California. J. Parasitol. 24:441-444.

1939. Myxosporidia from the tide pool fishes of California. J. Parasitol. 25:359-364.

1941. On distribution relationships betwen California tide pool fishes and their Myxosporidian (Protozoan) parasites. J. Parasitol. 27:409-415.

1943. Nuclear cycles in the protozoan parasite Myxidium gasterostei $\mathbf{n}$. sp. J. Morphol. 73:281-292.

1950. On a Myxosporidian (Protozoan) parasite of California trout. J. Parasitol. 36:457-460.

1961. The relations between Trichodina and metazoan parasites on gills of a fish. Progress in Protozoology, Proc: First Int. Conf. Protozool. Prague, Aug. 22-31, p. 521-523.

1966a. Myxosporida in deepwater fishes. J. Parasitol. 52:685-690.

1966b. The genus Encotyllable (Class Trematoda) with a description of a new species. Trans. Am. Microsc. Soc. 85:144-151.

1968. The flagellate Cryptobia in two species of deepsea fishes from the eastern Pacific. J. Parasitol. 54:720-724.

NOBLE, E. R., and S. B. COLLARD.

1970. The parasites of midwater fishes. In S. F. Snieszko (editor), A symposium of diseases of fishes and shellfishes, p. 57-68. Am. Fish. Soc. Spec. Publ. 5.

NOBLE, E. R., S. B. COLLARD, and S. N. WILKES.

1969. A new philichthyid copepod parasitic in the mucous canals of surfperches (Embiotocidae). J. Parasitol. 55:435-442.

NOBLE, E. R., and R. E. KING.

1960. The ecology of the fish Gillichthys mirabilis and one of its nematode parasites. J. Parasitol. 46:679-685.

NOBLE, E. R., R. E. KING, and B. L. JACOBS.

1963. Ecology of the gill parasites of Gillichthys mirabilis Cooper. Ecology 44:295-305.

NOBLE, E. R., and G. A. NOBLE.

1966. Amebic parasites of fishes. J. Protozool. 13:478-480.

NOBLE, E. R., and J. D. ORIAS.

1970. Aponurus californicus: new trematode from the deepsea smelt Leuroglossus stilbius. Trans. Am. Microsc. Soc. 89:413-417.

1975. Parasitism in the bathypelagic fish, Melanostigma pammelas. Int. J. Parasitol. 5:89-93.

NOBLE, G. A.

1975. Description of Nematobibothrioides histoidii (Noble, 1974) (Trematoda: Didymozoidae) and comparison with other genera. J.'Parasitol. 61:224-227.

NOBLE, G. A., and E. R. NOBLE.

1966. Monocercomonas molae n. sp., a flagellate from the sunfish Mola mola. J. Protozool. 13:257-259.

NUNES-RUIVO, L.

1956. Copépodes parasitas de peixes dos mares de Angola. An. Junta Invest. Ultramar 9:9-45.

1962. Copépodes parasites de poissons des côtes d'Angola. An. Junta Invest. Ultramar 33:67-86.

NUNES-RUIVO, L., and P. FOURMANOIR.

1956. Copepods parasites de poissons de Madegascar. Ser. A, Mém. Inst. Rech. Sci. Madagascar, Biol. Anim. 10:69-80.

OLSEN, L. S.

1952. Some nematodes parasitic in marine fishes. Publ. Inst. Mar. Sci. Univ. Tex. 2:173-215.

OLSON, A. C., Jr.

1972. Argulus melanostictus and other parasitic crustaceans on the California grunion, Leuresthes tenuis (Osteichthyes: Atherinidae). J. Parasitol. 58:1201-1204.

1975. Metacercaria of Bucephalopsis labiatus (Trematoda: Bucephalidae) in the California grunion, Leuresthes tenuis. J. Parasitol. 61:50.

1978. Lepocreadium manteri sp. n. (Trematoda: Lepocreadiidae) from the California grunion, Leuresthes tenuis, and its hyperparasitic microsporidan. Proc. Helminthol. Soc. Wash. 45:155-157.

OLSON, R. E.

1970. The life cycle of Cotylurus erraticus (Rudolphi, 1809) Szidat, $192 \hat{8}$

(Trematoda: Strigeidae). J. Parasitol. 56:55-63.

1972. An intense infection of Philometra americana (Nematoda) in an English sole (Parophrys vetulus). J. Parasitol. 58:188-189.

1978a. Parasites of silver (coho) salmon and king (chinook) salmon from the Pacific Ocean off Oregon. Calif. Fish Game 64:117-120.

1978b. Parasitology of the English sole, Parophrys vetulus Girard in Oregon, U.S.A. J. Fish Biol. 13:237-248.

OLSON, R. E., A. W. HANSON, and I. PRATT.

1970. Plectognathotrema hydrolagi sp. n. (Trematoda: Cephaloporidae) from the ratfish (Hydrolagus colliei), J. Parasitol. 56:724-726.

OLSON, R. E., and I. PRATT.

1973. Parasites as indicators of English sole (Parophrys vetulus) nursery grounds. Trans. Am. Fish. Soc. 102:405-411.

OTSURU, M., T. HATSUKANO, T. OYANAGI, and M. KENMOTSU.

1965. The visceral migrans of gastro-intestinal tract and its vicinity caused by some larval nematode. Jpn. J. Parasitol. 14:542-555.

OVERSTREET, R. M.

1969. Digenetic trematodes of marine teleost fishes from Biscayne Bay, Florida. Tulane Stud. Zool. 15:119-176.

1970. Spinitectus beaveri $\mathrm{sp}$. n. (Nematoda: Spiruroidea) from the bonefish, Albula vulpes (Linnaeus), in Florida. J. Parasitol. 56:128-130.

1971a. Neochasmus sogandaresi $\mathrm{n}$. sp. (Trematoda: Cryptogonimidae) from the striped bass in Mississippi. Trans. Am. Microsc. Soc. 90:87-89. 1971b. Some adult digenetic trematodes in striped mullet from the northern Gulf of Mexico. J. Parasitol. 57:967-974.

1973. Some species of Lecithaster Lïhe, 1901 (Digenea: Hemiuridae) and related genera from fishes in the northern Gulf of Mexico. Trans. Am. Microsc. Soc. 92:231-240.

OZAKI, Y.

1935. Two new trematodes of the family Opistholebidae Travassos. (Preliminary note). Proc. Imp. Acad. Tokyo. 11:244-246.

PALOMBI, A.

1943. Notizie elmintologiche. VIII. Le specie Italiane del genere Hexostoma Raphinesque (= Hexacotyle Blainville). Ann. Mus. Zool. R. Univ. Napoli. N.S. 7:1-8.

PAPERNA, I,

1964a. Parasitic crustacea (Copepoda and Branchiura) from inland water fishes of Israel. Isr. J. Zool. 13:58-68.

1964b. The Metazoan parasite fauna of Israel inland water fishes. Bamidgeh 16:3-66.

PAPERNA, I., and M. LAHAV.

1974. Mortality among gray mullets in a seawater pond due to caligiid parasitic copepod epizootic. Bamidgeh 26:12-15.

PAPERNA, I., and D. E. ZWERNER.

1974. Kudoa cerebralis sp. n. (Myxosporidea, Chloromyxidae) from the striped bass, Morone saxatilis (Walbaum). J. Protozool. 21:15-19.

1976. Parasites and diseases of striped bass, Morone saxatilis (Walbaum), from the lower Chesapeake Bay. J. Fish Biol. 9:267-281.

PAPPAS, P. W.

1970. The trypanorhynchid cestodes from Humboldt Bay and Pacific Ocean sharks. J. Parasitol. 56:1034.

PARISI, B.

1912. Primo contributo alla distribuzione geografica dei missosporidi in 
Italia. Atti. Soc. Ital. Sci. Nat. 50:283-291.

PARK, J. T.

1936. Two new trematodes Sterrhurus magnatestis and Tubulovesicula californica (Hemiuridae) from littoral fishes of Dillon's Beach, California. Trans. Am. Microsc. Soc. 55:477-482.

1937a. A new trematode, Genitocotyle acirrus gen. nov. sp. nov. (Allocreadiidae), from Holconotus rhodoterus. Trans. Am. Microsc. Soc. $56: 67-71$.

1937b. A revision of the genus Podocotyle (Allocreadiinae), with a description of eight new species from tide pool fishes from Dillon's Beach, California. J. Parasitol. 23:405-422.

PARKER, R. R.

1965. A review and redescription of Caligus gurnardi Krфyer, 1863 (Copepoda: Caligidae). Crustaceana 9:93-103.

1969. Validity of the binomen Caligus elongatus for a common parasitic copepod formerly misidentified with Caligus rapax. J. Fish. Res. Board Can. 26:1013-1035

PARKER, R. R., Z. KABATA, L. MARGOLIS, and M. D. DEAN.

1968. A review and description of Caligus curtus Müller, 1785 (Caligidae: Copepoda), type species of its genus. J. Fish. Res. Board Can. 25: 1923-1969.

PARKER, R. R., and L. MARGOLIS.

1964. A new species of parasitic copepod, Caligus clemensi sp. nov. (Caligoida: Caligidae), from pelagic fishes in the coastal waters of British Columbia. J. Fish. Res. Board Can. 21:873-889.

PARUKHIN, A. M.

1966. New trematodes from marine fish of Gulf of Tonkin. [In Russ.] Zool. Zh. 45:1462-1466.

1967. On the helminth fauna of the fish Echeneis naucrates from the South China Sea. [In Russ.] Uchen. Zap. Gorkdv. Gos. Pedagog. Inst. (66) (Gel'mint. Sb., No. 5):24-32. (From Helminthol. Abstr. 1970, Ser. A, 39 pt, 3:311.)

1968. Helminthofauna of fishes of South Atlantic. In V. A. Bodyanitsky (editor), Biology of the seas, p. 96-113. (Biologiia Maria) [In Russ.] "Naukova Dumka" Kiev 14.

1970. Acanthocephala of fish in southern seas. [In Russ.] Gos. Ped. Inst. Uch. Zap. 114:17-24.

PATASHNIK, M., and H. S. GRONINGER, Jr.

1964. Observations on the milky condition in some Pacific coast fishes. J. Fish. Res. Board Can. 21:335-346.

PEARSE, A. S.

1947. Parasitic copepods from Beaufort, North Carolina. J. Elisha Mitchell Sci. Soc. 63:1-16.

1951. Parasitic crustacea from Bimini, Bahamas. Proc. U.S. Natl. Mus. 101:341-372.

1952. Parasitic crustacea from the Texas coast. Publ. Inst. Mar. Scj. Univ. Tex. 2:5-42.

1953. A new copepod parasite from the grunion. J. Parasitol. 39: 332-333.

PEARSON, J. C

1964. A revision of the subfamily Haplorchinae Looss, 1899 (Trematoda: Heterophyidae). I. The Haplorchis group. Parasitology 54:601-676.

1968. Observations on the morphology and life-cycle of Paucivitellosus fragilis Coil, Reid \& Kuntz, 1965 (Trematoda: Bivesiculidae). Parasitology 58:769-788.

1973. A revision of the subfamily Haplorchinae Looss, 1899 (Trematoda: Heterophyidae). II. Genus Galactosomum. Philos. Trans. R. Soc. Lond., Serv, B, Biol. Sci. 266:341-447.

PEARSON, J. C., L. MARGOLIS, and N. P. BOYCE.

1978. The metacercaria of Galactosomum phalacrocoracis (Trematoda: Heterophyidae) from pink salmon, Oncorhynchus gorbuscha, from British Columbia waters. Can. J. Zool. 56:2235-2238.

PELLERDY, L. P

1965. Coccidia and coccidiosis. Akademiai Kiado, Budapest, 959 p.

PENNELL, D. A., C. D. BECKER, and N. R. SCOFIELD.

1973. Helminths of sockeye salmon (Oncorhynchus nerka) from the Kvichak River system, Bristol Bay, Alaska. Fish. Bull., U.S. 71:267-277.

PENNYCUICK, L.

1971. Seasonal variations in the parasite infections in a population of three-spined sticklebacks, Gasterosteus aculeatus L. Parasitology 63: 373-388.

PETRUSHEVSKI, G. K

1955. Changes in the parasite fauna of acclimatised fishes. In V. A. Dogiel, G. K. Petrushevski, and Yu. I. Polyanski (editors), Parasitology of fishes, p. 255-264. (Engl. ed. 1961). Oliver and Boyd, Lond, Russ. ed. Leningrad University Press.
PETRUSHEVSKI, G. K., V. M. MOSEVICH, and I. G. SHCHUPAKOV. 1949. Parasite fauna of fish of the Ob and Irtysh Rivers. [In Russ.] [ZV. Vses. Nauchno-Issled. Inst. Ozern. Rechn. Rȳb. Khoz. 27:67-96. (From Ind.-Cat. Med. Vet. Zool., Suppl. 16, p. 244.)

PETRUSHEVSKI, G. K., and S. S. SHULMAN

1955. The parasitic diseases of fishes in the natural waters of the USSR In V. A. Dogiel, G. K. Petrushevski, and Yu. I. Polyanski (editors), Parasitology of fishes, p. 299-319. (Engl. ed. 1961). Oliver and Boyd, Lond. Russ. ed. Leningrad University Press.

PILLAI, N. K.

1962. Copepods parasitic on South Indian fishes families Lernaeopodidae and Naobrachidae. J. Mar. Biol. Assoc. India 4:58-94.

1963. Copepods parasitic on South Indian fishes-family Caligidae. J. Mar. Biol. Assoc. India 5:68-96.

1964. A miscellaneous collection of copepods parasitic on South Indian fishes. J. Mar. Biol. Assoc. India 6:61-83.

1967. Three species of dichelesthiid copepods parasitic on South Indian sharks. Zool. Anz. 179:286-297.

PILLAI, N. K., and R. S. L. MOHAN,

1965. Observations on the genus Heniochophius (Copepoda) with a description of the type species. J. Mar. Biol. Assoc. India 7:270-276.

PINTNER, T.

1930. Tetrarhynchen aus Pacific Grove, California, U.S.A. Anz. Akad. Wiss. Wien, Math.-Nat. 67:26-28.

PODDER, T. N.

1937. On a new species of Neoechinorhynchus parasitic in Mugil cephalus Linn from the Chilka Lake. Rec. Indian Mus. (Calcutta) 39:129-131.

POGORELTSEVA, T. P

1964. New and little known species of monogenetic trematodes of fish in the Black Sea. [In Russ.] Tr. Ukr. Respubl. Nauch. Obshch. Parazit. 3:30-42.

POLYANSKI, Yu. I.

1955a. Ecology of parasites of marine fishes. In V. A. Dogiel, G. K. Petrushevski, and Yu. I. Polyanski (editors), Parasitology of fishes, p. 48-83. (Engl. ed. 1961). Oliver and Boyd, Lond. Russ. ed. Leningrad University Press.

1955b. Parasites of the fish of the Barents Sea. Izd. ANSSR MoskvaLeningrad 19. (Translated by Israel Program for Scientific Translations, Jerusalem, 1966.)

PRATT, I.

1970. Life cycle and host specificity of the marine digenetic trematode, Genitocotyle acirra Park. J. Parasitol. 56 (Sect. II, Part I):273-274.

PRATT, I., and L. E. ALDRICH, Jr.

1953. Megalocotyle trituba n. sp. (Trematoda: Monogenea). J. Parasitol. 39:535-537.

PRATT, I., and R. HERRMANN.

1962. Nitzschia quadritestes sp. n. (Monogenea: Capsalidae) from the Columbia River sturgeon. J. Parasitol. 48:291-292.

PRATT, I., and J. E. MCCAULEY

1961. Trematodes of the Pacific Northwest, an annotated catalogue. Oreg. State Monogr. Stud. Zool. il, 118 p.

PREFONTAINE, G., and P. BRUNEL.

1962. Liste d'invertébrés marine recueillis dans l'estuaire du Saint-Laurent de 1929 à 1934. Nat. Can. 89:237-263.

PRICE, C. E., and T. E. MCMAHON.

1967. The monogenetic trematodes of North American freshwater fishes. Riv. Parassitol. 28:177-220.

PRICE, E. W.

1934. New monogenetic trematodes from marine fishes. Smithson. Misc. Collect. 91:1-3.

1937a. A new monogenetic trematode from Alaskan salmonoid fishes. Proc. Helminthol. Soc. Wash, 4:27-29.

1937b. North American monogenetic trematodes. I. The superfamily Gyrodactyloidea. J. Wash. Acad. Sci. 27:114-130, 146-164.

1938a. North American monogenetic trematodes. II. The families Monocotylidae, Microbothriidae, Acanthocotylidae, and Udonellidae (Capsaloidea). J. Wash. Acad. Sci. 28:109-126

1938b. The monogenetic trematodes of Latin America. Livro Jubilar do Professor Lauro Travassos 1913-1938, p. 407-414

1939. North American monogenetic trematodes. III. The family Capsalidae (Capsaloidea). J. Wash. Acad. Sci. 29:63-92.

1942. North American monogenetic trematodes. V. The family Hexabothriidae n.n. (Polystomatoidea). Proc. Helminthol. Soc. Wash 9:39-56.

1943. North American monogenetic trematodes. VII. The family Discocotylidae (Diclidophoroidea). Proc. Helminthol. Soc. Wash. 10:10-15. 
1961a. North American monogenetic trematodes. VIII. The family Hexostomatidae. Proc. Helminthol. Soc. Wash. 28:4-9.

1961 b. North American monogenetic trematodes. IX. The families Mazocraeidae and Plectanocotylidae. Proc. Biol. Soc. Wash. 74:127-156.

1962a. A description of Tricotyla molae (Blanchard), with a discussion of the monogenetic trematodes of the sunfish (Mola mola L.). J. Parasitol. $48 \mathbf{8}: 74 \hat{8}-751$.

1962b. North American monogenetic trematodes. XI. The family Heteraxinidae. J. Parasitol, 48:402-418.

PRITCHARD, M. H.

1961. Notes on two species of Tristomella Guiart, 1938 (Monogenea: Capsalidae), from a South African black marlin. J. Parasitol. 47: 976-977.

1963. Studies on digenetic trematodes of Hawaiian fishes, primarily families Lepocreadiidae and Zoogonidae. J. Parasitol. 49:578-587.

1972. Neolabrifer bravoae gen. nov. sp. nov. and Labrifer secundus Manter, 1940 (Trematoda: Lepocreadiidae) from the California sheephead in the American Pacific. An. Inst. Biol. Univ. Méx. Ser. Zool. 41: 127-134.

PUTZ, R. E., G. L. HOFFMAN, and C. E. DUNBAR.

1965. Two new species of Plistophora (Microsporidea) from North American fish with a synopsis of Microsporidea of freshwater and euryhaline fishes. J. Protozool. 12:228-236.

QUAST, J. C.

1964. Meristic variation in the hexagrammid fishes. U.S. Fish Wildl. Serv., Fish. Bull. 63:589-609.

RAJU, G.

1962. New records of the giant trematodes of the genus Hirudinella Garcin from Indian waters. J. Mar. Biol. Assoc. India. 4:232-234.

RAMACHANDRAN, P.

1975. Philometra cephalus sp. $\mathrm{n}$. infecting the gonads of the striped mullet, Mugil cephalus L. from the Arabian coast of Kerala, India, with a note on its pathology. Zool. Anz. Jena 194:140-144.

RANEY, E. C.

1952. The life history of the striped bass, Roccus saxatilis (Walbaum). In E. C. Raney, E. F. Tresselt, E. H. Hollis, V. D. Vladykov, and D. H. Wallace (editors), The striped bass, Roccus saxatilis, p. 5-97. Bull. Bingham Oceanogr. Collect. 14.

RANGNEKAR, M. P.

1955. Caligus bombayensis sp. nov., a copepod parasitic on Mugil cephalis Linn. J. Univ. Bombay 24:55-59.

1956. Parasitic copepods from the marine fishes of Bombay. J. Univ. Bombay 24:42-65.

1957. Copepod parasites of the families Argulidae, Caligidae, Dichelesthidae, and Lernaeopodidae. J. Univ. Bombay, Sect. B, 26:8-20.

RAO, K. H., and R. MADHAVI.

1967. A record of Dionchus agassizi Goto, 1899 (Monogenea: Capsaloidea) from the sucker fish Echeneis naucrates Linnaeus from Bay of Bengal. Curr. Sci. 36:490-491.

RAO, T.

1951. On a new caligoid parasite, Gloiopotes zeugopteri, Lawson's Bay, Waltair. Proc. Indian Acad. Sci. Sect. B, 34B:248-255.

RATHBUN, $R$.

1884. Annotated list of the described species of parasitic Copepoda (Siphonostoma) from American waters contained in the United States National Museum. Proc. U.S. Natl. Mus. 7:483-492.

1887. Descriptions of new species of parasitic copepods, belonging to the genera Trebius, Perissopus and Lernanthopus. Proc. U.S. Natl. Mus. 10:559-571.

RAUSCH, R

1954. Studies on the helminth fauna of Alaska. XXI. Taxonomy, morphological variation and ecology of Diphyllobothrium ursi $\mathrm{n}$. sp. provis on Kodiak Island. J. Parasitol. 40:540-563.

RAWSON, M. V., Jr.

1973. Two new species of Gyrodactylus (Trematoda: Monogenea) from the Georgia Coast. Proc. Helminthol. Soc. Wash. 40:180-183.

1976. Population biology of parasites of striped mullet, Mugil cephalus L. I. Monogenea. J. Fish Biol. 9:155-194.

1977. Population biology of parasites of striped mullet, Mugil cephalus I. Crustacea. J. Fish Biol. 10:441-451.

REES, G.

1944. A new cestode of the genus Grillotia from a shark. Parasitology $35: 180-185$.

1970. Some helminth parasites of fishes of Bermuda and an account of the attachment organ of Alcicornis carangis MacCallum, 1917 (Digenea: Bucephalidae). Parasitology 60:195-221.

REID, W. A., W. H. COIL, and R. E. KUNTZ.

1965. Hemiurid trematodes of Formosan marine fishes. II. Subfamily Lecithochiriinae. Proc. Helminthol. Soc. Wash. 32:199-205.

RESHETNIKOVA, A. V.

1955. On the study of parasitic fauna of fishes of the Black Sea. [In Russ.] Tr. Karadah. Nauch. Stn. T. I. Vyazems'koho 13:105-121.

RICHARDSON, $\mathrm{H}$

1905. A monograph on the isopods of North America. U.S. Natl. Mus. Bull. 54,727 p.

RICHIARDI, S.

1877. Descrizione di due specie nuove di Lernaeenicus, con osservazioni intorno a questo ed ai generi Lernaeocera bl. e. Lernaeonema M.-Edw. Atti Soc. Tosc. Sci. Nat. 3:195-206.

RISER, N. W,

1948. Amphilina bipunctata n. sp., a North American cestodarian. J. Parasitol. 34:479-485.

1955. Studies on cestode parasites of sharks and skates. J. Tenn. Acad. Sci. 30:265-311.

1956. Observations on the plerocercoid larva of Pelichnibothrium speciosum Monticelli 1889. J. Parasitol. 42:31-33.

ROBERTS, L. S.

1963. Ergasilus nerkae n. sp. (Copepoda: Cyclopoida) from British Columbia with a discussion of the copepods of the $E$. caeruleus group. Can. J. Zool. 41:115-124.

1970. Ergasilus (Copepoda: Cyclopoida): revision and key to species in North America. Trans. Am. Microsc. Soc. 89:134-161.

ROBINSON, E. S.

1959a. Records of cestodes from marine fishes of New Zealand. Trans. R. Soc, N.Z. 86:143-153.

1959b. Some new cestodes from New Zealand marine fishes. Trans. R. Soc. N.Z. 86:381-392.

1961. Some monogenetic trematodes from marine fishes of the Pacific. Trans. Am. Microsc. Soc. 80:235-266.

ROBINSON, V. C.

1934. A new species of accacoelid trematode (Accacladocoelium alveolatum n. sp.) from the intestine of a sun-fish (Orthagoriscus mola Bloch). Parasitology 26:346-351.

RODELLA, T. D., and F. M. NAHHAS.

1969. Some digenetic trematodes of embiotocid fishes from Tomales and Bodega Bays, California. J. Parasitol. 55:758.

ROGERS, W. A.

1967. Polycithrum mugilini gen. et. sp. n. (Gyrodactylidae: Polyclithrinae subfam. n.) from Mugil cephalus L. J. Parasitol. 53:274-276.

ROKICKI, J.

1973. Helminths of certain Clupeidae, mainly of the herring Clupea harengus L. in South Baltic. Acta Parasitol. Pol. 21:443-464.

RUKAVINA, J., and S. DELIC.

1965. Endohelminths of salmonoid and some other fishes in Bosnia and Hercegovina. Veterinaria, Saraj. 14:289-294.

RUSSO, R. A.

1975. Notes on the external parasites of California inshore sharks. Calif. Fish Game 61:228-232.

SAITO, T., H. KITAYAMA, and Y. TANKAWA.

1970. Frequency of Anisakis larvae in marine fishes and cuttlefishes captured in the area of Hokkaido. Rep. Hokkaido Inst. Public Health 20: 115-122.

SANDEMANN, I. M., and J. H. C. PIPPY.

1967. Parasites of freshwater fishes (Salmonidae and Coregonidae) of insular Newfoundland. J. Fish. Res. Board Can. 24:1911-1943.

SANDERS, J. E., and J. L. FRYER.

1970. Occurrence of the myxosporidan parasite Myxidium minteri in salmonid fish. J. Protozool. 17:354-357.

SANDERS, J. E., J. L. FRYER, and R. W. GOULD.

1970. Occurrence of the myxosporidian parasite Ceratomyxa shasta, in salmonid fish from the Columbia River basin and Oregon coastal streams. In S. F. Snieszko (editor), A symposium of diseases of fishes and shellfishes, p. 133-141. Am. Fish. Soc. Spec. Publ. 5.

SANKURATHRI, C. S.

1977. Conispora renalis gen. nov. et sp. nov. (Myxosporida: Wardidae) from the kidney tubules of Pacific hake, Merluccius productus (Ayres 1855), in coastal waters of British Columbia. Can. J. Zool. 55:1147-1150.

SANO, T.

1970. Etiology and histopathology of several epizootics among fingerling salmonids. J. Tokyo Univ. Fish. 56:13-22. 
SANZIN, W. D

1965. Untersuchungen zur Oekologie, Artspezifität und variabilität von Hexamita salmonis Moore. Inaug-Diss. (Munchen), 103 p. (From Ind.Cat. Med. Vet. Zool. Suppl. 18, Pt. 7:162.)

\section{SASAKI, M.}

1973. Survey of parasites of the Alaska pollack, Theragra chalcogramma. Sci. Rep. Hokkaido Fish Exp. Stn. 30:14-34. (In Melminthol. Absit. 43:960.)

SAUNDERS, D. C.

1964. Blood parasites of marine fish of southwest Florida, including a new haemogregarine from the menhaden, Brevoortia tyrannus (Latrobe). Trans. Am. Microse. Soc. 83:218-225.

1966. A survey of the blood parasites of the marine fishes of Puerto Rico. Trans. Am. Microsc. Soc. 85:193-199.

SAWYER, R. T., A. R. LAWLER, and R. M. OVERSTREET.

1975. Marine leeches of the eastern United States and the Gulf of Mexico with a key to the species. J. Nat. Hist. 9:633-667.

SAWYER, T. K., J. G. HNATH, and J. F. CONRAD.

1974. Thecamoeba hoffmani sp. n. (Amoebida: Thecamoebidae) from gills of fingerling salmonid fish. J. Parasitol. 60:677-682.

SCHELL, S. C.

1972. Otodistomum hydrolagi sp. n. (Trematoda: Azygiidae) from the coelom of the ratfish, Hydroiagus coiliei (Lay and Bennett, 1839). J. Parasitol. 58:885-886.

1973a. Rugogaster hydrolagi gen. et sp. $\mathrm{n}$. (Trematoda: Aspidobothrea: Rugogastridae fam. n.) from the ratfish, Hydrolagus colliei (Lay and Bennett, 1839). J. Parasitol. 59:803-805.

1973b. Three new species of digenetic trematodes from Puget Sound fishes. Proc. Helminthol. Soc. Wash. 40:227-230.

1974. The life history of Sanguinicola idahoensis sp. n. (Trematoda: Sanguinicolidae), a blood parasite of steelhead trout, Salmo gairdneri Richardson. J. Parasitol. 60:561-566.

SCHILLER, E. L.

1954. Studies on the helminth fauna of Alaska. XVII. Notes on the intermediate stages of some helminth parasites of the sea otter. Biol. Bull. (Woods Hole) 106:107-121.

1956. Studies on the helminth fauna of Alaska. XXIX. Urinatrema aspinosum $\mathrm{n}$. sp. (Trematoda: Zoogonidae) from the urinary bladder of the greenling, Hexagrammos superciliosus (Pallas). J. Parasitol. 42: $531-532$.

SCHMIDT, G. D., and I. PAPERNA.

1978. Scterocollum rubrimaris gen. et sp. n. (Rhadinorhynchidae: Gorgorhynchinae), and other acanthocephala of marine fishes from Israel. J. Parasitol. 64:846-850.

SCOTT, A.

1904. Some parasites found on fishes in the Irish Sea. Proc. Trans. Liverpool Biol. Soc, 18:113-125.

SEKERAK, A. D.

1970. Parasitic copepods of Sebastodes alutus including Chondracanthus triventricosus sp. nov. and Colobomatus kyphosus sp. nov. J. Fish. Res. Board Can. 27:1943-1960.

SEKERAK, A. D., and H. P. ARAI.

1973. Helminths of Sebastes alutus (Pisces: Teleostei) from the northeastern Pacific. Can. J. Zool. 51:475-477.

1974. A revision of Helicometra Odhner, 1902, and related genera (Trematoda: Opecoelidae), including a description of Neohelicometra sebastis n. sp. Can. J. Zool. 52:707-738.

1977. Some metazoan parasites of rockfishes of the genus Sebastes from the northeastern Pacific Ocean. Syesis 10:139-144.

SEKI, N.

1975. Studies on helminth parasites of salmonid tishes in Hokkaido. Jpn. J. Vet. Res. 23:113. (In Helminthol. Abstr. Ser. A, 45:306.)

SEY, $O$.

1970a. Parasitic helminths occurring in Adriatic fishes. Part II (flukes and tapeworms). Acta Adriatica 13(6):3-15.

1970b. Parasitic helminths occurring in Adriatic fishes. Part III. (Nematodes, Acanthocephala). Acta Adriatica 13(7):1-16.

SHAFFER, E.

1916. Discocotyle solmomis nov. spec, ein neuer trematode an den kiemen der regenbogenforelle (Salmo irideus). Zool. Anz. 46:257-271.

SHAW, J. N.

1933. Some parasites of Oregon wild life. J. Am. Vet. Med. Assoc. 83: 599-603.

1947. Some parasites of Oregon wild life. Oreg. Agric. Stn. Bull. 11.
SHAW, J. N., B. T. SIMMS, and O. H. MUTH.

1934. Some diseases of Oregon fish and game and identification of parts of game animals. Oreg. Agric. Stn. Bull. 46:4-23.

SHEN, C. J.

1957. Parasitic copepods from fishes of China. Part II. Caligoida, Caligidae (1). Acta Zool. Sinica 9:351-377.

SHIGIN, A. A.

1965. Some of the results on the systematic study of metacercaria of the genus Diplostomum - cause of diplostomiasis of freshwater fish in SSSR. [In Russ.] Mater. Nauchn. Konf. Vsesoivz. Obschch. Gel'minthol. (1):261-265. (From Ind.-Cat. Med. Vet. Zool. Suppl. 18, Pt. 7:265.)

SHIINO, S. M.

1952. Copepods parasitic on Japanese fishes. 1. On the species of Caligus and Lepeophtheirus. Rep. Fac. Fish. Prefect. Univ. Mie 1:79-113.

1954a. A new fish-louse found on the mackerel-pike. Annot. Zool. Jpn. 27:150-153.

1954b. A new fish-louse found on Zenopsis nebulosa (T. et S.). Annot. Zool. Jpn. 27:154-156.

1954c. Copepods parasitic on Japanese fishes. 4. The family Euryphoridae. Rep. Fac. Fish. Prefect. Univ. Mie 1:273-290.

1954d. Copepods parasitic on Japanese fishes, 3. On two new species of the genus Anuretes. Rep. Fac. Fish. Prefect. Univ. Mie 1:260-272.

1954e. Copepods parasitic on Japanese fishes 5 . Five species of the family Pandaridae. Rep. Fac. Fish. Prefect. Univ. Mie 1:291-332.

1954f. Note on Caligus quadratus n. sp., a copepod parasitic on the fish, Neothynnus macroprerus (T. and S.). Bull. Jpn. Soc. Sci. Fish. 20:26-29.

1955a. Alebion echinatus Capart from Japanese waters, with observations on the newly found male form. Pac. Sci. 9:177-182.

1955b. A new piscicola copepod belonging to the genus Caligus from Matusima Bay. Bull. Biogeogr. Soc. Jpn. 16-19:135-140.

1955c. Copepods parasitic on Japanese fishes, 8. The Anthosomidae. Rep. Fac. Fish. Prefect. Univ. Mie 2:50-69.

1955d. Copepods parasitic on Japanese fishes. 9. Family Chrondracanthidae, subfamily Chondracanthinae. Rep. Fac. Fish. Prefect. Univ. Mie 2:70-111.

1957. Copepods parasitic on Japanese fishes. 13. Parasitic copepods collected off Kesennuma, Miyagi Prefecture. Rep. Fac. Fish. Prefect. Univ. Mie 2:359-375.

1959a. Neuer art name tï Japnische exemplare von Caligus bonito. Bull. Biogeogr. Soc. Jpn. 20:51-57.

1959b. On a new species of Caligus (Copepoda Parasitica) from the coast of California. Pac. Sci. 13:351-356.

1959c. Ostpazifische parasitierende Copepoden. Rep. Fac. Fish. Prefeci. Univ. Mie 3:267-333.

1959d. Revision der auf goldmakrele, Coryphaena hippurus L., schmarotzenden caligidenarten. Annu. Rep. Prefect. Univ. Mie, Sect. 2, 3:1-34. 1959e. Sammlung der parasitischen Copepoden in der Präfekturuniversitä von Mie. Rep. Fac. Fish. Prefect. Univ. Mie 3:334-374.

1960a. Copepods parasitic on fishes from Seto, Province Kii, Japan. Rep. Fac. Fish. Prefect. Univ. Mie 3:501-517.

1960b. Copepods parasitic on the fishes collected on the coast of Province Shima, Japan. Rep. Fac. Fish. Prefect. Univ. Mic 3:471-500.

1960c. Parasitic copepods of fishes from the Eastern Pacitic. Rep. Fate. Fish. Prefect. Univ. Mie 3:527-541.

1963a. Parasitic copepods of the Eastern Pacific fishes. 1. Records of the known species. Rep. Fac. Fish. Prefec1. Univ. Mic 4:335-347.

1963b. Parasitic copepods of the Eastern Pacific fishes. 2. Lïkenia elongata sp. nov. Rep. Fac. Fish. Prefect. Univ. Mie 4:349-356.

1963c. Parasitic copepods of the Eastern Pacific fishes. 4. On Clarellistes shoyoae gen, el sp. nov. Rep. Fac. Fish. Prcfec1. Univ. Mie 4:368-375.

1964. On two new species of the genus Acanthochondria Oakley (Crustacea, Copepodal found in Japan. 7.ö̈l. Meded. Leiden 39:30-36.

1965a. On Cecrops exiguus Wilson found in Japan. Rep. Fac. Fish. Prefect. Univ. Mie 5:381-390.

1965b. Parasitic copepods of the Eastern Pacific fishes. 5. Caligus. Rep. Fac. Fish. Prefect. Univ. Mie 5:391-420.

1965c. Parasitic copepods of the Eastern Pacific fishes. 6. Euryphoridae. Rep. Fac. Fish. Prefect. Univ. Mie 5:421-433.

1965d. Parasitic copepods of the Eastern Pacific fishes. 8. Lepeophtheirus. Rep. Fac. Fish. Prefect. Univ. Mie 5:44!-454.

SHIMAZU, T.

1970. Gonocerca oshoro sp. n. (Trematoda: Hemiuridae) from the ovary of the rat tail, Nematonurus pectoralis from the Gulf of Alaska. Jpn. J. Parasitol. 19:278-281. 
1975. A description of the adult of Nybelinia surmenicola with discussions on its life-history (Cestoda: Trypanorhyncha: Tentaculariidae). [Engl. abstr.] Bull. Jpn. Soc. Sci. Fish. 41:823-830.

SHIREMAN, J. V.

1964. Carassotrema mugilicola, a new haploporid trematode from the striped mullet, Mugil cephalus, in Louisiana. J. Parasitol. 50:555-556.

SHULMAN, S. S.

1955. Specificity of fish parasites. In V. A. Dogiel, G. K. Petrushevski, and Yu. I. Polyanski (editors), Parasitology of fishes, p. 104-106. (Engl. ed. 1961). Oliver and Boyd, Lond. Russ. ed. Lengingrad University Press.

1966. The Myxosporidia in the fauna of the USSR. Nauka MoscowLeningrad, p. 507.

SIDDIGI, A. H., and R. M. CABLE.

1960. Digenetic trematodes of marine fishes of Puerto Rico. N.Y. Acad. Sci. 17:261-369.

SILAS, F. G.

1967. Parasites of scombroid fishes. Part 1. Monogenetic trematodes, digenetic trematodes, and cestodes. Proc. Symp. Scombroid Fishes, Part 3, p. 799-875. Mar. Biol. Assoc. India, Mandapam Camp.

SIMHA, S. S., and R. S. PERSHAD.

1964. Echeneidocoelium indicum n. gen. n. sp. (Trematoda) from the sucker fish, Echeneis remora, from Vishakapatnam (A. P.) India. Riv. Parassitol. 25:21-24.

SIMMONS, D. C

1969. Maturity and spawning of skipjack tuna (Katsuwonus pelamis) in the Atlantic Ocean, with comments on nematode infestation of the ovaries. U.S. Fish Wildl. Serv., Spec. Sci. Rep. 580, 17 p.

SIMMS, B. T., C. R. DONHAM, J. N. SHAW, and A. M. McCAPES.

1931. Salmon poisoning. J. Am. Vet. Med. Assoc. 78:181-195.

SIMMS, B. T., and J. N. SHAW.

1931. Studies of the fishborne tapeworm Dibothrium cordiceps Leidy. J. Am. Vet. Assoc. 79:199-205.

SIMON, JMR, and F. SIMON.

1936. Philonema agubernaculum sp. nov. (Dracunculidae), a nematode from the body cavity of fishes. Parasitology 28:440-442.

SINDERMANN, C. J.

1957. Diseases of fishes of the western North Atlantic. V. Parasites as indicators of herring movements. Maine Dep. Sea Shore Fish. Res. Bull. $27,30 \mathrm{p}$.

SINDERMANN, C. J., and A. ROSENFIELD.

1954. Diseases of fishes of the western North Atlantic. I. Diseases of the sea herring (Clupea harengus). Maine Dep. Sea Shore Fish. Res. Bull. 18:1-23.

SINITSIN, B.

1931. Studien über die phylogenie der trematoden. IV. The life histories of Plagioporus siliculus and Plagioporus virens, with special reference to the origin of Digenea. Z. Wiss. Zool. 138:409-456.

SKINNER, R.

1975. Parasites of the striped mullet, Mugil cephalus, from Biscayne Bay, Florida, with description of a new genus and three new species of trematodes. Bull. Mar. Sci. 25:318-345.

SKRYABINA, E. S.

1963. Helminth fauna of marine fish of Kamchatka. Tr. Gel'mintol. Lab. Akad. Nauk SSSR 13:313-329.

SLINN, D. J.

1957. Erpocotyle abbreviata (Olsson, 1876) Price, 1942, a monogenetic trematode new to British waters. Nature (Lond.) 179:271-272.

SLUSARSKI, w.

1958. The adult Digenea from Salmonidae of the basin of the Vistula and of the South Baltic. [In Polish] Acta Parasitol. Pol. 6:247-528.

SMEDLEY, E. M.

1933. Nematode parasites from Canadian marine and fresh-water fishes. Contrib. Can. Biol. Fish. New Ser. 8:169-179.

1934. Some parasitic nematodes from Canadian fishes. J. Helminthol. 12:205-220.

SMIRNOVA, T. S., Y. A. STRELKOV, V. A. TIMOFEEV, and S. S. SHULMAN.

1964. Nasal cavaties of teleostei fishes as a site of dwelling of parasites. [In Russ.) Zool. Zh. 43:1649-1658. (From Ind.-Cat. Med. Vet. Zool. Suppl. 18, Pt. 7, p. 537.)

SMITH, H. D.

1973. Observations on the cestode Eubothrium salvelini in juvenile sockeye salmon (Oncorhynchus nerka) at Babine Lake, British Columbia. J. Fish. Res. Board Can. 30:947-964.
SMITH, J. W.

1967. Aporocotyle margolisi n. sp. (Digenea: Aporocotylidae) from Merluccius productus. J. Fish. Res. Board Can. 24:1763-1773.

SOBOLEV, A. A., and O. I. BELOGUROV.

1968. A representative of the genus Spinitectus in an oceanic fish. Parazity Zhivotnykh i Rastenii Izdat "Nauka" Moscow 4:59-62.

SOGANDARES-BERNAL, F.

1959. Digenetic trematodes of marine fishes from the Gulf of Panama and Bimini, British West Indies. Tulane Stud. Zool. 7:69-117.

SOGANDARES-BERNAL, F., and R. F. HUTTON.

1959. Studies on helminth parasites of the coast of Florida. I. Digenetic trematodes of marine fishes from Tampa and Boca Ciega Bays with descriptions of two new species. Bull. Mar. Sci. Gulf Caribb. 9:53-68.

SOKOLOVSKAYA, I. L.

1971. Acanthocephala of fish in Amur Basin. [In Russ.] Parazitol, Sb. $25: 165-176$.

SOLONCHENKO, A. I.

1968. Parasitofauna of mackerel Scomber colias Gmelin inhabiting the Atlantic Ocean near the southwestern coast of Africa. In V. A. Bodyanitsky (editor), Biology of the seas, p. 90-95. (Biologiia Moriia) IIn Russ.] "Naukova Dumka" Kiev 14.

SOUTHWELL, T.

1913. Notes from the Bengal fisheries laboratory, Indian Museum no. 1. Rec. Indian Mus. (Calcutta) 9:79-103.

1925. A monograph on the Tetraphyllidea. Liverpool School of Trop. Med. Memoir n. s. (2), Liverpool Univ. Press, 368 p.

1930. The fauna of British India, including Ceylon and Burma. Cestoda. I. Taylor and Francis, Lond., 391 p.

SPARKS, A. K.

1958. Some digenetic trematodes of fishes of Grand Isle, Louisiana. Proc. La. Acad. Sci. 20:71-82.

SPARKS, A, K., and K. K. CHEW.

1966. Gross infestation of the littleneck clam, Venerupis staminea, with a larval cestode (Echeneibothrium sp.). J. Invertebr. Pathol. 8:413-416.

SPECIAN, R. D., J. E. UBELAKER, and M. D. DAILEY.

1975. Neoleptus gen. $n$. and a revision of the genus Proleptus Dujardin, 1845. Proc. Helminthol. Soc. Wash. 42:14-21.

SPROSTON, N. G.

1946. A synopsis of the monogenetic trematodes. Trans. Zool. Soc. Lond. 25:185-600.

1948. On the genus Dinobothrium Van Beneden (Cestoda) with a description of two new species from sharks and a note on Monorygma species from the electric ray. Parasitology 39:73-90.

SRIVASTAVA, C.D., and R. K. GHOSH.

1968. On a new species of genus Fellodistomum Stafford, 1904 (Trematoda: Fellodistomidae), with redescription of F. agnotum Nicoll, 1909. Indian J. Helminth. 20:46-52.

STAFFORD, J.

1904. Trematodes from Canadian fishes. Zool. Anz. 27:481-495.

1907. Preliminary report on the trematodes of Canadian marine fishes. Contrib. Can. Biol. 1901-05:103-106.

STOKELL, G.

1936. The nematode parasites of Lake Elesmere trout. Proc. R. Soc. N.Z. 66:80-96

STRELKOV, Yu. A.

1960. Endoparasitic worms of ocean fish of East Kamchatka. [In Russ.] Tr, Zool. Inst. Akad. Nauk SSSR 28:146-196.

1971. Trematodes from the fishes of the Amur River basin. [In Russ.] Parazitol. Sb. 25:120-139.

STUNKARD, H. W.

1935. A new trematode, Probolitrema californiense from the coelom of the sting ray, Myliobatis californicus. J. Parasitol. 21:359-364.

1962. Caballerocotyla klawei sp. n. A monogenetic trematode from nasal capsule of Neothunnus macropterus. J. Parasitol. 48:883-890.

1974. Rhipidocotyle heptathelata $\mathrm{n}$. sp., a bucephalid trematode from Thynnus thunnina taken in the Red Sea. Trans. Am. Microsc. Soc. 93: 260-261.

SUBHAPRADHA, C. K.

1955. Cestode parasites of fishes of Madras Coast. Indian J. Helminthol. $7: 41-132$.

SUKHENKO, G. E.

1966. On some characteristics of distribution of parasitic crustacea in Ukrainian ponds. [In Russ.] Respublik. Mezhvedomstv. Sborn. Akad. Nauk Ukrain. SSR Problemy Parazitol. 6:67-74. (From Ind.-Cat. Med. Vet. Zool. Suppl. 18, Pt. 7:265.) 
SUTTERLIN, A. M., P. HARMON, and H. BARCHARD.

1976. The culture of brook trout in salt water. Environ. Can. Fish. Mar, Serv., Tech. Rep. 636, 21 p.

TASTO, R. N.

1975. Aspects of the biology of Pacific staghorn sculpin, Leptocottus armatus Girard, in Anaheim Bay. In E. D. Lane and C. W. Hill (editors), The marine resources of Anaheim Bay, p. 123-135. Calif. Fish Game, Fish Bull. 165.

THATCHER, V. E., and A. K. SPARKS.

1958. A new species of Dicrogaster (Trematoda, Haploporidae) from Mugil cephalis in the Gulf of Mexico. J. Parasitol. 44:647-648.

THOMAS, M. M.

1967. A new parasitic copepod, Caligus krishnai, from the mackerel tuna Euthynnus affinis affinis (Cantor). J. Mar. Biol. Assoc. India 9:137-140.

THOMPSON, N. F.

1916. A note on a sporozoan parasite of the halibut. Rep. Comm. Fish. Br. Columbia 1915:127-128.

THRELFALL, w.

1967. Some parasites recovered from the ocean sunfish, Mola mola (L), in Newfoundland. Can. Field-Nat. 81:168-172.

1968. A mass die-off of three-spined sticklebacks (Gasterosteus aculeatus L.) caused by parasites. Can. J. Zool. 46:105-106.

1969. Some parasites from elasmobranchs in Newfoundland. J. Fish. Res. Board Can. 26:805-811

TIAINEN, O. A.

1966. Diplostomum metacercariae (Trematoda, Diplostomatidae) as parasites in the eyes of trout and burbot in Finland. Ann. Zool. Fenn. 3:172.

TIMON-DAVID, J.

1963. Developpement experimental d'un trématode du genre Apophallus Lithe. Bull. Soc. Hist. Nat. Toulouse 98:452-458

TIMON-DAVID, P., and J. J. MUSSO.

1971. Les trematodes digènes du poisson-lune (Mola mola) dans le golfe de Marseille (Accacoeliidae, Didymozoidea). [in Fr., Engl, summ.] Ann. Parasitol. Hum. Comp. 46:233-256.

TOPP, R. W., and D. L. GIRARDIN.

1971. An adult louvar, Luvarus imperialis (Pisces, Luvaridae) from the Gulf of Mexico. Copeia 1971:181-182.

TOWNSEND, L. D.

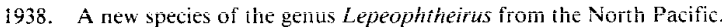
Ann. Mag. Nat. Hist., Ser. 11:599-604.

TRAVASSOS, L., J. F. T. FREITAS, and P. F. BÜHRNHEIM

1966. Trematodeos de peixes do littoral capixaba: Separogermiductus zeloticus sp. n., parasito de xaréu. Atas Soc. Biol. Rio de Janeiro 10: 63-64.

1967. Relatorio da excursāo do Instituto Oswaldo Cruz ao Estado do Espirito Santo em Novembro de 1964. Boln. Mus. Biol. Zool. 31, 54 p.

TRAVASSOS, L., J. F. T. FREITAS, and A. KOHN.

1969. Trematodeos do Brazil. Mem. Inst. Oswaldo Cruz 67:1-886.

TRIPATHI, Y. R.

1953. Studies on parasites of Indian fishes. I. Protozoa Myxosporidia together with a check list. Rec. Indian Mus. (Calcutta) 50:63-89.

TROFIMENKO, $\mathrm{V}, \mathrm{Y}$

1962. The helminth fauna of freshwater and related fish in Kamchatka. [In Russ.] Tr. Gel'mintol. Lab. 12:232-262.

TSIMBALYUK, A. K., and V. A. ROYTMAN.

1966. Bunodera mediovitellata (Bunoderidae) from sticklebacks on the Komander Islands. [In Russ.] Tr. Gel'mintol. Lab. 17:290-296.

TSIMBALYUK, E. M., and N. N. SEMESHKO.

1971. Cestodes from fish of the intertidal zone of western Kamchatka. [1n Russ.] Parazitol. 5:424-428.

TURNER, C. H., E. E. EBERT, and R. R. GIVEN.

1969. Man-made reef ecology. Calif. Fish Game, Fish Bull. 146:1-221.

UNNITHAN, R. V.

1957. On the functional morphology of a new fauna of Monogenea on fishes from Trivandrum and environs. Part 1. Axinidae fam. nov. Bull. Central Res. Inst. Univ. Kerala 5:27-122.

1962. On the functional morphology of a new fauna of Monogenoidea on fishes from Trivandrum and environs. Part Il. Opisthogynidae fam. nov. (Gastrocotyloidea) and Abortipedinae subfam. nov. (Protomicrocotyloidéa). Párásituóóogy 52:315-351.

1965. On a new monogenetic trematode Homostoma chura gen. et. sp. nov, from the marine fish Euthynnus affinis (Cantor) with a note on the family Hexostomatidae, Price, 1936. Treubia 26:329-336.

1968. On six species of monogenetic trematodes parasitic on the gills of marine fishes from the Indian Seas. Treubia 27:141-164.
UZMANN, J. R., and J. DOUGLAS.

1966. Clinostomum marginatum in steelhead trout (Salmo gairdneri) and cutthroat trout (Salmo clarki) in a western Washington lake. Trans. Am. Fish. Soc. 95:35-38

UZMANN, J. R., and S. H. HAYDUK.

1964. Larval Echinochasmus (Trematoda: Echinostomatidae) in rainbow trout, Salmo gairdneri. J. Parasitol. 50:586.

UZMANN, J, R., G. J. PAULIK, and S. H. HAYDUK,

1965. Experimental hexamitiasis in juvenile coho salmon (Oncorhynchus kisutch) and steelhead trout (Salmo gairdneri). Trans. Am. Fish. Soc. 94:53-61.

UZMANN, J. R., and H. J. RAYNER.

1958. Record of the parasitic copepod Lernaea cyprincacea L. in Oregon and Washington fishes. J. Parasitol. 44:452-453.

VAISSIERE, $\mathbf{R}$.

1959. Parasites de poissons de mer oeust-africains récoltés par J. Cadenet II. Copépodes. Bull. Inst. Fr. Afr. Noire, Sér. A, 21:534-553.

VALENTINE, D. W., and R. PHELPS.

1977. Parasitism of barred surfperch, Amphistichus argenteus, by Nerocila californica: a fit to the Poisson. Calif. Fish Game 63:129-132.

VAN CLEAVE, H.J.

1920. Acanthocephala of the Canadian Arctic Expedition, 1913-1918. Rep. Can. Arctic Exped. 1913-18, 9, Part E:1-11.

1931. Acanthocephala from Japan. II. Two new species of the genus Acanthocephalus. Annot. Zool. Jpn. 13:33-37.

1945. A new species of the acanthocephalan genus Illiosentis (Rhadinorhynchidae). J. Parasitol. 31:57-60.

1953. Acanthocephala of North American mammals. Ill. Biol. Monogr. 23:1-179.

VERVOORT, W.

1964. Notes on Bomolochidae (Copepoda). I. A redescription of Parabomolochus cuneatus (Fraser, 1920) and notes on its synonymy. Crustaceana 6:291-302.

1965. Three new species of Bomolochidae (Copepoda, Cyclopoida) from tropical Atlantic tunnies. Zoöl. Meded. Leiden 76:1-40.

VERVOORT, W., and H. P. ARAI.

1966. The rediscovery of Parabomolochus cuneatus (Fraser) from Cymatogaster aggregata Gibbons. Crustaceana 10:222-223.

VIGUERAS, I.

1935. Tristomum poeyi n. sp. (Trematoda), parasito de Makaira ampla Poey (Pisces). Soc. Cub. Hist. Nat. Havana Mem. 9:43-44.

VIK, R.

1954. Investigations on the pseudophyllidean cestodes of fish, birds, and

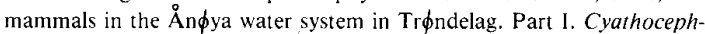
alus truncatus and Schistocephalus solidus. Nytt Mag. Zool. 2:5-51.

1963. Studies of the helminth fauna of Norway. IV. Occurrence and distribution of Eubothrium crassum (Bloch, 1779) and E. salvelini (Schrank, 1790) (Cestoda) in Norway, with notes on their life cycles. Nyt Mag. Zool. 11:47-73.

1965. Studies of the helminth fauna of Norway. V. Plerocercoids of $\mathrm{Di}$ phyllobothrium spp. from the Rössåga water system, Nordland County. Nytt Mag. Zool. 12:1-9.

VIK, R., O. HALVORSEN, and K. ANDERSEN.

1969. Observations on Diphyllobothrium plerocercoids in three-spined sticklebacks, Gasterosteus aculeatus L., from the River Elbe. Nytt Mag. Zool. 17:75-80.

VILLADOLID, D. V.

1927. The occurrence of Naobranchia occidentalis on the Pacific coast of the United States. J. Wash. Acad. Sci. 17:230-231.

VINNICHENKO, L. N., V. E. ZAIKA, V. A. TIMOFEEV, G. A. SHTEIN. and S. S. SHULMAN.

1971. Parasitic protozoa from the fishes of the Amur River basin. [In Russ.] Parazitol. Sb. 25:10-40.

WAGNER, E. D.

1975. A left-handed Grubea sp. from the Pacific Coast, Baja California, Mexico. Proc. Helminthol. Soc. Wash. 42:97-100.

WAGNER, E. D., and C. E. CARTER.

1967. Caballerocotyla gregalis sp. n. (Trematoda: Monogenea) from the gills of Sarda lineolata (Girard). J, Parasitol. 53:277-279

WALDER, G. L., and H. P. ARAI.

1974. The helminth parasites of embiotocid fishes. 1II. A new species of the genus Cucullanellus Törnquist, 1931 (Nematoda: Cucullanidac) from the shiner perch, Cymatogaster aggregata Gibbons. J. Fish. Res. Board Can. 31:205-209. 
WALES, J. $\mathrm{H}$.

1958. Two new blood flukes parasites of trout. Calif. Fish Game 44: 125-136.

WALES, J. H., and H. WOLF.

1955. Three protozoan diseases of trout in California. Calif. Fish Game 41:183-187.

WARD, H. I.

1953. A new genus and species, Floridonsentis elongatus of Neoechinorhynchidae (Acanthocephala). J. Parasitol. 39:392-394.

1954. Parasites of marine fishes of the Miami Region. Bull. Mar. Sci. Gulf Caribb. 4:244-261.

WARD, H. L., and H. A. WINTER.

1952. Juvenile acanthocephala from the yellowfin croaker, Umbrina roncador, with description of a new species of the genus Arhythmorhynchus. Trans. Am. Microsc. Soc. 71:154-156.

WARD, J. W.

1962. Helminth parasites of some marine animals, with special reference to those from the yellow-fin tuna, Thunnus albacares (Bonnaterre). J. Parasitol. 48:155.

WARDLE, R. A.

1932. The Cestoda of Canadian fishes. I. The Pacific Coast region. Contrib. Can. Biol. New Ser. 7:221-243.

1933a. The parasitic helminths of Canadian animals. I. The Cestodaria and Cestoda. Can. J. Res. 8:317-333.

1933b. The Cestoda of Canadian fish. III. Addition to Pacific coastal fauna. Contrib. Can. Biol. New Ser, 8:77-87.

WARDLE, R. A., and J. A. McLEOD.

1952. The zoology of tapeworms. Univ. Minnesota Press, Minneapolis, 780 p.

WARES, P. G.

1971. Biology of the pile perch, Rhacochilus vacca, in Yaquina Bay, Oregon. U.S. Fish WildI. Serv., Bur. Sport Fish., Tech. Pap. 57, 29 p.

WATERTOR, J. L.

1973. Incidence of Hirudinella marina Garcin, 1730 (Trematoda: Hirudinellidae) in tunas from the Atlantic Ocean. J. Parasitol. 59:207-208.

WELLBORN, T. L., Jr.

1967. Trichodina (Ciliata: Urceolariidae) of freshwater fishes of the southeastern United States. J. Protozool. 14:399-412.

WELLINGS, S. R., L. E. ASHLEY, and G. E. McARN.

1969. Microsporidial infection of English sole, Parophrys vetulus. J. Fish. Res. Board Can. 26:2215-2218.

WIERZBICKI, $\mathrm{K}$.

1962. Presence du nematode Sterliadochona tenuissima (Zeder 1800) dans le nord de la Pologne. Ann. Parasitol. Hum. Comp. 37:314-324.

WILKIE, D. W., and H. GORDIN.

1969. Outbreak of cryptocaryoniasis in marine aquaria at Scripps Institute of Oceanography. Calif. Fish Game 55:227-236.

WILLIAMS, H. H.

1958. Some phyllobothriidae (Cestoda: Tetraphyllidea) of elasmobranchs from the western seaboard of the British Isles. Ann. Mag. Nat. Hist. 1, Ser. 13:113-136.

1959. The anatomy of Phyllobothrium sinuosiceps sp. nov. (Cestoda; Tetraphyllidea) from Hexanchus griseus (Gmelin) the six gilled shark. Parasitology 49:54-69.

1966. The ecology, functional morphology and taxonomy of Echeneibothrium Beneden, 1849 (Cestoda: Tetraphyllidea), a revision of the genus and comments on Discobothrium Beneden, 1870, Pseudanthobothrium Baer, 1956, and Phoreobothrium Alexander, 1963. Parasitology $56: 227-285$.

1968. The taxonomy, ecology and host-specificity of some Phyllobothriidae (Cestoda: Tetraphyllidea), a critical revision of Phyllobothrium Beneden, 1849 and comments on some allied genera. Trans. R. Philos. Soc. Lond. Ser. B 253:231-307.

1969. The genus Acanthobothrium, Beneden 1849. (Cestoda: Tetraphyllidea). Nytt Mag. Zool. 17:1-56.

WILSON, C. B.

1905a. New species of parasitic copepods from the Massachusetts coast. Proc. Biol. Soc. Wash. 18:127-132.

1905b. North American parasitic copepods belonging to the family Caligidae. Part 1: The Caliginae. Proc. U.S. Natl. Mus. 28:479-672.

1907a. North American parasitic copepods belonging to the family Caligidae, 2: The Trebinae and Euryphorinae. Proc. U.S. Natl. Mus. 31: 669-720.

1907b. North American parasitic copepods belonging to the family Caligidae. Parts 3 and $4-A$ revision of the Pandarinae and the Cecropinae. Proc. U.S. Natl. Mus. 33:323-490. 1908a. North American parasitic copepods: A list of those found upon the fishes of the Pacific coast, with descriptiors of new genera and species. Proc. U.S. Natl. Mus. 35:431-481.

1908b. North American parasitic copepods: New genera and species of Caliginae. Proc. U.S. Natl. Mus. 33:593-627.

1911. North American parasitic copepods. Description of new genera and species. Proc. U.S. Natl. Mus. 39:625-634.

1912a. Description of new species of parasitic copepods in the collections of the United States National Museum. Proc. U.S. Natl. Mus. 42: 233-243.

1912b. Parasitic copepods from Nanaimo, British Columbia, including eight species new to science. Contrib. Can. Biol. 1906-1910:85-101.

1913. Crustacean parasites of West Indian fishes and land crabs with descriptions of new genera and species. Proc. U.S. Natl. Mus. 44:189-277.

1915. North American parasitic copepods belonging to the Lernaeopodidae, with a revision of the entire family. Proc. U.S. Natl. Mus. 47: 565-730.

1917. North American parasitic copepods belonging to the family Lernaeidae with a revision of the entire family. Proc. U.S. Natl. Mus. 53:1-150.

1919. A new species of parasitic copepod, with notes on species already described. Proc. U.S. Natl. Mus. 55:313-316.

1920. Report on the parasitic Copepoda collected during the Canadian Arctic Expedition, 19!3-18. Rep. Can. Arctic Exped. 1913-18, 7, part L:1-16.

1921. New species and a new genus of parasitic copepods. Proc. U.S. Nat1. Mus. 59:1-17.

1922. North American parasitic copepods belonging to the family Dichelesthiidae. Proc. U.S. Natl. Mus. 60(5), 100 p.

1923. Parasitic copepods in the collection of the Riksmuseum at Stockholm. Ark. Zool. 15, 15 p.

1924. New North American parasitic copepods, new hosts, and notes on copepod nomenclature. Proc. U.S. Natl. Mus. 64(17), 22 p.

1932. The copepods of the Woods Hole Region, Massachusetts. U.S Natl. Mus. Bull. 158, 635 p.

1935a. New parasitic copepods (Reports on the collections obtained by the first Johnson-Smithsonian deep-sea expedition to the Puerto Rican deep). Smithson. Misc. Collect. 91(3298), 9 p.

1935b. Parasitic copepods from the Pacific coast. Am. Midl. Nat, 16: 776-797.

1936. Parasitic copepods from the Dry Tortugas. Pap. Tortugas Lab. Carnegie Inst. Wash. 29:327-347.

1937a. Parasitic copepods taken during the third Hancock expedition to the Galapagos Islands. Allan Hancock Found. Pac. Exped. 2:23-30.

1937b. Some parasitic copepods from Panama Bay. J. Wash. Acad. Sci. 27:423-431.

1944. Parasitic copepods in the United States National Museum. Proc. U.S. Natl. Mus. 94:529-582.

WILSON, M. S.

1952. An emended diagnosis of the copepod genus Pupulina (Caligoida), with descriptions of new species and a redescription of the genotype. Proc. U.S. Nati. Mus. 102:245-263.

WINTER, H, A.

1955. "Capsala caballerol" sp. n., parasito de "Sarda orientalis," con un catalogo de los trematos monogeneos de los peces del Oceano Pacifico de las Americas. Rev. Bras. Biol. 15:9-32.

WOOD, J. W., and J. W. WALLIS.

1955. Kidney disease in adult chinook salmon and its transmission by feeding to young chinook salmon. Fish Comm. Oreg. Res. Briefs 6:32-40.

WOOD, R. A., and J. D. MIZELLE.

1957. Studies on monogenetic trematodes. XXI. North American Gyrodactylinae, Dactylogyrinae and a new host record for Urocleidus dispar (Mueller, 1936). Am. Midl. Nat. 57:183-202.

WOOTTEN, R.

1973a. Occurrence of the metacercariae of Cotylurus erraticus (Rudolph: 1809) Szidat, 1928 (Digenea: Strigeidae) in brown trout Salmo trutta L., and rainbow trout $S$. gairdneri Richardson, 1836, from Hanningfield Reservoir, Essex. J. Helminthol. 47:389-398.

1973b. The metazoan parasite fauna of fish from Hanningfield Reservoir, Essex in relation to features of the habitat and host populations. J. Zool. (Lond.) 171:323-331.

WOOTTON, D. M.

1957. The life history of Cryptocotyle concavum (Creplin, 1825) Fischoeder, 1903. (Trematoda: Heterophyidae). J. Parasitol. 43:271-279.

WYATT, E. J.

1978. A new host and site of infection for Myxobolus kisutchi and host record for Myxobolus insidiosus. J. Parasitol. 64:169-170. 
WYATT, E. J., and I. PRATT.

1963. Myxobolus insidiosus sp. n., a myxosporidian from the musculature of Oncorhynchus tshawytscha (Walbaum). J. Parasitol. 49:951-955.

YAMAGUTI, $S$.

1934a. Studies on the helminth fauna of Japan. Part 2. Trematodes of fishes, I. Jpn. J. Zool. 5:249-541.

1934b. Studies on the heiminth fauna of Japan. Part 4. Cestodes of fishes. Jpn. J. Zool. 6:1-112.

1936. Parasitic copepods from fishes of Japan. Part 3. Caligoida, II. Published by author, $21 \mathrm{p}$.

1938. Studies on the helminth fauna of Japan. Part 24. Trematodes of fishes. V. Jpn. J. Zool. 8:15-74.

1939a. Parasitic copepods from fishes of Japan. Part 4. Cyclopoida, II. Vol. Jub. Yoshida 2:39l-441.

1939b. Parasitic copepods from fishes of Japan. Part 5. Caligoida, III. Vol. Jub. Yoshida 2:443-527.

1939c. Parasitic copepods from fishes of Japan. Part 6. Lernaeopodoida, I. Vol. Jub. Yoshida 2:529-627.

1939d. Studies on the helminth fauna of Japan. Part 26. Trematodes of fishes, VI. Jpn. J. Zool, 8:211-230.

1939e. Studies on the helminth fauna of Japan. Part 29. Acanthocephala, I1. Jpn. J. Zool. 8:317-351.

1940. Studies on the helminth fauna of Japan. Part 31. Trematodes of fishes, VII. Jpn. J. Zool. 9:35-108.

1941. Studies on the helminth fauna of Japan. Part 33. Nematodes of fishes, II. Jpn. J. Zool. 9:343-396.

1951. Studies on the helminth fauna of Japan. Part 48. Trematodes of fishes. X. Arb. Med. Fak. Okayama 7:315-334.

1952. Studies on the helminth fauna of Japan. Part 49. Cestodes of fishes. II. Acta Med. Okayama 8:1-76.

1953a. Parasitic worms mainly from Celebes. Part 2. Monogenetic trematodes of fishes. Acta Med. Okayama 8:203-256.

1953b. Parasitic worms mainly from Celebes. Part 3. Digenetic trematodes of fishes, II. Acta Med. Okayama 8:257-295.

1954. Parasitic copepods from físhes of Celebes and Borneo. Publ. Seto Mar. Biol. Lab. 3:375-398.

1959a. Studies on the helminth fauna of Japan. Part 54. Trematodes of fishes, Xīîi. Publ. Seto Mar. Biol. Lab. 7:241-262.

1959b. Systema Helminthum. Vol. II. The cestodes of vertebrates. Interscience, N.Y., 860 p.

1961. Systema Helminthum. Vol. III. The nematodes of vertebrates. Part 1. Interscience, N.Y., $679 \mathrm{p}$.

1963a. Parasitic Copepoda and Branchiura of fishes. Interscience, N.Y., 1104 p.

1963b. Systema Helminthum. Volume IV. Monogenea and Aspidocotylea. Interscience, N.Y., 699 p.

1963c. Systema Helminthum. Volume V. Acanthocephala. Interscience, N.Y., 423 p.

1965a. New digenetic trematodes from Hawaiian fishes, 1. Pac. Sci. 19: 458-481.

1965b. New monogenetic trematodes from Hawaiian fishes, I. Pac. Sci. 19:55-95.

1966. New monogenetic trematodes from Hawaiian fishes, II. Pac. Sci. 20:419-434.

1968a. Cestode parasites of Hawaiian fishes. Pac. Sci. 22:21-36

1968b. Monogenetic trematodes of Hawaiian fishes. Contrib. Hawaii Inst. Mar. Biol. 262, $287 \mathrm{p}$.

1970. Digenetic trematodes of Hawaiian fishes. Keigaku, Tokyo, 436 p.

1971. Synopsis of digenetic trematodes of vertebrates. Vol. I. Keigaku Publ. Co. Tokyo, 1074 p.
YAMAGUTI, S. M., and MATUMURA.

1942. Two new species of fish trematodes from Hokkaido. Trans. Sapporo Nat. Hist. Soc. 17:117-122.

YASUTAKE, W. T., and E. M. WOOD.

1957. Some Myxosporidia found in Pacific Northwest salmonids. J. Parasitol. 43:633-637.

YOSHIHARA, K., and T. NAGASAKI.

1973. Studies on the parasites from fishes caught by tuna longline fishing. Bull. Coll. Agric. Vet. Med, Nihon Univ. (30):252-267. (In Helminthol. Abstr. Ser. A, 45:113.)

YOSHINO, T. P.

1972. Helminth parasitism in the Pacific killifish, Fundulus parvipinnis, from southern California. J. Parasitol. 58:635-636.

YOSHINO, T. P., and M. MOSER.

1974. Myxosporida (Protozoa) in macrourid fishes (Coryphaenoides spp.) of the northeastern Pacific. J. Parasitol, 60:655-659.

YOSHINO, T. P., and E. R. NOBLE.

1973. Myxosporida of macrourid fishes from southern California and Mexico. J. Parasitol. 59:844-850.

YOUNG, P. C.

1967. A taxonomic revision of the subfamilies Monocotylinae Gamble, 1896 and Dendromonocotylinae Hargis, 1955 (Monogenoidea: Monocotylidae). J. Zool. (Lond.) 153:38 i-422.

1970. The species of Monogenoidea recorded from Australian fishes and notes on their zoogeography. An. Inst. Biol. Univ. Mex. Ser. Zool. 41: 163-176.

YOUNG, R. T

1953. Postmonorchis donacis, a new species of monorchid trematode from the Pacific coast, and its life history. J. Wash. Acad. Sci, 43:88-93.

1954. Cestodes of sharks and rays in southern California. Proc. Helminthol. Soc. Wash. 21:106-112.

ZAIKA, V. E.

1968. Parasite protozoa of the Black Sea fishes. In V. A. Bodyanitski (editor), Biology of the seas, p. 41-46. (Biologiia Moriia) [In Russ.] "Naukova Dumka" Kiev 14.

ZERECERO Y DIAZ, M. C.

1960. Pyragraphorus caballeroi $\mathrm{n}$. $\mathrm{sp}$. (Trematoda de la subclase Monogenea Carus, 1863) en peces marinos del Oceano Pacifico del Norte. Libro Homenaje E. Caballero y Caballero, p. 345-351.

ZHUKOV, E. V

1960a. Endoparasitic worms of fishes in Sea of Japan and shallow water of South Kurile Islands. [In Russ.] Tr. Zool. Inst. Akad. Nauk SSSR 28:3-146.

1960b. On the fauna of parasites of fishes of the Chukotsk Peninsula and the adjoining seas. I. Monogenetic trematodes of marine and freshwater fishes. [In Russ.] Parazitol. Sb. 19:308-332.

1963. On the fauna of fishes of the Chukotsk Peninsula and the adjoining seas. II. Endoparasitic worms of marine and freshwater fishes. [In Russ.] Parazitol. Sb. 21:96-139.

1964. On the fauna of fishes of the Chukotsk Peninsula and the adjoining seas. III. The parasitic Protozoa of marine and freshwater fishes. [In Russ.] Parazitol. Sb. 22:224-262.

ZITNAN, R

1968. Nematoda, Acanthocephala und Hirudinea bei fishchen im Flusse Hron (CSSR). Stud. Helminthol. Year 1968, 2:21-32.

ZITNAN, R., and M. CANKOVIC.

1970. Comparison of the epizootological importance of the parasites of Salmo gairdneri irideus in the two coast areas of Bosna and Herzegovina. Helminthologia 11:161-166.

ZSCHOKKE, F., and A. HEITZ.

1914. Entoparasiten aus salmoniden von Kamtschatka. [In Ger.] Rev, Suisse Zool. 22:195-256. 


\section{FOOTNOTES}

${ }^{3}$ L. Margolis, Department of Fisheries and Oceans, Pacific Biological Station, P.O. Box 100, Nanaimo, British Columbia, pers. commun. August 1977.

${ }^{4}$ P. W. Pappas, Department of Zoology, Ohio State University, 1735 Nẹal Avenue, Columbus, Ohio, pers. commun. January 1977.

'Berê, R. 1930. Parasitic copepods from the Vancouver Island region. Fish. Res. Board Can. MS Rep. (Biol.) 259, 3 p.

'Kudo, R., and P. A. Meglitsch. In pre. Studies on Myxosporida. A synopsis of genera and species of Myxosporida.

'E. Burreson, Normandean Associates, Inc., Nashua Road, Bedford, N.H., pers. commun. July 1977.

'A. C. Olson, Jr., Department of Zoology, San Diego State University, San Diego, Calif., pers. commun. February 1977.

'S. B. Collard, Jr., Faculty of Biology, University of West Florida, Pensacola, Fla., pers. commun. April 1976.

${ }^{10} \mathrm{~F}$. M. Nahhas, Department of Biology, University of the Pacific, Stockton, Calif., pers. commun. May 1976.

"M. B. Mizrahi, Department of Zoology, San Diego State University, San Diego, Calif., pers. commun. March 1977.

${ }^{12}$ L. E. Aldrich, Jr., Department of Biology, Seattle University, Seattle, Wash., pers. commun. January 1976.

${ }^{13}$ Hoskins, G. E., G. R. Bell, and T. P. T. Evelyn. 1976. The occurrence, distribution and significance of infectious diseases and neoplasms observed in fish in the Păcific Regioni up to the end of 1974. Environ. Can. Fish. Mar. Serv., Res. Dev. Tech. Rep. 609, $37 \mathrm{p}$.

${ }^{14}$ Godfrey, H. 1968. Some observations on juvenile marine chum, chinook and coho salmon taken in waters adjacent to Georgia Strait in 1965. Fish. Res. Board Can., MS Rep. Ser. 955,19 p.

15Muldry, D. R., and R. S. Anderson. 1976. A bibliography of the literature relating to the parasites of freshwater fishes in Alberta, 1932-1975. Can. Wildl. Serv. Manuscr., $10 \mathrm{p}$.

${ }^{16}$ Robinson, D. G., W. E. Barraclough, and J. D. Fulton. 1968. Data record. Number, size composition, weight and food of larval and juvenile fish caught with a twoboat surface trawl in the Strait of Georgia June 5-9, 1967. Fish. Res. Board Can. MS Rep. Ser. 972, 109 p.

${ }^{17}$ M. E. Anderson, Virginia Institute of Marine Science, Rochester Point, Va., pers. commun. August 1976.

${ }^{18}$ L. A. Jensen, Department of Biological Sciences, University of Northern Colorado, Greeley, Colo., pers. commun. March 1977.

${ }^{19}$ M. Moser, Long Marine Laboratory, University of California, Santa Cruz, Calif., unpubl. data.

${ }^{20}$ A. Kuris, Department of Biological Sciences, Universisity ớ Câliforninia, Sântâ Bărbara, Calif., pers. commun. April 1976.

${ }^{21}$ R. Kliever, Moss Landing Marine Laboratory, P.O. Box 223, Moss Landing, Calif., pers. commun. April 1977.

${ }^{22}$ R. Baker, Alaska Department of Fish and Game, 333 Raspberry Road, Anchorage, Alaska, pers. commun. March 1976.

${ }^{23}$ E. Chapa, Department of Biology, University of the Pacific, Stockton, Calif., pers. commun. March 1976.

${ }^{24}$ Yamaguti, S. 1938. Studies on the helminth fauna of Japan. Part 21. Trematodes of fishes, IV. Published by author, $139 \mathrm{p}$.

${ }^{2 s}$ D. Schultze, California Department of Fish and Game, Long Beach, Calif,, pers. commun. June 1977.

${ }^{26}$ A. W. Hanson, Department of Biology, Whittier College, Whittier, Calif., pers. commun. June 1977.

${ }^{27}$ D. A. Hanan, California Department of Fish and Game, 350 Golden Shore Dr., Long Beach, Calif., pers. commun. April 1977.

${ }^{28}$ G. Robinson, Department of Biological Sciences, University of California, Santa Barbara, Calif., pers. commun. April 1977.

${ }^{29}$ P. Edmiston, Department of Biology, University of the Pacific, Stockton, Calif., pers. commun. April 1978.

${ }^{30}$ Robinson, D. G., W. E. Barraclough, and J. D. Fulton. 1968. Data record. Number, size composition, weight and food of larval and juvenile fish caught with a twoboat surface trawl in the Strait of Georgia, May 1-4, 1967. Fish. Res. Board Can., MS Rep. Ser. 964, 105 p.

${ }^{3}$ E. Noble, Department of Biological Sciences, University of California, Santa Barbara, Calif., pers. commun. April 1976.

${ }^{32}$ R. L. Haaker, California Department of Fish and Game, 350 Golden Shore Dr., Long Beach, Calif., pers. commun. April 1977.

${ }^{33}$ Yamaguti, S. 1937. Studies on the helminth fauna of Japan. Part 19. Fourteen new ectoparasitic trematodes of fishes. Published by the author, $28 \mathrm{p}$.

${ }^{34}$ Millemann, R. 1970. Laboratory manual for fish 490 - parasites and diseases of fish. Dep. Fish. Wildl., Oreg. State Univ., 161 p.

${ }^{35}$ J. C. Holmes, Department of Zoology, University of Alberta, Edmonton, Canada, pers. commun. September 1977. 


\section{HOST INDEX}

Acanthogobius flavimanus . . ..............253

Acipenser sp. ........................ 57

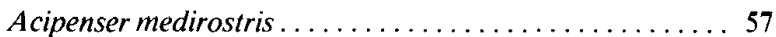

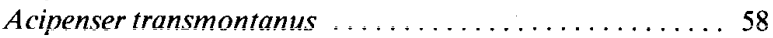

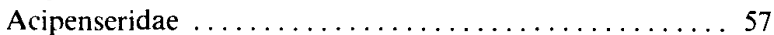

Agonidae ........................... 393

Agonopsis emmelane . .................... 393

Agonus acipenserinus . . . . . . . . . . . . . . . . 393

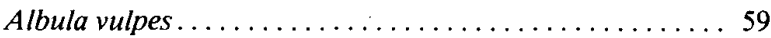

Albulidae . . . . . . . . . . . . . . . . . . . . . 59

Alepisauridae. . . . . . . . . . . . . . . . . . 127

Alepisaurus ferox . . . . . . . . . . . . . . . . 127

Alepocephalidae . . . . . . . . . . . . . . . 122

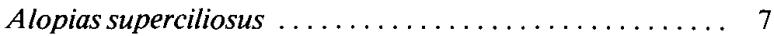

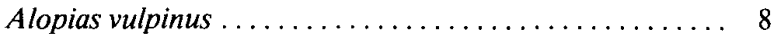

Alopiidae ....................... 7

Alosa sapidissima . . . . . . . . . . . . . . . . . . 62

Ammodytes hexapterus . ................ 252

Ammodytidae ........................ 252

Amphistichus argenteus ................. 215

Amphistichus koelzi ..................216

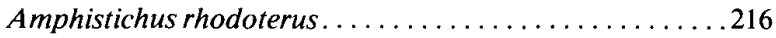

Anarhichadidae......................251

Anarrhichthys ocellatus . . . . . . . . . . . . 251

Anisotremus davidsoni . ................. 206

Anoplarchus purpurescens ................247

Anoplopoma fimbria . ................... 361

Anoplopomatidae ...................... 361

Antimora microlepis..................... 148

Apodichthys flavidus . . . . . . . . . . . . . . . 249

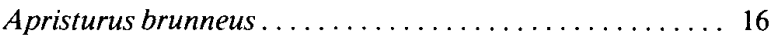

Aprodon cortezianus . ................... 145

Argyropelecus pacificus ................... 124

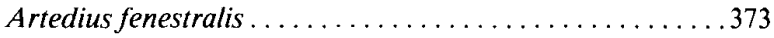

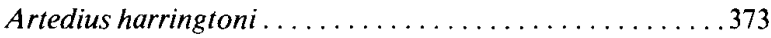

Artedius lateralis . . . . . . . . . . . . . . . . . 374

Artedius notospilotus . . . . . . . . . . . . . . . . . . 374

Ascelichthys rhodorus ..................... 374

Asterotheca infraspinata . . . . . . . . . . . . . . . . 394

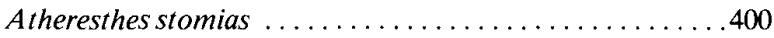

Atherinidae . ....................... 160

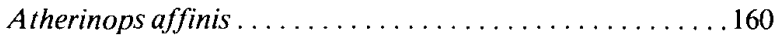

Atherinopsis californiensis. ............... 162

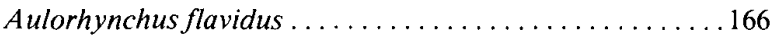

Auxis rochei ........................260

Auxis thazard ...................... 260

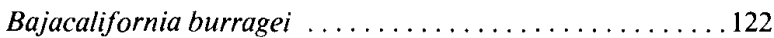

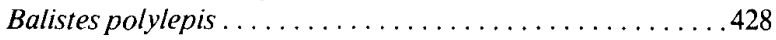

Balistidae . . . . . . . . . . . . . . . . . . 428

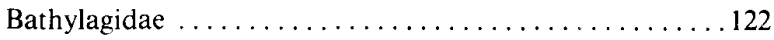

Bathylagus ochotensis . . . . . . . . . . . . . 122

Bathylagus wesethi . . . . . . . . . . . . . . . . 123

Bathymasteridae .................... 243

Batrachoididae ....................... 132

Blenniidae ........................ 246

Blepsias cirrhosus . . . . . . . . . . . . . . . . . . . 375

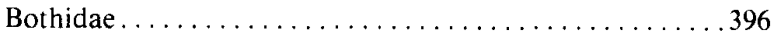

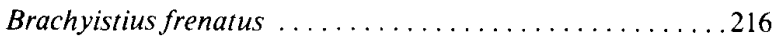

Brama japonica ................... 206
Bramidae ........................206

Branchiostegidae. . . . . . . . . . . . . . . . . . 189

Calamus brachysomus . . . . . . . . . . . . . 207

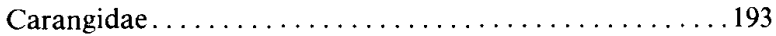

Caranx caballus ......................... 193

Caranx hippos . . . . . . . . . . . . . . . . . . 194

Carcharhinidae ................... 16

Carcharhinus leucas .................. 16

Carcharhinus longimanus ................ 18

Carcharhinus obscurus ........................ 19

Carcharodon carcharias .................. 10

Caulolatilus princeps . . . . . . . . . . . . . . 189

Cebidichthys sp. ..................... 247

Cebidichthys violaceus . . . . . . . . . . . . . . 247

Cephaloscyllium ventriosum ............... 16

Ceretoscopelus townsendi . . . . . . . . . . . . 127

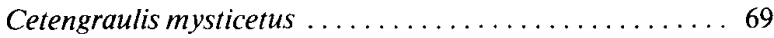

Cetorhinus maximus . . . . . . . . . . . . . . . 11

Cheilotrema saturnum . . . . . . . . . . . . . . . . . 207

Chilomycterus affinis . . . . . . . . . . . . . 430

Chimaeridae ..................... 56

Chitonotus pugetensis . . . . . . . . . . . . . 376

Chloroscombrus orqueta . . . . . . . . . . . . . . . . 197

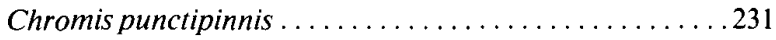

Citharichthys sordidus . . . . . . . . . . . . . . 396

Citharichthys stigmaeus . . . . . . . . . . . . . . . . 397

Citharichthys xanthostigma ................ 398

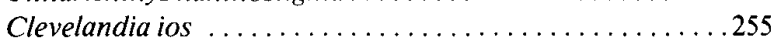

Clinidae $\ldots \ldots \ldots \ldots \ldots \ldots \ldots \ldots \ldots \ldots \ldots \ldots \ldots \ldots \ldots$

Clinocottus acuticeps . . . . . . . . . . . . . . . . 377

Clinocottus analis ....................... 377

Clinocottus embryum . . . . . . . . . . . . . . . . . . . 377

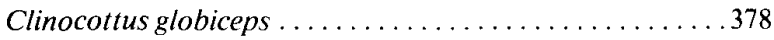

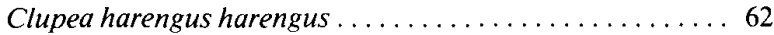

Clupea harengus pallasii . ................. 62

Clupeidae .........................6 62

Coelorinchus scaphopsis . . . . . . . . . . . . . . 149

Cololabis saira . . . . . . . . . . . . . . . . . . . 157

Coryphaena hippurus . . . . . . . . . . . . . 202

Coryphaenidae .......................202

Coryphaenoides sp. ......................149

Coryphaenoides acrolepis . . . . . . . . . . . . 149

Coryphaenoides armatus . . . . . . . . . . . . . . 151

Coryphaenoides filifer . ................... 153

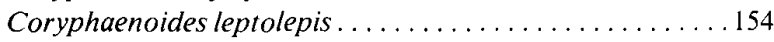

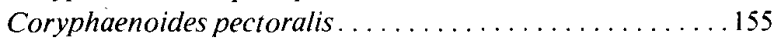

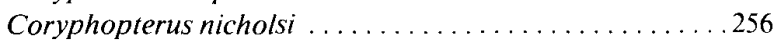

Cottidae .......................... 373

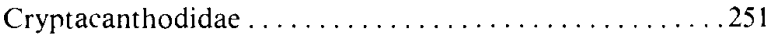

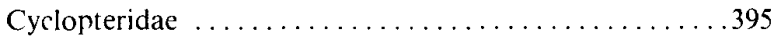

Cyclothone acclinidens . . . . . . . . . . . . . . 123

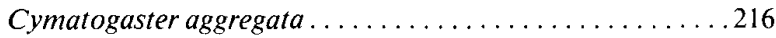

Cynoscion nobilis .......................207

Cynoscion parvipinnis . . . . . . . . . . . . . 209

Cyprinodontidae $=\ldots \ldots \ldots \ldots \ldots \ldots \ldots \ldots . \ldots \ldots$

Damalichthys vacca .................... 220

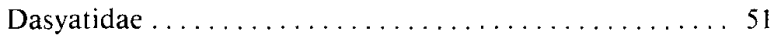

Dasyatis violacea . ................... 51 


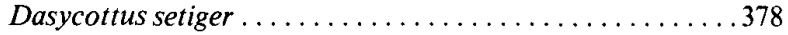

Delolepis gigantea ..................... 251

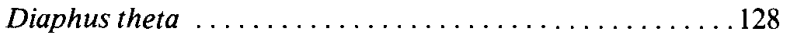

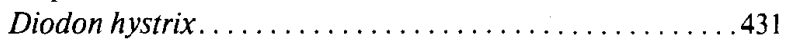

Diodontidae ........................4430

Dorosoma petenense ................... 67

Echeneidae............................. 189

Echeneis naucrates . . . . . . . . . . . . . . . . . . . 189

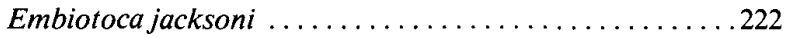

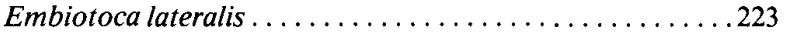

Embiotocidae ......................215

Engraulidae .......................... 69

Engraulis mordax . . . . . . . . . . . . . . . . . . . . 69

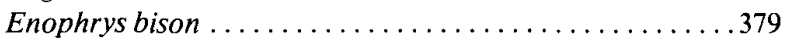

Eopsetta jordani . . . . . . . . . . . . . . . . . . . 402

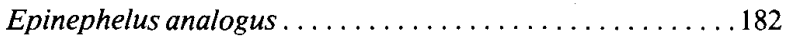

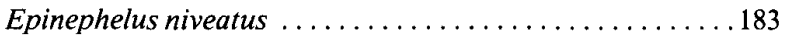

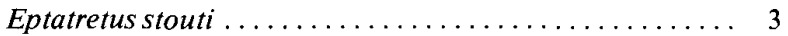

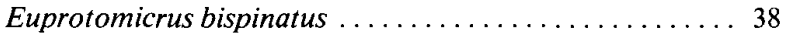

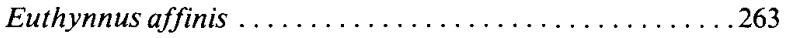

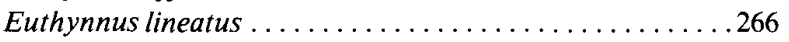

Euthynnus pelamis ................... 266

Fundulus parvipinnis ................. 159

Gadidae . . . . . . . . . . . . . . . . . . . . . . . 134

Gadus macrocephalus . . . . . . . . . . . . . . . . . 134

Galeocerdo cuvieri ................... 22

Galeorhinus zyopterus ................ 24

Gasterosteidae ........................ 166

Gasterosteus aculeatus . . . . . . . . . . . . . . . 166

Gempylidae .................................259

Gempylus serpens . . . . . . . . . . . . . . . . . . . . . . 259

Genyonemus lineatus . . . . . . . . . . . . . . . . . . . . 209

Gibbonsia sp........................243

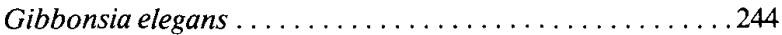

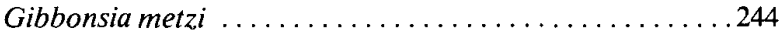

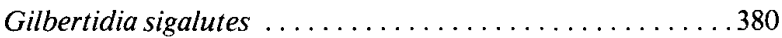

Gillichthys mirabilis ...................256

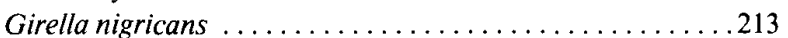

Glyptocephalus zachirus ...............4403

Gobiesocidae ......................133

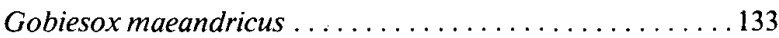

Gobiesox rhessodon ....................134

Gobiidae............................253

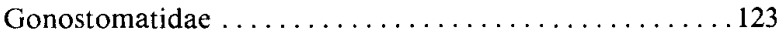

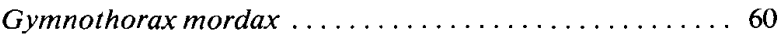

Gymnura marmorata .................... 52

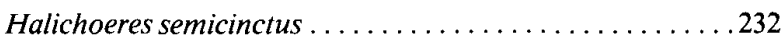

Hemanthias peruanus . . . . . . . . . . . . . . . . 184

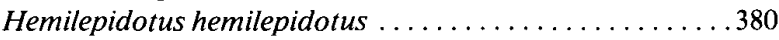

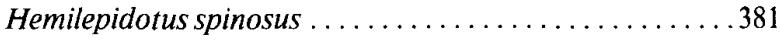

Heterodontidae ...................... 6

Heterodontus francisci $\ldots \ldots \ldots \ldots \ldots \ldots \ldots \ldots \ldots$

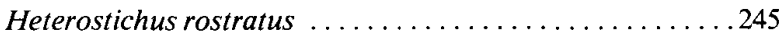

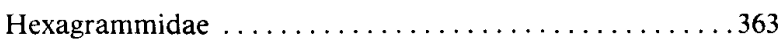

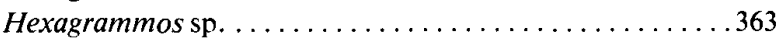

Hexagrammos decagrammus. . . . . . . . . . . 363

Hexagrammos lagocephalus . . . . . . . . . . . . . 364

Hexagrammos stelleri . . . . . . . . . . . . . 367
Hexagrammos superciliosus . . . . . . . . . . . . 364

Hexanchidae ...................... 4

Hexanchus griseus. . . . . . . . . . . . . . . . 4

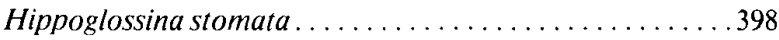

Hippoglossoides elassodon . . . . . . . . . . . . . 404

Hippoglossus stenolepis . . . . . . . . . . . . . . . 407

Hydrolagus colliei ...................... 56

Hyperprosopon anale . . . . . . . . . . . . . 224

Hyperprosopon argenteum ................225

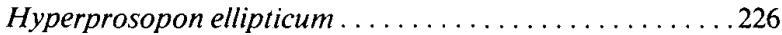

Hypomesus pretiosus . . . . . . . . . . . . . 120

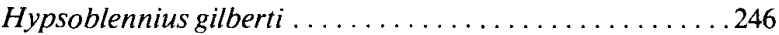

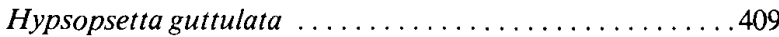

Hypsurus caryi .....................226

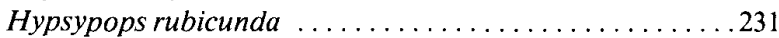

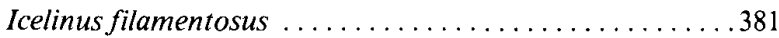

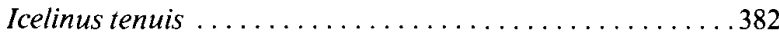

Icosteidae . . . . . . . . . . . . . . . . . . . . . . . 297

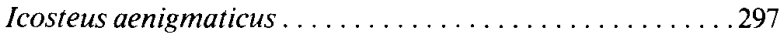

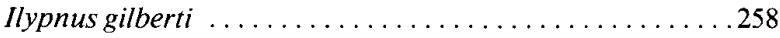

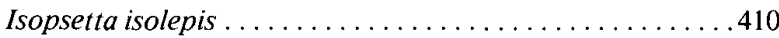

Istiophoridae . . . . . . . . . . . . . . . . . . . 293

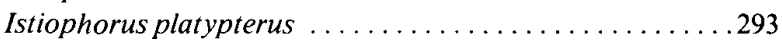

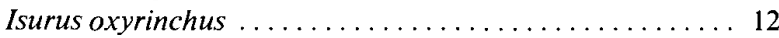

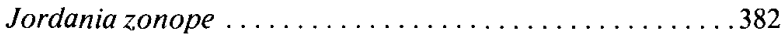

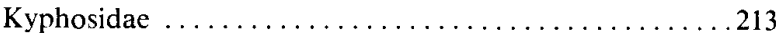

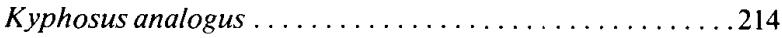

Labridae . . . . . . . . . . . . . . . . . . . . 232

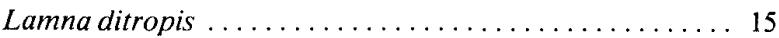

Lamnidae .......................... 10

Lampanyctus regalis . . . . . . . . . . . . . . . . . . . . 129

Lampanyctus ritteri . . . . . . . . . . . . . . . . . . . . . . 129

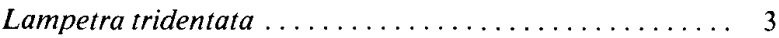

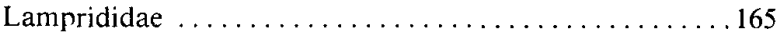

Lampris regius . . . . . . . . . . . . . . . . . 165

Lepidocybium flavobrunneum ...............269

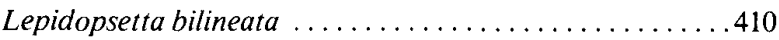

Leptocottus armatus . . . . . . . . . . . . . . . 382

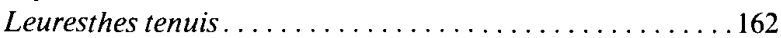

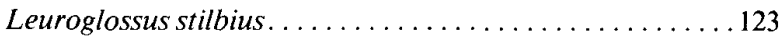

Liparis callyodon ....................... 395

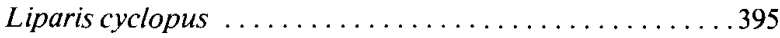

Liparis fucensis . . . . . . . . . . . . . . . . . . 396

Liparis pulchellus . . . . . . . . . . . . . . 396

Lumpenus anguillaris . . . . . . . . . . . . . . . 248

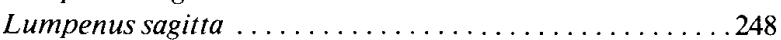

Luvaridae . . . . . . . . . . . . . . . . . . . . . . . . . 292

Luvarus imperialis . . . . . . . . . . . . . . . . 292

Lycodapus fierasfer . . . . . . . . . . . . . . . 146

Lycodapus mandibularis . . . . . . . . . . . . 146

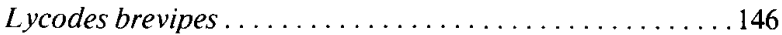

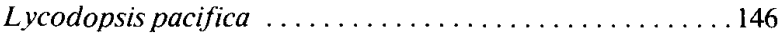

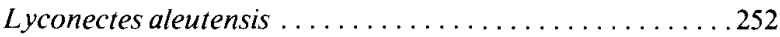

Lyopsetta exilis ........................414

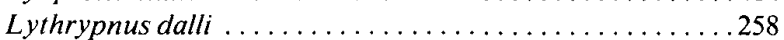

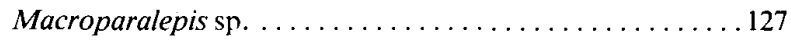

Macrouridae .......................149 
Makaira indica . . . . . . . . . . . . . . . . . . . . . 294

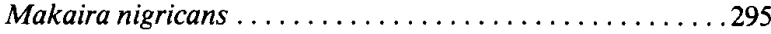

Malacottus kincaidii . . . . . . . . . . . . . . . . . 385

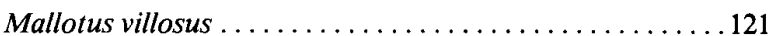

Maynea californica ....................... 147

Medialuna californiensis .................214

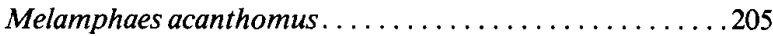

Melamphaidae........................205

Melanostigma pammelas . . . . . . . . . . . . . . . 147

Menticirrhus undulatus . . . . . . . . . . . . . . . 210

Merluccius productus . . . . . . . . . . . . . . . . . . 137

Microgadus proximus...................... 139

Micrometrus minimus . . . . . . . . . . . . . . . 227

Microstomus pacificus ....................415

Mobula lucasana ...................... 55

Mobulidae .......................... 55

Mola mola ..............................432

Molidae ..............................4432

Moridae ............................ 148

Morone saxatilis .......................... 177

Mugil cephalus .......................233

Mugilidae ..............................233

Muraenidae ........................ 60

Mustelus californicus .................... 25

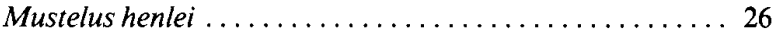

Mustelus lunulatus ..................... 27

Mycteroperca sp.......................... 184

Mycteroperca xenarcha ................... 184

Myctophidae ............................ 127

Myliobatidae ........................ 54

Myliobatis californica .................. 54

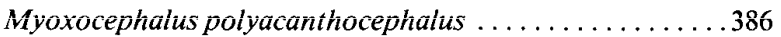

Myxinidae ....................... 3

Naucrates ductor........................ 198

Nautichthys oculofasciatus .................. 388

Nematistius pectoralis . . . . . . . . . . . . . . . . . . 198

Nemichthyidae $\ldots \ldots \ldots \ldots \ldots \ldots \ldots \ldots \ldots \ldots \ldots \ldots \ldots, 61$

Neoclinus blanchardi . . . . . . . . . . . . . . . . 246

Neoclinus uninotatus . . . . . . . . . . . . . . . . 246

Nezumia liolepis . ...................... 156

Nezumia stelgidolepis. . . . . . . . . . . . . . . 156

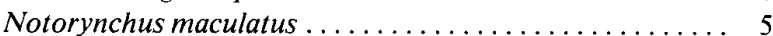

Odontaspididae $\ldots \ldots \ldots \ldots \ldots \ldots \ldots \ldots \ldots \ldots \ldots \ldots$

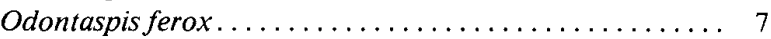

Oligocottus maculosus . . . . . . . . . . . . . . . . 388

Oligocottus snyderi . . . . . . . . . . . . . . . . . 389

Oligoplites saurus . . . . . . . . . . . . . . . . . . . 199

Oncorhynchus gorbuscha ............... 70

Oncorhynchus keta . . . . . . . . . . . . . . . . 77

Oncorhynchus kisutch . ................ 83

Oncorhynchus nerka ..................... 89

Oncorhynchus tshawytscha . . . . . . . . . . . . 97

Ophidiidae ..........................144

Ophiodon elongatus . . . . . . . . . . . . . . . . . 369

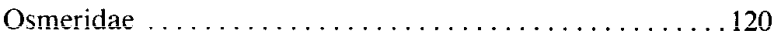

Ostraciidae. . ..........................429

Ostracion diaphanum . . . . . . . . . . . . . . . . . 429

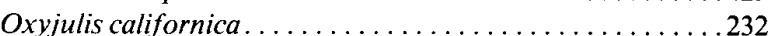

Oxylebius pictus ....................... 371

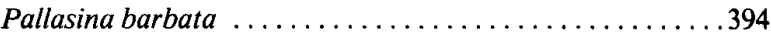

Parabassogigas grandis .................. 144

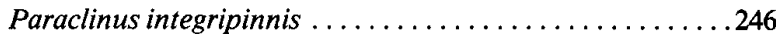

Paralabrax clathratus .................... 185

Paralabrax maculatofasciatus . . . . . . . . . . . . 186

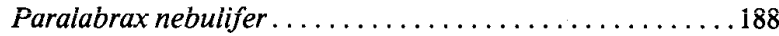

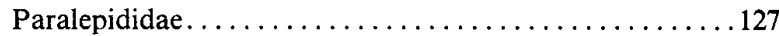

Paralichthys californicus . . . . . . . . . . . . . . . 398

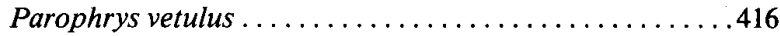

Parvilux ingens . . . . . . . . . . . . . . . . . . 130

Percichthyidae..........................177

Petromyzontidae..................... 3

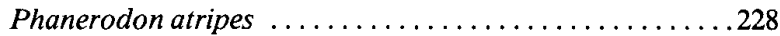

Phanerodon furcatus . . . . . . . . . . . . . . . 228

Pholidae ... . . . . . . . . . . . . . . . . . . . . . . . . . 249

Pholis laeta . . . . . . . . . . . . . . . . . . . . 250

Pholis ornata ..........................250

Pimelometopon pulchrum .................233

Platichthys stellatus ..................419

Platyrhinoidis triseriata ................ 45

Pleurogrammus monopterygius ................371

Pleuronectidae. . ......................400

Pleuronichthys coenosus ..................423

Pleuronichthys decurrens . . . . . . . . . . . . . . . .423

Pleuronichthys verticalis . . . . . . . . . . . . . 424

Polydactylus approximans . . . . . . . . . . . . . 242

Polydactylus opercularis . . . . . . . . . . . . . . . . 243

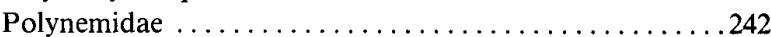

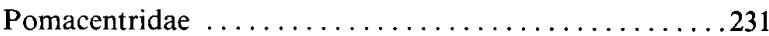

Pomadasyidae . . .......................206

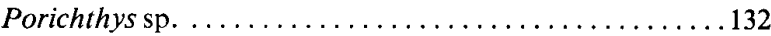

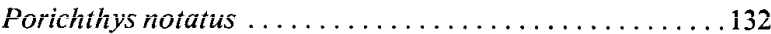

Prionace glauca ...................... 28

Psettichthys melanostictus . . . . . . . . . . . . . . . 424

Quietula $y$-cauda .....................258

Radulinus asprellus . . . . . . . . . . . . . . . . . . . . 390

Raja binoculata ........................47

Raja inornata...................... 49

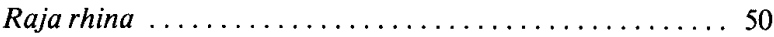

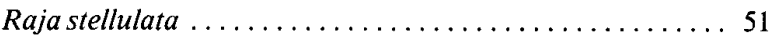

Rajidae .......................... 47

Ranzania laevis ........................436

Reinhardtius hippoglossoides . ...............425

Remora australis .......................191

Remora brachyptera.......................192

Remora remora ........................ 192

Rhacochilus toxotes . . . . . . . . . . . . . . . . . . 2229

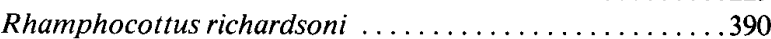

Rhincodon typus ..................... 7

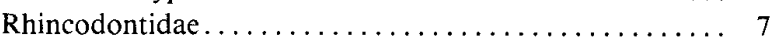

Rhinobatidae ....................... 45

Rhinobatos productus ................. 45

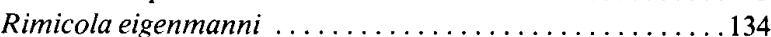

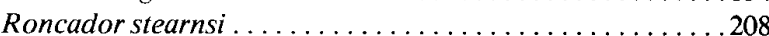

Ronquilus jordani . . . . . . . . . . . . . . . . . . . . 243

Ruvettus pretiosus. . . . . . . . . . . . . . . . 259

Sagamichthys abei...................... 126

Salmo clarki........................... 102

Salmo gairdneri.......................107 


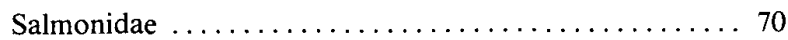

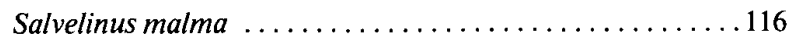

Sarda chiliensis . . . . . . . . . . . . . . . . . . 270

Sardinops sagax..................... 68

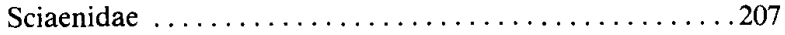

Scomber japonicus . .................. 271

Scomberesocidae. ........................ 157

Scomberomorus sierra . . . . . . . . . . . . . . . 275

Scombridae .............................260

Scopelogadus mizolepis bispinosus. . . . . . . . . . 206

Scorpaena guttata . . . . . . . . . . . . . . . . . . . . 297

Scorpaenichthys marmoratus .................391

Scorpaenidae . . . . . . . . . . . . . . . . . . . . . 297

Scyliorhinidae .................... 16

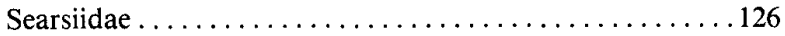

Sebastes sp. ..............................299

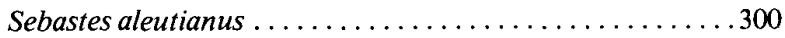

Sebastes alutus. . . . . . . . . . . . . . . . . . . . 303

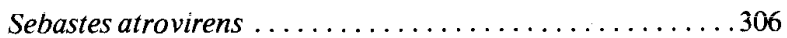

Sebastes auriculatus ...................... 308

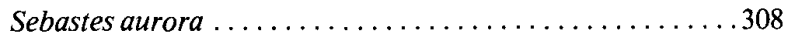

Sebastes babcocki ......................... 309

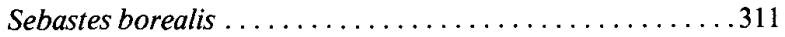

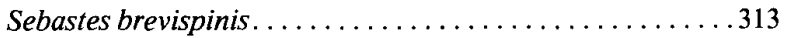

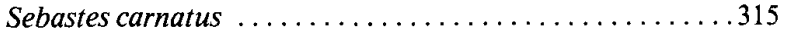

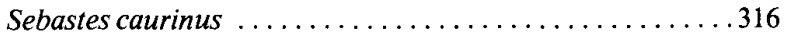

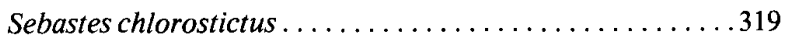

Sebastes chrysomelas .................... 320

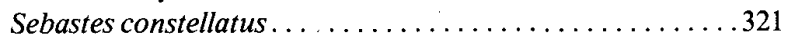

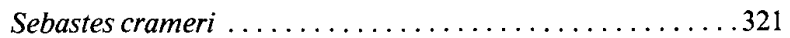

Sebastes dalli ........................ 323

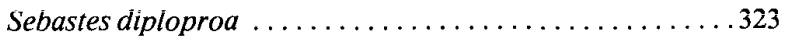

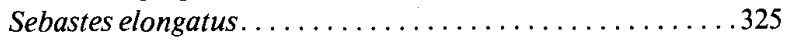

Sebastes ensifer . . . . . . . . . . . . . . . . . 327

Sebastes entomelas .................... 328

Sebastes eos ... . . . . . . . . . . . . . . . . . . . . . . . 329

Sebastes flavidus . . . . . . . . . . . . . . . . . . . . 329

Sebastes gilli............................331

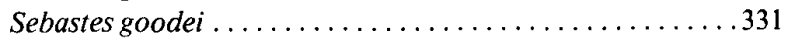

Sebastes helvomaculatus ................. 332

Sebastes hopkinsi ..................... 333

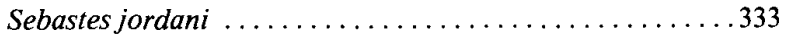

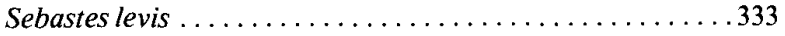

Sebastes macdonaldi. . . . . . . . . . . . . . . . . . . 334

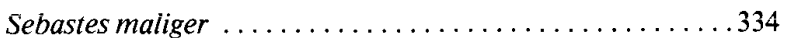

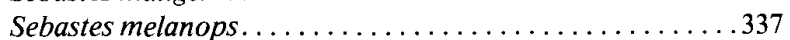

Sebastes miniatus . . . . . . . . . . . . . . . . . 338

Sebastes mystinus . . . . . . . . . . . . . . . 339

Sebastes nebulosus . . . . . . . . . . . . . . . 340

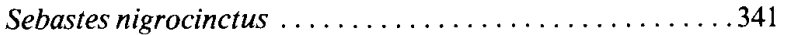

Sebastes ovalis ........................ 342

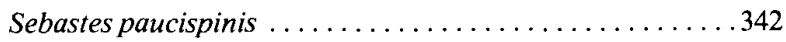

Sebastes phillipsi ........................ 345

Sebastes pinniger....................... 345

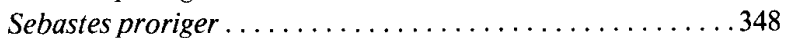

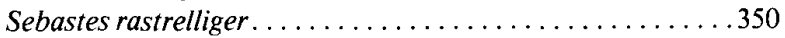

Sebastes reedi. . . . . . . . . . . . . . . . . . . . 350

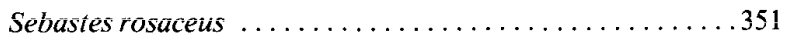

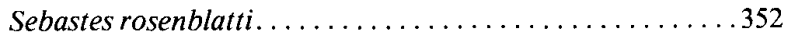

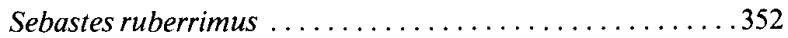

Sebastes rubrivinctus ................... 355

Sebastes rufus ......................... 355
Sebastes saxicola ........................ 355

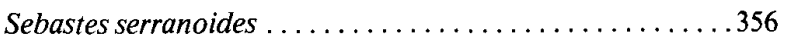

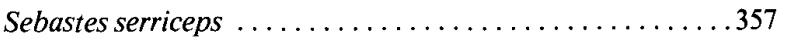

Sebastes umbrosus . . . . . . . . . . . . . . . . . . . . . 358

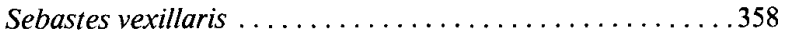

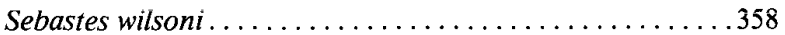

Sebastes zacentrus. . . . . . . . . . . . . . . . 359

Sebastolobus sp. ........................ 361

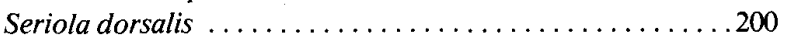

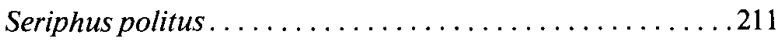

Serranidae .......................... 182

Serrivomer sector .....................61

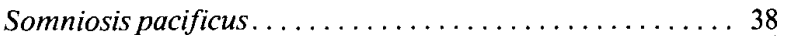

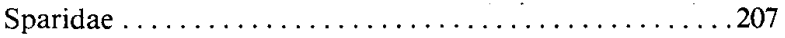

Sphoeroides sp. ............................429

Sphoeroides annulatus ....................429

Sphyraena argentea. ..................... 242

Sphyraenidae ........................242

Sphyrna lewini.................... 32

Sphyrna tiburo ....................... 33

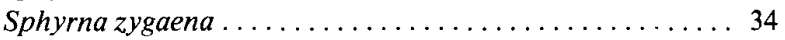

Sphyrnidae. . ................... 32

Spirinchus thaleichthys $\ldots \ldots \ldots \ldots \ldots \ldots \ldots \ldots \ldots \ldots 121$

Squalidae ......................... 38

Squalus acanthias ....................... 39

Squatina californica ..................... 44

Squatinidae ...................... 44

Stenobrachius leucopsarus. . . . . . . . . . . . . . 130

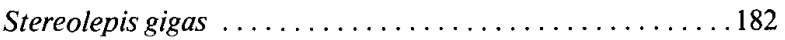

Sternoptychidae ......................... 124

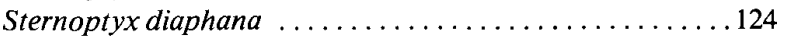

Stichaeidae . . ....................247

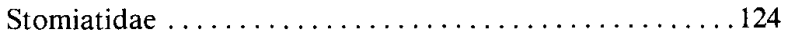

Stomias atriventer . . . . . . . . . . . . . . . . 124

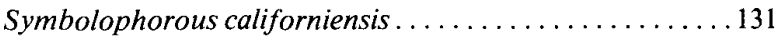

Synchirus gilli ....................... 392

Syngnathidae . . . . . . . . . . . . . . . . . 176

Syngnathus leptorhynchus ................ 176

Synodontidae............................ 124

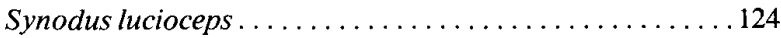

Tarletonbeania crenularis . . . . . . . . . . . 131

Tetraodontidae . . ...................... 429

Tetrapturus angustirostris . . . . . . . . . . . . 296

Tetrapiurus audax. . . . . . . . . . . . . . . 296

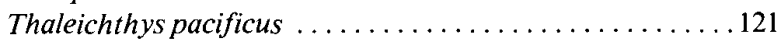

Theragra chalcogramma ................ 140

Thunnus alalunga ..................... 276

Thunnus albacares ...................... 279

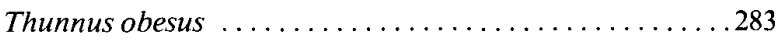

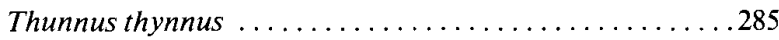

Torpedinidae ..................... 47

Torpedo californica .................. 47

Trachinotus rhodopus . . . . . . . . . . . . . . 201

Trachurus symmetricus .................201

Triakis semifasciata ................. 30

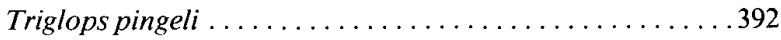

Triphoturus mexicanus . ...............13!

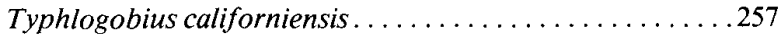

Umbrina roncador ....................212

Urolophus halleri ..................... 52 


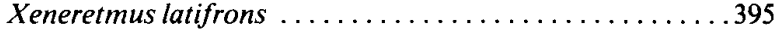

Xenistius californiensis.

Xiphias gladius

206

Xiphiidae

Xiphister atropurpureus.

289

Xiphisister mucosuss.
Zalembius rosaceus. . ...................230

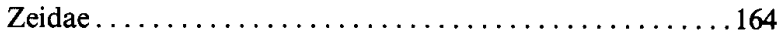

Zenopsis nebulosa . . . . . . . . . . . . . . . . . 164

Zoarcidae ........................... 145 


\section{ERRATA}

NOAA Technical Report NMFS SSRF-763: Climatology of surface heat fluxes over the California Current region, by Craig S. Nelson and David M. Husby.

Page 2, Figure 1 is upside down. Figure 1 should appear as below.

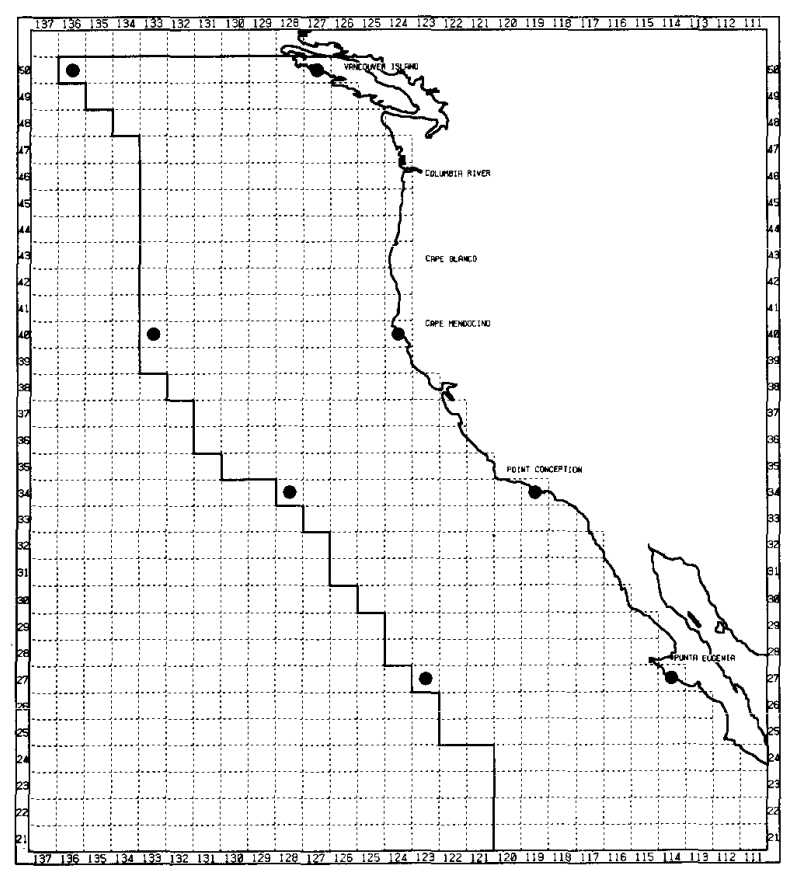

Page 29, Equation (11). The term to the left of the equal sign should read $\frac{\partial \bar{T}}{\partial t}$ rather than $\frac{\partial \bar{T}}{\partial \bar{t}}$

Page 30, Equation (12) should read $\quad \bar{w} \approx \frac{k}{\rho_{i} f}\left(\nabla \times \frac{\vec{\tau})}{} \quad\right.$ and the first line following the equation should read "where $\overrightarrow{k \bullet}(\nabla \times \vec{\tau}) \quad \ldots$ " 